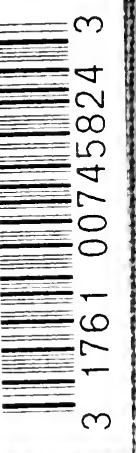




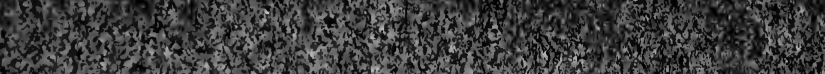

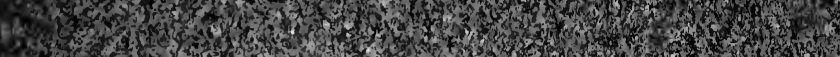

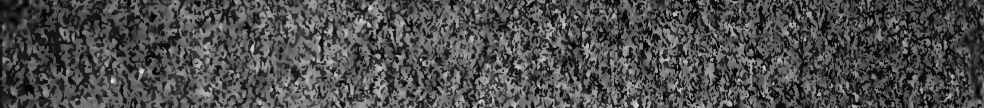

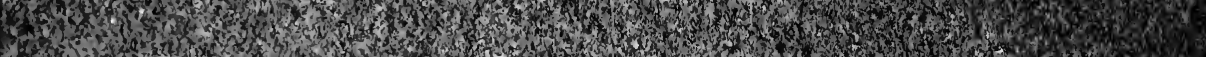

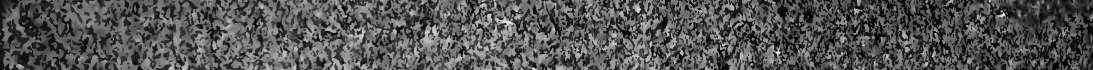

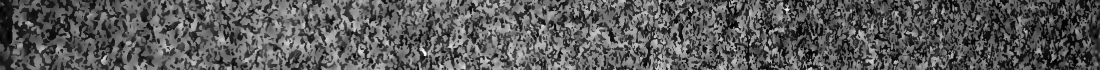
A.m.

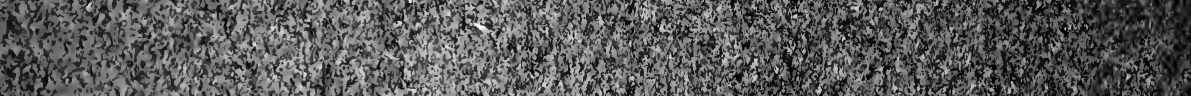
3. Th

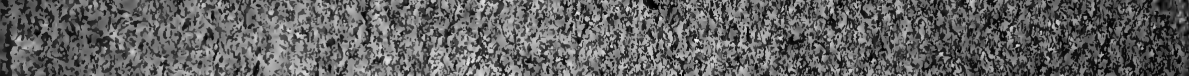

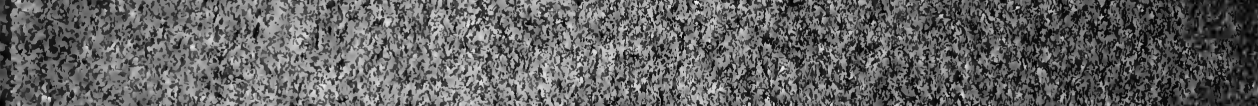

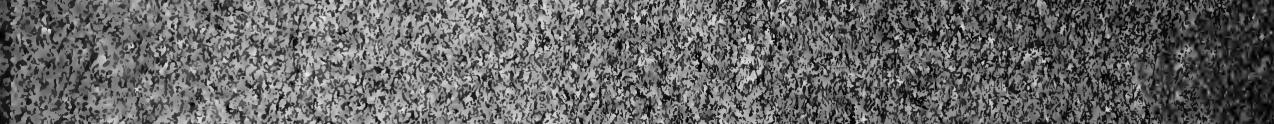

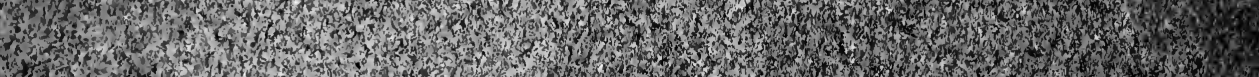

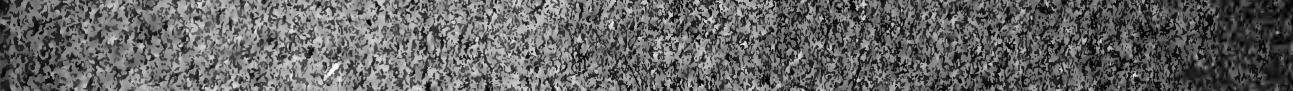
1.7. W.

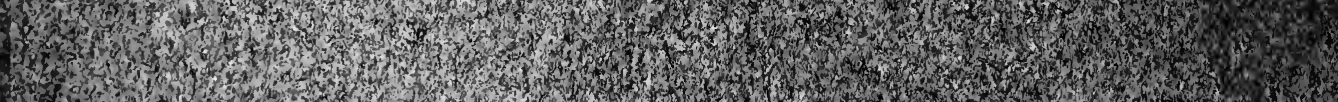

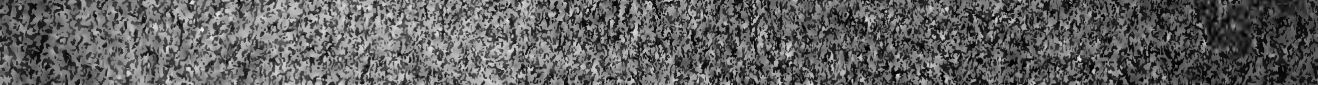

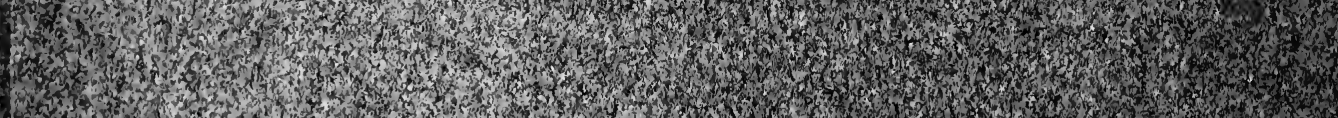

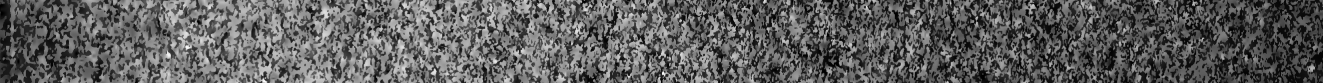
1.7.

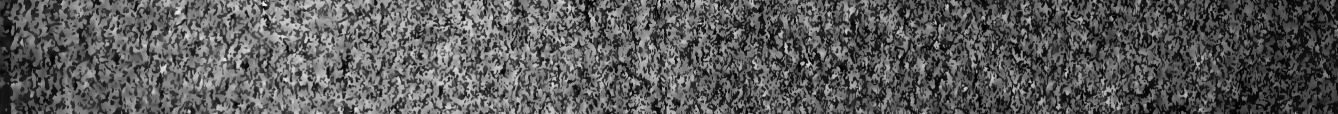
1.5. H.

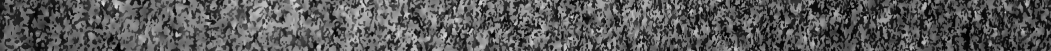
1.7.

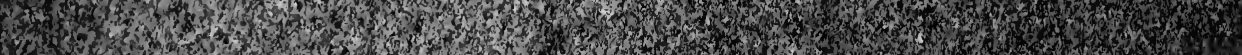

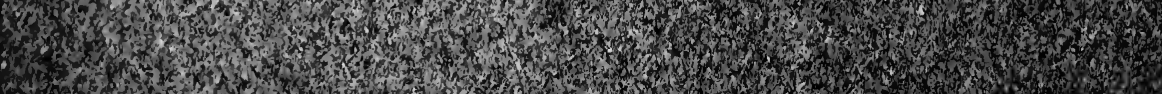

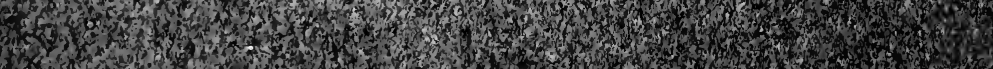

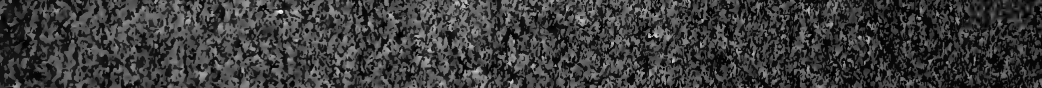

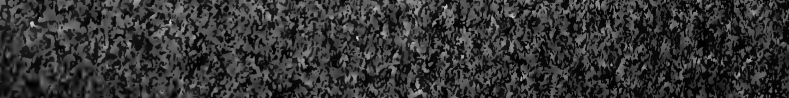

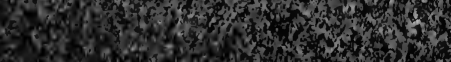

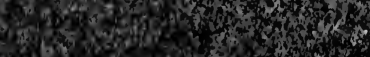


Digitized by the Internet Archive in 2007 with funding from Microsoft Corporation 


\section{MODERN ASPECTS}

OF THE

\section{CIRCULATION IN HEALTH AND DISEASE}

BY

CARL J. WIGGERS, M.D.

ASSISTANT PROFESSOR OF PHYSIOLOGY IN CORNELL UNIVERSITY MEDICAL COLLEGE, NEW YORK CITY

\section{ILLUSTRATED WITH 104 ENGRAVINGS}
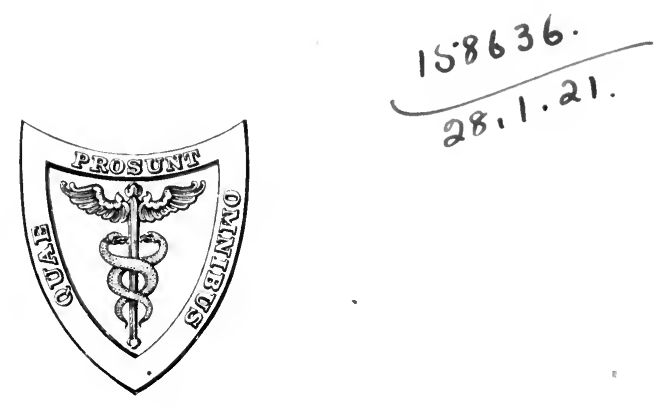

L E A F EBIGER

PHILADELPHIA AND NEW YORK 
Entered according to the Act of Congress, in the year 1915, by LEA \& FEBIGER, in the Office of the Librarian of Congress. All rights reserved. 
TO

OTTO FRANK,

IN APPRECIATIVE RECOGNITION

OF HIS MASTERFUL WORK,

THIS VOLUME IS

DEDICATED. 



\section{PREFACE.}

No one who pauses to reflect can fail to admire the master minds of medicine who, by simple signs and statistics, not only interpreted many disease processes correctly, but upon them built an admirable system of diagnosis. Nothing can be substituted for the power of accurate observation, either at the bedside or in the laboratory. Nevertheless, it happens that many phenomena of the circulation, normal and abnormal, remain undetected by our unaided senses. Consequently, various instrumental methods have been introduced which supplement our direct observations, either by recording the functions of the circulation graphically or by translating them into numerical terms which the mind can more definitely grasp.

This monograph deals with the ways in which the application of laboratory methods to the clinic has led to the elucidation of many obscure conditions, to the recognition of new diseases, and to the institution of new forms of treatment. In order to present the subject in the consecutive and logical manner necessary for academic purposes and still retain the convenient arrangement demanded of a reference medium, it has been divided into three sections.

The chapters of the first section attempt to present our most modern conceptions as to how the circulation is maintained in health. This is of fundamental importance, for no one can assay to discuss the abnormal conditions of the circulation from a modern standpoint without constant reference to the fundamental physiological conceptions. Unfortunately, in some respects, many of our current views must be revised to accord with the facts brought to light by the application of more improved methods in experimental investigation. Hence, it is desirable not only to review our new acquisitions of knowledge and fit these in with those long accepted, but to describe, to some extent, the principles of the newer apparatus by which they are obtained. 
The second section deals with the various instruments and procedures which are available for studying the circulation of man. Its purpose is more far-reaching than to serve as a mere catalogue of apparatus or a chronicle of "procedures" and "interpretations." It attempts, above all, to place a correct valuation upon different forms of apparatus and to point out their limitations and errors. To do this justly would be an impossible task were the question, as in the past, still a matter for individual opinion or preference. Fortunately, however, matters have recently changed. Through the painstaking analyses of men like Frank, Einthoven and their associates, the enormous value of whose contributions is still imperfectly appreciated, the principles underlying the construction and valuation of an instrument have been carefully evolved. Consequently, it is now possible, not only to construct mechanical apparatus along scientific lines, but also to ascertain definitely the defects and limitations of that already in existence.

In the chapters comprising the third section the effort has been made to correlate the data obtained by experimental investigation of abnormal conditions in the laboratory with the results derived from the application of instrumental methods at the bedside and then to relate these, in turn, with the simpler signs and symptoms of the clinic. Careful study has shown that such a comparison of different procedures in nowise diminishes the value of simple diagnostic criteria, but, on the contrary, often gives them an added significance or a clearer meaning.

In attempting such a correlation, reserve and caution must be exercised lest in our overzeal to use attractive clinical methods, we incorporate results from insufficiently tested or inaccurate apparatus, or, lest we transfer results obtained from experimental animals under one set of conditions, to the diseased human body in which conditions are entirely different.

Having thus delineated the scope and character of the separate sections, it seems appropriate to indicate briefly a few of the principles followed in their writing. It has been taken for granted that the reader of the first two sections has at least a general knowledge of the circulation as presented in current text-books of physiology, and that the reader of the last section is equally familiar with a description of circulatory disorders as found in text-books of medicine. The endeavor has been made, as far as consistent with 
the complicated nature of the subject, to present the subject material in a clear and simple way. It is a mistake, however, to define simplicity as synonymous with paucity of details or the avoidance of scientific modes of expression. The subject matter has been largely selected directly from the original sources and the chief references on each topic have been appended to the end of every chapter. It is probable that in the reading or digestion of so great a literature as has grown about the subject some errors of omission and commission have been made. For their correction, the author invites the coopperation of the reader. Attention may be called to the fact, however, that the original conclusions are not always accredited to the investigator in question when it has appeared to the author that they were unwarranted by the data or when they seem to read counter to facts. In general, it has been the endeavor to present the facts and data in preference to the "conclusions."

Throughout the book, the author has incorporated the results of his own investigations on the circulation extending now over a period of eleven years. Some of his more recent work appears here for the first time. Similarly, it has been possible, with their kind permission, to introduce several unpublished reports of the recent work of a number of colleagues.

The separate chapters have, with a number of exceptions, been submitted for approval or criticism to investigators or clinicians who were especially conversant with the subjects set forth in those chapters. It is a pleasure to acknowledge with gratitude their many kindly suggestions. To Mrs. Wiggers the author is especially indebted for many faithful hours of help rendered in preparing the manuscript.

New YoRk, 1915.

C. J. W. 


\title{
CONTENTS.
}

\author{
SECTION I. \\ - THE PHYSIOLOGY OF THE CIRCULATION. \\ CHAPTER I.
}

The Physiological Properties of the Heart and Their Control 17

CHAPTER II.

The Sequence of Cardiac Contraction and the Movements of the HEART

CHAP'TER III.

The Dycamics of the Heart Beat

CHAPTER IV.

The Mechanical Energy of the Heart Beat . . . . . . . 66

CHAP'TER V.

The Control of Blood Flow Through Organs . . . . . . 79

CHAPTER VI.

The Physiology of the Pulmonary Circuit . . . . . . 87

CHAPTER VII.

The Respiratory Variations of Arterial Pressure . . . . 95

SECTION II.

GRAPHIC METHODS FOR THE CLINICIAN.

CHAPTER VIII.

* The Arterial Pulse

CHAPTER $^{\circ}$ IX.

^ The Supraclavicular Venous Pulse or the Phlebogram . . . 126

CHAPTER X.

The Esophageal Pulse and the Esophagram . . . . . . . 146 
CHAPTER XI.

The Apex Beat and Cardigram . . . . . . . . . . . 150

CHAPTER XII.

The Electrocardiogram . . . . . . . . . . . . . . 154

CHAPTER XIII.

$\uparrow$ Heart Sounds and Murmurs-The Phonocardiogram . . . $\quad 174$

CHAPTER XIV.

Sphygmonanometry-The Clinical Estimation of Human BloodPRESSURE

CHAPTER XV.

The Volume Flow and Venous Pressure . . . . . . . 225

CHAPTER XVI.

The Radiograil and Orthodiagram . . . . . . . . . . 238

\section{SEC'TION III.}

DISEASES OF THE CIRCULATION.

CHAPTER XVII.

Affections of Heart Muscle Associated with Retrograde Changes,

Infiltrations, and Subsequent Repair . . . . . . . . 248

CHAPTER XVIII.

The Díanosis and Significance of Cardiac Arrhythmias . . . 268

CHAP'TER XIX.

Hypertrophic Compensation, Cardiac Insufficiency, and DilataTION . . . . . . . . . . . . . . . . . . 304

CHAPTER XX.

The Valvular lesions of the Heart . . . . . . . . 311

CHAPTER XXI.

Disturbances Due to Changes in the Circulating Volume • • 331

CHAPTER XXII.

Mechanical Impairment of Cardiac Action . . • . . . . 344

CHAPTER XXIIl.

Affections of the Arteries 


\title{
CIRCULATION IN HEALTH AND DISEASE.
}

\section{SECTION I.}

\author{
CHAPTER I.
}

\section{THE PHYSIOLOGICAL PROPER'TIES OF THE HEAR'T AND THEIR CONTROL.}

HEarT tissue is endowed with four fundamental properties: Rhythmicity, the power of originating its impulse; conductivity, the power of transmitting the impulse; irritability, the power of responding to an impulse or irritant, and contractility, the power of changing its shape and length in response to excitation. 'To these may probably be added a fifth function, tonicity, or the power of sustained partial contraction by virtue of which it resists stretching.

\section{METHODS OF STUDYING THE HEART BEAT.}

The Mechanical Reaction of the Heart.-The registration of the mechanical contraction and relaxation of the heart has been of great value. By recording the rapidity and amplitude of contraction the phenomena of contractility and irritability have been studied, the latter, on the assumption that if the impulse may be regarded as of constant intensity, then the amplitude is an index of irritability. By recording the rate and extent of active relaxation, the tonicity has been investigated. By estimating the time difference between the beginning of contractions recorded from several different regions, the rate of conductivity has been studied. Thus, the difference between the beginning of auricular and ventricular contractions (the so-called $A s-V s$ interval) has frequently been used as a measure of the conduction time. By recording and, still better, by careful observation of the sequence and priority of contraction in separate portions of the heart, the region in which rhythmicity predominates has been mapped out. It is apparent, therefore, that the accurate recording of the contractions from different regions of the heart is of great importance.

The simplest procedure by which this may be accomplished consists in fastening one end of a thread to any point of the heart 
and attaching the other to a simple lever of such efficiency that it follows, with a reasonable degree of accuracy, the movements of that point of the heart to which it is attached. Unfortunately, sufficient attention has not been paid to the efficiency of levers. This is evident from an inspection of the levers in use in many laboratories, as well as from a study of published records.

The factors that determine the efficiency of a lever may be briefly stated by the formula $G=\frac{3}{\mathrm{vL} \mu}$, in which $G$, the efficiency, is inversely proportionate to $\mathrm{v}$, the magnification; $\mathrm{L}$, the length of the lever; and $\mu$, its unit mass (Frank). In other words, the best lever is theoretically the one with the lowest magnification, the shortest length, and the lightest material. These qualifications have a practical limit. On account of the great ares written, a lever less than $6 \mathrm{~cm}$. in length is not feasible. On account of a certain required rigidity, an infinitely light lever is also impossible unless beams of light which record photographically are used.

In the selection of ponderable levers, one should choose, whenever possible, short, hollow straws tipped with light yet flexible pointers, and the magnification should be reduced to the lowest figure. It may be pointed out in this connection that physiologists have usually been too greatly concerned with obtaining records of large amplitude, and have been unaware that such have been secured at the expense of accuracy. Provided that friction between drum and pointer are minimized or, better, entirely avoided by photographic registration, there is no reason why records 10-15 $\mathrm{mm}$. in amplitude are not adequate for most purposes. In case larger records are needed, it would be far better to secure them by subsequent photographic enlargement, which can be done without a sacrifice of accuracy.

Instead of transmitting the movements of a point on the heart directly to a lever, it has been found more convenient to transfer them to a receiving capsule closed with rubber dam and then communicate the change by air transmission to a recording capsule of the Marey type. Although exceedingly convenient, such devices are always less efficient for the same amplitude of record than direct lever-registrations. Their extensive use has therefore not added to the accuracy of the study of heart functions.

In determining the adequacy of the apparatus for the purpose in hand- $i$. $e$. , in satisfying oneself that the lever, on account of resonance effects and interference waves, does not distort the contour and height of curves and that the rise starts promptly-the physical principle is made use of that the inherent vibrationfrequency of the apparatus must definitely exceed the highest rate of vibrations it attempts to record. Thus, it is clearly impossible to make a second's pendulum vibrate 15-30 times per second. 
Feats no less ridiculous from a physical standpoint have, however, been attempted by physiologists. Thus, a lever with one or two inherent vibrations per second would be adequate to record the slow beats of a frog's or a tortoise's heart, but entirely inadequate to record the contractions of a rabbit's or cat's heart beating at a rate of $200-250$ per minute.

Given a lever or tambour-system of such efficiency that the punctate movements of the heart are accurately recorded, the question still remains whether the movement of the point on the heart is an index of the amplitude of contraction. Obviously, this depends entirely on conditions. If the opposite point is fixed or stationary, the movements of the chosen point will correspond well with variations in the length of the muscle fibers. This is the case of the perfused heart, in which the base is fixed by a cannula in the aorta and the movements of a point on the apex of the left ventricle are recorded. On the other hand, if the opposite point moves or the heart, as a whole, is dislocated, then the variations of any point communicated to the most ideal lever give no reliable information as to the variations in the length of cardiac fibers.

To obviate this disadvantage in punctate registration, various forms of myocardiographs (Roy, Cushny) have been introduced. These instruments consist of two arms fastened by hooks or by stitches to the heart. One arm is stationary and the other movable. The movements of the latter are communicated either directly to a lever (Roy, Cushny) or to a recording tambour (Wiggers). The latter has the advantage that the oscillations can be led any distance by rubber tubing. The cardiograph (Fig. 1) attached to a Lombard pattern of a recording tambour can be fitted up so that it has a vibration frequency of 16 per second, while it remains sensitive enough to record the beat of the cat's heart. In this apparatus the lever moves up in systole, while in Cushny's myocardiograph the lever is raised during relaxation by a light spring and drawn down by the shortening of the heart. Since the entire instrument swings freely in all directions as the heart shifts its position, the actual shortening between two points is obtained independent of all secondary movements of the heart. To attain this end most efficiently, however, it is desirable to so attach the points that the distance between their attachments is not influenced passively by volume changes within the heart. It is more desirable, for instance, to attach the two arms to the anterior surface of the ventricles than to the lateral aspects; in which case the varying volumes of the left and right ventricles might modify the excursions. The construction of these instruments has so far necessitated the introduction of such considerable mass that it is questionable whether the recorded waves are not distorted and whether a reaction of the entire mass of the instrument upon the heart is not enough 
to render its action unnatural. ${ }^{1}$ This disadvantage has been recently overcome in a miniature form of myocardiograph designed and now being used by the author in connection with optically recording capsules. The total weight of the myocardiograph is less than 2 grams.

The Electrical Record of the Heart Beat.-Since every initiation of activity is accomplished by the development of a condition of electrical negativity and the spread of the impulse is accompanied by a similar state of negativity, it has been sought by this means

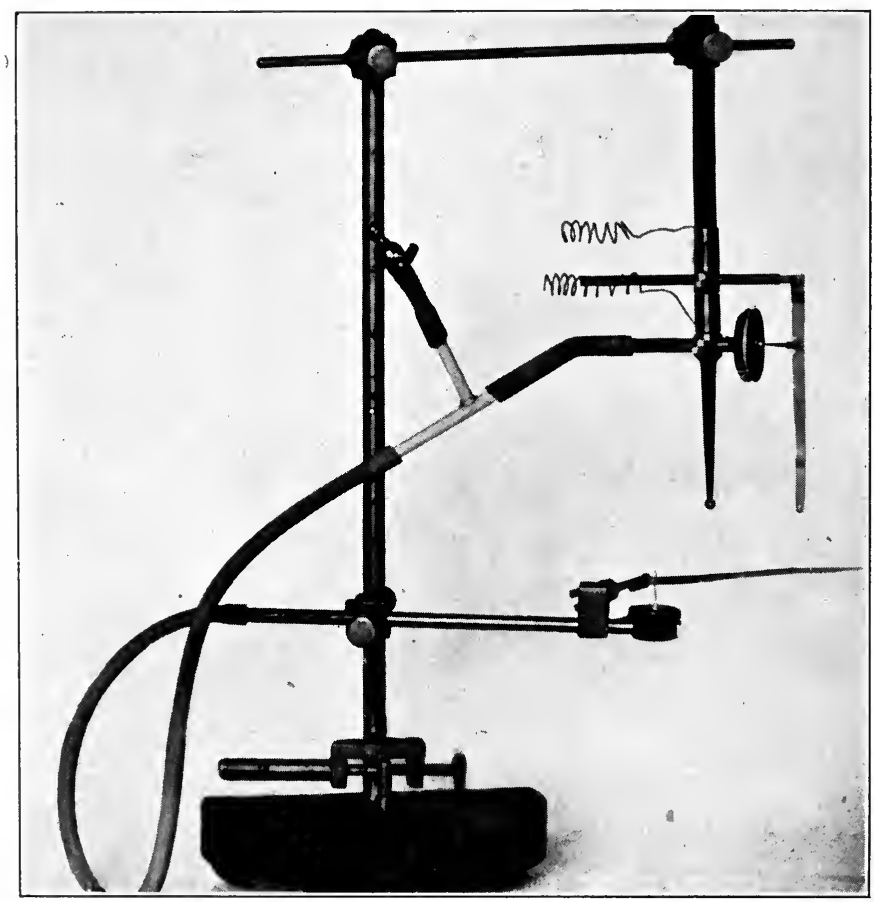

FIG. 1.-Cardiograph and recording tambour.

to determine the origin and propagation of the cardiac impulse more definitely than is possible by mechanical registration. This has been given a new impetus by the introduction of the Einthoven string galvanometer. The principles, details, and critique of this apparatus have been reserved for a later chapter ( $c f$. page 154). For experimentally investigating the rhythmicity and conductivity of hearts, the poles of the string galvanometer are connected to some

${ }^{1}$ For a full consideration of the qualities of levers and the methods of registering the heart beat, consult Frank, in Tigerstedt's Handbuch der physiologischen Methodik, 1911, I, part IV, p. 17; and II, 1913, part IV, p. 175. 
form of non-polarizable electrodes. - From their non-polarizable surface a worsted thread is suspended so that its tip lies on or, better still, is fastened to the heart. When the base is connected to one pole and the apex to another, a curve is recorded that contains three distinct waves, $P, R$, and $T$, the latter being frequently a downward wave. Although there is some evidence to the contrary (page 160), the interval between the rise of the $P$ and $R$ waves is usually considered equal to the time required for the impulse to pass from its point of origin to the ventricular musculature. Any variation of this interval is therefore an indication of an altered conduction time. In addition to determining the relative electrical variations between the base and apex of the heart (page 163), the string galvanometers have been employed to determine the initial activity in more restricted areas, for when two such points are connected, the one showing the first sign of negativity is primarily active. The study of the point of initial activity has been furthered by the application of differential electrodes to mammalian hearts (Garten and his pupils). The method permits one not only to determine the existence of initial negativity, but indicates the direction that the excitation process takes.

\section{THE PHYSIOLOGICAL PROPERTIES OF THE HEART.}

Rythmicity.-Stannius, by his classical experiment, established almost conclusively that the beat of the cold-blooded heart starts in the sinus venosus. His experiment consisted in tying a ligature about the sino-auricular junction, whereupon the beat of the auricle and ventricle ceased while that of the sinus continued. Inasmuch as the human heart is evolved from an embryonic organ in which a sinus venosus region is present (His), it seems logical to assume that the remains of the sinus tissue probably constitutes the rhythmic region.

The question naturally follows, What becomes of the sinus region in the mammalian heart? Studies in comparative anatomy show that, while the cavities of the sinus, auricle and ventricle are in direct communication the musculature of the auricle is not interspersed between sinus and ventricle but rather superimposed upon the connecting band, known in comparative anatomy as the canalis auricularis (MacWilliams). In the mammalian heart the sinus tissue has become submerged in the musculature forming the posterior portion of the auricle between the openings of the superior vena cava above, and the coronary sinus below. Within this region lies a special knot of heart tissue, the sinus node (Keith and Flack). This node, situated in the groove called the "sulcus terminalis" is a club-shaped masš, its headlike enlargements sending a few strands up to the superior vena cava, its lower end terminating 
midway between the superior vena cava and the coronary sinus (Fig. 2).

Histologically, this node is composed of delicate, fusiform, interlacing muscle fibers embedded in a densely packed connective tissue. The fibers are pale in color, faintly striated and contain elongated nuclei. The node is not entirely a muscular structure, as was originally supposed, but contains many nerve fibers and fibrils and a few ganglion cells (Oppenheimer and Oppenheimer). Keith and Flack suggested that this node represents the remains

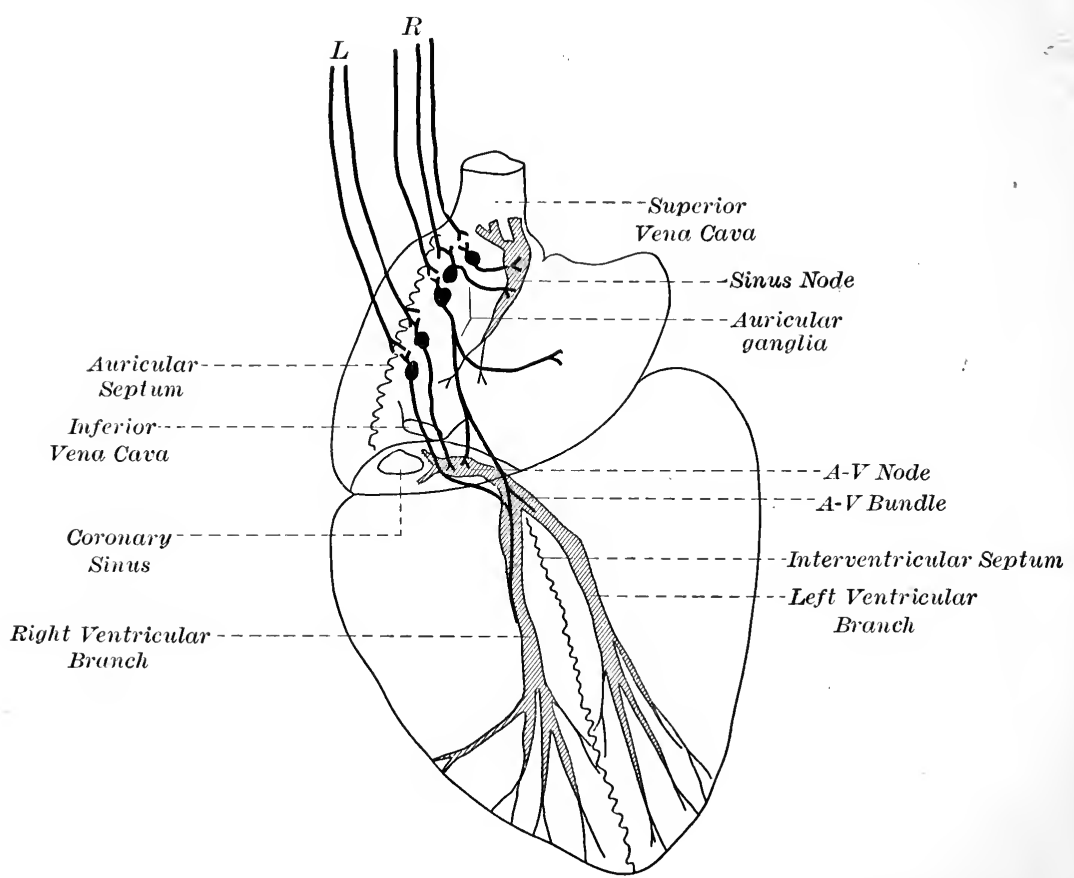

Fig. 2.-Schematic figure showing relations of sinus node, $A-V$ node, $A-V$ bundle, His-Tawara branches as well as probable relations of ganglion cells and extrinsic nerve fibers. $R$, right vagus; $L$, left vagus.

of sinus tissue, and Lewis has designated it the "pacemaker" of the heart. The experimental evidence in favor of this view is: (1) that excision of this node generally causes a cessation or slowing of the auricle (de Jäger, Hering, Cohn, Kessel and Mason); (2) that of the tissue in this vicinity, the node alone is sensitive to temperature changes (Brandenburg and Hoffman, Ganter and Zahn, Zahn); and (3) that the node becomes electrically negative before the rest of the sinus region (Wybauer, Lewis, Lewis, Oppenheimer and Oppenheimer, Eyster and Meek, Garten and Sulze). 
It is interesting to note that the sinus node is by no means the only locality in which the impulse actuating the heart beat can originate, but that the seat of impulse formation may shift for a longer or shorter time to some other portion of the conducting system. Thus, if the sinus node is either depressed or destroyed (formalin, cold, inflammation) or, if the irritability of the $a-v$ node is raised, the latter assumes the role of controlling the heart beat. By heating or cooling the $a-v$ node by a specially constructed thermode, it has been possible to show that when the impulse originates here the $A s-V s$ interval is not necessarily reduced, as commonly supposed; but that such a shortening occurs only when the impulse originates from the middle region (Zahn). It is extremely probable that, owing to the nervous mechanisms which modify the relative irritability of these structures, there is normally more or less shifting of the pacemaker; and that some cases of cardiac acceleration are due, not to a hyperactivity of the sinus node, but to the fact that the pacemaker has shifted toward the $a-v$ node.

Conductivity.-By what paths and in what manner are the impulses propagated to the auricles and ventricles so that they beat in definite sequence? By extensive histological investigations (His, Tawara, Braeunig, Retzer, and De Witt) it has been shown that a definite conducting system lies enclosed within its own sheath beneath the endocardium. This may be spoken of as the II isTawara system. It begins as a few strands of muscle fibers in the region of the coronary sinus. These converge toward a thickening spoken of as the auriculo-ventricular node (Tawara) or, more correctly, perhaps, the sino-ventricular or supra-ventricular node. From this node a thin strand passes across the auriculo-ventricular septum forming the bundle first described by IIis and bearing his name. It courses downward and forward reaching the membrano-muscular junction of the interventricular septum. Here it divides into two branches which pass subendocardially, one to the left, the other to the right ventricle. These, in turn, divide and redivide, some strands crossing the cavity of the ventricle as "false cords," others dividing more and more until they join the muscle cells forming the ventricle walls (Fig. 2).

The muscle cells composing this system differ in their general appearance in various regions. The cells of the auriculo-ventricular node resemble those of the sinus node, but as the strand passes into the bundle of His their character changes. Their striations are less marked and the nuclei are large; in short, they assume the characteristics long ascribed by Purkinje to the peculiar cardiac fibers that bear his name. It still seems questionable whether, in man, communication with the cardiac muscle cells is made through such Purkinje cells in the ventricular walls or whether a direct union occurs (Fahr). The His-Tawara system, even more than the 
sinus node, is a neuro-muscular structure for not only are separate nerve fibers distributed through it, but distinct bundles and trunks are also present. In addition, ganglion cells are found (Wilson, Engle, DeWitt). Returning to the other end of the conducting system, it may be stated that the exact morphological connection between the sinus node and the auriculo-ventricular node has not been definitely established.

The anatomical paths by which impulses reach the auricle are not certainly known. Three possibilities exist: (1) the impulses may pass from the sinus node to the $a-v$ node, and from this place may stimulate the auricles as well as the ventricles; (2) the impulses may be sent to the auricular tissue first, and from this to the $a-v$ node, and so to the ventricles; or (3) the auricles and ventricles may be stimulated by separate impulses. Opposed to the second view is the evidence that the $a-v$ node becomes negative about 0.015 seconds before the auricle does (Fig. 75, A) (Eyster and Meek). In favor of the last view is the fact that in the embryo a direct communication between the sinus tissue and the ventricle exists without intervention of auricular tissue, and also that the wave of negativity spreads from the sinus node in all directions at the rate of 800 to $1200 \mathrm{~mm}$. per second (Lewis, Hering).

The impulse is not conducted to the ventricle through the HisTawara system at a constant velocity, for if this were so we should expect it, on the basis of its velocity in ventricular Purkinje muscle, to travel a greater distance in the same time interval (Erlanger). Lewis ${ }^{1}$ has recently placed the rate of conductivity of intact ventricular cardiac muscle at 2000 to $3000 \mathrm{~mm}$. per second. It is quite evident that a delay occurs somewhere in the conduction from sinus to ventricle. Upon the basis of Gaskell's experiments on the tortoise, the delay has generally been attributed to the fact that the impulse must pass over the narrow $a-v$ bundle. The experiments of Erlanger, however, make this seem improbable by showing that the impulse has a very considerable velocity in very narrow tendons of Purkinje tissue (at least $750 \mathrm{~mm}$. per second). It is more probable that the delay occurs in the $a-v$ node for not only is this region most susceptible to complete block (Lewis, White, and Meakins), but the delay can be increased by cooling and decreased by warming the node, procedures that seem to have less influence on the rest of the conducting system. According to Zahn, it is possible to show by finer methods that the delay actually occurs in the middle portion of the $a-v$ node. Having passed this node, the impulse spreads at a uniform rate so that the entire ventricular musculature is apparently excited at almost the same time (Garten and his pupils). Very careful measurements, however,

${ }^{1}$ Harvey Lecture, New York, October 24, 1914 (unpublished). 
have shown that the slight difference of a few thousandths of a second in the time of the appearance of negativity over the middle right ventricle and over the apex of the left may be attributed to the thinness of ventricular muscle at the former place and the closer approximation of the Purkinje tissue to the surface (Lewis).

The probability that impulses reach the ventricle entirely and necessarily through the His-Tawara system is generally regarded as established by the fact that when the bundle of His is cut or crushed, the impulses fail to reach the ventricle. Kent, however, has described other muscular paths running especially from the posterior-auricular wall on the right side to the ventricular septum ("right lateral connection"). The existence of such fibers has been confirmed by subsequent studies of His and Mall, and a functional use was demonstrated by Kent. He showed that if the auricles and ventricles were severed so that only these bridges remained the impulses continued to be sent to the ventricle. 'The practical significance of these tracts, however, remains unsolved.

Contractility and Irritability.-The question arises whether the amplitude of cardiac contraction is determined by the intensity of the stimulus received through the Purkinje system. Apparently no direct observation upon this subject has been made but the reaction of the ventricle to artificial stimuli is opposed to such an assumption. It has long been known that heart muscle responds by a maximal contraction to any stimulus that is not subliminal (Bowditch), a reaction which may probably be attributed to the extensive intercommunication in the muscle svncitium rather than to any peculiarity of cardiac tissue (Lucas, Gotch).

The reaction of "all or none" as it is frequently termed after Ranvier, in no way implies that the heart cannot alter the amplitude of its contraction, but, on the contrary, presupposes that the irritability has not altered. Thus, after repeated stimulation, chemical action, etc., the amplitude may vary considerably when the stimulus remains unchanged. In short, the irritability of cardiac muscle and not the strength of the impulse determines the amplitude of contraction.

Unlike skeletal striated muscles, the heart is not irritable during activity. Thus, while a stimulus applied during its relaxation causes a second contraction, one applied during contraction elicits no response, $i . e$., the heart is said to be in its refractory period (Marey). In explanation of this phenomenon, it is generally supposed that the irritability of the heart is built up during relaxation and rest and is suddenly released at the onset of contraction, just as a gradually filling pail may be suddenly upset. Recent experiments have raised the issue, however, whether the refractory phase may be regarded as-a function of irritability (Schultze), for it persists in unaltered relation to the length of systole, even 
when the irritability is raised by salts and temperature. Schultze therefore concludes that the muscle fails to respond during the refractory phase because neither the chemical reaction nor the physical rearrangements of colloidal particles has been completed. Inasmuch as the hearts of crustaceæ in which ganglion cells and nerve fibers are situated extracardially do not exhibit a refractory period, the question has arisen whether the refractory state in vertebrate hearts is a function of the muscular tissue or of the ganglion cells contained within the muscle. The fact that the refractory period can be abolished (Rohde) or abridged (Schultze) would seem to favor such a view, especially since in the limulus heart this drug acts by preference on the nerve cells (Carlson). It may be pointed out, however, that the admission that nerve cells determine the refractory phase in no way compromises the view that the impulse is of myogenic origin.

Tonus.-Although there is a certain amount of evidence that variations in the fundamental contractions are related to changes in tonus. it is probable that the two functions are separate and independent. Thus, it has been shown by Porter that, unlike fundamental contractions of the heart, tonus contractions are proportional to the strength of stimulus, have no refractory period, may be superimposed and develop into "tetanus of tonus," meaning by this the fused series of tonus contractions upon which the fundamental contractions are placed. Porter points out that the great and permanent contraction into which the heart can go upon repeated stimulation is not a tonus due to fusion of fundamental contractions but a fusion of tonus contractions. It seems that a certain amount of tonus is favorable to the occurrence of vigorous fundamental contractions. Thus, it has been shown that the tonus as well as the amplitude of contraction is simultaneously decreased by the presence of $\mathrm{CO}_{2}$ and increased by oxygen (Ketcham, King and Hooker). On the other hand, the influences that increase the one may decrease the other. This is the case of adrenalin which diminishes the tonus but augments the fundamental contractions.

The part played by tonus in the efficiency of the mammalian heart beat has been frequently discussed by clinicians and investigated by laboratory workers. ${ }^{1}$ Unfortunately, the subject has been complicated by the fact that the conception which regards tonus as a state of sustained partial contraction by virtue of which it resists stretching has not been strictly adhered to and the term has been loosely applied to any volume change at the end of diastole (Henderson, Cameron, etc.). Thus, if a glass oncometer (page $56)$ is placed about the heart and the diastolic volume decreases

${ }^{1}$ Compare also pages 63 and 306. 
it is said to indicate an increased tonus. It is evident, howiever, that the diastolic volume is dependent on the duration of diastole as well as on the passive distensibility which depends on the distending pressure. We are therefore obliged to conclude that the significance and importance of true tonus for the efficiency of the mammalian heart has not been sufficiently investigated.

\section{THE INFLUENCE OF CARDIAC NERVES.}

Anatomical and Histological.- The heart is supplied by two sets of nerve fibers, those passing from the medulla in the vagus trunk and those arising from the upper segments of the thoracic cord, to pass by white rami fibers to the stellate ganglion, thence by the fibers of the Annulus of Vieussens to the inferior cervical ganglion, from which point fibers intermingle with those of the vagus in complex fashion. Fig. 3 shows the details as found in the dog, in which animal the relations resemble those in man (Dogiel). By special treatment the nerve fibers can be seen to form plexuses about the larger arteries and on the posterior surface of the auricles, some sweeping over the auriculo-ventricular groove to the ventricles. Between the fibers knob-like masses are everywhere evident in the basal plexuses of the heart (Böhm, Lim Boom Keng, Dogiel, Müller, Marchand and Meyer, etc.). The nerve supply is essentially homolateral although there is some overlapping of the right fibers on the anterior aspect of the ventricle.

The most interesting question in connection with the nerve supply has been the relation of the extracardiac fibers to the fibers of the sinus node, the $a-v$ node and the His bundle with its branches. Although many technical difficulties have prevented histologists from actually following the connections, the relation is becoming gradually clearer as a result of the studies of Dogiel, De Witt, Keith and Flack, Meiklejohn, Morrison, Oppenheimer and Oppenheimer, Wilson, Marchand and Meyer. It appears that the vago-sympathetic strands which pass sub-epicardially to the posterior region of the auricle apparently end around the multipolar and monopolar cells situated in the sinus region or on those in the interauricular septum. That they are truly relay stations for vagus impulses is evident from the fact that local application of nicotine abolishes the inhibitory vagus action (Marchand and Meyer). The ganglion cells around which medulated fibers presumably of vagus origin end are small. They contain many processes and their nonmedulated axones can be traced to muscle cells (Dogiel). The relation of another type of ganglion cell which is larger and ends in a long medulated axone has-not been discovered. Within the sinus node itself, ganglion cells are absent or very scarce, but nerve fibers sometimes aggregated into a small nerve trunk enter the sinus node 
from above and divide into delicate plexuses from which varicose fibers terminate in simple or complex fashion around the nuclei

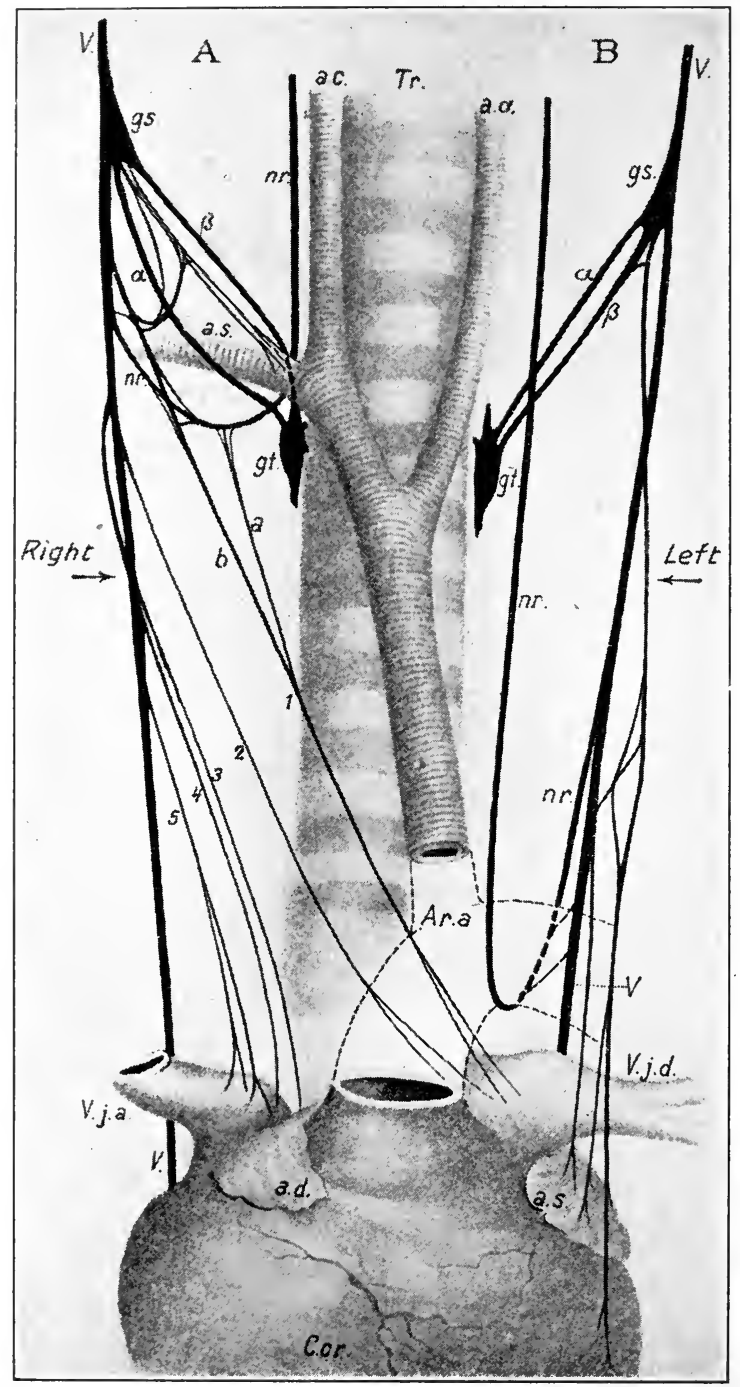

FIG. 3.-Nerve supply of the dog's heart. $v$, vagus nerve; $g s$, inferior cervical ganglion; $a, \beta$, annulus of Vieussens; $g t$, superior thoracic or stellate ganglion; $n r$, recurrent laryngeal nerve; $1,2,3,4$ and 5 , vago-sympathetic filaments to heart. (After Dogiel.)

of the muscle cells. Similarly, fibers from the ganglion cells of the interauricular septum enter the $a-v$ node and pass in bundles 
and plexuses into the ramifications of the His-Tawara system. Some of these fibrils end around nuclei of the muscle cells, while the termination of others apparently remains unknown. It is impossible from histological evidence to decide whether these fibers passing to the ventricle belong to the sympathetic or the vagus system.

Action of Vagus and Sympathetic Fibers.-The vagus and sympathetic fibers exert an antagonistic action on the functions of the heart. "Thus, as is well known, stimulation of the vagus decreases the rate, while stimulation of the sympathetic accelerates it. This antagonism is generally regarded as not restricted to the effect on rate alone, but the vagus is supposed to exert a negative and the sympathetic a positive influence on all the functions of the heart. (Engleman). Moreover, according to some investigators, these functions are mediated by separate nerve fibers (Pawlow). The earlier work supporting this idea is reviewed extensively in Nagel's Handbuch. It will be our purpose to investigate to what extent recent work upholds this hypothesis.

1. Influence on Rhythmicity and Conductivity (Chronotropic and Dromotropic Effects).- It is well known that if the divided vagus is stimulated by a weak tetanizing current, the heart rate is slowed and the interval between auricular and ventricular contractions, the $A s-V s$ interval, is increased (Hering). If the stimulus is stronger, the heart may be entirely stopped for a time, but eventually either the auricle or the ventricle separately, or both together break away from the inhibition.

While it has long been known that the right vagus frequently exerts a more pronounced inhibitory action, it has only recently been shown that a qualitative difference occurs as well (Cohn, Ganter and Zahn). Thus, when slowing of the heart occurs from stimulation of the right vagus, no alteration in the conduction time is apparent either from the $A s-V s$ interval of myograms or from the $P-R$ interval of the electrocardiogram. The suggestion made by Cohn and Rothberger and Winterberg that this indicates a direct influence of the vagus on the sinus node has been further substiantiated by Ganter and Zahn who found that, if during stimulation the node was warmed, the normal rate was restored. Stimulation of the left vagus, on the other hand, may cause a slowing of the ventricles without a corresponding change in auricular rate. It acts therefore largely by blocking the conductivity and can be counteracted by raising the temperature of the $a-v$ node. Although these changes are not manifest in all animals, it appears that in the majority of cases the right vagus fibers communicate largely with the ganglion cells sending fibers to the sinus node while the left vagus fibers terminate chiefly around cells, the fibers of which are distributed to the $a-v$ node and the His bundle. When 
the ventricle escapes from inhibition the impulses originate on one side before the other. This is probably due to the establishment of an unequal degree of block in the distributing branches of the His-Tawara system (Fig. 2).

Apparently there is a similar difference in the distribution _of accelerator fibers (Rothberger and Winterberg). Stimulation of the right stellate ganglion causes an increase in auricular and ventricular rhythm without a change in conduction time. Upon stimulating the left stellate ganglion, the heart not only accelerates but the auriculo-ventricular conduction time is reduced or rendered negative (Hering, Rothberger and Winterberg) suggesting that the nerve terminals end around the $a-v$ node and by increasing its irritability make this the pacemaker of the heart. If now the right stellate ganglion is stimulated, a further increase in rate occurs owing to the generation, at the sinus node, of more frequent impulses.

2. Influence on Irritability and Contractility (Bathmotropic and Inotropic Effects).- It is frequently found that stimulation of the vagus diminishes the recorded contraction of the mammalian auricle although the ventricular contraction usually augments due to the long intervening rest. Occasionally, however, the amplitude of ventricular contractions has also been reported diminished while the duration of systole is prolonged (Klug). Such experiments have led to the conclusion that in the auricle, at least, the function of contractility is depressed by vagus stimulation. On the other hand, stimulation of the sympathetic causes coincident with or apart from an acceleration, an augmentation of the auricular and ventricular amplitude (Pawlow). Hence, it is generally assumed that the sympathetic contains augmentor as well as accelerator fibers for the ventricle.

Englemann has pointed out that a change in the amplitude of contraction may result either from a modification of irritability or of contractility and his experiments seem to demonstrate that, in the frog, stimulation of the vagus decreases the irritability of the heart apart from its influence on contractility and that this is responsible for the reduced amplitude.

All recent work seems to indicate, however, that the vagus influence is not exerted upon the irritability or the contractility of the mammalian ventricle, for, aside from fibers distributed to the conducting system, the bulk of ventricular musculature probably receives no fibers in communication with the vagus ( $c f$. Cullis and Tribe for literature). Since it is possible, however, to influence the amplitude of ventricular contraction even after the $a-v$ node had been severed, the conclusion seems probable that the irritability and contractility of the ventricle may be influenced through the sympathetic fibers passing from auricle to ventricle outside the 
bundle of His. From studies on the hearts of cats, Henderson concludes that no such augmentor effect is obtained except when the ventricle is beating with considerably diminished vigor and the arterial pressure is low. Even then he found it to be of little consequence, incapable of causing a stroke that more than compensated for the abbreviated diastole caused by the more rapid rate.

There is no clear evidence that the nerve fibers exert any influence on the tonus of the heart. Thus, Frank demonstrated on the frog's heart that if the initial tension is maintained high and constant so that the heart is passively maximally stretched, vagus stimulation causes no further relaxation. That the increased relaxation of the mammalian heart in diastole is similarly governed passively by venous pressure is evident from the volume curves of the ventricles (Henderson).

\section{INFLUENCES DIRECTLY AFFECTING THE HEART BEAT.}

The functions of the heart may be influenced not only through nervous channels, but also by influences brought into play through the blood stream. They may be classed as thermal, mechanical, and chemical.

The Perfusion Method.-To study the direct influences, the perfusion method is frequently used, for, in this way, the factors may be separately varied and controlled with greater precision than is possible in the intact heart. In principle, perfusion aims to pass, through the vessels of the heart, a stream of nutrient fluid, under normal conditions of temperature and pressure in such a manner that both ventricles are adequately distended in diastole. The right ventricle is usually distended by fluid returning through the coronary veins but unless precautions are taken, the left ventricle remains empty and goes into a state of tonic contraction. This is frequently the case with the procedure of Martin and Langendorf in which a cannula is placed in the aorta and the semilunar valves are closed. The writer has therefore preferred for general work the modification of Schaefer which consists in pushing the cannula beyond the valves into the left ventricle and depending for the coronary supply upon the fluid forced around it.

The forms of apparatus are so numerous and the choice of solutions so great that it would carry us beyond our scope to enlarge upon them. ${ }^{1}$

The Effect of Temperature on the Mammalian Heart.-An increase in temperature causes an increase in rate until an optimum has been

1 For a description of devices, see Tigerstedt's Handbuch der Physiologischen Methodik, II, Part 4, p. 144. For several recent forms of apparatus, see Eyster and Lövenhart, Jour. Pharmacol. and Exper. Therap., 1913, v, 57; Dresbach, Quart. Jour. Exper. Physiol., 1914, viii, 73. 
reached. The writer is not aware that any investigation has been made to determine whether, in this case the sinus node remains the pacemaker or whether the $a-v$ node assumes that function. If the temperature is raised further the rate diminishes and, finally, $\left(44^{\circ}-45^{\circ}\right)$ the beat ceases entirely (Martin). The rate decreases as the temperature falls and at temperatures varying from $13^{\circ}-19^{\circ}$ the beat again ceases. Rhythmicity is latent, however, and upon subsequent warming, a coördinated beat returns. The amplitude of contraction is increased by heat and decreased by cold, a change which is no doubt dependent on the altered excitability due to the different rates of chemical reactions; for, after increasing the temperature, the heart reacts to stimuli that before were ineffective. Cold increases, heat decreases tonus. The duration of the contraction is also dependent on the temperature, variations from 0.13 to 10-12 seconds being possible (Langendorf). Heat augments, cold decreases conductivity. Experiments in which similar effects are obtained by local application of heat to portions of the HisTawara conducting system, seem to indicate that the effect of a general change in temperature must be referred to these structures.

Effect of Pressure in the Coronaries.-To a certain extent, an increased flow through the coronaries increases the amplitude of cardiac contraction. That this is due, not entirely to an increased supply of nutrient fluid to the capillaries of the heart, but, in part at least, to the increased intravascular pressure, which accompanies such a change is indicated by the observation that, if the coronary vessels are perfused with neutral oil, the beat of the heart may for a while be maintained (Sollman). Porter and his pupils have shown that an increased intraventricular tension, although tending to decrease the coronary flow, increases the height of contraction. A certain amount of mechanical stretching of the walls is apparently favorable for a large amplitude, just as in the case of striated muscles, the addition of a slight weight yields a greater contraction.

Effect of the Composition of the Perfusion Fluid.-The composition of the perfusion fluid has a marked influence on the beat of the heart, indeed upon certain of its constituents the beat is absolutely dependent. In the earliest experiments, defibrinated blood diluted with equal volumes of isotonic saline was used and found to satisfactorily maintain the heart beat. When defibrinated blood is replaced by serum, the beat is not maintained unless the serum is charged with oxygen under pressure, indicating the absolute necessity of oxygen for the maintenance of irritability. That oxygen is not the only requirement for the heart to continue beating can be readily demonstrated by perfusing the heart with an oxygenated isotonic sodium chloride solution. The beat promptly ceases. (Kronecker and Starling).

The question then arises: What constituents are essential to the 
heart beat? Naturally the idea first suggested itself that the "nutritive blood-proteins" were necessary, a view which apparently received confirmation when it was shown that the addition of various proteins (blood-proteins, egg-proteins or milk-proteins) to an oxygenated saline solution favored or restored the beat. This view became less probable again when it was found that the proteins could be replaced entirely by a non-protein substance such as gum-arabic. Hence, it was believed that the proteins exerted no nutritive function but that they favored the beat by virtue of the viscosity which they imparted to the solution Albanese). Subsequently, it was also shown that the proteins were not utilized by the beating heart (Howell and Cooke). The same is true of amino acids. It was found by Miss Wishart and the author that instead of being absorbed, the amino acids already present in the heart are washed out by and added to the perfusion fluid.

It has long been known (Ringer) that the frog's heart can beat on an inorganic mixture which contains sodium, calcium and potassium, while the beat is very much improved by the further addition of sodium bicarbonate. It was but a step to show that upon an oxygenated Ringer's solution ${ }^{1}$ the mammalian heart executes rhythmic beats. The store of energy-yielding material in the heart must be restricted, however, for the effect of such a solution extends over only a short interval of time. By adding dextrose as an available form of energy, Locke found that the heart perfused with Ringer's solution improved, and, on subsequent investigation, it has been shown to be metabolized and destroyed by the beating heart (Locke and Rosenbaum).

Summing up the evidence, it appears to be demonstrated that while oxygen and energy yielding material are essential to supply the liberated energy, and a degree of alkalinity is required to wash away depressing acids formed during activity, the substances essential in eliciting the beat are the salts of sodium, calcium, and potassium. The manner in which it is presumed that they act, will be discussed later.

It is now desirable to note the effect of an excess of any one of these constituents or the presence of other chemical substances, which we can conceive of as existing in the normal circulating blood. This at once excludes all reference to drugs. Calcium is absolutely necessary to the beat of the heart. In excesss, it causes

1 The formula for Ringer's solution used in the mammalian heart, based upon the analysis of blood, is as follows:

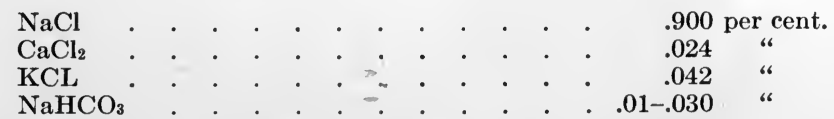

To this is added 0.1 per cent. of dextrose in Locke's formula. 
an increased tonus which may become so extreme as to entirely prevent the fundamental contractions. Similar tonic contractions are produced by small quantities of the other alkaline earths (barium, strontium, etc.). Potassium ions are apparently not absolutely necessary to rhythmicity, hearts having been made to beat on perfusion with a mixture of sodium and calcium ions alone. When present, however, potassium favors relaxation and tends to reduce the irritability to artificial stimuli. In excess, it causes the heart to stop in extreme relaxation.

The importance of $\mathrm{CO}_{2}$ has been variously interpreted. According to Starling and Henderson, small quantities increase the amplitude of contraction; but larger quantities act as a depressant, The observation of Ketcham, King and Hooker, on the other hand, indicate that $\mathrm{CO}_{2}$ in any concentration depresses both contractility and tonus. A similar effect is exerted by lactic acid (Backman, Burridge, etc.).

The activity of the heart is apparently increased by the addition of various nitrogenous extractions; for example, urea (Eyster) and small doses of indol and skatol (Danilewsky). Alanine seems to have no effect on a vigorously beating heart, but when the contractions become weak, it may cause a revival in strength (Lausana, Hasegovna). Witte's peptone, foreign proteins and, especially in sensitized rabbits' hearts, horse serum exert a depressant and toxic action (Demel, Popielski, Friedberger and Meta). These results should be applied cautiously to anaphylactic questions. Cushny and Gunn have recently shown that the same reaction follows when the serum from the same animal is added to the saline perfusion fluid; the preliminary augmented and accelerated contractions being followed by a stage of slowing and depression during which heart block frequently occurred. The results are interpreted to mean that the heart perfused with Ringer's solution represents a hypodynamic organ rather than that the sera have a toxic action.

The addition of bile slows the rhythm, augments the tonus and diminishes the expansion of the heart (Berti-Malesani), extracts of tissue organs (brain, nerves, etc.), depress the rate and amplitude of cardiac contraction either because of their cholin content or because they contain a large amount of potassium (Macleod). Of the internal secretions which are presumably found in the normal blood in small quantities, the actions of epinephrin and pituitrin are best known. Epinephrin or adrenalin, the active principle extracted from the suprarenal glands, causes an increase in the rate and amplitude of the perfused heart, the tonus being reduced. Pituitary extracts have the reverse effect; they slow the heart, decrease the amplitude, and increase the tonus. 


\section{THEORIES AS TO THE CAUSE OF THE HEART BEAT AND THE MECHANISM OF NERVOUS CONTROL.}

It is well known that there are two views held as to the cause of the heart beat-the muscular or myogenic and the nervous or neurogenic. Our statements of these views must, in the case of mammalian hearts, be more definite than was necessary before the details of the structure and functions of the heart tissue became so well understood.

According to the myogenic theory, the impulse is initiated in the peculiar muscle cells of the sinus node unless their irritability is exceeded by that of some other portion of the His-Tawara system (e. g., in the $a-v$ node), in which case the muscle cells of this region become the rhythmic centre or "pacemaker." The impulse is conducted by the peculiar muscle fibers of the His-Tawara system to the fibers of the auricle and ventricle. The irritability and conductivity of these muscle fibers is under the control of vagus and sympathetic fibers relayed through ganglion cells and their axones.

According to the neurogenic hypothesis, it is necessary to assume that the inner stimulus originates in the ganglion cells in the vicinity of the sinus node or in those of the inter-auricular septum. It is transmitted by axones to the cells of the sinus node, of the $a-v$ node, the His-Tawara system or the auricular and ventricular muscles themselves. Moreover, it is not impossible to conceive that one system generates the impulses and the other conducts them, or again that both the nerve and muscle fibers of the HisTawara system share the property of conduction.

It may be recorded at once, that, without question, the majority of physiologists favor the myogenic view of impulse initiation and conduction. We may, therefore, briefly examine the validity of this belief.

When the existence of a muscular connection between the auricle and ventricle was revealed (Kent, His, Jr.) and it was subsequently shown that this represented but a portion of an extensive conducting system of peculiar muscle fibers; when, moreover, a structure in the form of a sinus node was demonstrated which was apparently the homologue of the sinus venosus, and when this was finally shown to be the seat of initial negativity in the heart, it seemed to many conclusive of the muscular origin of the heart beat. With the subsequent discovery that the sinus node and the $a-v$ node contain or are related to ganglion cells in their immediate proximity, and that the entire His-Tawara system contains many bundles of nerve fibers as well as ganglion cells, the absolute guarantee that nerves are not concerned is not afforded by morphological studies alone. It is necessary, therefore, to seek other evidence. 
Greatly in favor of the myogenic hypothesis is the fact that the embryonic heart beats before the nervous elements have wandered in from their extra-cardial position (Pflüger, His, Jr.). The fact that individual cells grown in artificial media (Burrows) pulsate is corroborative. Important as this fact is, it cannot be regarded as conclusive. Its value depends, of course, on the probability that a tissue that once assumes a function in early life retains this function to the end. That this is not necessarily the case has been shown by the fact that in the limulus heart the beat is originally muscular in character although later the function is undoubtedly transferred to nerve cells (Carlson).

The demonstration that the sinus node shows the first evidence of activity combined with the fact that very few ganglion cells are situated here speaks in favor of a muscular origin of the beat. The only question that can possibly arise is whether with the most careful technic it is possible to so apply the electrodes that they may not be placed on ganglion cells in the vicinity, or provided such placement is possible, whether the initial current does not reach the surface more readily at the more exposed sinus tissue. The careful experiments of Eyster and Meek with very sensitive galvanometers and the application of differential electrodes by Garten and his pupils apparently negative such an objection.

Experimental evidence does not favor the view that the nerve fibers in the His-Tawara system transmit impulses to the ventricle for (1) histological examination has shown that many fibers terminate in the muscle cells of this tissue directly; (2) conductivity fails to occur after recovery from crushing, when muscle tissue does not regenerate but nerve fibers do so (Erlanger); and (3) application of cocaine which paralyzes nerve fibers does not produce $a-v$ block (Cullus and I)ixon).

While the evidence seems clearly to favor the muscular origin of the heart beat, the cause and nature of the "inner stimulus" is less clearly established. It may be logically assumed that some stabile chemical compound is dissociated or rendered unstable and that the energy liberated acts as the "inner stimulus." What is this chemical substance and what the reaction concerned in its transformation? Why does it occur in specific regions of extremely limited areas and what form of energy is liberated? To these questions no final answer can be given. It seems that the inorganic salts, Na, Ca, K, in proper proportions cause spontaneous beats of muscle cells. As to their action, several views are held. Lingle and Loeb believe that sodium ions act as an excitant while calcium antagonizes the poisonous effects of sodium. Howell believes that calcium converts the stable material into an unstable compound in which process potassium is liberated. According to this view, the salts are not direct excitants but liberate energy of some form 
which acts in this role. Martin has elaborated the idea further: He thinks that while calcium converts the stable substance into an unstable one, sodium or some other agent is necessary to force this substance to liberate its energy.

Closely related to the question of the source of the "inner stimulus" is that relating to the mechanism by which vagus and accelerator nerves modify the heart. For the maintenance of nerve action calcium is also essential (Howell and Duke, Busquet and Pachon, Hagan and Ormond). The function of calcium in producing inhibition apparently consists in aiding the transmission of vagus impulses rather than in producing an inhibitory effect on the heart muscle. The latter function is attributed to potassium by Howell and Duke, who regard vagus and potassium inhibitions as similar conditions.

The most commonly accepted version of nerve action is that advanced by Gaskell, who regards the vagus nerve as anabolic or trophic and the accelerator nerve as catabolic or motor in character. It may be pointed out that recent work has adduced evidence in favor of his views. In the first place, it has been possible after many failures to corroborate by the delicate string galvanometer the observations of Gaskell that the auricle in the terrapin becomes increasingly positive with respect to an injured part when the vagus is stimulated (Meek and Eyster). The failure to obtain this response in mammals is probably accounted for by the fact that it is impossible to use sufficiently sensitive strings in their investigation. In the second place, it has been shown that the beat of a stopped heart may be revived by stimulating the accelerator nerves (Hering), while premature systoles have been produced in the beating heart in the same manner.

Still other theories as to vagus action evolved as a result of the investigation of different phases of cardiac activity have been suggested by Carlson, Erlanger and Schmiedeberg for which the original articles may be consulted (see literature).

\section{LITERATURE.}

Articles Dealing with Properties of Heart Muscle.

Braeunig. Arch. f. Anat. u. Physiol., 1904, Suppl. Bd., 1.

Brandenburg and Hoffman. Med. Klin., 1912, viii, 16.

Cameron. Johns Hopkins Hosp. Reports, 1911, xvi, 549.

Carlson. Am. Jour. Physiol., 1906, xvi, 67; 1907, xviii, 71.

Cohn. Heart, 1909, i, 167.

Cohn, Kessel, and Mason. Heart, 1912, ii, 311.

Danilewsky. Arch. f. d. ges. Physiol., 1905, cix, 602.

De Witt. Anat. Rec., 1909, iii, 475.

Engle. Beitr. z. path. Anat. u. z. allg. Path., 1910, xlviii, 499.

Erlanger. Arch. Int. Med., 1913, xi, 334. 
Eyster and Meek. Heart, 1914, v, 119, 137.

Amer. Jour. Physiol., 1915, xxxvi, 367.

Fahr. Virchow's Arch. f. path. Anat., 1907, clxxxviii, 562.

Garten. Skan. Arch. f. Physiol., 1913, xxix, 114.

Henderson. Am. Jour. Physiol., 1913, xxxi, 361.

His. Deutsch. Arch. f. klin. Med., 1899, lxiv, 316.

Humblet. Arch. internat. de physiol., 1904, i, 278; 1905, iii, 330.

Kent. Quart. Jour. Exper. Physiol., 1913-14, vii, 193.

“ Jour. Physiol., 1893, xiv, 233; 1914, xlviii, 57.

Keith and Flack. Jour. Anat. and Physiol., 1907, xli, 172.

" “ " $\quad$ Lancet, 1906, ii, 359.

Ketcham, King, and Hooker. Amer. Jour. Physiol., 1912, xxxi, 64.

Koch. Deutsch. med. Wchnschr., 1909, xxxv, 429; 1910, xxxvi, 688.

Lewis. Heart, 1910-11, ii, 23.

Lewis, Oppenheimer, and Oppenheimer. Heart, 1910-11, ii, 147.

Lewis, White, and Meakins. Heart, 1914, v, 289.

Lhannon. Am. Jour. Anat., 1912, xiii, 55,

Mall. Am. Jour. Anat., 1912, xiii, 284.

Martin. Am. Jour. Physiol., 1912, xxx, 182.

Oppenheimer and Oppenheimer. Jour. Exper. Med., 1912, xvi, 613.

Porter. Am. Jour. Physiol., 1905, xv, 1.

Retzer. Arch. f. Anat. u. Physiol., 1904 (Anat. section), 1.

Rohde. Arch. f. exper. Path. u. Pharmacol., 1905, liv, 104.

Schultz. Am. Jour. Physiol., 1906, xvi, 483; 1908, xxii, 133.

Tawara. Das Reizleitungssystem des Saugetierherzens, 1908.

Wilson. Proc. Roy. Soc., 1909, lxxxi, B, 151.

Wybauw. Areh. internat. de physiol., 1911, x, 79.

Zahn, Zentralbl. f. Physiol., 1912, xxvi, 495. Arch. f. d. ges. Physiol., 1913, cli, 247.

\section{Articles Dealing with the Nerve Supply of the Heart}

Boehm. Arch. f. exper. Path. u. Pharmacol., 1875, iv, 351.

Cohn. Proc. Soc. Exper. Biol. and Med., 1912, x, 8.

Jour. Exper. Med., 1912, xvi, 732; 1913, xviii, 715.

Cohn and Lewis. Jour. Exper. Med., 1913, xviii, 739.

Cullis and Tribe. Jour. Physiol., 1913, xlvi, 141.

Dogiel. Arch. f. d. ges. Physiol., 1911, cxlii, 109.

Arch. f. mikros. Anat., 1899, liii, 237.

Einthoven and Wieringa. Arch. f. d. ges. Physiol., 1912, cxlix, 48.

Engleman. Arch. f. Physiol., 1900, 315; 1902, 1.

Ganter and Zahn. Arch. f. d. ges. Physiol., 1913, cliv, 492.

Garrey. Am. Jour. Physiol., 1912, xxx, 451.

Hagen and Ormond. Am. Jour. Physiol., 1912, xxix, 105.

Henderson. Am. Jour. Physiol., 1913, xxxi, 297.

Hering. Zentralbl. f. Physiol., 1905, xix, 129.

Arch. f. d. ges. Physiol., 1906, cxv, 354.

Ken Kure. Ztschr. f. exper. Path. u. Therap., 1913, xii.

“ Deutsch. Arch. f. klin. Med., 1912, cvi, 33.

Mansfield. Arch. f. d. ges. Physiol., 1910, exxxiv, 598.

Marchand and Meyer. Arch. f. d. ges. Physiol., 1912, cxlv, 401.

Meek and Eyster. Am. Jour. Physiol., 1912, xxx, 271.'

Heart, 1914, v, 227.

Meiklejohn. Jour. Anat. and Physiol., 1913, xlviii, 1.

Morison. Jour. Anat. and Physiol., 1912, xlvi, 319.

Robinson, Canby, and Draper. Jour. Exper. Med., 1911, xiv, 217.

Rothberger and Winterberg. Arch. f. d. ges. Physiol., 1911, cxli, 343.

"

“ “ Zentralbl. f. Physiol., xxiv, 247, 305, 789.

Wybauw. Arch. internat. de physiol., 1905, ii, 198. 
Articles Dealing with Influences Modifying the Heart Beat.

Albanese. Arch. f. exper. Path. u. Therap., 1893, xxxii, 29.

Backman. Compt. rend. soc. de biol., lxii, 218.

Benedict. Am. Jour. Physiol., 1905, xiii, 192.

Berti, Malesani. Arch. ital. biol., 1910, liv, 101.

Bohlman. Arch. f. d. ges. Physiol., 1907, cxx, 400.

Carlson. Am. Jour. Physiol., 1906, xv, 357.

“ Am. Jour. Physiol., 1907, xviii, 149.

Cushny. Heart, 1911-12, iii, 257.

Cushny and Gunn. Jour. Pharmacol. and Exper. Therap., 913, v, 1.

Danilewsky. Arch. f. d. ges. Physiol., 1908, cxxv, 349.

Demel. Ztschr. f. Immunitätsforch., 1910, iii, 1046.

Arch. ital. biol., 1910, i, 54, 141.

Deneke and Adam. Ztschr. f. exper. Path. u. Therap., 1906, ii, 491.

Einis. Biochem. Ztschr., 1913, lii, 96.

Eyster. Science, 1910, xxxi, 236.

Friedberger and Mita. Ztschr. Immunitätsforch., 1911, x, 216.

Gorham and Morrison. Amer. Jour. of Physiol., 1910, xxv, 419.

Guthrie and Pike. Am. Jour. Physiol., 1907, xviii, 14.

Hasegovna. Arch. internat. de physiol., 1912, xii, 79.

Hoffmann. Nagel's Handbuch der Physiol. des Menschen, 1909, i, 223.

Howell and Cooke. Jour. Physiol., 1893, xiv, 198.

Knowlton and Starling. Jour. Physiol., 1912, xliv, 206.

Kronecker and Stirling. Ludwig's Festschrift, 1874, 173.

Langendorf. Arch. f. d. ges. Physiol., 1895, lxi, 291; 1897, lxvi, 355, 384.

Ergebn. der Physiol., 1902, I2, 298.

Leontowitsch. Arch. f. d. ges. Physiol., 1912, cxlvii, 473.

Locke. Jour. Physiol., 1895, xviii, 332.

Lusana. Arch. internat. de physiol., 1910, ix, 393.

Macleod. Am. Jour. Physiol., 1907, xix, 426.

Macnider and Mathews. Am. Jour. Physiol., 1908, xx, 323.

Martin. Studies from Biological Laboratory of Johns Hopkins University 1881, ii, 119 .

Martin and Applegarth. Studies from Johns Hopkins University, 1890, iv, 275.

Müller. Deutsch. Arch. f. klin. Med., 1911, ci, 421.

Popielski. Arch. f. d. ges. Physiol.; 1909, exxx, 394.

Snyder. Am. Jour. Physiol., 1906, xvii, 350.

Sollman. Am. Jour. Physiol., 1905, xv, 121.

\section{Articles Relating to Causes of Beat and Nature of Nerve Action.}

Burrows. München. med. Wchnschr., 1912, 1473.

Carlson. Science, 1905, N. S., xxi, 889 .

" Am. Jour. Physiol., 1905, xiii, 217.

“ Am. Jour. Physiol., 1908, xxi, 1.

Cullis and Dixon. Jour. Physiol., 1911, xlii, 156.

Erlanger. Am. Jour. Physiol., 1909, xxiv, 375.

Am. Jour. Physiol., 1909, xxv, xvi.

His, Jr. Med. Klin., 1893, j, 14.

Howell and Duke. Jour. Physiol., 1906, xxxv, 131.

Howell. Am. Jour. Physiol., 1906, xv, 280.

" Jour. Am. Med. Assn., 1906, xlvi, 1665, 1749.

“ Harvey Lectures, 1905-06, 305.

Lingle. Am. Jour. Physiol., 1900, iv, 265.

Martin. Am. Jour. Physiol., 1913, xxxi, 165.

Schmiedeberg. Arch. Anat. u. Physiol., 1910, 173. 


\section{THE SEQUENCE OF CARDIAC CON'TRACTION AND THE MOVEMENTS OF THE HEART.}

\section{THE CARDIAC CYCLE.}

Although the term systole has been defined in various ways, it seems preferable to apply it to that portion of the auricular or ventricular cycle extending from the beginning to the end of mechanical contraction. Similarly, diastole covers the period of mechanical relaxation. In this application we may pass the biological question as to whether all fibers of a chamber simultaneously cease contracting or whether the mechanical shortening stops because a balance has been struck between relaxing and contracting fibers.

It has long been recognized that the contraction of the auricle precedes that of the ventricle and it is therefore often designated as the pre-systole of the heart. In spite of the fact that no definite expression concerning it is found in any current text-book of physiology, the opinion seems to be widespread that in the mammalian heart the systole of the auricle terminates before that of the ventricle begins, $i$. e., that a short inter-systolic period exists. 'There is no conclusive evidence of this fact, however. Although many thousands of simultaneous records of auricular and ventricular beats have been recorded, the question is still an open one. The writer has carefully compared a large number of published tracings of various investigators and has also made accurate measurements of his own records. This survey has shown that in the majority of cases examined, auricular systole terminated before ventricular contraction began; in other cases it continued somewhat into this period. The differences are probably to be accounted for by the variable efficiency of the recording levers used. It is frequently supposed that the existence of a complete $a$ wave in the venous pulse before the occurrence of other waves evidently associated with ventricular systole indicates that auricular contraction and relaxation have taken place. If, however, the recent work of Ewing is accepted the entire rise and fall of the $a$ wave may take place during auricular contraction. Similarly, it is uncertain whether the $P$ wave of the electrocardiogram and the auricular waves present in the esophagram, the central arterial pulse and the intraventricular pressure curves coincide in time with the duration of auricular 
systole, or whether they are the effect of systole succeeded by diastole. This makes it difficult to determine accurately either the relation between auriculàr diastole and ventricular systole or the duration of auricular systole alone. The average value of 0.1 second so commonly given as representing the length of auricular systole is apparently a daring guess. Using the upstroke of the $a$ wave of the venous pulse as a criterion, the writer found in 58 records taken with optical capules that the auricular systole varied from 0.038 to 1.04 second but that in 87 per cent. of cases the systole fell within the range of $0.063-0.075$ seconds.

The duration of auricular and ventricular systoles varies greatly in different animals and in the same animal under different conditions. The chief interest lies in the variations in man. The duration of ventricular systole has been determined with approximate accuracy in man by several methods: as (1) by establishing the interval between the beginnings of the two heart sounds; $(2)$ by estimating the interval between the rise of the primary and of the dicrotic wave of the arterial pulse; (3) by measuring the interval between the rise of the $c$ wave and the fall of the $v$ wave in the venous pulse; (4) by estimating the interval from the $Q$ to the end of the $T$ variations of the electrocardiogram. None of these procedures are absolutely correct. The closest approximation is probably afforded by determining the interval in the arterial pulse extending from the onset of the preliminary oscillation to the beginning of the incisura (Figs. 27 and 34). (For the details see subsequent chapters.) The average duration of human systoles estimated by various studies are incorporated in the following table:

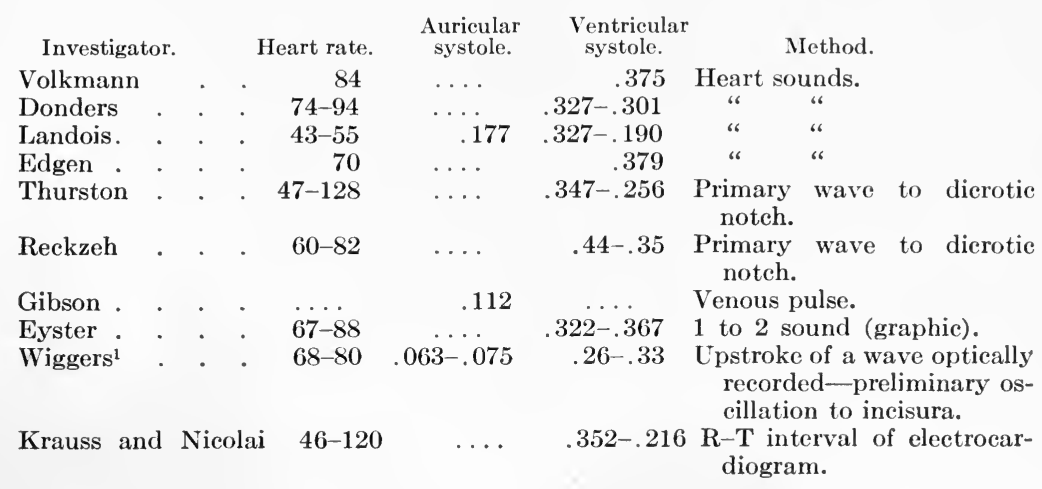

The Spread of the Contraction Wave over the Ventricle.-It is generally regarded as an established fact that the contraction,

1 Unpublished results. 
like the excitation, spreads over the heart as a peristaltic wave. According to the generally accepted view the interventricular walls contract first and the base contracts before the apex. With the demonstration that all points on the outside of the ventricle become negative at the same time (Clement, Erfmann, Lewis, etc.), some doubt may be felt as to whether the mechanical apparatus by which the difference in time has usually been measured was adequate to determine such variations and whether, on the other hand, the entire ventricular muscle does not contract at once.

Of special interest has been the relative time of contraction of the papillary muscles. The earlier work (Roy and Adami, Fenwick and Overand) indicated that the papillary muscles start their shortening later and end it earlier than the rest of the ventricular muscles. The results of other investigators indicate, however, that they precede the contraction of the conus arteriosus by 0.015 to 0.031 seconds (Hering, Saltzmann). Inasmuch as such studies have for technical reasons been restricted to excised and perfused hearts, it would be premature to lay undue stress upon the transfer of such results to the intact heart (Saltzmann).

\section{THE MOVEMENTS OF THE HEART.}

During ventricular systole, as the period of mechanical contraction of the heart is called, all its diameters decrease and the position of the entire organ changes. These changes are due in part to the spiral arrangement of muscle bands in the heart, in part, also, to its fixation and support. The anatomical researches of MacCallum and their subsequent extension by Mall have shown that the two ventricles are made up of a series of museular bands passing from the base to the apex in the form of a scroll. Mall divides these fibers into four groups, viz.: (1) the superficial bulbospiral fibers, (2) the superficial sino-spiral fibers, (3) the deep bulbospiral fibers and (4) the deep sino-spiral fibers.

The superficial bulbo-spiral fibers arise from the conus arteriosus and left side of the aorta and auriculo-ventricular ring, and pass down obliquely to the apex where they form a spiral and thus reach the interior of the ventricle to terminate in the interventricular septum and posterior papillary muscle. The superficial sinospiral fibers arise from the posterior portion of the $a-v$ ring, run obliquely to and over the anterior surface of the right ventricle, and, after reaching the apex, pass by a spiral turn inward to end in the anterior papillary muscles of the left heart. The deep bulbospiral fibers are attached to the dorsal side of the aorta and encircle the left ventricle. The deep sino-spiral fibers encircle the right ventricle in similar fashion.

When the heart is exposed in the open chest and the pericardium 
is removed, or, when it is suspended from the large vessels in per: fusion experiments, the effect of these muscular bands is brought into play. The base remains practically stationary but the apex moves upward. The entire heart is caused by the oblique fibers to rotate from left to right and the apex is lifted forward.

If, however, the movements of the heart are studied when only the upper three-fourths of the sternum is resected so that pericardial attachments remain intact, and if, in addition, the severed sternocardial bands are fastened to a wire substituted for the sternum, the base descends, the apex rotates anteriorly and in so doing moves downward slightly. If the animal is placed on the left side so that the heart is definitely in contact with the thoracic wall, it is evident that the slight downward movement of the apex is largely responsible for the impact felt externally as the apex beat. (Personal observations.). These differences between the movements of the free and the intact heart are due to the restraining influence which the pericardium exerts. This covering is attached to the large vessels at the base of the heart, stretches upward into the cervical fascia and joins the central tendon of the diaphragm below.

It appears from $x$-ray studies that the movements in man are similar to those directly observed in the dog's chest by special technic. These observations favor the view that the slight downward movement of the apex, its slight rotation and consequently its closer apposition to the chest wall account for the major part of the shock felt in the fifth intercostal space. It seems very questionable, indeed, whether changes in the volume of the heart or variations of intraventricular pressure are in the least concerned in the production of the cardiogram obtained by graphically recording the variations at the apex.

The heart shifts its position, not only in systole and diastole, but also during phases of respiration, a change accounted for by the intimate relation of the heart and diaphragm.

Fluoroscopic examination, instantaneous $x$-ray plates, orthodiagrams, as well as the Röntgen kinematograph, have combined to show that the entire heart moves downward and that the apex is rotated clockwise during inspiration. The shadow of the entire heart lengthens and becomes narrower (Fig. 4). Corroborative evidence that the cardiac axis changes has also been given by the electrocardiograph (Waller, Einthoven), it having been shown by computation of the electrocardiac angle from the height of ventricular waves, that expiration causes the heart to become more horizontal.

There is further evidence in dogs that the apex descends in inspiration. The writer has frequently found that when a sound is accidentally placed deep in the ventricular chamber, the end is 
open throughout systole only during those beats occurring in inspiration, while, during expiration, either no pressure changes are recorded, or a flat-top type of curve is obtained which is well known to be due to blocking of a sound by ventricular contraction.

The exact mechanism by which the position of the heart is changed in inspiration has been studied by attaching strings to five or six different points of the heart intact within the pericardium and connecting these simultaneously to recording levers (Wiggers). The following observations were made:

1. During the descent of the diaphragm, the posterior portions of the heart and the venæ cavæ descend more than the anterior portion of the base while the anterior aspects of the apex move forward and as a result often slightly upward. 'The right and left borders of the ventricle move toward the right.

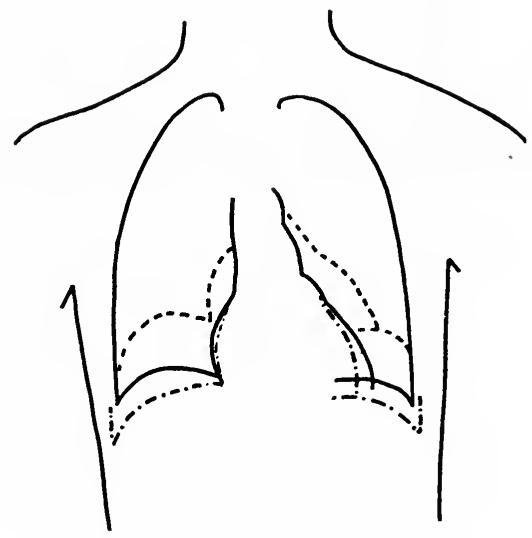

FIG. 4.-Orthodiagram showing the effect of deep inspiration and expiration on the positions of the heart and diaphragm. Broken lines, expiration; dots and dashes, deep inspiration; continuous line, shallow respiration. (After Claytor and Merrill.)

2. The movement of the heart to the right does not occur after severing the left phrenic nerves nor upon stimulating the right phrenic nerve. It is, therefore, due to a traction of the left sheath of the pericardium.

3. A descent of the base of the ventricles, the auricles and the venæ cavæ occurs after the entire pericardium is severed from the diaphragm. Direct experiments show that this is due in part to a traction upon the inferior vena cava making it longer and narrower.

4. In the dog the descent of the base of the heart persists after the vena cava is clamped and divided. This results from a traction upon the ligamentum pulmonale (a double fold continuous with the pleura pulmonalis, and passing downward from the root of the lung to its vertebral and diaphragmatic attachments) which 
causes the roots of the lungs, the pulmonary vessels and through these, the base of the heart to move downward.

Studies with the $x$-rays and electrocardiograph indicate that the position of the heart is not as fixed as has often been supposed but that, on the contrary, it undergoes considerable shifting with the position of the diaphgram and changes in different positions of the body. The heart is displaced to the left when the left lateral position is assumed and the apex approximates the chest wall more firmly, a point of some significance in recording tracings from the apex beat. A change from the supine to the prone position causes a compression of the abdominal viscera and so pushes the diaphragm up, which causes the heart to assume a more horizontal angle. Sitting acts in a similar way but to a lesser degree by compressing the abdominal contents.

\section{BIBLIOGRAPHY.}

MacCallum. Contributions to Medical Science Dedicated to W. H. Welch, 1900, 307.

Mall. Am. Jour. of Anat., 1911, xi, 211.

Wiggers. Proc. Soc. Exper. Biol. and Med., 1914, xi, 107.

Hering. Arch. f. d. gesam. Physiol., 1909, exxvi, 225.

Saltzman. Skan. Arch. f. Physiol., 1908, xx, 233.

For literature dealing with the $x$-ray and electrocardiographic investigation of cardiac position, consult references at the end of their respective chapters. 


\section{CH A P T E R I I.}

\section{THE DYNAMICS OF THE HEAR'T BEA'T.}

THe dynamics of the heart beat is concerned with the mechanisms by which the heart transfers the blood from the venous to the arterial side under sufficient pressure to insure its circulation around the body. It involves a study of (1) the mechanism of the valve action, (2) the pressure relations at different times in the different chambers, and (3) the passage of blood to and from the ventricles.

\section{THE MECHANISM OF VALVE ACTION.}

The atrio-ventricular and aortic openings of the two ventricles are guarded by exceedingly efficient sets of valves.

The mitral and tricuspid valves arise from the atrioventricular ring. They are divided by deep incisions into two flaps on the left side and into three flaps on the right. At their origin the valves are fairly thick and contain strands of muscle tissue but toward their free edges they become exceedingly thin. In fact such thin structures can resist pressure only because they approximate in a lateral fashion. Into the free ventricular surfaces of the valves are inserted the chordæ tendinæ arising from the papillary muscles which project into the ventricles (Fig. 5, A). Their function is apparently to prevent or guard against a possible inversion of the valves into the auricles. During diastole these tendinæ are somewhat relaxed, but as the valves close they are rendered taut. This is assisted by the contraction and shortening of the papillary muscles.

It is generally regarded as established (Baumgarten) that the valves in diastole do not lie in close proximity to the heart wall but float together as blood rushes in. This is, no doubt, partly accounted for by their extreme lightness, but is aided also by the formation of eddies behind them during the inrush of blood which takes place in diastole.

The semilunar valves guarding the pulmonary artery and aorta consist essentially of three small pockets, the free edges of which, when the pockets are expanded form a complete closure of the aortic opening. Their shape and the support offered by their attachment are such that no sustaining chordæ are required. Exactly at the centre of the rim of each pocket is a tiny nodule, 
the corpus Arantii. These together fill the small central gap that would otherwise be left when the pockets expanded. Like the semilunar valves, they are floated away from the wall even during systole owing to the fact that some fluid remains within them constantly and that eddies are formed behind them.

The closure of the valves normally takes place without the least regurgitation and is instantaneous. Following the conception advanced by Krehl, it is generally held that closure is brought about by the eddies set up behind the valves when fluid rapidly passes through their openings. When the flow ceases the eddy circles enlarge, thus pushing the valves together as soon as the pressure on the concave side becomes a trifle higher than on the other (Fig. 5, B).

As a result of recent studies Henderson and Johnson advance another theory. According to these investigators, the sudden

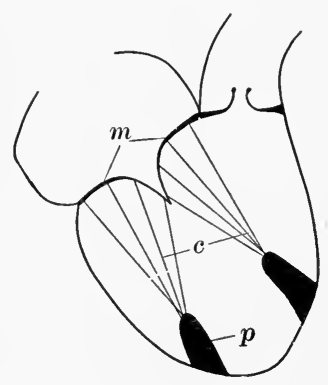

$A$

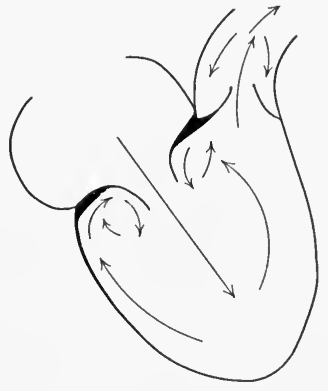

$B$

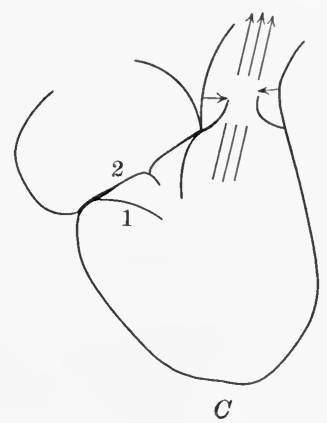

Fig. 5.-Three diagrams showing mechanisms concerned in valve closure. $A$, relations of papillary muscles and chordæ tendine to valve flaps; $B$, partial closure due to eddy formation; $C$, closure by hinge movement and breaking of a jet.

cessation of flow through an opening causes a breaking of the jet and leaves a small area of negative pressure "much as in the wake of a ship." From each side fluid is drawn in and this movement closes the valves (Fig. 5, C). They also believe that a similar mechanism is responsible for the closure of the $a-v$ valves provided blood is still rushing in at the beginning of ventricular contraction. This occurs when the heart rate is rapid. When the heart rate is slow, however, so that the filling has been completed before the beginning of systole and the auricles are not beating then the valves do not unfurl but swing into place like doors on hinges (Fig. 5, C). This mode of closure, which necessarily involves some leakage does not occur normally because the contracting auricle forces a small quantity of blood into the ventricle and the sudden cessation of this current is responsible for the valve closure. It may be added parenthetically that if, as most of the evidence indicates, auricular systole terminates a short interval before 
ventricular systole begins, then the Henderson and Johnson view necessitates the assumption that the $a-v$ valve closure and the first heart sound precede ventricular systole. Of this there is no evidence either in the pressure curves taken from the ventricle or in the most delicate records of heart sounds (Fahr.)

\section{THE PRESSURE CHANGES IN THE HEART.}

Apparatus.-The variations of pressure in the chambers of the heart and aorta occur so rapidly that, as has long been recognized, the mercury manometer is incapable of following the changes accurately. This is due to the inertia of the mercury, $i$. $e$., the physical property by virtue of which it resists being set in motion when at rest, and strives to remain in motion after the acting force has ceased. This causes the apparatus to record an amplitude which does not correspond to the pressure changes actually involved. If a high pressure is suddenly communicated to a manometer and then released the mercury rises above and falls below the true level. The amplitude of the curves is larger than the true variation. If the variations recur rapidly neither the highest nor the lowest pressures are approached and the recorded amplitude is less than the change which actually occurs. This is the case when the mercury manometer registers pressures within the heart and large vessels. Not only are the extremes of pressure incorrectly recorded, but the rise starts later and lags behind the pressure change. In other words the inertia is determined by the low vibration frequency inherent in the instrument. We return, therefore, to the physical fact that an apparatus, in order to record the oscillations correctly must have an inherent frequency that is more rapid than that of the swiftest oscillations to be recorded.

Recognizing the limitations of the mercury manometer, attempts have been made by physiologists to devise membrane or spring manometers capable of correctly following the details and height of the pressure curves. In this they have been relatively unsuccessful, however, until Frank worked out the mathematical and theoretical basis for the construction of adequate types. Frank's critique constitutes, therefore, the most important contribution yet made to the study of the dynamics of the circulation.

Theory of Manometers.-According to Frank the "manometer" constitutes the entire pressure recording system extending from the rubber capsule and recording point to the mouth of the cannula in the artery. The efficiency of any instrument (Güte) is expressed by the formula $G=\frac{v \gamma \epsilon E^{\prime}}{M^{\prime}}$ where $v$ is the magnification of the lever, $\gamma \epsilon$ the sensitiveness of the membrane, $E^{\prime}$ is the volume elasticity coefficient and $M^{\prime}$ is the effective mass. By increasing the 
first three and decreasing the last factor, the efficiency of an instrument may be improved. The effective mass $M^{\prime}$ is dependent directly on the length and the specific gravity of the fluid column and is inversely related to the cross section, $i . e ., M^{\prime}={ }_{Q}^{l} s$ in which $l$ equals the length; $Q$, the cross-section, and $s$, the specific gravity. It is apparent that the efficiency of the apparatus is augmented whenever the length of the manometer decreases and the width of the tube increases. The sensitiveness, is the ratio of membrane excursion to pressure change, $i . e ., \gamma \epsilon=\frac{f}{p}$. The excursion $f$ for a definite pressure change and hence the sensitiveness of the instrument may be increased either by decreasing the tension of the membrane, increasing its diameter or by decreasing the diameter of the supported plate. This is indicated in the formula $f=\frac{p}{s} \cdot \frac{r^{2}-q^{2}}{4}$ in which $p$ is the pressure; $s$, the tension of the rubber; $r$, the radius of the rubber and $q$, the radius of the plate. The volume elasticity coefficient $E^{\prime}$ represented by the ratio of the pressure change to the volume change, on the other hand, may be increased by increasing the tension or by decreasing the size. This is shown in the formula $E=\frac{\triangle p}{\triangle v}=\frac{8 s}{r^{4}\left(1-d^{4}\right) \pi}$. In this formula $\triangle p=$ pressure increase, $\Delta v=$ volume increase, $s=$ the tension and $r$, the radius of the membrane. It is apparent that the increase in efficiency which can be gained by increasing the sensitiveness of the membrane is limited for in so doing the volume-elasticity coefficient is simultaneously reduced. Furthermore, the sensitiveness of the membrane must remain small in order that an adequate vibration period may be retained, for upon this depends the ability to record pressure changes accurately.

The inherent vibration frequency, $N$, is the reciprocal of the period of single vibration, or, $N=1 / T$. In a simple manometer system $T=2 \pi \sqrt{\frac{M^{\prime}}{E^{\prime}}}$. From this it is apparent that the quality is increased, not only by reducing the effective mass, but also by increasing the volume-elasticity coefficient. Both are usually necessary to obtain an instrument capable of recording pressure variations.

It is apparent that every increase in quality resulting from a higher volume-elasticity coefficient occurs at the expense of its sensitiveness, hence it becomes necessary to magnify these movements. When this can be brought about by a beam of light the efficiency of the apparatus is improved, for in this way $v$ is increased without reducing the quality. When, however, the movements of the membrane are magnified by ponderable levers there must be taken into consideration, (1) the reduced mass of the lever $(\mathrm{m})$ 
(i.e., the mass which applied at the spot where the force is exerted, would have the same effect on the system as the lever) and (2), the linear elasticity coefficient $\nu(i . e$. , the distorting effect of pressure on the membrane). In other words, $T$ becomes $2 \pi \sqrt{\frac{M^{\prime}}{E^{\prime}}+\frac{m}{\eta}}$. The reduced mass of the lever may be calculated by the formula, $m=\frac{\eta^{2} l \mu}{3}$. Here $\eta$ is the magnification, $l$ is the length and $\mu$, the specific mass. It follows that the vibration period becomes longer, and the frequency per second, less when the length or the specific mass of the lever increases with the magnification.

The application of Frank's guiding principles has enabled several investigators to construct manometers of high vibration frequency to meet the demands of special problems. Frank, himself, con-

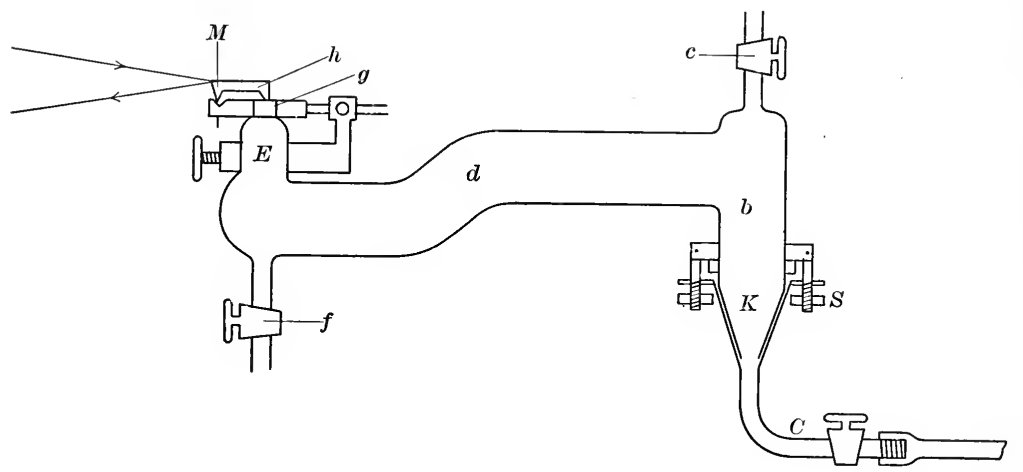

FIG. 6.-Diagram of Frank's manometer.

structed the first apparatus for estimating arterial pressure, and other forms for studying pressures in the heart have been added by Straub, Piper and Wiggers.

Frank's Arterial Manometer (Fig. 6).-The body of the instrument consists of two vertical glass tubes, $b$ and $e$, communicating by a bent horizontal tube, $d$. The vertical tube, $b$, is prolonged downward into a metal cone, $K$, over which the cone of the arterial cannula, $c$, is rigidly attached by the screws, $S$. The vertical projection, $E$, terminates in a small end with a manometer capsule three millimeters in diameter. The apparatus is filled to the exclusion of the smallest air bubble by allowing fluid to enter slowly by stop-cock, $f$, the air being allowed to escape at $c$. When completely filled, a rubber dam is tensely stretched and tied over the opening at $g$, and a tiny glass circlet cemented to it. The very slight excursions of this tense and small membrane are communicated to a tiny mirror $(M)$ by a small well-balanced lever, $h$, pivoting on free 
axes. As the membrane rises the mirror tilts and causes the slight movement to be magnified by reflecting a band of light from an optical lamp. ${ }^{1}$

Manometers for Recording Pressures in the Heart.-Several forms of intraventricular pressure manometers have been devised. The trocar-manometers devised by Straub and Piper have the disadvantage that, as the plunger is withdrawn, the apparatus fills with blood which is liable to coagulate and change the damping. The manometer devised by the author consists (Fig. 7) of a vertical glass tube, $d$, surmounted by a brass cylinder, $e$. This contains a stop-cock, $g$, with a conical lumen, the truncated cone of which comes into apposition with a damping plate, $a$, having a lumen $2 \mathrm{~mm}$. in diameter. Above the damping plate the cylinder ends in a segment capsule, $b, 3 \mathrm{~mm}$. in diameter and covered with rubber dam. Upon this a small piece of celluloid carrying a little Zeiss mirror, $c$, is fastened so that it pivots on the chord side of the segment capsule. Above the recording mirror is mounted a reflecting mirror, $f$, adjustible about a horizontal axis by a screw. The reflection of the light band to a horizontal plane occurs as shown in Fig. 7 . The lower end is fitted by a conical joint with several styles of cannulæ adapted for recording auricular and ventricular pressures respectively.

To record ventricular pressures the short, straight cannula with a slightly conical shape, as shown in the diagram, is inserted directly through the heart walls in a place where the movement is slight. The entire manometer is then rigidly clamped. To record auricular pressure, a short curved cannula is slipped through the earof the auricle and tied.

Contour of Pressure Curves.-The normal pressure curves in the auricles, ventricles and carotid have been studied by Frank, Straub,

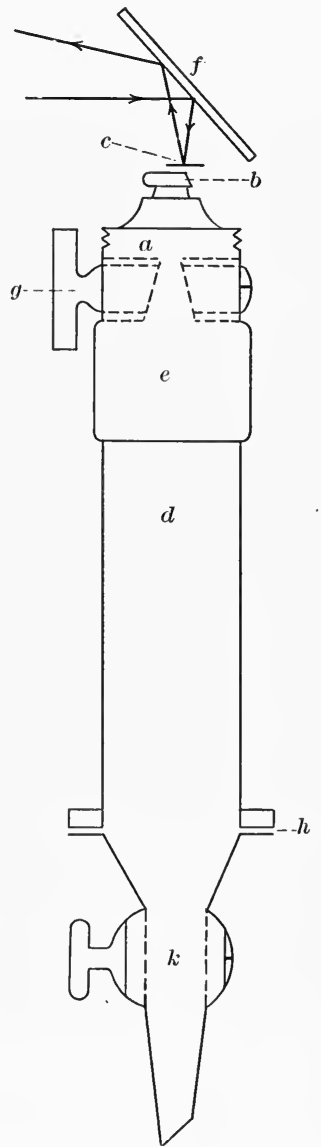

FIG. 7.-Optical manometer for recording intracardiac pressures. (Author's model.) Piper, C. Tigerstedt, and the author. Although minor differences capable of explanation occur, it is desirable to limit the description to the records that may be regarded as typical.

${ }^{1}$ For a description of a Nernst light arrangement, see page 102. For an arrangement utilizing a small automatic are light, see Wiggers, Arch. Int. Med, 1915, xv, 79. 
The Intraventricular Pressure Curves on the two sides do not differ in their essential features. The top of the left curve is broader, that of the right more rounded or often 'peaked (compare curves of Figs. 8 and 10). The curves of different investigators at the first glance seem very dissimilar but closer study shows that the differences are unessential and largely to be attributed to the use of instruments, differing in their sensitiveness and damping. The curve (Fig. 8) is inaugurated by a small elevation (1 to 2 ) due to auricular contraction. This is followed by the large ventricular wave divided into three periods: (a) the isometric period, $(b)$ the

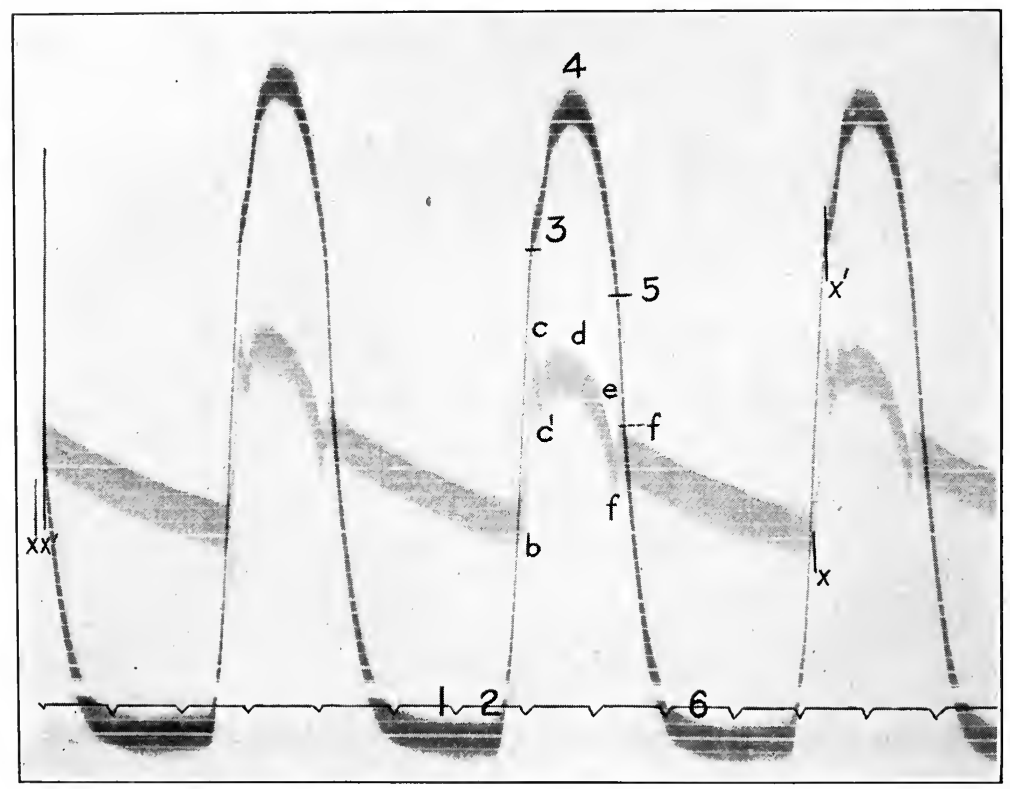

Fig. 8.- Simultaneous intraventricular and subclavian pressure curves. $x-x^{\prime}$, relative positions; $T$, time in $\frac{1}{5}$ second; $1-2$, auricular rise; $2-3$, isometric period; 3 , opening of semilunar valves; $3-4$, ascending limb of ejection period; $4-5$, relaxation; $b-c$, primary oscillation; $d$, systolic summit; $e$, incisura; $f$, valvular oscillation.

ejection period and (c) the relaxation period. At the onset of the ventricular wave the pressure rises sharply ( 2 to 3 ). Since the semilunar as well as the auriculo-ventricular valves are closed during this rise, the heart does not alter its diameters but its fibers develop tension. This period is called isometric, since the condition of contraction resembles the contraction of a muscle against a stiff spring. As soon as the pressure within the ventricle has overpowered that in the aorta, and the semilunar valves open (3), blood is ejected into the aorta and the ejection period begins (3 to 5). After several confused and usually merged vibrations 
(3), the pressure first rises ( 3 to 4 ) but less steeply than during the isometric period. Having attained its maximum height (4), it gradually decreases ( 4 to 5 ). At the beginning of ventricular diastole (5) the pressure falls rapidly but the gradient is less steep than during the isometric rise. During the remainder of diastole, the pressure remains unaltered until the next auricular systole causes another wave.

The Aortic Pressure Curve shows its chief changes during the ejection period of the ventricle. Synchronous with the change in contour of the intraventricular curve (Fig. $8, x-x^{\prime}$ ) the arterial pressure rises sharply $(b-c)$ and falls again $\left.c-c^{\prime}\right)$. This primary wave is due to the sudden ejection which throws the aortic column of blood into vibration. It is more pronounced in the pulmonary artery where there may be two primary oscillations (Fig. 9, 1). During the rest of the ejection period $(d-e)$ the curve follows that of the ventricle, since the two chambers now constitute a common cavity. With the onset of diastole the pressure falls rapidly $(e)$ with that of the ventricle, a fall that is accompanied by a backward movement of the column of blood. It has been called the incisura (Frank). The fall is suddenly checked by the closure of the semilunar valves and is followed by one or several oscillations due to their after vibration $(f)$. During diastole, the pressure falls gradually in the systemic aorta but more rapidly in the pulmonary circuit (Fig. 9, $A$ ).

In addition to these grosser features, several smaller preliminary waves can be made out in perfect curves. They are well shown in Fig. 9, $A$. The auricular rise of intraventricular tension by causing a bulging of the semilunar valves causes the first of these waves $(a)$. The second wave $(b)$ occurs during the isometric rise of intraventricular tension and is also due to a bulging of the semilunar valves. It varies from 0.0166 to 0.03 second in duration.

The Intra-auricular Curve (Fig. 9, $B$ ) shows no essential variation on the two sides of the heart. The first and chief rise $(a-b)$ is caused by auricular systole and its chief fall $(b-c)$ by diastole. The author believes this interpretation warranted from the fact that when, on stimulating the left vagus, the ventricle is inhibited without the auricle, such waves are the sole variation occurring within the auricle.

At the beginning of ventricular systole, another short, positive rise $(c-d)$ followed by a rapid negative drop $(d-e)$, occurs. It still remains debatable whether this corresponds to a preliminary upward, and later a downward movement of the $a-v$ valves and the $a-v$ floor. Sometimes a positive wave only occurs and, at other times, the negative wave only is present.

During ventricular systole, the auricular pressure rises $(f)$ slowly due to stasis. The rise continues into the early part of diastole, 
that is, until the $a-v$ valves open. At this point the venous pressure falls more or less. Sometimes it is a very distinct drop, as shown in Fig. 9; in many records, however, no evidence of a drop

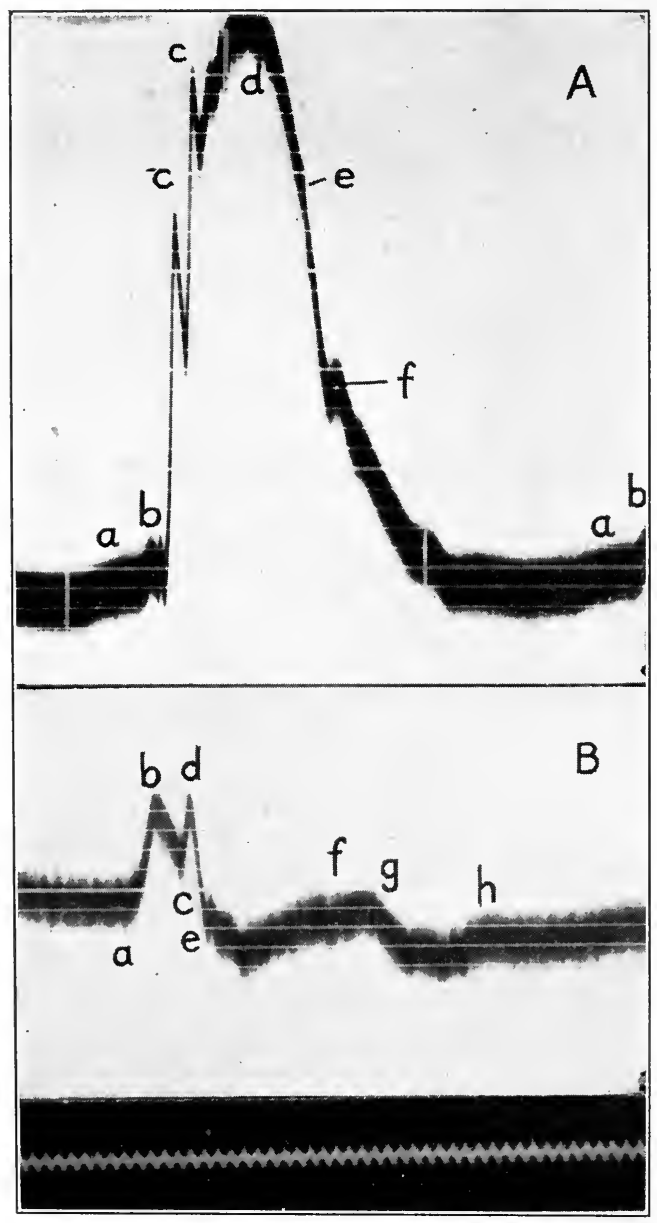

Fio. 9.-Pressure changes in pulmonary artery (upper) and right auricle (lower). Upper record: $a$, auricular wave; $b$, preliminary vibrations; other letters as in Fig. 8. Lower record: $a-b$, auricular systole; $b-c$, early part of auricular diastole; $c-d-e$, early systolic vilbration; $f$, small noteh at end of systole; $q$, early diastolic fall, opening of tricuspids; $h$, mid-diastolic wave, oceasionally present.

is obtained. The time relations of these curves are plotted in detail in the chart of Fig. 25.

Atypical Curves.-Two influences, variations in arterial pressure and in venous pressure, are capable of changing the contour of the 
curves. The aortic curve is frequently abnormal because the diastolic pressure is low, either because the peripheral vessels are relaxed or the tonus of the larger vessels is abolished. The primary oscillation is then extremely large but the real systolic summit is much lower and slopes rapidly downward instead of rising. The bottom of the incisura occurs nearer to the diastolic pressure level.

The intraventricular pressure curve possesses the contour described as characteristic only when the effective auricular pressure is normal. When venous pressure, for any reason, falls, the distending pressure of the ventricle or the initial tension, as it has been tersely called, also decreases. The intraventricular curves then change their contour. This is shown by the series of curves in

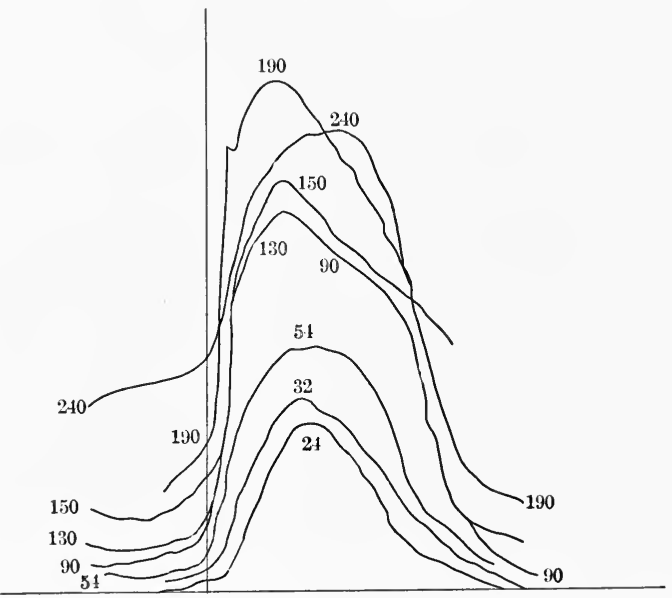

FIg. 10.-Effect of auricular pressure on the initial tension and contour of the pressure curve of the right ventricle. Numbers refer to auricular pressure, in $\mathrm{mm}$. of water.

Fig. 10. As the auricular pressure decreases, the curve gets lower. The steepness of the isometric limb becomes less. The contour of the ejection period changes; the onset is indistinguishable from the isometric rise and the top is quickly reached. The descending limb of the ejection period merges imperceptibly with the diastolic fall, giving the curve a very simple and rounded contour. Many curves published in the past to show the simple contour of the intraventricular pressure change, clearly owe their existence to the fact that a normal pressure was not maintained in the auricle during the experiment.

It is possible that the height of the pressure curve and the steepness of its rise are not entirely governed, as such results indicate, by the initial tension exerted upon the muscle fibers. This applies 
particularly during the range of low initial pressures. Thus, it has been found (Patterson, Piper, and Starling) that if the arterial pressure against which the discharge occurred was maintained high, the intraventricular pressure might rise higher and more steeply without any alteration of initial pressure. In such events, the relaxation curves were always more rapid, however, indicating a greater diastolic inflow without an increase of initial pressure. These observations indicate that as long as the venous pressure is low, the initial pressure in the ventricle depends more on the rate of relaxation of the ventricle than on variations of pressure in the inferior vena cava. It seems questionable, however, whether these results are sufficient to warrant the further conclusion which has sometimes been made, namely, that the efficiency of the beat depends, not on the tension exerted on the individual muscle fibers, but rather upon the extent to which they are elongated by active or passive pressures (Patterson, Piper, and Starling).

\section{THE VOLUME CURVES OF THE VENTRICLES.}

We have seen that after the opening of the semilunar valves, the isometric period terminates and the ejection of blood into the aorta takes place. This ejection is naturally accompanied by a reduction in the volume of the ventricles. When the ventricles subsequently relax and the auriculo-ventricular valves open, blood flows into the ventricles and their volume again increases. A record of these volume changes may be obtained by enclosing the heart in a glass plethysmograph, snug closure being made around the auriculo-ventricular groove by a rubber diaphragm. This cardiometer, as the plethysmograph is specifically called, communicates by a projecting tube with a recorder (Fig. 11). As the volume of the ventricles decreases during systole, the slight diminution of pressure within the system is transmitted to a tambour or volume recorder. The method has been used by François, Frank, and Tigerstedt, but we owe its development to Henderson.

If the resistance to the ejection of blood were constant, the volume curve so obtained would represent an isotonic contraction of the heart, that is, a contraction in which the lengths of the individual fibers change but the tension remains the same. The arterial pressure during ejection is not constant, however, and the volume curve, therefore, only approximately isotonic. Its careful study is of importance, nevertheless, for it permits a consideration of the ejection period by itself, gives a quantitative estimate of the systolic volume discharged and offers a means of directly studying the diastolic filling of the ventricles.

Critique of Apparatus.-To accomplish these results, it is of course necessary that the apparatus employed should be adequate 
to record the variations in volume accurately and at the same time not interfere with the natural beat of the heart. The most satisfactory cardiometer consists of a glass sphere containing an opening with a small flange over which a rubber diaphragm is tied (Fig. 11). In this is cut a round opening slightly smaller than the circumference of the cardiac base. After it is applied to the heart, the cardiometer must be so supported that the heart itself does not move in and out of the rubber and that no impact from the auricle is imparted to it.

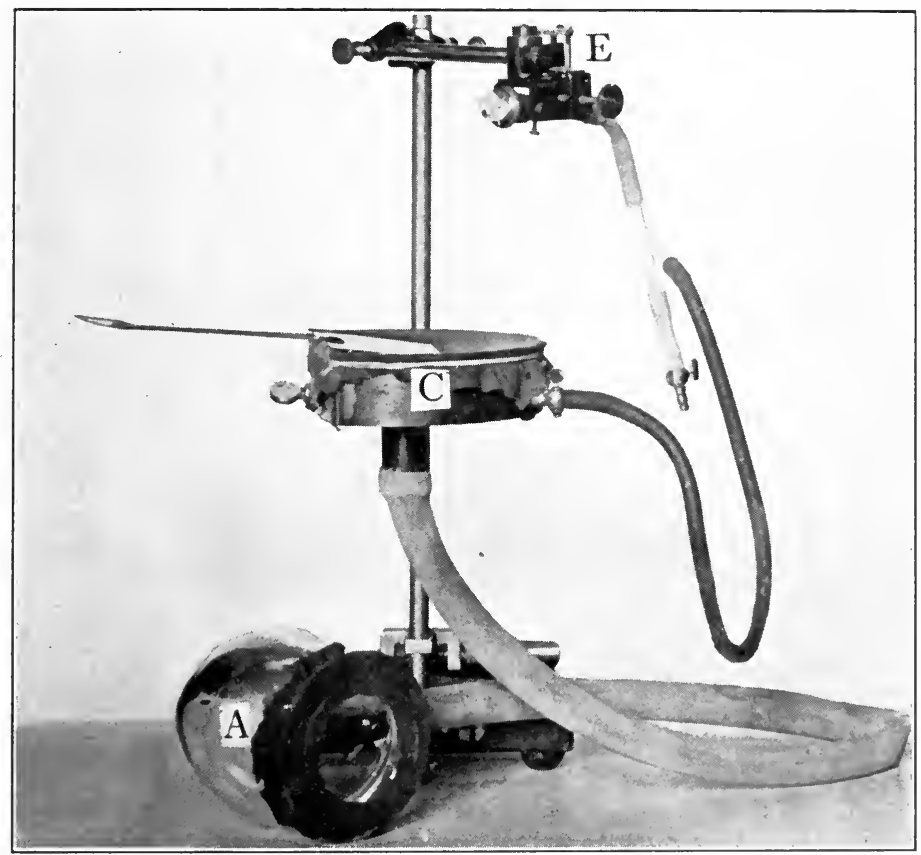

FIG. 11.-Cardiometer $A$ and tambour $C$ used by writer for recording ventricular volume curves. To take an optical record the slight pressure changes within the large tambour are communicated to a Frank's segment capsule $(E)$.

To record volume variations with a minimum pressure change in the system, Henderson used a large Marey tambour, twelve centimeters in diameter, which was covered by unstretched rubber. This instrument was connected by large tubing to the cardiometer to reduce the damping. Straub has maintained that such recording devices are not capable of accurately following the volume changes, because the curves are necessarily distorted, either by the inertia of the writing system, or by the damping necessary to overcome it.

Relevant to this discussion, the following remarks may be made: No one who is familiar with the critical analysis of apparatus and 
the principles laid down by Frank for the construction of efficient instruments, will feel convinced by the dynamic tests carried out by Henderson that it is possible to construct an instrument with as great masses as are used in his own and retain a degree of efficiency sufficient for quantitative work. Fortunately, however, the requirements for qualitative records are not as great as the discussion of Straub would lead us to suppose.

In the dog, at least, the filling and ejection occur in a relatively slow and smooth fashion which an instrument with quite a low period can follow with a fair degree of accuracy.

The objection that an ordinary tambour cannot be used because too great a tension develops within the system and so prevents a normal contraction is by no means established. In the first place, as shown in Fig. 12, the heart within the thorax is subjected,

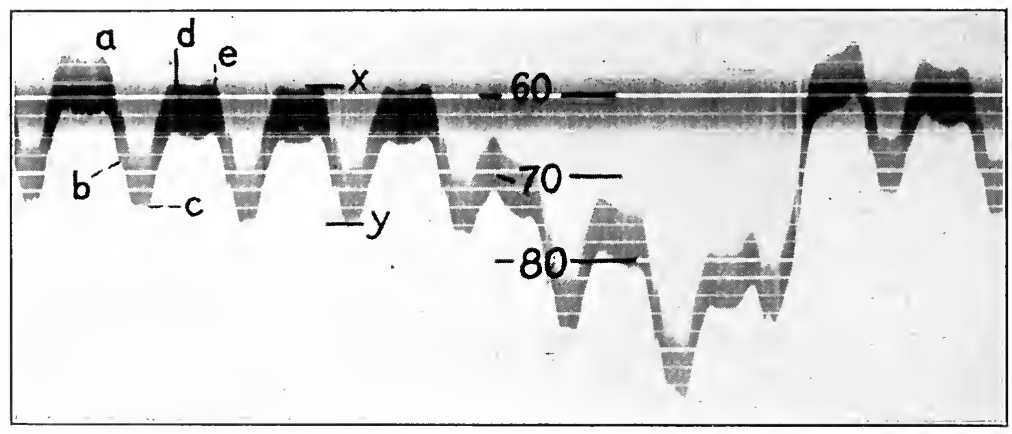

FIg. 12.-Cardiac and respiratory variations of intrathoracic pressure. Cardiac contractions caused a total decrease in pressure $(x-y)$ equal to $16 \mathrm{~mm}$. of water, when the total respiratory variation equalled $63 \mathrm{~mm}$. $a-c$, ventricular systole; $c-d$, elastic diastole; $d-e$, diastasis.

not only to varying negative pressures during inspiration and expiration, but also to the cardiac variations of intrathoractic pressure, equal, in the dog, to from 9-15 mm. of water. In other words, the heart is normally enclosed within a plethysmographic cavity (the chest) within which greater variations of pressure occur than in any instrument. If this factor were of any importance, it would be desirable to allow the ventricle to contract against a slight negative pressure, rather than to make special attempts to avoid any change. As a matter of fact, it still remains to be demonstrated that such variations exert any effect.

The discussion of accurate registration being put aside, the question remains whether the volume curves give any accurate indication of the systolic output of the heart. Henderson recognized that the decrease in volume during systole was the combined effect of ventricular discharge and flow through the coronary vessels but 
he believed that the latter factor was small and fairly constant. Patterson, Piper, and Starling have recently presented experiments, however, indicating that the coronary flow may be decidedly variable and the volume curve a very inaccurate measurement of systolic flow. Their results can hardly be regarded as conclusive, however, when critically considered. Their method of determining the output of the heart by the flow from the vessels peripheral to an artificial constriction is accurate only as long as arterial pressure does not alter. The faultiness of their method is shown by the fact that there was no outflow in certain cases while the heart volume was decreasing perceptibly. Could their explanation of this observation be accepted, viz., that the entire flow in such cases passes through the coronary vessels, it would in itself be an admission of the inadequacy of their method. Using their own figures one must conclude that normally from 25 to 30 per cent. of the blood volume ejected by each systole passes through the coronary vessels. Such conclusions can scarcely be accepted and the results must be attributed to experimental error.

It has also been suggested that during systole the auriculoventricular floor moves toward the auricle, and that consequently the volume changes between the inner and outer walls do not accurately represent the entire volume change of the ventricles. From optical records of right auricular pressure, the writer has not been convinced that this occurs to any appreciable extent. The objection is theoretical or based on experiments with inadequate apparatus. There is, however, a more serious difficulty in preventing a certain amount of slipping of the ventricle into and out of the cardiometer membrane, a difficulty one cannot feel sure is obviated by the best technic. On the whole it seems probable that the volume curve, when recorded by efficient apparatus, can give quantitative evidence of the total output per beat. It is even possible that combined strohmuhr experiments and volume curves may be utilized, not to check each other, but to estimate the volume flow through the intramural vessels of the heart (Rothberger).

Nature of the Ventricular Volume Curve.-The volume curve may be divided into three periods: systolic discharge, early diastolic filling (active diastole) and slow, passive filling toward the end of diastole. The systolic discharge begins at the opening of the semilunar valves. During early systole the rate of discharge is constant but toward the end it diminishes so that, for a brief period, little or no blood is ejected. This period corresponds to the descending limb of the pressure curve during the ejection period.

Filling does not begin immediately in diastole for the $a-v$ valves are opened a trifle later than the semilunars close. The increase in size occurs at a rapid rate during the first part of diastole. Both the rate and duration of filling depend on the height of venous 
pressure and on the tonus of the heart. When the heart cycle is fairly long it ends smoothly or occasionally sharply with a shoulder at which the curve becomes more inclined to the horizontal, thus initiating the period of diastasis. The duration and even the existence of this period depends on the heart rate. As the heart cycle becomes shorter, this period is encroached upon and in accelerated heart entirely fails to occur. When present, it is terminated by auricular systole, which adds a distinct but very small increment of blood to the ventricular filling. This is indicated in the curves recently published by Straub, Patterson, Piper and Starling, as well as those of Henderson and his co-workers (Figs. 13 and 14).

Effect of Rate.-The amplitude of the systolic discharge but not the contour of the curves is modified by the heart rate (Hender-

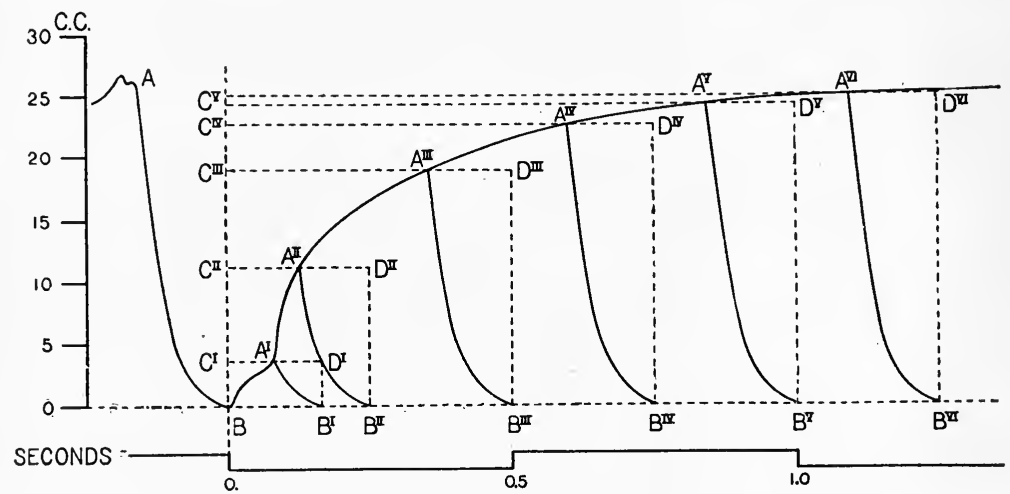

FIG. 13.-Scheme showing the volume eurves at different length of cardiac cyeles. (After Henderson.) $A B$ represents the maximal output during systole; $B D^{\mathrm{VI}}$ the filling in a prolonged diastole. Ares, $A^{\mathrm{I}} B^{\mathrm{I}}, A^{\mathrm{II}} B^{\mathrm{II}}$, etc., show amplitude of systolie stroke when systole begins at different periods of active diostole or diastasis.

son). At different rates the curves are practically superimposable (Henderson), i. e., the smaller ares inscribed at more rapid rates may be exactly fitted over similar portions of larger arcs obtained at slow rates in the manner that angles are compared in geometry (Fig. 13). In stating this law of "uniformity in behavior" (Henderson) of the heart, it should be emphasized that it applies only as long as the venous pressure effective in filling the ventricles remains constant. When this condition is complied with, it appears that the systolic discharge is only very slightly reduced by the increased rate as long as the period of active diastole is not encroached upon. When the cardiac cycle has a duration of 1 second the amplitude of beat is almost maximal. Therefore, at all rates up to 60 per minute the volume ejected per minute is proportional to the heart rate. It is, in fact, almost proportional to the rate up to 80 per 
minute. When the cycle shortens to 0.5 second, however, $i$. e.; when the heart beats 120 times per minute, the active diastole is abbreviated and the output per beat reduced in a pronounced fashion. Although the output per minute still increases, it is not in proportion to the increase in heart rate.

The amplitude as well as the contour of the volume curve is affected by the height of venous pressure. At low pressures the filling is more gradual early in diastole but at higher pressures the slope of the filling curve is more rapid (Fig. 14). According to Henderson and Barringer, this holds good only when lower venous pressures prevail. They believe, for example, that any pressure above $50 \mathrm{~mm}$. water, which they designate as a "critical pressure" will not modify the contour of the volume curve and hence the systolic output of the heart. The writer, upon repeating these

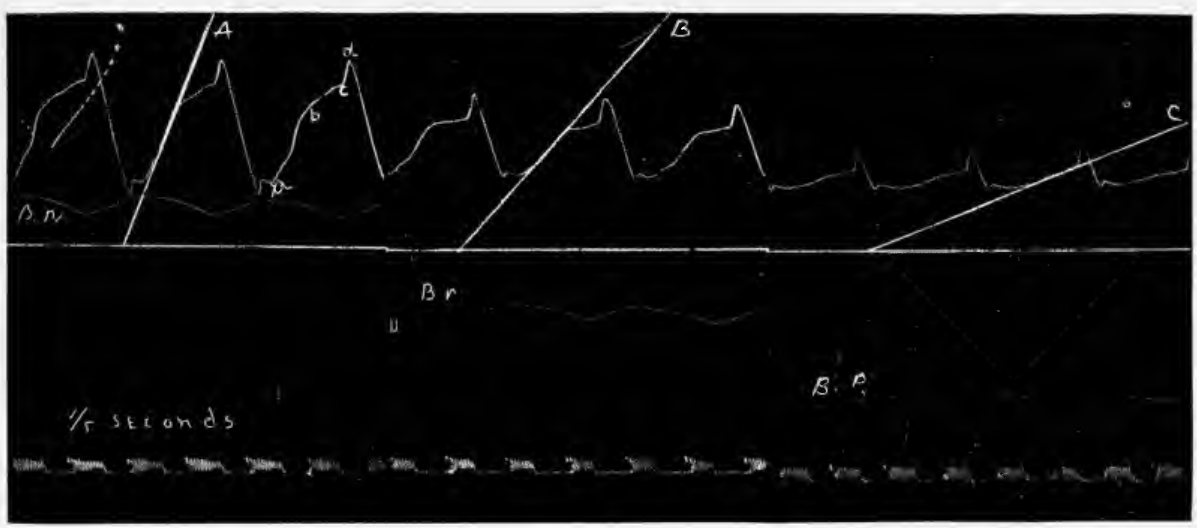

Fig. 14.-Effect of diminishing the venous pressure on the rate of ventricular filling. $A$, normal; $B$, after moderate hemorrhage; $C$, after severe hemorrhage.

experiments, found, however, an increasingly rapid filling and output up to pressures as high as $150 \mathrm{~mm}$. of water and believes that there exists a venous pressure level ( 40 to $70 \mathrm{~mm}$. of water), which may be regarded as relatively critical, in the sense that venous pressures above such a level may still modify to a small extent the output per beat, while far above this, an absolutely critical pressure is found beyond which the heart no longer increases its discharge but dilates and decreases it. The writer places this level at about $150 \mathrm{~mm}$. of water. Recent observations would indicate that a pressure of 200 to $250 \mathrm{~mm}$. of saline may be regarded as absolutely critical in some cases (Patterson and Starling).

The Effective Venous Pressure,-It is of interest to inquire whether the beats at different rates in the body are normally influenced by or independent of variations in venous pressures. In discussing 
this question, it is essential to bear in mind that when the heart is surrounded by a pressure less than atmospheric, as is the case in the closed chest, the pressure effective in filling the ventricle is not the pressure actually measured in the auricle but the algebraic difference between intrathoracic and intra-auricular pressures (measured, to be exact, at the $a-v$ valves immediately before ventricular diastole). This pressure difference has been termed the "effective pressure" (Henderson and Barringer). Thus, if a venous pressure of $+10 \mathrm{~mm}$. of water and an intrathoracic pressure of $-40 \mathrm{~mm}$. exist, then the effective pressure would be $50 \mathrm{~mm}$. of water.

How great is the effective pressure in unanesthetized animals and man? Henderson and Barringer state that the presence of the venous pulse in the neck indicates that the effective pressure must be greater than intrathoracic, and, hence, safely but not far above the "critical" level for man. Since the heart rate variations in man did not seem to account for the variations of systolic and diastolic pressures, the writer preferred to believe that the effective venous pressure is not great enough to insure superimposible beats during inspiration and expiration. In locally anesthetized, but quietly breathing dogs, the writer found the effective pressure in the right auricle to average $63.1 \mathrm{~mm}$. during inspiration and 43.6 $\mathrm{mm}$. of water during expiration.

We may conclude from all recent work (Patterson and Starling, Wiggers) that the heart within the thorax can respond with a variable systolic output, independent of a variation in rate. It is difficult, however, for any one who has observed the relative importance of these two factors in normal animals to be convinced that it is physically possible that the increased venous pressure during exercise, for example, could be so effective in increasing the filling that it overbalances the tendency of the heart rate and output to decrease the systolic discharge. Such an assumption would have to be made, however, in order to explain the large volume of blood which has been calculated to be used during exercise upon the basis of the oxygen capacity of the blood, the total oxygen consumption and the pulse rate (Zuntz, Plesch, and Krogh). The recent experiments of Patterson and Starling are published as proof that this is physically possible. They found, for example, that under the best conditions of venous pressure and venous inflow, a perfused heart weighing 56 grams could throw out 3000 c.c. per minute or 22.3 c.c. per beat. They calculated that upon this basis a human heart might eject 18 liters of blood per minute, a figure approaching that estimated in man during exercise by the gasometric method. It may be pointed out, however, that no such enormous output per minute was obtained in intact hearts by these investigators, and, furthermore, that these results were not accompanied by simultaneous acceleration of the heart, as is the case in exercise. It 
therefore still remains questionable whether their results obtained in perfusion experiments and when conditions could be adjusted for maximal efficiency, may be applied without reserve to the intact circulation.

Effect of Tonus.-It is apparent that the degree of true tonus, $i$. $e$., the partial contraction by virtue of which a pressure change is resisted, will determine to a considerable extent the filling and output apart from changes in venous pressure and heart rate. A heart with high tonus may empty itself more effectively during systole than it normally does, but during diastole the filling will be prevented to such an extent that the output per beat at the slowest rate and highest venous pressure may be very small. On the contrary, when tonus is low, relaxation will be very rapid and filling very susceptible to changes in venous pressure. For a considerable range the ventricle in low tone will respond to high venous pressures by a greater output before it becomes fatigued and dilated so that the fundamental stroke no longer expels all the blood. The degree of tonus is very hard to reckon with in cardiometer experiments and is still less controlable in perfusion experiments, such as Patterson and Starling performed.

Sequence of Events in the Filling and Emptying of the Ventricles Having determined accurately the pressure changes of the cardiac chambers and the aorta, as well as the volume changes of the ventricles, it is possible to picture the mechanics of the heart beat in detail (cf. Fig. 25). The mechanisms of the two sides are not different. The right auricle during its diastole collects the blood which is used for filling the right ventricle. It should be borne in mind that the right auricle is not the only reservoir but is aided in the accommodation of blood by the large veins and the vascular channels of the liver so that a luxus supply of venous blood is normally present in the venous reservoirs. With the beginning of ventricular diastole the pressure within the ventricles falls rapidly. When it has become lower than the auricular pressures, the $a-v$ valves open and blood rushes quickly into the ventricles. In this way a considerable filling occurs with little rise of pressure. What proportion of the entire filling is thus completed depends on the height of venous pressure as well as the rate of ventricular relaxation or tonus. If the venous pressure is high or cardiac tonus low, filling may be almost completed with the active relaxation of the ventricle. As soon as auricular and ventricular pressures are equalized, filling proceeds at a slower rate, being then governed by the passive rise of venous pressure resulting from the continued flow of blood into the auricle. In short, the filling of the ventricle during any period of diastole is determined by the difference in pressure existing in the auricle and ventricle.

At the time that auricular systole occurs, the pressures in the 
auricles and ventricles are probably about equal, since these cavities are still in free communication. The rise of auricular pressure during auricular systole is then transmitted to the ventricle with very little transfer of blood, its importance for the vigor of the succeeding ventricular beat consisting in the fact that it raises the initial pressure within the ventricle. The writer explains the results of Gesell in this way and does not believe that they demonstrate necessarily that auricular systole aids materially in filling the ventricle.

When the ventricle contracts the pressure rises and very quickly the $a-v$ valves close. Since the semilunar valves are also closed, the ventricle contracts isometrically. This causes frequently a slight bulging of the $a-v$ valves, followed apparently by a descent of the auriculo-ventricular floor. The aortic valves also bulge and transmit a preliminary vibration to the aorta. As soon as intraventricular pressure has overcome the aortic pressure the valves open and blood is ejected into the aorta. The sudden entrance of a considerable quantity of blood into the aorta causes the blood column and the arterial walls to undergo a sudden oscillation which is quickly damped, however. This oscillation may extend into the ventricle and cause a slight notch in the curve. The fact that it is always small and often absent, being then marked merely by a change in slope is probably accounted for by the fact that the oscillation is damped at the aortic orifice.

After the primary vibration, ventricle and aorta are in free communication and their curves have the same contour. The pressure continues to rise as long as the flow of blood into the aorta per unit time exceeds the outflow from the peripheral vessels. As soon as the reverse occurs the pressures fall. Apparently the time in which the summit is reached is governed by both factors. Thus, a sharp and early summit with a rapid decline may be due to a small output or a low peripheral resistance.

At the end of ventricular relaxation the pressure in the ventricle falls sharply and quickly closes the semilunar valves. As they close, a negative pressure is created at the mouth of the aorta which is responsible for the sharp incisura which is accompanied by an actual though slight back movement of the blood column. The oscillation of the blood column is, however, quickly damped out. After the closure of the semilunar valves, the pressure in the arteries falls slowly and in proportion to the flow from the peripheral arteries.

Finally, it may be pointed out that the systole of the ventricle extends in the central arteries from the beginning of the preliminary oscillations to the beginning of the incisura drop and not, as is commonly assumed by studies with less efficient apparatus, from the rise of the primary wave to the bottom of the dicrotic notch so 
recorded. Furthermore, the sudden fall of auricular pressure ${ }^{\text {- }}$ does not precisely mark the end of systole but occurs early in diastole.

\section{BIBLIOGRAPHY.}

\section{Articles Dealing with Valve Action.}

Baumgarten. Arch. f. Anat. u. Physiol., 1843, 464, 466.

Henderson and Johnson. Heart, 1912-13, iv, 69.

Hering. Zentralbl. f. Physiol., 1907, xxi, 719.

“ Arch. f. d. ges. Physiol., 1909, exxvi, 225.

Krehl. Abhandl. d. sächs. Gesellsch. d. Wissensch., 1891, xvii, 349.

Saltzmann. Skan. Arch. f. Physiol., 1908, xx, 233.

\section{Articles Dealing with Apparatus and Pressure Curves.}

Frank. Ztschr. f. Biol., liii, 429; xlv, 464; xlviii, 489; 1, 309.

Patterson, Piper, and Starling. Jour. Physiol., 1914, xlviii, 465.

Piper. Arch. f. Physiol., 1912, 343.

Straub. Deutsch. Areh. f. klin. Med., 1914, exv, 531.

Arch. f. d. ges. Physiol., 1911, cxliii, 69.

C. Tigerstedt. Skan. Arch. f. Physiol., 1912, xxviii, 36.

Wiggers. Am. Jour. Physiol., 1914, xxxiii, 1; 1914, xxxiii, 382.

\section{Articles Dealing with Volume Curves.}

Frank. Ztschr. f. Biol., 1895, xxxii, 370 .

François, Franck. Travaux du lab. de Marey, 1877, cxi, 321.

Arch. de Physiol., 1890, 395.

Gesell. Am. Jour. Physiol., 1911, xxix, 32.

Heitler. Ztschr. f. exper. Path. u. Therap., 1910, vii, 763.

Henderson. Am. Jour. Physiol., 1906, xvi, 325; 1909, xxiii, 345.

Henderson and Prince. Heart, 1914, v, 217.

Krogh. Skan. Arch. f. Physiol., 1912, xxvii, 100, 126, 227.

Patterson and Starling. Jour. Physiol., 1914, xlviii, 357.

Plesch. Zentralbl. f. Physiol., 1912, xxvi, 89.

Rothberger. Arch. f. d. ges. Physiol., 1907, cxviii, 353.

Straub. Jour. Physiol., 1910, xl, 378.

Tigerstedt. Ergebn. der Physiol., 1907, vi, 269.

Wiggers. Arch. Int. Med., 1910, vi, 281.

Jour. Exper. Med., 1914, xix, 1.

Zuntz. Ztschr. f. klin. Med., 1912, lxxiv, 347. 
CHAPTER IV.

\section{THE MECHANICAL ENERGY OF THE HEAR'T BEAT.}

\section{BLOOD-PRESSURE AND BLOOD FLOW.}

As soon as intraventricular exceeds arterial pressure and the semilunar valves open, a certain quantity of blood is suddenly ejected into the aorta due to the difference of pressure developed in the ventricle and that present in the arteries. Since the arteries are already distended at the time of this systolic discharge, room must be made, either by moving the column of blood onward or by increasing the capacity of the arterial system. In the former case, the pressure energy generated by the heart is transformed to kinetic energy, or energy of flow; in the latter case, it is stored as potential energy by further distending the arterial walls. Normally, about one per cent. of the total pressure energy is immediately converted into flow, but the actual ratio of flow to pressure energy is determined by the distensibility of the arterial walls or is inversely proportional to the volume elasticity coefficient of the entire arterial system, $i$. $e$., the ratio of the pressure increase to the volume increase. This coefficient varies (1) with the degree of pressure in the artery, (2) with the degree of tonus and (3) with the physical characteristics of the arterial wall. Thus, an arteriosclerotic artery has a higher coefficient of elasticity than a normal artery (cf. MacWilliams). The greater the coefficient becomes, the less fluid will be accommodated and hence a greater conversion of potential energy into kinetic energy takes place.

The potential energy stored by the distended walls during systole as pressure is reconverted into kinetic energy during diastole when blood ceases to be injected into the aorta, thus insuring practically a constant flow through the smaller vessels during diastole as well as systole. Hence, the arterial pressure reaches its maximum in systole and its minimum during diastole. These pressures have been termed systolic and diastolic pressures, respectively. Their difference is called the pulse pressure (Figs. 15 and 16).

The fundamental factors determining the actual and relative heights of these pressures under different conditions may be conveniently studied by some form of artificial circulation machine. This not only has the advantage that the factors can be adequately varied and controlled; but also, that the pressure changes 
occur slowly enough to enable an ordinary mercury manometer to follow them fairly accurately, which it cannot do in animal experiments.

When the pressure variations in the tubes representing the arteries are recorded (Fig. 15), the pressure rises during systole and falls during diastole. If now the peripheral resistance is increased, both systolic and diastolic pressures (and consequently the mean pressure) rise. This is brought about by the fact that less blood is able to pass from the arterial system during each cardiac cycle. Since the

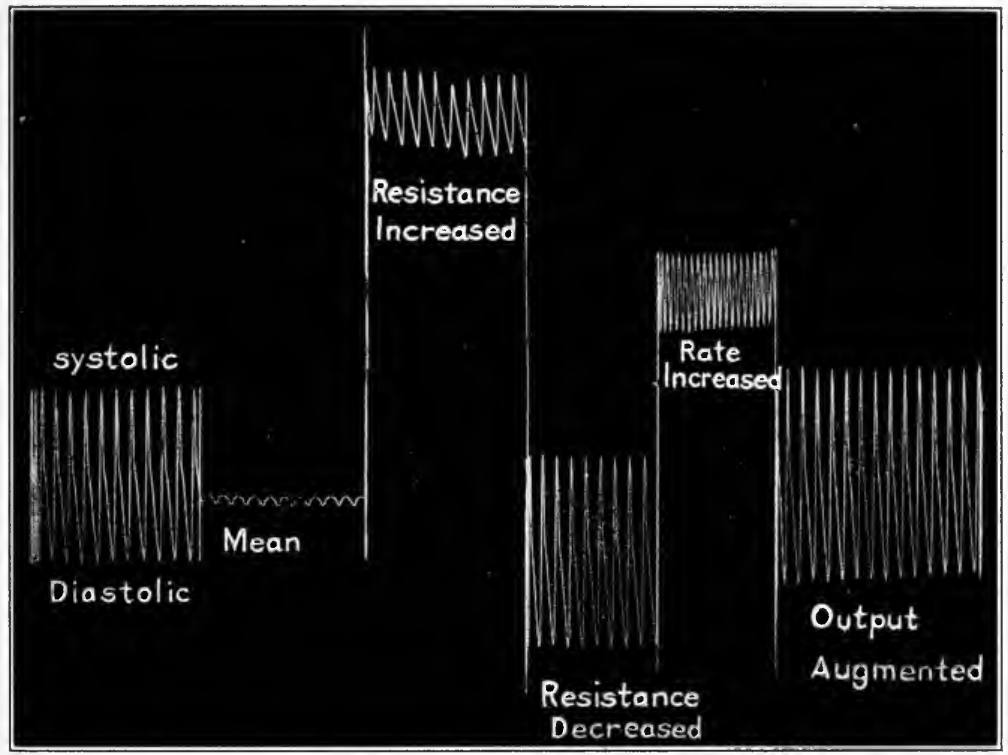

FIG. 15.-Arterial pressure variations taken from an artificial circulation machine under different conditions, described in text.

flow or kinetic energy is thus decreased, a larger share of the total energy liberated by the heart must be stored as potential or pressure energy.

Careful examination shows that the systolic pressure rises less than the diastolic and that the pulse pressure is accordingly decreased. This is accounted for by the fact that the period of diastole is so much longer than systole, hence a larger share of the potential energy is converted into energy of flow during this phase. In other words, the diastolic rise is greater than the systolic by an amount of energy not converted into flow during diastole. The reverse occurs when the peripheral resistance is diminished. Systolic and diastolic pressures both fall, the latter more than the former, however, and so the pulse pressure increases. 
If, while the resistance remains decreased, the rate of the heart increases without changing the systolic discharge, the systolic and diastolic pressures will also increase. This occurs because the minute volume, $i . e$., the amount of blood pumped out per minute, is augmented. Of this increased output only a portion succeeds in escaping through the peripheral arterioles into the veins, hence the larger share remaining becomes effective as an increased arterial pressure. The diastolic pressure rises more than the systolic and the pulse pressure is smaller, largely because the conversion of potential energy to the kinetic form is curtailed only during diastole, which is shortened when the rate increases.

If, finally, while the peripheral resistance and heart rate remain constant, the systolic output increases, both systolic and diastolic pressures rise, owing to the fact that, as in the case of an increased rate, the minute volume injected exceeds the minute outflow from the arteries. The systolic pressure, however, rises more than the diastolic in this case, making the pulse pressure greater, because, with the higher pressure at the end of systole and the same resistance peripherally, a larger quantity of potential energy (pressure) is converted to energy of flow during a diastole of the same length.

I. Summary.

Inereased peripheral resistance .

Decreased peripheral resistence .

Increased heart rate

Decreased heart rate

Increased systolic discharge .

Decreased systolic discharge

\section{Significance of Increased Pulse Pressure.}

Due to:

Decreased resistance

Decreased rate

Increased output

$$
\begin{aligned}
& \text { Decreased systolic and } \\
& \text { diastolic pressures } \\
& \text { Decreased systolic and } \\
& \text { diastolic pressures } \\
& \text { Increased systolic and } \\
& \text { diastolic pressures }
\end{aligned}
$$

Heart rate same.

Heart rate slower.

Heart rate same.

\section{Conceptions and Relations of Systolic and Diastolic Pressures.-} The pressure within the arteries of an animal and in man do not vary in so simple a fashion as is indicated by artificial circulation apparatus, hence it is desirable to establish precise conceptions of circulatory changes.

In the first place the pressure rises and falls during systole. Furthermore, in the central vessels the systolic top is represented by a peak and a summit, the former sharp (primary oscillation), the latter more rounded and corresponding to the true systolic level of pressure 


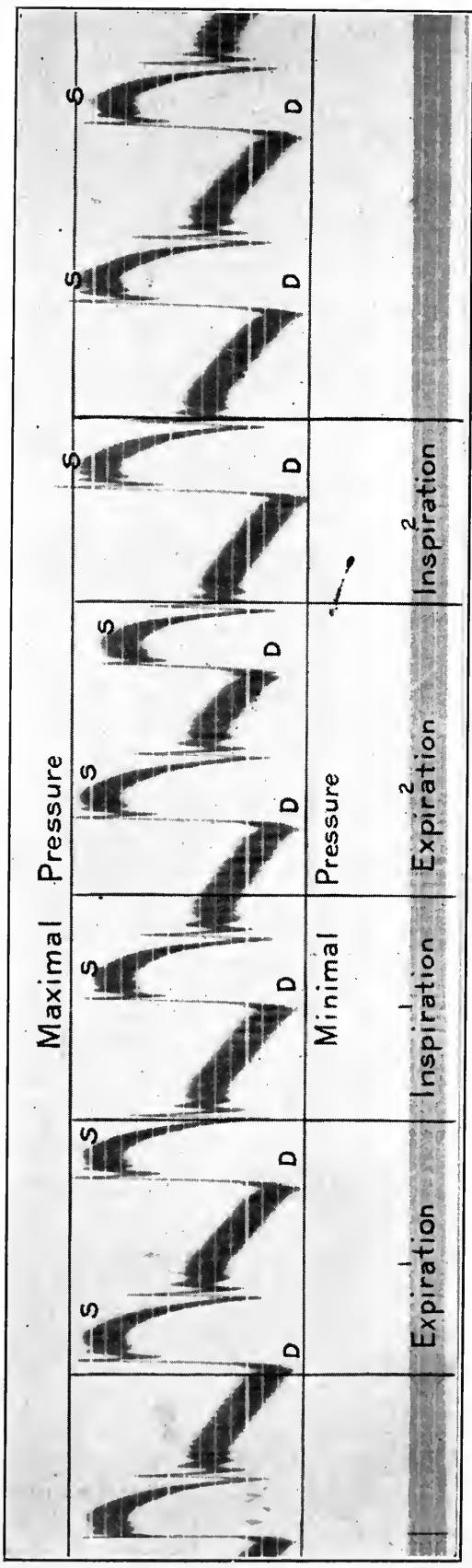

氶

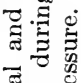

范

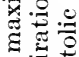

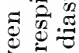

$+i$

80

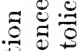

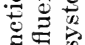

要,

$\exists \frac{\pi}{5}$

F.

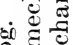

- 0

$4+\frac{0}{0}$

룰

芯氖

สี कू

.

蛋.

औ

. $\Xi$

을

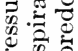

कै कै

응

䠉

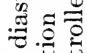

․ㅡㄹ

¿

穷

足

ㄴ.

象.

用

.

个通

0.0

$\rightarrow$.

ن்

蛋.

a. 
in the ventricle. When resistance is fairly. high the summit constitutes the highest point both in animals and in man (Figs. 8, 9, 27 , and 34). It not infrequently happens, however, that the preliminary peak is the highest point (Fig. 16). Inasmuch as the summit corresponds to the actual systolic pressure within the ventricle, it would seem desirable to limit the term systolic pressure to the highest point on the summit. The term diastolic pressure may likewise be accurately limited to the pressure reached in diastole before the preliminary oscillations occur.

In the second place, the systolic and diastolic pressures of consecutive beats fluctuate with the phases of respiration and change with the duration of consecutive cycles (Fig. 16). It is apparent that systolic and diastolic pressures are not equivalent to the maximum and minimum pressures reached, nor is the pulse pressure equal to the maximal-minimal pressure difference.

That this distinction between systolic and maximal pressures, on the one hand, and diastolic and minimal pressures, on the other, is of more than academic interest is shown by the fact that in dogs the systolic pressure varies from 7-28 $\mathrm{mm}$. during the act of respiration, while the diastolic pressure varies from 2-30 $\mathrm{mm}$. The average pulse pressure of consecutive beats equaled from 66 to 98 per cent. of the maximal-minimal pressure difference (Wiggers, Eberly and Wenner). Although impossible to determine quantitatively, it is probable that these differences are great enough in man to warrant a distinction.

The Pressor Influence of Respiratory Movements.-If respiration ceases for a time both systolic and diastolic pressures fall until a new pressure level is reached. It is possible to estimate the "pressor effect" of respiratory movements by taking an average of the systolic and diastolic variations and comparing these with the pressure during apnea temporarily induced by stimulating the central vagus for 20-30 seconds with a weak current. Under such conditions, vasomotor reactions are delayed long enough in the dog so that the mechanical effect of respiration can be determined. The following data in which the figures represent $\mathrm{mm}$. of mercury illustrate the method:

Average systolic pressure.

91.3

88.4

2.9
Average diastolic pressure.

$\begin{array}{ll}44.3 & \text { During natural respiration. } \\ 38.9 & \text { During apnea. }\end{array}$

5.4

In this case the normal act of respiration elevated the average systolic pressure $2.9 \mathrm{~mm}$., or 3.1 per cent., while it depressed the diastolic pressure $5.4 \mathrm{~mm}$., or 8.4 per cent. In dogs this respiratory pressor factor, as the percentile figure may be termed, is responsible 
for 2-3 per cent. of the average systolic and 3-4 per cent. of the diastolic variation. Its importance consists, not only in the small share it plays in thus maintaining normal blood-pressure, but also in the fact that when arterial pressure is low, deep respirations contribute a relatively larger share of blood-pressure variations (Wiggers, Eberly, and Wenner).

Rhythmic variations of the cardiac cycle, such as occur normally due to a rhythmic activity of the vagus centre or such as occur in abnormal rhythms of the heart (page 216) also modify the bloodpressures. We might anticipate from the hemodynamics evolved from an artificial circulation apparatus that a shortened cycle (i. e., an increased rate) would elevate both systolic and diastolic pressures, while lengthened cycles would depress them. We must bear in mind, however, that such a change occurs only after a state of pressure equilibrium has been established and when the systolic discharge is unaltered. Actual records with optically recording manometers (Fig. 16) show that when fleeting variations in the cardiac cycle are concerned a shortened cycle (fifth wave, Fig. 16) is followed by a decreased systolic and increased diastolic pressure. A lengthened cycle ( $c f$. sixth wave, Fig. 16), on the other hand, causes an elevation of systolic and a fall of diastolic pressure in the next wave.

The variations of systolic pressure deviate from this rule only when the alteration of the cardiac cycle does not change the filling (i.e., in very slow hearts) and when a change in rate lasts for a considerable period so as to allow the pressure to become stable at a new level (Wiggers).

In the large arteries the pressures vary little from those in the aorta. It is not until the smaller arteries are reached-such, for example, as enter into the formation of the Circle of Willis - that the mean pressure is conspicuously decreased (Poiseuille). The minimal pressure is also constant in the larger arteries, but the maximal pressure declines somewhat in arteries of such caliber as the thyroids (Hürthle, Dawson).

Relative Pressures at Different Points of the Vascular System.As we pass to the smaller arteries both maximal and minimal pressures diminish, the former more than the latter. This reduction continues until, in the smaller arteries, a constant pressure exists during systole and diastole. The most accurate idea of the actual pressures in the human arterioles, capillaries and veins may be obtained by directly examining the vascular loops of the skin under a microscope. To accomplish this, a drop of glycerine is placed on the epidermis which is then illuminated by a strong light. The intravascular pressure is ascertained by applying pressure to the skin and observing the amount required to obliterate any particular vessel or group of vessels under observation (Lombard). The 
following values, expressed in $\mathrm{mm}$. of mercury were obtained by Lombard:

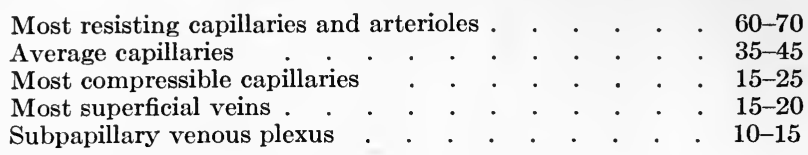

Apparently there is a fall of blood-pressure in the passage from the small arteries through the capillaries to the veins of $40-50 \mathrm{~mm}$. mercury, which indicates that a great resistance is encountered in the capillary field. It may be noted in passing that the capillaries are not the only fields where resistance is offered, for, if we accept $90 \mathrm{~mm}$. as a mean aortic pressure in man, the fall from the aorta to the arterioles examined by Lombard is nearly as great, showing that the arterioles themselves offer considerable resistance to the flow of blood.

The Velocity of the Blood Flow.-By the velocity of flow is meant the rate at which a certain cross-section of the blood column passes a definite point, and is determined by the difference in pressure between two places at any time.

Methods.-The average velocity in any vessel may be calculated from the volume flow per minute, according to the formula $L=\frac{V}{\pi r^{2}}$ in which $L$ equals the length of the blood column passing per unit time and hence represents the velocity, $V$ equals the volume flow per unit time determined by a strohmuhr, ${ }^{1}$ and $r$, the radius of the artery. This, however, gives only the mean velocity and not the variations occurring during systole and diastole. To establish these variations the hemodromograph was devised by Chaveau. This instrument, described in most modern text-books, has proven of little real value. In the first place, its use is restricted to large animals, the horse being apparently the only animal in which it has been used. Secondly, coagulation interferes with its prolonged use in any experiment. Lastly, the movable mass of the apparatus is so great that it is not capable of following rapid changes of velocity accurately.

Still another method of studying variations in velocity is by the use of Pitot's tubes. The principle of their use is as follows: If into a larger tube, in which fluid under pressure is passing, two tubes are introduced in such a way that the opening of one is directed against and the other with the stream, a difference of pressure in the two tubes takes place which, at any time, is proportional to the velocity. This principle was applied by Cybulski to study varia- 
tions in the velocity of the blood stream, but the heavy mass of the mercurial column of his manometer rendered it incapable of accurately following the pressure changes. The same principle has recently found application in the laboratory of Frank (Fig. 17). He inserts a double-barrelled cannula with lateral openings pointing in opposite directions, into a side branch of the vessels in which the velocity is to be measured. This is connected with a double chamber, the cavities of which are separated by a rubber membrane and filled with fluid. In a recent form of apparatus, Frank has sought to record the movements of this differential membrane by cementing a small mirror to it and reflecting a beam of light through

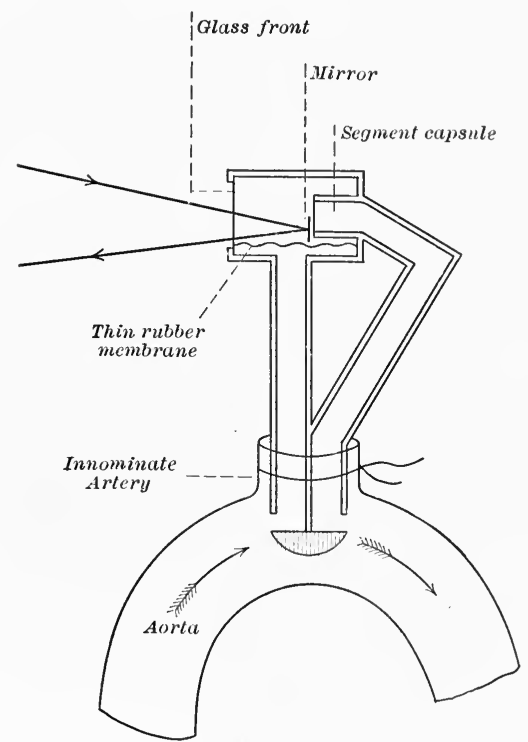

FIg. 17.-Diagram showing the principle of Frank's differential manometer for recording variations in velocity.

the glass side of the chamber. To prevent the upward diffusion of blood and thus the interference with the passage of the light beam, he interposes a delicate membrane between the outer chamber and the artery. The principle of this apparatus is shown in Fig. 17.

The velocity flow in man was at first deduced from the volume curve of an arm enclosed within a plethysmograph, recorded by some form of volume recorder (Fick). The idea upon which this was founded assumes that the venous outflow is constant. The first differential quotient of the volume curve is, then, proportional to the velocity. Garten has improved upon this method, by substituting a soap bubble as a registering 'mechanism. To obviate 
the determination of the differential quotient of the volume curve, v. Kries devised the tachograph to record the velocity. This apparatus in which the variations of a gas flame were photographed, had obvious disadvantages. It has been adopted and improved by Frank in the following manner: The arm is placed in an arm plethysmograph. The plethysmograph instead of being kept closed communicates with the outside air by a second opening. The capsule, therefore, records, not the volume change but its differential quotient which gives the velocity change directly. It is important to keep the chamber as small as possible and the external communication as large as is permitted by the sensitiveness of the recording mechanism. This appliance records the velocity changes accurately only when the variations are not too extreme and rapid. If this is the case, the records may become mixtures of volume curves and tachograms, as the records of velocity are called.

Velocity Curves.-By these methods, it has been shown that the variations of velocity change according to the distance of the artery from the heart. Thus, in the aorta near the valves, the velocity is great during systole, while, during diastole, the blood remains practically at rest. As we move slightly from the valves, we obtain both a systolic and a diastolic flow (Hürthle). The flow accelerates markedly early in systole but in the latter part diminishes rapidly and this slower rate continues into diastole. As the smaller arteries are approached, the velocity becomes less and less during systole but remains greater during diastole than in the larger arteries.

Trustworthy quantitative values for these variations are still not available. Figures yielded by the hemodromograph indicate that in the horse the flow may attain a maximum velocity of 520 $\mathrm{mm}$. per second and fall to $150 \mathrm{~mm}$. during diastole (Lorlet, quoted by Tigerstedt). The average velocity of flow has been established more satisfactorily in a quantitative way. In dogs, this has been placed at $260 \mathrm{~mm}$. (Vierordt) and $241 \mathrm{~mm}$. per second (Tschuewsky). This average velocity decreases as the smaller vessels are approached. The rate of flow in the veins is more constant, increasing somewhat in the larger vessels. Thus, the flow in the jugular equals $147 \mathrm{~mm}$. per second, while in the femoral it is 61.6 and in the renal $63 \mathrm{~mm}$. (Burton-Opitz).

We may therefore picture the average velocity as gradually decreasing from the heart to the capillaries and increasing slowly as the blood is returned to the heart. This is due to the fact that the total stream bed widens as the periphery is approached and narrows again as blood is collected and returned to the heart.

The Volume Flow of Blood.-By volume flow is meant the volume of blood passing through a vessel or organ in a definite time, independent of whether its velocity is great or small. The volume 
of blood passing through the aorta measures the total volume passing through all the organs with the exception of the heart walls. The volume flow per minute has been termed the minute volume. This is equal to the product of the pulse volume (i.e., the quantity flowing during one heart cycle) and the heart rate per minute.

The magnitude of the minute volume and also of the pulse volume has been determined in various ways in animals, namely:

1. By perfusing a heart in such a way that fluid passes in normal fashion into and through the cardiac chambers and measuring the outflow (Stolnikow, Howell and Donaldson, Patterson and Starling).

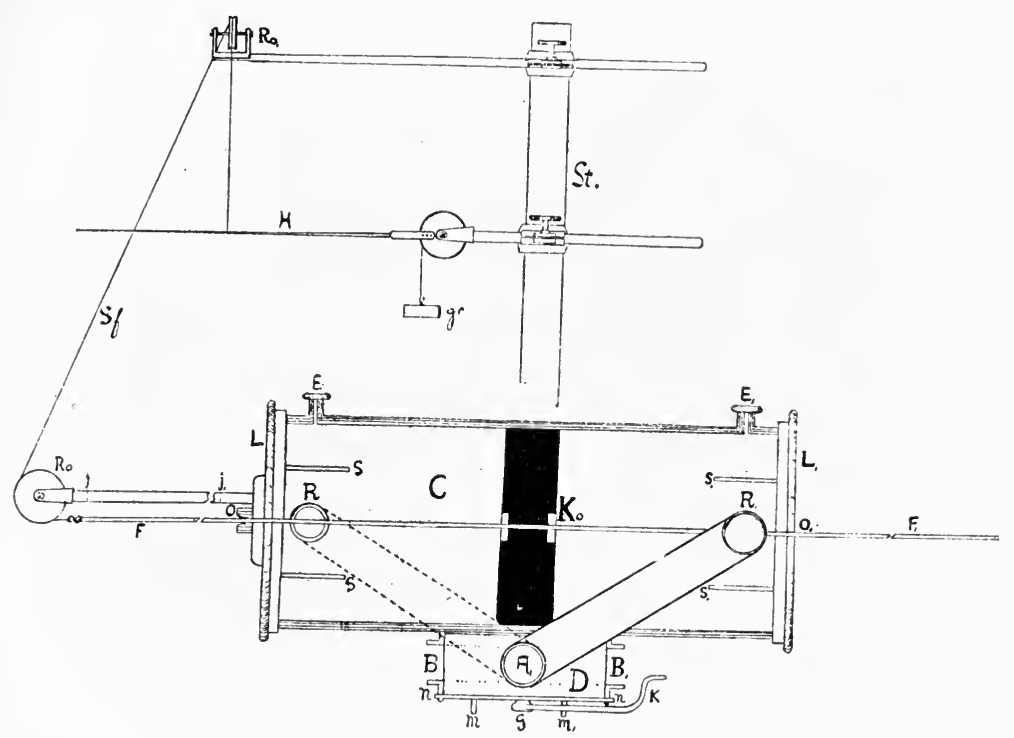

Fig. 18.- Recording stromuhr. Fluid entering the right compartment of cylinder (C) via $A R$, pushes the plunger $(K)$ to the left and causes a downward movement of the lever $(H)$. On reversing the plate $(D)$ by means of a handle $(\mathrm{k})$ blood flows via $A R$ into the left compartment and pushes the plunger to the right, thereby raising the lever $(H)$. (After Burton Opitz.)

Theoretically the aortic outflow should be directly measured, but as this causes the heart to act without arterial resistance, the flow beyond an arterial resistance has been used (Patterson and Starling). This is obviously only an accurate estimate of volume flow as long as the arterial pressure remains unaltered.

2. By inserting a strohmuhr into the aorta after temporary interruption of the current (Tigerstedt).

Various forms of strohmuhr have been devised and are described in current text-books of physiology. ${ }^{1}$ Fig. 18 shows the principle

1 For a detailed description of various forms see Tigerstedt's Handbuch der physiologische Methodik, 1909, II, part 4, 105. 
of a graphically recording form devised by. Burton Opitz which has the advantage that hydrostatic variations are avoided by the employment of a horizontal barrel.

3. By the blood dilution method (Stewart). Salt solution is allowed to slowly enter for a definite number of seconds into the left ventricle through a tube passed down the carotid. A sample of the mixture of blood and salt solution is collected from a femoral branch where its arrival is detected by a change in electrical resistance. The quantity of blood with which the solution was mixed in the ventricle can be determined by adding to a sample of blood previously drawn the same saline solution until its conductivity is the same as that of the solution collected in the experiment.

4. By the gasometric method (described on page 227).

5. By determining quantitatively the cardiometric variations of the ventricle and calculating the minute volume.

By these different methods the following average results have been obtained in dogs by various investigators:

\begin{tabular}{|c|c|c|c|c|}
\hline Investigator. & $\begin{array}{l}\text { Pulse volume } \\
\text { c.c. }\end{array}$ & $\begin{array}{l}\text { Minute volume } \\
\text { c.c. }\end{array}$ & $\begin{array}{l}\text { Flow per minute } \\
\text { per } 100 \mathrm{G} .\end{array}$ & $\begin{array}{l}\text { Heart rate } \\
\text { per min. }\end{array}$ \\
\hline Stolnikow (1) & $11-55.0$ & 1815-2200 & $2.5-34.6$ & $33-200$ \\
\hline Stewart (3). & 52.6 & 3892 & $13.9-22.2$ & $74-84$ \\
\hline $\begin{array}{c}\text { Howell and } \\
\text { Donaldson (1) }\end{array}$ & $\ldots$ & $\ldots$ & 19.7 & \\
\hline $\begin{array}{c}\text { Grehant and } \\
\text { Quinquaud (4) }\end{array}$ & $\ldots$ & $591-2614$ & $8.4-14.5$ & \\
\hline $\begin{array}{l}\text { Tigerstedt (rab- } \\
\text { bits) (2) }\end{array}$ & 5.1 & 984.3 & $\cdots$ & 193 \\
\hline Henderson (5) & $13.5-32.5$ & $810-1950$ & $1.3-2.6$ & 60 \\
\hline & $13.1-26.5$ & $1576-3188$ & $2.0-3.9$ & 120 \\
\hline $\begin{array}{l}\text { Murlin } \\
\text { Greer (4) }\end{array}$ & $8.6-26.1$ & $1204-4386$ & $8.9-14.6$ & \\
\hline Rothberger (2) & $1-3$ & $55.4-466.8$ & & \\
\hline$($ cats) (5) & $1-3$ & $55.5-447.5$ & $1.8-14$ & $54-157$ \\
\hline $\begin{array}{l}\text { Patterson and } \\
\text { Starling (1) }\end{array}$ & $4.2-22.3$ & $560-3000$ & & $126-135$ \\
\hline
\end{tabular}

It is apparent from this compilation that the systolic output of the heart and consequently the minute volume may vary considerably under different conditions. For this reason a consideration of the factors that determine the minute volume is important. It is generally concluded that an increase in the heart rate is accompanied by an increased minute volume. According to Henderson, it is proportional to an increase in rate only so long as the next systole falls during diastasis and does not shorten the elastic diastole (cf. Fig. 13). Henderson and his co-workers believe it is impossible for an increased systolic discharge to occur synchronously with an increased rate, but recently Knowlton and Starling have reported that the perfused heart, at least, is capable of throwing out the 
same systolic volume per beat even when the rate is very much accelerated.

It is generally agreed that the filling of the heart, its systolic discharge and, therefore, the minute volume are dependent on the height of venous pressure. As the pressure rises the output increases. There is some difference of opinion, however, as to the extent to which this increase may be carried. According to Henderson and his co-workers, the heart attains its maximum output per beat when the effective pressure in the auricle reaches about $50 \mathrm{~mm}$. of water in the right auricle and about $80 \mathrm{~mm}$. in the left. Pressures above these levels are supposed by him to increase the output per beat very little, hence they have been termed "critical pressures." Inasmuch as pressures of such magnitude are effective in filling the heart within the thorax, it would follow on this hypothesis that an increase in yenous pressure beyond these limits could not appreciably affect the output of the heart. This has not been the impression gained by subsequent experimenters, however. It appears that the optimum venous pressure level is much higher (125-250 $\mathrm{mm}$.) both for the heart intact within the thorax (Wiggers) and the perfused heart as well (Patterson and Starling). Upon this concep-. tion any increase in venous pressure such as might result from augmented breathing, etc., could produce a larger or an equal discharge per beat even at very high rates.

As the minute volume varies with the size and weight of the animal, much more satisfactory figures may be obtained by comparing the blood flow per minute per 100 grams body substance. The results of investigators show that in dogs this varies from 15-30 c.c., while in man the average appears to be about 6 c.c. (For details, see page 231.)

The Vascularity of Different Organs.-Such calculations of blood flow per 100 grams substance should not lead to the inference that all organs are equally well supplied with blood, which is utilized by the different tissues in various ways. In the nervous system and muscles, for instance, it essentially supplies the material for maintaining their activity, and offers an avenue for the disposal of waste products. Other organs, however, modify or regulate the composition of the blood, either, as in the lungs or intestines or glands of internal secretion, by adding new substances to it, or, as in the kidney, by removing waste products of metabolism. A knowledge of the relative and absolute blood supply of different organs is not only of interest from the viewpoint of the circulation, but also in understanding the metabolism of the body, since it may be assumed that the share taken by different organs in the total metabolism is proportional to their blood supply, per unit time, per 100 grams of that organ. The following table, copied, in the main, from Burton Opitz, gives an idea of the relative vascularity 
of different organs of the body as determined by inserting a strohmuhr into the arteries furnishing blood to the organs.

\section{Minute Volume per 100 Grams Substance.}

\begin{tabular}{|c|c|c|c|c|c|}
\hline Posterior extre & mity & $y$ & 5.0 & c.c. & Tschuewsky. \\
\hline Skeletal muscle & & . & 12.0 & c.c. & \\
\hline Heart . . & & . & 16.0 & c.c. & Bohr and Henriquès. \\
\hline Head. & & . & 20.0 & c.c. & Bohr and Henriquès. \\
\hline Stomach & & & 21.0 & c.c. & Burton Opitz. \\
\hline Liver (arterial) & & & 25.0 & c.e. & Burton Opitz. \\
\hline Portal organs ( & $(\mathrm{com})$ & abined) & 30.6 & c.c. & Burton Opitz. \\
\hline Intestine & . & . . & 31.0 & c.e. & Burton Opitz. \\
\hline Spleen . . & . & . & 58.0 & c.c. & Burton Opitz. \\
\hline Liver (venous) & & . & 59.0 & c.c. & Burton Opitz. \\
\hline Pancreas & . & . & 80.0 & c.c. & Burton Opitz. \\
\hline Liver (total) & & . & 84.0 & c.c. & Burton Opitz. \\
\hline Brain & . & . & 136.0 & c.c. & Jenson. \\
\hline Kidney & & & 150.0 & c.c. & Burton Opitz. \\
\hline Thyroid & . &.$\quad$. & 560.0 & c.c. & Tschuewsky. \\
\hline
\end{tabular}

It is of interest to notice that the liver with its large mass and varied functions takes a place subordinate to such organs as the brain, kidney and thyroid, when calculated on a basis of unit mass.

\section{LITERATURE.}

Bohr and Henriquès. Skan. Arch. f. Physiol., 1895, v, 232.

Burton Opitz. Am. Jour. Physiol., 1902, vii, 435; 1903, ix, 161.

" " Arch. f. d. ges. Phy siol., 1908, 123, 553; 1908, 124, 469.

Cybulski. Zentralbl. f. Physiol., 1890, v, 834.

Dawson. Am. Jour. Physiol., 1906, xv, 244.

Fick. Verhandl. d. Physiol. med Gesellsch, in Würzburg, 1886, xx, 33.

Frank. Tigerstedt's Handbuch der Physiol. Methodik, 1913, II, part 4.

“ Ztschr. f. Biol., 1898, xxxvii, 1; 1907, 1, 303.

Henderson. Am. Jour. Physiol., 1906, xvi, 325; 1909, xxiii, 345.

Henderson and Barringer. Am. Jour. Physiol., 1913, xxx, 288, 352.

Howell and Donaldson. Phil. Tr. Roy. Soc. London, 1884, 139.

Hürthle. Arch. f. d. ges. Physiol., 1890, xlvii, 176; 1905, cx, 42; 1912, cxlvii, 561.

Garten. Arch. f. d. ges. Physiol., 1904, civ, 351.

Jenson. Arch. f. d. ges. Physiol., 1904, ciii, 191.

v. Kries. Arch. f. Physiol., 1887, 254.

Lombard. Am. Jour. Physiol., 1911, xxix, 335.

Patterson and Starling. Jour. Physiol., 1914, xlviii, 357.

Rothberger. Arch. f. d. ges. Physiol., 1907, cxviii, 353.

Stewart. Jour. Physiol., 1894, xv, 1.

Stolnikow. Arch. f. Physiol., 1886, 1.

Tschuewsky. Arch. f. d. ges. Physiol., 1903, xcvii, 386.

Wiggers. Am. Jour. Physiol., 1914, xxxiii, 13.

" Jour. Exper. Med., 1914, xix, 1.

Wiggers, Eberly, and Wenner. Jour. Exper. Med., 1912, xv, 174. 


\section{THE CONTROL OF BLOOD FLOW THROUGH ORGANS.}

The quantity of blood passing through different organs is not constant from time to time but alters, as a rule, with the conditions of activity. Thus, when the salivary or other glands secrete, when muscles contract and perhaps when increased nervous activity takes place, the blood flow augments. This may be brought about either by an alteration in the general pressure or by a change in the total peripheral resistance of the organ in question.

The Supplying Pressure.-When the total resistance in an organ remains constant and the pressure supplying that organ is intermittent, the mean pressure does not entirely determine the flow; but, on the contrary, the flow is proportional to the amplitude of the pressure variation (Hooker, Hamel, Hoffiman, Gesell, etc.). The recent work of Gesell raises the question whether organs left intact within the body follow such simple dynamic rules, for he found that the flow through the kidney did not alter when the pulse pressure was reduced and the mean pressure remained the same. On the contrary, it sometimes slightly increased when the pulse pressure was reduced and the mean pressure fell. It is concluded from these results that the vascular resistance of intact organs alters to compensate for the change in pulse pressure.

The Total Resistance.-The total resistance in an organ is determined by the resistance offered to the flow by the venous pressure and by the friction between the blood and the vessel walls. This friction depends on the viscosity of the blood and the size of the vessels, as controlled, actively by vasomotor influences, and passively by extravascular support or pressure.

Thus, a high intraventricular or intracranial pressure may interfere with the flow through the heart or brain, respectively, and a great tonic contraction in muscular organs (for example, intestines and uterus) may entirely obliterate the vascular channels by compression. This is often the determining method of modifying peripheral resistance in muscular organs. In many organs devoid of such a mechanism (e.g., glands) the active vasomotor constriction and relaxation of vessels determines the peripheral resistance. The presence of vasomotor fibers has been demonstrated for all organs, but their efficiency in controlling the blood flow is not equally 
developed in all regions. In fact, their activity has been so overshadowed by other factors that their presence in some organs has until recently been questioned. This has been particularly so in the case of the cerebral and coronary vessels. ${ }^{1}$

The evidence that the cerebral vessels are supplied with vasomotor fibers is partly histological and partly physiological. The vessels contain muscular elements. Nerve fibrils have been traced as far as their terminations in the cerebral vessels (Obersteiner, Huber, Gulland). That they are probably vasomotor rather than sensory fibers is evidenced by the following investigations of the writer:

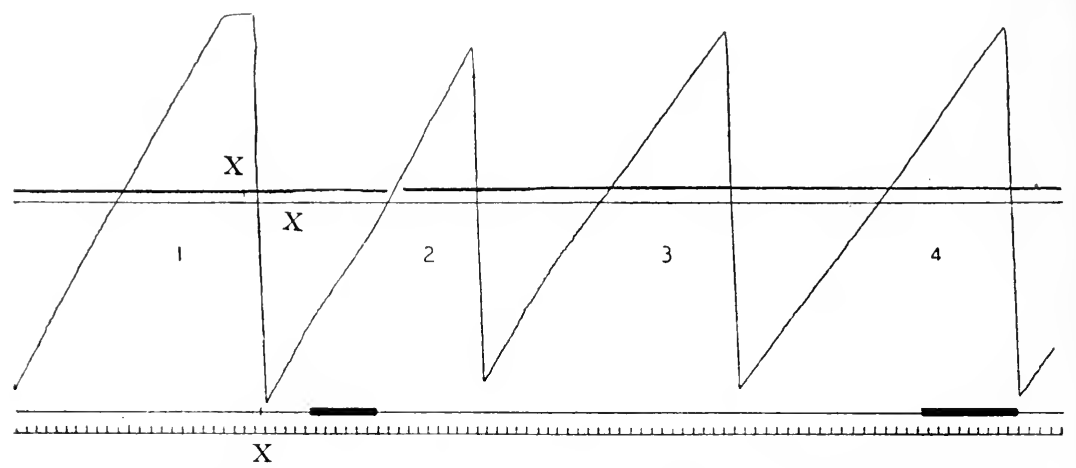

FIG. 19.-Curve showing by its change in slope the effect of stimulating the carotid sheath on the flow through the isolated hrain.

1. When the isolated brain is perfused with a pulsating stream, the addition of adrenalin which presumably acts on the sympathetic nerve terminals (Brodie and Dixon, Elliott, Dale) causes a diminution of flow through the organ. The criticism of the method made by Dixon and Halliburton has been answered by the writer, recently; and, moreover, it has been possible to show that when the identical method of these investigators is used, adrenalin still causes its typical constriction.

2. If the nerve plexuses surrounding the carotid sheaths are stimulated at a time when the physiological condition of nerves and technic are favorable, a decrease in flow through the brain vessels follows, as shown in Fig. 19. These results give direct evidence of a nerve control over the cerebral vessels, a conclusion foreshadowed by histological studies and the reaction to adrenalin. It may be pointed out that, owing to technical difficulties, negative evidence cannot counterbalance evidence of a positive character unless it

1 For review of literature, consult Howell, Text-book of Physiology, 1913, 612: Bayliss, Ergebnisse der Physiol., 1906, v2, 319. 
can be shown that the procedures by which the latter was obtained were faulty. So far this has not been done.

Evidence of a similar kind favors the existence of a vasomotor supply to the coronary vessels. In the first place, fibers have been traced to the bloodvessels of the Purkinje system (Dogiel and others). In the second place, a diminution of outflow from the right ventricle of a perfused heart has been observed on vagus stimulation (Porter and Maas). These experiments, however, furnish "probable rather than quite conclusive proof" of a vasomotor influence. The factors affecting coronary vessels are so many and the communications of the vessels with the ventricle so numerous that any decrease or increase in outflow can be accepted as demonstrating vasomotor change only when it can be shown that (1) the pressure supplying the coronaries remains the same and (2) the size of the right chambers existing as intermediary reservoirs is constant, and (3) the massaging effect of cardiac contractions on the intramural vessels has not altered. Recently, Porter has suggested another method of more accurately gauging the flow through the coronaries which is entirely free from these objections and should yield trustworthy results on stimulation. In the third place, adrenalin when perfused through a quiescent heart preparation invariably diminishes the coronary flow (Wiggers). A similar result has more recently been obtained by Rabe. In the case of the beating heart, most investigators find that the flow is augmented (Schäfer, Wiggers, Morawitz and Zahn and F. Meyer). 'This, the writer has attributed to the fact that the amplitude and rate of beat increase, a factor that Porter has shown to augment the flow. Brodie and Cullis found a similar reaction with large doses of adrenalin but in the case of the smaller doses a decreased flow preceded this increase; and this they attributed to the constriction brought about by adrenalin.

It remains to consider the contradictory evidence offered by the fact that adrenalin usually dilates strips of coronaries immersed in Ringer's solution. 'This reaction has been repeatedly obtained in strips of the larger coronaries from the sheep, ox, goat, calf, etc. (Langendorff, Pal, Cow, Eppinger and Hess, Barbour, etc.). Barbour, however, found that the coronaries of man obtained at autopsy contracted. In harmonizing the constrictor action exclusively obtained in perfusing the resting heart with the dilator action found by the ring contraction method, it may be pointed out that the two methods test the reaction of different parts of the coronary system to adrenalin. The perfusion method tests essentially the reaction of the arterioles, the ring method that of the larger arteries. The results are not necessarily antagonistic but offer presumptive evidence that the larger vessels are supplied only with dilator fibers, while the smaller ones-and these are principally 
concerned with determining the blood flow through the organs-are supplied with both constrictor and dilator fibers. More direct evidence that the coronaries are influenced by vasomotors exists. Stimulation of the vagus nerve causes in the atropinized $\operatorname{dog}$ a decreased outflow from a wounded heart vein. This persists in spite of the fact that changes in the blood-pressure, the contraction of auricles and ventricles and back flow do not occur (Fig. 20).

The Cerebral Circulation and its Control.-The brain is enclosed within the bony cranial cavity, through certain openings of which arterial blood is admitted and venous blood passes away. Except for a few cubic centimeters of cerebrospinal fluid that may be

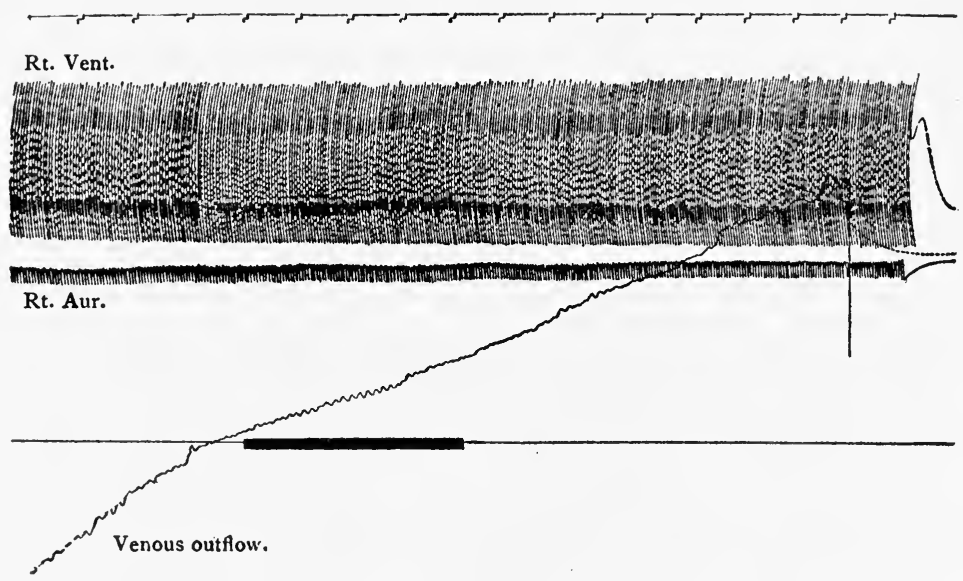

Fig. 20.-Effect of stimulating vagus (weak current) on the venous outflow from the coronaries when no change in the contraction of the auricle and ventricle was present.

displaced from the subarachnoid space into the spinal cavities, the brain is limited in its expansion. Hence the quantity of blood entering at any time cannot greatly exceed the outflow from the veins. This principle first stated by Monro in 1783 has generally been designated as the Monro-Kellie doctrine.

The brain is supplied by the two vertebrals and the two internal carotid arteries which form the basal anastamosing complex, known as the Circle of Willis. Recent evidence indicates that the functional anastamosis is less complete than the anatomical studies of Willis. would indicate. Colored solutions injected into one vessel are distributed only to that section of the brain directly tributary to this artery, while drugs, such as chloroform, which are harmless when injected into the carotid act very powerfully on the respiratory and vasomotor centres when injected through the 
vertebral (Kramer). This is evidently due to the fact that the pressures are so evenly balanced that little or no intermingling occurs.

The blood returning from the brain substance is collected into the venous sinuses which are contained between folds of the dura and rest in grooves on the inner surface of the cranial bones. These sinuses drain largely through the lateral sinuses into the internal jugular vein, although numerous other collateral routes exist.

The cerebrospinal fluid surrounding the brain and large vessels is normally under a pressure equal to that of the veins (Bayliss and Hill). Under abnormal conditions, it may rise considerably. When this occurs the veins are not obliterated as might be expected and a self-strangulation does not occur. The flow is not reduced until a pressure nearly equal to intra-arterial is attained. This is probably due to the protection which is afforded to the veins by the inelastic dura. It is only when the pressure becomes high enough to compress the unprotected arteries that the flow is reduced. Were it not for this protection, an increase in general blood-pressure by expanding the cerebral arteries and raising intracranial pressure might compress the veins and reduce the outflow, as was at one time claimed by Geigel and Grashney.

It has been conclusively demonstrated that the cerebral circulation in experimental animals follows in every essential phase the variations of the general arterial pressure. When the arterial pressure rises the volume tends to increase if a trephine opening is made, the intracranial, intra-arterial and intravenous pressures increase and a larger flow of blood through the brain occurs (Roy and Sherrington, Bayliss and Hill, Gaertner and Wagner, etc.). There is no reliable evidence that the vasomotor fibers demonstrated to be present are powerful enough to overcome any considerable variation of general arterial pressure. Of this one can convince oneself directly by perfusion experiments. If a constriction is induced by adrenalin it requires only a very slight elevation of the perfusion pressure $(5 \mathrm{~mm}$. often suffices) to annul it, whereas, in other organs, as the kidney, a pressure rise of $40-50 \mathrm{~mm}$. will not overcome the restriction. These nerves clearly play very little part in modifying the blood flow through the brain. Their function is as yet unknown. The possibility exists, as Howell suggests, that they may serve to adjust the distribution of blood within the cerebral areas, by directing blood in larger quantities to those regions undergoing special activity. It is not impossible that they are in some way associated with or perhaps responsible for sleep, for, according to Shepard, the volume of the brain increases during this act.

The Coronary Circulation and its Control.-The heart muscle receives its blood supply from the two coronary arteries which 
break up into intramuscular capillaries. From these regions blood is collected by venules and veins which finally empty into the coronary sinus of the right heart. There are, however, direct communications with the ventricles by means of the Thebesian vessels, which, as Pratt has shown, are efficient vascular channels in the nutrition of the heart.

The flow through the coronary system is dependent to a marked extent on the arterial pressure. The height of blood-pressure, as a rule, overbalances any active vasomotor changes. Thus, if a general vasoconstriction of which the coronary vessels partake (for example, after adrenalin) is induced and the blood-pressure is raised in this way, the flow through the heart will increase. This has been shown in perfusion experiments, as well as in hearts intact within the body. 'The flow is modified also by the activity of the organ itself, that is, by its rate and amplitude of contraction, its tonus and the height of intraventricular pressure (Magrath and Kennedy, Hyde).

With each beat blood can be seen to be forced from a cut vessel. This is due to the fact that, during systole, muscular contraction compresses the intramural vessels and forces blood onward in the direction of least resistance; and, during diastole, the emptied vessels are quickly refilled by the greater pressure in the central arteries. Distention of the ventricle and increased tonus diminish the flow by increasing the total resistance.

The Circulation Through the Liver and its Control.-On account of its very important functions associated with digestion and nutrition, the liver is favored with an exceedingly great blood supply. With the exception of the lungs, the liver receives the largest total supply of any organ in the body. It has been estimated (Burton Opitz) that a liver weighing 500 grams in a dog weighing 15 kilos, receives 420 c.c. of blood per minute; or, roughly stated, an amount of blood equal to the entire amount in the body traverses the liver every three minutes.

It receives this supply from two sources, the hepatic artery and the portal vein. 'The arteries not only supply the walls of the bile ducts and the portal vessels, the connective tissue capsule and trabeculæ but also communicate with the radicals of the portal system so that a mixture of arterial and venous blood reaches the capillaries. The arterial and venous branches join at an acute angle (Gad) forming a sort of valve which may shift in accordance with the pressure on each side.

Strohmuhr experiments (Burton Opitz) indicate that more than two-thirds of the total liver flow comes from the portal side, which, in turn, derives its greatest supply in the order named from the intestines, stomach, spleen, and other splanchnic organs. This relation exists in spite of the fact that the hepatic artery has a 
pressure not materially lower than that in the aorta, while the portal vein has a pressure equal to about $10 \mathrm{~mm}$. of mercury.

It is evident that the flow through the liver may be passively modified, not only by variations of arterial pressure, but also by changes in the portal pressure, which is determined by the flow through the abdominal organs. Thus, a marked constriction of the vessels of the intestines, spleen, etc., causes an elevation of pressure in the aorta and hepatic artery but a decrease in portal pressure. It is desirable to determine the result of such vasoconstriction on the arterial and venous flow through the liver. Inasmuch as the splanchnic nerves apparently are not connected with the postganglionic vasomotor fibers for the liver, (Burton Opitz) it is possible to do this by stimulation of the splanchnic nerves. When this is done, the portal flow first increases and the pressure rises due to the onward movement of blood into the central portal veins. This is soon followed by a reduced flow and a fall of portal pressure. This is compensated for, however, by an increased inflow passively brought about by the rise of pressure in the aorta. The hepatic artery serves as a compensatory mechanism in such cases insuring an adequate supply to the liver.

A different situation results, however, when the portal flow is mechanically shut off or reduced. The arterial flow then does not increase in a compensatory manner as might be expected but is likewise reduced. The reason for this is that when the portal supply is shut off, less blood is returned to the heart and in consequence the arterial pressure falls. The flow through the arterial channels of the liver is then determined largely by the height of the arterial pressure.

The question remains whether the pressure within the portal vessels and the flow may also be modified by veno-motor nerves. It is generally held, after the experiments of Mall, that the portal vein is supplied with such a special vasomotor control which may regulate the flow independent of other organs. While some experiments have not favored the idea that such a control exists (Velich, Mares), its existence and significance have been definitely established by the recent work of Burton Opitz.

Having recognized the dependence of the liver volume on arterial and portal pressure, it is necessary to consider the arterial vasomotor regulation. It has been shown that stimulating the nerve strands, passing from the celiac ganglia with the hepatic trunks to the liver causes a marked rise of arterial pressure and a reduction in arterial flow. In the case of some fibers, this amounts to almost a complete cessation. While the arterial inflow is thus reduced due to vasoconstriction, the portal flow suffers only a slight reduction, which indicates that the fibers distributed to the venous radicals act feebly as compared to those supplying the arterial branches. 
Since nerve stimulation does not itself modify the flow of portal blood, it seems that this flow is largely dependent on the vasomotor mechanism in the organs tributary to the portal vein. The fact, however, that the flow diminishes after the introduction of adrenalin indicates that a contraction of the intrahepatic radicals of the portal vein occurs. The exact location of this mechanism is, however, as yet undetermined (Burton Opitz).

\section{LITERATURE.}

Gesell. Am. Jour. Physiol., 1913, xxxii, 70.

Hamel. Ztsehr. f. Biol., 1889, xxv, 474.

Hoffman. Arch. f. d. ges. Physiol., 1903, c, 242.

Hooker. Am. Jour. Physiol., 1910, xxvii, 24.

“ Arch. Int. Med., 1910, v, 491.

\section{Articles Dealing with Cerebral Vessels.}

Bayliss and Hill. Jour. Physiol., 1895, xviii, 334.

Dixon and Halliburton. Quart. Jour. exper. Physiol., 1910, iii, 316.

“ " Jour. Physiol., 1913, xlvii, 233.

Elliott. Jour. Physiol., 1905, xxxii, 401.

Gaertner and Wagner. Wien. med. Wohnsehr., 1887, 602, 639.

Hill. The Physiology and Pathology of the Cerebral Cireulation, 1896.

Kramer. Jour. Exper. Med., 1912, xv, 348.

Roy and Sherrington. Jour. Physiol., 1890, xi, 85.

Shepard. Am. Jour. Phy siol., 1909, xxiii, xii.

“ The Circulation and Sleep, 1914.

Wiggers. Am. Jour. Physiol., 1905, xiv, 452; 1907, xx, 206; 1908, xxi, 454. Jour. Physiol., 1914, xlviii, 109.

\section{Articles Dealing with Coronary Vessels.}

Barbour. Jour. Exper. Med., 1912, xv, 404.

Brodie and Cullis. Jour. Physiol., 1911, xliii, 313.

Cow. Jour. Physiol., 1911, xlii, 125.

Eppinger and Hess. Ztschr. f. exper. Path. u. Therap., 1909, v, 622.

Hyde. Am. Jour. Physiol., 1898, i, 215.

Langendorff. Zentralbl. f. Physiol., 1907, xxi, 551.

Maass. Arch. f. d. ges. Physiol., 1899, lxxi, 291.

Magrath and Kennedy. Jour. Exper. Med., 1897, ii, 13.

F. Meyer. Arch. f. Physiol., 1912, 223.

Morawitz and Zahn. Zentralbl. f. Physiol., 1912, xxvi, 465.

Porter. Boston Med. and Surg. Jour., 1896, i, 39.

“ Am. Jour. Physiol., 1912, xxix, 31.

“ Am. Text-book of Physiol., 1900, i, 179.

Pratt. Am. Jour. Physiol., 1898, i, 86.

Rabe. Ztsehr. f. exper. Path. u. Therap., 1912, xi, 175.

Wiggers. Am. Jour. Physiol., 1909, xxiv, 391.

\section{Articles Dealing with the Hepatic Vessels.}

Burton Opitz. Quart. Jour. Exper. Physiol., 1910, iii, 297; 1911, iv, 93, 103, $113 ; 1912$, v, 83, 189, 197, 309, 325, 329, 913; vii, 57 .

Arch. f. d. ges. Physiol., 1909, exxix, 189; 1910, exxxv, 205, 245; 1910, cxlvi, 344 .

Am. Jour. Physiol., xxxvi, 325.

Gad. Dissertations, Berlin, 1873.

Mall. Arch. f. Physiol., 1892, 409.

Mares. Areh. f. d. ges. Physiol., 1903, xcvii, 567.

Velich. Arch. f. d. ges. Physiol., 1903, xcv, 264. 


\section{THE PHYSIOLOGY OF THE PULMONARY CIRCUIT.}

The Pressure Variations in the Pulmonary Circuit.-The mean pressure existing within the pulmonary artery has been measured by a number of investigators. Most of the experiments have been carried out under artificial respiration after opening the thorax. A cannula was inserted into the central end of an arterial branch going to one of the smaller lobes and the lateral pressure in the main vessel was thus ascertained. In this way, values ranging from 8 to $34 \mathrm{~mm}$. of mercury were obtained; the average, $20 \mathrm{~mm}$., being usually given as the mean pressure in the pulmonary circuit. It is quite obvious that pressures so obtained are not necessarily identical with those measured in naturally breathing animals, especially since we now know that the pressure within the right auricle and, hence, the output of the right heart tend to diminish unless special precautions are taken. The mean pressure during natural respiration has also been registered (Plumier, Wiggers). The procedure first suggested by Fredericq is usually followed with some modifications. A thoracic trocar connected by an air-tight system with a recording tambour is first inserted through an intercostal space and the negative pressure during inspiration and expiration recorded for a short time interval. Under artificial respiration the fifth to the eighth ribs are resected on the right side so that the lower lobe can be withdrawn with gentle traction and the arterial branch to the small lobule accessary to this lower lobe dissected from the lung tissue. Ligation of this vessel cuts off so small a part of the entire pulmonary circuit that its closure by a clamp even during natural breathing is without any influence on recorded arterial pressures. After applying a clamp which can subsequently be operated by a screw projecting from the repaired chest, a short-necked waxed cannula is securely tied into this vessel and a connecting rubber tube is pulled through a small intercostal incision in such a way that, with the lung reduced, a straight line is formed with the arterial branch. This proves exceedingly important for, if the least tendency to kinking of the pulmonary artery exists, it increases when the ribs are raised in inspiration. If this occurs the lumen of the vessel is reduced during inspiration and the true pressure variations in the large pulmonary arteries are not accurately reproduced. To prevent the elevation 
of the ribs from compressing the rubber tube during inspiration and so introducing an artificial pressure change, a waxed glass tube, $8 \mathrm{~mm}$. in internal diameter, is slipped into the rubber connection until it approximates the cannula. This tube is similarly connected with the pressure recording apparatus. The thoracic wall is then surgically repaired and rendered air-tight. After stoppage of artificial respiration, which has just previously been increased so that the animal is in apnea, a negative pressure equal to that existing during expiratory quiet previous to operation is restored by gentle suction through a T-tube of the trocar tambour system.

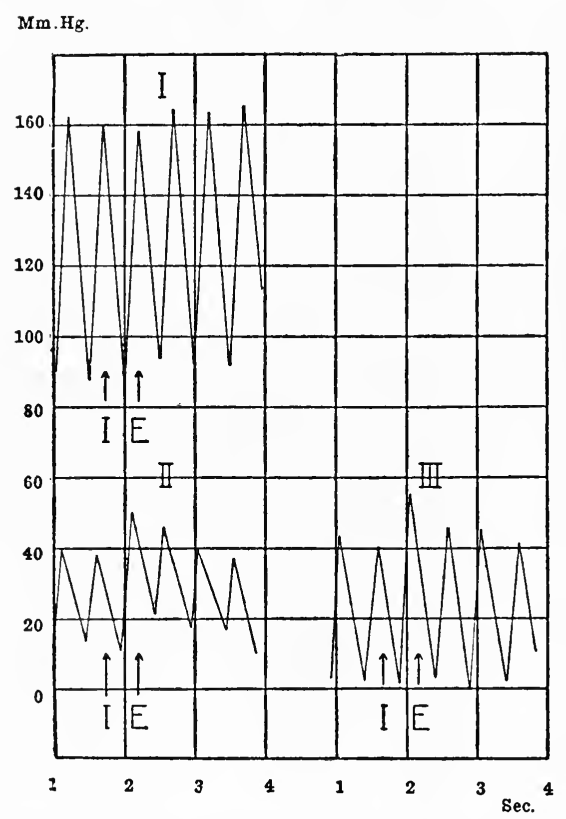

FIG. 21.-Diagram showing the respiratory variations of systolic and diastolic pressure in the carotid artery $(I)$, pulmonary artery $(I I)$, and right ventricle $(I I I)$.

Utilizing this method, Plumier found that the mean pressure averaged $18 \mathrm{~mm}$., but that this level was elevated and depressed rhythmically by respiration. The writer using maximal- and minimal-valved manometers, found that the maximal pressure averaged $31.3 \mathrm{~mm}$. and the minimal pressure averaged $5.9 \mathrm{~mm}$., giving a mean pressure of about $19 \mathrm{~mm}$. In a second series of experiments in which greater pains was taken to have a normal venous pressure, and in which a different form of pulse pressure instrument was employed, the average maximal pressures ranged somewhat higher, namely, $43.3 \mathrm{~mm}$., while the minimal averaged $11.9 \mathrm{~mm}$. The average variation of the systolic pressure during 
respiratory phases was $12 \mathrm{~mm}$., the average diastolic variation, $8 \mathrm{~mm}$.

Comparing these results (Fig. 21) with the variations in the systemic circuit, it is evident that while both systolic and diastolic pressures range much lower in the pulmonary vessels, the respiratory fluctuations are actually as great and in some instances larger. The mechanical action of respiratory movements contributes approximately 32 to 40 per cent. to the height of the maximal pressure and depresses the minimal pressures 10 to 20 per cent. In other words, during apnea the maximal pressure falls and the minimal increases. Presumably the mean pressure is little affected.

The rate of the heart modifies the pressures only within certain limits. As the cardiac cycle lengthens to approximately 0.8 second, the diastolic pressure falls somewhat but the systolic pressure undergoes no change or displays only a slight tendency to decrease. When the cycle becomes longer than one second, the diastolic pressure falls no further but the systolic exceeds that at slower rates. These studies lead to the inference that variations in the length of the cardiac cycle between 0.6 and 0.9 seconds, such as occur normally from beat to beat, are practically without influence in modifying the pulmonary diastolic pressure, while their influence on systolic pressure is very slight.

The Nature of the Pressure Variations.--As in the systemic circuit, the variations in pressure may be described as cardiac and respiratory.

The contour of the cardiac variation resembles that obtained by Frank in the aorta, the chief difference being that the summit is reached relatively early in systole (Fig. 22). 'The wave $A-B$ evidently corresponds to the auricular contraction. In many cases this is followed by an elevation $(B-C)$ before the negative wave $(C-D)$ supervenes. This, in turn, is followed by the short vibration $(D-E-F)$, after which the main rise occurs. A study of curves enlarged and transcribed to millimeter paper after a method similar to that described by Broemser leads to the conclusion that the true isometric period falls during the waves $C-D-E-F$ and that the wave $B-C$, when present, is concerned with auricular pressure changes. The negative wave $(C-D)$ present in the aortic curve only during low pressure is always clearly distinguishable in the pulmonary arterial record. Although the diastolic pressure in the pulmonary artery is much lower than that in the aorta, the isometric period is not materially different from that in the left ventricle.

Following the preliminary vibrations and the opening of the semilunar valves, the pressure rises suddenly to $G$ and rapidly falls to $H$. Since the vibration period of the primary pressure wave 
of $G$ equals about 0.03 second and the inherent period of the instrument was 0.00636 , it must evidently be attributed to a vibration of the blood column existing within the artery and not to an instrumental vibration. In all the records the amplitude of this vibration is much greater than in the systemic circuit. Following the primary wave, the record assumes a rounded top which may be regarded as the true systolic summit and then rapidly falls to the incisura. In the experiment from which the waves of Fig. 22 were taken, the right auricular pressure was $14 \mathrm{~mm}$. of water during expiration and $-15 \mathrm{~mm}$. during inspiration, pressures which may be regarded as normal, inasmuch as they agree favorably with pressures found in unanesthetized animals. In order to study the effect of increasing the venous pressure, saline was slowly infused by the

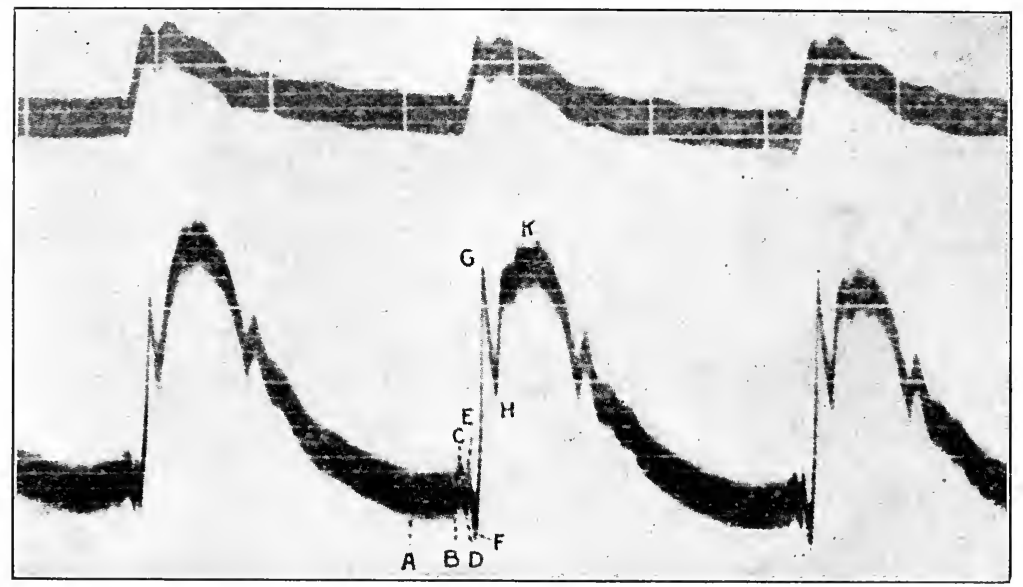

FIG. 22.-The details of the pressure changes in the pulmonary artery during inspiration and expiration.

jugular. When the pressures reached $68-80 \mathrm{~mm}$. above intrathoracic a pronounced increase in amplitude occurred, and the curve assumed the form shown in Fig. 9, $A$.

In naturally breathing animals important changes, not only in the height, but also in the details of contour occur during inspiration and expiration. Their essential character is shown in the consecutive waves of Fig. 22, the first of which occurs during expiration and the last two during inspiration. Inspiration at its beginning causes a descent of the diastolic portion of the second curve which is followed by a proportionate fall of systolic pressure (measured at the height of the rounded curve) so that the pulse pressure is not greatly reduced. In the third wave, however, occurring at the height of inspiration, the systolic pressure is 
markedly lower and the pulse pressure is much reduced. Corresponding changes in the height of the carotid curve take place. The contour of the inspiratory pressure wave (second wave) differs from that of expiration (first wave). During inspiration the auricular wave is less prominent but the amplitude of the preliminary vibration $(D-E-F)$ becomes much greater. The time relations remain unchanged. The primary upstroke $(G-H)$ also becomes larger and sharper in its rebound and after its completion returns more rapidly to the top. The systolic summit which was smoothly rounded in expiration approximates a short ascending or horizontal plateau. These changes can be explained by a decreased resistance in the pulmonary vessels.

More extended observations show, however, that systolic and diastolic pressures fall in this manner in inspiration only when not more than two beats occur during that phase. Should a third beat occur, the systolic pressure increases again in spite of the fact that the diastolic pressure remains low. A study of the intraventricular pressure curve indicates that this increase is accounted for by an increased systolic discharge.

During expiration both systolic and diastolic pressures are increased, the diastolic often during the period when the semilunars are closed. This is apparently traceable to an increased pulmonary resistance in the deflating lungs and also to an augmented systolic discharge. Further experiments are necessary to ascertain the causes of the variations of output during inspiration and expiration and to analyze more fully the factors involved in modifying the total pulmonary resistance during acts of respiration.

The variations of pressure in the pulmonary veins have not as yet been satisfactorily investigated in the closed chest. In the opened chest the mean pressure equals only a few millimeters of mercury. Probably the pressure in the closed chest differs only a trifle from that in the left auricle. Experiments have shown that the actual left auricular pressure is usually negative during inspiration ( -2 to $-50 \mathrm{~mm}$. saline), but, as a rule, becomes positive during expiration $(+6$ to $+38 \mathrm{~mm}$. saline). The effective pressure, $i$. e., the difference between left auricular and intrathoracic is slightly greater in inspiration than in expiration, $51.1 \mathrm{~mm}$. saline as compared with $46.4 \mathrm{~mm}$.).

Influences Modifying the Pressure and Flow in the Pulmonary Vessels.-It is conceivable that the pressures in the pulmonary artery may rise when either the heart rate, the systolic discharge or the total peripheral resistance increases. It has already been pointed out, however, that within normal ranges, the cardiac variations cause little change in the systolic and diastolic pressures within the pulmonary circuit. " This does not signify, however, that the volume flow is unaltered, but, on the contrary, the flow from 
the pulmonary veins decreases, as shown in Fig. 23, when the heart is slowed by vagus stimulation or by drugs acting on the vagus

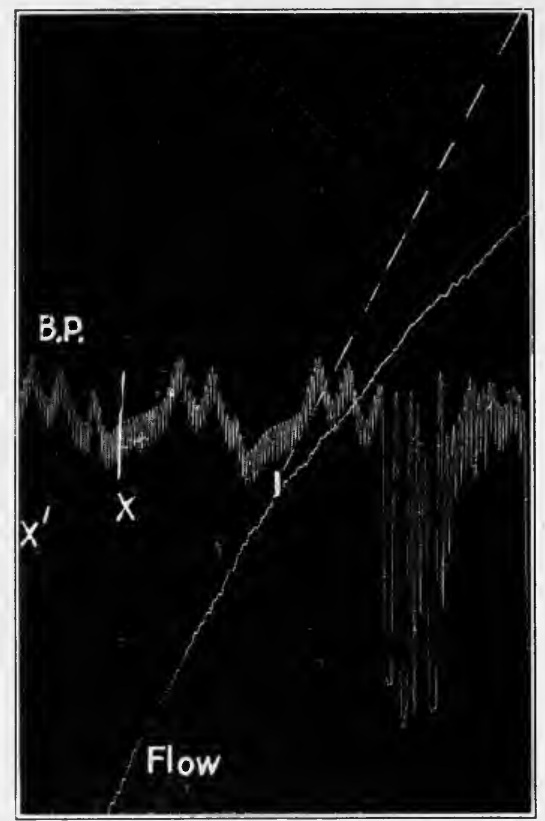

FIG. 23.-Effect of decrease in heart rate eonsequent to vagus stimulation on the blood flow through the pulmonary vein. $x-x^{\prime}$, relative positions of points.

centre. The output of the heart directly dominates the pressure within the pulmonary arteries and also the flow through the capillar-

$A$

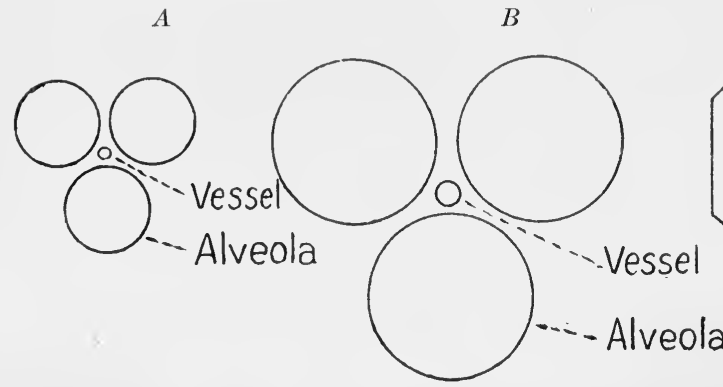

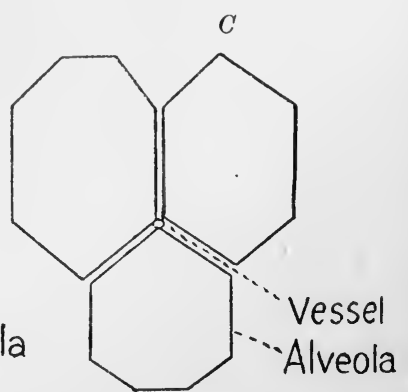

FIG. 24.-Diagrams illustrating the effect of lung inflation on the alveoli and lumen of the intrapulmonary vessels. $A$, collapsed lung; $B$, moderately inflated lung eausing larger lumen' by radial traction; $C$, marked inflation causing compression by virtue of polygonal shape of alveoli. (After Cloetta.)

ies and veins. Whenever the output of the right heart increases, whether due to a different venous pressure or to an inherent influence 
modifying the contraction of the heart, the pressures increase in the pulmonary artery and the flow augments. This increase in pressure and flow also extends to the pulmonary veins even when the heart rate is somewhat slowed and the lung vessels tend to actively constrict as they do after adrenalin. It appears that a change in the total peripheral resistance is practically without influence on the pulmonary circuit when the change opposes the cardiac output in its effect. When, however, rate and output are constant, then the total peripheral resistance may modify the flow through the lungs; an increase in resistance, raising both systolic and diastolic arterial pressures and reducing the volume flow.

By the total pulmonary resistance we mean the sum of all the resistances that impede the flow through the lungs. It is governed (a) by the degree of lung expansion, $(b)$ by the effect of negative pressure on the extrapulmonary vessels, $(c)$ by vasomotor variations and $(d)$ by an altered vis a fronte caused by impaired action of the left heart. We may take these factors up separately. It has recently been pointed out (Cloetta) that as the lung expands two opposing factors act upon the intrapulmonic vessels. When first the alveoli expand, they tend to exert a radical traction upon the small vessels and capillaries and so enlarge them, but as the enlargement proceeds and the alveoli acquire a polygonal shape, they tend to compress the intrapulmonic ressels (Fig. 24). Furthermore, as the lungs enlarge, they necessarily cause a linear extension of the bloodvessels and thereby further reduce their caliber. From this it appears that a moderate distention of the lungs causes a diminished resistance, but an extreme distention an augmented resistance.

It can readily be shown that the capacity of the large extrapulmonary vessels may increase when the surrounding pressure becomes more negative. This influence is exerted more upon the large pulmonary veins than upon the arteries (de Jager). It is probable that the mechanical variations of resistance of the intrapulmonary as well as the extrapulmonary vessels, play an important role in creating the respiratory variations in pressure in the pulmonary circuit.

Some doubt has long existed whether or not the lung vessels possess vasomotor fibers. The direct evidence that seems to favor their existence is as follows: (1) The histological demonstration of nerve fibers and plexuses surrounding these vessels (Retzius, Berkley, Karsner); (2) the demonstration by perfusion experiments that adrenalin constricts the lung arterioles (Plumier, Wiggers). 


\section{LITERATURE.}

Berkley. Johns Hopkins Hosp. Reports, 1894, iv, 240.

Cloetta. Arch. f. exper. Path. u. Pharm., 1911, lxvi, 409.

“ Arch. f. exper. Path. u. Pharm., 1912, lxx, 407.

Fredericq. Travaux du Lab. de Loon Fredericq, 1880, i, 3.

de Jager. Arch. f. d. ges. Physiol., 1879, xx, 426.

Karsner. Jour. Exper. Med., 1911, xiv, 322.

Plumier. Arch. internat. de Physiol., 1904, i, 176.

Retzius. Biolog. Untersuehungen, 1893, v, 41.

Tigerstedt. Ergebn. der Physiol., 1903, $\mathrm{II}_{2}, 528$.

Wiggers. Am. Jour. Physiol., 1912, xxx, 233; 1914, xxxiii, 1, 13; 1914, xxxv, 124. " Jour. Pharmacol. and Exper. Therap., 1909, i, 341. 


\section{H A P'T E R VI.}

\section{THE RESPIRATORY VARIA'TIONS OF AR'TERIAL PRESSURE.}

The observation that blood-pressure undergoes periodic variations with inspiration and expiration dates from the experiment of Stephen Hales in 1733. The relations of the pressure changes to inspiration and expiration, as well as their causes, though frequently reinvestigated, still remain a subject for research and discussion. In the following table have been gathered, after the clever manner suggested by Lewis, the findings of various observers on different animals.

Respiratory Variations in the Dog.

Year. Investigator. Insp. Exp.

1860 Einbrodt. +

1881 de Jager. $\quad++\quad+-$

$\begin{array}{llll}1882 & \text { Fredericq. } & -+ & + \\ 1908 & \text { Lewis } & - & + \\ & & -+ & + \\ & & +- \\ 1910-12 & \text { Wiggers. } & + & - \\ & & -+ & +-\end{array}$
Respiratory Variations in the Rabbit.

$\begin{array}{cccr}\text { Year. } & \text { Investigator. } & \text { Insp. } & \text { Exp. } \\ 1882 & \text { Fredericq. } & - & + \\ 1882 & \begin{array}{c}\text { Moreau } \\ \text { and }\end{array} & - & +\end{array}$

Lecrenier

$\begin{array}{cccc}1883 & \begin{array}{c}\text { Legros and } \\ \text { Griffe } \\ \text { de Jager }\end{array} & - & + \\ 1886 & \cdot & -+ & +- \\ & & +- & -+\end{array}$

Respiratory Variations in Man.

\begin{tabular}{|c|c|c|c|c|}
\hline Year. & Author. & Insp. & Exp. & Method. \\
\hline 1855 & Vierordt & - & + & Sphygmograph. \\
\hline 1865 & Wolff & - & + & “ \\
\hline 1872 & Landois & - & + & “ \\
\hline 1876 & Riegel & - & + & “ \\
\hline 1877 & Sommerbrodt & - & + & “ \\
\hline 1877 & Klemensiwicz & $\begin{array}{r}+ \\
-+\end{array}$ & +- & $\begin{array}{l}\text { Sphygmograph and respira- } \\
\text { tory tracing. }\end{array}$ \\
\hline 1881 & Marey & $\overline{+}$ & \pm & $\begin{array}{l}\text { Sphygmograph and respira- } \\
\text { tory tracing. }\end{array}$ \\
\hline 1881 & Schweinburg & $\overline{+}$ & \pm & $\begin{array}{l}\text { Sphygmograph and respira- } \\
\text { tory tracing. }\end{array}$ \\
\hline
\end{tabular}




\begin{tabular}{|c|c|c|c|c|}
\hline & RESPIRATORY & VARIAT & NS in & (Continued.) \\
\hline $\begin{array}{l}\text { Year. } \\
1883\end{array}$ & $\begin{array}{l}\text { Author. } \\
\text { Le Gros and } \\
\text { Griffè }\end{array}$ & $\begin{array}{c}\text { Insp. } \\
-\end{array}$ & $\stackrel{\text { Exp. }}{+}$ & $\begin{array}{l}\text { Method. } \\
\text { Sphygmograph and respira- } \\
\text { tory tracing. }\end{array}$ \\
\hline 1889 & $\begin{array}{l}\text { Wertheiner } \\
\text { and Meyer }\end{array}$ & - & + & $\begin{array}{l}\text { Sphygmograph and respira- } \\
\text { tory tracing. }\end{array}$ \\
\hline 1895 & Mosso & + & - & Sphygmomanometer. \\
\hline 1902 & Mackenzie & - & + & Sphygmograph. \\
\hline 1908 & Lewis & $\begin{array}{r}+ \\
+ \\
+ \\
-+ \\
+-\end{array}$ & $\begin{array}{r}\overline{+} \\
-\overline{-} \\
+\overline{+}\end{array}$ & Suspended sphygmograph. \\
\hline 1910 & Groedel & - & + & Uskoff sphygmomanometer. \\
\hline 1912 & $\begin{array}{l}\text { Erlanger and } \\
\text { Festerling }\end{array}$ & - & + & Erlanger sphygmomanometer. \\
\hline 1914 & $\begin{array}{l}\text { Wiggers } \\
\text { Systolic } \\
\text { Diastolic }\end{array}$ & $-\overline{+}$ & $\begin{array}{l}++ \\
+-\end{array}$ & $\begin{array}{l}\text { Erlanger sphygmomanometer } \\
\text { and optical registration. }\end{array}$ \\
\hline
\end{tabular}

It is evident from this tabulation that there is no great uniformity of opinion as to the nature of the respiratory variations of pressure in different or even in the same species of animal. As Lewis has pointed out, current text-books unjustifiably present the results obtained by Einbrodt in the dog and Klemensiewicz in man. The great discrepancy is probably traceable to several factors: (1) that the mercury manometer does not always follow the changes in trend of pressure promptly, thus increasing some oscillations by resonance and making others smaller by interference of waves, (2) that these variations are due to different causes in different animals and man.

Many investigators, observing that variations in the cardiac cycle occur from beat to beat, have attributed the changes exclusively to this factor, while others have assigned the chief effect to the mechanical influence of the phases of respiration. It is more probable that both play a part. Fredericq was probably the first to describe clearly the relative importance of each of these two factors.

He pointed out that variations in blood-pressure occurred in the dog during inspiration and expiration whether the heart was rhythmic or arrhythmic, with this difference: in the former case, the blood-pressure fell during inspiration, while in the latter it rose.

He also pointed out that in animals in which no cardiac variations occur (rabbit) the pressure always falls during inspiration.

He therefore concluded that the mechanical influence of respiration tends to reduce arterial pressure during inspiration, but that in the dog these changes are often overbalanced by an inspiratory 
acceleration of the heart. Inasmuch as he observed a similar variation of rhythm in his own pulse, he believed that cardiac rhythm determined the direction of human blood-pressure. This seems to be essentially correct. In the rabbit, observers generally report a simple fall during inspiration and a rise during expiration. In a total of 97 experiments on dogs analyzed with reference to this point, the writer has found that, without exception, both systolic and diastolic pressures fall during inspiration and rise during expiration $^{1}$ as long as the successive heart cycles are of equal length.

Since these variations disappear promptly during temporary apnea and reappear during the first respiratory movement, it seems safe to assert that they are caused by the mechanical influence of respiration. When marked variations in cardiac rhythm are present, however, so that the heart accelerates during inspiration, then the mean pressure rises during this phase and falls during expiration. In such cases the change in heart rate overbalances the contrary effect on arterial pressure and dominates the mean pressure curve. This may be a complete or partial domination, as shown in the following scheme:

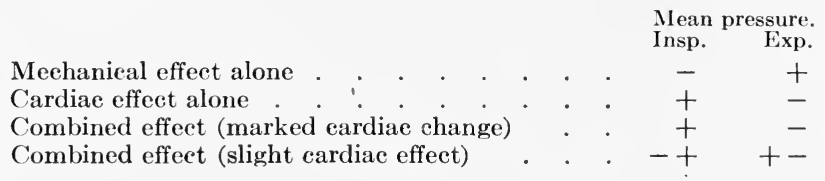

This, however, only imperfectly relates the pressure effects due to the heart rhythm variations, as the inference might be drawn that both systolic and diastolic pressures follow the directional change of mean pressure, which is not the case. A shortening of a cycle as shown in Fig. 16 increases the diastolic and decreases the systolic pressure of the beat following. A lengthening of a cycle causes an elevation in the systolic pressure and a fall in the diastolic pressure of the next beat.

It seems, however, that when the mechanical effect of inspiration dominates the pressure, both systolic and diastolic pressures fall (Fig. 16), whereas, if the acceleration of the heart is predominant during inspiration, the diastolic pressure rises and the systolic falls.

The results of most investigators seem to support the idea that in man a simple fall of pressure occurs in inspiration. This, together with the observations that, in a large series of healthy individuals the rhythmic variations of heart rate are not very great indicate

${ }^{1}$ In these experiments the pressures were recorded in part with Hürthle membrane or spring manometers and partly by optically recording manometers. 


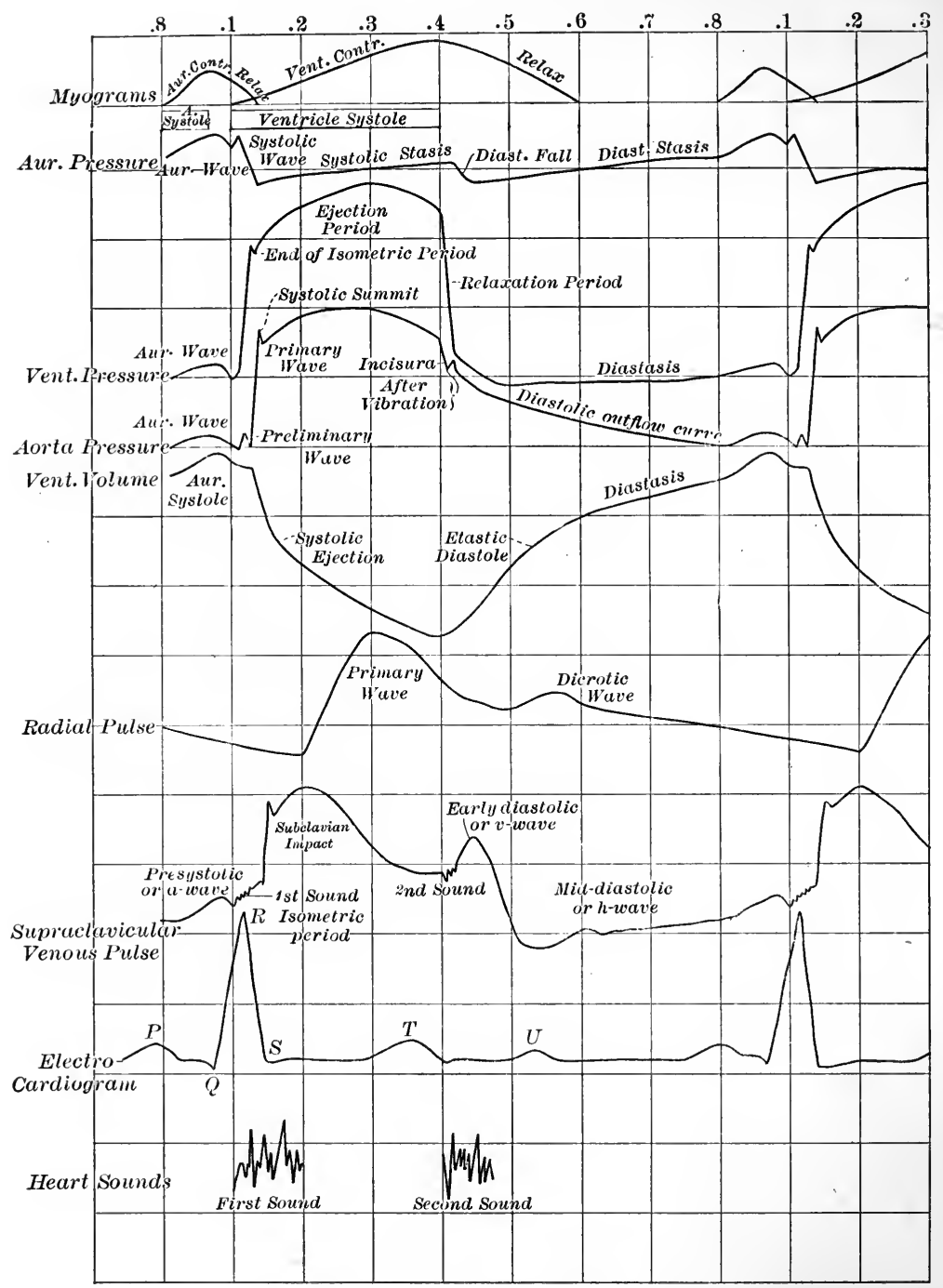

FIg. 25.-Composite figure, showing temporal relations of events in the cardiac cycle, based on most recent work. 
that the arterial pressure is largely influenced by the mechanical action of respiration and but little by the variation in heart rate. As different polyphasic relations have often been obtained, however, it is necessary to assume that in man, too, the variations in rate may disturb the rhythm established by the mechanical influence of respiration. No attempt to separate these influences can be made unless we are capable of obtaining evidence of the variations of systolic and diastolic pressures in consecutive beats. Such qualitative variations, the writer believes, can be secured by the method proposed by Erlanger and Festerling. Applying this, the writer has found that variations in cardiac rate play some part in determining variations of systolic and diastolic pressure in man. In the minority of cases, they are the dominant influence, the majority showing that the mechanical effect of respiration controls the inspiratory fall of systolic and diastolic pressures. There is, in short, no standard type of respiratory variation in man. The cases range from those in which respiration governs the change of pressures entirely, through those in which more or less complicated mixtures of heart rate and respiratory influences intermingle, to those in which extreme cardiac variations alone determine the pressure changes.

The reason that a temporary shortening of the cardiac cycle causes a fall of systolic and a rise of diastolic pressure is readily found, for, while the output is decreased in systole the time of peripheral outflow from the arteries is decreased most in diastole.

Why inspiration should cause a fall of both systolic and diastolic pressures when the heart rate is constant is not entirely clear. Only two possibilities exist: (1) that the decreased negative pressure within the thorax lowers the pressures within the large intrathoracic arteries and (2) that the output of the left ventricle is decreased. Since no absolute proof is available as to which of these two factors is concerned a few probabilities may be weighed. If the output per beat is lessened such a change occurs in spite of the fact that the effective pressure in the left auricle is slightly increased (Wiggers). It can then only be attributed to the restraining effect of a negative pressure on the contraction of the ventricle. This seems scarcely probable since the pressure curves of the right heart give no evidence of a decreased output during this respiratory phase (Wiggers), and the volume curves of the ventricles are not influenced by slight changes of pressure around them. With the evidence before us, it seems that the fall of pressure during inspiration is probably produced in a very simple fashion, namely, by an influence of the greater negative intrathoracic pressure on the intrathoracic arteries. 
Such an effect is physically possible, the old idea that the thickwalled vessels are not susceptible to pressure changes having been shown erroneous by the fact that, if the aorta is tied above and below, slight variations of pressure within the closed chest are transmitted to the lumen of the artery.

\section{LITERATURE.}

Fredericq. Arch. de biol., 1882, exi, 55.

Hales. Statical Essays, 1731-33, ii, 33.

Lewis. Jour. Physiol., 1908, xxxvii, 213, 233.

Tigerstedt. Ergebn. der Physiol., 1903, $\mathrm{II}_{2}, 560$.

Wiggers. Jour. Exper. Med., 1914, xix, 1.

" Am. Jour. Physiol., 1914, xxxiii, 13. 


\section{SECTION II.}

CHA P TER VIII.

\section{THE AR'TERIAL PUISE.}

Definition.- The pulse in any part of the arterial system may be defined, as the intra-arterial pressure variations during systole and diastole, transmitted to the elastic walls, and producing in them an expansion and elongation.

When blood is suddenly thrown into the aorta during systole, it is accommodated, partly by moving the entire arterial column onward at a greater velocity, but largely by distending the arterial wall under the pressure generated by the ventricle. As this increase in pressure is transmitted toward the periphery, the arterial distention is also communicated from one segment of an artery to the next peripheral segment in the form of a wave, the velocity of which is entirely independent of the velocity of the blood flow. This is evident from the fact that the pulse wave is transmitted peripherally at a velocity of from six to ten meters per second, while the average velocity of the blood flow is probably about threetenths of a meter per second. The relation of the pulse wave and the blood flow may be compared to an impact transmitted through a train of cars in motion; the rate at which the impact travels is entirely independent of the speed of the train and may, in fact, occur in an opposite direction.

The Conversion of the Central to the Peripheral Pulse.-As the variations of pressure in the aorta and central arteries follow each other rapidly and require manometers of high vibration frequency to record them, so also the oscillations of the walls of the central arteries are complicated and difficult to record. In fact, it is only since the introduction of the "segment capsule" by Frank that it has been possible to record the details of these pressure variations. This useful recording device is shown in the lower right hand part of Fig. 26. It consists of a capsule, $S$, covered by a rubber membrane. The edge over which the rubber is stretched is not circulàr but has one straight side, representing a chord of the circle. Upon the rubber is fastened a small trapezoidal plate which moves upon the chord side of the capsule and on this, in turn, is cemented 
a tiny mirror, $R$, so that its diameter also pivots upon the chord side of the capsule. The entire capsule is held in a mount, $T$, resembling a miniature cannon carriage which permits a lateral adjustment by the screw, $V$, and a vertical adjustment by the screw, $W$.

That these capsules may be rendered available for bedside service in the hospital, they have been mounted, together with a Nernst projection lamp and a photokymograph, on a heavy oak table (Fig. 27) which can be wheeled around the ward and supported on felt strips while the records are taken. The capsules are placed on an upright support in the same vertical plane as the slit of the

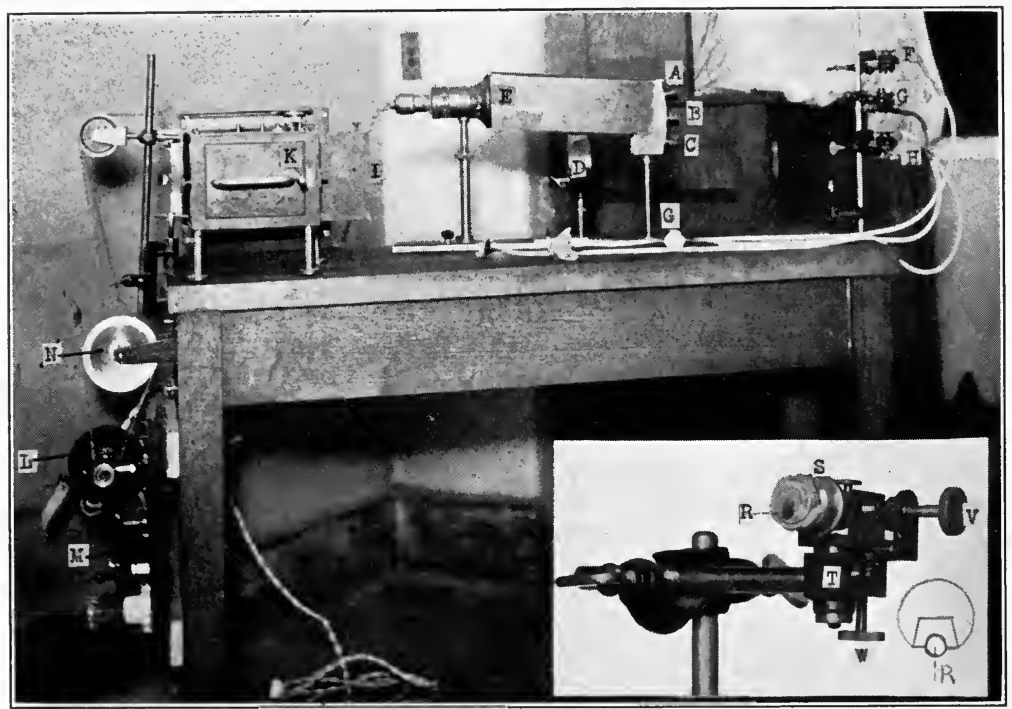

Fig. 26.-Photograph showing the mounting of Frank's segment capsules with photokymograph and Nernst projector for hospital use. Small photograph in lower right hand corner shows details of segment capsules.

photokymograph. Upon the mirrors of these three capsules $(F, G, H)$ a band of light supplied by the filament of the Nernst lamp $(E)$ is focused by three lenses $(A, B, C)$ and reflected to the lens of the photokymograph $(K)$. The bromide paper within the kymograph is moved by a quietly running Eck motor $(L)$, the speed of which is controlled by a rheostat $(M)$.

The records thus obtained from the large central arteries, such as the subclavian, show all the essential details already described as characteristic for the aortic pressure curve (pp. 52, 54, Figs. 8 and 9). The "central pulse," as the pulse in the large arteries near the heart is called, shows (Fig. 27) first two preliminary vibrations; 
one due to auricular systole $(a-b)$, the other, to the isometric rise of tension in the ventricle $(b-c)$. These are followed by a sharp primary oscillation, $(c, d, e) 0.013$ to 0.02 in duration and beginning at the ejection of blood into the aorta. It is due to the fact that in consequence of the sudden ejection the arterial column has been set in vibration. After this vibration, the arterial curve follows the intra-ventricular pressure for now ventricles and arteries are a common cavity. First, the pressure rises $(e, f)$, thereafter reaches a more or less definite summit and then falls gradually during the rest of systole $(f, g)$. At the beginning of the ventricular relaxation (diastole), there is a rapid backward movement of the blood toward the heart causing the pressure to fall suddenly, thus creating the "incisura" of the central pulse $(g, h)$. Several after vibrations of the valves and blood column $(k)$, recognized over the chest as heart sounds, follow. The pressure then falls smoothly except for a few slight oscillations due, no doubt, to reflections from the peripheral bifurcations of arteries (Frank).

These complicated series of pressure variations present in the aorta and large arteries are modified in their peripheral transmission by friction and interference with reflected waves (Frank). Or, one may state the case differently by saying that the vascular system represents a manometer system the ability of which to transmit the pressure variations in the central arteries faithfully to the periphery becomes less and less as the length of the column increases (Weber).

The changes actually noted as we pass step by step to the peripheral vessels are: (1) The preliminary and primary oscillations are damped and obliterated, the sharpness of the incisura is reduced and finally replaced by a rounded dicrotic dip and elevation; (2) the upstroke is delayed and becomes more gradual, the tops are more rounded and the amplitude smaller until in the smallest arteries and capillaries the pulse is entirely obliterated. The changes occurring in the pulse in its transmission from the subclavian to the radial artery are well shown in Fig. 27.

The part that reflected waves play in the production of the dicrotic notch and wave in the peripheral pulse has been much discussed, and while it is still open for debate, we are much nearer a solution as a result of recent investigations. To consider the question clearly, it should be recalled that whenever the fluid in any branching system is set in motion, reflected waves are sent back from the periphery. That such reflections are probably present in the arterial system can "a fortiori" be inferred from the fact that the presence of the blood corpuscles favors such a reflection. Without entering into the contradictory evidence of the past (for which see Tigerstedt), it may be said that it has been firmly established that reflected waves cannot primarily be respon- 
sible for the dicrotic notch and wave, for in optical records of the aortic pressure, the incisura goes hand in hand with valve closure and when reflected waves appear at all they occur much later in diastole. It would seem, therefore, that the dicrotic notch and wave are the representatives of the incisura and after-vibration in the peripheral pulse.

Frank, however, finds difficulty in interpreting the dicrotic wave as merely the incisura and after-vibration modified in transmission, for the dicrotic wave becomes larger toward the periphery and all other waves submitted to the same frictional influence, become smaller. He, therefore, believes that its amplitude is augmented

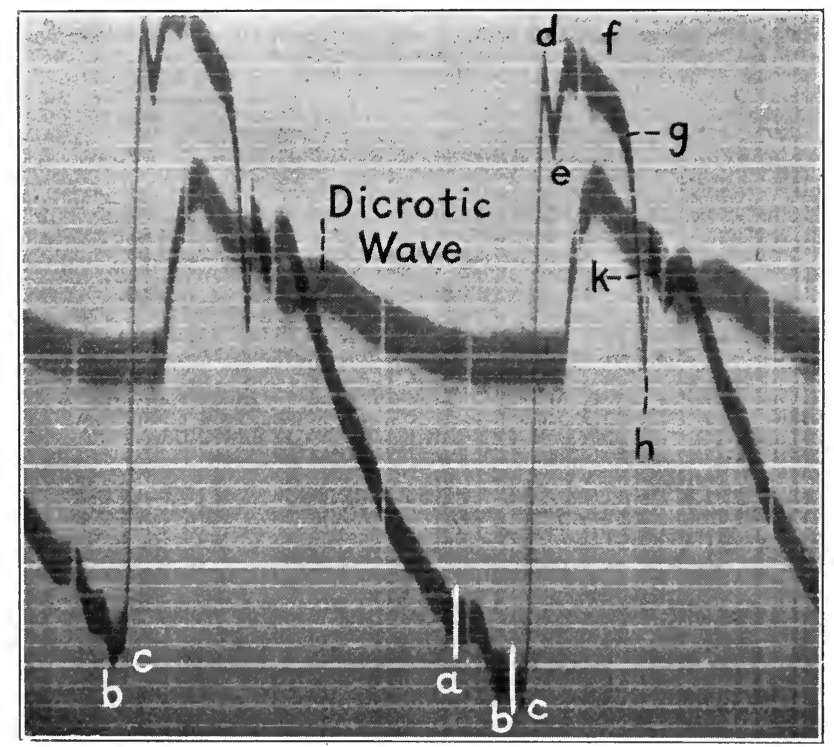

Frg. 27.- Tracing showing the difference between the subclavian (central) and radial (peripheral) pulse in man. The delay of the radial is well shown.

by a resonance effect with other vibrations reflected from the periphery.

Out of this discussion grow two very important practical facts: (1) We must distinguish between the central pulse, as it is recorded from the subclavian or the lower carotid arteries, and the peripheral pulse as it is recorded from the radial artery; (2) on account of the poor transmitting ability of the arterial system, the peripheral pulse reproduces only inaccurately the pressure changes established in the aorta and consequently it may be anticipated that its diagnostic value is correspondingly less. The study of the carotid and subclavian pulses should, on the other hand, receive greater attention than has hitherto been accorded it. 


\section{METHODS OF RECORDING THE PULSE.}

The methods devised for registering the pulse in various regions may be reviewed according to the principles upon which they are built.

Wrist Sphygmographs.-A sphygmograph is an instrument in which a button is pressed on the skin over an artery by a spring, the movements of which are communicated by a lever system to moving paper. It is apparent that in principle the sphgymograph is a tension recording device and comparable to a membrane manometer, the elastic arterial wall corresponding to the membrane.

Patterns of Wrist Sphygmographs.- The first sphygmograph utilizing a spring was that devised by Marey (1857-1860). In

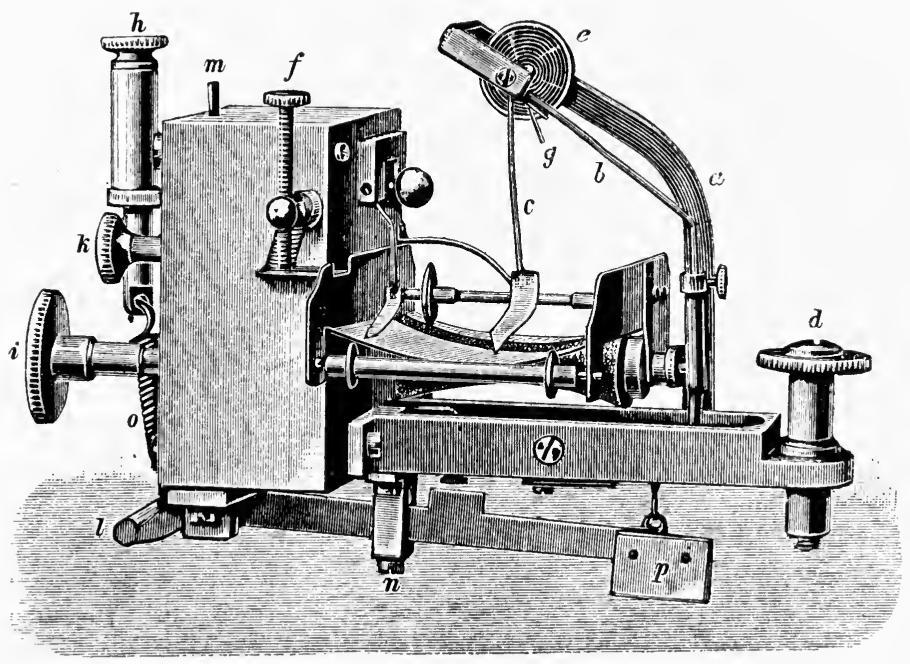

FIG. 28.-Perspective view of Frank-Petter's sphygmograph.

this instrument the movements communicated to the spring are recorded by a simple lever. Von Frey subsequently improved upon this type of sphygmograph by a better construction of the lever. It remained for Dudgeon to enhance the compactness of the instrument, as well as its sensitiveness by supplanting the simple with a compound lever which writes without an arc on a horizontal surface. The subsequent instrument of Jacquet based on the same principle was a practical advance in the sense that the instrument is more firmly supported on the wrist and has a very good time marker.

The flat spring remained in use until Frank and Petter (1908) devised their sphygmograph (Fig. 28). In this apparatus the button 
$(p)$ is pressed upon the artery by a spiral spring (o) which is applied near the axis of the lever $(l)$. The tension of the spring and hence the pressure of the button can be regulated by the screw $(h)$. The movement of the button is transmitted to a double lever $(b c)$. The arm $b$ pivots on needle points, but the arm $c$, which is held in fixed conical bearings, is equipped with a spiral spring under slight tension so directed that it pulls the lever forward as soon as the button moves up. The paper moves on a plate curved to counteract the arcs written by lever $c$.

In 1910, Jacquet entirely rebuilt his sphygmograph utilizing all the essential ideas of the Frank-Petter apparatus except that the old lever system in fixed bearings and the straight paper lead were retained. In describing tracings it is therefore desirable to designate whether they were taken with the old or the new form of Jacquet sphygmograph so that their value may be correctly estimated.

Critique of Wrist Sphygmographs.--Since the sphygmograph is based upon the same principle as the spring manometer, it is possible to analyze its efficiency upon the same basis. The ability with which it accurately follows the pressure variations is determined by its inherent vibration period $\left(T^{\prime}\right)$ or its frequency $(N=1 / T)$ and by its damping which may be estimated from the logarithmic decrement of the vibrations. The periodicity is determined by the formula $T=2 \pi \sqrt{\frac{M^{\prime}}{E^{\prime}+e}}$ in which $M^{\prime}$ equals the calculated effective mass $E^{\prime}$, the elasticity coefficient of the spring and $e$, that of the tissues and artery. The efficiency of an apparatus depends not only upon its inherent vibration rate, however, but also upon the magnitude with which the pressure changes are reproduced. Frank has therefore expressed the efficiency by the formula $G=\varepsilon N^{2}$ where $\varepsilon$ represents the sensitiveness of the writing point and $N$ the vibration frequency.

By applying the theoretical formulæ evolved, Petter was able to determine the relative value of the different instruments. The figures shown in the following table, extracted from a more complete compilation by Petter, show that in none of the sphygmographs constructed upon experimental principles does the vibration rate reach the required level of thirty-two per minute (Frank). It is further of interest to note that, with the exception of von Frey's, each successive model of the sphygmograph following Marey's, in becoming more convenient for practical use, has decreased in efficiency. The apparatus of Frank and Petter alone seems to have the required vibration frequency. Its efficiency, as compared with the Jacquet (old form), is as $3000: 150$. These facts are indicated in the following table: 


\begin{tabular}{|c|c|c|c|c|}
\hline Apparatus. & $\begin{array}{l}\text { Efficiency, } \\
\text { in } \mathrm{G}=10^{-5} \text {. }\end{array}$ & $\begin{array}{c}\text { Vibration. } \\
\text { N. }\end{array}$ & $\begin{array}{l}\text { Damping } \\
\text { constant. } \\
\mathrm{K} \text { in } 10^{3} .\end{array}$ & $\begin{array}{l}\text { Magnification } \\
\text { of lever. }\end{array}$ \\
\hline Marey (first model) & . 300 & 13.0 & $6-20$ & 50 \\
\hline von Frey . & 360 & 11.0 & $9-35$ & 90 \\
\hline Dudgeon & 150 & 9.2 & $4-20$ & $50^{\circ}$ \\
\hline Jacquet (old) & . 150 & 7.0 & $35-100$ & $100-140$ \\
\hline Frank-Petter. & . 3000 & 32.0 & $.5-3$ & 50 \\
\hline
\end{tabular}

The records obtained with all instruments except the FrankPetter model therefore require correction. The distortion produced by different instruments was also investigated by Petter through the use of artificial pulses of known form. Thus, the curves recorded by the Dudgeon (Fig. 29, A) and the curve of known form rose almost simultaneously from the base line and returned practically together. For time determinations the Dudgeon would therefore be sufficiently exact were it equipped with a time recorder. Neither the height nor the contour, however, are correctly reproduced.

$A$

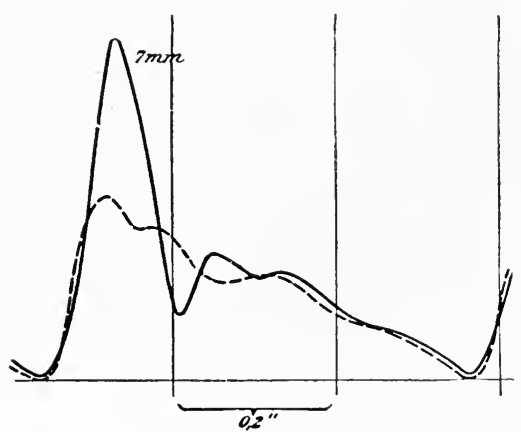

$B$
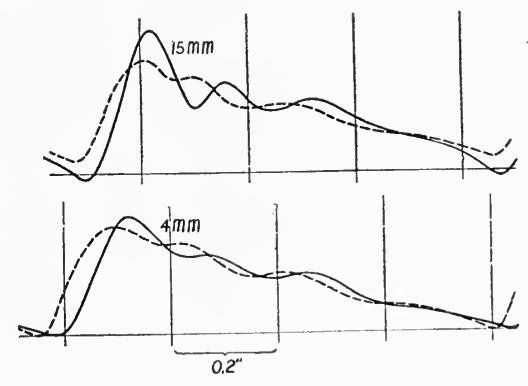

FIg. 29.-Experimental tests of the efficiency of sphygmographs. A, Dudgeon record; $B$, Jacquet records eompared with true eurves (broken line). (After Petter.)

The dicrotic notch appears relatively too low and the elevation begins too late. This is due to the fact that its vibration period is low and its damping very slight. (See Table.) It is evident that the Dudgeon apparatus is not reliable in giving the correct shape of the radial pulse.

The records obtained with Jacquet's apparatus (old model) are even worse (Fig. 29, B). No true pulse curves are recorded. The rise and return to the base line are delayed, the rise is steeper and exceeds the true height. The dicrotic wave is not recorded at all, but is replaced by a series of after-vibrations, which have deceived investigators since the instrumental period happens to correspond closely to that of the dicrotic wave.

In testing the Frank-Petter apparatus, the curves were found to differ only in that a few inherent vibrations were present in the upstroke. 
There is, however, a simpler and more practical way of testing the efficiency of an instrument which every user of an apparatus can readily apply. This procedure, the introduction of which we owe to von Frey, consists in recording pulse tracings with different pressures of the button. If the apparatus reproduces the pulse curve accurately, only the amplitude and not the shape of the curve should alter, whereas, if this is not the case, the curve will have a different form with every pressure. The latter, it is almost universally recognized, is the case with the instruments in common use. The writer has applied von Frey's criterion to the new form of Jacquet. In the case of this instrument it is found that the degree of pressure determines the character of the pulse form. This, in agreement with the findings of Veil and Noltenius indicates that by incorporating the old form of fixed axis bearings and a different style of lever, the efficiency of the instrument still remains low.

Technic of Taking Sphygmograms.-Owing to the fact that pulse curves of different form, as well as amplitude, have been obtained by different pressures of inadequate sphygmographs, it has frequently been debated at what pressure the most correct curves are obtained. From what has preceded it is evident that the shape and contour of curves recorded with an accurate instrument do not change with varying pressure. Ohm has succeeded in recording true curves without any pressure by cementing a small mirror over the radial artery. Those forms of sphygmographs, the vibration frequencies of which are too low, do not correctly reproduce the oscillations under any pressure. Therefore, the only question of interest is, at what pressure are the records most nearly correct in contour? It is generally believed that the button applied to the artery follows the pressure variations best when the intra- and extra-arterial pressures approximate each other. It is during such pressure relations that the largest oscillations occur, hence it is generally assumed that the greatest amplitude of oscillation is accompanied by the curve of most correct form. This, however, is fallacious since, as the excursions become larger, the incidence of lever throw and inherent oscillations become greater and the deviation from the correct curve, more pronounced. This is clearly shown in the two tracings of Fig. 29, $B$, in the upper curve of which the amplitude of the original record was $15 \mathrm{~mm}$. and that of the lower $4 \mathrm{~mm}$. The truer form is given when the records are of smaller amplitude. Taking curves of larger amplitude is therefore not to be encouraged with instruments of low vibration frequency.

The question as to the proper tension of the sphygmograph band has also come up for discussion among investigators. Thus, Hirschman pointed out the danger from venous stasis if the band was too tight. Mackenzie believes that the use of the rigid fixation for a sphygmograph is entirely wrong in principle and has used 
instead an elastic band. Lewis has sought to obviate the defect of the ordinary sphygmograph by substituting for it a suspended sphygmograph. The reason for selecting such a fixation lies in the fear that the varying filling of the venæ comites may affect the pulse curve (Hirschman, Hill, and others). It is questionable in view of the work of Frank and Petter, whether the variation in the venous volume plays any role in determining the form of the curve. However this may be in the rigid fixation, the elastic fixation or suspended application has the decided drawback that the body of the apparatus itself may be set in motion and add to the extraneous oscillations. It is, in fact, theoretically desirable, as Petter emphasizes, to adjust the apparatus so that the body is absolutely rigid as compared to the artery beneath. According to Petter, even the elasticity of the ordinary bands of some forms of sphygmographs adds materially to their inaccuracy.

Transmission Sphymographs. - In the transmission sphymograph (Fig. 30) the movement of a spring and button similar to those of the direct sphygmograph is communicated to a larger tambour covered with rubber and the compression of the air thus produced is communicated to a second tambour patterned more or less after the well-known form of Marey. Marey, Grumach, Knoll, Edgren and Mackenzie have employed apparatus of this kind. The practical advantage consists in the fact that the pulsations can be recorded simultaneously with other pulsations and that it is adapted to determine the velocity of the pulse waves.

No scientific critique of these forms of apparatus has been evolved. It may be said, however, that with the best possible construction the efficiency of the transmission apparatus is less than that of the apparatus with direct lever transmission. The inertia of the recording tambours is the chief drawback. In the Mackenzie polygraph, the lever of which is armed with an ink pen, the tambour and lever have a vibration frequency of 4.5 per second. In the tambour supplied with the portable polygraph of Zimmerman, the inherent frequency was found to be 5.8 per second. In the Jacquet polygraph the vibration frequency is 6.5 per second. (Personal Tests.)

Optical Transmission Sphygmographs.-To improve the efficiency of this form of sphygmograph, Frank substituted a segment capsule (see page 101) for the recording mechanism. With this is connected a piece of soft rubber tubing tied on one end and fastened over the artery by a pressure clamp. By this apparatus the nature of the pulsation is reproduced in trustworthy fashion.

The writer has found the sphygmograph shown in Fig. 30 very satisfactory. A small button $A$, attached to an $\mathrm{L}$-shaped lever, is pressed upon the radial by an adjustible, spiral spring $B$. The lever works in free axes, being held by the spring very much as 
in the Frank-Petter sphygmograph. As the button rises, the movements are transmitted to a capsule $C$, covered with very light rubber and communicating with a segment capsule $D$. It has the advantage over the method proposed by Frank that the pulsation is obtained from a single spot and that definite tension can be employed.

This list by no means includes all the tension devices described for recording the radial pulse. Their number is legion. The attempt has here been made to present only those instruments which are in common use or the employment of which is likely to be considered in practical work. At the end is given a partial

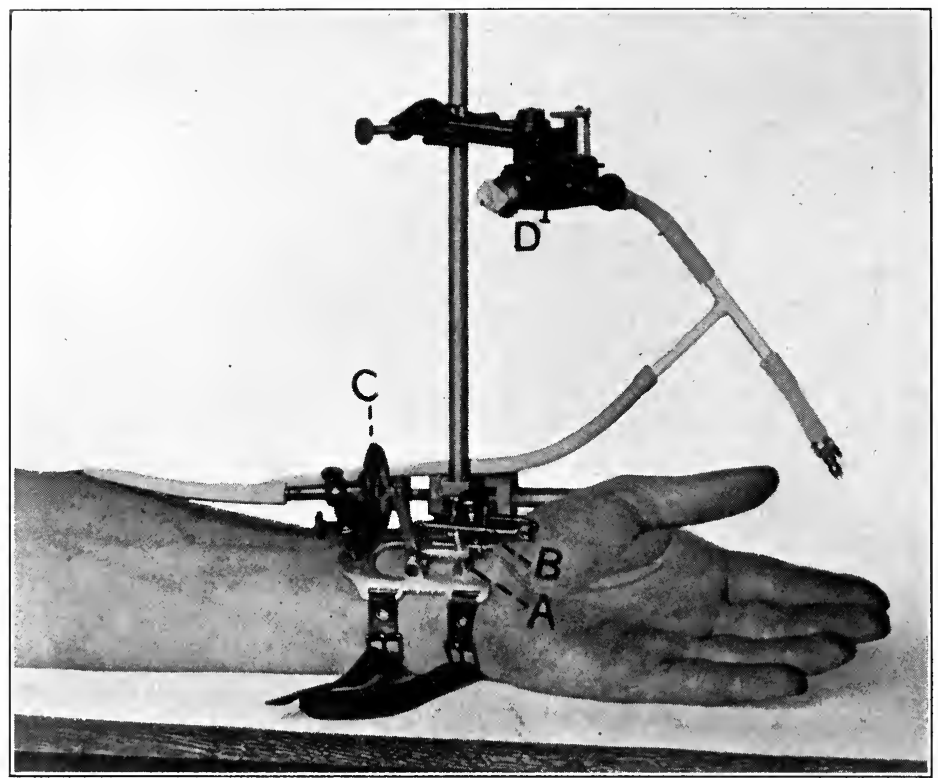

Fig. 30.-Radial Transmission-sphygmograph and Frank's segment capsule.

list of references dealing with some of the rarer forms of pulse instruments not considered in this volume.

Volumetric Pulse Registration.-In contrast to the methods which register the pressure variations in the artery are those which measure the volume changes of the arterial wall during systole and diastole. Theoretically, such apparatus should be so adapted that its application causes no changes in the oscillations.

Cup-Tambour Method.-When an open cup, or better, one loosely covered with thin rubber dam is firmly pressed to the skin over an artery as the carotid or subclavian and connected to a recording tambour, a pulse wave is obtained which represents the expansion 
of the artery below. This method is commonly employed in polygraphic work. In determining the transmission time of the pulse it is often combined with the radial transmission sphygmograph. It is applicable to the subclavian, carotid, and temporal arteries. Since the accuracy of the method depends largely upon the recording tambour, and since the vibration period of the ordinary tambour is inadequate to record central pulse details, a segment capsule has been substituted for the tambour by various workers. By this apparatus, Frank, Frieberger, Veil and the writer have obtained pulsations from the subclavian which reduplicate all the details obtained from the aorta by optical manometers, thus establishing experimentally the efficiency of the apparatus (Fig. 27). In obtaining these pulses it is necessary in order to avoil complication with venous tracings to exert some pressure so that venous blood may not enter or leave the space covered by the receiver.

Finger Plethysmograph. - If a finger is inserted through a properly fitting rubber cuff into a small glass tube, and this in turn is connected to a delicate tambour, piston recorder or preferably an optically recording capsule, a curve of the peripheral pulse may be readily obtained, the contour of which gives evidence that a correct registration has taken place.

Sphygmoscopic Pulse Tracings.-The sphygmoscope is a device in which a high oscillatory pressure is prevented from acting upon a delicate membrane by interposing a heavy membrane or ball, enclosing this in a chamber and allowing its pulsations to be transmitted to the recording tambour. The arm-bag, rubber ball, and tambour of the Erlanger and Uskoff apparatus (see page 200) represent such a sphygmoscope system.

Sphygmomanometer Pulse Tracings.-Tracings are frequently taken, especially in polygraph work, from the brachial artery by applying a cuff of an Erlanger sphygmomanometer or an Uskoff sphygmotonograph, inflating it by a variable pressure and recording the pulse so obtained. The shape of the curve varies with the external pressures and consequently cannot be considered to be accurate at any pressure (cf. Fig. 58). The principle of construction of both of these apparatuses is opposed to their yielding at any pressure an accurate picture of the pulse form. In the first place, the periods of the recording tambours are entirely inadequate. This is not the only drawback, however, as has been shown to the writer by substituting a segment capsule for the recording mechanism. The method cannot therefore be used for determining the contour of pulse tracings, but may be of value in roughly establishing the time relations. 


\section{COIMPARATIVE VALUE OF PALPATION AND SPHYGMOGRAPHIC STUDY OF THE PULSE.}

The relative value for the physician of recording or palpating the pulse has been frequently discussed, to the disadvantage, it must be said, of the recording method. For palpation the radial artery is usually selected. In favor of palpation may be brought forward the advantages: (1) that it is simple in execution as compared with the employment of pulse tracing machines; (2) that it allows an estimate of the character of the arterial wall; ${ }^{1}(3)$ that it permits as true an estimate of the amplitude as the sphygmograph which has no quantitative value but is governed entirely by the adjustment of the instrument; (4) that it allows a truer determination of a collapsing pulse than the sphygmograph, which often indicates the presence of one where none exists, or, vice versa, shows the presence of a normal pulse when a collapsing pulse is present. In favor of the sphygmographic tracings it has been argued: (1) that they are indispensable in establishing accurately the variations in successive cardiac cycles, which it is necessary to know in order to recognize the nature of irregularities, and (2) that they show detailed changes of the ascending limb, top and dicrotic wave which are associated with characteristic disturbances. Among these may be mentioned the anacrotic pulse in stenosis, the sustained top in high tension, etc. On the whole, however, the use of the sphygmograph, except for time determinations, has not been considered of great value either by clinicians or physiologists. Cabot speaks of the apparatus as "an interesting little toy," Mackenzie states that it was expected to give information of a kind that it was incapable of supplying, von Frey regarded the practice, as commonly carried out, as "an interesting pastime," while Tigerstedt in 1893 stated that the diagnostic utilization could not be definitely determined." Mackenzie and likewise Lewis have used it to establish temporal relations.

It is evident that this common sentiment against the employment of the sphygmograph, aside from determining time relations by its aid, has grown out of the recognized inaccuracy of the apparatus. It is therefore to the greater credit of Frank and Petter that they have evolved a theoretical critique of the apparatus, in which the principles determining accuracy were defined, the shortcomings of the old form revealed and the path for the construction of better forms suggested. This resulted in introducing several forms of apparatus for recording pulse tracings accurately. This contribu-

\footnotetext{
1 The fallacy of this assumption is again well illustrated by the recent report of Lande who found no histological grounds for many diagnoses of thickened arteries made previous to death by no less a diagnostician than Romberg.
} 
tion, as yet relatively unknown, among clinical men, may be considered one of the greatest offerings from a laboratory man to the clinician in the last ten years.

In consideration of these improved forms of apparatus, it becomes necessary to reconsider our pessimistic ideas concerning the value of graphic pulse tracings.

\section{CLINICAL SIGNIFICANCE OF RADIAL PULSE TRACINGS.}

We may now analyze the ways in which the radial pulse curves are of value in determining the condition of the heart and circulation and how their study may, in turn, aid in the art of palpation. For practical purposes we may divide the results into two. classes: (1) those dealing with time relations of the pulse and (2) those dealing with its conformation.

Temporal Relations of the Pulse.-Rate.-One of the important points to be determined about the pulse is its rate. In doing this the graphic record has only the advantage that very small pulses which are not palpable may be recorded. This is because the sensation transmitted to the finger is determined largely by the suddenness of the impact, consequently, the smaller waves which are much more rounded and gradual in their rise make no impression on the tactile sense.

The pulse rate usually corresponds to the heart rate. Frequently, however, a deficit in the pulse beats exists. This occurs when the ventricles give contractions too feeble to open the semilunar valves or when the pulse wave is so weak that it does not reach the wrist (cf. Fig. 86). Such a pulse deficit, as the condition is termed, may be suspected when the pulse beats are irregular in size or uneven in rhythm and when the pulse count is low. The exact deficit may usually be determined by comparison with the apex beats when palpable, or by auscultation for the heart sounds. In this case it should be borne in mind that only a single sound occurs in cases of weak systoles unaccompanied by the opening of the semilunar valves; whereas two sounds are audible when the pulse deficit is due to an impaired transmission. A source of error readily occurs in interpreting the first sound of a feeble contraction occurring early in the diastole of the preceding beat as a reduplicated sound.

Since the pulse rate, with these exceptions, corresponds to the rate of cardiac contraction, it can give information as to the condition of the latter. To recognize clearly the nature of the information given, it is necessary to bear in mind that the bulk of evidence indicates that the beat of the heart originates within the sinus node, is transmitted thence by the His-Tawara system to the ventricles and causes their excitation. The rapidity with which 
impulses are initiated, however, and the speed with which they are conducted are modified by the central nervous system through two types of antagonistic fibers, the inhibitory and the accelerator fibers. The former pass in the vagus trunk and tend to slow the rate, the latter run in the sympathetic chain and lend an accelerating influence ( $c f$. page 27 ).

It is apparent that the heart rate may be modified as follows:

(a) Through stimulation or inhibition of the vagus system.

(b) Through stimulation or inhibition of the accelerator system.

(c) Through inherent change in the rhythm production or impulse propagation in the heart itself.

Causes of Rate Variations.-In any text-book of diagnosis are to be found tabulations more or less complete, of conditions and diseases which are accompanied by abnormal pulse rates. These need not be reconsidered here. The reasons for many of these disturbances are not as yet proven experimentally but rest largely on a theoretical basis. In a few cases experimental relations between pulse rate and other factors have been determined and the table appended represents some of these in classified form. Slowing of the heart may be due to:

A. Stimulation of the vagus centre,

1. Directly by

(a) Mechanical means, as

Rise of intracranial pressure.

Intracranial tumor pressure.

Increased blood supply.

(b) Toxic effects, as

Bile.

Lead, digitalis, adrenalin.

Products of asphyxia.

2. Indirectly by

(a) Influences from higher cerebral areas (epilepsy, hysteria).

(b) Reflexes from the digestive tract (via vagus).

(c) Reflexes from the bloodvessels and heart (via depressor).

B. Stimulation of the vagus nerve.

Pressure in neck, vagus tumors, vagus neuritis.

C. Alteration in cardiac muscle.

Toxic myocarditis (diphtheria, etc.).

Heart block.

D. Paralysis of the sympathetic (rare).

Thoracic cord lesions, pressure. 
Acceleration of the heart occurs:

A. Stimulation of the sympathetic system (goitre).

B. Depression of the cardio-inhibitory centre, resulting from:

(a) Decreased blood supply:

Anemia.

Hemorrhage.

Splanchnic dilatation.

Intestinal disturbances, baths.

(b) Drugs, toxins.

(c) Reflexes:

Exercise.

(d) Cerebral influence:

Emotion.

Fright.

C. Depression or paralysis of the peripheral mechanism, e. g., atropine.

D. Stimulation of the heart itself.

(a) Mechanical means (failing compensation).

(b) Thermal means (fevers).

(c) Toxic products (infections, drugs).

(d) Functional disturbances (tachycardia).

Pulse Rhythm.- - If the time intervals occupied by consecutive cycles are approximately equal, as in the normal pulse, where they vary only slightly with the phases of respiration, we speak of a rhythmic pulse. If they are not equal, however, the pulse is said to be arrhythmic. Although other and simpler aids now exist for clearly determining the causes of irregular heart action, it is frequently possible, when a single disturbance exists, to obtain evidence of the nature of the irregularity by a careful study of the arterial curves alone (Wenckebach).

Arrhythmias of the heart have been divided on the basis of arterial pulse curves into $(a)$ the allorhythmias and $(b)$ the complete arrhythmias. In the former there is a distinct order or periodicity in the recurrence of certain wave groups while in the latter no such order exists.

The following are some of the most common and typical forms of irregularities which are illustrated in the curves with corresponding letter in Fig. 36. The nature and causes of these irregularities as well as others of rarer and more complicated character are discussed in a later chapter.

I. Regular Arrhythmias (Allorhythmias).-A. Sinus Arrhythmia, due to a variation and alternate influence of vagal inhibition and excitation of the "pacemaker." They are characterized by a progressive increase and decrease in the length of the cardiac 
cycle. Occasionally the long pauses are so lengthened that we may speak of a temporary cardiac stand-still. When the variations occur so that acceleration falls approximately during inspiration and retardation mainly during expiration, it is spoken of as respiratory irregularity. When the variations occur regularly, or irregularly without regard to the respiratory activity, it may be designated as phasic sinus arrhythmia.

B. Premature ventricular contractions or extra-systoles - due to an excessive irritability of the ventricle or the presence of abnormal stimuli. They are characterized by the presence of a premature small wave, $x$ or the absence of a wave, $x^{\prime}$. In either case the extra contraction is followed by a compensatory period of such length that the normal and premature waves approximately equal in length two normal waves $(2 T)$. Such a pulse group is termed a full bigeminus. Each smaller wave and compensatory pause is followed by a large arterial beat $(P)$.

C. Premature Auricular Systole - due to the origin of a premature impulse in the auricle which, when transmitted to the ventricles gives a premature ventricular contraction as well. The premature ventricular contraction is characterized by a small wave $x$ or, when it occurs very early, by a pause as shown in curve $D$ at $x$. The auricular origin of the extra stimulus is evidenced by the fact that the interval following is not so long that with the preceeding beat it equals two pulse cycles $\left(i\right.$. $e$., $\left.T<T^{\prime}\right)$. Such a group has been termed a shortened bigeminus.

D. Paroxysmal Tachycardia-A sudden acceleration of the heart preceded by a few extra-systoles of auricular origin $(e . g$., at $x$. $\left.T_{2}>T x\right)$.

E. Incomplete Sino-ventricular Block (usually called $a-v$ block).This condition can be most definitely determined from the arterial pulse when the ventricle regularly fails to respond to a sinus stimulus. The rhythm is then regular but slow. When the block is irregular (e. g., $2: 1,3: 1)$ it is usually found that some short pulse wave is contained in the longer waves a definite number of times. Thus $m$ is contained twice in $n$ and three times in $o$.

II. Irregular Arrhythmias.-F. Auricular Flutter. A condition in which the auricle contracts at a very rapid rate but the ventricle responds irregularly. The arterial pulse in this condition is on the border between an allorhythmia and complete arrhythmia. On superficial inspection of the registered arterial pulse it appears quite rapid and completely irregular both in rhythm and sequence of beats. In some instances a scheme of regularity may, however, be made out, for the long wave lengths are often multiples of the shortest waves but the correspondence in these cases is not very exact so that it seems preferable to class this with the completely irregular pulses. 
G. Complete Heart Block-Due to the complete blocking of impulses from the sinus region and the establishment of an independent ventricular rhythm. On superficial inspection the pulse appears to be slow but rhythmic. Careful measurements show, however, that the intervals vary considerably and that it really represents a totally arrhythmic condition. Its likeness to a perfect rhythm is enhanced by the fact that the waves are almost of equal height.

H. Auricular Fibrillation-A fibrillation of the auricles in which the ventricles beat in a rapid and absolutely irregular manner. The pulse is irregular in size and rhythm and the amplitude of beats bears no relation to the previous diastoles as is the case in extrasystoles.

I. Pulsus Alternans-Due to an impaired contractile power of the ventricles of such a nature that every second beat is smaller. The time relations are equal or if any difference exists the smaller beat has the shorter period, a fact which seems to differentiate a true alternans from premature systoles in which the reverse is the case.

Variations in Conformation.-The changes in the conformation of the radial pulse consist in alterations in size and outline.

Amplitude.- The amplitude of the pulse waves is determined in a considerable measure by adjustment of the apparatus which, even through it may record faithfully the variations in pressure, does so without the abscissæ. Nevertheless it is found by practice that when the best possible adjustment is made, each instrument records a tracing which is quite constant in amplitude in normal individuals. For three different instruments used upon students the following averages were obtained:

Jacquet, old pattern.

$9 \mathrm{~mm}$.

48 cases
Jacquet, new model.

$7.3 \mathrm{~mm}$.

23 cases
Dudgeon.

$13.3 \mathrm{~mm}$.

23 cases

A large oscillation may be due, therefore, either to a large output, a slow rate, a low peripheral resistence, a poor filling of the arteries, as in hemorrhage or a low degree of arterial tonus. A small oscillation may occur, on the other hand, when the output is small, the rate rapid, the resistance high, the arterial tonus great or the elasticity impaired by arteriosclerotic changes (cf. Fig. 105).

When the size of the consecutive pulse beats varies, we speak of an unequal pulse. The normal pulse waves are not exactly equal. This is due, in part, to the fact that the mechanical effect of respiration modifies the ventricular output. When the pulse is regular the amplitude usually decreases-during inspiration and increases during expiration. When, however, the rhythm varies during the respiratory phases so that the cycles becomes shorter in inspiration 
and longer in expiration, the amplitude decreases in inspiration and increases in expiration (see page 69). Variations in size may occur, however, in perfectly regular pulses, as is the case when the filling of the heart or the output is periodically obstructed; for example, in pericardial adhesions, tumors, etc. Lastly, it may occur when the vigor of cardiac contraction is inherently modified, as in the alternating pulse described above.

Pathologically, irregularities in size are associated with irregularities in rhythm. As a rule, the longer a certain cycle, the larger the pulsation following and vice versa. This is due, in part, to the fact that the output of the heart depends upon the interval of diastolic filling, but also, to the fact that the diastolic pressure is allowed to fall more during the longer interval.

Form of the Pulse.-In forming an estimate of the clinical value of the pulse form, it must be remembered that the instruments in common use are utterly incapable of recording the pressure variations present in the arteries. Any deductions drawn from such studies are entirely fallacious. It is, therefore, not to be wondered at that different clinicians have reached very different conclusions as to the diagnostic value of the pulse shape. The records taken with optically recording sphygmographs are as yet exceedingly few in number. We shall confine ourselves, however, entirely to the variations in form of the peripheral pulses so obtained. In the study of variations in form we are concerned with the rise, the fall, and the position and size of the dicrotic wave.

Variations in the Rise.- It can readily be shown by an adjustible artificial circulation scheme that the gradient of the rise is determined $(a)$ by the rate of discharge, $(b)$ the volume of discharge per beat and $(c)$ by the size of the aortic opening (Fig. 89). Furthermore, it is dependent on the height of the diastolic pressure. 'It is also possible to show this in experimental animals and more especially to prove that in conditions such as aortic stenosis the wave rises slowly, is rounded or flat-topped. It does not necessarily follow, however, that the radial pulse in man will, in conditions of stenosis, for example, give such a typical form by which the condition may be recognized. In the first place, the pulse wave has to be transmitted a long distance and due to friction the steepness of the rise progressively diminishes in the arteries until, as we approach the periphery, any slight diminution in rise which was evident in the central pulse becomes quite indistinguishable. In other words, a pulse of diminished steepness in the central artery may, especially when the diastolic pressure is low, be transmitted to the periphery so that the angle of its upstroke does not differ essentially from that of a normal pulse wave.

In the second place, the production of a stenotic lesion in man is gradual and accompanied by compensatory changes in the ventricles 
so that an excessive stenosis is necessary to produce a diminished steepness, even in the central pulse. At all events, the presence in the radial of a sufficiently slow rise to warrant a diagnosis of aortic stenosis would be rather surprising. The records so interpreted ( $c f . e . g$. , Broadbent) were all obtained with inadequate instruments. The writer is unfamiliar with any such records reported from improved methods. The infrequency of its occurrence as a simple lesion undoubtedly adds to the difficulty of diagnosing it by pulse records alone. In 1912, Freiberger and Veiel reported what they believed evidence of a modification of the central pulse recorded by Frank's capsules. In twelve arteriosclerotic cases from patients over forty years of age, they recorded curves both from the central and from the radial pulse in which the steepness of rise was less and in which a rounded top and ascending systolic plateau replaced the descending plateau found in normal pulses. The better transmission of the central changes to the peripheral vessels in arteriosclerotic cases may be explained, perhaps, by the fact that the arteries more nearly resemble solid tubes.

Anacrotism.-Occasionally it is found that instead of a smooth, ascending limb, a break or jog occurs on the ascent which is designated as the anacrotic notch. It has been observed in cases in which the artery was partially compressed centrally (for example, by a tumor), in cases of aortic stenosis, arteriosclerosis, aortic aneurysm, and occasionally in aortic insufficiency. Various explanations have been forthcoming. It has been regarded as due to an "irregular" or "jerky" contraction of the ventricle (Nicolai), to a high arterial pressure (Sahli), to the nature of the arteriosclerotic vessels (Lüthje), to a rapid reflection of peripheral waves, etc. A general theory to explain its occurrence in different conditions has been offered by Schonewald, recently: Whenever the volume elasticity of the aorta or large arteries is so changed (for example, in aneurysm, compression, arteriosclerosis or high pressure) that further expansion is not possible at the onset of systole, the ejected blood is moved onward to the periphery, causing the rise of the radial curve. As the pressure continues to rise, the aorta finally dilates toward the end of systole and the pressure is distributed less slowly to the periphery, causing the notch and slower rise. It is possible, however, as Lewis has shown to produce anacrotism by peripheral factors alone so that its diagnostic value remains in doubt.

Variations in Descending Limb.-The descending limb of the pulse wave may vary greatly in its slope. The significance of this is greater than of the variation in the rise. It is readily shown by pulse tracings taken on an artificial circulation model that the gradient of the pressure fall becomes steeper when the peripheral resistence is lower, when the arterial wall is thicker and when an insufficiency of the aortic valves is created (Fig. 89). Similarly, 
it can be shown experimentally (Borne, Biandet and Wechman), that, when the peripheral resistance is decreased by depressor influences or nitrites, the slope of the descending limb of the carotid becomes more rapid; while it becomes more gradual after the intraarterial injection of adrenalin or after clamping of the abdominal aorta. Also, it can be shown that the descending limb drops more rapidly after the induction of aortic insufficiency in experimental animals (Fig. 95). As before pointed out these results cannot be directly transferred to man nor can the assumption be made that the peripheral pulse at the radial will undergo similar changes. There are reasons, however, for believing that in this case the peripheral pulse in man directly follows the changes in the central pulse. If amyl nitrite is administered to a subject the changes in the shape of the radial pulse become evident even with the less accurate forms of sphygmographs. As shown in an optical tracing of Fig. 73 the descending limb frequently becomes steeper at the same time that the amplitude increases. Similarly, Wirth has shown that the steepness of the descending curves taken optically could be modified by varying the resistance and tonus of the arteries by baths, direct applications of heat and reflex effects from the other hand.

There seems to be no question that a rapid slope of the descending limb of the radial is often associated with a low diastolic pressure and, when present, no doubt often indicates such a pressure. More accurately stated, however, the rapid decline signifies that diastolic pressure is relatively low, as compared with the systolic pressure. Thus, it may occur when the diastolic pressure remains unaltered but the systolic pressure is high, as happens when rigid arteries are insufficiently distended during systole or when the systolic output from a hypertrophied heart is great and rapid so as to produce a higher systolic pressure and a more rapid peripheral flow. The low diastolic pressure present explains the rapid drop found in aortic insufficiency, when it is so marked that it can generally be appreciated by palpation (Figs. 95 and 97). It may be added, however, that some cases of aortic insufficiency in which the pulse gives a distinct collapsing impression to the finger, are found, on investigation, by optical sphygmographs, not to possess such a fall, the palpating finger being evidently misled by the sudden impact (Fig. 97).

Variations in Dicrotism.-The significance of the depth and prominence of the dicrotic notch and wave has given rise to extensive discussion. . Before attempting an analysis of dicrotism, it is necessary to realize that a pulse may appear dicrotic when it is not really so from a physical viewpoint (Lewis). Thus, if we place dicrotic notches of the same magnitude on three pulse skeletons, 
as shown in Fig. 31, no real dicrotism occurs (that is, $a b, a^{\prime} b^{\prime}$, and $\dot{a}^{\prime \prime} b^{\prime \prime}$ are equal), but the middle curve apparently displays an increased dicrotism. It is obvious that this form of apparent dicrotism to which clinicians obviously refer is determined by the relative position of the dicrotic notch on the descending limbbeing increased whenever the descending limb falls more rapidly and diminished when it falls slowly.

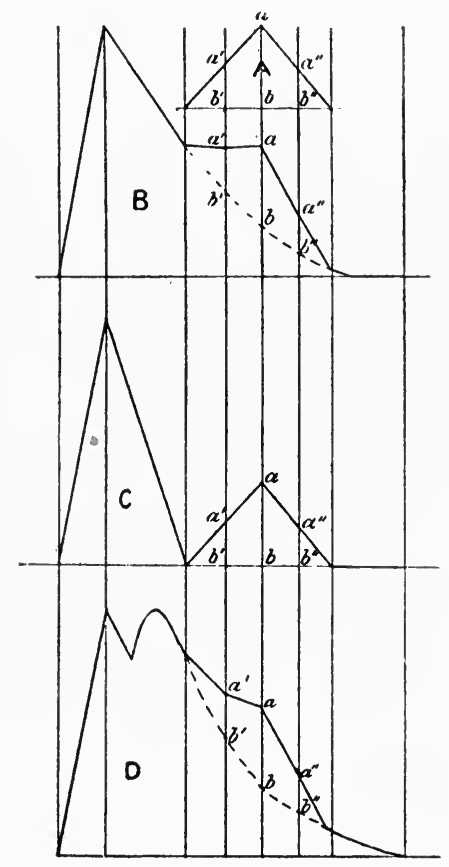

FIG. 31.-Sehematic figure illustrating "apparent dicrotism" when real dicrotism does not exist. Dicrotic wave $(A)$ superimposed upon the deseending limbs of three primary waves, $B, C, D$, so that $a b, a^{\prime} b^{\prime}, a^{\prime \prime} b^{\prime \prime}$, etc., are equal. (After Lewis.)

Since a rapid fall, as before pointed out, occurs frequently when the diastolic pressure is low in consequence of low peripheral resistance, a large dicrotic is usually found in such conditions (amyl nitrite and typhoid pulse). The dicrotism may be real instead of apparent. 'This is due in part to the fact that the arterial walls oscillate at a larger amplitude at lower pressures and in part to the fact that the interference or resonance with peripherally reflected waves is altered ( $c f$. Figs. 73, 74, and 101).

Increased dicrotism like the rapid diastolic collapse is not neces- 
sarily associated with low diastolic pressure or relaxed arteries, as is evident from its occurrence in conditions of high systolic pressure (for example, arteriosclerosis and nephritic hypertension). It occurs here in association with a rapid fall because the rigid arteries are imperfectly distended by the rapid systolic output from a hypertrophied ventricle and, in consequence, a systolic pressure relatively high, as compared to diastolic, is created. From this analysis, it seems that increased dicrotism should be the invariable accompaniment of a more rapid fall of the pulse wave. Such, however, is not exactly the case, for it must be remembered that its position and amplitude are governed, not entirely by the depth of the incisura in the central pulse, but partially, also by resonating reflections. Any influence tending to increase this resonating effect likewise increases the amplitude of the dicrotic wave and vice versa, any diminishing agent acts accordingly upon the amplitude. Finally, it should be remembred, in interpreting dicrotism in the clinic, that many of the ordinary forms of sphygmographs employed actually record no dicrotic notch but instead show mere after-vibrations of the lever in the descending limb. When it happens, for example, that, after amyl nitrite, the pulse accelerates, the requirements of the instrument are increased and the lever is thrown into greater oscillations so that the so-called dicrotic notch markedly increases in amplitude owing to greater lever throw. This, in a large measure explains why the pulse records taken by various observers during fevers and aortic insufficiency, when the pulse is large, are sometimes accompanied by great and sometimes by little dicrotism, and why the significance of dicrotism has received such varied interpretation.

Transmission Time and Velocity of the Pulse Wave.-The transmission time of the pulse is estimated by the difference in time between two pulses as the radial and brachial or the radial and the subclavian (Fig. 27). By obtaining the distance as accurately as possible, the velocity may be computed. The velocity of the pulse has been used as a functional test for determinating the elasticity of the artery. 'This is based on the formula of Moens that the propagation $M=K \sqrt{g \frac{e a}{\Delta d}}$ in which $K$ is a constant, $g=$ the acceleration due to gravity, $e=$ the elasticity coefficient, $a=$ the thickness of the arterial wall, $d=$ its diameter and $\Delta=$ the density of the fluid. In this formula the variations in the specific gravity may be neglected. Thus, if the specific gravity of the blood at 1.005 yields a pulse velocity of ten meters per second, the time of transmission at 1.035 specific gravity would be 10.09 meters per second. It is evident then that the velocity of the pulse wave becomes an index of the elasticity of the artery and it has therefore 
been much used as a functional method of determining the condition of the arteries. Thus, if the velocity in a given case be seven meters, and upon the application of ice over the artery it increases to nine meters, we may infer that an increased contraction of the arterial wall has occurred. The transmission rate in the old is greater than that in the young and is still greater in cases in which arteriosclerotic changes are present. Since the formula contains no factor involving the force of the heart, it is possible to compare only those cases in which the force is equal. While it is suited, therefore, to the study of peripheral changes in the same individual it is difficult to compare pathological conditions since every change in the arterial wall probably involves some change in the force of the cardiac output. It has, therefore, been sought to obviate this error (Friberger) by comparing only cases which have the same systolic pressure, upon the assumption that the adaptation of the heart to the vascular system is indicated by the pressure and that upon this the elasticity coefficient is really dependent.

Determination of the Isometric Period of Ventricular Contraction (Erroneously Termed the Presphygmic Period).-A determination of the interval elapsing between the beginning of ventricular contraction and the ejection of blood after the opening of the valves is frequently desired. This period corresponds to the isometric period of the ventricle, and since it was supposed by the use of older methods to be that interval of systole which caused no effect on the arterial pulse it was designated as the presphygmic period. In view of the work of Frank, showing that no such early systolic period of inertia exists, but that the rise of intraventricular tension is transmitted to the aorta, this term seems inappropriate. This period has usually been measured by the somewhat uncertain method of comparing the time difference between the first evidence of ventricular activity, as shown in the apex beat and the rise of pressure in some artery, after allowing approximately for transmission time. (For literature, see Robinson and Draper.) More accurate estimates have recently been made by comparing the curves with the electrocardiograms and heart sounds. This period may be more exactly, more directly and more easily established by the duration of the preliminary vibrations of the central pulse as suggested by Frank. 


\section{Results of Various Observers and their Methods.}

By Cardiogram and Carotid Pulse Corrected for Transmission.

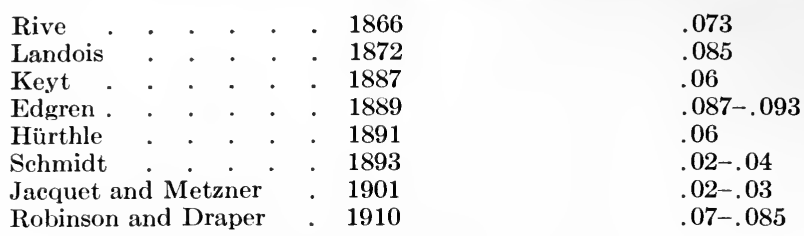

By Differences Between Heart Tone at Apex and Base.

Einthoven and Geluk 1894

.06

By Preliminary Oscillations of Central Pulse.

$\begin{array}{llllllll}\text { Tigerstedt } & . & . & . & . & . & 1908 & .051 \\ \text { Wiggers } & . & . & . & . & . & 1914 & .058-.085\end{array}$

Clinical Significance.-It is apparent that while the normal isometric period of the ventricle varies considerably, it averages about 0.07 to 0.085 . Under pathological conditions it may vary markedly. The period is said to be shortened in some cases of arteriosclerosis and aortic insufficiency (Pezzi). A lengthening indicates an impairment of ventricular function due to valvular, muscular or arterial disease. In arrhythmia very unequal isometric periods naturally occur.

\section{BIBLIOGRAPHY.}

Books AND Monograms.

Broadbent. The Pulse, Philadelphia, 1890.

von Frey. Untersuchung des Pulses, Berlin, 1892.

Hirschfelder. Diseases of the Heart and Aorta, Philadelphia, 2d edition, 1910.

Landois. Die Lehre von Arterienpuls, Berlin, 1872.

Mackenzie. The Study of the Pulse, New York and London, 1902.

“ Diseases of the Heart, 3d edition, London, 1913.

Marey. La Circulation du Sang, Paris, 1881.

Mosso. Die Diagnostik des Pulses, Leipzig, 1879.

Tigerstedt. Physiologie des Kreislaufes, Leipzig, 1893.

\section{Papers.}

v. Born. Skan. Arch. f. Physiol., 1910, xxiv, 127.

Curl. Lancet, 1906, i, 1091.

Frank. München med. Wchnschr., 1903, 1, 1909.

“ Ztşchr. f. Biol., 1905, xlvi, 44.

Frank and Petter. Ztschr. f. Biol., 1907, xlix, 70.

Friberger. Deutsch. Arch. f. klin. Med., 1912, cvii, 268, 280.

Friberger and Veiel. Deut. Arch. f. klin. Med., 1912, cvii, 268.

Grantström. Ztschr. f. klin. Med., 1908, lxvi, 146.

Hill, Barnard and Sequeira. Jour. Physiol., 1897, xxi, 148.

Hirschman. Arch. f. d. ges. Physiol., 1894, lvi, 389. 
Hoke and Rihl. Wien. med. Wchnschr., 1913, xxvi, 1149.

Jacquet. Cor-Bl. f. schweiz. Aerzte, 1910, iii.

Levy. Ztschr. f. klin. Med., 1910, lxx, 429.

Lewis. Jour. Physiol, 1908, xxxvii, 212, 233.

“ Jour. Physiol., 1906, xxxiv, 391.

“ Lancet, 1906, ii, 714.

“ Brit. Med. Jour., 1907, i, 918.

Lombard and Budgett. Arch. internat. de physiol., 1905, ii, 121.

Müller and Weiss. Deutsch. Arch. f. klin. Med., 1912, cv, 105.

Ohm. München. med. Wchnschr., 1910, lvii, 343.

Petter. Ztschr. f. Biol., 1908, li, 335, 354.

Robinson and Draper. Arch. int. Med., 1910, v, 168.

Tigerstedt. Skan. Arch. f. Physiol., 1908, xx, 249.

“ Ergeb. der Physiol., 1909, viii, 593.

Veiel. Deutsch. Arch. f. klin. Med., 1912, cv, 249.

Veiel and Noltenius. München. med. Wchnschr., 1910, lvii, 782.

Weber. Ber. d. k. sächs. Geselsch. d. Wisschen., 1850, i, 164.

“ Deutsch. Arch. f. klin. Med., 1912, cviii, 311.

\section{New Forms of Sphygmographs.}

Amblard. Compt. rend. Soc. de biol. Paris, 1908, lxv, 618 (sphygmométroscope).

Bernd. Wien. klin. Wehnschr., 1906, xix, 39 (equitension sphygmograph).

Berkeley. Med. Rec., New York, 1910, lxxvii, 140 (simple polygraph).

Brugsch. Ztschr. f. exper. Path. u. Therap., 1912, xi, 169 (sphygmotonograph).

Castagna. Wien. klin. Wehnschr., 1901, xliv (onychograph).

François Franck. Compt. rend. Soc. de biol., 1908, lxv, 226 (sphygmopalpeur).

Glover. Lancet, 1911, clxxx, 384 (Band for sphygmograph).

Herz. Zentralbl. f. Physiol., 1896, x, 143 (onychograph).

Koziczkowsky. Berl. klin. Wehnschr., 1907, xliv, 369 (turgo-sphygmograph).

Kreidl. Zentralbl. f. Physiol., 1902, xvi, 257 (nagelpuls).

Kronecker. Ztschr. f. biol., Tech. u. Method., 1911, ii, 110 (kapillar sphygmograph.)

Kronecker. Zentralbl. f. Physiol., 1910, xxiv, 828.

Morelli. Ztschr. f. exper. Path. u. Therap., 1912, xi, 477 (new sphygmograph).

Pal. Zentralbl. f. inn. Med., 1906, xxvii, 121 (sphygmoscope for determining pulse pressure).

Rheinboldt. Berl. klin. Wchnschr., 1907, xliv, 161 (sphygmoscope).

Sommer. Berl. klin. Wehnschr., 1903, xl, 1169.

“ Zentralbl. f. Physiol., xxi, 502 (sound sphygmoscope).

Strauss and Fleischer. Berl. klin. Wchnschr., 1908, xlv, 1087 (turgosphygmograph).

Vasquez. Compt. rend. Soc. de biol., lxv, 226 (sphygmosignal).

" Compt. rend. Soc. de biol., lxv, 226 (sphygmopalpeur).

" Compt. rend. Soc. de biol., lxv, 681 (sphygmometroscope).

“ Arch. ital. d. biol., xlix, 418 (bitemporal angiograph).

\section{Miscellaneous Articles.}

Biandet and Wechman. Skan. Arch. f. Physiol., 1913, xxviii, 278.

Landé. Deut. Arch. f. klin. Med., 1914, cxvi, 295.

Pezzi. Journal de physiol. et de path. gen., 1913, xv, 1178.

Schoenewald. Zentralbl. f. Herz. u. Gefässkrankheit, 1914, vi, 249. 


\section{THE SUPRACLAVICULAR VENOUS PULSE OR THE PHLEBOGRAM.}

IF the cervical veins in the supraclavicular region are observed, two distinct types of pulsation caused by their variable filling are evident. These are designated as the respiratory and cardiac venous pulses. With every inspiration blood is aspirated into the thorax, and the veins diminish in volume; with every expiration the flow is impeded and the veins increase in size. If the breath is held during expiratory quiet, however, the cardiac variations normally super-imposed upon the respiratory waves, are more clearly discerned. During every systole of the ventricle, as gauged by the apex beat, the veins collapse, thus giving rise to what has long been known as the "negative pulse." This is distinguished from the pathological or "positive venous pulse," which shows a systolic swelling of the veins. Unless otherwise qualified, the term "venous pulse" designates the cardiac venous pulse, although the precautions are not always taken to study its variations during apnea, hence a mixture of the two pulsations has often been obtained.

\section{TECHNIC OF RECORDING VENOUS PULSE TRACINGS.}

The cardiac variations in the jugular vein can be studied in greater detail by taking graphic records of their volume changes. The apparatus used for this consists of an open receiving cup connected by rubber tubing with a delicate recording tambour, or better with a segment capsule recording optically (cf. page 102 and Fig. 26).

The following technic is usually followed: The subject lies for several minutes with the neck and head supported by a single pillow. The receiving tambour is applied snugly, but without undue pressure, to the right supraclavicular fossa, either over, or to the right border of the sterno-cleido-mastoid muscle. The effort should be made to relax the neck muscles by keeping the face directed forward or turning the head slightly toward the right- the involuntary tendency of the subject to turn the head to the left must be resisted.

When a place has been found where a satisfactory pulsation (at least 5 millimeters) is obtained, another cup also connected with a recording tambour is firmly pressed over the carotid or subclavian 
area and, after marking the relative position of the two lever points, a tracing of these two pulses is simultaneously recorded, together with some form of time record. In place of the carotid pulse, the radial pulse from a transmission sphygmograph is sometimes used.

\section{NOMENCLATURE, TIME RELATIONS, AND INTERPRETATION OF VENOUS PULSE TRACINGS.}

The cardiac venous pulse contains at least three, and occasionally four waves for each heart cycle. Upon these, accidental wavelets or notches may be superimposed. Since the contours of the different waves are often not distinctive in polygraph tracings it is necessary to check them by comparison with the accompanying arterial
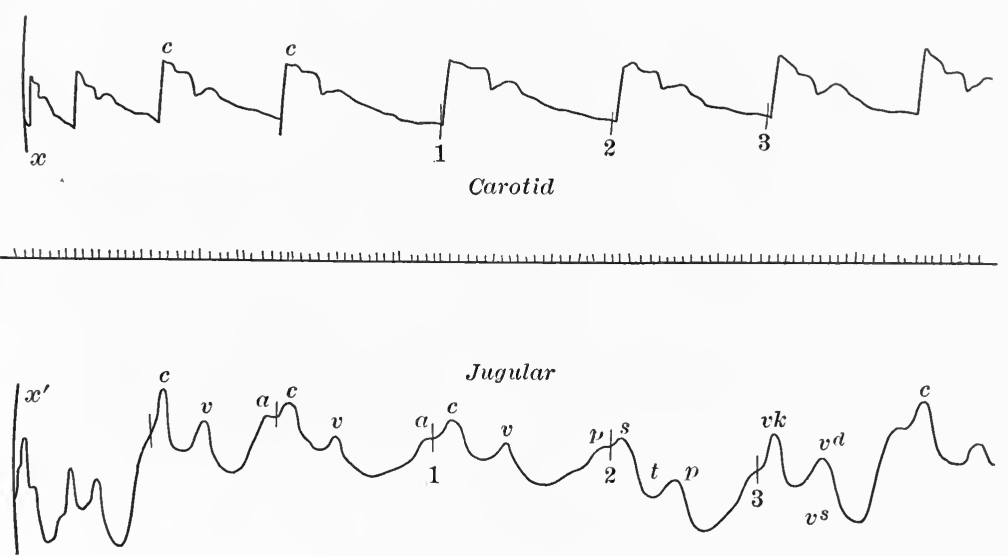

FIG. 32.- Simultaneous earotid and venous pulse tracings taken with a sensitive form of Lombard's tambour. $x-x^{\prime}$, relative position of writing points with ares. On the record is illustrated the method of marking $c$-waves as well as the different terminologies found in the literature.

curve. This, however, can be dispensed with in optical records after one has become familiar with the characteristic wave shapes. The checking is accomplished in the following manner (Fig. 32): One point of a pair of dividers is applied to the line indicating the starting position of the arterial lever $(x)$ and the other to the rise of the primary wave of any arterial pulse $(1,2,3)$. Now, without changing the dividers, one point is applied similarly to the starting point of the venous tracing $\left(x^{\prime}\right)$ and the wave cut by the other point determined $(1,2,3)$. This is the second or systolic wave. Its contour and prominence is variable. In case the radial pulse is used as a standard, 0.1 second is allowed for transmission time. Care should be taken to make all measurements between the 
line of points and the waves in the same horizontal line so as to take into account the ares of levers.

Nomenclature.-Various names have been applied to these waves. Following Mackenzie, the first wave is most commonly designated as the $a$ wave to denote its association with auricular systole. Since it is presystolic in relation to ventricular systole, it has also been called the presystolic or $p$ wave (Bard).

The second wave is usually labeled the $c$ wave, also after Mackenzie, to designate the fact that it owes its origin to a carotid impact. Since it is systolic in time, it has been called the systolic or $s$ wave (Fredericq, Bachmann, Ohm). Morrow speaks of it as the ventricular wave, while Hering and Rihl have designated it as the ventricular valve wave or the $v k$ wave.

The third wave is generally named, after Mackenzie, the ventricular or $v$ wave. Morrow speaks of it as the first onflow wave. A number of investigators have noted that the ascending limb of this wave is divided by a notch or bend and hence different names have been attached to these two portions. Thus, Bard speaks of a tele-systolic or proto-diastolic wave $(t+p$ wave), Wenckebach of an $i+v$ wave, and Rihl of a $v^{s}$ and $v^{d}$ wave.

The fourth wave has been designated as the $h$ wave by Hirschfelder, the $b$ wave by Gibson, and the stasis or $s$ wave by Rihl.

Time Relations of Different Waves.-Some discrepancies, the reasons for which are now readily explained, have arisen in establishing the temporal relations of these four different waves. It is quite generally considered established, on the basis of myocardiographic studies, that the first, or $a$ wave begins to rise with auricular systole and falls with diastole. Indeed, the synchronism reported by several investigators is almost incredibly exact. Ewing, however, denies that the auricular systole terminates at the crest of the $a$ wave, but believes that it continues throughout its entire fall. This question is of importance, for if the crest terminates auricular systole and the beginning of the rise indicates its onset, its duration is shorter than is commonly supposed. As a result of the measurement of fifty-three records taken from medical students by optically recording capsules, I have found that if the rise of the $a$ wave alone is taken as the span of the auricular systole, its duration is 0.063 to 0.075 seconds, while if the up and down strokes are taken, it is considerably longer than is usually acknowledged ( 0.14 to 0.2 second). In neither case is the generally accepted interval of 0.1 second found.

The writer's animal experiments do not support the view that auricular systole continues through both the rise and fall of the $a$ wave, for (1) a distinct interval is usually found between the end of auricular and beginning of ventricular systole, and (2) when the heart is inhibited by vagus stimulation so that the auricle 
alone responds, the pressure in the superior vena cava rises during systole and falls during diastole exactly as in the normal curve.

The second wave undoubtedly begins to rise early in systole. How clearly it actually establishes the very beginning of systole has been open to question. Mackenzie regarded its rise as caused by, and, hence, synchronous with the carotid impact. Bard, however, found that it preceded the carotid wave by 0.01 to 0.02 second, a fact given greater significance, when Morrow subsequently showed that the waves were transmitted more slowly in the veins than in the arteries (1-3 meters as compared with $7-10$ meters). Bachmann, subsequently, recorded curves in which this wave similarly preceded the carotid rise but occurred synchronously with the apex rise attributed to ventricular systole. Furthermore, Eyster demonstrated that the first sound occurs on an average 0.03 to 0.07 second before the $c$ wave rises but may be coincident with it.

In 1911, Edens optically recorded the venous pulse with Frank's capsules and showed that the so-called systolic wave is made up of two portions, a first wave due to valve closure followed after an interval varying from 0.018 to 0.048 second by a true $c$ wave. The first elevation occurs precisely at the onset of the first sound, whereas the true $c$ wave is somewhat delayed. Ohm simultaneously recorded the heart sounds and the venous pulse by his optically recording gelatine capsules. These records also show that a short though uncalculated interval elapses between the beginning of systole and the true $c$ wave. The optical records of the writer obtained in 1913 and 1914 are in exact agreement with these results (Fig. 34). They show that the true $c$ wave rises an interval of 0.058 to 0.085 second after the onset of ventricular systole and that this period, moreover, corresponds to the isometric period calculated from the subclavian pulse ( $c f$. page 124).

All investigators seem agreed that the drop of the third or $v$ wave occurs synchronously with the opening of the tricuspid valves. The summit is therefore commonly regarded as approximately coinciding with the onset of diastole. Thus, Rautenberg, by comparing esophageal and venous tracings, came to the conclusion that the entire rise was systolic and the summit marked the beginning of diastole. On the other hand, Wenckebach believed that the foot of the rise was synchronous with the diastolic onset. According to a more recent statement by Mackenzie, Gotwald, Knoll and Hering, the rise begins during the end of systole and continues into early diastole. According to this view the beginning of diastole occurs somewhere on the ascent of this wave and is often marked by a notch or jog. This view is substantiated by optical tracings and simultaneous records of sounds and waves. Thus, Eyster found that the second heart sound begins on the average a few 
hundredths of a second after the beginning of the $v$ wave, although this sound has been observed as much as 0.045 second before and 0.15 second after its rise. The records taken by Edens and the writer with Frank's capsules, as well as those of Ohm taken with gelatine membranes show that the third wave is essentially, and often entirely, a diastolic one, provided we take the closure of the semilunar valves as establishing the end of systole and the beginning of diastole (Fig. 34).

The fourth wave when present is distinctly diastolic in time. Hirschfelder, Thayer and Gibson all related it to the third heart sound (see page 174). This is confirmed to a certain extent by the work of Eyster, who shows that the third sound precedes the beginning of this wave by a few hundredths of a second. This writer also corroborates the work of Einthoven, however, that the two are not necessarily associated since a sound may occur without evidence of a wave, and vice versa.

Interpretation of Waves.-Before bringing forward the evidence that the use of optically recording apparatus has produced to aid in the interpretation of the venous pulse, it may be well to briefly review the different views held concerning the cause of the chief venous pulse waves. In doing this we may designate the waves from their time relations as presystolic, systolic, early diastolic and mid-diastolic, appending the most widely used letters $a, c, v$, and $h$ to these waves respectively.

\section{Pulse :}

Summary of Views Concerning Causes of Chief Waves of the Venous

Presystolic or a wave.

Rise: Auricular systole causing a stasis of blood or a slight regurgitation into the veins.

Fall: (a) Auricular diastole, inflow of blood and collapse of vein.

(b) Ventricular systole, drawing down floor of and enlarging capacity of auricle.

(c) Decrease in intrathoracic pressure at onset of ventricular systole causing suction.

(d) Rebound of blood during auricular systole (Ewing). Systolic or c wave.

Rise: (a) Impact from underlying carotid or subclavian (Mackenzie).

(b) Impact from large intrathoracic arteries upon large veins or auricle.

(c) Jar or tug due to change in position of contracting ventricle.

(d) Bulging or closure of tricuspid valves at onset of ventricular systole (François Frank, Fredericq, Hering, Rihl, Bard, Bachmann, etc.).

(e) Increase in auricular pressure due to sudden ejection of blood from coronary sinus (Sewall, Hirschfelder). 
Fall: (a) Continuation of the fall of the $a$ wave.

(b) Superimposed descending limb of an arterial impact.

Early Diastolic or v wave.

Rise: (a) End of stasis-rise in auriculo-venous system while tricuspid valves remain closed.

(b) Elevation of $a-v$ septum at onset of ventricular diastole. Fall: (a) Opening of tricuspid valves and onflow of blood into ventricle.

Mid-diastolic or h wave.

(a) Change from a flow to a stasis of blood in ventricle, auricle and veins.

(b) Approximation of $a-v$ valves.

These varied opinions as to the factors concerned in the production of the different waves are based either upon practical views that can be harmonized with the time relations of the waves; or upon actual records of the right auricular pressure, the variations of which are regarded as equivalent to those in the jugular pulse. The first process is subject to error in making appropriate time allowances for wave-transmission. The latter is questionable, because the assumption that the right auricular variations and those of the venous pulse are identical, has been shown to be erroneous by the use of optical methods. A suggestion of the dissimilarity is shown in the synchronous venous and esophageal pulse tracings taken by the segment capsules of Edens. His curves indicate that, although both vein and auricle have three main waves, the similarity ceases with this fact, for neither their time relations nor contours correspond exactly. Thus, the auricular wave from the esophagus precedes that in the jugular by 0.06 to 0.08 second, while the second esophageal wave is related to the first small elevation in the jugular and not to the main $c$ wave.

During ventricular systole the jugular curve mainly falls while the esophageal tracing mainly rises. It is evident also that while in the esophageal curve the main rise of the $v$ wave is systolic, in the jugular it is largely diastolic.

Since we cannot be absolutely certain that such esophagrams represent variations in auricular pressure alone (see page 148), they cannot finally determine the question. Simultaneous registration of the right auricular and venous pressures by optical means led Van Zwaluenberg and Agnew to conclude that in dogs the curves were different. They found that the rise of the $v$ wave was delayed more than the $a$ wave, and that all the finer details of the auricular tracing were lost in the jugular record, a fact attributed by them to the interposition of venous valves.

The writer has made an effort to trace carefully the transformation of the intra-auricular pressure curve into the venous pulse as 
commonly recorded. This work was done partly in association with Frank and Broemser in Munich, and partly independently through subsequent experiments in Cornell Medical College. Reserving the details for future communication, the facts may be briefly outlined in connection with the diagram of Fig. 33:

1. If the intra-auricular pressure from an animal with an active heart is recorded by a sensitive optically recording manometer of adequate efficiency, we find, similar to the results of Straub and Piper that the pressure variations in the auricle are composed of the following typical series of waves (Fig. 33, $I$, also Fig. 9, B).

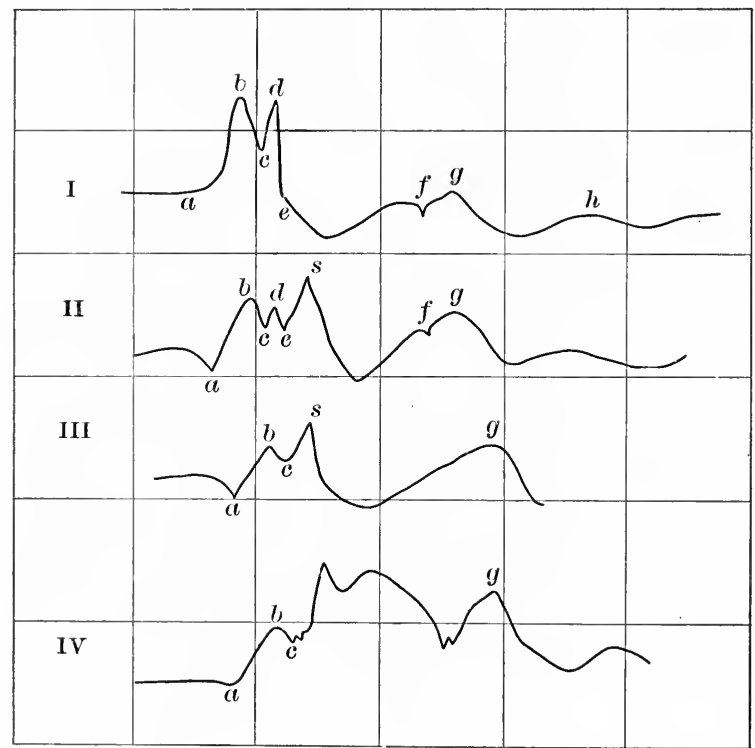

FIg. 33.-Four curves showing transition of right aurieular pulse to supraclavicular venous pulse. $I$, pressure changes in right auricle; $I I$, pressure changes in extrathoracic veins; $I I I$, volume changes of neck vein; $I V$, supraclavicular pulse taken with tambour.

A rise and fall $(a, b, c)$ synchronous with auricular systole and diastole.

A sharp positive rise and negative fall $(c, d, e)$ apparently associated with valve closure and downward movement of the auricular floor.

A slow stasis rise continuing throughout systole $(e, f)$ and marked at the closure of the semilunars by a notch $(f)$.

A diastolic rise $(f, g)$.

A diastolic drop $(g)$ due to the opening of the tricuspid valves.

A mid-diastolic oscillation $(h)$, when the heart is slow. 
2. If a jugular vein is tied and the pressure within is recorded low in the neck with the same optical manometer, the following series of changes are found (Fig. 33, $I I$ ):

A rise and fall $(a, b, c)$ due to auricular systole and diastole. This rise occurs a few hundredths of a second later than the rise in the auricle but is preceded by a negative depression which is apparently accounted for by a slight traction of the contracting auricle on the veins.

A small notch $(c, d)$ corresponding to a similar rise in the auricle but not followed by the sharp drop $(d, e)$ since it is cut short by

A prominent elevation $(s)$ apparently due to a systolic impact from some intrathoracic artery.

A late systolic rise (to $f$ ).

A diastolic rise $(f, g)$.

A diastolic depression $(g)$.

To sum up, the pressure waves have changed to the extent that the steep fall $(d, e)$ is largely replaced by a second positive wave $(s)$; and that the stasis rise during systole starts later.

3. If the jugular vein low in the neck is dissected free, but not tied, so that blood flows from the periphery; and the variations in venous volume are directly recorded by methods of unquestionable accuracy, then the three main waves persist, but all evidence of the variations due to valve closure or movements of the auricular floor $(c, d, e)$ have vanished-probably they have been damped out by the presence of valves or the onflowing blood (Fig. 33, III).

4. If a supraclavicular tracing is taken as in man by placing a small tambour over the skin of the venous region, the curve again contains three waves, but the systolic wave assumes greater prominence and gives distinct evidence of the detailed contour of a central arterial wave. The $v$ wave becomes almost entirely a diastolic affair (Fig. 33, IV).

To summarize, we may say: (1) there exists in the right auricle a small systolic wave due to the movement of valves or the auriculoventricular floor; (2) there exists in the central veins (occasionally in the auricle) another distinct wave due to an intrathoracic impact; (3) the latter is, and the former is not transmitted to the veins of the neck; (4) it is difficult to record either of these systolic vibrations when a small tambour simultaneously covers an arterial area, for then the period of systole is obscured by a direct arterial impact. The venous pulse is therefore not, as current text-books state, an index of intra-auricular pressure. Only during presystole and diastole does it follow the auricular pressure in any measure. 


\section{THE DETAILS AND INTERPRETATION OF THE SUPRA- CLAVICULAR VENOUS PULSE IN MAN.}

Since the pulse tracings taken from the right supraclavicular fossa represent a mixture of the pulsations from the subclavian artery and the jugular bulb the term supraclavicular venous pulse seems a more distinctive term. This region has several advantages over those venous regions more remote but freer from systolic arterial impact, for the venous column is physically so poor a conducting system that every increase in the distance from the heart makes the record resemble less and less the true intra-auricular pressure curve.

It is generally supposed that the waves of the supraclavicular venous pulse have no distinctive contour, as is shown in the case of the arterial pulse. This is due to the fact that it is imperfectly recorded by the transmission methods in common use. The records taken with optical capsules are so distinctive that no comparison with other records is necessary. Furthermore, the variations in contour are of diagnostic importance. It is therefore proper to analyze the details of such curves. In Fig. 34, two simultaneous tracings from the supraclavicular region are shown: the upper record was obtained through a tambour lightly pressing over the region of the jugular bulb, and the lower from a cup firmly pressed over the supraclavicular space external to the first. The first is a record in which the venous elements predominate and the arterial elements are reduced to a minimum; while the latter is almost a clear subclavian pulse.

The details of the latter correspond to those already described as characteristic of the central arterial pulse. Since the relations of its detailed features to the cardiac cycle have been carefully delineated, such a record offers an aid in the interpretation of the supraclavicular venous tracing not hitherto supplied in the less accurate tracings obtained from more distant arterial pulsations by polygraph tambours of low vibration frequency. Briefly recalled, such a subclavian pulse shows the following details: $1-2$, a wave due to auricular systole; $2-3$, the preliminary vibrations due to the bulging of the semilunar valves during the isometric period of systole (Anspannungszeit); 3-4, the primary shock of the entire blood column due to ejection; $4-5$, the ascending and $5-6$, the descending phases of the more constant ejection period; 6 , the "incisura" or sudden drop in pressure at the beginning of ventricular relaxation followed by several valve vibrations at $s^{2}$. After this the pressure gradually falls in diastole.

The supraclavicular venous tracing obtained by lighter pressure may, at once, be divided into presystolic, systolic and diastolic periods, using the term "ventricular systole" to indicate the period that 
the cardiac muscle actually remains in a state of contraction. To accomplish this division, the vibrations resulting from the closure of the tricuspid and semilunar valves $\left(s^{1}\right.$ and $s^{2}$ ) which are transmitted to the neck and directly inscribed on the curve, may be used to mark approximately the beginning and end of ventricular systole. It is evident that in each period one wave reaches its crest. Hence, we may designate them very simply as presystolic, systolic, and diastolic. Upon the basis of animal experiments before quoted the following interpretation of the details is proposed:

Presystolic Wave.-The wave occurring during this period ( $a$ wave of Mackenzie) consists of a rise $(a, b)$ and a fall $(b, c)$. The rise is unquestionably associated with, and caused by, auricular systole. The fall is commonly attributed to the onset of auricular diastole, a view with which the writer agrees.

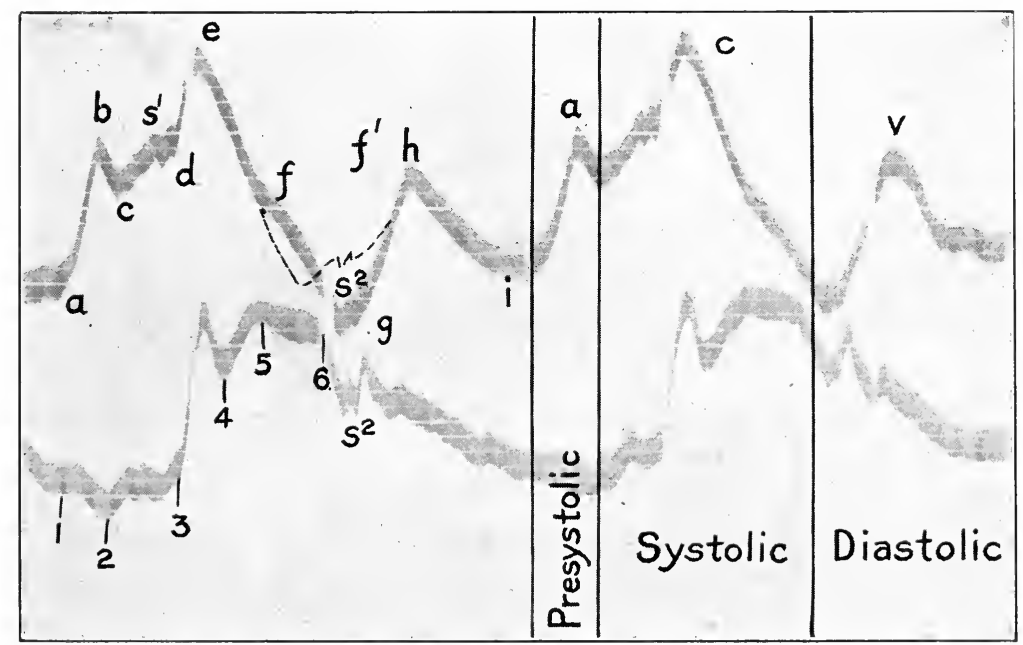

FIG. 34.-Supraclavicular venous pulse (upper) and subclavian pulse (lower) recorded by Frank's capsules.

Systolic Wave.-The onset of ventricular systole is characterized by a preliminary rise of pressure $(c, d)$, synchronous with the isometric rise of the subclavian record $(2,3)$. Whether this rise is to be interpreted as due to the closure of the tricuspid valves, or is merely the transmitted preliminary vibration of the aorta, as at 2,3 , cannot be definitely stated. Whichever interpretation is adopted, there can be no question that this represents the isometric period, or the period of rising intraventricular tension. Absolutely synchronous with the rise of the subclavian pulse (at 3) occurs the rise of the supraclavicular pulse $(d, e)$ obtained with 
light pressure. It is therefore possible to ascribe this only to a direct impact of the underlying artery.

After reaching its summit at $e$, the pressure sharply falls until it changes its slope at $f$, or, in other cases, as shown at $f^{1}$, begins to ascend. The contour of the arterial curve is not exactly followed because the impact was poorly recorded, on account of the light pressure of the tambour. The lighter the pressure becomes, the less perfectly it follows the arterial pressure contour being then transformed, as is shown in the venous record of Fig. 47, into a simple oscillation. The subclavian drop in pressure entirely overshadows the systolic rise in pressure simultaneously occurring in the auricles only rarely, as at $f^{1}$, is the rise due to stasis (telesystolic rise of Bard) obvious during the systole of healthy subjects. Similar relations are evident in the recent optical curves of Edens and Ohm.

Diastolic Wave.-The wave $g, h, i$, ( $v$ wave) (Fig. 34), as pointed ont, is, as a rule, largely a diastolic affair. Its fall after $h$ occurs at the opening of the tricuspid valves. Its rise after the vibrations of the second heart sound at $s^{2}$ is so sudden that it remains questionable whether it can be attributed entirely to a continuation of the systolic stasis wave in the auricle. It is possible that the upward movement of the ventricles and auricular floor at the beginning of diastole may account for the increase in the volume of the vein.

\section{CLINICAL ASPECTS OF THE VENOUS PULSE.}

The Efficiency of Polygraphs.-Although optical records have been taken from patients by Edens and Ohm, and Frank's apparatus has been arranged in a convenient and portable form for hospital use by the writer, ${ }^{1}$ the convenient forms of polygraphs will no doubt continue to be favorites for taking supraclavicular venous pulsations. The clinical aspects, therefore, closely involve the question: To what extent are the records taken with these forms of apparatus reliable - for it must be admitted that all of them have an exceedingly low vibration frequency and many are in no sense aperiodic? That they are incapable of recording the intra-auricular pressure variations faithfully cannot be questioned.

If, however, we apply the principle of judging an instrument by its results, we must admit that a most wonderful advance in cardiovascular diagnosis has come about through the use of such inferior instruments, and that the ideas formulated upon the basis of such records have been recently confirmed by more accurate studies with the string galvanometer. The reasons for this are not difficult to discover. In the first place, the veins are themselves transmitting

1 See Fig. 26 and description in Jour. Amer. Med. Assoc., 1915, lxiv, 1305. 
systems of low efficiency so the intra-auricular waves are considerably distorted, annulled, or hidden before reaching the neck. Hence, the demands made upon an apparatus to record the main venous waves are not very exacting. 'The chief mistake made is to assume that the right auricular pressure can be actually recorded by such an apparatus. In the second place, the diagnoses read from the records by men like Mackenzie, Wenckebach and Lewis have been tempered with extreme foresight and reason which counts for so much in establishing any diagnosis. Furthermore, the true facts have gradually unfolded themselves to these investigators. The novice who wishes to read from tracings all the details described in the literature must needs be familiar with the characteristic effects of all the different cardiac disturbances on the tracing. To him, more than to the experienced diagnostician a recognition of the possible errors incurred by the apparatus is also necessary. For this reason we may briefly consider the ways in which the records may be distorted by the use of polygraph tambours and how disturbed cardiac action may modify the venous pulse waves.

In polygraph tracings the heart sound vibrations as well as the isometric interval are not recorded at all. Hence, the exact beginning and end of systole cannot be directly established on the records, and the true $A s-V s$ conduction time cannot be accurately determined. The relative amplitude of the waves is not correctly reproduced, and the relative prominence of the waves may be entirely wrong. The curves (in the periodic forms of apparatus) are often complicated by waves which do not exist in the vein at all but are due to the inherent oscillation of the lever. ${ }^{1}$

The polygraph tracings establish very roughly (1) the beginning of auricular systole ( $a$ wave rise), (2) the beginning of ventricular ejection ( $c$ wave rise), (3) very roughly the $A s-V s$ interval ( $a-c$ interval), and (4) the opening of the tricuspid valves (drop of $v$ wave), which occurs a few hundredths of a second after the beginning of diastole.

In abnormal cardiac activity they may also give evidence of the presence or absence of an auricular contraction, the presence and sequence of supernumerary auricular contractions, and, finally, detect the presence of auricular stasis and tricuspid regurgitations.

In order to recognize the value and limitations of the ordinary polygraph in determining these abnormal variations, it is proposed to present side by side a number of common effects, as recorded by' Frank's capsules and Mackenzie's polygraph levers. 'To economize space, this will be done in diagrammatic form, the curves

1 For curves illustrating these changes see Wiggers, Jour. of Amer. Med. Assoc., 1915, lxiv, 1485. 
being copied from records obtained during the last year in the wards of the second medical division of Bellevue Hospital. ${ }^{1}$

Significance of the Systolic Waves.-It is of importance in polygraph curves, for purposes of orientation to first check up the systolic or $c$ wave after the manner described on page 127 . Hence, we may consider its importance in diagnosis first. The systolic or $c$ wave in optical tracings is normally very prominent; in polygraphic tracings it may be scarcely indicated. Its chief characteristic is that it falls during the major part of systole (Fig $35, I$ ). The relative time of the termination of this fall indicates in some fashion the degree of stasis in the right auricle. If the stasis is greater than

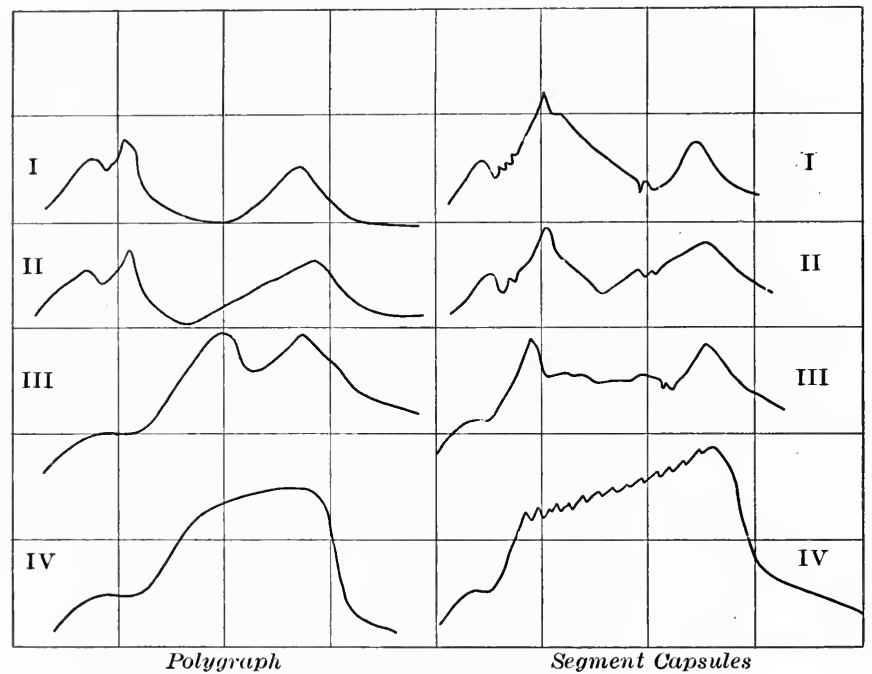

FIG. 35.-Comparative diagrams illustrating the effect of a consecutive increase in stasis $(I, I I, I I I)$ and of regurgitation $(I V)$ on the venous pulse curves as recorded by optical apparatus and polygraph tambours.

normal the rise occurs during the end of systole. Further stasis causes the wave to remain high as shown in Fig. 35, III. In such cases the systolic or $c$ wave and the diastolic or $v$ wave are always distinctly separated by a notch in optical tracings. Aided by lever throw, these notches may become very deep in polygraph curves and give the effect of a bifurcated wave. If a tricuspid regurgitation occurs, however, during systole, as in $I V$, the venous pressure rises throughout systole and the systolic and diastolic waves merge into one with a broad plateau. In such cases, the optical records indicate clearly the vibrations of the murmur

1 A detailed publication will be made when a greater collection of curves becomes available. 
accompanying the regurgitation. Since, in this form of pulse, the systolic waves are exceedingly conspicuous, it has been designated the "ventricular type" of venous pulse (Mackenzie).

Optical tracings of these pulsations have been reported by Ohm and the writer. One of the curves shown in Fig. 88 and taken from a case of auricular fibrillation, illustrates that, when the heart beats are irregular, different types of waves may follow each other. Thus, in the group labeled $I$, a distinct impact or $c$ wave occurs which is followed somewhat prematurely by a rise of the $v$ wave, indicating stasis. In groups labeled $I I$, we have the entire systole filled by an ascending or horizontal plateau in which vibrations due to an accompanying murmur are vaguely discernible. Here the $c$ and $v$ waves are merged.

A prominent systolic wave may also occur when auricle and ventricle contract simultaneously. This may occur occasionally or for continued intervals in paroxysmal tachycardia (Mackenzie). In such an event, a sustained top is not found, however, but the curves resemble a large $a$ wave. To my knowledge, optical tracings of such a condition have not been reported. On the other hand, it is questionable whether such an interpretation is warranted from the nature of the curves in many cases in which it is made. We have here an example of an explanation that conveniently fits the diagnosis being given to the record instead of allowing the record itself to supply the evidence.

A sharp, distinct systolic wave may be entirely lacking when an ineffective systole occurs, that is, one that fails to open the semilunar valves and to send a pulse to the arteries. In such cases the registration of heart sounds alone will determine the presence of a ventricular systole. An example is shown in Fig. 84. The ventricle contracted at 1 as is indicated by the sound at $S^{\prime}$. No impact wave occurred, but owing to the fact that the entire contraction was isometric - that is, occurred without valve opening - a stasis wave $2-3$ resulted in the auricle during systole. When the tricuspid valves subsequently opened at $3 a$, the blood entered the ventricle but at a gradual rate since the ventricle was already filled before relaxation had set in. It becomes apparent, then, that while the waves of the systolic interval give us no indication of intra-auricular phenomena in normal venous pulse, on account of the fact that they are overbalanced by arterial impact phenomena, in cases of stenosis and regurgitation they become so pronounced that they overshadow the arterial impact. Hence, the recognition of the time at which the curve begins to rise is of extreme importance.

The Significance of the Presystolic Wave.-The auricular wave is of importance in establishing the existence or absence of auricular contractions. If a distinct wave is present from 0.08 to 0.2 second before the curve rises, the assumption is generally made that it 
represents the presence of a presystolic wave. If the interval is longer it should be regarded suspiciously. Occasionally even in optical records it becomes exceedingly difficult to determine whether a certain wave is due to auricular systole. We may examine the conditions under which this occurs: In the first place the ventricular systole may be so weak that no carotid or radial beat is recorded and, hence, the identity of $a$ waves becomes questionable. Optical tracings often have the advantage in such cases because the beginning and end of systole are directly indicated by the heart valve vibrations, superimposed on the venous pulse; but in many instances, this, as well as a direct record of sounds from the chest, fails, either because the sounds are not sufficiently intense to be recorded, or, to judge from animal experiments, are not generated at all.

Again, it not infrequently happens that the $a$ wave is entirely absent in records taken with the breath held (Edens). This probably occurs when the contractions are weak or the venous pressure unusually low. In such cases, a regular rhythm warrants, a closer investigation into the existence of the $a$ waves, for their absence throughout a record usually occurs only with an irregular ventricular beat.

Even in cases with irregular hearts, however, the $a$ waves may apparently be absent when they occur synchronously with carotid systoles or during the $v$ waves. According to Lewis we may suspect such a coincidence of the $a$ and $v$ waves when the $v$ wave projects distinctly into diastole. This criterion is of doubtful value. Many of the interpretations of synchronous $a$ and $c$ or $a$ and $v$ waves should be questioned unless verified by other means.

The $a$ waves are truly absent when the auricle is extremely distended or in a state of fibrillary contraction.

The $a$ wave may determine the abnormal sequence or the excessive recurrence of auricular contractions. In deciding that the presence of certain waves indicates an excessive rate of auricular contraction, it is necessary that all the other possible causes of such waves be excluded. The $h$ waves may recur so as to be equidistant from two regular $a$ waves and, hence, mistaken for extra contractions of the auricle. If such a wave is less prominent than the recognized $a$ waves, and if no other evidence of conduction is present, it is probably an $h$ wave. Again, $a$ waves may be confused with $v$ waves. Lewis proposes to differentiate by determining whether the drop comes after the dicrotic notch. This method however, is of little value especially in polygraphic tracings, for the $v$ wave begins to fall after the dicrotic notch. Furthermore, most of the tambours on the polygraphs record no dicrotic notch but merely an inherent after-vibration (cf. page 107). When the heart is very irregular, the curve may be further complicated by the fact that ineffective ventricular systoles cause small waves in 
the auricle which simulate those due to auricular contraction. Thus, it is very questionable whether all the small waves found in curves of auricular flutter can be referred to contraction of the auricle and its tempo so determined ( $c f$. Fig. 81). Furthermore, in cases undoubtedly established as auricular fibrillation, presystolic waves closely resembling $a$ waves are not infrequently found ( $c f$. wave $4,5,6$, Fig. 84 ).

The a-c Interval.-The time interval from the rise of the presystolic or $a$ wave to the systolic or $c$ wave is called the $a-c$ interval. It is generally regarded as giving an estimate of the time interval between auricular and ventricular contraction ( $A s-V s$ interval), and so permits a determination of the conduction time from the auricle to ventricle. This interval is about 0.1 second longer than the $P-R$ interval of the electrocardiogram, which may be due to the fact that the $a$ and $c$ waves are transmitted with irregular velocity to the neck, or, to the fact that the records represent different phenomena (see page 161, Electrocardiogram).

According to Lewis it has been established by electrocardiographic studies that whenever this interval is longer than 0.3 second it may be assumed to be due to an increase in conduction time.

The idea that the $a-c$ interval may be used as a criterion of the conduction time presupposes, first of all, that the venous $a$ wave and the arterial $c$ wave are conducted to the neck at a constant velocity. It is evident from optical tracings that this is not so, especially in cases of irregularity in which the isometric period of the ventricle lengthens and the rate of arterial transmission is slower. Passing this source of error by, however, one may examine what this interval really represents. It embraces $(a)$ the systole of the auricle, $(b)$ the period of rising tension in the ventricle, and $(c)$ the ejection plus the transmission of the arterial pulse to the neck.

It was formerly assumed that the same impulse that set off the auricle passed on and stimulated the ventricle. Hence, it was reasoned that if we assumed the systole of the auricle, the period of rising tension and the transmission time to be equal-in itself somewhat daring in the case of irregularities in which experiments show them to be prolonged - then the $a-c$ interval represents the time taken for the impulse to be conducted from auricle to ventricle.

Recent work, however, has shown it to be improbable that the impulse is conducted to the ventricle via the auricle, ${ }^{1}$ but directly from the sinus, hence the $A s-V s$ interval is not necessarily related to the sino-ventricular conduction period. As diagrammatically illustrated in Fig. 75, the sino-ventricular conduction period $\left(C-F^{\prime}\right)$ is longer than the $A s-V s$ interval $(B-F)$, and the latter serves as an estimate of the former only when we may suppose that the conduc-

1 The term "auricle" is not used here as synonymous with "atrium" but designates the main body of the contraeting auricle. 
A

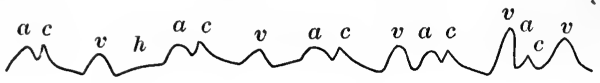
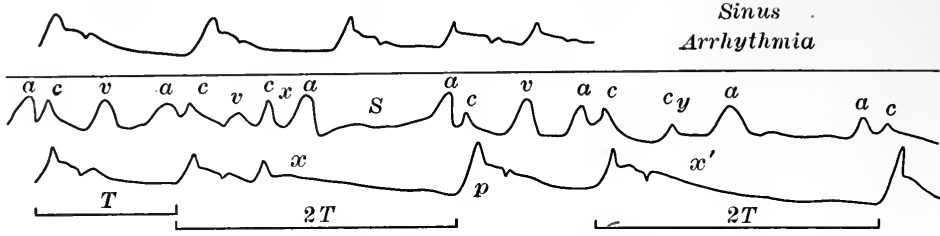

$B$

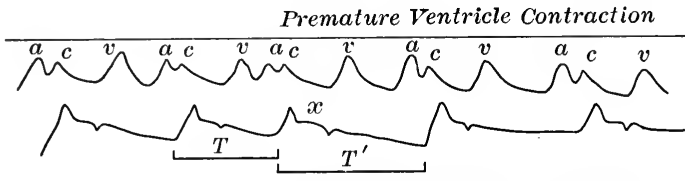

Premature Auricle Contraction

D

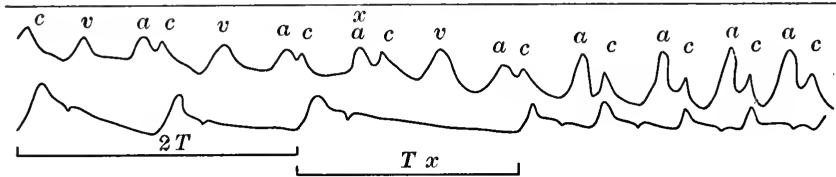

\section{Paroxysmal Tachycardia}

E

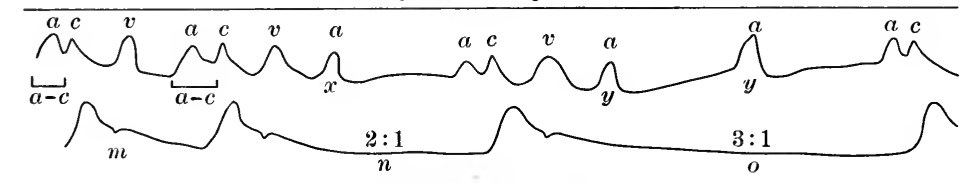

Partial Heart Block

F

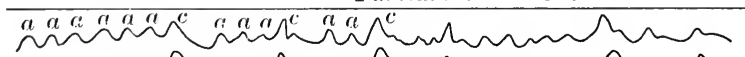

$F$

G

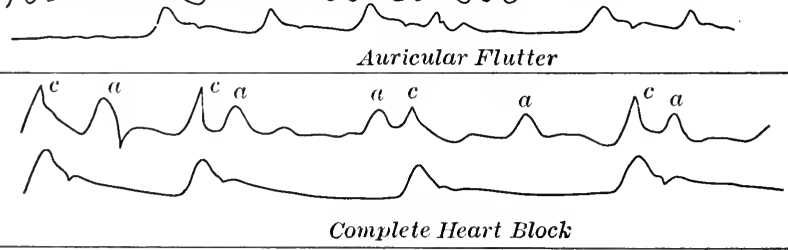

II

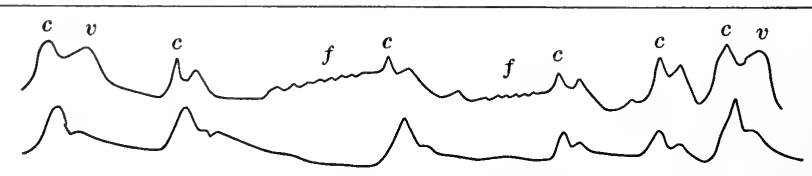

Auricular Fibrillation

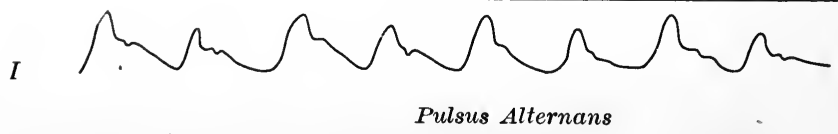

FIG. 36.-Scheme showing the effect of various forms of arrhythmias on the arterial and venous pulses. For description of the arterial pulses see page 115; of venous pulses, page 143 . 
tion from sinus to auricle $(A-B)$ is also constant. It must be admitted that for many reasons the $a-c$ interval cannot be implicitly regarded as an index of sino-ventricular conduction time. That it does correspond so well with the electrocardiogram $P-R$ intervals is in fact extremely remarkable.

Cardiac Arrhythmias. - The most characteristic variations of the supraclavicular venous pulse in some of the common forms of irregularity are indicated in Fig. 36. A more detailed exposition of the various forms of irregularity will be found in a subsequent chapter.

A. Sinus Arrhythmia. - The venous pulse is usually characterized by a regular sequence of the $a, c, v$ waves and a normal $a-c$ interval (0.1-0.2 second). The irregularity consists in the early or late occurrence of each group. Individual variations may arise in which the length of cycles varies markedly. Thus, in the long cycles an $h$ wave may occur while in the shorter ones the $v$ wave may be combined with the next $a$ wave. In extreme cases the $a-c$ interval may also vary but it cannot be definitely accepted that this represents an altered conduction time.

B. Premature Ventricular Contraction.-The venous pulse is characterized by a premature $c$ wave as at $x$. This is followed by an auricular contraction $(a)$. Since this stimulus finds the ventricle in its refractory phase, it is followed by no $c-v$ complex (cf. Fig. 79). For this is substituted a stasis rise $(S)$ which fills the compensatory period. The premature systole may occur so early $(y)$ that it replaces the $v$ wave. Such a systole is usually so weak, however, that the semilunar valves fail to open and $a$ wave fails to appear in the arterial tracing taken from the wrist. It may, in fact, happen that no extra $c$ wave appears in the venous pulse, the presence of the extra contraction being then recognized only by the heart sounds in optical tracings.

C. Premature Auricular Contraction.-The venous tracing is characterized by a normal $a, c, v$ sequence, for an entire $a, c, v$ group occurs prematurely $(x)$. The $a-c$ interval is increased due, partly, perhaps, to the changed sino-ventricular transmission time and the altered length of the isometric period of ventricular contraction.

D. Paroxysmal Tachycardia.-The venous curve shows, previous to onset, one or several auricular extra systoles, $i$. e., premature $a, c, v$ wave groups followed by an interval with a stasis rise. At the onset of tachycardia the $a, c, v$ sequence is disturbed only by the fact that the $v$ wave becomes merged with the succeeding auricular contraction.

E. Incomplete Heart Block.-The earliest stages are said to be indicated by a lengthening of the $a-c$ interval alone. Such curves should be corroborated, however, by other evidence before diagnosing an impaired conductivity. Similarly, impaired conductivity 
between sinus and ventricle may occur with an $a-c$ interval, entirely normal. The diagnosis may be made, however, when a long $a-c$ interval or a progressively increasing interval is followed occasionally or regularly by a dropped arterial beat. In such cases, illustrated at $x$ and $y$, the $a$ wave recurs regularly but is not followed by the $c-v$ complexes.

F. Auricular Flutter.-The venous pulse, when the arterial rate is slow, shows a rapid succession of small $a$ waves which are occasionally interrupted by waves of ventricular origin ( $f f$. also Fig. 81). When the ventricle beats rapidly, however, and tricuspid regurgitation supervenes all evidence of auricular contraction may be obscured and the ventricular form of venous pulse alone predominate. In such cases the venous pulse is not diagnostic.

G. Complete Heart Block.-The venous pulse shows a regular succession of $a$ waves, interrupted at irregular intervals by $c$ and $v$ waves of ventricular origin. These groups fall between the $a$ waves or are superimposed upon them.

H. Auricular Fibrillation.-The venous pulse is characterized by an entire absence of $a$ waves and the presence of ventricular $c-v$ complexes. These have a varying comformation and recur very irregularly but at a rather rapid rate. Between these systolic groups are long stasis periods upon which small undulations attributed to auricular fibrillations are evident. Other smaller waves due perhaps to ineffective systoles of the ventricles and other causes as yet unknown are common.

\section{BIBLIOGRAPHY.}

\section{BOOKS AND MONOGRAPHS.}

Gibson. Diseases of the Heart and Aorta, London, 1907.

Hirsehfelder. Diseases of the Heart and Aorta, Philadelphia, 2d ed., 1910.

Lewis. The Mechanism of the Heart Beat, London, 1911.

Mackenzie. Diseases of the Heart, 3d ed., 1913.

Ohm. Der Venenpuls, 1914.

\section{PApers.}

Bachmann. Amer. Jour. Med. Sci., 1908, cxxxvi, 674.

Bard. Jour. Physiol., et Path. gèn., 1906, viii, 454, 466.

Cushny. Jour. of exp. Med., 1899, iv, 327.

Edens. Deutsch. Arch. f. klin. Med., 1910, c, 221.

" Deutsch. Arch. f. klin. Med., 1911, ciii, 245.

Edens and Wartensleben. Deutsch. Arch. f. klin. Med., 1911 civ, 552.

Ewing. Am. Jour. Physiol., 1914, xxxiii, 158.

Eyster. Jour. Exper. Med., 1911, xiv, 594.

François Franck. Arch. de physiol., 1889, i, 70.

Fredericq. Arch. de biol., 1888, viii, 497.

" Arch. internat. d. physiol., 1907, v, 1.

“ Zentralbl. f. physiol., 1908, xxii, 297.

“ Jahresb. der Physiol., 1908, 99, 122.

" Arch. internat. d. Physiol., 1906, iv, 57.

“ Arch. internat. d. Physiol., 1907, v, 1. 
Freidreich. Deutsch. Arch. f. klin. Med., 1866, i, 241.

Gibson. Lancet, 1907, ii, 1380.

Gottwalt. Arch. f. d. ges. Physiol., 1881, xxv, 1.

Grosh and Cushny. Jour. Am. Med. Assn., 1907, xlix, 1254.

Hering. Deutsch. med. Wehnschr., 1895, xxxiii, 1.

" Arch. f. ges. Physiol., 1912, cxlix, 594.

“ Arch. f. ges. Physiol., 1904, cvi, 1.

Hirschfelder. Am. Jour. Med. Sc., 1906, exxxii, 378.

"Johns Hop. Hosp. Bul., 1907, xviii, 262.

Keith. Jour. Anat. and Physiol., 1908, xlii, 1.

Knoll. Arch. f. d. ges. Physiol., 1898, lxxii, 317.

Lian. Jour. physiol, path. gén., 1912, xiv, 569.

Mackenzie. Am. Jour. Med. Sc., 1907, cxxxiv, 12.

" Jour. Path. and Bacteriol., 1893, i, 53.

" Jour. Path. and Bacteriol., 1894, ii, 84.

Mathieu and Watrin. Compt. rend. Acad. d. Sci., 1912, lxxii, 1103.

Morrow. Arch. f. d. ges. Physiol., 1900, lxxix, 442.

“ Brit. Med. Jour., 1906, ii, 1807, 1119.

Ohm. Deutsch. med. Wehnschr., 1912, xxxviii, 2260.

“ Deutsch. med. Wchnschr., 1913, xxxvii, 970, 1493.

“ Ztschr. f. exper. Path. u. Therap., 1912, xi, 526.

“ Zentralbl. h. Herz. u. Gefäss., 1913, v, 153.

Piersol. Am. Jour. Med. Sci., 1908, exxxv, 812.

Piper. Arch. f. Physiol., 1913, 385.

Pramberger. Wien. klin. Wehnschr., 1888, p. 13.

Rautenberg. Deutsch. Arch. f. klin. Med., 1907, xci, 251.

“ Ztschr. f. klin. Med., 1908, lxv, 106.

“ Deutsch. med. Wehnschr., 1913, xxxix, 1033.

Riegel. Deutsch. Arch. f. klin. Med., 1882, xxxi, 1.

Rihl. Ztschr. f. exper. Path. u. Therap., 1904, i, 43.

“ Ztschr. f. exper. Path. u. Therap., 1909, vi, 619.

"Wien, klin. Wchnschr., 1907, xx, 931.

“ Berl. klin. Wchnschr., 1907, xliv, 825.

Straub. Arch. f. d. gesam., Physiol., 1911, cxliii, 69.

Verdon. Lancet, 1913, clxxxiv, 239, 1486.

Wenckebach. Ztschr. f. klin. Med., 1899, xxxvi, 181.

"Ztschr. f. klin. Med., 1899, xxxvii, 475.

"Ztschr. f. klin. Med., 1900, xxxix, 293.

“ Arch. f. Anat. u. Physiol., 1906, p. 277.

Wichrew. Russk. Vrach, vii, 578.

Wiggers. Jour. Am. Med. Assoc., 1915, xliv, 1305, 1485.

Van Zwaluenberg and Agnew. Heart, 1913, iii, 343. 


\section{CHAP'TER X.}

\section{THE ESOPHAGEAL PULSE AND THE ESOPHAGRAM.}

IF an esophascope is inserted into the esophagus, pulsations are visible in two places. The upper pulsations, which are the weaker, are seen at about the level of the tracheal bifurcation or about 26-30 centimeters from the teeth (Rautenberg). The lower pulsations which are more vigorous occur somewhat lower down, 32-36 centimeters from the teeth (Rautenberg). A study of the anatomical relations shows that in this region the left auricle is in direct contact with the esophagus for a distance of 5 to 6 centimeters, hence the pulsations are evidently transmitted directly from it to the esophagus.

Registration.-'The idea of obtaining esophagrams, as the records may be termed, occurred first to Luciani and later to Fredericq and his pupils who employed the method in animals. To Rautenberg and Minkowski, however, we owe the clinical application of the method.

To obtain an esophagram the following technic is usually followed: After cocainization of the pharynx, a lubricated rubber tube about 5 millimeters in diameter and 75 centimeters in length, which is equipped below with a small soft rubber bag, is swallowed to a distance of about 38 centimeters. The bag is then gently inflated and gradually drawn out either until a distinct pulsation is obtained in the tambour with which it is connected, or until the heart sounds heard through a connected stethescope become loudest. The tendency to swallow saliva must be counteracted by suitable means, either by allowing it to drip into a receptacle or by employing one of the various drying appliances used by dentists for similar purposes. The records are preferably taken in the sitting posture; but if it is necessary to recline, the lateral or abdominal position should be assumed.

Nature and Time Relations of the Records.-As can readily be understood, the records necessarily differ according to the part of the esophagus from which they are recorded (Young and Hewlett, Edens). With the most exact placement over the left auricular area, three waves regarded as similar in character to those obtained from the venous pulse, are recorded (Fig. 37). They have been designated as the $A s, V s$, and $D$ waves by Rautenberg. As has been pointed out by Young and Hewlett as well as by Edens, how- 
ever, a distinct $A s$ wave is not always present, but in the upper and lower portions of the auricular area, it is replaced by a negative drop. The time relations of these waves have been compared with the waves of the jugular pulse in a previous section of this book. It is only necessary to repeat that they precede the corresponding jugular pulse waves by irregular intervals. Their exact relations to the waves of the electrocardiogram have been established by Rautenberg, Kahn and more recently by Benjamin. The $A s$ wave rises about 0.015 second after the rise of the $P$ wave and the fall continues until the $Q$ depression of the electrocardiogram. 'The rise of the $R$ wave also precedes the mechanical elevation, $V s$, by a similar interval. The $D$ wave drops a distinct interval after the end of the $T$ wave.

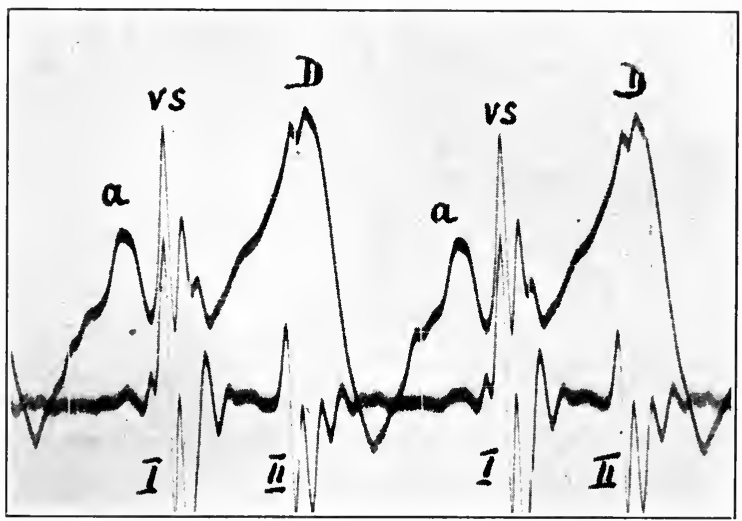

Fig. 37.-Esophagram and heart sounds recorded with segment capsules. $a$, auricular wave; $v s$, systolic wave; $D$, diastolic peak. Lower records show first and second sounds. (After Edens.)

Interpretation of the Esophagram.- The interpretation of the esophagram involves the question as to what form of cardial activity is actually transmitted to the esophagus. The anatomical proximity of the left auricle to the esophagus has led to the natural inference that the intra-auricular pressure variations are transmitted through the elastic walls of the auricle and esophagus. Van Zwaluenberg and Agnew have supported this view by showing that the waves in the auricle and those recorded from the esophagus by optical apparatus correspond exactly. This fact, however, does not conclusively indicate that the two sets of waves are due to exactly the same influence. It has been suggested that the changing position of the auricle during its systole and diastole, as well as the traction influence of the ventricular systole, must directly modify the intra-esophageal pressure. Attention has likewise been called to the effect of cardiopneumatic variations in intra- 
thoracic pressure upon the esophagram: Luciani, as early as 1877 , reported that the esophagram taken from an intact thorax differed essentially from one recorded when the thorax was opened. Too much significance, however, should not be attached to such observations, for not only is a great risk incurred of directly altering the position of the sound, but, in addition, the entire circulation phenomena are known to change when the chest is opened so that one should expect a difference in the esophagram apart from that caused by negative pressure. The results of Meltzer and Auer lead us to believe that the importance of the part played by cardiopneumatic phenomena is doubtful, since they have shown that the negative pressure variations within the thorax were very poorly transmitted to the posterior mediastinal area below the bifurcation of the trachea.

In interpreting the esophagram as an index of left auricular activity, it should be remembered that it represents a combined influence of auricular position change and intra-auricular pressure phenomena. For this reason, different types of curves may be obtained from different regions of the esophagus. The $A s$ wave when present is undoubtedly associated with a contraction of the auricle and so is presumably caused by its central bulging as the pressure rises. When, instead of this wave, a negative drop appears, it is probably because the contracting auricle draws away from the esophagus in the place where the record is taken. The $V s$ wave, attributed by Rautenberg to a change in the length of the heart as a result of ventricular systole, is shown by optical methods (Edens) to be a more complicated phenomenon. It seems very probable from the nature of the oscillations present that the vibrations of the mitral valves are concerned. Following these vibrations the pressure rises due to stasis, very much as in the intra-auricular pressure curve. As at the opening of the tricuspid valves the auricular pressure rapidly falls and the position of the heart changes as well, the curve drops quickly. It is evident that with proper allowances for a possible predominating influence of cardiac movement, the esophagram follows the changes in the intra-auricular pressure closely (Edens, Van Zwaluenberg and Agnew).

\section{CLINICAL VALUE OF THE ESOPHAGRAM.}

The ways in which the esophagram has been considered useful in clinical cases may be briefly enumerated:

1. It establishes the existence of auricular activity-but in this capacity it merely supplements information gained from records of the jugular pulse.

2. It gives evidence of dissociated activity of the two auricles. Edens published a rare case in which the left auricle was apparently 
paralyzed due to distention, while the activity of the right was unimpaired.

3. It may give evidence of mitral regurgitation much as the ventricular form of venous pulse gives evidence of tricuspid regurgitation (Minkowski). Many of the curves, however, which were published as characteristic of this condition showed no deviation that might not be found in perfectly normal tracings (Young and Hewlett). Edens, also, after a rather extensive experience with optically recorded esophagrams, found no typical type of curve in these conditions.

4. It has been suggested that the method might give evidence of adhesive pericarditis, especially in cases in which the systolic negative apex beat is absent. Edens, however, found no characteristic tracings in this condition.

It is evident that the method only supplements the information given by the jugular tracing in cases in which the interpretation of the latter is in doubt. That the esophageal pulse more nearly approximates the pressure variation in the auricle than the jugular pulse is evident, but its usefulness is limited by the fact that the movements of the heart may, under special circumstances, become its predominant factor.

\section{BIBLIOGRAPHY.}

\section{Papers.}

Benjamins. Arch. f. d. ges. Physiol., 1914, clviii, 158.

Edena. Deutsch. Arch. f. klin. Med., 1910, c, 241.

Fredericq. Arch. de biol., 1887, vii, 238.

Janowski. Ztschr. f. klin. Med., 1910, lxx, 211.

Kahn. Ergebn. der Physiol., 1914, xiv, 1.

Luciani. Physiol. des Menschen., 1905.

Minkowski. Ztschr. f. klin. Med., 1907, Ixii, 371.

Rautenberg. Deutsch. Arch. f. klin. Med., 1907, xci, 251.

" Deutsch. med. Wehnsehr., 1907, xxxiii, 364.

Van Zwaluenburg and Agnew. Heart, 1913, iii, 343.

Young and Hewlett. Jour. Med. Research, 1907, xvi, 427. 


\section{THE APEX BEA'T AND CARDIOGRAM.}

IF the finger is placed over that region of the chest corresponding to the apex, an impact is felt that is spoken of as the apex beat. Although much discussion has arisen as to its cause, it has always been recognized as representing the beginning of ventricular systole. The graphic record of this impulse is termed a cardiogram.

\section{METHODS OF RECORDING.}

For reasons of convenience, the direct registration of cardiograms by levers has been almost entirely replaced by that of air transmission systems. The simplest procedure consists in applying a round or horizontal box-shaped receiver over the impulse area and transmitting the movements of the skin through tubing to a recording tambour. This method is at once the oldest, simplest, and best. As in the transmission sphygmograph, the chief desideratum for accurate work is a recording mechanism having a sufficient vibration frequency. This no tambour recording on a smoked surface or with ink pens can possibly have. Use has, therefore, been recently made of Frank's optical capsules (Frank and Hess, Wiggers) and the interference bands of the micrograph (Crehore).

A more complicated transmission occurs in the use of the cardiograph first devised by Marey and subsequently modified by a number of investigators (Kronecker). The cardiograph of Marey consists of a tambour covered by heavy rubber in the centre of which is a button brought into apposition with the impact area. In some forms, as that by Edgren, a spring is placed inside of the capsule making this instrument, in contrast to the funnel receiver, not a mere volume recorder but a tension apparatus. A tension recording instrument would be necessary if the recorded curve represented a record of intraventricular pressure. A sane consideration of physical and anatomical facts, however, makes it difficult to conceive how this can be the case, for the intraventricular pressure variations not only fail to be transmitted through the thick apical and thoracic musculature but the relation of the apex to the chest wall changes during the cardiac cycle. Even if the cardiogram could be shown to possess a contour identical with that of the intraventricular curve-which has not been accomplished 
-it would not be proof that they represented the same thing. We may assume with reasonable surety that the apex beat represents only the varying pressure of the cardiac apex on the thoracic tissue; and this is solely governed by the shifting of the heart's position and its degree of filling. In recording these movements through the skin and intercostal muscles, nothing is gained by a tension recording device of such a construction as the Edgren cardiograph.

Nature of Cardiograms Obtained by Recording Tambours of Adequate Efficiency.-Cardiograms taken with reliable forms of apparatus have not been extensively published. Frank and Hess reported a diagram of what they consider a typical cardiogram in

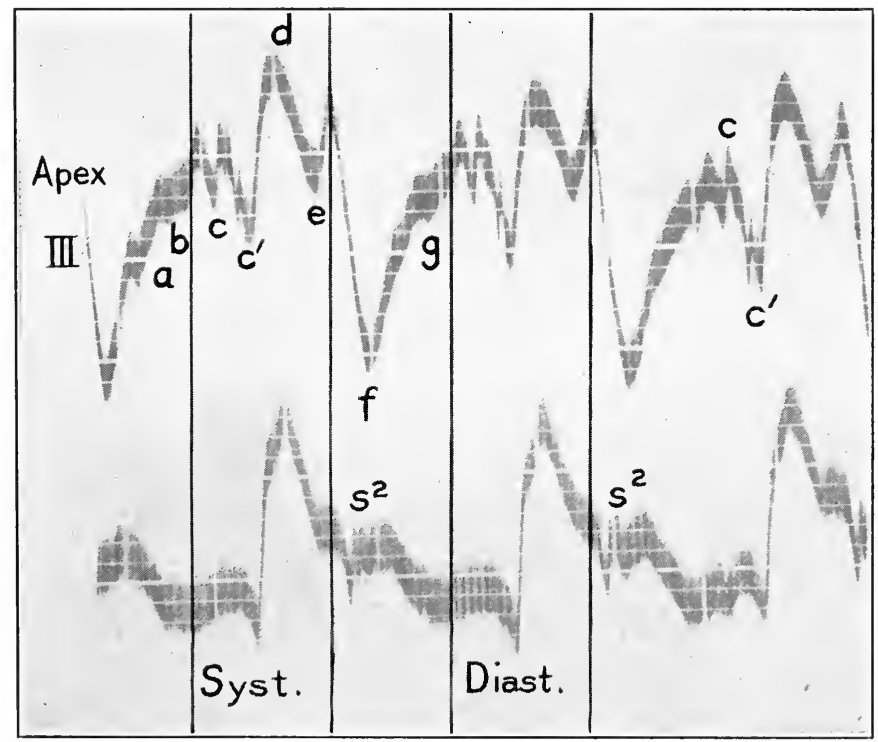

Fig. 38.-Optical tracings of cardiogram and subclavian pulse. $a-b$, auricular systole; $c$, initial heart sound vibrations; $c^{\prime}$, valve vibrations of first sound: $s^{2}$, vibrations of second sound indicated on subclavian pulse ( $c f$. with Fig. 47).

the dog. Records not unlike this have been obtained by the author in man (Fig. 38). More than a tentative explanation of such records is impossible at present. The curves begin with an oscillation $a-b$ that may conceivably be due to auricular contraction. Ventricular systole is inaugurated by a series of sharp vibrations due to the first sound. There are apparently two groups of these vibrations, an initial set of longer period $(c)$ and a final set of shorter period $\left(c^{\prime}\right)$. Their explanation is still in doubt. They last at least through the isometric period. As soon as the ejection period begins, as shown by the subclavian rise, the curve slowly rises to its maximum $(d)$ and then slowly recedes again $(e)$. This can be 
explained by assuming that, early in ejection, the heart continues to move downward and presses more firmly against the chest wall, but during its later phases, the apex gradually moves away, owing to the diminution in ventricular volume. At the beginning of diastole (probably) a rapid drop to $f$ takes place. This can be explained by assuming that the apex moves upward in the beginning of diastole. Finally, however, as the ventricle fills, the apex is again pressed against the chest wall more firmly and occasions the slow diastolic rise $(g)$.

It is incorrect to designate such a curve as a "typical cardiogram." The writer is inclined to believe that there is no "typical curve." If the receiving tambour be moved only $1 \frac{1}{2}$ centimeters to the right, the contour of the curve changes. The heart sound vibrations may still be recognized but the systolic curve is directed downward indicating that that particular part of the heart moved away during the entire systolic phase (negative cardiogram). If the tambour is displaced 1 centimeter to the left of its original position, the curve becomes entirely positive but of different contour (Fig. 47). After the typical heart sound oscillations $(c)$, the curve rises sharply to a peak $(d)$ and then slowly draws away during the rest of systole.

It is evident that slight variations in the position of the receiving tambour modify in pronounced fashion the contour of the cardiogram-probably because the relation of the heart to different points on the chest wall varies greatly.

Similar conclusions have been arrived at by Crehore who reported the details of cardiograms reconstructed from the micrograph interference bands. His curves also give evidence of preliminary oscillations similar to $c-c^{\prime}$. He concluded that the ejection period occurred somewhere within this group of waves rather than after it, as interpreted by the writer.

\section{CLINICAL VALUE OF CARDIOGRAMS.}

In estimating the clinical value of cardiograms it is desirable to consider separately those taken with polygraph tambours and those recorded with apparatus of adequate efficiency. The former have been used for the following purposes: (1) to determine the $A s-V s$ interval, (2) to mark exactly the onset of systole, (3) to determine by its conformation the presence of hypertrophy, (4) to distinguish right-sided from left-sided hypertrophy and (5) to determine whether a pulse-deficit is present.

In none of these capacities may the ordinary cardiograms be considered of any value. Optical tracings indicate that the onset of systole is frequently not accompanied by an immediate rise of the curve. If we add the delay in the rise of the tambour lever, it is apparent that the onset of systole can be only approximately 
established in this way. Since this is the case neither the presphygmic period nor the $A s-V s$ conduction time can be accurately estimated by comparing other pulses with the apex tracing.

Tracings taken by means of optical apparatus also indicate that curves of exceedingly varied contour may be obtained from perfectly normal subjects; that from the same subject, in fact, either positive or negative tracings may be obtained by shifting the tambour slightly. The latter are, therefore, of no diagnostic significance.

Although useful in a didactic way to demonstrate the existence of a pulse deficit, the cardiogram supplies no evidence not obtainable by auscultation for the heart sounds. It may be pointed out, in this connection, that every ventricular systole may not give a typical cardiogram. Due to the varying volume of the ventricles, the varying approximation to the chest wall and the changing force of systole, it is sometimes impossible to determine whether two consecutive waves are associated with the same systole or belong to two rapidly following beats. On the other hand, a feeble systole often gives no record at all.

Optical tracings of the apex beat have so far been shown of value in only one respect, namely, that they incorporate the heart sounds and so allow an exact establishment of systole and diastole. Whether any other significance may be attached to pathological tracings further investigation alone can determine.

\section{BIBLIOGRAPHY.}

PAPERS.

Crehore. Jour. Exper. Med., 1911, xiv, 339, 351, 520.

Frank. Tigerstedt's Handb. der Physiol. Method., 1913, II, Part 4, 182.

Frank and Hess. Verhandl. d. Cong. f. inn. Med., 1908, xxv, 285.

Kronecker. Compt. rend. Soc. de biol., 1901, liii, 390. 


\section{THE ELECTROCARDIOGRAM.}

\section{APPARATUS, TECHNIC, AND CRITIQUE.}

TuE fact has long been known in physiology, that whenever a tissue is active, the active portion becomes electrically negative to the resting portion. Hence, if an external conductor connects these portions, an electric current flows, the electromotive force of which is determined, as in a battery, by the degree of activity but the ampèrage of which is measured, according to Ohm's law, by the external resistance as well. This is called the current of action. If the tissue is so connected that, first the portion under one electrode, and later that under another is active, we obtain a current that flows first in one direction and then in the other, that is, a diphasic current.

To measure the difference of potential or the electro-motive force, the capillary electrometer was first employed. A description of this instrument is given in all modern text-books of physiology. It has certain advantages, two of which are: that it is relatively inexpensive, and lends itself to the detection of minute currents (0.013 millivolt) which it records without a latent period. The excursions are proportional to the electromotive force, when the capillary is of even caliber, and are not influenced by variations in external resistence. It is therefore possible to use this instrument as was done by Kölliker, Müller, Waller and Bayliss and Starling to detect the presence of rapidly recurring electrical variations in the hearts of animals and of men. In order to maintain a sufficient degree of sensitiveness, however, the apparatus must be so adjusted that neither the contour nor the size of the rapidly recurring electrical variations are correctly reproduced, and the curves obtained demand reconstruction. This can be done after the scheme formulated by Garten. The most practical objection to the use of the capillary electrometer-and the one no doubt that has limited its application in clinical work-is the fact that the instrument so readily becomes disarranged. For this reason it has been almost entirely supplanted by the string galvanometer in recording electrocardiac phenomena.

The String Galvanometer.-The string galvanometer, the development of which in its modern form is almost entirely due to the 
ingenuity of Einthoven, is based upon the principle that, when a conductor carrying electric current is placed at right angles to a magnetic

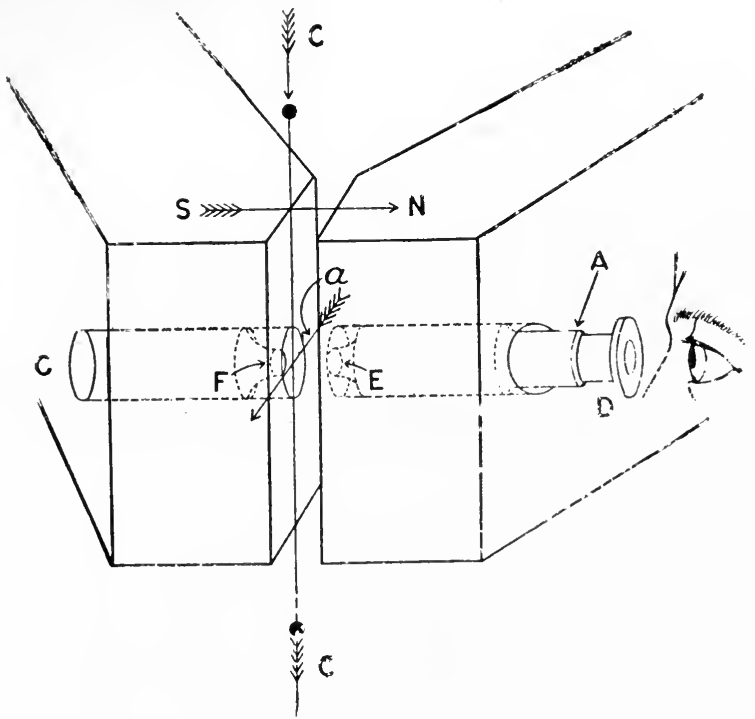

FIG. 39.-Diagram illustrating the principle of the string galvanometer. (After Lewis.)

field the current moves in a direction at right angles to both the field and the conductor. Thus, as is shown in Fig. 39, if a current passes

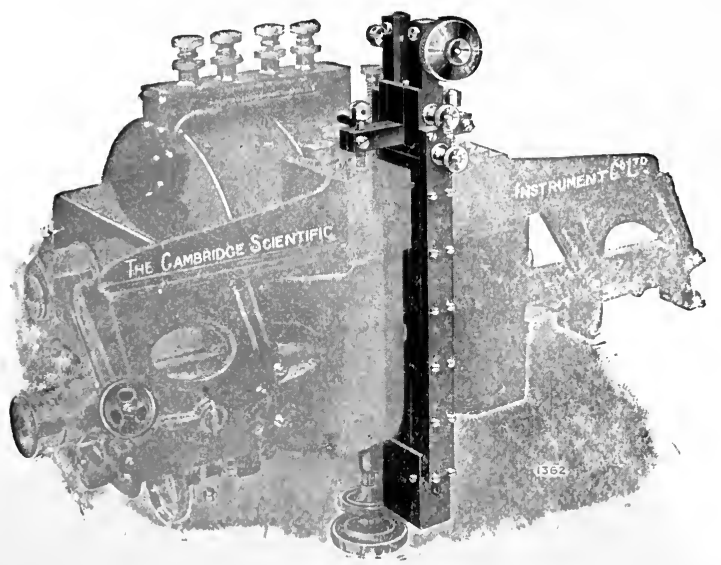

FIG. 40.-Cambridge model of Einthoven's string galvanometer.

down a very light fiber of silvered quartz $(C)$ placed in the field of two electro-magnets, $S$ and $N$, it will move in the direction shown 
by the arrow, $a$. In the best Einthoven galvanometers the string is made of silvered quartz and varies from 2-4 microns in diameter and is consequently visible to the naked eye only-in very intense light (Fig. 40). The tension of the string may be adjusted by the milled head which is fitted with a safety stop to prevent overtightening. The electro-magnets are excited by connecting them with a storage battery yielding $10-20$ volts. The movements of the string may be observed by a microscope (Fig. 39, $A$ ), fitted through openings in the two magnets, or the shadow of its movements may be projected to the lens of a photokymograph by concentrating the heat-free rays of a quiet arc lamp upon the fiber.

Procedure in Recording Electrocardiograms.-A procedure similar to the following is generally employed to obtain electrocardiograms

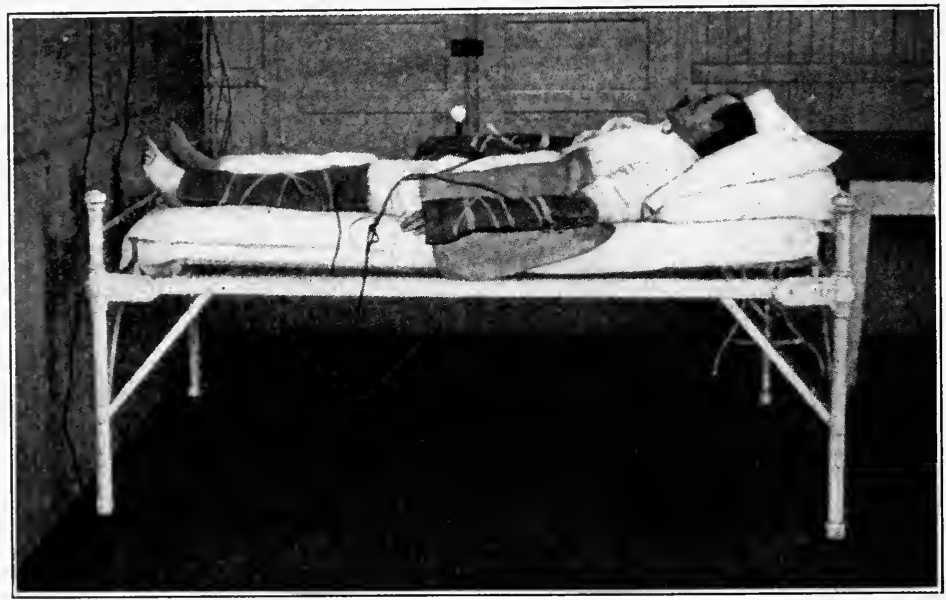

F1G. 41.-Photograph illustrating Williams' electrodes in use. (After James and Williams.)

from patients: Three electrodes, each consisting of a vessel of saline, or, more conveniently, of a metal strip covered with absorbent material soaked in saline (Williams) are applied to the two arms and the left leg of the subject (Fig. 41). By connecting any two of these electrodes in such a way that the photographed shadow moves up when the base of the heart is negative, it is possible to tap the heart currents in three different places or, as generally worded, we obtain three different leads, namely:

Lead I.-Right arm, left arm.

Lead II.-Right arm, left leg.

Lead III.-Left arm, left leg.

When any one of these leads is connected with the galvanometer, a deflection of the string in one direction occurs' even when the 
patient lies thoroughly relaxed. This so-called "body current," probably due to the activity of innumerable glands, must be neutralized or "compensated" by introducing into the circuit a corresponding counter-current from a cell. With both body and galvanometer in the circuit the sensitiveness of the apparatus is so adjusted that, after the custom introduced by Einthoven, one millivolt causes a deflection of one centimeter in the shadow of the fiber, a procedure spoken of as the "standardization." The combined resistance of the patient's body and the electrodes may be determined by substituting an artificial resistance sufficient to again cause a shadow deflection of one centimeter when one millivolt is passed through the circuit. A distinction should be made between the real and the apparent resistance (Einthoven). 'The apparent resistance is determined by the use of a direct current, the real by the use of an alternating one. In the body the apparent resistance may be more than twice as great as the real resistance. Einthoven has recently pointed out that the apparent and real resistances should not differ from each other by more than four hundred ohms. This may be brought about by immersing the extremities deeply into a 20 per cent. saline bath.

All of these procedures, that is, standardization, compensation for body currents, estimating external resistance, are accomplished by convenient electrical devices, the details of which do not properly belong here. ${ }^{1}$ The electrocardiogram is then recorded by allowing the projected shadow of the string to be focused by a cylindrical lens in the camera on a sensitive bromide paper, film or plate. A millimeter scale drawn on the lens projects horizontal lines on the paper and a rotating device, regularly interrupting the light may be added to conveniently give short time intervals as intersecting vertical lines. Such devices called episcotisters have been cleverly devised by Garten, Einthoven, Bull, and Williams.

Critique.-The impression seems wide-spread that the use of a string galvanometer to record electrical variations is in itself a guarantee of their trustworthy character. No idea could be more erroneous. It is quite possible to obtain curves-and, unfortunately, many such curves have been obtained and published-which are in no way superior to records obtained with the capillary electrometer. $^{2}$

1 For description of details consult Einthoven, Arch. f. d. ges. Phys., 1908, exxii, 517; Lewis, Clinical Electrocardiography, 1913, p. 4.

2 We refer only to the accuracy of the electrical variations and not to the inartistic and foggy records that often appear, although in this respect it is hoped some improvement may soon be made. Few investigators have published records that approach the excellent standard set by Einthoven, Lewis and Williams. Those who experience difficulty in this direction may perhaps with profit read Garten's excellent exposition of the subject of photographic registration in Tigerstedt's Handbuch der physiologischen Methodik, Band I, part 2, pages 65-124. 
It is important to remember that the deflection for any given string is proportional to the ampèrage and not the voltage. It varies also with the length, diameter, composition, preparation, and tension of the string. To utilize the apparatus as a voltmeter the standardization before explained is necessary. In order to accomplish this with a variable external resistance either the resistance of the string must be altered or its tension changed. The latter is the more convenient procedure and the one generally employed. It is obvious, furthermore, that the relation between the actual deflection of the string and its shadow on the photokymograph depends on the optical magnification (that is, the lens magnification and the distance from the photokymograph). It is quite evident that those investigators, who, on account of inadequate illumination or inferior lenses, are forced to work with a magnification of 100-200 require a much greater actual deflection, and hence must employ a string under less tension than those who are able to utilize a magnification of 600 to $1000 .{ }^{1}$ Unfortunately, however, as the string is slackened, the deflection time, which is the time interval between the zero position and the final level is increased. This is well shown in the following table compiled from the results of Samojloff:

\begin{tabular}{lccc}
\multicolumn{2}{c}{ Sensitiveness. } & Magnification. & Deflection time. \\
1 millivolt $=1 \mathrm{~cm}$. & 800 & 0.024 second \\
1 millivolt $=1 \mathrm{~cm}$. & 400 & 0.060 second \\
1 millivolt $=2 \mathrm{~cm}$. & 400 & 0.100 second
\end{tabular}

This leads to the discussion of the qualifications of a reliable instrument and the methods by which they may be determined. As Einthoven has recently pointed out, a perfect galvanometer has not yet been constructed, but the error can be reduced to a point where it is negligible. Fahr, who has recently made a theoretical analysis of the string galvanometer, has shown that the string must have an inherent vibration rate, at least five times as great as the rate of vibration to be recorded if the error is to be reduced to 4 per cent.; whereas, it is necessary that the inherent rate of the string should exceed the vibration ten times if the error is to fall to 2 per cent. Fahr very practically points out that, since the tension of the average string 10 centimeters long cannot be safely increased to give more than a thousand vibrations per second, the electrical variations recorded should not exceed two hundred per second.

Given an instrument with a vibration rate five times as great as the vibration to be recorded, we may be certain that it is reliable for prac-

\footnotetext{
${ }^{1}$ This is the ehief defect of the less expensive models, the installation of which is often urged upon those institutions and individuals, who are unable to afford higherpriced apparatus. The time is at hand, however, to discourage such installations.
} 
tical purposes, provided the combined air and electromagnetic damping is sufficient to render the string aperiodic.

There is, however, another and more practical way in which the efficiency of an instrument may be tested, namely, by determining experimentally the longest deflection period permissible without causing any alteration in the height and contour of the curves. Samojloff, Einthoven, and Lewis have made such determinations. The curves of Lewis show that when the deflection time becomes greater than 0.02 second, a distortion of the electrocardiogram waves occurs. Briefly stated, the amplitude of the $R$ wave is too small and that of the $T$ wave is relatively too large. An evident failure on the part of some clinicians to comply with this requirement discounts seriously any significance they have attributed to slight variations in the height of these waves. If it can be shown by supplementary tests that the string is aperiodic and the deflection time is 0.02 second or less, it may be inferred that the instrument is sufficiently accurate for all practical purposes.

\section{THE NATURE OF THE RECORDS AND THEIR INTERPRETATION.}

Terminology.-The normal electrocardiograms, as the records are termed, are characterized by three or four positive variations and generally by two sharp negative waves (Fig. 42). Different terminologies have been used to designate these waves. Bayliss and Starling, who first recorded a triphasic current with the capillary electrometer, call the second and third variations the "initial and terminal phases" of the ventricular current. They also applied the term "spike" to the large, sharp wave. Realizing that the electrocardiogram could be open to different interpretations, Einthoven, in 1895, introduced the alphabetical designation $(P, Q, R$, $S, T$ and recently $U$ ), a terminology that has been almost universally followed. To indicate the lead in which the wave occurred, the Roman suffix is usually added in descriptions. 'Thus, $R$ II means the $R$ wave obtained by lead $I I$, etc. Krauss and Nicolai, partly, because they believe the letters should in some way indicate the cause of the waves and, partly, because they think that the letters used by Einthoven have in pathological cases been used to designate waves of diverse origin, suggest a nomenclature of their own, namely, $A(=P)$, the atrial wave, $J(=R)$ the initial ventricular wave, and $F(=T)$ the final variation. The interval between $A$ and $J$ is indicated by $h$ since this is considered the time that elapses in passing the His bundle. The nomenclature of these authors has, however, been received with little favor and seems entirely unnecessary for the reasons given by them.

Time Relations.-The generally accepted relations of the waves to auricular and ventricular systoles may first be stated: $P$ is asso- 
ciated with auricular systole. The $R$ wave very slightly precedes active ventricular systole or is coincident with it. 'The $T$ wave occurs at the end of ventricular systole, diastole following its termination. The small $U$ wave when present is early diastolic in time. The interval between the rise of the $P$ wave and the $R$ wave (the $P-R$ interval) is usually supposed to represent the conduction time through the Purkinje system.

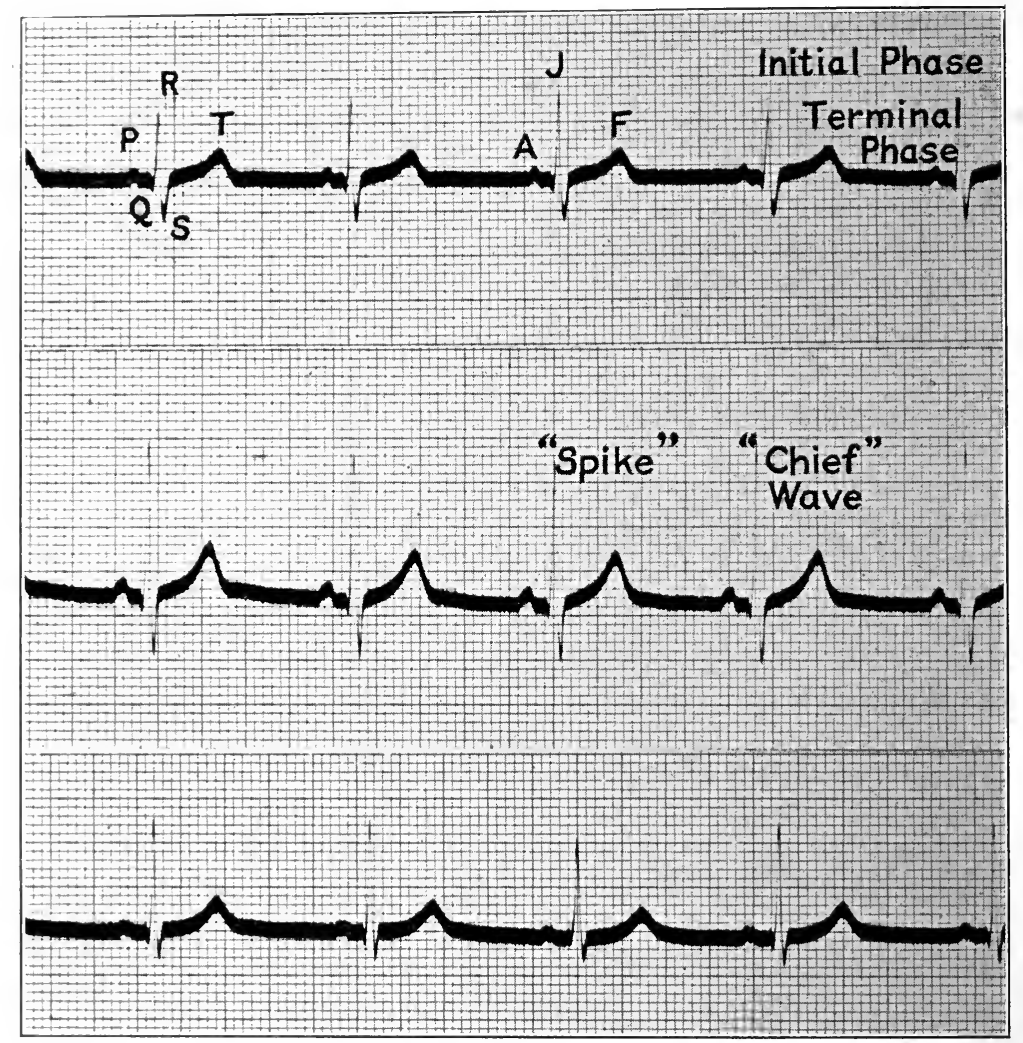

FIG. 42.-Normal electrocardiograms of a healthy young man-three leads; abscissæ 1 div. $=0.04$ sec.; ordinates 1 div. $=10^{-4}$ volt. The terminologies suggested by various investigators are indicated. (Courtesy of Dr. H. B. Williams.)

Recent work has tended to question the details of these relations (Kahn, Meek and Eyster). The $P$ wave apparently precedes the rise of right auricular pressure in animals and the $a$ wave of the venous pulse in man. The mechanical auricular response recorded by the suspension method also occurs about 0.07 second later than the rise of the $P$ wave, obtained by lead $I I$. On the other hand, the $P$ wave rises almost synchronously with the develop- 
ment of negativity in the sinus region (Meek and Eyster). These investigators therefore believe that the beginning of the $P$ wave is synchronous with the activity of the sinus tissue and that the wave itself expresses activity of the sinus plus auricle.

Kahn has presented records to support the idea that the $R$ wave is entirely completed before a rise of intra-ventricular pressure takes place; that it, likewise, precedes any mechanical change that can be recorded by levers from the right ventricles, and, lastly, that the first heart sound comes after the completion of the $R$ wave. The second sound follows closely after the $T$ wave.

Eyster and Meek found the $P-R$ interval in lead $I I$ shorter than the corrected $a-c$ interval of the venous pulse in four normal men, and noted similar differences between the $P-R$ interval and the $A s-V s$ interval determined from direct records of the heart. Hence, they conclude that the $P-R$ interval does not give a correct estimate of the $A s-V s$ interval.

It seems desirable that many of these results should be substantiated in other ways, however, before they are finally accepted. Thus, it becomes questionable whether the mechanical apparatus equals the electrocardiograph in efficiency and reaction time. No investigator has given data as to the efficiency of the mechanical apparatus employed. That the $a-c$ interval of the jugular pulse, even when corrected with reference to uncertain apex tracings, can give no exact data in problems of this kind, has been pointed out before (see page 141). That the intracardial curves recorded by Kahn were obtained by entirely inadequate manometers requires but a beginner in physiology to see.

Evidence of a more definite nature has been furnished by Fahr, who showed that the vibrations of the first heart sound occur on the upstroke of the $R$ wave, a conclusion confirmed by Williams and shown in Fig. 49 (personal communication). If we select the observations that seem to be reliably established, viz., that PII rises synchronously with initial sinus negativity (Meek and Eyster), that the rise of the $R$ wave begins slightly before the initial vibrations of the first sound and that the second sound comes after the completion of the $T$ wave (Kahn, Fahr and Lewis), we may adopt the tentative time relations for an electrocardiogram by lead $I I$, as shown in Fig. 25.

Interpretation of the Electrocardiogram Waves. - In spite of extensive efforts to elucidate the electrocardiogram waves, their cause is still in doubt. To begin with, the fundamental cause of electric currents in muscle tissue is also in doubt. Although most physiologists believe that the bulk of evidence supports Herman's theory, namely, that a difference in potential in living muscle only accompanies activity, while the "rest current" is really due to injury, there still remain some who choose to believe that, since difference 
in electrical potential is expressive of metabolism, and metabolism occurs during rest, the "currents of rest" are not due to a condition of injury.

Furthermore it has not been clearly demonstrated whether the condition of negativity passing over the heart accompanies the contraction or whether it develops in association with conduction of the impulse. There are those who doubt whether a slowly contracting muscle can generate a difference of potential except at the stage when the contraction of a part is beginning to pass off. The problem has also been complicated in its final analysis by the fact that it is difficult to determine the detailed activity in the heart by direct leads. The currents led from base and apex differ exceedingly in the number, height, and time relation of the waves. When a triphasic variation was repeatedly obtained, it was supposed to represent the correct change in electrical variation in the heart, especially as the waves bore some resemblance to the waves of the human electrocardiogram. Such a curve has frequently been explained to accord with the developmental history of the heart. According to this idea, first suggested by Gotch, the base of the heart is first negative due to auricular contraction ( $P$ wave), returning to a normal potential as the auricles relax ( $Q$ wave). As the excitation spreads to the ventricle, the base again becomes negative ( $R$ wave) and when, subsequently, the apex has become negative, the $S$ depression occurs. According to this view the base of the ventricle around the aorta contracts last and is responsible for the $T$ wave. The recent experiments of Neek and Eyster, however, tend to show that no such state of late negativity can be demonstrated in the region of the aorta by a very sensitive galvanometer.

That the generation of negativity in the heart is very much more complex than generally assumed is shown by experiments of Noyons, Straub, Bakko and Eiger, who showed that isolated auricles, sinus tissue, and even the conus arteriosus are able individually to give a triphasic current when a sensitive galvanometer is used.

It is evident that out of the whole series of triphasic variations we lead off from the entire heart three waves corresponding, perhaps, to the largest of these, or at least to waves very similar in character. This is due to the fact that the current within the heart not only spreads by external circuits but is also short-circuited more or less through the intraventricular fluid. 'Thus, the type of electrical variation led off from the surface depends, as Seeman has shown, upon the quantity of fluid within the ventricle.

Partly upon experimental evidence and partly upon the interpretation of normal and pathological electrocardiograms in man, it has been attempted to explain the cause of the electrocardio- 
gram waves. No explanation can be harmonized with all the available data, however; hence it remains impossible at the present time to decide between different explanations. Broadly considered, two views are held: The first that all wares accompany the excitation and contraction of heart muscle; the second that most of the waves are due to electrical changes accompanying impulse conduction, the contraction of the ventricle accounting for the $T$ wave, only.

The conceptions as to how contraction of the ventricle is capable of explaining, according to the first view, the ventricular waves, $Q, R, S$ and $T$, vary with different investigators. Einthoven believes that, as far as the electrocardiogram records are concerned, the right ventricle may largely be regarded as the cardiac base and the left ventricle largely as the apex. Whenever negativity predominates in the right heart an upward movement occurs; whereas, whenever it predominates in the left ventricle, a downward movement takes place. Upon this conception, the rise of the $R$ wave is attributed to systole of the right heart, the fall $S$ to the systole of the left ventricle; while in the $S-T$ interval the negativity at the base and apex are neutralized. The $T$ wave is explained as due to the fact that the contraction of the basal right ventricle outlasts that of the left.

Other investigators draw the line of isopotential more definitely between the anatomical base and apex and believe that negativity spreads in paths corresponding to the anatomical conduction paths. These investigators believe that the curve rises when the combined bases of right and left sides are negative and falls when the apices of the two are negative. According to this conception, the rise $R$ is due to the contraction of the two ventricular bases; the fall to the negativity of both apices; the interval intervening, to a contraction of the different systems so that currents neutralize each other, and the $T$ wave to a late contraction of the muscles around the aorta. This idea, which has here been expressed in very general terms, has been made much more explicit by Gotch, Kraus and Nicolai as well as by Eppinger and Rothberger, by assigning each variation to different systems or layers of ventricular muscle. For the details of this conception the original sources must be consulted.

In contrast to these views Hoffmann has suggested the idea that the electrocardiogram is essentially a picture of the electrical variations accompanying impulse conduction rather than contraction.

Upon this hypothesis, the $P$ wave accompanies conduction over the auricle. The $P-R$ interval is accounted for by the slow conduction across the His bundle. If the papillary muscles of the apex receive the impulse first, a $Q$ depression results. The rise of the $R$ wave occurs when the bases of the ventricles are excited and its 
fall when the impulse subsequently spreads to the apex. The $S-T$ interval without variations of potential, occurs during ventricular contraction because no further impulse conduction takes place, and the slowly contracting heart muscle yields no current until a part has begun to relax. When this happens, the $T$ wave appears.

\section{SUMMARY.}

The following summary expresses the different interpretations that have been given to the different waves:

$P$ wave accompanies $(a)$ contraction of auricle, $(b)$ conduction through auricle, $(c)$ activity of sinus region plus conduction through auricle.

$R$ wave (rise) accompanies (a) predominant contraction of right ventricle, $(b)$ contraction of basal portions of both ventricles, $(c)$ impulse conduction from base to apex of ventricles.

$R$ wave (drop) accompanies $(a)$ predominant contraction of left ventricle, $(b)$ contraction of apical portion, $(c)$ conduction from apex to base.

$S-T$ interval results from $(a)$ a balance of potential between left and right hearts, $(b)$ a balance of potential between base and apex, $(c)$ a balance between different layers of the heart, $(d)$ an absence of further conduction because all muscle is already excited and contracting.

$T$ wave, due to $(a)$ a change in the position of the heart (Usoff), (b) a return of the contraction wave to fibers around the aorta (Gotch, Nicolai), (c) a continued negativity of base outlasting that of apex (Bayliss and Starling and Einthoven), $(d)$ the contraction of no particular part but the entire expression of the electrical wave accompanying the contraction of the ventricle (Samojloff, Straub, Hoffman), (e) a diminution of the intracardial shortcircuiting at the end of systole when the ventricle is empty.

$U$ wave, a diastolic event, due to $(a)$ the last relaxation of the fibers of the ventricle (Einthoven), $(b)$ the electrical variation of the arteries (Hering).

Factors Determining the Size and Duration of the Electrocardiogram Waves. - The algebraic sum of all the differences in potential generated in the heart must be conducted in different directions through the tissues, but the leads, as usually applied tap only the currents that flow in the plane of the leading off points. Hence, whether we consider the electrical variations as primarily due to conduction or contraction-whether we regard the variation in negativity as resulting from alternate predominance of the right and left ventricular systole, or the anatomical base and apex contractions-the essential fact remains that the size of the deflection obtained with any lead depends on the relation between the current 
developed in the heart and the direction of the line of lead. This is shown in a simple manner in the diagram of Fig. 43. If the original current is represented in direction and quantity by the arrow, $x y$, the amount of current recorded by each lead may be measured by projecting the arrow perpendicularly to the lines of the triangle indicating the directions of the three leads. Thus, the distances $x_{1} y_{1}, x_{2} y_{2}, x_{3} y_{3}$ represent the magnitude of the recorded curve. According to this conception, we should expect the waves recorded by lead II to be larger than those from lead I and these, in turn, larger than those from lead III. That this is, as a rule, actually the case

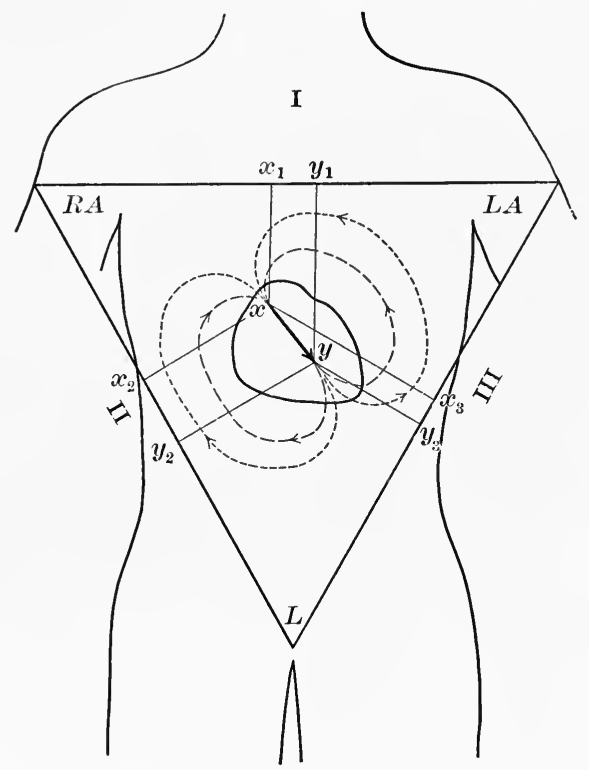

Fig. 43.-Diagram showing relations of the electrical axis of the heart to the three leads. $R A$., right arm; $L A$, left arm. Roman numerals refer to leads. (After Pardee.)

in normal hearts, is shown in the three leads of Fig. 42. Einthoven and Fahr have shown mathematically that lead $I I$ equals lead $I$ plus lead $I I I$, provided account is taken of the fact that the waves in leads $I I$ and $I I I$ start earlier than those in lead $I$, while the summits are recorded later than in lead $I$.

The direction of the waves in any lead depends on the direction that the current flows in the heart. If $x-y$ represents (Fig. 43) the direction of the flow in the heart and the dotted lines its distribution and direction in the tissues, then the flow in lead $I$ will be from $L A$ to $R A$, in lead $I I$ from $L$ to $R A$, in lead $I I I$ from $L$ to $L A$. 
Now if the current represented by $x y$ flows in a horizontal direction, as shown in Fig. 44, $A$, then, as normally, the current would flow in lead $I$ from $L A$ to $R A$, in lead $I I$ from $L$ to $R A$ as is normally the case; but in lead $I I I$, from $L A$ to $L$.

Again, if the current represented by the line $x y$ flows downward parallel to lead $I I I$, then (Fig. 44, B) the current will flow in lead $I I$ from $L$ to $R A$ and in lead $I I I$ from $L$ to $L A$ as is normally the case; but in lead $I$ it will flow from $R A$ to $L A$. Since in left- and right-sided hypertrophy the axis of the heart is changed; it is possible on the basis of such applications to diagnose these conditions (cf. Fig. 87).

A

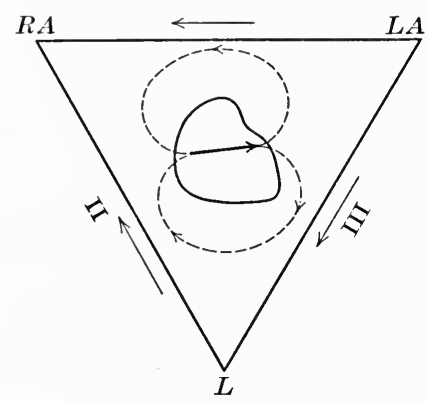

B

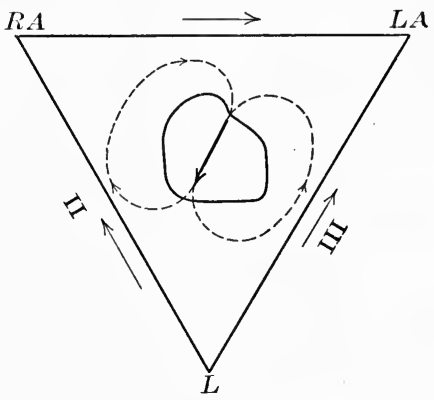

FrG. 44.-Diagrams showing how abnormal "electrical axes" in the heart cause different deflections in various leads, lettering same as before. (Modified slightly after Pardee.)

\section{CLINICAL ASPECTS OF THE ELECTROCARDIOGRAM.}

In spite of the fact that electrocardiography has been introduced into clinical use within comparatively recent years, its field of activity is no longer restricted to scientific and experimental work, but it has become an important and often indispensible aid in the diagnosis and management of cardiac disorders. This is due to the fact that we possess sufficient results, from which it is possible to state with a considerable degree of surety that certain pathological disturbances are associated with definite variation in the size, contour, and time relations of the individual waves.

Normal Variations. - Before it is possible to interpret the waves characteristic of pathological conditions it is desirable to know what variations may be considered within the range of normal. Various investigators, among whom may be mentioned Linetzky, Lewis and Gilder, and Strubell, have made such studies in normal persons. As a result it has been found that the electrocardiagram 
does not change in the same individual for long periods of time. The contour and time relations of the different waves are generally so characteristic that they can be picked out at a glance. Certain variations in the different waves, however, occur in different individuals.

The $P$ wave is rounded in contour and averages one-tenth the height of the $R$ wave. Occasionally it is negat.ve and sometimes bifurcated. Lewis found this type present in seventeen cases out of fifty-nine in leads $I$ and $I I$. Hering, who also observed this double wave, suggests a dual cause, attributing the first peak to sinus activity and the second to auricular contraction.

Kraus and Nicolai state that the $Q$ depression was present in approximately one-third of their cases. Out of the fifty-nine cases examined, Lewis and Gilder observed its presence in thirty-seven cases in lead $I$, forty-five cases in lead $I I$, and in thirty-six cases in lead III. Its average depth, measured from the zero level, was somewhat greater than the height of the $P$ wave. The $R$ wave is characterized by its steep rise and its sharp point and is a useful guide to early ventricular events. Its rise begins 0.12 to 0.18 second after the rise of the $P$ wave. The $P-R$ interval is theoretically the most exact estimate we have of the time taken for the impulse to be conducted from the sinus to the ventricle. It varies considerably, however, in different leads due to the fact that corresponding waves do not rise simultaneously. This is well illustrated in curves recently published by Williams in which $P_{\mathrm{I}} R_{\mathrm{I}}$ $=0.13 ; P_{\mathrm{II}} R_{\mathrm{II}}=0.17$ and $P_{\mathrm{III}} R_{\mathrm{III}}=0.18$ sec. The $S$ depression is sometimes lacking especially in lead $I I I$, where Lewis and Gilder observed its presence in thirty-one cases out of fifty-nine as compared with fifty out of fifty-nine in leads $I$ and $I I$. In some cases, apparently normal, it may be unusually deep. Kraus and Nicolai believe that such a deep depression is found especially in neurotic cases and have designated it the "neurasthenic wave." The entire $Q R S$ group usually occupies a time interval of 0.08 to 0.09 second. Certain variations of this group occur. Occasionally it is very small (not more than one-third the size of $T$ ) and in other cases it is split. Lewis and Gilder observed this split more frequently in lead III. It is difficult to give an exact interpretation of these bifurcated waves nor is it possible to determine what portion corresponds to the $Q R S$ constituent.

The $T$ wave is rounded in character and varies from one-fourth to two-fifths the size of the $R$ wave in lead $I I$. In lead $I I I$ the variation may be negative.

The $U$ wave following the $T$ ' wave was present in three-fourths of Lewis and Gilder's cases, and in fully one-half of the records obtained by Einthoven. It is most frequently present in lead $I I$, and is always very small. 
The following table taken from Lewis and Gilder, showing the average height of the waves expressed in millivolts, may be of interest:

\begin{tabular}{rrrrrrr} 
Lead. & P. & Q. & \multicolumn{1}{c}{ R. } & S. & T & U \\
I. & 0.52 & 0.51 & 5.16 & 2.06 & 1.93 & 0.07 \\
II. & 1.16 & 0.73 & 10.32 & 2.23 & 2.46 & 0.16 \\
III. & 0.81 & 0.86 & 6.61 & 1.73 & 0.61 & 0.06
\end{tabular}

The following percentile relation in lead $I$ given by Linetzky is a rougher statement of the relative height but a convenient one to bear in mind.

$$
\mathrm{P}: \mathrm{R}: \mathrm{S}: \mathrm{T}=10: 100: 5: 25 .
$$

Effect of Respiration.-The actual and relative size of the waves may vary with the phase of respiration. Einthoven believes that this is due neither to an action current developed by the contraction of the respiratory muscles, nor to the changing resistance occasioned by enlargement of the chest. The nature of the changes depends on whether or not the heart is rhythmic. When the length of the cycle varies greatly it determines the effect on the individual waves. In the long cycles, the $P$ wave is smaller, especially in lead $I I I ; R$ is larger and $T$ smaller in leads $I$ and $I I$; but $R$ is also smaller in lead $I I I$ (Hoffmann).

When cardiac variations are absent, there may or may not be variations in the amplitude of waves. When variations are present, the waves $R, T$ are smaller during inspiration in lead $I$ but larger in lead $I I I$. This is due to the changing position of the heart, causing the electrical axis to become more vertical. This is illustrated by the following figures calculated from these respiratory variations by Waller:

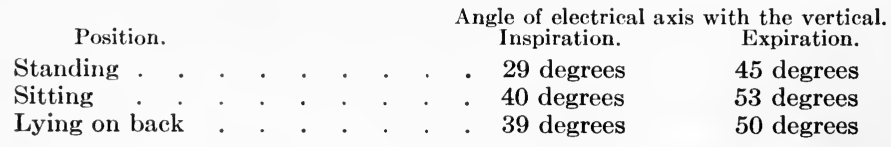

Effect of Body Position.-The position of the body modifies the waves. The $S$ wave becomes larger when the body is shifted from the left to the right side. A change from a dorsal to a stomach position induces the same effect as expiration, probably because the diaphragm is pushed up. Slight changes in the waves often follow a change from a reclining to an upright sitting position. Sitting acts similarly to expiration by compressing the abdomen and causing an upward movement of the diaphragm. No difference in the record occurs between the "lying down" and the "propped up" positions in bed. 


\section{PATHOLOGICAL VARIATIONS.}

Changes in Arrhythmia.-The detailed changes found in various types of heart irregularities will be more fully considered when each condition is taken up. In general, three types of electrocardiogram records help to differentiate the different forms of irregularities: (1) The waves may be present in their normal sequence but with altered time relations. Thus, it is possible to ascertain the presence of a slowing of conduction time from sinus to ventricle when the $P-R$ interval in lead $I I$ is greater than 0.2 second and, similarly, a slowing of conduction through the ventricle is indicated by a lengthening of the $Q R S$ group. During infections, a lengthening of the $P-R$ interval may be the first evidence

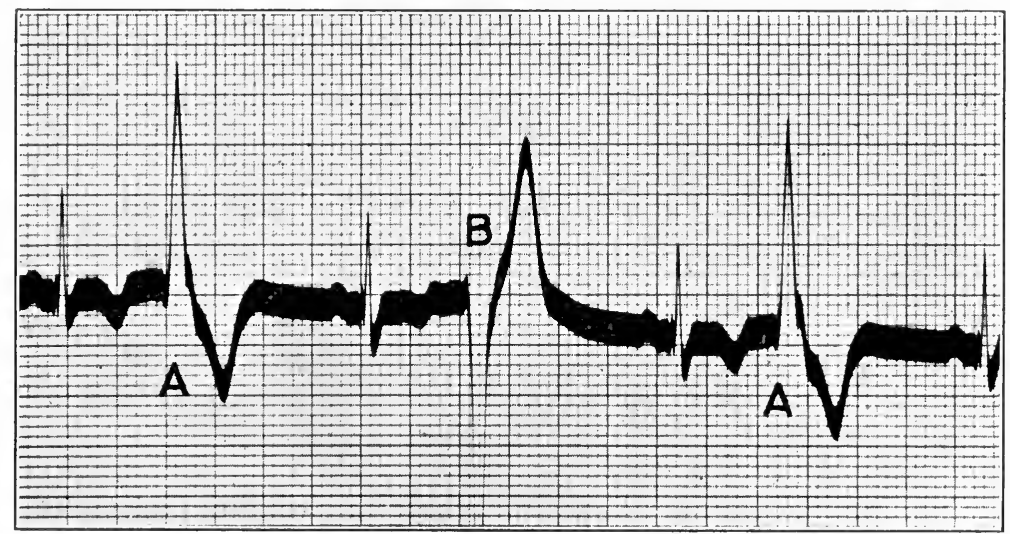

Fig. 45.-Electrocardiogram (lead $I I I$ ) showing two premature right-sided systoles $(A)$ and a premature left-sided systole of the ventricle $(B)$ interposed between normal complexes. (Kindness of H. B. Williams.)

of involvement of the cardiac muscle. In sinus irregularities and tachycardias, the waves follow in proper sequence but their rhythm or rate is altered. (2) Certain waves may be absent or present in excessive number. Thus, in auricular flutter, when the auricle may attain a rate of 200 or 300 per minute, the number of $P$ waves between the preceding $T$ and following $R$ are greatly increased, while in auricular fibrillation, the typical $P$ waves are absent. In auricular extrasystoles the $P, R$ and $T$ waves may be entirely normal except for the fact that they occur prematurely. (3) Waves of irregular and unusual contour may be present. In auricular extrasystole, the $P$ wave may be small, indented or negative. When the latter occurs it is believed to be due to a contraction originating in the left auricle or in the coronary sinus region. The chief forms of aberrant waves, however, are those 
that result when one ventricle is excited before the other. These waves in their general appearance resemble $R$ waves but differ in that they are larger, occupy a longer time interval and are followed by a marked negative depression (Fig. 45). When the right ventricle is excited before the left, the waves are directed upward in leads $I I$ and $I I I$ but downward in lead $I$; while, if the left ventricle is excited first, they are positive in leads $I$ and $I I$ and negative in lead IIII. Waves of such form are found in ventricular extrasystoles, in heart block involving only one branch of the His-Tawara system and frequently with auricular extrasystole associated with a one-sided block. Finally, during auricular fibrillation, there appear, in place of the regular $P$ waves, a series of characteristic movements of high frequency (300-800 per minute). These waves are again superimposed, in many cases, on undulations of longer period (50-60 per minute). (Fig. 85.)

Other Changes Due to Heart Involvement.-It has already been indicated that a lengthening of the conduction time gives an early indication of the involvement of heart muscle. Aside from this phenomenon associated with irregular heart action, certain waves appear to be related to definite pathological processes. An enlargement of the $P$ wave is an indication of auricular hypertrophy. In cases of mitral stenosis when such waves are often one-fifth instead of one-tenth as high as the $R$ wave, they are prolonged and often have a flattened or bifurcated top. A similar large $P$ wave is observed when dilation or weakening of the left ventricle occurs.

The nature of a cardiac enlargement determined by percussion may be better established by the electrocardiogram (Fig. 87). In hypertrophy of the right ventricle the $R \mathrm{I}$ is usually directed downward while $R_{\mathrm{II}}$ is smaller than $R$ III. In hypertrophy of the left ventricle, on the other hand, the $R \mathrm{III}$ is directed downward while $R_{\mathrm{II}}$ is smaller than $R_{\mathrm{I}}$. 'This is not due to the fact that any difference exists in the mode of generation of the current by the lead, but to the fact that when one ventricle enlarges there is a change in the electrical axis of the heart and consequently a change in the line of isopotential occurs. That this is the explanation is indicated by the fact that a change of the cardiac axis due to other causes gives the same result. Thus, in deep and forced respiratory movements, inverted $R$ waves may be obtained and in authentic cases of situs inversus viscerum, in which the axis is reversed, the electrocardiogram is also exactly reversed. This inversion of waves in hypertrophy serves a useful purpose in differentiating in cases in which doubt exists as to whether dulness is really due to hypertrophy. Since lateral displacements of the heart, such as accompany pleural or pericardial effusions, pneumothorax and lung involvements and which also give areas of increased dullness, do not 
usually alter the angle of the heart; they give a normal type of electrocardiogram.

A certain amount of caution should, however, be exercised in interpreting the inverted waves as due to hypertrophy, for any change in the angle of the heart caused by upward pressure of the diaphragm brought about by an inflated stomach, abdominal growths or fluid, as well as by abnormal positions of the body may likewise give inverted waves.

The amplitude of the $R$ wave is no doubt occasionally related to the amount of current generated and, hence, to the degree of activity. Thus, in weak and dilated hearts this wave is often very small. That the amplitude of the $R$ wave is, on the whole, not a reliable index of the vigor of cardiac contraction, however, is indicated by the fact that small $R$ waves are often found in individuals who, we have every reason to believe have strong heart action. Furthermore, extrasystoles, well-known to be weak contractions, give larger $R$ waves than are normally found. Finally, there is some reason to think that the $R$ wave is in no way associated with muscular contraction, at least with the contraction of those muscles which are concerned in the production of effective systoles.

The size of the $T$ wave has, with perhaps more reason, been regarded as an index of the cardiac power. Thus, Lewis points out that a large $T$ wave occurs in cases which possess vigorous heart action (e.g., in goitre cases and in animals after asphyxia). The fact that the $T$ wave is small after weakening influences as chloroform, poisons or hemorrhage, has led some investigators to consider a small $T$ wave an indication that a weak action of the ventricle exists. Kraus and Nicolai state that if this wave is negative it means in 80 per cent. of cases that a dangerous condition is present. The fact (Samojloff, Lewis) that a negative wave sometimes occurs in apparently normal men, however, lessens its diagnostic significance. Lewis, however, points out the significant fact that a negative wave is never found normally in leads $I$ or $I I$, but is common in lead $I I I$.

Use of the Electrocardiogram to Detect Valvular Lesions.Obviously the electrocardiogram can give no direct evidence of the existence of valvular lesions. It has been shown, however, that hypertrophy of the left ventricle is usually associated with known cases of aortic insufficiency and arteriosclerosis, whereas right-sided hypertrophy accompanies congenital pulmonary lesions and mitral involvement. By detecting the presence of these hypertrophies, the electrocardiogram may draw attention to unrecognized valvular lesions as well as confirm a diagnosis previously made by auscultation - This is very important, as it is a well-known fact that generally no presystolic murmur occurs in mitral stenosis when the auricles are fibrillating. It is also often 
valuable in clearing up a doubtful interpretation of murmurs. For instance, suppose a patient has a diastolic aortic murmur combined with a presystolic murmur at the apex, as is so common. The question arises whether the presystolic murmur is due to a condition described by Flint, or whether an actual mitral stenosis exists. If a right-sided hypertrophy is shown by the electrocardiogram the diagnosis of mitral stenosis can quite safely be made. Again, suppose a murmur is heard in the second interspaces of a child's chest in which sounds are widely transmitted. It is difficult to ascertain by auscultation whether the murmur is produced in the pulmonary or aortic area. The type of hypertrophy indicated by the electrocardiogram would clinch the diagnosis. (Lewis.)

One of the most important services of the electrocardiogram consists in its ability to supply evidence as to the condition of the heart muscle, for it is well understood that heart failure depends as much on the condition of the cardiac muscle, brought about by the same toxic or infectious agent that is responsible for the leak, as on the dynamic changes produced by the leak itself.

If, in cases of valvular disturbance, the electrocardiogram shows a normal conduction time, if the chambers react in normal sequence and the waves are of relatively normal amplitude, the cardiac muscle may be considered as practically uninjured and the prognosis is good. If, on the other hand, the conduction time is lengthened, the impulses blocked and the heart beat irregular, the efficiency of the circulation is impaired and the probability of an efficient compensation is less.

\section{LITERATURE.}

\section{Literature Dealing with Capillary Electrometer.}

Bayliss and Starling. Month. Internat. Jour. of Anat. and Physiol., 1892, ix, 256. Garten. Areh. f. d. ges. Physiol., 1902, lxxxix, 613.

Kölliker and Müller. Verhand. d. physiol. med. Gesellsch. in Würzburg, 1856, vi, 528.

Waller. Jour. Physiol., 1887, viii, 227.

\section{Books and Pamphlets Dealing with the Electrocardiogram.}

Hoffmann. Die Electrocardiographie, 1914, Bergman, Wiesbaden.

Kahn. Das Electrocardiogram, Ergebnisse der Physiol., xiv, 1914.

Kraus and Nicolai. Das Electrokardiogram des Gesunden u. Kranken Menschen, Leipsig, 1910.

Lewis. Mechanism of the Heart Beat, 1911, London.

“ Clinical Electrocardiography, 1913, London.

“ Lectures on the Heart, 1915, New York.

Samojloff. Das Electrocardiogram, Samling Anat. u. Physiol., Verträge, F. Fischer, Jena. 
Bibliography Dealing with the Construction, Methods and Principles of Using the Electrocardiograph.

Clements. Ztschr. of Biol., 1912, lviii, 110.

Einthoven. Arch. f. d. ges. Physiol., 1908, cxxii, 517.

“ Ibid., 1907, cxvii, 461.

“ Ibid., 1909, exxx, 287.

“ Arch. internat. de Physiol., 1906, iv, 132.

“ Lancet, 1912, i, 853.

Fahr. Ztschr. f. Biol., 1914, lxiv, 61.

Garten. Tigerstedt's Handbuch der Physiol., Methodik, i, 65, 1911.

Lewis. Phil. Tr. Roy. Soc. London, ser. B, ccii, 377.

\section{Records and Interpretations (Normal and Pathological).}

Eiger. Arch. f. d. ges. Physiol., 1913, cli, 1.

Einthoven. Arch. f. d. ges. Physiol., 1908, exxii, 517; 1909, cxxx, 287; 1910, cxxx, $139 ; 1912$, exlix, 65 .

Einthoven, Waart, Fahr. Arch. f. d. ges. Physiol., 1913, cl, 275.

Eyster and Meek. Arch. Int. Med., 1913, xi, 204.

Hering. Arch. f. d. ges. Physiol., 1913, cli, 111.

Hoffman. Arch. f. d. gesam. Physiol., 1910, exxxiii, 552.

Lewis. Lancet, $1909, \mathrm{i}, 382$.

“ Heart, 1909, i, 262; 1910, ii, 750.

“ Brit. Med. Jour., 1910, i, 570.

Meek and Eyster. Amer. Jour. of Physiol., 1912, xxxi, 31.

Mines. Jour. of Physiol., 1913, xlvi, 188.

Mines and Dale. Jour. of Physiol., 1913, xlvi, 319, 349.

Pardee. Jour. Amer. Med. Assn., 1914, lxii, 311.

Pribram and Kahn. Deutsch. Arch. f. klin. Med., 1910, xcix, 479.

Redfisch. Berl. klin. Wochnschr., 1910, i, 1186.

Samojloff and Steshinski. München. med. Wehnschr., 1909, lvi, 1942.

Seemann. Ztschr. f. Biol., 1912, lix, 53.

Steriopulo. Ztschr. f. exper. Path. u. Therap., 1909, vii, 467.

Waller. Proc. Roy. Soc. of Lond., 1912-13, ]xxxvi, 507.

Williams. Amer. Jour. Physiol., 1914, xxxv, 292.

\section{Clinical Uses of the Electrocardiograph.}

Eppinger and Stoerk. Ztschr. f. klin. Med., 1910, lxxi, 157.

Herzig. Deutsch. Arch. f. klin. Med., 1912, cv, 234.

Lewis. Lancet, 1909, i, 382; Heart, 1909, i, 262.

“ Heart, 1910, ii, 23.

Linetzke. Ztschr. f. exp. Pathol., 1911, ix, 669.

Pribram and Kahn. Deutsch. Arch. f. klin. Med., 1910, xcix, 479.

Strubell. Zentralbl. f. Herz. u. Gefass-Krankh., 1912, Nr. 5.

“ Verh. d. XXVI Kongr. f. inn. Med., Wiesbaden, 1909, p. 623.

v. Wyss. Deutsch. Arch. f. klin. Med., 1911, ciii, 505. 


\section{HEAR'T SOUNDS AND MURMURS-THE PHONOCARDIOGRAM.}

The Three Heart Sounds.- Since the time that the two heart sounds were first recognized by Harvey and their use in diagnosis suggested by Laënnec, they have offered a fruitful field of investigation and study for physicists, biologists, and clinicians alike. The first sound as is well known is deeper in pitch and more booming in character, also of longer duration than the second; and, according to Haycraft, separated from it by a musical interval of a minor third. Recent investigation of the sounds has shown, however, that this must be an auditory delusion and, according to Frank, such statements should be promptly deleted from current textbooks. Einthoven also has pointed out the impossibility of giving the sounds a position in a musical scale since they are composed of vibrations of irregular frequency, and therefore belong in the same category as murmurs.

For years clinicians have recognized that in pathological conditions three sounds may be heard due to a splitting or reduplication of the first or second sound. Only recently, however, Gibson in England and Thayer in America have independently reported that in normal subjects a third sound can be detected. This sound, though clear, is of lower pitch than the second and much softer than the first (according to Einthoven 200 times). Einthoven, Thayer and others believe its occurrence is quite frequent in young adults ( 65 per cent. of cases, Thayer) but it is audible only at the apex and when the patient lies upon the left side. The observations of other clinicians (personal communications) and the records of other investigators (Lewis) make it seem probable that its presence is not as common as at first supposed.

The Relation between Cardial and Thoracic Sounds and Murmurs. - Passing over a discussion as to the cause of the sounds, we may briefly consider, first, the probable relation existing between the vibrations actually produced within the heart and those heard over and registered from the thoracic wall. Physically, heart sounds are vibrations of the heart walls, valves, blood columns, and arteries. In accordance with physical laws, these vibrations are transmitted best by solid contact, less well by liquids and least efficiently by gases. Other factors being equal, they are heard 
best where direct contact with the chest wall occurs (apex) or where the least air-space intervenes (base). For this reason, also, they are transmitted through the fluid in the veins and arteries to the supraclavicular spaces and may be here recorded (Figs. 27, 34, 38). The intensity also varies with the distance from the origin of the sound, hence, the second sound clearly originating at the semilunar valves, is louder in the second interspaces and very soft or scarcely appreciated by the ear in the supra-clavicular fossæ. Lastly, the intensity of the sound is largely determined by the vibration periods and damping of the structures which transmit them. Structures in which the period of vibration corresponds closely to that of the sounds are thrown into resonant vibration and accordingly transmit them more loudly. Before the vibrations recognized as heart sounds are auscultated they are transmitted to the chest wall. In what manner the chest wall, by the addition of its inherent or other mechanical vibrations, may modify the recorded frequency or pitch; or, how it may change the amplitude or intensity by its resonance, are questions that cannot be answered satisfactorily at present. That the thoracic wall has a pronounced modifying influence, however, even if we regard it as sufficiently damped to add no vibrations of its own, is probable from the one fact alone that its own period is probably not high enough to transmit cardiac vibrations faultlessly (Ohm). In support of this, the writer has found that the vibration frequency of sounds recorded from the supraclavicular region in normal and pathological cases does not correspond exactly with those taken from the chest. It is questionable, therefore, whether the vibrations recorded from the chesteven when the direct contact of the receiver is avoided-or the sounds and murmurs heard by the ear directly are identical with the vibrations originating in the heart.

The Possibilities and Difficulties of Sound Perception in Auscultation.-The heart sounds transmitted to the chest wall are made up of vibrations, the period of some of which lies not far above that to which the ear mechanism is incapable of responding. Owing to the fact that their amplitude is also small and the intensity thereby greatly reduced it is in fact questionable whether some of them are audible (Einthoven). It should of course be recognized that auditory acuteness varies not only in different individuals and depends on the bodily or physical condition (Winkelman); but, also, that it can be improved by training. At its best, however, the average ear is not able to distinguish fine differences in tone (Gerhartz) and when sounds recur rapidly it is not always able to give their correct temporal relation. When several types of vibration, as those causing a sound or murmur, intermingle, the ear, according to inclination or training, tends to pick out the one and fails to hear the other (Lewis). It must therefore be recognized that, although 
long strides can be made by practice and training, the ear alone is far from being a perfect instrument.

Various attempts have been made by the invention of stethoscopes to intensify the sounds and so aid the ear in sound perception and differentiation. In the simple form of binaural stethoscope in which a funnel-shaped or bell-shaped cup is applied tightly over the skin, a series of sound reflections occur from the smooth walls, but the effect does not materially intensify the sound vibration. Such an instrument is simply a convenience for the auscultator, not a sound intensifier. It has been attempted to intensify the sounds by giving the receiver a parabola shape upon the physical principle that the sound waves vibrating parallel to the axis are concentrated at the focus and so conducted without appreciable loss. Stethoscopes with such receivers have not been found satisfactory as intensifiers of heart sounds. According to Gerhartz, this is due to the physical fact that the sounds or murmurs are not transmitted parallel from their point of origin, but are deflected in the process of passing through the blood and tissues to the point of auscultation.

The most effective method employed in increasing sound vibration consists in the use of a receiver closed by an unyielding disk as in the phonendoscope and the Bowles stethoscope. This converts the system into a resonator and the sounds are accentuated because the air space is set in vibration. The inherent vibration of these instruments is high and, according to $\mathrm{Ohm}$, they have a favorable decrement. So from a physical view-point, their construction seems advantageous. Einthoven, Frank and $\mathrm{Ohm}$, all authorities in matters pertaining to sound registration, have employed them to intensify sounds before registration. The apparatus has made an unfavorable impression among clinicians, however, partly because it often fails to pick up high tones which are perfectly evident to the ear, and partly because it does not reproduce the quality of the original sound. This is due to the fact that the amplitude of some vibrations is increased out of proportion to that of others (Gerhartz). The original vibrations of small amplitude may have their amplitude increased while those of larger amplitude may be almost suppressed.

The heart sounds may also be intensified by an instrument. combining a microphone receiver in circuit with a telephone earpiece. The microphone-telephone apparatus works upon the well-known principle that when loose carbons are brought into contact with a membrane and a current passes through them, the resistance is modified as the contacts change with the membrane oscillations. These variations in the current, affect the magnetic field in a telephone receiver and so, by altering the attraction of the magnet for the disk, cause it to vibrate. These instruments, however, totally destroy all the qualities of heart sounds, and 
it is questionable whether they are reliable to determine more than the presence and time relations of sound phenomena.

The clearness with which sounds and murmurs over the chest are heard in auscultation depends also on the application of the stethoscope bell (Sewall, Emmerson). It has been already indicated that the resonant vibrations of the chest wall add materially to their intensity. If the stethoscope is firmly pressed against the chest wall it is damped and certain sounds and murmurs may become inaudible. This principle is better recognized in ausculting for fetal heart sounds than in listening over the thorax.

\section{REGISTRATION OF HEART SOUNDS AND MURMURS.}

In the registration of heart sounds it is the endeavor to supplant the ear-drum by some mechanism which is more sensitive and at

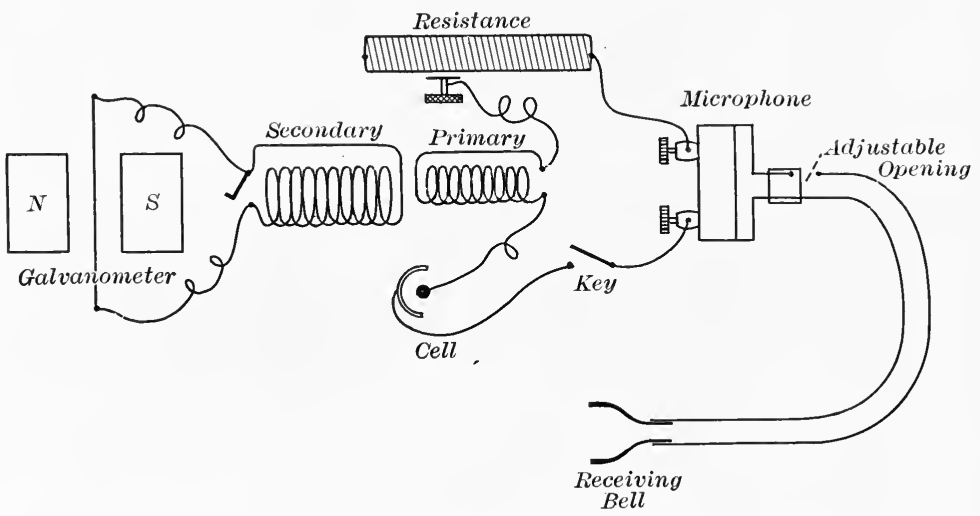

FIG. 46.-Diagram showing the arrangement of the electrocardiograph and microphone to record heart sounds by Einthoven's method.

the same time gives a permanent and objective record of the sounds. Of the numerous devices that have been designed to accomplish this, it will be possible to describe, briefly, only those that have been most generally accepted.

Einthoven's Phonocardiograph (Fig. 46).-Einthoven registered the sounds by placing a microphone in circuit with a string galvanometer. In detail, the bell of a stethoscope fastened to the chest by adhesive is connected by rubber tubing about 75 centimeters long to a suspended microphone. In the system an adjustable opening guarded by a valve is introduced. By opening the valve to a certain degree the system operates on the principle of the tachygraph (Frank) eliminating the relatively slower oscillations of the apex while the more frequent sound vibrations continue to be transmitted. The transfer of microphone oscillations to 
the string galvanometer is accomplished by placing in circuit with the microphone and battery a primary coil without a core and a rheostat of approximately $70 \mathrm{ohms}$ resistance. The current induced in the secondary is communicated to the galvanometer. The intensity of this current can be varied by the distance between the primary and secondary coils and also by the resistance introduced by the rheostat. The registration of the string movements occurs as described for the electrocardiogram. Instead of the string galvanometer a suitable form of oscillograph may be substituted (Watson and Wenyss).

Critique.-The oscillations obtained by Einthoven's method are so characteristic and variable as to create the impression that they are recorded in a perfect manner. There can be no question that the string used by Einthoven had a vibration period far in excess of that necessary to accurately record heart sounds. This, however, does not follow in the case of heart sound records taken by every string galvanometer. Fahr has recently shown that the vibration frequency of the string must be ten times greater than that of the vibrations recorded if the error is to be not greater than 2 per cent. It is apparent, therefore, that with a string vibrating 500 times per second the vibrations exceeding 50 per second will be reproduced less accurately. Furthermore, Frank pointed out that the vibration frequency of the microphone system which is also concerned in sound transformation, in no way equals that of the string. Furthermore, the transmutation of direct electrical variations to those of the secondary circuit where changes in intensity play a predominant role, introduces a possible source of error in the absolute registration of sounds.

These shortcomings, however, are minimal as compared with those of other methods at present available and, hence, this procedure remains the best when its physical as well as its practical aspects are considered. The only practical drawbacks are the expense, the bulk of the apparatus involved, and the technic required for its manipulation.

Registration by Frank's Segment Capsules (formerly called Herzton Capsules).-Frank sought to record the sounds directly from the chest wall and accordingly endeavored at first to reconstruct a registering capsule upon the physical principles of the human eardrum. With this instrument he was partly successful in directly recording sounds transmitted by a stethoscope bell and tubing. Upon práctical grounds, the instrument was finally given the definite form of a segment capsule the shape and mounting of which has already been described (Fig. 26). The capsules are covered by very thin rubber dam or, as was suggested by Weber, with mesentery from a guinea-pig. Upon this membrane is fastened a mirror connected to a light trapezoid plate which moves upon the chord 
side of the capsule. The records are obtained by photographing on a moving film the band of a Nernst light focused on the mirror. This capsule is directly connected by a piece of rubber tubing (about $70 \mathrm{~cm}$. long) having an adjustable side opening, to a stethoscope bell or preferably a phonendoscope applied directly to the chest. When the side tube is closed there is recorded a large record of the apex beat superimposed upon which are the heart sound vibrations (Fig. 47). In some respects this represents the ideal form of record for in this way the time relations of the sounds to the apex beat are directly established and the sounds heard by a closed system represent the sum total of impact and sound oscillations. To rule out the larger oscillations the procedure of Einthoven is made use of, namely, to partly open the system to the external

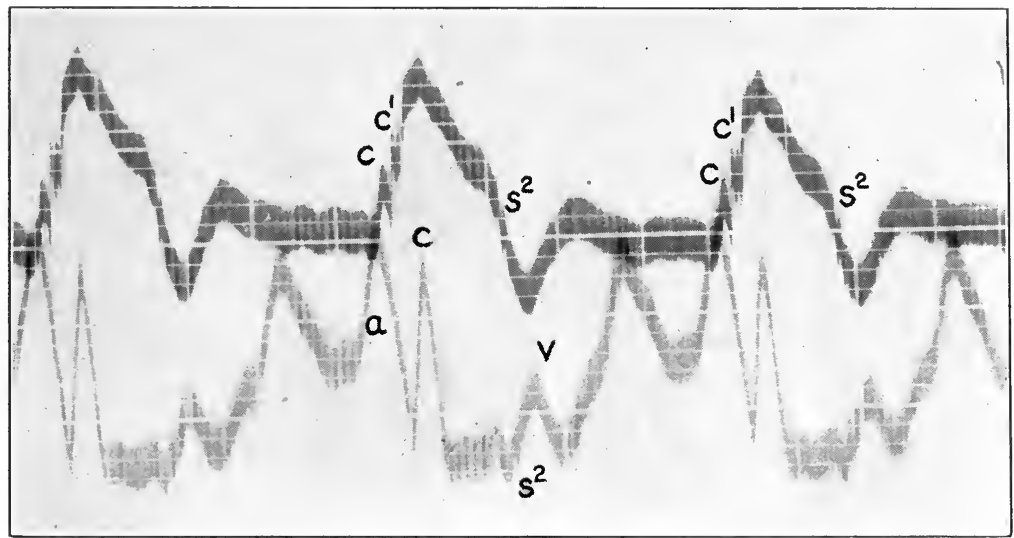

FIG. 47.- Heart sounds superimposed on apex tracing (upper) recorded simultaneously with supraclavicular venous pulse (lower). $c$, initial vibrations; $c^{1}$, valve vibrations; $s^{2}$, second sound also shown transmitted to jugular.

air. By this procedure records have been obtained by Frank and Hess as well as Edens which resemble in many details those obtained by the string galvanometer and leave no doubt from their general character that they are true heart sounds (Fig. 37).

In 1913, Bröemser and Frank described a new apparatus for recording sounds. It consists of a capsule covered with a very thin piece of isinglass, upon the most expansible part of which a tiny mirror ( $1 \mathrm{~mm}$. diameter) is placed. Damping is secured by placing very close to the internal surface of the membrane a damping plate with a very tiny opening. The adaptability or superiority of this apparatus for recording heart sounds has not been reported upon.

Critique.-The vibration frequency of the capsules themselves is sufficiently high and their theoretical analysis has been carried 
out so that they themselves are not open to criticism. The chief drawback consists in the fact that, when the system communicates with the outside air the use of a phonendoscope is necessary, for otherwise the capsules are not sufficiently sensitive to respond to the heart sound vibrations. The use of a phonendoscope is justified by $\mathrm{Ohm}$ on the ground that its inherent vibration is high and it has a favorable decrement. Gerhartz, however, points out that it acts as a resonator and so its employment is fraught with some suspicions as to its accuracy in depicting heart sounds. Gerhartz, further questions whether, by opening the communicating tube partially to the outside air, it is possible, to obtain sound records that are absolutely free from mechanical vibrations. Whether or not it is possible to do this depends on the sensitiveness of the recording membrane and the size of opening permitted in the side

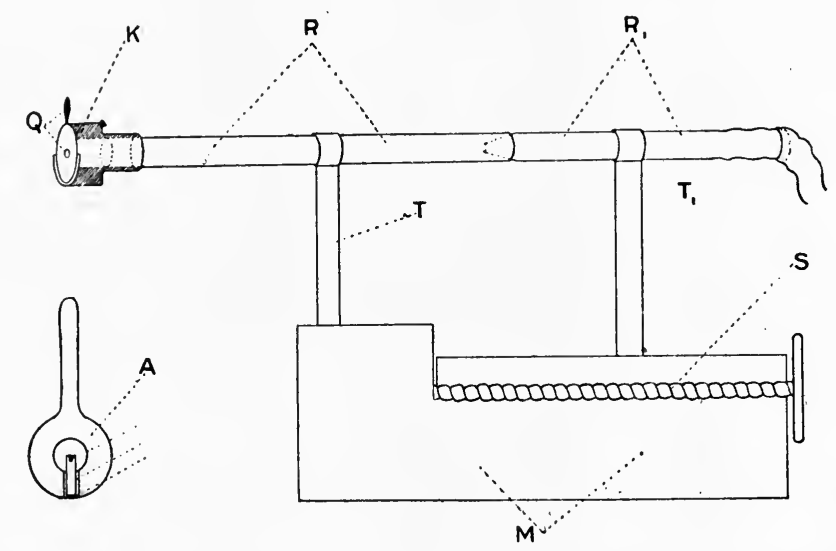

Fig. 4\$.-Diagram showing Ohm's apparatus for recording heart sounds.

tube. That it is possible to obtain such records when the sounds are loud and clear in thin-chested individuals is evidenced by the fact that they rise from the same base-line and oscillate to each end of this line (Fig. 37). On the other hand, there is no doubt that this cannot be accomplished when the sounds or murmurs are weak and distant or when the chest wall is even moderately thick. In order to obtain any record in these cases, it is necessary to close the side tube considerably in which case mixtures of mechanical impacts and sound vibrations are evidently recorded.

Registration with Ohm's Gelatine Membranes.-The apparatus devised by Ohm is shown in Fig. 48. The vibrations are reproduced by a gelatine film made by dipping a ring $(Q)$ into a gelatine solution and allowing it to cool. A small mirror which reflects a band of light, as in the case of Frank's capsules, is mounted centrally on 
the gelatine by a narrow strip of very thin paper fastened peripherally to the ring. The damping of the membrane may be varied by slipping the chamber $K$ over the closed tube $R$ and so varying the depth of the capsule chamber. The membrane is protected by approximating the movable tube $R \mathrm{I}$ to the tube $R$. Tube $R_{\mathrm{I}}$ is connected by tubing with a phonendoscope which is not directly in contact with the chest wall but mounted upon a hard wooden base $\frac{1}{2}$ centimeter in thickness. The idea is to eliminate all the coarser vibrations due to the cardiac contraction and kick.

Critique.-Since the apparatus is familiar to the writer only through its description, it is difficult to give a critical analysis, especially since no details as to its parts are given. The fact that it has (according to $\mathrm{Ohm}$ ) a vibration frequency of two hundred and a sensitiveness sufficient to yield records of such amplitude as are reproduced by Weber and Wirth are in its favor. The arrangement of the mirror is theoretically less desirable than that of Frank's capsule.

Registration with Gerhartz's Apparatus.-In the apparatus of Gerhartz an attempt is made to prevent the cardiac impact by the use of a conical receiver closed below by a wooden diaphragm (4 millimeters thick) which is perforated with very small holes. The vibrations are transmitted by rubber tubes without the employment of a phonendoscope to a collodium membrane, 20 millimeters in diameter. The oscillations are transmitted by a bamboo splinter resting in a holder to a tiny steel platelet, oscillating by two needle points in holes of the magnet poles. Upon this plate a tiny mirror is fastened and by varying the relation of the two electro-magnetic poles, the position of the mirror is altered. Since the electromagnet causes its return to a position determined by the lines of magnetic force, its movements are electromagnetically damped. The movements of the mirror are photographed by reflecting a band of light as shown in other apparatus.

Critique.-Gerhartz gives no data as to the constants of his apparatus nor has its inherent vibration frequency apparently been established. One would judge its period to be low from the appliance used to communicate the membrane movements to the mirror. No details are given as to the grade of damping. Much that applies to the discussion of the apparatus of Weiss very possibly also applies to this apparatus. It has furthermore the serious drawback, which Gerhartz himself points out, that the oscillations are recorded in insufficient amplitude for careful study, while the records obtained are technically so poor that they cannot, as a rule, be reproduced in illustrations. Gerhartz' illustrations are therefore nearly all copies, in the making of which, extreme care was evidentlı not always exercised. 
Registration with Weiss' Phonoscope.-The central portion of this apparatus consists of a metal box, on one side of which is a plate containing in its centre an opening. This is covered by a film of soap. The roof supports a delicate lever of silvered glass the vertical arm of which is bent horizontally. The end of the horizontal arm terminates in a small loop which is brought into contact with the soap film by an adjustment screw. The vibrations of the soap film are transmitted to the horizontal arm of the glass lever and the similar movements of the vertical arm are recorded by projecting its shadow upon a photokymograph. The heart sound vibrations are transmitted to the membrane from a funnel which is not in direct contact with the chest but, to avoid the cardiac impact, is suspended by a holder fixed to the chest so that its free end is separated from the chest wall.

Critique.-The apparatus is evidently very sensitive and, as used by Weiss and Joachim, Bull, and others, has yielded records of very respectable amplitude. Even fetal heart sounds have actually been recorded by Hofbauer and Weiss. The introduction of this instrument has given rise to the liveliest discussion, not only as to its ability to reproduce heart sounds, but also as to the principles upon which sound registry apparatus should be constructed. Inasmuch as these principles are of general application, the discussion may be briefly reviewed.

Frank, who has studied the subject most thoroughly, has emphasized the fact that the sound vibration can be accurately reproduced only by an apparatus, the inherent vibration frequency of which exceeds that of the sound to be recorded. When this requirement is not fulfilled, the curves are distorted by friction and inertia and must be corrected by laborious procedures. When it is fulfilled and the instrumental period is known, damping is not essential or should be present only to such a degree that the instrument is not quite aperiodic. If the damping is greater assurance must be had that the deflection period remains less than the interval required for the smallest deviation to take place. Frank believes that the records obtained by Weiss' apparatus are nothing more than the inherent vibrations of the glass lever which has a frequency of 22 per second. In support of this he cites the fact that the periods of different sound vibrations are nearly the same and show only a small decrement. Weiss has replied to this that, in the first place, the period of the lever is calculated too low by Frank, and in the second place, that this is of no great importance, since the instrument was damped more than sufficient to render it aperiodic and its deflection time was 0.01 of a second.

Herman pointing to the success of the phonograph, has opposed Frank's contentions that a vibration frequency in excess of that of the oscillations is necessary, provided the instrument is damped 
enough to render it aperiodic. According to this doctrine it would be possible to increase the sensitiveness and obtain larger oscillations in the recording membrane through resonance, by selecting a membrane the inherent frequency of which approximates that of the process to be recorded. Frank has subsequently definitely denied the physical soundness of this principle and has pointed out that nothing is gained, as far as accuracy is concerned by an extreme degree of damping. Furthermore, he states that an examination reveals the fact that this principle has never been successfully applied to any sound recording device, neither the phonograph nor the ear-drum being modeled upon it.

We may conclude, therefore, that the most that can be hoped for from Weiss' phonoscope is that it records with approximate correctness the occurrence and time relations of the different sounds. Upon the period and amplitude of the vibrations no reliance can be placed.

A much better principle of rendering soap film vibrations visible was devised by Garten, who supported a tiny splinter of steel on a very tiny soap bubble, centred it by a magnetic field and recorded the movements of its shadow on a photokymograph. Its practical value in registering heart sounds has not been established.

\section{NATURE AND TIME RELATIONS OF PHONOCARDIOGRAMS.}

The records of heart sounds may be designated as phonocardiograms. Reliable information concerning their detailed nature is still restricted to the relatively few contributions of phonocardiograms with the apparatus of Einthoven, Frank, and Ohm.

The two most unanticipated facts regarding the heart sounds which the phonocardiogram reveals are (1) that they are not sounds in a musical sense and (2) that their vibration frequency is very low. The records of Einthoven, Frank, Fahr, Lewis, and others, all show that in each sound, vibrations of different periods and amplitudes occur, indicating that it is a mixture. As Einthoven points out, these composite vibrations differ in different individuals but recur exactly from one cycle to the other in any one person. Gerhartz calculated the average frequency per second from Einthoven's curves to be 39.4 for the first sound, 47.5 for the second, and Einthoven gives 50 for the third. Frank and also Lewis find the average vibration frequency to be near 40 per second, although the latter observer has noted a vibration frequency of 70 per second in the first sound and one as high as 86 in the second. According to Lewis, sounds have, as a rule, a lower frequency than murmurs, which vary from 41 to 107 per second. Fahr reports that the regular vibrations of the first sound are definitely preceded by others of slower frequency and lesser amplitude, which he 
designates "initial vibrations." The writer has observed similar vibrations in sound records superimposed on apex tracings ( $c f$. page 151 , Figs. 38 and 47 ).

As regards amplitude, the vibrations composing the first sound average larger than those of the second, although it is often difficult, on account of their irregularity, to express an off-hand opinion upon this point. Lewis states that the oscillations composing the first sound generally increase in crescendo fashion and later decrease, while in the record of the second sound they are entirely decresendo in character. This is often, though not always true in the records taken with Frank's capsules (cf. Figs. 37 and 49).

The following table indicates the average duration in seconds, found by different observers for the two heart sounds recorded from the apex:

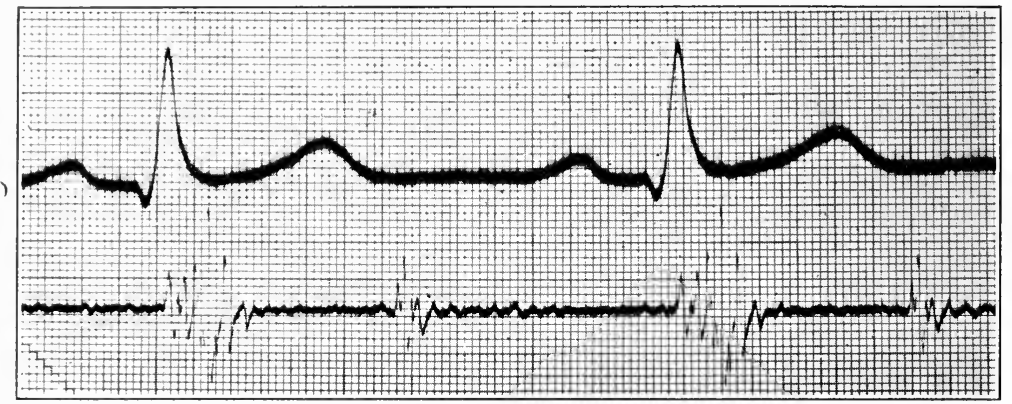

FIG. 49.-Simultaneous records of electrocardiogram and heart sounds showing relation of latter to waves of the electrocardiogram. (Courtesy of Dr. H. B. Williams.)

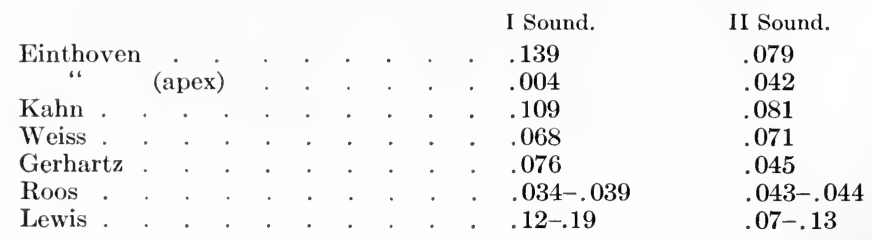

In comparing the records taken from the second intercostal space and the apex, investigators have found that the first sound is usually shorter in the first locality, and that a delay varying from 0.02 to 0.06 second usually occurs before the sound begins at the second intercostal space. Fahr suggests that this delay is due to the fact that the initial vibrations can usually not be detected in the aorta or pulmonary areas, either because the current in the microphone circuit cannot be made sufficiently intense or because they cannot be separated from certain accidental vibrations nearly always present. 
The time relations of the sounds to the carotid rise, the intraventricular pressure (in experimental animals) and to the waves of the electrocardiogram have been frequently investigated. The results obtained by several men are gathered in the following table:

\begin{tabular}{|c|c|c|c|c|c|}
\hline \multirow[b]{2}{*}{ Author. } & \multirow[b]{2}{*}{$\begin{array}{l}\text { Before } \\
\text { carotid } \\
\text { rise. }\end{array}$} & \multicolumn{2}{|c|}{ I Sound. } & \multicolumn{2}{|c|}{ II Sound. } \\
\hline & & $\begin{array}{l}\text { After rise } \\
\text { of } R \text { wave, } \\
\text { electrocard. }\end{array}$ & $\begin{array}{l}\text { After rise of } \\
\text { intravent. } \\
\text { pressure curve. }\end{array}$ & $\begin{array}{l}\text { After } \\
\text { carotid } \\
\text { rise. }\end{array}$ & $\begin{array}{l}\text { After end of } \\
\mathrm{T}, \text { electro- } \\
\text { card. }\end{array}$ \\
\hline Einthoven (man) & .16 & - & - & .12 & - \\
\hline Weiss (man) . & $\begin{array}{r}.067 \\
.075\end{array}$ & - & - & .2 & - \\
\hline Frank (dog) & .059 & - & - & .106 & \\
\hline Gerhartz (man) & $\begin{array}{r}. .042 \\
.133\end{array}$ & .06 & - & .214 & .048 \\
\hline Kahn (man) . & .067 & .028 & - & - & .031 \\
\hline Wiggers (man) &. .116 & - & - & $.24-.035$ & \\
\hline Lewis (man) . & · & $\begin{array}{l}.022 \\
.026\end{array}$ & - & - & .028 \\
\hline Lewis (dog) & . - & $.01-.03$ & - & - & Before end. \\
\hline Fahr (man) & . - & $.02-.03$ & - & - & $.01-.02$ \\
\hline Bull (man) & - & $.03-.04$ & - & - & $.005-.01$ \\
\hline Wirth (dogs) . & . - & - & .025 & - & \\
\hline $\operatorname{Roos}(\operatorname{man})$ & $.06-.09$ & - & - & - & - \\
\hline
\end{tabular}

As may be anticipated the relations of the sounds to the carotid rise are very variable. The most satisfactory method of determining this relation and the one which is automatically corrected for conduction time is to record them by Frank's capsules, superimposed upon the venous and arterial pulses from the supraclavicular region (Figs. 27, 34, and 47). The average of a number of such records indicates that the first sound occurs before the subclavian rise by an interval of 0.116 second, while the second sound begins 0.24 second after the primary rise and 0.035 second after the beginning of the incisura or beginning of diastole. The comparison with the intraventricular pressure, notably as carried out by Wirth and Weber, shows clearly that the first sound begins very shortly after the beginning of the isometric period and continues into the time of the ejection period. The second sound, however, does not begin until the relaxation has been under way for a short interval.

Of extreme interest has been its relation to the electrocardiogram (Fig. 49). Investigators are agreed that in man the sound begins a short interval $(0.01-0.03)$ after the $R$ wave begins to rise. This has been interpreted to mean either that the early contraction of the ventricle causes no sound, or that the $R$ wave is not an accompaniment of actual contraction but of conduction. The relation of the sounds to the intraventricular pressure distinctly favors the former view.

\section{THE CAUSE OF THE HEART SOUNDS.}

Before discussing in what directions the registration of heart sounds by modern methods has aided in interpreting their cause, 
it is desirable to recall the accepted conclusions as to their origin. The views generally held are those propounded before the British Medical Society by the appointed committees. These are: that the first sound is due to the combined vibrations of the contracting ventricle and auriculo-ventricular valves and possibly also the semi-lunar valves; that the second sound is entirely due to vibrations of the closing semi-lunars. ${ }^{1}$ These views have been many times attacked by physicists, physiologists, and clinicians alike.

In regard to the first sound, discussion still centres about the question as to whether the muscular contraction per se is really concerned in its production ( $c f . e . g$. . Pezzi, Quain, etc.). It has been pointed out that the heart muscle does not give a tetanic contraction and is therefore incapable of producing a sound; that the experiments of Ludwig and Dogiel and others of a similar nature are fallacious since the sound recorded may have been produced in some cases by the friction of the heart with the fluid in which it is suspended, in other cases by its direct frictional effect upon the stethoscope (Quain).

Much help is obtained from the registration of heart sounds in answering these questions which are of practical as well as scientific interest, since upon them hinges the question as to whether the intensity of the first sound aids in ascertaining the condition of the cardiac muscle. From the established facts: (1) that the first sound begins early in systole (electrocardiogram), (2) that it follows closely upon the closure of the semilunar valves, (3) that it begins shortly after the rise of tension in the ventricle and gains its maximum amplitude (Wirth and Weber) during this period, (4) that it becomes decrescendo during the ejection period-all discussions as to the exact time relations of the sound are settled. Opinions such as those expressed by Pezzi, that the first sound begins late in systole together with the ejection period, hence is largely valvular, can no longer be accepted.

A greater field of usefulness still awaits the phonocardiogram in answering fundamental questions such as: whether heart muscle is able to give a sound in contracting and whether, in excised hearts, the vibration frequency is really modified when valves are damaged and when pressure relations are changed.

In regard to the second sound, two questions have been discussed, namely, is it a late systolic or an early diastolic event and is it produced by the closure of the valves itself? Exact registration has shown that the second sound occurs at a distinct interval after the $T$ wave, $i$. $e$, when all evidence of muscular contraction has passed and also appreciably later than the sudden diastolic drop in the intraventricular (Piper), aortic (Frank), and supraclavicular

\footnotetext{
${ }^{1}$ For a clear presentation of the evidence upon which these views are based, see Tigerstedt Physiol. des Kreislaufes, Leipsig, 1893, p. 55.
} 
curve (Wiggers). It is plain, therefore, that the second sound is not a systolic event, as even recently claimed by Bocci, but is distinctly an early diastolic affair.

Three possibilities as to the cause of this sound have been discussed: (1) that the reflection of blood behind the valves and the elastic recoil of the aorta late in systole cause the valves to vibrate (Faivre and Chaveau), (2) that after their closure the sudden diastolic tension throws the valves and the column of blood into vibration, and (3) that the valves themselves are a minor factor but that the blood column vibration following the closure is the principal cause.

It is highly probable in the light of the reported time relations that the closure of the valves itself is accomplished without a sound but that the vibration of the blood column and valves following the sudden closure are concerned largely in the sound production.

Even if elementary facts and theoretical considerations must probably always combine in moulding our views concerning the heart sounds, it may be pointed out that the greater the definite information we obtain, the more exacting will be the facts with which theoretical ideas must harmonize before they are elevated to the plane of the plausible. This may be illustrated by a recent occurrence. Hürthle and Weiss believed that they recognized early vibrations in their records which they explained as due to auricular systole. Fahr who obtained more definite evidence of their existence concluded that, as they occurred only 0.02 to 0.04 second before the others, they could not be attributed to an auricular event occurring from 0.12 to 0.18 second before. He therefore suggests that they are associated with the isometric rise of tension in the ventricle and are probably synchronous with the "Vorschwingung" of the aortic pulse. In making this suggestion, Fahr was at the disadvantage of not having intraventricular tracings as a guide, for they show that the vibrations due to valve closure appear in the records before any appreciable elevation of pressure has occurred. Hence this explanation is also untenable. It is more probable, however, that they are in some way associated with the early shifting in position of the heart, or, possibly they are due, as Fahr alternately suggests, to the vibrations of the chordæ tendinæ.

We are now in a position to postulate a view as to the cause of the two first heart sounds which is, as far as the writer knows, in accord with the recent facts established by the graphic method.

When the ventricle begins its contraction its position changes and the papillary muscles contract. Either of these events may produce the initial vibrations of the first sound, which, if audible, are extremely feeble. As soon as the ventricular pressure is raised to an equivalent of a few millimeters of mercury, the $a-v$ valves 
close synchronously without a sound. Following this closure and during the isometric period when all valves are closed, vibrations from their closure are set up within the ventricle. They are added to by the vibrations of muscular contraction and by the vibrations created when the tension is suddenly directed against the semilunar valves. The vibrations from these different sources which have different periods intermingle and increase in intensity until the end of the isometric period. They are transmitted to the entire heart and through the semilunar valves to the aorta. When the period of rising tension passes over into the ejection period the oscillations are modified in that they are of lower frequency and amplitude because a longer column is thrown into vibration.

When ventricular contraction ceases and the pressure within the aorta and ventricle quickly falls, it results in a noiseless closure of the semilunar valves. The after-vibrations of the closed valves together with those of the arterial column cause the second sound. These oscillations rapidly decrease in amplitude and continue only for a short interval because they are damped by the friction of the blood and, as the pressure falls, their vibration frequency tends to become less.

The third sound which by registration is shown to come 0.13 second after the beginning of the second, consists of vibrations of a more constant character. Gibson and Thayer think that they discovered this sound in association with a wave ( $b$ wave, $h$ wave) in the venous pulse and have accordingly attributed it to vibrations of the tricuspid valves when they are floated into position by the inrushing blood. Einthoven has, however, failed to find such waves in the subjects from whom he recorded a definite third sound. In a case reported by Lewis this sound occurred 0.18 second after the second sound, and, as it occurred during auricular systole, it could not have been due to the cause assigned by Gibson and Thayer Gibson and Ewald have suggested that it is due to a systole of the auricle and resembles in cause the presystolic murmur of mitral stenosis. Neither the nature of the vibrations nor the time of the occurrence of the sound, however, favors this etiology. The possibility that it is due to the closure at unequal times of the pulmonary and aortic semilunars is discouraged by Einthoven on the ground that the interval between the aortic and pulmonary second is not great enough to account for this variation. Einthoven would therefore regard it as due to after-vibrations of the aortic valves, the transmission of which to the apex was interrupted. As reasons for this view, Einthoven states: (1) that the duration of the second aortic tone $=0.18$ second, of the second apex tone plus the third tone $=0.16$ second; and (2) that the vibration frequency corresponds to the last vibration of the second pulmonic tone. 


\section{THE CLINICAL SIGNIFICANCE OF THE PHONOCARDIOGRAM.}

Clinically the registration of heart sounds serves the purposes enumerated below:

1. The exact time relations of events in the cardiac cycle may be determined. This is especially valuable in cases of heart irregularities in which it is often impossible to distinguish the sounds of extra contractions from reduplications. The interpretation of venous pulse waves, also, becomes uncertain when ineffective systoles occur which do not raise the intraventricular pressure sufficiently to produce waves in the arteries. In such cases, the heart sound records offer a simple means of differentiation. An instance of this is shown in the segment of a record of Fig. $84^{1}$ which was taken by the author from a patient in the wards of Bellevue Hospital. In this patient the diagnosis of mitral stenosis had been made and auricular fibrillation supervened. An early diastolic murmur followed by a mesodiastolic rumble was audible. The upper record shows the supraclavicular venous pulse, the lower that of the heart sounds. 'They differentiate clearly between the true diastolic waves and those due to ineffective systoles. Thus, were it not for the additional heart sounds recorded at $s^{1}$, no one would suspect that the wave $2-3$ did not have the same diastolic position as the $h$ wave of the preceding cycle.

2 . While the intensity of various sounds cannot be compared in different records on account of the fact that no satisfactory calibration has so far been devised, in the same record, the relative intensity of sounds can be definitely computed by comparing the squares of the product of their frequency and amplitude $\left(I_{\mathrm{a}}=N A^{2}\right)$. Thus, suppose the amplitude of sound $a$ averaged $14 \mathrm{~mm}$. and that of sound $b, 2 \mathrm{~mm}$. The relation of $a$ to $b$ would be as 7 to 1 . Now, suppose the vibration frequency of $a$ averaged eighty and that of $b$, forty, then again their relations would be as 2 to 1 . Computing $(7 \times 2)^{2}:(1 \times 1)^{2}$ we obtain $196: 1$; or sound $a$ is 196 times as loud as sound $b$. Since the vibration period frequently does not alter greatly, the amplitude may be taken as a rough index. Einthoven has indicated how it may be possible in doubtful cases to determine by the relative amplitude of sounds whether a pulmonary accentuated second sound is present.

Lewis has experimentally determined that the amplitude of oscillation is not affected by raising the pressure even by clamping the aorta, whereas, it is diminished when an increase in heart rate occurs. The amplitude is largely dependent upon the air content of the thorax for, when it is opened and antomical relations are destroyed, no sounds can be recorded from the chest wall. In

\footnotetext{
1 In the reduction necessary to reproduce this record page size the clearness of the sound vibrations has necessarily suffered.
} 
cases with heart irregularities, Lewis observed extreme variations in amplitude. In premature systole, for example, the amplitude is often reduced $\frac{1}{10}$ and the length of the sounds is curtailed. If the extrasystole occurs early in diastole and the aortic valves do not open, due to inefficient systoles, the second sound disappears. In the beats following an extrasystole, which are of distinctly larger amplitude, the first sound is louder and of longer duration while the second is unaffected. Cases of this kind almost force one to the conclusion that the muscular element is concerned in the production of the first sound and, furthermore, that the intensity of the sound in some manner corresponds to the force of contraction. In confirmation of this it may be noted that Kahn who has studied the sounds produced during experimental pulsus alternans (glyoxylic acid) finds the time interval between the two sounds is shortened in the smaller beats while the amplitude of the sounds during these beats is also smaller.

3. The phonocardiogram offers a means of analyzing and sometimes explaining reduplicated sounds. It is commonly recognized that reduplicated first sounds may be of such a character that the first sound is preceded by a presystolic element or followed by a midsystolic sound. 'The second sound may be immediately followed by a third sound (protodiastolic) or this may fall in mid-diastole. ${ }^{1}$

Reduplications of the first sound have been assigned to irregular action of the two ventricles during block of one branch of the His bundle. In this case one ventricle is supposed to receive the impulse through the other. Lewis found the first sound presystolic in all his cases. Hence it is impossible that it was occasioned by asynchronous action of the ventricles but was more probably due to a twice repeated closure of the $a-v$ valves. The records show two types of vibrations: (1) those in which two sets of vibrations of like frequency follow each other successively, and (2) those in which the amplitude of the first is distinctly smaller. A reduplicated second sound (early diastole) is often attributed to an unbalanced pressure relation between the aorta and the pulmonary artery. While no observations on the effect of a rise in pulmonary pressure are at hand, it has been shown that raising the aortic pressure does not increase the amplitude.

4. The time relations and character of murmur may be determined by a phonocardiogram. It should be clearly borne in mind that murmurs are not essentially different from heart sounds, hence, no phenomenal difference in the vibrations occurs. As is well known, they are usually produced when fluid passes through narrow openings. Eddies and whirls, of themselves, do not create sounds or murmurs but do so only when some structure is set in 
vibration by them. The vibrations are set up from several sources, as a rule, and are modified and reflected before being transmitted to the ear. They are, consequently, like the sounds, irregular in their sequence and amplitude. The vibration frequency is determined by the size of the opening at which the murmurs originate. On the average, though not always, their frequency is greater than that of heart sounds. Their amplitude is determined by the vigor with which fluid is sent through a narrowed or leaky orifice. Hence they are sometimes of smaller and sometimes of larger amplitude than the heart sound vibrations to which they are related. The presence of murmurs is indicated in the records only by their relative position in the cardiac cycle and to differentiate them from heart sounds it is necessary that the record be accompanied by some other index of the circulation, as the carotid pulse or the electrocardiogram. In this connection it is essential to bear in mind that while the phonocardiograph records more exactly the time relations of the heart sounds, no mechanical apparatus can supply the power of differentiating the finer variations in the quality of the sounds or murmurs as does the human ear. Hence, it is desirable to correlate the recorded oscillations with the auscultatory findings.

Systolic Murmurs.-Systolic murmurs may replace or follow the first sound. A blowing murmur, largely replacing the first sound, is usually associated with mitral insufficiency. In such cases it is sometimes difficult to record the distant murmur (Joachim, Lewis). In the tracings successfully obtained the vibrations occupy a large part of systole but are distinctly separated from the second sound (Fig. 90). In other cases the vibrations constituting the first sound are present but smaller vibrations are superimposed. When the murmur occurs in mid-systole, the vibrations follow the sound and recur at a frequency ranging from 112 to 140 per second. Gerhartz gives the duration of the systolic murmur as 0.45 second when it replaces the first sound.

Systolic murmurs, especially when they follow the first sound, are not always associated with mitral regurgitations but are frequent accompaniments of arteriosclerosis and aortic insufficiency. ${ }^{1}$ 'These murmurs are diminuendo in character and have a frequency of about 80 per second (Lewis). According to Weiss and Joachim, the relatively rare murmur of aortic stenosis differs from that of mitral insufficiency in that it does not begin until the onset of the ejection period.

Diastolic Murmurs.-According to the phase of diastole in which they occur, murmurs are designated as proto-, meso- and telediastolic, or presystolic.

An early diastolic murmur is usually associated with aortic insufficiency and is erroneously attributed to a regurgitation of

${ }^{1} C f$. Fig. 97 , in which the vibrations are superimposed on subclavian tracings. 
blood. Lewis found, in experimental animals, that the second sound still persisted after destruction of one cusp but it was followed by diminuendo vibrations having a frequency of 100 to 120 per second. A presystolic Flint murmur was also present. In patients with these murmurs two types of oscillations appear. If the murmur is of the rough to-and-fro variety, it is shown in the phonocardiograms as a systolic and diastolic murmur, diminuendo in character and having a vibration frequency of about 80 per second. In the case of the musical murmurs, the vibrations last long (0.24 second), are of greater frequency (138), and their amplitude may exceed that of the first sound (Fig. 50).

An early diastolic murmur continuing to the middle and sometimes to the end of diastole is the accompaniment of mitral stenosis combined with auricular fibrillation. In this case the auricular

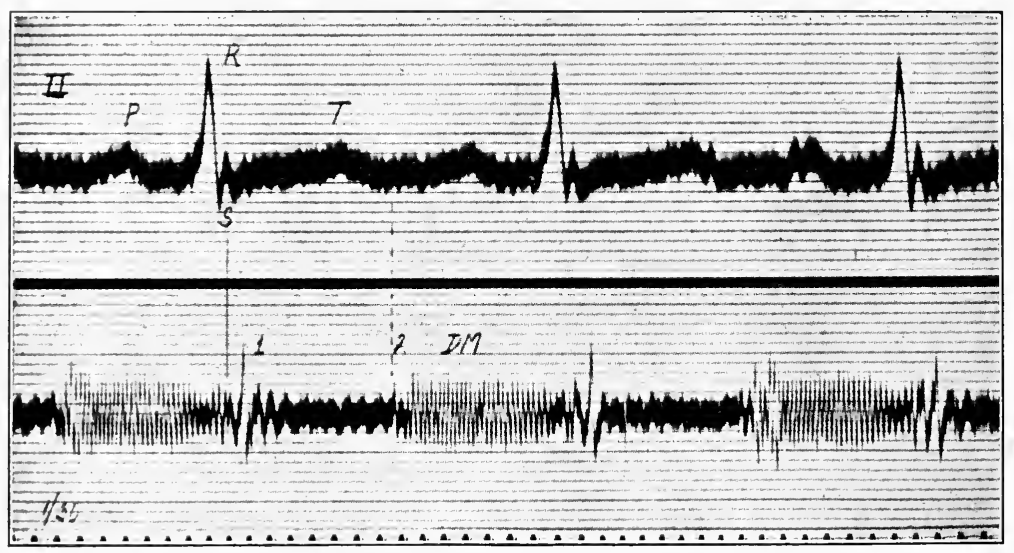

FIG. 50.-Phonocardiogram of a musical aortic murmur. (After Lewis.)

contraction is in abeyance and the filling occurs rapidly due to high venous pressure during early diastole. Records show that if the stenosis is mild, filling will be completed relatively soon, if severe it may extend throughout diastole (Mackenzie, Lewis).

A presystolic murmur is a characteristic accompaniment of mitral, and occasionally tricuspid, stenosis as long as the auricles are beating. In the former lesion it is often limited to the apex, is purring or rumbling in character, and terminates in a clear snap. Its vibrations are of large amplitude and coarse, so that it can usually be recognized on palpation as a thrill. Phonocardiograms taken from the apex show vibrations of large amplitude preceding the systole of the ventricle. They exceed slightly in frequency (41-107 per sec.) those of the first sound, with which they merge (Fig. 93). (Einthoven, Lewis.) The latter, however, do not exactly 
resemble those due to the first sound in normal cases, but are of larger amplitude and begin somewhat later. They probably correspond to the characteristic snap heard on auscultation and have been attributed to the recoil vibrations occurring when blood is ejected by the ventricle (Gerhartz). Sometimes the presystolic vibrations are preceded by mesodiastolic vibrations similar to those observed when the auricle is fibrillating.

A presystolic rumbling murmur is often associated with a diastolic murmur in aortic insufficiency. This murmur described by Flint is most satisfactorily explained as due to the fact that the regurgitated blood impinges on the anterior mitral valve and causes a moderate narrowing effect. Graphically, it may be distinguished from the murmurs due to mitral stenosis in that its vibrations are of greater frequency and that an interval never exists between it and the meso-diastolic murmur preceding it.

\section{BIBLIOGRAPHY.}

Books.

Gergèl. Sejtfaden der diagnost. Akustik, Stuttgart, 1908.

Gerhartz. Die Registrierung des Herzschalles, Berlin, 1911.

\section{Papers.}

Bocci. Zentralbl. f. Physiol., 1910, p. 1515.

Brömser and Frank. Sitzungsbericht d. morph.-physiol. Gesellschaft, München, 1913, xxvii, 46.

Einthoven. Arch. f. ges. Physiol., 1907, cxvii, 461.

Arch. f. ges. Physiol., 1907, cxx, 31.

Einthoven and Geluk. Arch. f. ges. Physiol., 1894, lvii, 617.

Fahr. Heart, 1912-13, iv, 147.

Frank. München. med. Wehnschr., 1904, p. 953.

" Ztschr. f. Biol., 1905, xlviii, 489.

“ Ztschr. f. Biol., 1907, 1, 341 .

“ Tigerstedt's Handbuch der Physiol. Methodik u. Tecknic, Leipzig, II, part

4, 185."

Frank. Berl. klin. Wehnschr., xlv, 1159.

" Ztschr. f. Biol., 1911, lv, 530.

" Ztschr. f. Biol., 1914, lxiv, 125.

“ Ztschr. f. Biol., 1913, lxi, 264.

Garten. Ztschr. f. Biol., 1911, lvi, 41.

Geigel. Arch. f. Path. Anat., 1895, cxli, 1-28.

“ Arch. f. Path. Anat., 1895, cxl, 385-395.

Gerhartz. Ztschr. f. exper. Path. u. Therap., 1908-9, v, 105.

“ Arch. f. d. ges. Physiol., cxxiv, 526.

" Arch. f. d. ges. Physiol., cxxxi, 509.

“ Arch. f. d. ges. Physiol., 1912, cxlvii, 437.

“ Deutsch. Arch. f. klin. Med., 1907, xc, 50.

Gibson. Lancet, 1907, ii, 1380.

Hart. Med. Rec., New York, 1911. Ixxx, 2.

Harvey. Du Motu Cortis, Chap. v. -

Haycraft. Jour. f. Physiol., 1890, xi, 4886.

Herman. Arch. f. d. ges. Physiol., 1913, cl, 92.

Heitler. Wein. klin. Wchnschr., 1911, xxiv, 851. 
Henschen. Deutsch. med. Wchnschr., 1909, xxxv, 1505.

Hess. Deutsch. med. Wchnschr., xxxiv, 161.

Hofbauer and Weiss. Zentralbl. f. Gynäk., 1908, xxxii, 429.

Hoffman. Arch. f. d. ges. Physiol., 1912, cxlvi, 295.

“ Jahrb. d. ges. Med. Schmidt's, cccii, 126.

Holowinski. Ztschr. f. klin. Med., 1901, xlii, 186.

Hürthle. Arch. f. ges. Physiol., 1895, lx, 263.

Kahn. Arch. f. d. ges. Physiol., 1910, cxxxiii, 597.

“ Arch. f. d. ges. Physiol., 1911, cxl, 471.

Laennec. de l'Auscultation, 1819, ii, 2110.

Lederer and Stolte. Zentralbl. f. Physiol., 1911, xxv. 376.

Lewis. Heart, 1912-13, iv, 241.

“ Quart. Jour. Med., 1913, vi, 441.

Ohm. Ztschr. f. exper. Path. u. Therap., 1912, xi, 138.

“ Deutsch. med. Wchnschr., 1911, xxxvii, 1432.

Pezzi. Ztschr. f. klin. Med., 1912, lxxv, 102.

Quain. Proc. Roy. Med. and Chir. Soc., 1897, lxi, 331.

Roos. Deutsch. Arch. f. klin. Med., 1908, xcii, 314.

Shaw. Proc. Roy. Med. Soc. of London, 1905, Ser. A, lxxvi, 350, 360.

Thayer. Boston Med. and Surg. Jour., 1908, clviii. 713.

“ Arch. Int. Med. 1909, iv, 297.

Watson and Wenyss. Edinburgh Med. Jour., 1913, N. S., xi, 121.

Weber and Wirth. Deutsch. Arch. f. klin. Med., 1912, ci, 562.

Weiss. Arch. f. d. ges. Physiol., 1909, exxvii, 74.

“ Ztschr. f. biol. Tech. u. Method., i, 121.

“ Samml. anat. u. physiol. Vort. Aufsätze, 1909, Heft 7, Jena.

“ Arch. f. d. ges. Physiol., 1910, cxxxii, 544.

Weiss and Joachim. Ztschr. f. klin. Med., 1911, Ixxiii, 240.

" " Ztsehr. f. biol. Tech. u. Method., 1908, i, 49-57, 121-125.

Wiescl. Deutsch. Arch. f. klin. Med., cii. 552.

Wyss. Deutsch. Arch. f. klin. Med., 1910, ci, 1. 


\section{SPHYGMOMANOMETRY-THE CLINICAL ESTIMATION OF HUMAN BLOOD-PRESSURE.}

\section{APPARATUS, TECHNIC, AND CRITIQUE.}

ALL forms of blood-pressure apparatus in common use have three essential parts: the compressing cuff, the manometer and the inflating bulb or pump, together with a fine exhaust. If we desire to determine the diastolic pressure as well, some form of oscillometer is necessary.

The Compressing Cuff.-The arm-piece applied to the upper arm is composed of a rubber bag surrounded by an unyielding cuff. The bag should be made of fairly heavy rubber of good elastic quality. It should have a width of 12 centimeters. A narrow bag, as originally used by Riva Rocci is generally supposed to yield too high readings. 'This is due ( $\mathrm{v}$. Recklinghausen) to the fact that pressure applied to a small area must overcome, not only the intra-arterial tension and the resistance of the arterial wall but also the tension of the tissues; whereas, if the same pressure per unit area is applied over a wider space, only the outer pressures are concerned in overcoming tissue resistance, while the central pressure is transmitted directly to the arterial wall. This is illustrated by the diagram of v. Recklinghausen (Fig. 51). The bag may entirely encircle the arm, as is the case in most models of sphygmomanometers, or it may be a small bag 12 by 16 centimeters, as in the case of the Erlanger apparatus. It should be applied over the brachial artery on the inner aspects of the arm. Theoretically, the smaller bag, on account of its smaller volume of air is preferable.

The arm-bag should be surrounded by an unyielding cuff made preferably of leather and fastened by suitable clasps. Some of the recent instruments have an outer covering of linen which is not sufficiently unyielding to prevent an outward loss of pulsation.

Critique.-It has not been universally accepted that a wide cuff is theoretically more desirable (Sahli). There can be no question that v. Recklinghausen's contention would apply if it were desired to obliterate an artery containing a constant pressure or, in words of the physicist, if "statics" alone were concerned. Such is not the case, however. The systolic pressure which we desire to measure 
exists only for a moment during each systole. Can an artery collapsed for a considerable distance by a continued external pressure just equal to systolic be opened by the momentary equalization of intra-arterial and extra-arterial pressures? It appears from the results of arteriograph and physical experiments (Erlanger and Hooker) that this dynamic effect does not come into play enough to appreciably affect the readings, even when the artery is compressed for a considerable distance.

The Manometer.-The manometer may be a single limb, or U-shaped mercury manometer, a compressed air manometer, or an aneroid pressure gauge. The one-limb mercury manometer consists of a reservoir of mercury into which a graduated tube
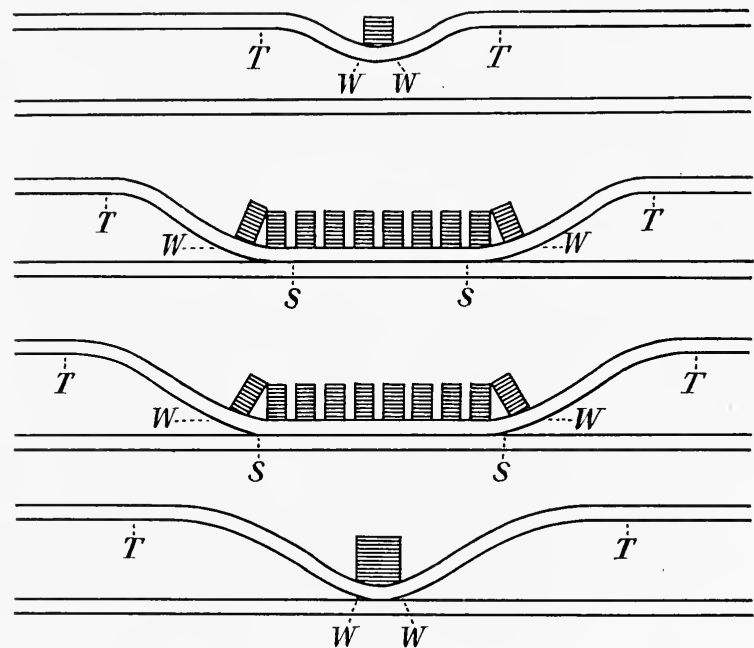

FIG. 51.-Scheme showing effect of equal pressures per unit area when applied over small and wide area of a collapsible tube. (After v. Recklinghausen.)

dips or with the bottom of which it communicates. It was used on Riva Rocci's instrument and has been employed on many of its modifications (Cook, Stanton, Hill, Nicholson) (Fig. 52).

As the level of mercury changes or when it rises in the manometer tube, the scale must be corrected slightly, otherwise the reading is incorrect. In many of the earlier forms it was difficult to determine the exact zero level, owing to the depression of the mercury in the capillary tube, but this has been obviated in recent models.

The 'U-shaped mercury manometer has been utilized in many models (Janeway, Erlanger, Faught) (Fig. 55). The rise in one limb actually equals one-half the pressure change since the mercury in the opposite limb is depressed an equal amount. The scale is therefore less sensitive than the single limb manometers. It has 
the advantages of an easily determined zero level and of requiring only a small quantity of mercury.

The manometer tube should be made of heavy glass, of even caliber and should have a length of forty centimeters. It should have sufficient damping so that the mercury does not oscillate, for otherwise the reading cannot be accurately made. This damping may be accomplished by reducing the caliber of the manometer tube to two millimeters, as in the Erlanger apparatus, or by introducing an artificial resistance, as in the modified Uskoff apparatus made by Zimmerman. This does not apply, of course, to those

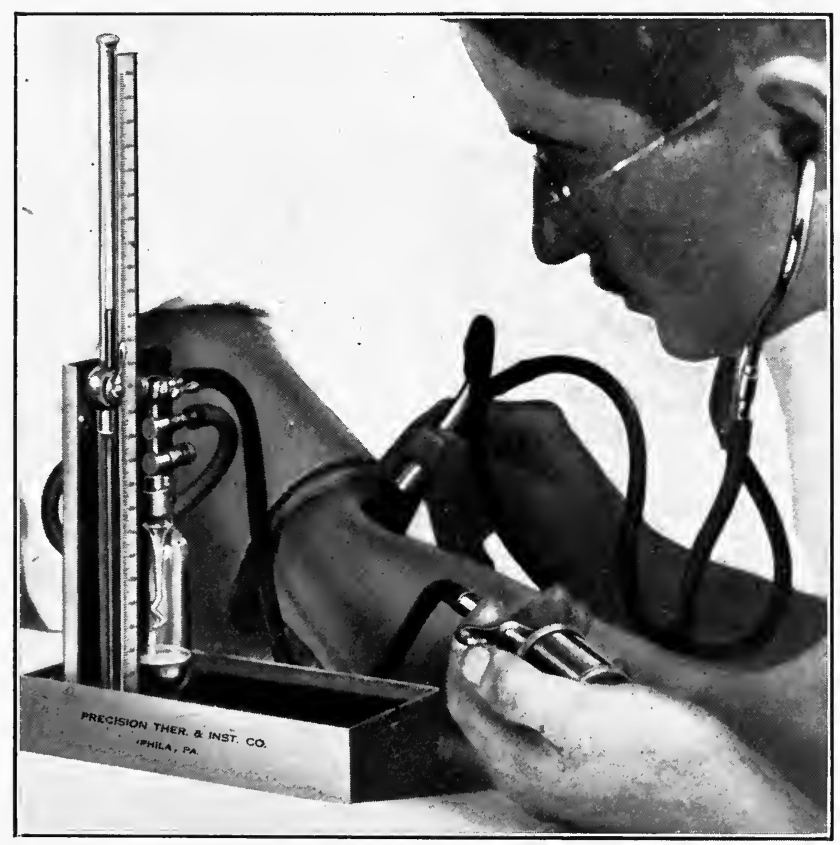

FIG. 52.-Nicholson's pattern of a one-limb mercury manometer. Method of auscultatory determination of blood-pressure. (After Norris.)

instruments in which the mercury is used to record the amplitude of oscillation as well (Janeway, Gibson). In this case a lumen of three or even five millimeters is necessary.

Compressed air manometers consist of a sealed tube containing a globule of mercury or other fluid. As pressure is applied, the air enclosed is compressed and the globule serves as a guide on the calibrated scale. The principle has been used by Gaertner, Oliver, Hertz, etc. The effect of temperature changes must be obviated by compensatory adjustments or by enclosing the apparatus in 
a vacuum tube of clear glass, as in Oliver's instrument. Their chief advantage is their lightness and compactness; their chief

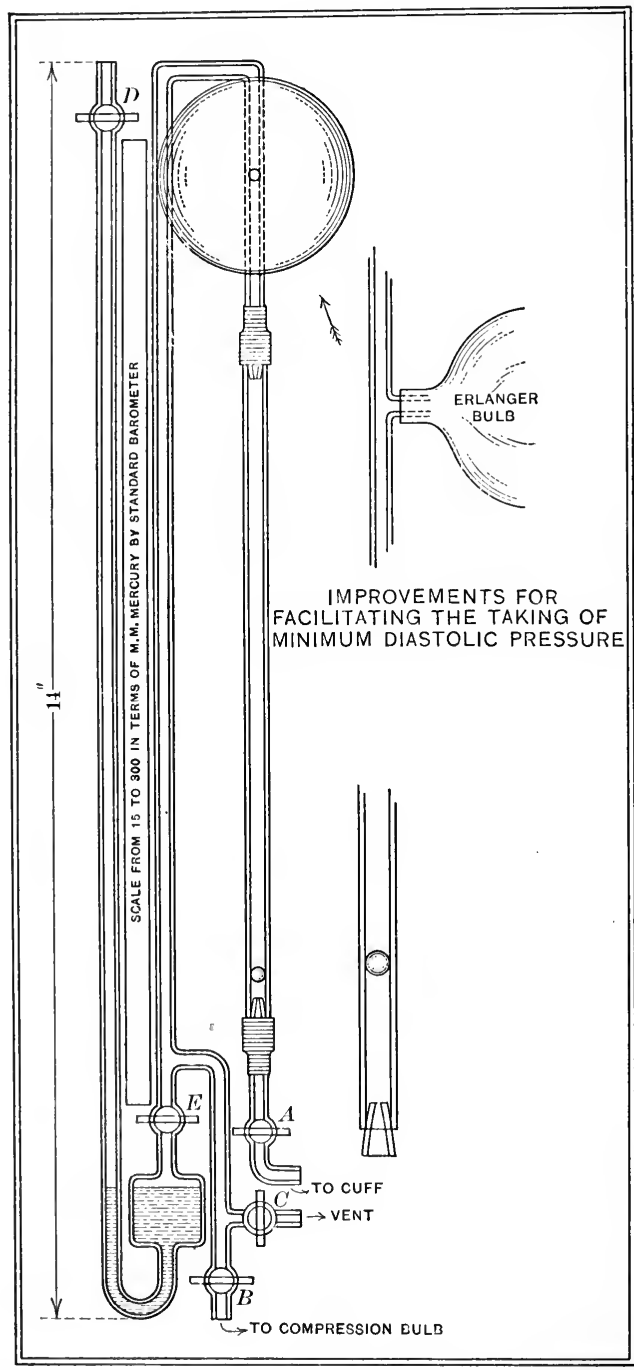

FIG. 53.-Diagram showing principle of one limb mercury manometer, and of the Feddé pith-ball oscillometer. (After Feddé.)

criticism, that they are not sufficiently sensitive, $i$. $e$., that only small movements of the index occur for considerable pressure variations. 
Aneroid (i. e., without fluid) manometers are of two types. They may consist of a chamber of corrugated metal which expands and acts upon a set of cog-wheels actuating the hands which move over a calibrated dial (Fig. 54). To this class belong the Pachon, Tycos, Tagliabue and Pilling aneroids and the recording Jacquet sphygmotomograph.

A second type is made with a curved and hollow Bourdon spring which tends to straighten when pressure is applied internally. The v. Recklinghausen tonometer is based upon this principle.

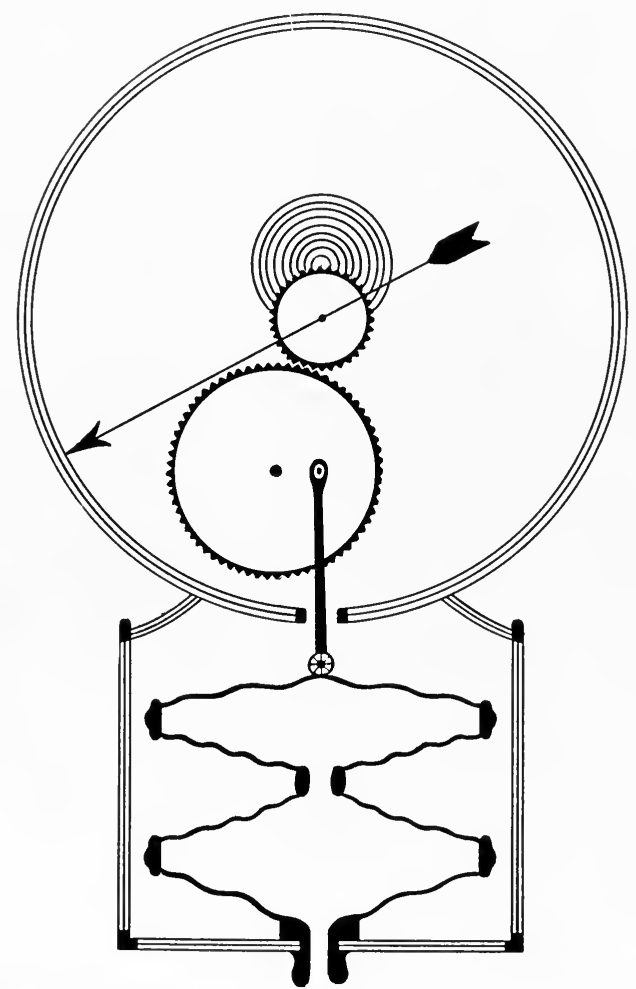

FIG. 54.-Diagram illustrating the construction of an aneroid instrument. (After Norris.)

The pressure is increased in the system by introducing air with an ordinary atomizer bulb, a cautery bulb, a Politzer bulb or a metal pump. The large and clumsy pump used by v. Recklinghausen has never appealed to Americans. The pressure is reduced by letting out air by means of a fine leak. This may consist merely of a small opening over which the finger is placed, of a longitudinal slit, a screw adjustment or a special stop-cock. All tubing should be heavy walled and as inelastic as possible. 


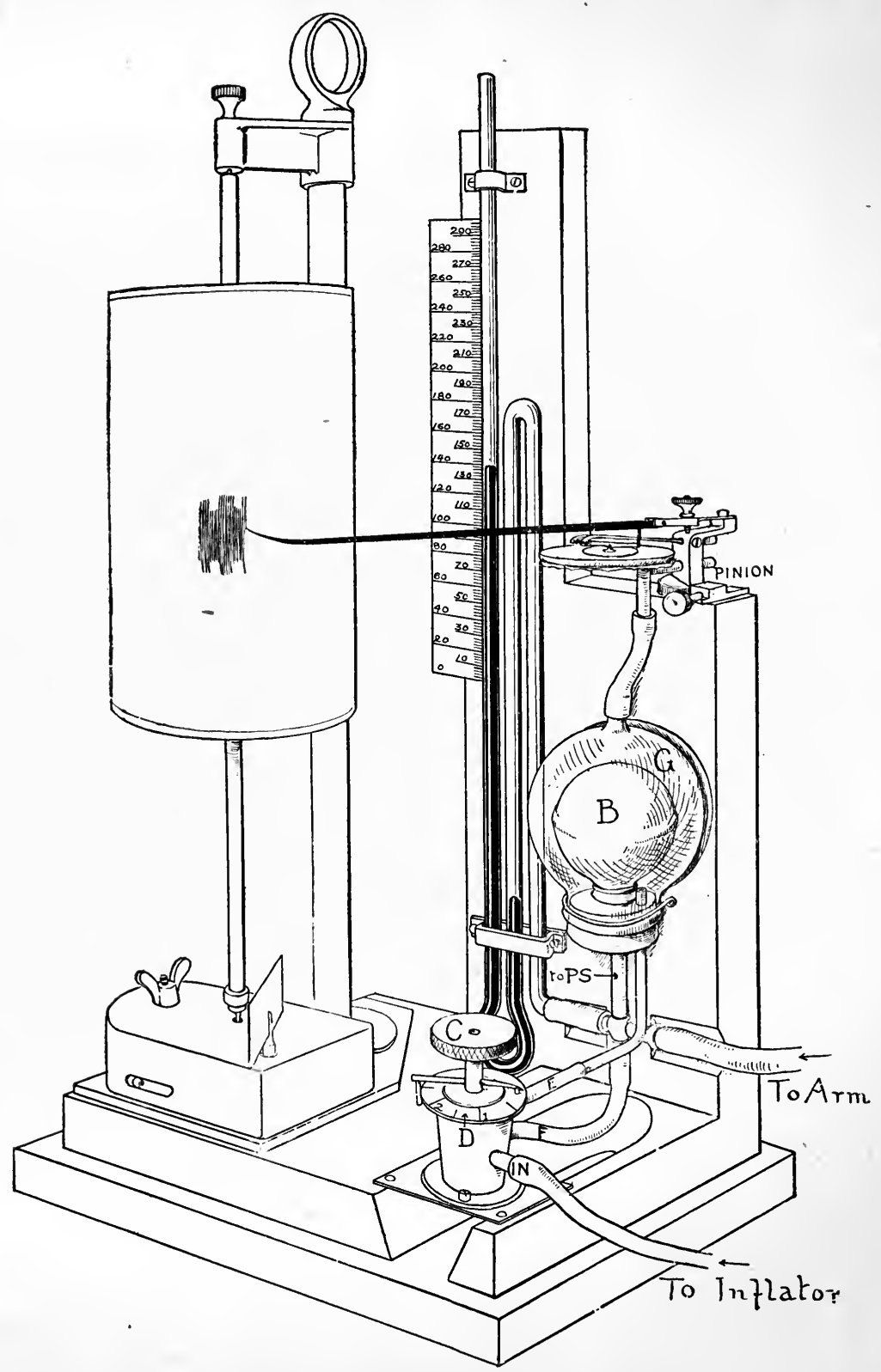

Fic. 55.-Diagram showing Erlanger's sphygmomanometer. (After Erlanger.) 
Oscillometers.-Devices for the determination of variations in amplitude with the sphygomanometer are designated as oscillometers. The mercury or aneroid manometers recording the pressure may themselves fulfill this purpose, or a special device may be shunted into the circuit.

As already indicated, when the mercury manometer is employed to register oscillations, a wider tube than is otherwise necessary must be used. In many instruments the variations in amplitude are directly estimated (Janeway, Stanton). In others, a float as in laboratory manometers has been added (Gibson, and Brugsch, Silberman). Similarly, the needle oscillations of an aneroid may be read off and the regions of largest excursions estimated.

Various forms of oscillometers have been shunted into the sphygmomanometer system. Thus, v. Recklinghausen constructed a recording tonograph by utilizing a Bourdon spring which could be calibrated. Erlanger (Fig. 55) used a sphygmoscope system in

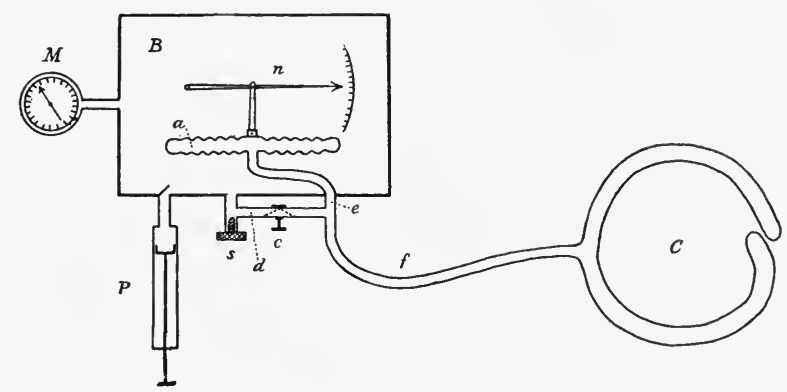

FIg. 56.-Diagram illustrating the construction of Pachon's aneroid oscillometer. (After Norris.)

which the pressure was prevented from acting on a delicate tambour by a rubber bulb $(B)$ enclosed in a glass chamber $(C)$. Similar devices are used by Muenzer, Wybauer, and Uskoff.

The Fedde oscillator is a visual indicator of the amplitude. It consists of a tube containing a loosely fitting pith-ball which drops by its own weight during diastole and during systole is sent up with an excursion proportionate to the amplitude of the pressure change in the system (Fig. 53). The light weight of the ball and the absence of inertia add to the value of the instrument.

Oscillations have also been visually estimated by the movements of a drop of fluid in a horizontal tube and in some forms the oscillations are transmitted from a separate cuff (Bing, Pal).

A separate aneroid oscillator is included in Pachon's instrument. As shown in Fig. 56, the air-tight box, $B$, communicates with the air-bag by tubes $f$ and $d$. The pressure applied to the arm is measured by the manometer, $M$. The oscillations are transmitted 
to an expansile chamber, $a$, the pressure of. which is maintained equal on two sides. The movements of this box are transmitted to the needle.

Critique of Oscillometers.-The oscillations within the sphygmomanometer system occur because the sudden increase in volume of the arteries under the bag causes a temporary increase in pressure within the arm-bag. We should not, therefore, be deceived into thinking, as is commonly done, that a constant extra-arterial pressure is playing on the arteries at any time. The pressure within the apparatus rises during systole and falls during diastole just as in the artery. The relation existing between the magnitude of these oscillations and those within the artery need not concern us here. It is pertinent to inquire, however, with what degree of accuracy the various oscillations of the arterial wall are faithfully recorded in amplitude by different oscillometers.

The mercury manometer is generally suspected of following the variations in amplitude very imperfectly. Its great inertia, indicated by its long vibration period, makes it a very inadequate mechanism. The practical dilemma usually arises, moreover, that the oscillations are either so small (which is the rule) that they do not serve a differential purpose, or so large (which is rare) that an accurate reading of the pressure is impossible. Were the manometer reliable, the lowest level would obviously represent the pressure exerted during diastole, but since the lower level is the resultant of two tendencies - the tendency of the instrumental inertia to place it higher and the combined tendencies of resonance and artificial reduction of pressure to draw it down-it can be considered no more than a wild guess to locate the pressure exerted on the artery during the largest oscillation.

The statements made concerning the oscillatory mercury manometer apply, though with less force, to the aneroid patterns the inertia of which is very much less. They are less apt, on account of their somewhat higher vibration rate, to undergo resonance effects. Of these the Pachon instrument, however, demands separate consideration, for in this apparatus, oscillations are recorded by a separate aneroid (Fig. 56). This oscillatory aneroid is supposed to be constructed on ideal principles for it purports to maintain the pressure on the internal and external surfaces of the drum (a) equal. In this way a very sensitive drum can be utilized and the sensibility is supposed to be constant at all pressures. Neither the designer nor Bacchman, who recently endorsed this apparatus, seems to have been aware of the fact that the sensitiveness is necessarily modified whenever air enters or leaves the system (see page 203) nor do they seem to recognize that the balancing of pressures during diastole is only approximate, a fact that could probably be remedied by introducing a minimal valve at $C$. The manometer, 
$M$, would then indicate the lowest pressure applied to the artery and the recording oscillometer would show the changes in amplitude. 'That it would do this very accurately, however, is questionable, since the lever has a vibration frequency of only 7 per second. The last statement also applies to the recording tonograph of v. Recklinghausen, which purports to record the form of the intra-arterial pressure curve. A mere inspection of the curve shows that this is not the case, however (Frank).

The oscillometer methods so far considered attempt to measure the qualitative pressure variations in the bag directly. In the case of the Erlanger, Uskoff and allied instruments, the oscillations are recorded through the intervention of a restraining ball or sphygmoscope system. In order that the oscillations of the lever shall correspond in amplitude to the pressure variations in the bag, it is necessary, first of all, that the ball shall expand by equal volumes for every equal increase of pressure. As Erlanger has shown, this occurs fairly evenly in the bulb utilized in his instrument, but it does not occur when a softer bulb restrained by a mesh-work is used, as in the Uskoff apparatus. The result is more unfortunate since the sensitiveness of the instrument increases precisely in the pressure field where the largest excursions are likely to occur.

Another factor, apparently not recognized by Erlanger, operates to change the sensitiveness at different pressures. As the pressure falls and air escapes, the elasticity coefficient of the air-containing bag decreases and the sensitiveness of the system gradually diminishes (Frank). Furthermore, the lever, the vibration frequency of which is only 6.5 per second, is inadequate to accurately pick up the variations transmitted by the rubber ball. The advantages of the Erlanger apparatus are that oscillations of considerable amplitude are recorded and their sudden variations more clearly marked in the average run of cases than in other similar patterns. Furthermore, the damped mercury manometer allows an approximate estimate of the pressure applied to the artery during diastole. A more accurate measure of this could perhaps be obtained by the interposition of a minimal valve during the period in which the pressure is falling.

The Feddé pith-ball oscillometer is based on an entirely different principle, namely, that a light particle in a tube within which pressure changes occur rapidly, will move in proportion to the force exerted by the pressure change. On account of its lightness, it should prove a very accurate measure of the pressure changes within the bag. The error arises when the ball becomes rough or is no longer round or the tube causes it to adhere by friction. A careful and expert adaptation of the ball to the tube is imminently desirable. The chief draw-back is that its movements cannot be recorded. 


\section{CRITERIA FOR ESTIMATING SYSTOLIC PRESSURE.}

The Peripheral Pulsation Method.-Riva Rocci Method.-The procedures now commonly employed consist: (1) in applying a circular arm-piece snugly to the bare arm, so that the bag lies on the inner aspect (2) in inflating the system until the radial pulse disappears (3) in gradually allowing air to escape through a fine leak and palpating for the first return of the pulse at the wrist. The reading of the mercury pressure at this moment is taken as systolic pressure. The procedure may be modified by recording the radial pulse by a sphygmograph instead of by palpation, but it is questionable whether the former is more sensitive. Or the brachial artery may be palpated instead of the radial, a procedure that has a theoretical advantage, since weak pulse beats are possibly smoothed out before they reach the radial artery. The advantage is probably offset by the fact that the brachial artery lends itself less easily to palpation. To make use of the advantage and negative the disadvantage, a smaller cuff has been snugly applied below the compressing cuff and, after inflating with moderate pressure, it is connected to some form of oscillometer (Bing, Pal, Hoobler). By this method it is possible to recognize pulsations peripheral to the bag at pressures from 3 to $10 \mathrm{~mm}$. higher than by radial palpation. This procedure is especially valuable in children (Hoobler). The actual discrepancy between the two methods, of course, depends on the acuteness of the tactile sense, as well as on the sensitiveness of the oscillometer.

Principle.- The principle of using the first evidence of a returning pulsation peripheral to the compressing bag as a criterion of systolic pressure, is based on the supposition that, as long as a pressure greater than systolic is exerted on the artery, it remains collapsed during systole as well as during diastole; but, as soon as extra-arterial pressure equals or falls slightly below intra-arterial systolic, it allows blood to pass during systole and gives rise to a peripheral pulse. This dictum presupposes: (1) that the arterial wall offers no appreciable or at least a constant resistance to compression; (2) that the artery compressed for a considerable length can be opened by the systolic pressure which acts only momentarily and (3) that the first blood passing into the peripheral vessels can cause a pulse wave. All of these suppositions have been questioned. The second has already been discussed. The question has frequently been discussed whether sclerosed vessels offer more resistance to compression (Russell) than normal arteries. Experiments indicate that while a thickening or calcification does not appreciably increase the resistance of vessels to compression (Janeway and Park, MacWilliam and Kesson) a high state of tonic contraction may offer considerable resistance, and by reduc- 
ing the lumen of the vessels may also interfere with the peripheral transmission of the systolic pressure wave (MacWilliam).

In regard to the third supposition, it can readily be demonstrated that when the forearm is enclosed in a plethysmograph, its volume begins to increase before pulsations are visible either in the plethysmograph recorder or in a second armbag. It would appear, therefore, that the application of an equi-systolic pressure to the arm causes merely a stenosis through which blood may seep at the height of systole but in amounts not sufficient to cause a pulsation. It appears likely, therefore, that a return of a peripheral pulsation large enough for recognition occurs only when the extra-arterial pressure is less than intra-arterial systolic. Comparison with the plethysmograph method indicate that this error may be equal to 10 or 15 millimeters of mercury in man and comparisons with the direct pressures obtained in animals also indicate that the systolic pressure is greater than that estimated (Fellner and Rudinger); Erlanger, Wiggers, Eberly and Wenner). Comparisons of these pressures with the intra-arterial pressures in man directly estimated

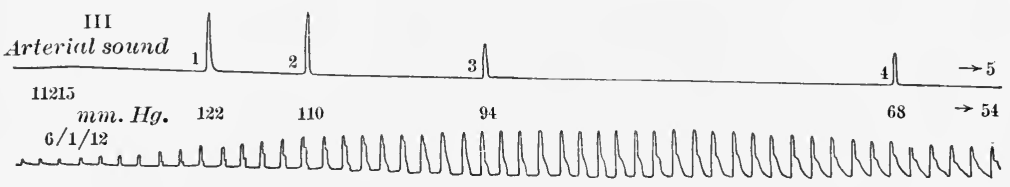

FIG. 57.-Curve showing the relation of amplitude of oscillation to different phases determined by the auscultatory method. (After Norris.)

by the Hürthle manometer indicate an opposite relation, namely, that the intra-arterial systolic is less than that estimated by palpation (Müller and Blauel). Too great stress should not, however, be placed upon these results which were obtained, apparently, on three individuals by types of direct manometers themselves not free from errors.

The Auscultatory Method.-The method of auscultation suggested by Korotkow (Fig. 52), which utilizes the appearance of a sharp sound peripheral to the bag as a criterion of systolic pressure, is based on the principle that when a small quantity of blood passes beneath the cuff into the relatively relaxed arteries below, it causes a sound. Upon detailed examination it seems that, when the upper arm is compressed by an arm-bag, the venous efflux from the forearm is first prevented and, later, the arterial inflow. While this compression persists, the arterial and venous pressures tend to equalize (Hill and Flack), and, consequently, some blood passes from the arteries leaving them relatively empty. The sudden distention by even a small quantity of blood is evidently sufficient, when aided by the resonant bag, to produce a sound. Actual 
comparisons, therefore show, as was to be expected, that the first sound is heard at pressures from 5 to 15 millimeters higher than those which permit a pulse to pass to the wrist (Korotkoff), yet it occurs almost synchronously with the graphic criterion for systolic pressure (Fig. 57).

5 The Oscillatory Method.-The pressure at which oscillations in the manometer system first occur has been suggested as a criterion of systolic pressure on the supposition that they appear only after blood has been forced under the bag (Pachon, Uskoff). It has been established, however, that such oscillations occur at pressures far in excess of systolic, due to the "ram action" of the occluded artery upon the bag (v. Recklinghausen, Erlanger). Evidently their incidence is no criterion for systolic pressure, the relative time of occurrence depending entirely upon the sensitiveness of the apparatus.

When blood actually passes under the bag and the heaving sensation is noted, the recorded waves become larger and change their character at the same time that the base line rises. The abrupt and simultaneous occurrence of these changes has therefore been suggested by Erlanger as a criterion of systolic pressure (Fig. 58). Practically, neither the rise of the base line which depends on the size of the leak in the tambour, nor the sudden increase in amplitude has met the expectation as a criterion. Frequently the increase in size is either not sudden, but gradual and progressive, or several abrupt changes in amplitude occur. The change in the shape of the curve is, however, a valuable criterion. The change in the conformation of the wave (Fig. 58) consists in the fact that a wave (dicrotic) becomes supported on the descending limb which gives an effect, when written on the slowly moving drum as if the bases had been separated.

What is the explanation of these changes? The rise in the base line is accounted for by the fact that, when more blood rushes under the bag, the base line is elevated before the pressure in the tambour can be equalized through the small leak. The amplitude is increased because a stretch of artery pulsates under the arm-bag. The change in shape demands a fuller explanation. As long as the oscillations are due to a "ram action," the recorded pressure is the expression of a sudden shock, rising and falling rapidly together with a small reflected wave. As soon as blood passes under the bag, the fall occurs more slowly because blood must be squeezed out during its fall (Erlanger). Experience indicates that the systolic pressure determined by this criterion averages from 5 to $15 \mathrm{~mm}$. higher than that determined by palpation, while it is synchronous with the first sound heard on auscultation (Fig. 57). 


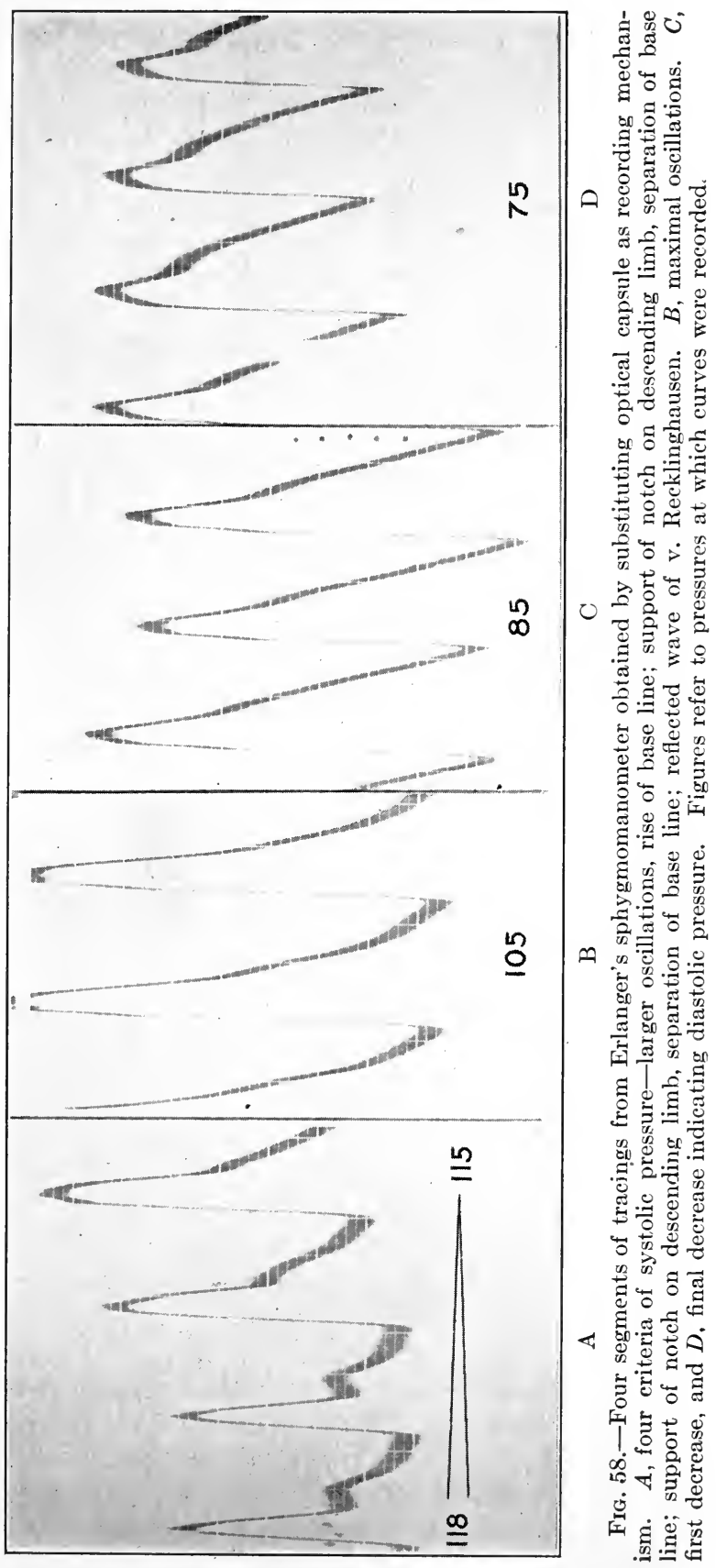




\section{CRITERIA FOR ESTIMATING DIASTOLIC PRESSURE.}

Amplitude of Oscillations Peripheral to the Bag.-If the pressure is gradually released from the arm-bag, and the radial pulse palpated (Strassburger) or better, if the radial pulse is recorded by a sphygmograph (Janeway, Massing, Sahli, Jacquet), it will be found that the amplitude increases until a certain maximum is reached after which no further increase in size occurs. A similar increase in oscillations up to maximal may be observed in the excursions of an oscillometer which is connected to a second armbag applied below the compressing bag (Janeway, Clark, Bing,

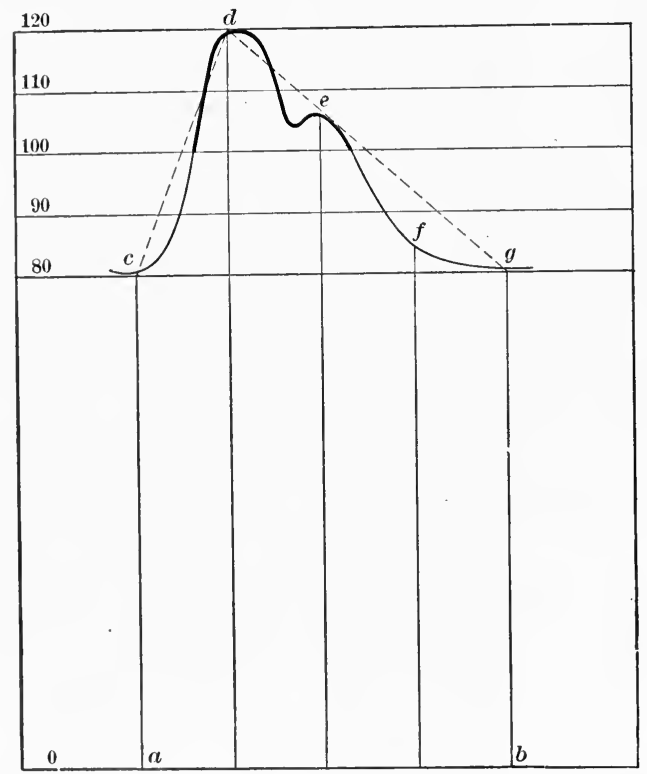

FIG. 59.-Diagram illustrating the utilization of the amplitude of the peripheral pulse as a criterion of diastolic pressure.

Vasquez, Oliver, etc). The point at which the oscillations peripheral to the bag become maximal has been used as a criterion of diastolic pressure.

This criterion is based on the principle that the quantity of blood which is allowed to pass through the arm-bag and, hence, the pulse amplitude, depends on the compression of the artery. Thus, if the extra-arterial pressure were $100 \mathrm{~mm}$. (Fig. 59), the artery presumably would not open until the intra-arterial pressure during systole had passed $100 \mathrm{~mm}$. It remains open and allows a pulse wave to pass peripheral to the bag so long as the pressure remains above 
this level during systole and subsequent diastole. For this reason, a small pulse wave is recorded as indicated by the heavy lines.

As the extra-arterial pressure gradually falls and the intra-arterial diastolic pressure is approached (say $80 \mathrm{~mm}$.), the artery beneath the bag remains open during the entire interval of diastole and allows a maximum pulse wave to be propagated. Since a further reduction in the external pressure could not lengthen the interval that the artery remains open, no further increase in the amplitude occurs.

The criterion is open to an essential criticism, however, viz., that the amplitude of the peripheral pulse is determined, not only by the amount of blood passing under the bag, but also by the volume elasticity coefficient of the artery which depends largely on the volume of blood in the peripheral arteries. When both venous efflux and arterial influx have been shut off, the blood tends to pass from the higher arterial to the lower venous pressure and, hence, tends to leave the arteries so that the pressure within them becomes low. As soon as blood reënters the forearm and causes pulsations of a small amplitude, it raises the peripheral pressure during every beat and decreases the volume elasticity coefficient. The effect is that for equal volumes of blood entering, there is always less and less excursion of the artery. There are, then, two forces at work, the greater filling of the artery tending to reduce the amplitude, and the greater volume entering per beat tending to increase it. For a long time the latter overpowers the former. It is quite possible, however, that, when the arm-bag contains an extra-arterial pressure somewhat above actual diastolic, these two forces counter-balance. Then, no further increase in amplitude occurs. This may explain why the readings of the diastolic pressure thus obtained are always considerably above those obtained by other methods. The varying pressure of the sphygmograph button which exists when the artery fills also seriously affects the accuracy. It is well known that the amplitude of excursion recorded by the sphygmograph depends on the relation of intra-arterial pressure to extra-arterial tension. As the former gradually rises, and the arterial volume increases, this relation may be improved or made worse, depending on the adjustment of the apparatus. It is therefore not surprising that widely differing results have been obtained and the method has not been generally accepted as reliable.

Amplitude of Oscillations Within the Arm-bag. - If, while the pressure is slowly falling within the manometer system, the oscillations from the arm-bag are graphically recorded, as in the Gibson, Erlanger, Uskoff and similar apparatuses, or are observed in the swing of a mercury column, an aneroid pointer, or any other oscillometer, the oscillations will be seen to increase in amplitude, remain at a maximum for a considerable time and then gradually 
(more rarely abruptly) decrease. The extra-arterial pressure at which the oscillations first become smaller is taken as a criterion of the diastolic pressure. It is quite generally stated that the principle on which this criterion depends is that of Marey. It was Marey who first laid down the principle that an artery oscillates with greater amplitude as the pressure increases up to a certain level. He assumed that when the internal and external pressures are equalized the arterial wall is relieved of tension and in a condition to oscillate freely. Such an equalization of tension causes a greater excursion according to Frank, because the volume elasticity coefficient $\left(\frac{\Delta p}{\Delta v}\right)$ of the wall is reduced and, hence the sensitiveness of the entire recording system, of which the arterial wall is essentially a part, is thereby increased.

The mechanical experiments upon excised arteries, rubber tubes and arteries in connection with the circulation (Mosso, Howell and Brush, Erlanger) have been considered as demonstrating conclusively that the largest oscillations may be used as a criterion of diastolic pressure. All of these investigators, no matter how careful their technic, have been obliged to register the actual pressure variations by the apparatus available at their respective periods. Thus, Mosso was forced to content himself with the mercury manometer, Howell and Brush with the Fick spring-manometer, while Erlanger used maximum and minimum valved manometers. All of these are now known not to be devoid of error. It cannot, therefore, be accepted as demonstrated beyond doubt that the largest oscillations do occur when an exact equilibrium is reached between intra- and extra-arterial pressure.

MacWilliams and Melvin, who have recently investigated the question conclude that this view is erroneous. They find that with normal elastic arteries the maximal oscillations occur when the external pressure is sufficient to produce what they term a "half-flattening" of the artery, $i$. $e$., a state in which the short diameter is roughly one-half that of the distended tube. The natural inferences from such experiments is that the pressures at which largest oscillations occur must be considerably above diastolic, while the point at which subsequent reduction in amplitude occurs more accurately corresponds to diastolic pressure. In agreement with this view is the theoretical exposition of Christen. He points out that whenever a tube of an elastic nature is expanded by equal pressure increments, it will be found that at first the expansion is small, then becomes suddenly greater and, after the distensibility has remained constant, grows smaller again. The tube may be said to pass through three elastic fields: the first, in which the distensibility is small, the second, in which it is great, and the third, in which it decreases again. 
Arteries in their normal condition are always in the third field of elasticity, which accounts for the fact that the excursion of the wall is so slight. When an extra-arterial pressure is applied, however, which somewhat exceeds the intra-arterial diastolic pressure, the artery shifts to the second field of elasticity, $i . e$., the one in which the volume elasticity coefficient is small and the oscillations large. When the extra-arterial pressure is lowered to the point where it exactly falls below the intra-arterial pressure, the arterial wall enters the third field of elasticity again and the oscillations become smaller. 'The suddenness of the change is determined by the nature of the artery - the more resistant the vessel, the more gradual the change in amplitude.

This also accords with the results of animal experiments in which the pressure is applied to the leg of a dog by a conical cuff prevented from slipping by special devices (Lang and Manswetura, Wiggers (unpublished) ). It may be added, however, that when the pressure is applied directly to an artery by an arteriograph, the largest oscillations occur at pressures only $3-4 \mathrm{~mm}$. from intra-arterial minimum (Erlanger, Wiggers, Eberly and Wenner). It is not possible to give a final explanation of these discrepancies. It is possible that pressure cannot be applied without loss to the conical leg of a dog (Erlanger, Müller and Blauel), but with this idea apparently not all are agreed (Janeway, Lang and Manswetura, Wiggers). It is very probable that the progressive reduction in sensitiveness which occurs when air escapes during the fall of pressure (Frank) causes a reduction in the amplitude of the lever oscillations before a diminution in arterial volume occurs. This unavoidable instrumental error may be greater when a large arm-bag is used in dog or man than when an arteriograph of small capacity is employed.

The Auscultatory Method.-If the chest-piece or bell of a stethoscope is applied over the brachial artery, below the cuff of any bloodpressure apparatus (Fig. 52), a series of sounds can be heard as the pressure gradually escapes. The changes in the sounds may be divided into five phases, the relations of which to the oscillatory changes are shown in Fig. 57.

1st phase. The sudden appearance of a clear sound lasting for a fall of approximately $14 \mathrm{~mm}$. of mercury. This has already been described as a criterion of systolic pressure.

$2 \mathrm{~d}$ phase. The acquisition of a murmurish character, lasting while the pressure falls approximately $20 \mathrm{~mm}$. more of mercury. It is attributed to the stenosis of the vessel produced under the cuff at this stage.

$3 \mathrm{~d}$ phase. The replacement of the murmur by a sound becoming progressively louder and lasting during the next $25 \mathrm{~mm}$. of pressure fall. This is attributed to the oscillation of the arterial wall aided by the resonance of the bag. 
4th phase. The muffling of the sounds lasting while the pressure falls 5-6 mm. more. The cause of this muffling has been variously interpreted as due $(a)$ to the absence of diastolic collapse, $(b)$ to the slowing of the stream under the cuff, $(c)$ to the decreased resonance in the deflated cuff, and $(d)$ to a lack of flattening of the arterial wall (MacWilliams and Melvin).

5 th phase. The disappearance of all sound.

Although Korotkoff and his followers at first utilized the disappearance of all sound as a criterion of diastolic pressure, some doubt has arisen as to whether the entire disappearance of sound or the fourth phase first described by Ettinger, in 1907, represents the correct criterion. Numerous investigations have been instituted to determine this question. With a few exceptions these have consisted in comparing the pressures read at the fourth and fifth phases with other criteria. The work may be summarized in the following table:

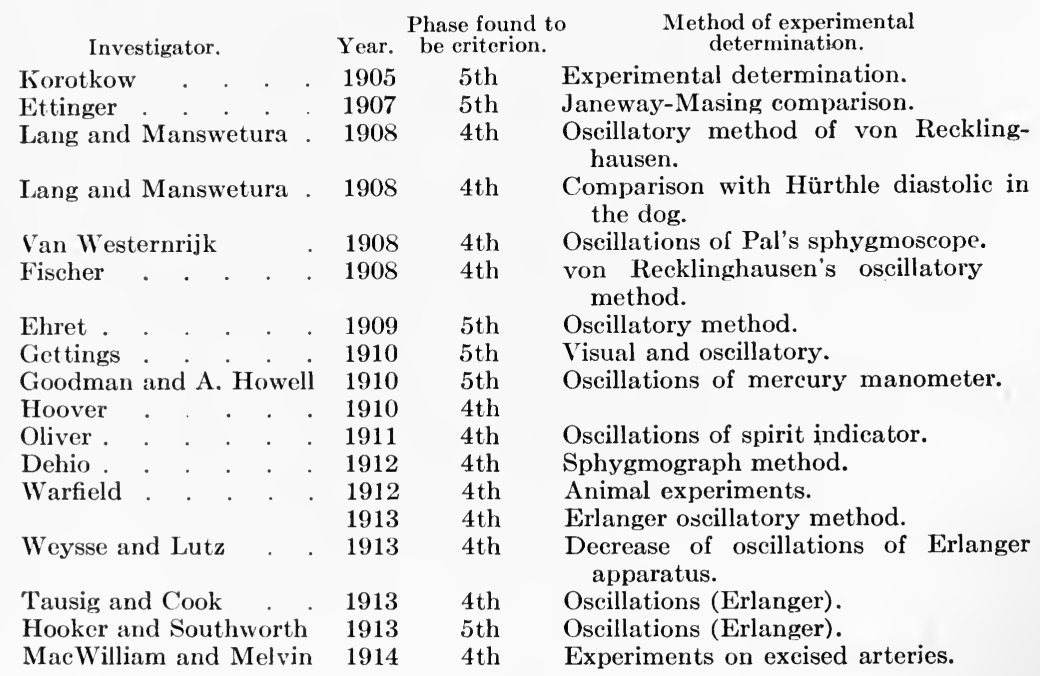

The perusal of the foregoing table indicates that the consensus of opinion favors taking the fourth phase as a criterion of diastolic pressure. This has a distinct advantage in clinical work since in many cases a total disappearance of sound does not occur $(e . g$., aortic insufficiency).

The method has been further studied in relation to the changes that occur during abnormal conditions of the circulation. Thus, it has been pointed out that the duration of the phases may vary under pathological conditions (Goodman and A. Howell). When the second or murmur stage is absent or short and disappears after exercise, a weak or dilated heart is suspected. It is variable in 
duration and intensity when arrhythmia exists. A prolonged or intense murmur phase indicates vigorous action of the heart.

The duration of the third phase depends upon the strength of the systole. A phase of long duration is present during vigorous action of the heart and in arteriosclerosis. A short and indistinct phase occurs during weak cardiac action. During arrhythmias the variation in the strength of succeeding systoles may thus be studied and compared with pulse tracing amplitudes. "The fifth phase is filled by a continued sound in cases of aortic insufficiency (Taussig and Cook).

\section{PRACTICAL ASPECTS OF BLOOD-PRESSURE DETERMINATION.}

Choice of Apparatus.-Before selecting any form of apparatus, the principles it is desired to utilize should be chosen. This applies largely to the estimation of diastolic pressure. For general practice the auscultatory method is in all respects the best. It may be carried out with any form of apparatus. The oscillatory method of recording diastolic pressure is peculiarly adapted to hospitals and office work. Among the forms of apparatus utilizing this criterion those employing a direct recording spring manometer (as those of v. Recklinghausen and Jacquet) are probably preferable to those using a sphygmoscope system, as the Erlanger, but the latter, in turn, is more accurate in construction than the Uskoff. Visual oscillatory methods are less satisfactory because the point at which large oscillations cease can be only approximately gauged. Those forms of apparatus in which the largest oscillations occur are best. The Feddé and Pachon oscillators are therefore preferable to those of Hill, Bing, etc. Instruments that read the oscillation with the manometer recording the pressure, as the Gibson, Janeway and other mercury models, as well as the spring aneroids, are not to be recommended for determining diastolic pressure by the oscillatory method.

Having selected an instrument adapted to operate upon the principle chosen, attention should next be directed to its accuracy and workmanship. 'The scale should be correct and adjustable; the zero level readily verifiable. The system should be free from leaks; the tubing, of heavy quality; the bag elastic, $12 \mathrm{~cm}$. wide and not too long, but above all protected by a rigid unyielding cuff.

Portability is a great consideration, but accuracy should never be sacrificed on its account. Aneroid manometers, while convenient, are liable to become inaccurate and to need recalibration.

The Patient as a Factor in Accurate Determination.-The pressure should always be taken in the same position, recumbent or sitting, and if in the latter, the arm-bag should be placed on a level with the 
costosternal angle. The arm-bag should be applied loosely so that a small volume of air remains within when the manometer is at zere, but tightly enough so that this contained volume of air is not too great. It is desirable to make the determination as rapidly as possible in order to avoid or minimize a direct mechanical or reflex effect on the general blood-pressure. If meals, warm drinks, alcohol, tea or coffee have been partaken of shortly before the bloodpressure measurement, or if exercise or smoking have been indulged in, their tendency to elevate pressure should be taken into account, as should the psychic reaction of the patient. The following sequence of procedures suggested by Norris probably adequately guards against psychological errors on the parts of both examiner and patient.

"In order to minimize all these sources of error the following procedures should be adopted: (1) Discard the results of the first reading, using it simply to demonstrate the harmless and painless character of the procedure; and, when possible, make subsequent readings after some little time has elapsed. (2) Avoid making blood-pressure observations when the patient is excited, anxious or worried, as a result of an examination, etc. (3) Make several consecutive readings and if they correspond more or less closely, take the arithmetic mean. (4) Make the observations as quickly as is consistent with accuracy; do not look at the manometer until the pulse is felt (or a certain phase of sound heard on auscultation) - at this point the air escapement should be tightly closed until the reading is made. (5) Allow the pressure to fall to zero between observations, and permit sufficient time to elapse between readings for the venous pressure (stasis) to fall to the normal level."

\section{CLINICAL VALUE OF BLOOD-PRESSURE DETERMINATION.}

Normal Variations.-Before it is possible to attribute pathological significance to blood-pressure readings, it is desirable to ascertain the normal variations. It should always be borne in mind that a perfect form of blood-pressure apparatus has not, as yet, been devised, and that our readings are only approximately correct (i. e., perhaps within $5 \mathrm{~mm}$. if proper apparatus and technic are used). With a wide cuff, the average systolic pressure by palpation may be placed at 100 to 110 , while with auscultation and oscillatory methods it may be from 5 to $15 \mathrm{~mm}$. higher. A somewhat higher pressure $(10 \mathrm{~mm}$.) may be expected after middle life. Janeway regards a persistent pressure above 135 in the young or 145 after middle age as suspicious and life insurance statistics indicate that the mortality in individuals with pressure above 150 is 35 per cent. greater (Fischer). The average diastolic pressure may be placed between 65 and $75 \mathrm{~mm}$. During ten years that the writer has 
checked the pressures taken by students in the physiological laboratory by Erlanger's method, the pressure was rarely found to vary beyond these limits $(i . e$., if we except about 20 per cent. of cases in which it is difficult to establish a diastolic reading from the Erlanger curves). This gives a pulse pressure, as the difference between systolic and diastolic pressure is called, which varies from 35 to $50 \mathrm{~mm}$.

Permanent Alterations of Pressure.-Practically, the measurement of blood-pressure is used to determine whether the pressure is permanently high or low. The conditions giving rise to permanent hypertension and hypotension will be discussed in detail in other sections but may be here classified for convenience, after the plan of Janeway:

Hypertension:

1. Functional:

(a) Physiological (i. e., due to excitement, exertion, labor, pain reflexes, etc.).

(b) Toxic (as morphine, lead and nicotine poisonings, gout uremia, and rheumatic affections.

(c) Cerebral anemia and asphyxia.

2. Essential (i.e., accompanied by organic change and indicating derangement of regulating power):

(a) Arteriosclerosis.

(b) Nephritis.

Hypotension:

1. Functional:

(a) Diarrhea, excessive sweating, etc.

(b) Hemorrhage.

(c) Shock.

(d) Acute infections (except meningitis).

2. Essential:

(a) Cachectic (in tuberculosis, carcinoma, general paralysis).

(b) Enteroptotic (relaxed abdominal walls, enteroptosis of varying degree).

Rhythmic Variations of Blood-pressure.-In establishing a certain figure for systolic and diastolic pressures in man, it should be borne in mind that these figures represent only approximately the highest and lowest pressures in the artery. This is due, in the first instance, to the fact that the intra-arterial systolic and diastolic pressures vary with rhythmic changes in the heart cycle and with the acts of respiration. ${ }^{1}$ These variations are of such a nature that, in the majority of normal individuals, both pressures are lower in inspiration than during expiration. The variations of systolic pressure are often great enough to be detected by different criteria. 
Thus, if the pressure is allowed to fall slowly and auscultation is carefully carried out, it is often found that the sound is first limited to the expiratory beats, while after the pressure has fallen 3-5 $\mathrm{mm}$. more a sound is present for every beat. Similarly, in the Erlanger tracing, the supported wave in the descending limb often occurs at first on one of two waves of a respiratory group before it becomes mounted on all waves. In these ways we may estimate the highest and lowest systolic pressures.

While these variations of normal pressure are not of extreme importance, a determination of the highest and lowest systolic pressures may be of great diagnostic significance. Thus, it happens that pericardial adhesions or tumors may be so situated that they exert a traction or compression upon the heart or large vessels during one respiratory phase. The difference between inspiratory and expiratory pressures may then be so great as to be of diagnostic value.

Blood-pressure in Extreme Cardiac Irregularity.-When the heart is markedly irregular or the auricles are fibrillating, the pressure varies considerably during consecutive beats, in fact, some beats may entirely fail to reach the instrument, thus showing a pulse deficit. To determine systolic and diastolic pressures is often impossible with the available criteria. Some idea of the systolic pressures may be obtained in such cases by estimating the "average systolic pressure" according to the procedure suggested by James and Hart. The pressure in the cuff is raised until all pulsations are obliterated. It is then lowered $10 \mathrm{~mm}$. at a time, and each time the number of pulse beats that pass peripheral to the cuff are counted. An assistant counts the apex beats at the same time. The average syrstolic pressure is then obtained by multiplying each different pressure tested by the number of beats passing per minute. These products are added and the sum divided by the number of apex beats per minute. The quotient gives the average systolic pressure.

A simpler and, in some respects, a preferable method, which the author employs consists in writing the highest and lowest systolic and the highest and lowest diastolic pressures, the pulse having first been counted. The cuff is inflated until the pulse is obliterated. Upon gradually deflating the system of air, the pressure is noted when the first sound is heard peripheral to the bag. This is the highest systolic pressure. The pressure is then released until the sounds for all waves are audible. This is the lowest systolic pressure. The estimation of any diastolic pressure is, however, very unsatisfactory. The oscillation method is of less value in these cases, for the theory of this criterion presupposes that the pulse pressure remains at least approximately constant or varies rhythmically as during natural respiration. It is, however, possible to obtain an approximate idea.in many cases when groups of waves remain 
permanently smaller. There would be little question, for example, that the diastolic pressure was approximately 85 in the record shown in Fig. 60. At about the same level the sounds of most of the beats disappeared on ausculation.

The Value and Limitations of Pulse-pressure Determinations in Disease.-Blood-pressure determinations are sometimes employed to follow the changes in the circulation during disease processes or to test the effect of medication, it being sought not only to determine whether the circulation is changed but also what part is at fault. In this capacity it has been used largely in the acute febrile affections and in chronic valvular lesions. In neither group have the pressure determinations given the results anticipated; partly, no doubt, because the readings have not been taken at sufficiently close intervals and partly because the systolic and diastolic pressures and their differences, the pulse pressures, were not studied in relation

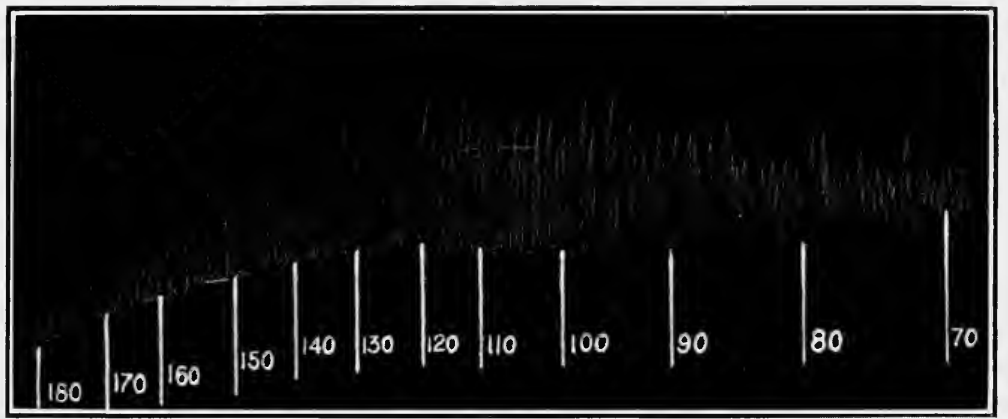

FIG. 60.-Sphygmomanometer tracing from case of auricular fibrillation, showing irregularity in amplitude of consecutive oscillations and the difficulty of determining either systolic or diastolic pressure by the oscillatory method.

to the heart rate. If information concerning the changes in the circulation is desired, it is just as important to take hourly readings of pressures as it is to make the temperature and pulse-rate charts.

We may first of all inquire what information the pulse-pressure gives us concerning the systolic discharge of the heart. The pulse pressure is an index of the volume of cardiac discharge only when it may be assumed that neither the viscosity of the blood, nor the diameter of the peripheral vessels nor the cubic increase in volume for definite pressure increments varies. It may, therefore, be at once set down that pulse pressure cannot be an exact measure of the systolic discharge and the only question of interest is, whether, recognizing its limitations, it may be a sufficiently exact criterion. The experimental investigation (Dawson and Gordon, Henderson, Wiggers) indicates that, although there is a general corre- 
spondence between the amplitude of pulse pressure and the output of the heart-in the sense that both increase and decrease synchronously so that the course of one may be prophesied from the otherthere is no quantitative relation. The same pulse pressure may at different times correspond to systolic discharges of very different volumes. Unless subsequent reinvestigation, by more accurate methods, should speak to the contrary, the clinician is not warranted in assuming that, because the pulse pressure is equal to one obtained some days or weeks previous, therefore, the output of the heart has not altered; or, because the pulse pressure does vary, that the output has necessarily changed. The pulse pressure is not a quantative measure of systolic output, but when compared to some not too remotely preceding observation in the same individual, it may be used as an indication of the direction in which the discharge varies. Thus, by following the pulse pressure during periods of an acute febrile process or during the presence of a valvular lesion or a myocarditis hour by hour, one may obtain valuable information as to whether the output is improving or deteriorating, and this, as indicated by experimental investigations, occurs in spite of considerable variation in viscosity or peripheral resistance.

One is not warranted in assuming any correspondence whatever between pulse pressure and systolic output in different individuals in whom the viscosity of the blood, the volume and the vascular elasticity and tonicity are different by an unknown amount due to some diseased condition such as arteriosclerosis, infections, cardiac disease, etc.

Investigators have established that the systolic output is directly dependent on the heart rate and, in such a way, that an increase in rate results in a decrease in output per beat. This may frequently be indicated by studying the product of the heart rate and pulse pressure. Under normal conditions this product tends to remain constant (Erlanger and Hooker) or, if any change occurs, the tendency is toward an increase as the heart accelerates. When the output of the heart is primarily affected, as in hemorrhage and shock through a reduction of its blood supply, or by a diminution in its contraction amplitude, as occurs in depressing toxic action or in valvular insufficiency, the product decreases. It is obviously possible, however, that even when hourly estimations of pulse pressure are made that their comparison with preceding observations may be erroneous when considerable variation in the tonus of the peripheral vessels, the vicosity of the blood or the total blood volume occurs. In order to decide whether the output of the heart or the peripheral resistance or viscosity is the chief variable, a comparison of the pulse pressure with the height of systolic and diastolic pressures has been suggested. Erlanger and Hooker have constructed the following table as indicating the relations: 


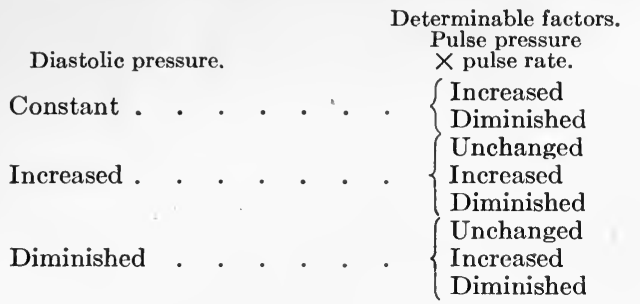

$\begin{array}{lc}\begin{array}{c}\text { Causative factors. } \\ \text { Energy }\end{array} & \begin{array}{c}\text { Peripheral } \\ \text { resistance. }\end{array} \\ \text { Increased } & \text { Diminished } \\ \text { Diminished } & \text { Increased } \\ \text { Increased } & \text { Increased } \\ \text { Increased } & \text { Unchanged } \\ \text { Unchanged } & \text { Increased } \\ \text { Diminished } & \text { Diminished } \\ \text { Unchanged } & \text { Diminished } \\ \text { Diminished } & \text { Unchanged }\end{array}$

It is evident that, in general, a decrease in the product of the heart rate and pulse pressure indicates that the heart output is unchanged when it is accompanied by an increased diastolic pressure; but that an increased product likewise indicates an unchanged output when the diastolic pressure is diminished. The practical value of such a guiding table has been frequently questioned. Thus, Norris points out, as an example, that in nephritic hypertension both an increase in diastolic pressure and the product occur which would be interpreted as due to an increased cardiac output and an unaltered peripheral resistance-a conclusion evidently erroneous. This, however, should be cited not as an example of a defect in the table, but of its misapplication; for, as variations in the pulse pressure can reasonably be expected to be of prognostic value only when compared in the same individual with observations preceding at not too distant intervals, so this table is restricted in its applicability to consecutive observations in the same case.

A similar though less accurate procedure has been evolved by Strassburger who compared the relation between pulse pressure and systolic pressure as a quotient (blood-pressure quotient) -B. P. Q. = $\frac{\text { pulse pressure }}{\text { systolic pressure }}$. This formula is less reliable than that offered by Erlanger and Hooker, partly because the maximal pressure follows the mean pressure less accurately than does the diastolic; but chiefly because it contains no factor allowing for heart rate variation.

It may be pointed out, in conclusion, that this most promising field, say hourly or two-hourly determinations of pulse pressures and heart rates during infectious disease or critical stages of cardiovascular lesions has not been adequately invaded. Clinicians have too often been content with occasional and haphazard determinations which have yielded results of no importance and hence can probably be dispensed with entirely. Whether the efficiency of the circulation is improving or whether failure is threatened can only be determined by frequent and systematic studies of pulse pressure and heart rate.

Methods Designed to Estimate the Mechanical Energy or Work of the Heart.-Two attempts have been made to estimate the mechanical energy of cardiac action by procedures related 
to blood-pressure determinations, viz., by the application of the sphygmobolometer or sphygmobolograph of Sahli and the energometer of Christen.

The theory upon which these measurements are founded is briefly as follows: During every cardiac systole, a certain amount of mechanical energy is liberated. About 1 per cent. is utilized in moving the blood onward (kinetic energy), the rest is transformed to elastic tension of the wall (potential energy). The energy utilized in filling and expanding the arteries and so creating the entire pulse wave (pulse energy) is not directly measurable. It is possible, however, that the energy required to produce the pulse in a limited area of artery (pulse impact energy) can be estimated, and upon the assumption that this gives information concerning pulse energy, the methods rest.

The Sahli Sphygmobolograph.-Sahli attempts to estimate the energy of the pulse impact by the product of the distance that the wall expands and the compressing force. His sphygmobolometer first devised for this purpose is probably less exact and more difficult of application than the sphygmobolographic method. The sphygmobolograph is essentially a Jacquet sphygmograph (new model) in which the two tiny rollers usually pressing the paper against the propelling roller actuated by clock-work have been replaced by nine tiny rollers which write a series of abscissæ lines upon the paper as it passes. These rollers are not equidistant but so placed that the distances between them represent a movement of $0.05 \mathrm{~mm}$. In this way the excursion of the arterial wall can be estimated from the pulse tracing. The pressure exerted by the spring is determined by reading the number on the dial and, by reference to an accompanying table, the pressure can be estimated in grams.

It is apparent that a single computation at any one pressure is not sufficient since every different tension will yield a different product. The product is therefore determined during the entire gamut of pressures. The largest product is chosen as a standard for comparing the energy of pulse owaves of different individuals and of the same individual at different times.

Leaving the question as to the accuracy of the principle out of consideration, the method is at best a crude one in consideration of the limitations of the Jacquet sphygmograph. How correctly the gram readings on the instrument correspond to the pressure exerted on the vessel wall, depends on its adjustment. How truly the amplitude of the button movement in this instrument, which has an inadequate vibration frequency, corresponds to the actual movement of the artery depends largely on the vigor of the pulse beat. Finally, how accurately the movement of the button under pressure as an index of the energy exerted by the entire pulse wave, 
is determined by the elasticity and tonus of the radial under the button.

Energometer Method.-In some respects the energometer method of Christen is an improvement. This apparatus is shown in Fig. 61. Its cuff is applied to the arm or thigh and brought to the proper

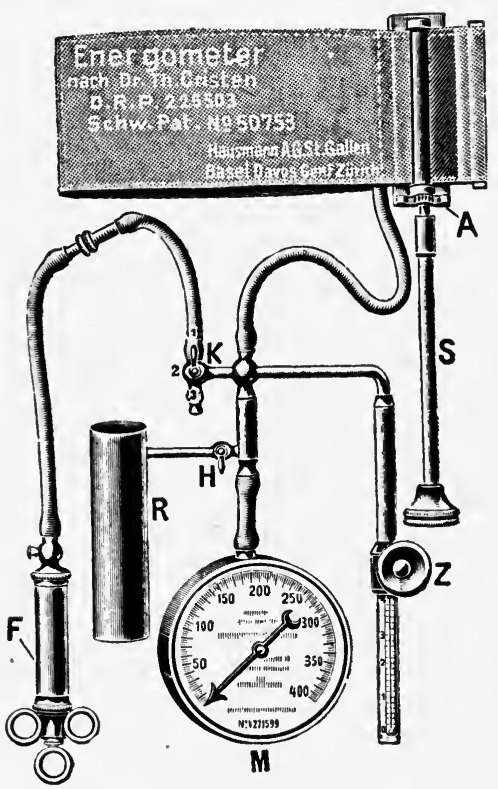

Fig. 61.-The energometer. (After Christen.)

tension by tightening the key $S$. The position of the volume syringe is placed at zero by means of the screw $Z$, and stop-cock $I I$ is closed. Air is then pumped into the system by the pump $F$ and the stop-cock $K$ is closed. The mean pressure is read either in terms of $\mathrm{cm}$. of water pressure or in $\mathrm{mm}$. of atmospheric pressure at the mid-point of the oscillations of the spring manometer (which according to Christen) has an inherent vibration frequency of 20 per second. If the oscillations are too large the sensitiveness is diminished by increasing the capacity of the system by opening stop-cock $H$, and so adding the reserve space $R$ to the system. The piston of the volume pump is now moved in by screw $Z$, so that the pressure before read at the upper excursion now becomes the lower pressure. The volume is then read off. The product of the mean pressure and volume change is expressed in gram centimeters, as shown in the following table: 


$\begin{array}{ccc}\text { Pressure. } & \text { Volume. } & \begin{array}{c}\text { Pressure } \times \text { vo } \\ \text { Energy of puls }\end{array} \\ 30 & 0.2 & 6 \\ 50 & 0.25 & 12.5 \\ 70 & 0.3 & 21 \\ 80 & 0.5 & 40 \\ 100 & 0.9 & 81 \\ 110 & 1.4 & 154 \\ 130 & 1.7 & 221 \\ 150 & 1.5 & 225 \\ 170 & 1.2 & 204 \\ 190 & 1.0 & 190 \\ 200 & 0.8 & 160 \\ 220 & 0.6 & 132\end{array}$

\section{$V \cdot 4,0 \mathrm{~cm}^{3}$}

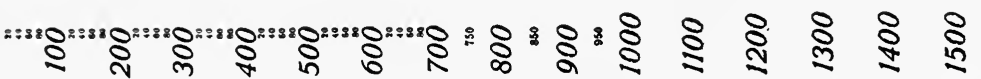

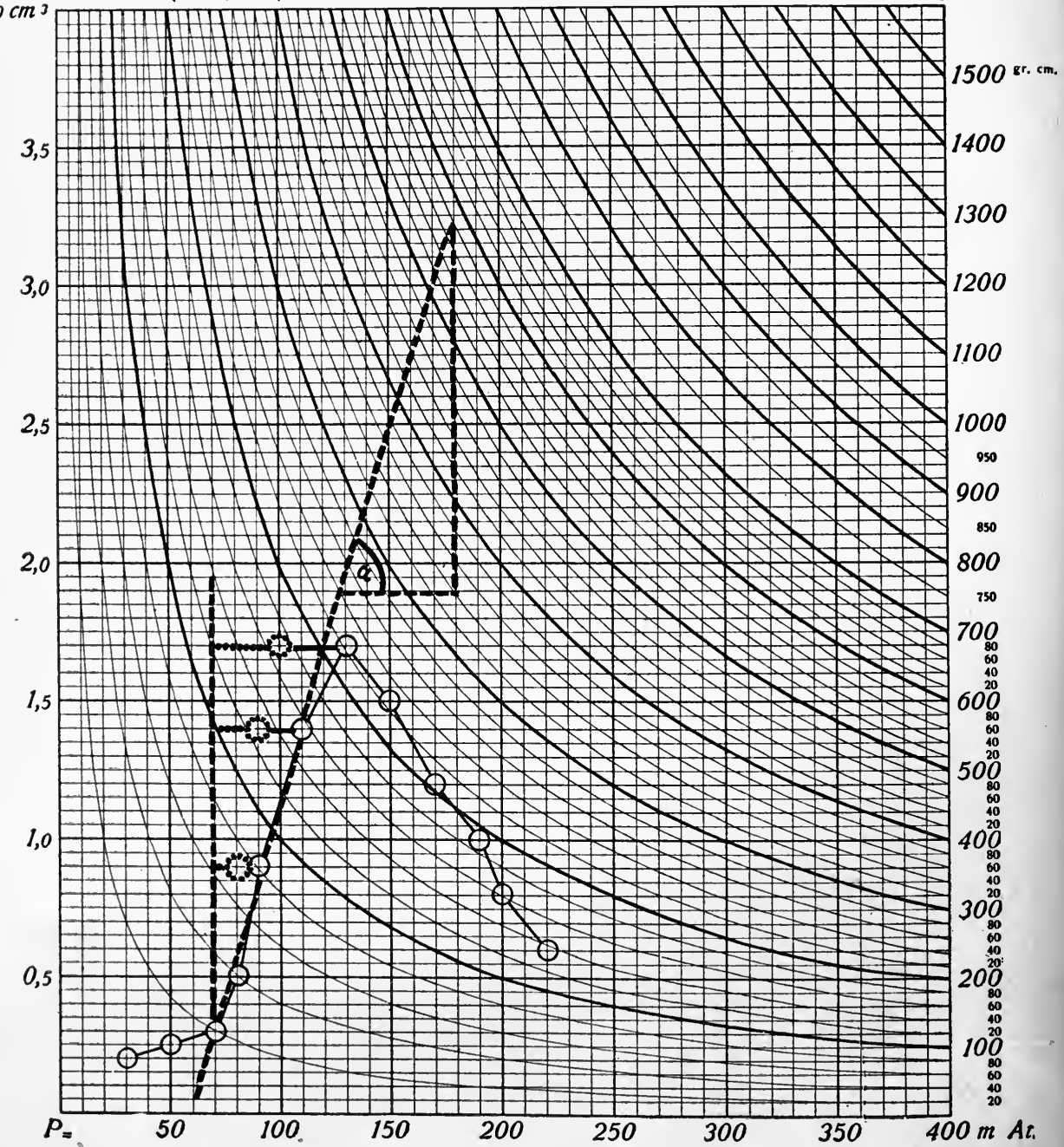

Fig. 62.-Dynamic diagram. (After Christen.) Discussion in text. 
The largest product is evidently equal to 225 gram centimeters. To obviate calculations, Christen has constructed a hyperbolic scheme (Fig. 62). By laying off the volume reading on the abscissæ and the pressure reading on the intersecting ordinates the product is found by following the hyperbolic curve to the right or upper margin. The product so obtained represents the gross energy of the pulse impact, $i$. e., the sum of the energy contributed by the pulse impact and the wall elasticity. $P . V .=A+A^{\prime}$, in which $P=$ the pressure change, $V=$ the volume change, $A=$ energy of the pulse impact and $A^{\prime}$ the energy of the elastic wall.

The net energy due to the impact can be determined by subtracting the latter. The net value can be ascertained from the diagrams by drawing back horizontal lines to the point where the bend in the ascending curve occurs and taking a point (heavy circle) in the middle of this line. The hyperbole upon which this falls represents the net energy of the pulse.

For the limited clinical application, Christen's original monograph should be consulted. Criticism or commendation must be tentatively withheld until its possibilities can be further tested out.

\section{LITERATURE.}

Bоокs.

Bishop. Heart Disease, Blood-pressure and the Nauheim Treatment, New York and London, 1914.

Christen. Dynamische Pulsuntersuchungen, Leipsig, 1914.

Faught. Blood-pressure from the Clinical Standpoint, Philadelphia, 1913.

Frank. Der Blutdruck bein Menschen, Tigerstedt's Handbuch der physiol., Methodik, II, part 4, p. 216.

Janeway. The Clinical Study of Blood-pressure, New York and London, 1904.

Norris. Blood-pressure-Its Clinical Applications, Philadelphia and New York, 1914.

Sahli. Klinische Untersuchungmethoden, 1911, vi ed.

Articles Dealing with Apparatus, Construction and Principles.

Amblard. Compt. rend. Soc. de biol., lxv, 682; Arch. gén. de méd., lxxxix, 3.

Bing. Berl. klin. Wehnschr., 1907, lxiv, 690.

“ Berl. klin. Wehnschr., 1907, lxiv, 1650.

Brugsch. Ztschr. f. exper. Path. u. Therap., 1912, xi, 169.

Christen. Zentralbl. f. Heilk. u. Gefässkr., 1914, vi, 225.

Dehow, Dulus, Heitz. Compt. rend. Soc. de biol., 1912, lxxii, 789.

Erlanger. Johns Hopkins Hosp. Report, 1904, xii, 52.

Feddé. Med. Rec., New York, 1910, lxxviii, 105.

Proc. Soc. Exper. Biol. and Med., 1910, viii, 35.

Fellner and Rudinger. Ztschr. f. klin. Med., 1905, lvii, 125.

Gaertner. Wien. Med. Wchnschr., 1899, xlix, 1412.

Gibson. Quart. Jour. Med., 1907-08, i, 103.

“ Proc. Roy. Soc., Edinburgh, 1908, xxviii, 333.

Herz. München. med. Wchnschr., 1909, lvi, 1899.

Hoobler. Med. Rec., New York, 1911, lxxx, 1323.

Jacquet. München. Med. Wchnschr., 1908, lv, 445.

Müller and Blauel. Deutsch. Arch. f. klin. Med., 1907, xci, 517.

Münzer. München. med. Wchnschr., 1907, liv, 1809. 
Pachon. Compt. rend. Soc. de biol., 1909, lxvi, 776, 955, 733.

Pal. Zentralbl. f. inn. Med., 1906, xxvii, 121.

v. Recklinghausen. Arch. exper. Path., 1906, lvi, 1; lv, 375.

Rocci. Gazz. med. di Torino, 1896, 1, 51.

Schultze. Arch. f. d. ges. Physiol., 1908, cxxiv, 592.

Silberman. Med. Klin., 1908, IV $2,1347$.

Uskoff. Ztschr. f. klin. Med., 1908, lxvi, 90.

Wybauw. Ztsehr. f. klin. Med., 1911, lxxiii, 214.

\section{Articles Dealing with Principles of Bi.ood-Pressure Measurement.}

Benczùr. Deutsch. med. Wehnschr., 1910, xxxvi, 1030.

Clark. Am. Jour. Physiol., 1905, xiii, 27.

Dehio. Abh. d. kaiserl, Leop.-Carol., Akad. d. Naturf., 1912, xevii, 1.

Ehret. München. med. Wchnschr., 1909, lvi, 606; lviii, 243.

Erlanger. Am. Jour. Physiol., 1908, xxxi, 24.

Ettinger. Wien. klin. Wehnschr., 1907, xx, 992.

Fischer. Ztschr. f. diätet. u. physik. Therap., 1909, xii, 389.

Gittings. Arch. Int. Med., 1910, vi, 196.

Goodman and Howell. Am. Jour. Med. Sci., 1911, exliii, 334.

Herringham and Womack. Brit. Med. Jour., 1908, ii, 1614.

Hill and Flack. Brit. Med. Jour., 1909, i, 272.

Hooker and Southworth. Arch. Int. Med., 1913, xiii, 384.

Hoover. Jour. Am. Med. Assn., 1910, lv, 815.

Howell and Brush. Boston Med. and Surg. Jour., 1901, cxlv, 146.

Jaequet. München. med. Wchnschr., 1908, lv, 445.

Janeway. Proc. Soc. Exper. Biol. and Med., 1909, vi, 108.

“ New York Bul. Med. Sciences, 1901, i.

Janeway and Park. Proc. Soc. Exper. Biol. and Med., 1910, vii, 156.

Korotkoff. Vrach. Gaz., 1906, p. 128.

“ Tr. Imp. Mil. Med. Acad. St. Petersburg, 1905, xi, 365.

Lang and Manswetowa. Deutsch. Arch. f. klin. Med., 1908, xciv, 441.

MacWilliam and Kesson. Heart, 1913, iv, 279.

MacWilliam and Melin. Heart, 1914, v, 153.

Masing. Deutsch. Arch. f. klin. Med., 1902, lxxiv, 253.

Mosso. Arch. Ital. de Biol., 1895, xxiii, 177.

Mummery. Jour. Physiol., 1905, xxxii, 33.

Oliver. Quart. Jour. Exper. Physiol., 1911, iv, 45.

Russel. Brit. Med. Jour., 1912, i, 659.

Sahli. Deutsch. Arch. f. klin. Med., 1904, lxxxi, 493.

Sterzing. Deutsch. med. Wehnschr., 1874, p. 35

Strassburger. Ztschr. f. klin. Med., 1904, liv, 373.

Taussig and Cook. Arch. f. Int. Med., 1913, xi, 543.

Tormai. Ztschr. f. diätet u. physik. Therap., 1909, xiii, 504.

deVries Reilingh. Ztschr. f. klin. Med., 1913, lxxvii, 67.

Warfield. Arch. Int. Med., 1912, x, 258.

Van Westenrijk. Wien. med. Wehnschr., 1908, lviii, 2363.

Ztsehr. f. klin. Med., 1908, lxvi, 465.

Weysse and Lutz. Am. Jour. Physiol., 1913, xxxii, 427.

Wiggers, Eberly and Wenner. Jour. Exper. Med., 1912, xv, 174. 


\section{THE VOLUME FLOW AND VENOUS PRESSURE IN MAN.}

By volume flow is understood the quantity of blood flowing through an organ or the entire body in unit time. It is to be distinguished from the velocity of the blood flow which means the rate at which blood moves in unit time. The latter depends on the diameter of the tube as well as on the volume flow; hence, one may be altered without the other. This distinction which is not always made is of more than academic interest. The important question is often raised whether the circulation functionates in such a way that an adequate volume of blood - upon which a proper interchange of waste products, nourishment, etc., depend-is passing through the organs. It is of less concern whether it flows through the narrow arteries at a great velocity or through large arteries at a lesser velocity, provided only that the supply is sufficient. We shall therefore concern ourselves only with the question of volume flow and refer back to the question of velocity to chapters in the first section.

\section{METHODS OF ESTABLISHING THE VOLUME FLOW.}

Plethysmographic Method.--This method depends on the principle demonstrated by Brodie, that if the vein from an organ is suddenly occluded, the volume increases constantly for a short interval before venous congestion has occurred. In the plethysmographic methods (Müller, Hewlett and Van 'Zwaluenburg) (Fig. $63)$, the forearm is placed in a plethysmograph $(P)$ connected with a sensitive manometer and float (Müller) or with some form of volume recorder $(V)$ (Hewlett and Van Zwaluenburg). A narrow pressure cuff $(C)$, such as is used in determining blood-pressure, is placed around the arm above the plethysmograph with the lever of the volume recorder writing horizontally on a revolving drum. The pressure in the cuff is raised quickly to about fifty millimeters of mercury by opening a stop-cock communicating with a large flask $(A)$ containing air under such pressure. This pressure is sufficient to prevent venous outflow but presumably does not affect arterial inflow. For a short time the arm swells at a constant rate but soon diminishes because blood is dammed up in the capillaries and later still, when the venous pressure has risen extensively, 
blood is forced out under the cuff. Only the first part of the curve can therefore be used to determine the normal arm flow. By subsequently closing off the plethysmograph $(Z)$ and allowing a definite quantity of water to flow from the burette $(Y)$ into the flask, the lever record can be calibrated and the quantity of blood flowing into the arm calculated. By simultaneously recording the time in seconds, the flow per second is determined. The volume of the forearm enclosed within the plethysmograph is ascertained by determining the volume of water displaced by thrusting it into a vessel of water to the same mark at which it entered the plethysmograph. The flow may then be calculated in terms of cubic centimeters per minute per hundred grams arm substance. ${ }^{1}$

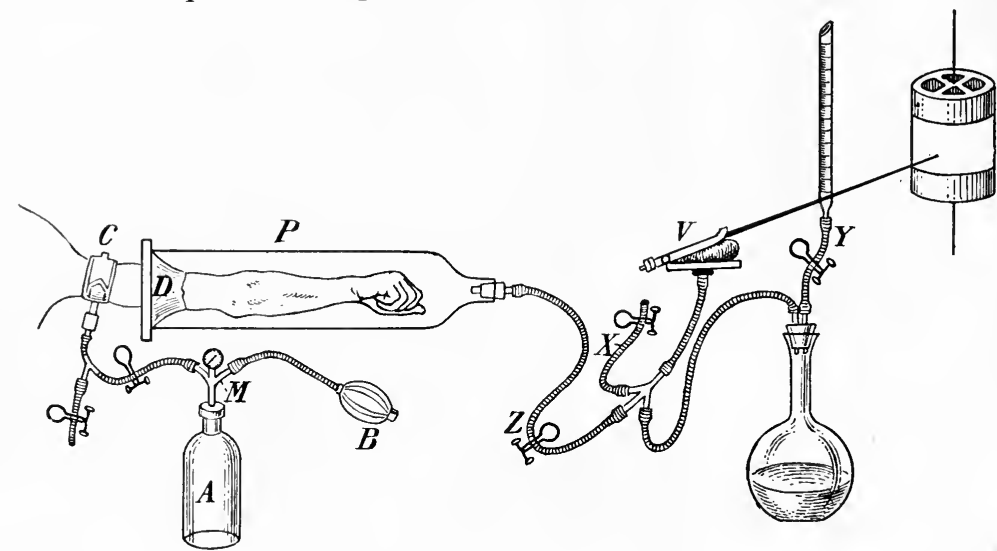

FIG. 63.-Diagram showing Hewlett and Van Zwaluenberg's method for estimating the rate of blood flow in the arm. (After Hewlett and Van Zwaluenberg.)

Calorimetric Method.-This method, devised by Stewart, depends on the principle that the heat given off by the hand at rest is derived almost entirely from the circulating blood. Knowing the temperature of the arterial blood, the temperature of venous blood and the number of calories given off in a given time, the amount of blood that must have flowed in a given area can be calculated by the following formula:

$$
\mathrm{Q}=\frac{\mathrm{H}}{\mathrm{T}-\mathrm{T}^{\prime}} \times \frac{1}{\mathrm{~s}} .
$$

In this formula $Q$ is the blood volume; $I I$, the heat eliminated (in calories); $T$, the temperature of arterial blood; $T^{\prime}$, the temperature of venous blood, and $s$ is the specific heat of blood which equals 0.9 . The volume of the hand is determined as before by measuring the amount of water displaced.

\footnotetext{
${ }^{1}$ By substituting a segment capsule as a volume recorder, Hewlett and Van Zwaluenberg have recently been able to calculate accurately the "pulse flow," i.e., the arm flow during each pulse beat.
} 
The calorimeter into which either a hand or foot is placed consists of an interior copper vessel containing a known amount of water. Into this the hand is inserted through an opening in the lid, heattight closure being obtained by a collar of felt. The interior vessel is packed in cork to prevent loss of heat or irregular cooling, when exposed to draughts. The heat lost by the calorimeter is estimated separately and added to the results of experiments. The hand is immersed in water at the temperature of the calorimeter previous to an experiment for ten minutes. The temperature within the calorimeter is read to hundredths of a degree.

The temperature of the incoming arterial blood is considered to be $0.5^{\circ}$ lower than rectal temperature, that of the incoming venous blood as equal to the average temperature of the calorimeter during an experiment.

It may be pointed out that both of these methods which measure the volume flow through a peripheral organ do not necessarily give an indication of a similar flow through other organs, since it is well known that a sort of reciprocal relation exists between the splanchnic and limb circulation in many conditions.

Gasometric Method.-The gasometric method is calculated to supply a way for estimating the volume flow of the entire circulation or the minute volume, as it is termed. If we count the pulse rate as well, the average volume of blood flowing through the peripheral vessels during the interval of a single pulse beat, that is, the pulse volume may be determined. Although the method is very complicated and ill adapted for ordinary clinical usage, the results obtained with it are of such great importance that a brief description of the essential principles and processes may be given.

The method is based on the principle first enunciated by Fick in 1870, that if we know first, how much oxygen is consumed by the tissues per minute, and second, how much oxygen is required to arteriolize, say 100 c.c. of venous blood, we can calculate the blood flow required to deliver this quantity of oxygen to the tissues. The amount of oxygen consumed may be determined by measuring the quantity taken from the air by the lungs in a minute. The volume of oxygen neceśsary to convert venous into arterial blood is determined by taking the difference between the volume per cent. of oxygen in the venous and in the arterial blood. Then the following proportion can be made:

$$
\text { Minute volume : oxygen used }=100: \begin{gathered}
\text { volume per cent. difference between } \\
\text { arterial and venous blood. }
\end{gathered}
$$

Thus, if the oxygen content of arterial blood is 20 volume per cent. that of venous blood 13 volume per cent., the difference will 
be 7 volume per cent. If now 200 c.c. of oxygen were used during that minute,

$$
\underset{\text { volume }}{\text { Minute }}=\frac{200 \times 100}{7}=2857 \text { c.c. }
$$

If this is divided by the number of heart beats, for example 70 per minute, the pulse volume of 40.8 is found.

Method of Application.-In order to apply this principle, it is evidently necessary to know:

(a) The volume per cent. of oxygen of the arterial blood as it leaves the heart.

(b) The volume per cent. of oxygen of the venous blood in the right heart.

(c) The volume of oxygen consumed per minute.

To determine the volume per cent. of oxygen in the arterial and venous bloods, it is obviously impossible in man to make direct blood-gas analyses as in animals. It is therefore necessary to utilize indirect methods.

To Determine the Volume Per Cent. of Oxygen in Arterial Blood.Since the oxygen tension of arterial blood is presumably the same as that of alveolar air, the volume per cent. of oxygen in the arterial blood may be determined by the following series of procedures (Plesch):

1. The tension of alveolar oxygen is determined according to the formula:

$$
\mathrm{T}=\frac{\left(\mathrm{a} \times \mathrm{O}_{2} \%-29.3\right)(\mathrm{P}-49)}{\mathrm{a}-\mathrm{S} .}
$$

in which $a$ equals the tidal volume determined by a gasometer, $\mathrm{O}_{2}$ the percentage of oxygen determined by direct analysis of a sample of expired air; $P$ the barometric pressure. The figure, 29.3 , represents the oxygen in the dead air-space; the figure, 49, the water vapor tension of the expired air at $37^{\circ} \mathrm{C}$., and $S$, the volume of the dead air-space (equal to about 140 c.c.).

2. The percentage oxygen saturation of arterial blood is calculated from the alveolar tension by the following table:

\begin{tabular}{|c|}
\hline $\begin{array}{l}\text { Saturation of art } \\
\text { per cent. ox }\end{array}$ \\
\hline 86 \\
\hline 88 \\
\hline 90 \\
\hline 92 \\
\hline 94 \\
\hline 95 \\
\hline 98 \\
\hline
\end{tabular}

Alveolar tension
mm. mercury.
70
75
80
85
90
95
100

3. The volume per cent. of oxygen in arterial blood is then determined by multiplying this figure by the saturation capacity 
of the blood, as determined either by blood gas analysis or hemoglobin estimation for the blood of the individual under examination.

To Determine the Volume Per Cent. of Oxygen in Venous Blood.The principle consists in increasing the total lung space by breathing and rebreathing the air from a sensitive rubber bag. The air in the bag soon reaches the composition of the alveolar air, the oxygen tension of which is assumed to be identical, within 40 seconds, with that of the venous blood. Since, however, the analysis of this mixture of gases fails to take cognizance of the oxygen available in the residual air, it is desirable to dilute this residual oxygen by breathing (after a preliminary deep expiration) into a bag of nitrogen gas of 10 liters capacity during two respirations, then, by quickly turning a stop-cock, breathing for 5 to 15 seconds into an empty bag of 3 liters capacity.

1. Having determined the composition of this rebreathed air, the tension of oxygen in renous blood is calculated by multiplying the percentage volume by the existing barometric pressure corrected for aqueous vapor tension at $37^{\circ} \mathrm{C}$.

2. The percentage oxygen saturation of the blood is estimated by the dissociation curve of Bohr, Hasselbach and Krogh, shown in Fing. 64. By following the line corresponding to the oxygen tension up until it strikes the curve of $\mathrm{CO}_{2}$ tension (as found in alveolar air) and reading the figure of this abscissa on the left hand side, the percentage oxygen saturation can be determined for venous blood.

3. The volume per cent. of oxygen in venous blood may then be determined by multiplying this figure by the oxygen capacity of that individual.

The Oxygen Consumed Per Minute--To determine the oxygen consumption, either the more complex apparatus of Benedict, or the simpler Douglas bag may be used. 'This consists of a large bag into which the patient breathes the expired air. After a clip has been applied to the nose the patient breathes through the mouth-piece communicating with a valve permitting an intake of atmospheric air and allowing the expired air to pass only to the bag. The time that air is breathed into the bag is noted, its volume subsequently measured and the difference between its oxygen content and the corrected oxygen content of atmospheric air determined. The corrected oxygen content $(X)$ may be calculated by the proportion:

$\% \mathrm{~N}$ in air : $\% \mathrm{~N}$ in bag $=\% \mathrm{O}$ in air : $\mathrm{X}$.

The Mass Movement and the Recoil Curve.-While the aforegoing method offers a means of determining the minute volume of the circulation and the systolic output of the ventricle, it gives no details of the mass movements and distribution of the blood 
during systole and diastole. This, Henderson has sought to do by recording the recoil curve of the body while the breath is held.

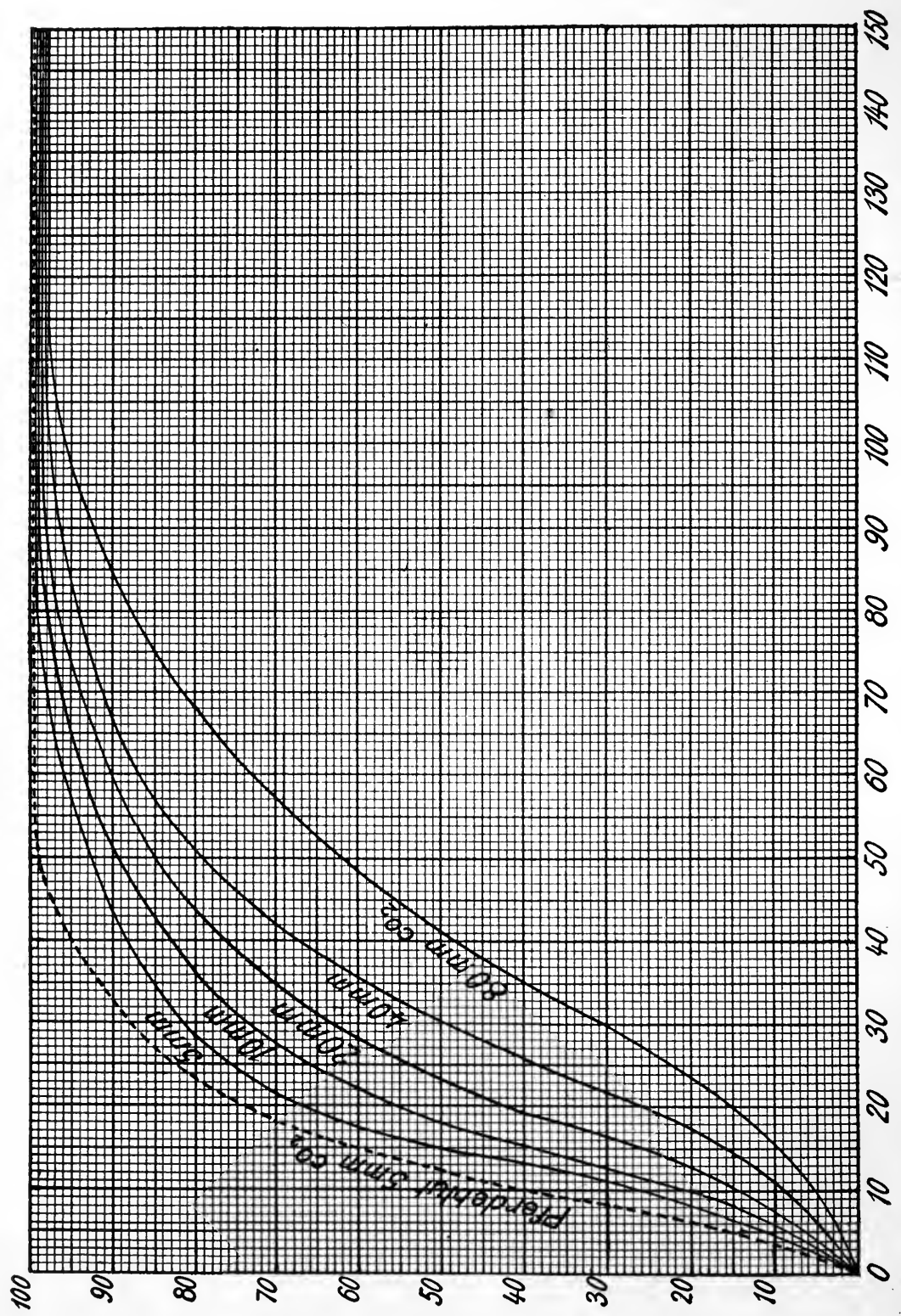

Fig. 64.-Plotted curve showing tension in $\mathrm{mm}$. of mercury (vertical figures) as related to percentage oxygen saturation (horizontal lines) at different $\mathrm{CO}_{2}$ tension. (After Bohr, Hasselbach, and Krogh.) 
To accomplish this the subject lies on a horizontal board which is suspended from a frame by four music wires and prevented from undergoing lateral movements by pointed steel pins resting in conical cups on the frame. The horizontal movements magnified 100 times are recorded on a smoked surface.

The principle of the apparatus is briefly as follows: When a subject is supported on a swinging table, every mass movement of blood headward or feetward is associated with an equal movement of the body in an opposite direction. If a headward and feetward movement occur simultaneously, the movement of the body depends on their algebraic sum and the curve gives a record of the relative distribution of blood above and below the centre of body gravity. Thus, during systole, the algebraic mass movement occurs first headward to a slight degree, than markedly feetward, and again markedly headward. 'The second or feetward movement occurs synchronously with the carotid rise and has been interpreted as indicating the ejection of blood into the aorta. The last or headward movement is intrepreted as due to the movement of blood beyond the arch of the aorta.

Henderson believes that the volume of the systolic discharge per unit body area may be obtained by dividing the distance that the body has moved by the height of the individual. The clinical applicability of such a device must abide further investigation.

\section{CLINICAL ASPECTS OF BLOOD-FLOW DETERMINATION.}

Normal Cases. - The minute volume, according to Pletsch, normally varies from 3732 c.c. to 5334 c.c., an average of 4359 c.c. The average pulse volume was found to be 58.74 e.c. No proportionality was found between minute volume and pulse rate. Calculated per 100 grams of body substance, the volume flow varied from 3.9 c.c. to 8.8 c.c. per minute, an average flow of 6.12 c.c. per minute. These figures agree closely with those obtained by Lowey and Schrötter who found an average flow of 6.4 c.c. per 100 grams per minute, and also with the figures for the volume flow in the extremities. Thus Hewlett and his co-workers found a variation between 2 and 8 c.c. per 100 grams of arm substance per minute and Stewart obtained figures ranging from 3.5 to 14 c.c. of blood flow per 100 grams per minute.

Pathological Cases.-In many pathological conditions of the circulation the volume flow is practically unchanged, indicating that the circulation is efficiently carried on in spite of pathological processes. This is frequently the case where there is distinct evidence of valvular lesions. Pletsch found the average flow per 100 grams actually increased in some instances. When decom- 
pensation occurs, however, the volume flow decreases, especially in the limbs.

The reduction in the peripheral volume flow is greatest in cases of heart irregularities and where there is other evidence of myocardial impairment. This occurs even when no proof of valvular lesions exists. This emphasizes the fact insisted on by Mackenzie, that impairment of cardiac power and not back pressure from valvular insufficiency is responsible for the ill-effects in cardiac cases.

The peripheral flow is markedly reduced in arteriosclerosis and no further reduction of flow is obtained by dipping the opposite hand in cold water.

The minute volume, as also the peripheral volume flow is distinctly increased in cases of exophthalmic goitre. Pletsch found an average minute volume of 5286 c.c. with a flow per 100 grams of 9.73 c.c. per minute. Stewart also found an exceptionally large flow in the hands (14.18 c.c. per 100 grams per minute).

In anemias a curious relation between the minute volume and the peripheral flow exists. Pletsch found a large increase of minute volume averaging 9083 c.c., giving a flow of 13.23 c.c. per 100 grams per second. The increase is greater than is accounted for by the heart rate since the pulse volume equaled 142.9 c.c. The peripheral volume flow, however, is diminished in anemias, less in chlorosis than in other forms. Stewart regards this as a compensatory endeavor on the part of the peripheral mechanism to divert the blood from the periphery to the more vital organs, in this way assisting the return of blood to the heart and lungs by shortening the circulation time.

\section{BIBLIOGRAPHY.}

Papers.

Benedict. Am. Jour. Physiol., 1908, xxiv, 345.

Douglas. Jour. of Physiol., 1911, xlii, 17.

Henderson. Arner. Jour. Physiol., 1905, xiv, 287.

Hewlett. Am. Jour. Med. Sci., 1913, cxlv, 656.

Loewy and Schrötter. Ztschr. f. exper. Path. u. Therap., 1905, i, 197.

Plesch. Ztschr. f. exper. Path. u. Therap., 1909, vi, 462.

Stewart. Harvey Lecture, Nov. 23, 1912.

\section{VENOUS PRESSURE MEASUREMENTS.}

Methods and Technic.-Method of Gaertner.-This method which was primarily suggested but not developed by Frey, is based on the principle that the veins of the extremities represent a manometer column in communication with the auricle; hence, if the arm is raised they collapse when the hydrostatic pressure within them counterbalances the pressure in the right auricle. The following 
procedure is usually followed. With the subject seated erect before the measuring apparatus, the position of the centre of the costo-sternal angle is first ascertained. The experimenter then supports the relaxed arm of the subject by the elbow and the hand, bending the arm at an angle of $120^{\circ}$. With a good illumination on the back of the hand, the whole arm is raised to such a level that the veins collapse. The distance between this level and the point fixed on the chest is then measured by the aid of a meter rule or by a special set of pointers. It represents the auricular pressure in $\mathrm{mm}$. of blood which approximates water in its specific gravity sufficiently closely so that it may be translated without correction.

v. Recklinghausen has suggested the following convenient though rougher modification for bedside use. The one hand of the patient in recumbent posture is allowed to rest by his side on the bed, the other is placed on the thigh. If the veins of the latter collapse while those of the former fill, normal pressure relations exist. If the veins of both collapse venous pressure is low. If the veins of both fill, venous pressure is high.

Method of v. Recklinghausen and its Modification (Fig. 65).- The principle first suggested by v. Recklinghausen consists in exerting a pressure over some superficial vein by a transparent device through which the collapse of the vein can be observed. The essential parts of the apparatus consist of a water manometer for measuring the pressure, a bulb for increasing or decreasing it, and, in addition, a device for applying it to the vein. This pressure box has been given several forms. In v. Recklinghausen's apparatus it consists of a small rubber bag $(5.5 \mathrm{~cm}$. in diameter) with an opening in the centre. Over the top of this a glass plate is laid. Eyster and Hooker substituted for this a small glass pressure box covered below with rubber in which an opening had been eut. The writer has used for three years an ordinary thistle tube, the stem of which was cut off and bent and the flange of which was covered with rubber dam with an opening in the centre. Recently, Hooker has similarly employed a light glass cup, $20 \mathrm{~mm}$. in diameter, which may be used without the rubber dam.

The ring of the v. Recklinghausen apparatus is fitted air-tight to the skin coated with glycerine and the top is covered with a glass plate. The older pressure box of Eyster and Hooker as well as the thistle tubes are similarly rendered air-tight but are held on by tapes or straps. In the recent modification of Hooker the chamber is only temporarily held with a rubber band encircling the hand. It is then cemented to the skin by a film of collodion allowed to fill in the angle formed between the skin and the glass cup.

The technic consists in applying this chamber to the vein of a passively resting arm and raising the pressure within the system until the vein under good illumination collapses. Some degree of 
judgment is required to decide this point exactly. According to Hooker, the most consistent results are obtained when the reading is made at the point where slight oscillations of pressure cause the vein shadows to come and go quickly. The pressure reading thus obtained must be corrected by adding or subtracting the hydrostatic column between the level of the vein and the mid-point of the sternal angle which is arbitrarily taken as the right auricular level, as in Gaertner's method.

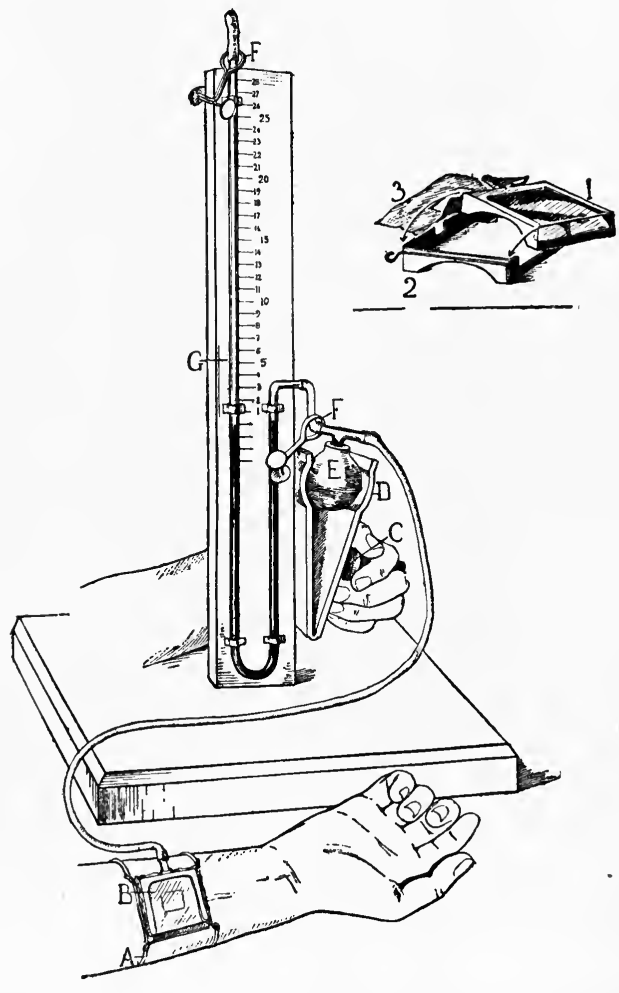

FIG. 65.-Hooker and Eyster's venous pressure apparatus. (After Hooker and Eyster.)

Venous Pressure Gauges.-If the blood is stroked out of a superficial vein in the direction of the heart, the vein collapses as far peripherally as the next venous valve. Both Oliver and Sewall have devised instruments called pressure gauges which replace the finger and exert a variable pressure on the vein. By totally compressing it and then releasing the button until the blood just flows through, the pressure exerted by the peripheral venous blood may be estimated by a spring guage. To obtain accurate comparisons, 
the readings are corrected by the hydrostatic differences between the level of the vein and the costo-sternal angle, as in the methods of Gaertner and v. Recklinghausen.

Plethysmographic Method.-Two cuffs are applied, one to the upper arm and the other to the forearm. The forearm cuff serves as a plethysmograph and hence is very slightly inflated so as to exert a very little pressure (not more than $1 \mathrm{~cm}$. water). It may be connected to a water manometer or some form of recording tambour (Frank and Reh). The upper cuff is then very slowly inflated until the volume and pressure in the lower cuff increase due to the damming back of blood, when the veins of the upper arm are compressed.

Direct Measurement.-The direct measurement of venous pressure is carried out, after the description of Moritz and Tabora, by aseptically inserting a needle into the median vein of the forearm and allowing a sterile saline solution to slowly enter from a burette graduated by a millimeter rather than a centimeter scale. When the inflow ceases, the level of the saline above the level of the heart represents the venous pressure in $\mathrm{mm}$. of water (Fig. 66).

Clinical Value of Accurate Venous Pressure Determination.-The procedures for determining the venous pressure so far described have been largely used to study its height and variation in normal cases. Thus, it has been determined by Hooker and Eyster that the corrected auricular pressure varies from 2 to $16 \mathrm{~cm}$. of water, although Hooker obtained somewhat higher figures with his newer apparatus. During exercise the pressure rises to 18 or $22 \mathrm{~cm}$. of water. The venous pressure undergoes diurnal variations, rising from 10 to $20 \mathrm{~cm}$. during the day and decreasing by a corresponding amount during the night, the average pressure during the day being about $15 \mathrm{~cm}$. The venous pressure is regulated by the intrathoracic pressure. When this approaches atmospheric the venous pressure rises and vice versa.

Local dilations and contractions such as are induced by heat and cold may not affect the venous pressure, thus showing that a relation does not necessarily exist between the venous pressure and the caliber of the vein. This leads to the conclusion that the prominence of the veins is not always an index of the venous pressure.

Clinically the value of accurate venous pressure measurements has not been sufficiently developed to warrant a statement concerning their possible diagnostic significance. Eyster and Hooker report a few cases showing a higher pressure in pathological conditions, and Sewall believes that more information may be gained by comparing the ratio of venous and arterial pressures. He divides the clinical cases with disturbed venous pressures into the following classes: 
(a) Those in which the ratio of arterial to venous pressure is increased. This is due to a positive elevation of arteral pressure in such disorders as vasomotor spasm, increased viscosity of the blood, and arteriosclerosis.

(b) Those in which the ratio of arterial to venous pressure is decreased. This is due to a positive elevation of the venous pressure caused either by physical obstruction of the output of the right heart or by functional changes of intermediary metabolism.

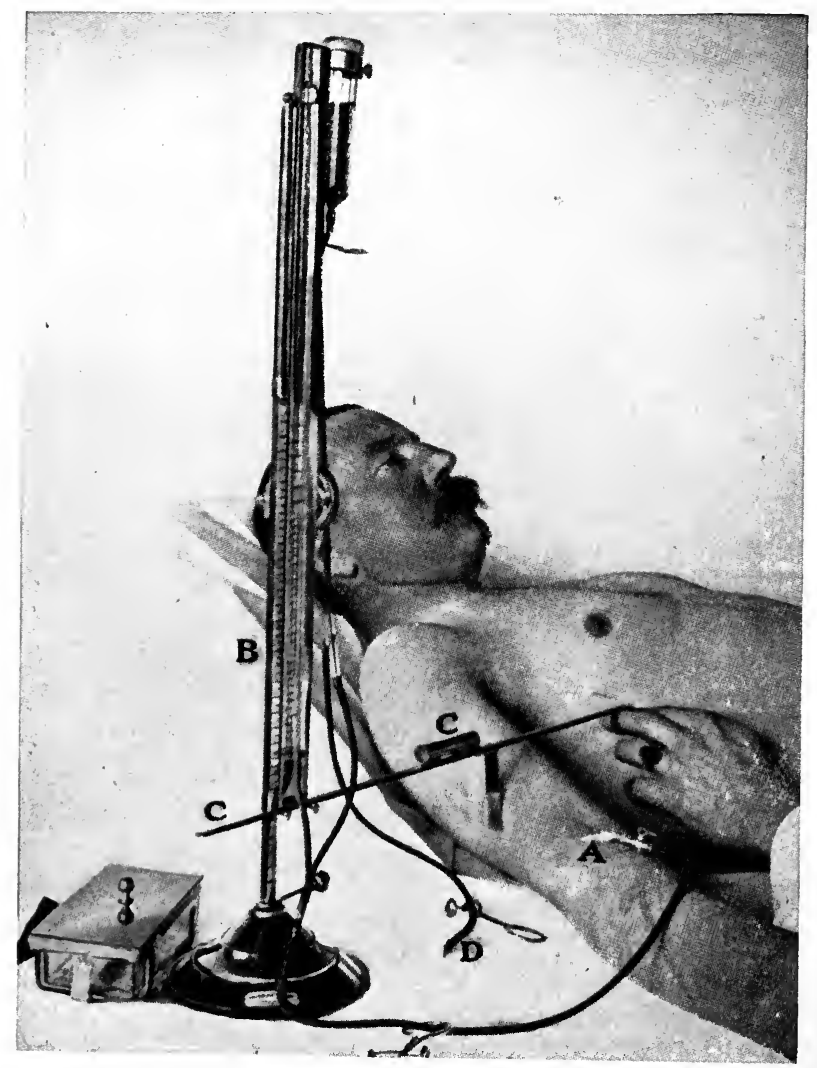

FIG. 66.-Mortiz-Tabora's method of estimating venous pressure. (After Hoffman.)

(c) Those in which the ratio of arterial to venous pressure is not materially changed but both are abnormally high. In these conditions a general plethora exists.

(d) Those in which the ratio of arterial to venous pressure is not materially changed but both are low. In these a condition of asthenia or decompensation is imminent. 
'The following table compiled by Austin in Norris' volume on blood-pressure, summarizes the variations in venous pressures obtained by different observers:

\begin{tabular}{|c|c|c|c|c|c|}
\hline & & At heart level & Normal & Pat & $\operatorname{logic}$ \\
\hline Venous pressure. & & cm. of $\mathrm{H}_{2} \mathrm{O}$ & mm. $\mathrm{Hg}$. & $\mathrm{cm} . \mathrm{H}_{2} \mathrm{O}$. & mm. $\mathrm{Hg}$. \\
\hline Sewall . . . . & . & 4.6 to 5.2 & 3.4 to 3.8 & & \\
\hline von Basch . . . & & 8.8 & 6.5 & & \\
\hline von Recklinghausen & (filled) & 14 to 22 & & & \\
\hline (veins empty) & . . & 20 to 26 & & & \\
\hline Hooker-Eyster. & . & 3 to 10 & 2.2 to 7.3 & & \\
\hline Frank and Reh & . & 1 to 6 & 7 to 4.4 & to 17 & to 12.5 \\
\hline Howell . . . & . & 4 to 13 & 2.9 to 9.5 & 7 to 25 & 5.1 to 18.4 \\
\hline Moritz and Tabora & . & 1.1 to 8.7 & 0.8 to 6.4 & & \\
\hline Summ & . & 1 to 13 & 0.7 to 9.5 & up to 25 & up to $18+$ \\
\hline
\end{tabular}

The Effective Venous Pressure.-The significance and clinical value of exact venous pressure measurements is no doubt minimized by the fact that the filling of the heart is not determined, as in the opened chest, by the actual venous pressure but by the "effective venous pressure," as the difference between intrathoracic and venous pressure is called (cf. page 61). It follows, that any increase in intrathoracic pressure may increase the extrathoracic venous pressure without any disturbance of cardiac activity. Unless it can be assumed that intrathoracic pressure remains unchanged, or unless some simple and safe method for its simultaneous determination can be found, the value of venous pressure measurements in pathological cases is not evident.

\section{BIBLIOGRAPHY.}

S. v. Basch. Wien. klin. Rundschau, 1900, pp. 549, 572.

Frank and Reh. Ztschr. f. exper. Path. u. Therap., 1912, x, 241.

Frey. Deutsch. Arch. f, klin. Med., 1902, lxxiii, 511.

Gaertner. München. med. Wehnschr., 1904, p. 212; 1903, pp. 2038, 2080.

A. A. Howell. Arch. Int. Med., 1912, ix, 148.

Hooker. Am. Jour. Physiol., 1914, xxxv, 73.

Hooker and Eyster. Bull. John's Hopkins Hosp., 1908, xix, 274.

Moritz and v. Tabora. Deutsch. Arch. klin. Med., 1909, xeviii, 475.

Oliver. Jour. of Physiol., 1898, xxiii, p. v.

v. Recklinghausen. Arch. f. exper. Path. u. Pharmakol., 1906, lv, 468.

Sewall. Jour. Am. Med. Assn., 1906, xlvii, 1279. 


\section{CHAPTER XVI.}

\section{THE RADIOGRAM AND ORTHODIAGRAM.}

\section{PHYSICS, APPARATUS AND TECHNIC IN ROENTGEN RAY EXAMINATION OF THE HEART AND LARGE VESSELS.}

WHEN an electric current of high voltage sparks from the anode to the cathode of a tube exhausted to a high vacuum, a stream of molecular particles called cathode rays is repelled from the cathode. Upon striking a solid substance, as a "target" or anticathode (Fig. 67), it produces certain rays known after their discoverer as Roentgen or $x$-rays. It is clearly the function of an $x$-ray tube to transform the energy of the electric current to a new form of energy known as Roentgen rays. These rays differ from ordinary light rays in that they are themselves invisible, penetrate obstacles in accordance with their atomic weight and not according to their molecular arrangement, are not refracted by prisms, concentrated by lenses nor reflected from the highest polished surfaces so far made. They resemble light in that they are a form of transverse vibration of the ether (though of much higher frequency) and also produce chemical effects upon photographic plates and animal tissues. Their value in studying the intrathoracic organs lies in the fact that the penetrating power of the rays depends on the density of the structures through which they have to pass. 'The heart and large vessels, for example, on account of their high atomic weight, absorb a larger number of rays than the air-containing lungs which, by contrast, allow the rays to pass better. By placing on one side of the chest an $x$-ray tube and on the other a photographic plate or a fluoroscopic screen which is rendered luminous by the penetrating rays, the shadows of the heart and large vessels can be clearly distinguished from the lungs.

The definition shown in a radiogram, as the photographic plate or print is termed, depends on the character of the tube used and its adaption for the object to be transillumined. $X$-ray tubes are classified as "hard" and "soft" in accordance as their vacuum is high or low and the penetrating power of the rays generated, great or small. It is possible to obtain tubes of such hardness that even the bones are readily penetrated. In obtaining radiograms of the intrathoracic organs, medium hard tubes seem to be preferable, for in radiograms so obtained the shadows of the sternum and vertebræ are less prominent and the heart shadow is consequently clearer. 
The only known form of energy which is at present capable of being transformed into $x$-rays is the electric current. The ideal current is one that is uni-directional, of high voltage, of uniform intensity, and evenly interrupted. The static machine, at first almost entirely used, supplies such a current but has proven unsatisfactory in radiographic examination of the thoracic organs since the current is not of sufficient intensity to permit as short an exposure as desired. The induction coil which generates a current of greater intensity and high voltage has therefore been brought largely into service. As is well known, this consists of a primary coil and some special form of automatic interrupter supplied by a constant current from an electric light circuit or storage battery. The current produced in the secondary coil by induction has the disadvantage of being alternating in character, and the reason that it can be used with $x$-ray tubes at all lies in the fact that, owing to

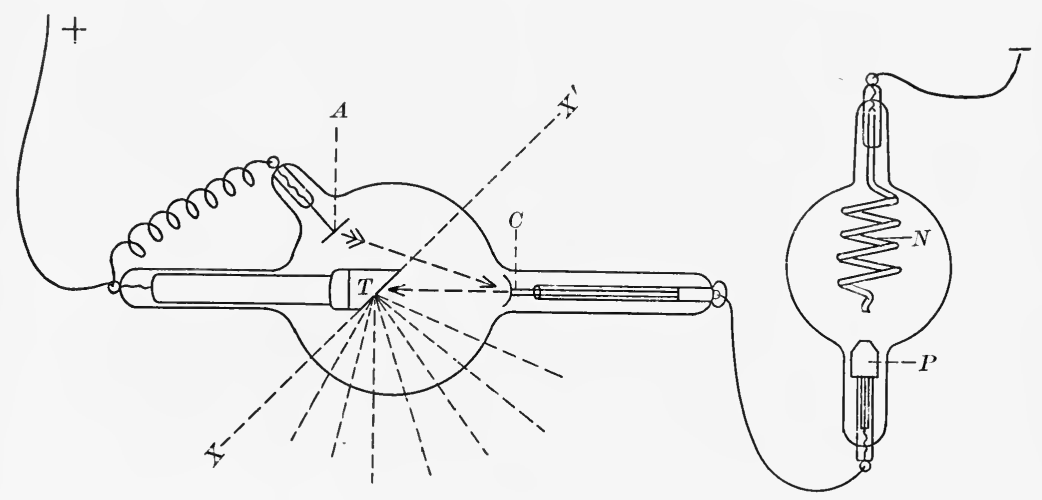

Fig. 67.-Diagram showing $x$-ray tube and ventril tube in series: $A$, anode;

$T$, target; $C$, eathode; $P$, aluminum electrode, and $N$, spiral electrode.

self induction in the primary on the "make," the current in one direction is far stronger than in the other, thus giving a current predominating in one direction. This inverse current, as the make current is called, is deleterious, however, both because it gives rise to stray $x$-rays and also because it shortens the time a tube remains usable. Various devices for preventing the entrance of the inverse current into the tube or for converting it into a current of opposite direction have been devised. The simplest procedure is to place the tube in series with a spark gap across which the inverse current cannot leap. Its passage is more satisfactorily prevented, however, by placing a ventril tube or valve tube in series. This device (Fig. $67)$ consists of a vacuum tube with two electrodes, one of which $(P)$ is a small aluminum affair $r_{2}$ the other, $(N)$ a long spiral of greater surface. When the direction of the current is such that the spiral $(N)$ is the cathode, the current passes readily, whereas its passage 
is entirely prevented when it becomes the anode. These valve tubes are even more needed when the current supplying an $x$-ray tube is generated by a transformer, for an alternating high voltage current so generated, unlike that from the induction coil, is equal in each direction and is itself entirely useless unless the current in one direction is suppressed.

The technic of $x$-ray examination need be only very briefly indicated. The tube grasped in a suitable holder and enclosed in a lead and rubber lined box which permits rays to escape only in a forward direction is placed about $60 \mathrm{~cm}$. from one side of the chest. The plate within its light protecting envelope is placed in as close contact with the chest wall as possible. The alignment between tube and plate is such that the middle of the target is perpendicularly in line with the middle of the plate (Fig. 68, $c-c$ ).

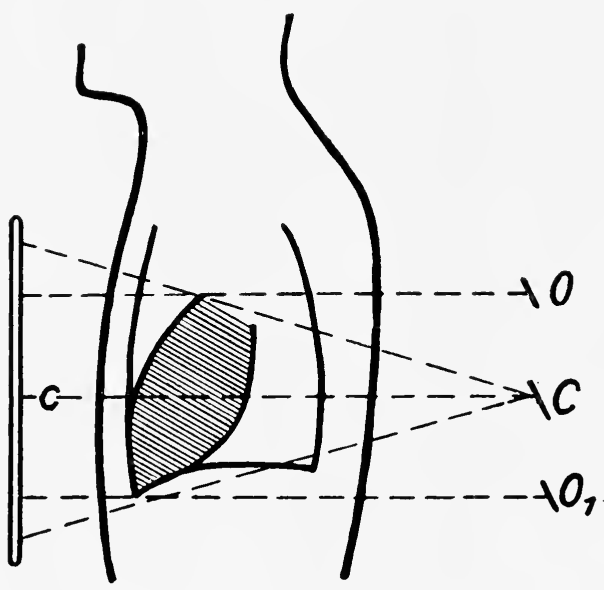

FIG. 68.-Diagram illustrating the difference in the size of the shadow when protected by divergent and parallel rays.

The transillumination of the chest is usually made in a dorsoventral (tube behind, plate in front) or a ventrodorsal (tube in front, plate behind) direction because the best definition can be so obtained. Exposure in lateral or diagonal planes are sometimes necessary, however, for supplementary information. In taking radiograms of the heart it is desirable to make the exposure so brief that it can be obtained at the height of a natural inspiration. By proper selection of the tubes and plate and by covering the plate with an intensifying screen, it has been found possible to obtain radiograms in a fraction of a second. These intensifying screens are plates coated with some substance such as calcium tungstate, which allows all the $x$-rays to pass readily and, in addition, becomes luminous and so more readily affects the plate. 
Since the $x$-rays emanating from the anti-cathode are divergent, it becomes evident that the heart shadow must be larger than the actual size of the heart (Fig. 68), furthermore, that the degree of enlargement depends on $(a)$ the proximity of the plate and tube to the chest and $(b)$ on the proximity of the heart to the tube. Thus, the base of the heart which lies posterior to the apex is enlarged more than the apex. It follows that only by keeping the plates and tubes at exact distances from the chest in different subjects or in subsequent observations upon the same subject, can even an approximately correct idea of the heart area be obtained. Even so, the radiogram should be regarded as qualitative and not quantitative. Various attempts have been made to overcome this artificial enlargement of the heart area. All efforts to convert the divergent rays into parallel rays have failed. If, however, the tube is removed to a greater distance from the chest, the rays become more nearly parallel and the error is greatly diminished. Only recently has it been possible to use tubes at a distance of two meters and obtain radiodiagrams within an exposure time of one and one half to two seconds (Grödel). Plates so obtained may be termed teleradiograms.

In 1900, Moritz devised an apparatus, the orthodiagraph, which, as improved upon by subsequent workers, offers the most exact method of determining the heart area. The principle of the orthodiagraph may be explained by reference to Fig. 68 . If the target of an $x$-ray tube be shifted so that the central rays $(c c)$ emanating from it at right angles to the body axis touch the heart boundaries tangentially as at $O$ and $O_{1}$, the heart shadow will be projected by parallel rays upon the screen. It should be borne in mind, however, that on account of the sloping position of the heart, neither the dorso-ventral nor the ventro-dorsal orthodiagram, as the record is termed, gives its correct anatomical length. Fortunately, however, the correct breadth is more nearly projected.

The technic of operating an orthodiagraph can be only briefly described in this book. For a more comprehensive description, the small volume by Grödel may be recommended. Fig. 69 illustrates one form of this apparatus which may be used either in horizontal or vertical posture. The patient lies upon a table beneath which the $x$-ray tube $(A)$ is held. Fastened to the same vertical arm $(X)$ is a small screen $(B)$. Upon this screen is a mark centred so that it is directly over the middle of the anticathode. By a special mechanical device these two points move together in various directions so that the same central rays pass between them. Any point of the heart border during diastole which is visible upon the screen is made to coincide with the mark upon the screen. By tracing this mark around the heart and lung borders they may be outlined and graphically recorded upon a parallel plane $(C)$ 
below the table by a pencil $(D)$ which is in line with the target and mark. The record so obtained is called an orthodiagram. Since an interval of time must naturally elapse before the heart can be outlined, it is necessary to allow the patient to breathe quietly. These respiratory movements and the movements of the heart itself are factors which interfere slightly with a correct projection of the heart, and, together with the personal equation of the operator, constitute the greatest source of the error incurred in orthodiagraphic work. Various procedures, as stereoscopic pictures and kinematographic pictures of the heart are being tested with the hope of obtaining still better methods of outlining the heart and studying its action within the closed chest.

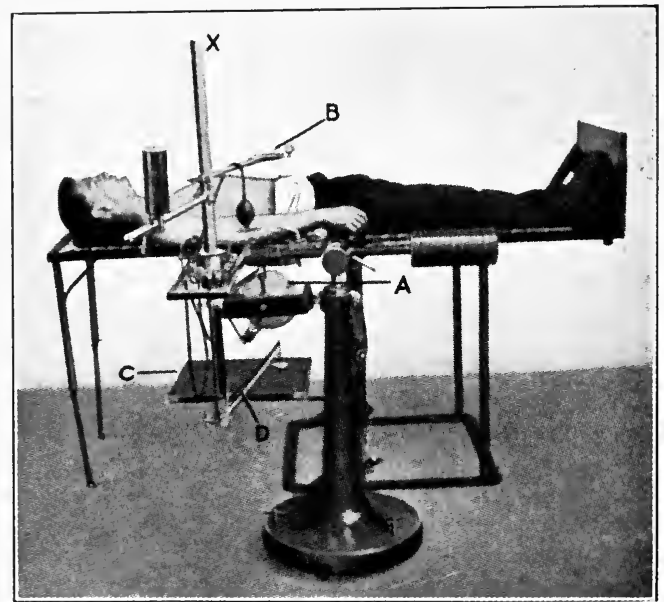

FIG. 69.-The Orthodiagraph. (After Grödel.)

\section{THE NATURE AND SIGNIFICANCE OF NORMAL RADIOGRAMS AND ORTHODIAGRAMS.}

In Fig. 70 is shown a radiogram taken in the dorsoventral diameter (tube behind). Aside from the shadows of the bony structures which do not concern us, the positive print shows two lateral light areas in each of which a fringed shadow indicates the roots of the lungs. Central to this appears the flask-shaped darker shadow of the heart and large vessels. The shadow is not equally dense throughout, but, owing to the air-containing trachea is distinctly lighter in its upper portion. Upon fluroscopic examination, it is observed that the right portion (Fig. 70, A) undergoes a very slight presystolic pulsation, while the right upper border at $B$ often shows a systolic enlargement. The lower left border $(C)$ shows a systolic decrease in size while the upper border $(D)$ slightly later shows a 
systolic enlargement. In the orthodiagram (Fig. 71) we recognize two distinct arcs on the right and three on the left boundary of the

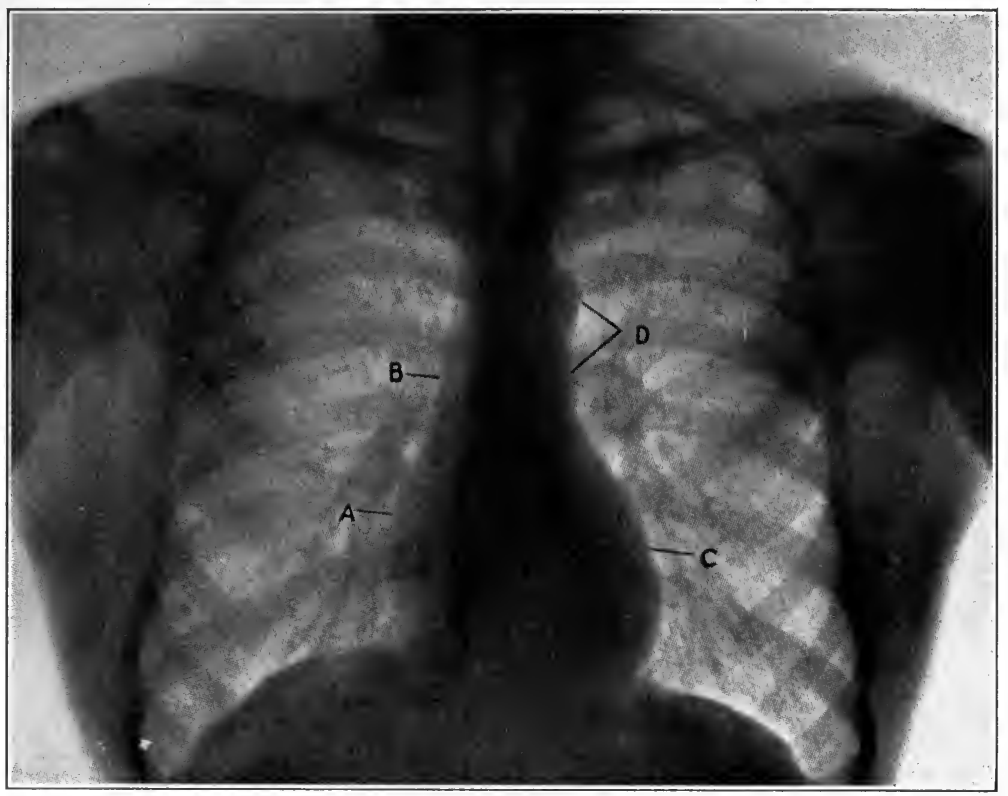

FIG. 70.-Normal radiogram of heart during inspiration. (After Groedel.)

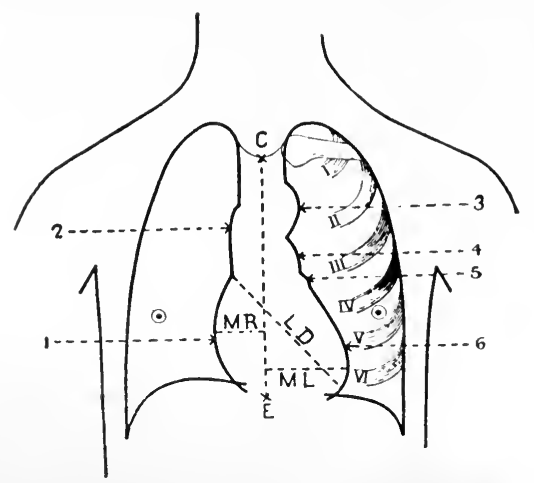

FIG. 71.-Orthodiagram of normal heart. 1, right auricular curve; 2 , great vessels curve; 3 , aortic curve; 4 , pulmonary curve; $5-6$, left ventricular curve. $M R$, median right diameter; $M L$, median left diameter; $T$. $D$., sum of $M R$ and $M L$; $L D$, longitudinal diameter; $C E$, central line.

heart. By the nature of the pulsations just described, as well as by postmortem examination, it has been established that the upper right arc bounds the aorta, (occasionally the vena cava) while the 
lower encircles the right auricle. On the left side the upper arc marks the boundary of the aorta, the second that of the pulmonary artery while the lower arc indicates the left ventricle. The shadow of the right ventricle merges with that of the diaphragm below.

In a normal orthodiagram, it is possible to make definite measurements and so quantitative comparisons. According to the system introduced by Moritz, it is customary to measure (Fig. 71) the greatest distance to the right and left of the median line ( $M R$ and $M L)$, the total transverse diameter $T(=M R+M L)$ and the diagonal length $(L D)$. The measurements collected by various observers show a variation in different individuals. They may be said to increase in proportion to body weight and size, they alter with age and occupation, they differ in the two sexes; but very little difference seems to exist between those made in vertical and in horizontal positions. In children the measurements do not vary in accordance with size or weight and sexual differences are absent until puberty. The following table compiled from the combined results of Dietlen, Grödel and Verth gives average variations at different ages and in individuals of different body weight and size.

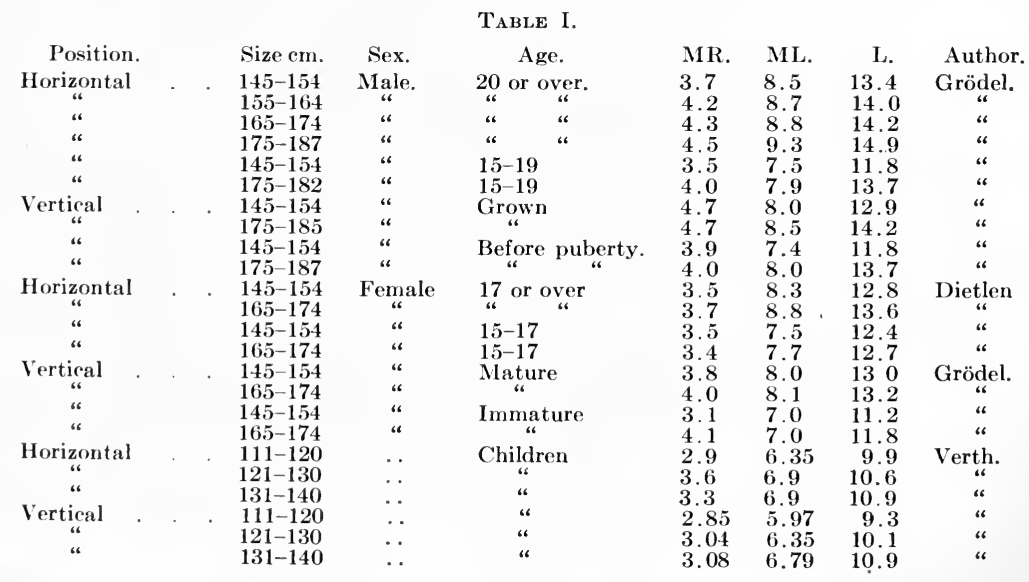

A perusal of this table and others published by these investigators shows that certain measurements may vary under different conditions as much as three centimeters, a fact which makes questionable the value of the orthodiagram as a means of detecting slight variations in the size or form of organs due to disease or treatment.

\section{CLINICAL SIGNIFICANCE OF X-RAY EXAMINATION.}

Combined fluoroscopic, radiographic and orthodiagraphic examinations of diseased conditions give evidence of the nature of the 
affection through the occurrence of changes in $(a)$ the character of the pulsation, $(b)$ the position of the heart and $(c)$ the size and form of its outline.

In fluoroscopic examinations the lower right border (right auricle) expands extensively during systole in some cases of tricuspid regurgitation. The upper left border (aorta) gives strong systolic expansions in aortic insufficiency. Whenever the pulsations of the left auricle become visible, as in mitral lesions, they are differentiated by being presystolic in time. Strong pulsations of the pulmonary artery are evident on the left side in cases of persistent ductus arteriosus, or more frequently, when a severe stasis due to mitral lesions is present. They have been observed when an aneurysm ruptures into the pulmonary artery, but this event is rare. Abnormal rhythms, as heart block and pulsus alternans, have been studied and diagnosed by fluoroscopic methods, but this procedure possesses no obvious advantages over auscultation.

A change in the position of the heart may occur (a) from congenital causes, as situs inversus vicerum, $(b)$ from pleural and pericardial adhesions, or $(c)$ from changes in the intrathoracic volume. These last are very common. Even normal variations are caused by the varying position of the diaphragm. In longchested individuals the cardiac shadow is long and narrow, the axis being more vertical, while in short-chested individuals or in those in whom the diaphragm is pushed up by abdominal distention, it is broad and assumes a more horizontal axis. Its position changes as the diaphragm ascends and descends in respiration. It should be remembered that normally the heart is subject to considerable shifting and undue weight should not be attached to slight variations in position. If for any pathological cause (e. g., enlargement of the liver) the right dome of the diaphragm is pushed upward, the heart shadow will be displaced to the left. Pulmonary affections such as atalectasis, tuberculosis and pneumothorax cause a traction on the heart toward the side of the lesion. On the contrary, pleural effusions, tumors, etc., push the heart toward the opposite side.

Changes in the size of the heart shadow or orthodiagram when sufficiently marked to be safely beyond the limits of normal variation, are of the greatest practical importance when obtained by guarded technic. The heart shadow probably decreases whenever the heart accelerates (e.g., in exercise, tachycardia, after atropine), although the results obtained concerning this point have been discordant. During asthmatic attacks the heart is also reduced in size. A condition simulating asthma, as far as the effect upon the circulation is concerned, ean be produced by the well-known experiment of Valsalva which consists in taking a deep inspiration and then, with closed glottis, making a forced expiration. This 
diminishes the blood content of the heart.which accounts for its decreased size. In tuberculosis the shadow is decreased, and here it is often assigned, but without good reasons, to an actual hypoplasia of the heart muscle.

The heart outline increases after continued hard labor or exercise, pathologically also in nephritis and arteriosclerosis. In these . cases an actual hypertrophy resulting from the greater strain to which the heart has been continually subjected is usually the cause. The increase in size (often temporary) associated with acute infections, such as diphtheria, scarlet fever and polyarthritis is no doubt accounted for by a dilatation of the heart.

The details of the enlargement are of the greatest importance in heart lesions in which case it is due either to dilatation or to hypertrophy and hence accompanies only lesions of considerable duration and severity. The nature of the dilatation or hypertrophy determines the direction of the enlargement and the contour of the shadow. A left ventricular enlargement takes place to the left. Dilatation or hypertrophy of the right ventricle displaces the shadow partly to the right but also, to a marked degree, upward and to the left. In typical cases of aortic insufficiency the heart shadow is enormously increased toward the left and the contour resembles a horizontal oval or is sometimes called "shoe-shaped" (Fig. 96). The aortic shadow is increased in width and the apex is never merged with the shadow of the diaphragm. Aortic stenosis causes very similar though less pronounced changes in the radiographic outline. In mitral stenosis the heart shadow which is relatively small, resembles more nearly a vertical oval. The enlarged left auricle becomes prominent on the left margin (Fig. 92) and above it the pulmonary artery bulges, thus giving the entire left border a step-like appearance. In mitral insufficiency (Fig. 91) the enlargement tends to be uniform in all directions giving the shadow the appearance of a poorly rounded circle. The right auricular border is distinctly enlarged to the right and the pulmonary artery dilated. The left ventricular shadow is increased toward the left.

Aside from its service in the diagnosis of cardiac affections, the $x$-ray has given valuable information concerning extra-cardial conditions, as pericardial effusions, aneurysms, etc., which will be discussed in chapters dealing with these conditions.

\section{BIBLIOGRAPHY.}

BOOKS AND MONOGRAPHS.

Beck. Roentgen Rays, Diagnosis and Therapy, 1914, New York.

Christie. Manual of X-ray Technic, Philadelphia, 1913.

Franke. Orthodiagraphie, München, 1906.

Gocht. Lehrbuch der Roentgenuntersuchungen, Stuttgart, 1903.

Groedel. Orthoroentgenography, München, 1908. 
Grödel. Grundriss und Atlas der Roentgendiagnostik, München, 2nd ed., 1914. Pusey and Caldwell. The Roentgen Rays in Therapeutics and Diagnosis, 1904, 2d ed., Philadelphia.

Schwenter, Leitfaden der Momentaufnamen, Leipzig, 1913.

Tousey, Medical Electricity and Roentgen Rays, Philadelphia, 2nd ed., 1915.

\section{Articles Dealing with the Examination of Thoracic Organs.}

Beclère. Buletin de la Soc. Radiog., Paris, 1897.

Claytor and Merrill. Amer. Jour. Med. Sciences, 1909, cxxxviii, 549.

Grödel, Münch. Med. Wochenschr., 1906, liii, 826.

“ Arch. Roentg. Rays, 1907, xii, 150, 180, 303.

Kohler. Verhandl. d. deut. Roentgengeselschaft, 1907, iii, 165.

Koranyi and Elischer. Ztschr. f. Roentgenkundeu. Radiumforsch., 1910, xii, 265.

Levy-Dorn and Möller. Deut. Med. Wochenschr., 1900, xxvi, 565, 584.

" " Ztschr. f. klin. Med., 1911, Ixxii, 563.

Moritz. Münch. med. Wochenschr., 1902, lxix, 1.

\section{Articles Dealing with Normal Heart.}

Dietlen. Deut. Arch. f. klin. Med., 1906, lxxxviii, 55, 286; 1909, xcvii, 132.

“ Ergebnisse der Physiol., 1910, x, 624.

Franze. Edinburgh med. Jour., 1906, xix, 223.

Grödel. Zeitschr. f. klin. Med., 1910, lxx, 47.

“ Zeitschr. f. klin. Med., 1911, lxxii, 310.

Grunmach. Deut. med. Wochenschr., 1904, xxx, 459.

Mortiz. Deut. Arch. f. klin. Med., 1905, lxxxii, 1; 1904, lxxxi, 31.

\section{Heart in Pathological Conditions.}

Dietlen. Münch. med. Wochenschr., 1908, lv, 2077.

Grödel and Grödel. Arch. of Roent. Ray, 1908, xii, 303.
“
Deut. Arch. f. Klin. Med., 1911, ciii, 413.

Otten. Deut. Arch. f. Klin. Med., 1912, cv, 370.

Van Zwaluenberg and Warren. Arch. Int. Med., 1911, vii, 137. 


\title{
SECTION III.
}

\author{
CHAPTER XVII.
}

\section{AFFECTIONS OF HEART MUSCLE ASSOCIATED WITH RETROGRADE CHANGES, INFILTRATIONS, AND SUBSEQUENT REPAIR.}

When the nutrition of the heart cells is impaired, or they are submitted to chemical or infectious influences of a toxic nature, their metabolism is altered. The intracellular chemical processes and perhaps the "molecular movements" are disturbed. Many chemical agents also increase or decrease the rate, amplitude, and vigor of cardiac contractions. Thus, drugs like adrenalin and digitalis stimulate and others such as alcohol, chloroform, ether, chloretone, chloral and pituitary extract depress the contractile function.

Still another class of substances, as the bacterial toxins, or the split products of bacterial proteins (typhoid) act at once to stimulate the heat production and affect the rate, amplitude and vigor of contractions (Cleghorn). Since it is possible to cause such functional disturbance before histological changes have had time to develop, it may be assumed that when the heart is submitted to these influences in disease, disturbances of function generally precede parenchymatous changes, the latter occurring when the deleterious influences persist for a longer time. They are therefore effects rather than causes of disturbances in function (Aschoff).

The parenchymatous changes set up vary with the nature of the toxic influence, its period, and intensity of action; but they are probably not fundamentally different in the various infections (Krehl). They consist in milder cases (e.g., acute infections, typhoid) of granular degeneration, in more severe or prolonged cases of fatty, hyaline and calcareous degeneration (e. g., after diphtheria), and in cases of circulatory disturbances of hydropic or fatty change. Toxic agents and particularly those due to infections are not restricted in their action to muscle cells alone but either simultaneously or secondarily affect the vascular system causing hyperemia with exudation of serum, lymphocytes, and leukocytes. If the infectious organisms (e.g., pus cocci) themselves lodge in the heart, the attraction of polymorphonuclear leukocytes is great and their extravascular massing may give rise to circum- 
scribed abscesses or more diffuse suppuration. Such inflammatory infiltration may by pressure or disturbance of parenchymatous tissue be the cause of added functional disturbances. If, as frequently happens, these infiltrations and abscesses form in regions such as the auriculo-ventricular septum or the papillary muscles, they may involve the conducting system of Tawara and so be the cause of serious or fatal derangements of the heart.

When the climax of retrograde changes has passed, repair sets in. This means the resorption of necrotic tissue by endothelial leukocytes, the proliferation of fibroblasts and the formation of new connective tissue. When, subsequently, the scar tissue contracts, it may either compress really vital regions of the heart, or shut off the blood supply to certain regions. In either case it may be regarded as the cause of disturbances in function.

\section{DISTURBANCES OF THE CORONARY CIRCULATION.}

Disturbances of the coronary blood supply may be considered as of two kinds: (a) those due to occlusion of vessels (thrombosis, embolism) and $(b)$ those due to dynamic derangements of the circulation (coronary sclerosis).

Pathological Physiology of Coronary Occlusion.-Although the existence of an anastamosis between the branches of the right and left coronary systems has been anatomically established (Spalteholz, Merkel) and a further communication exists by the Thebesian vessels with the ventricular cavities (Pratt), it is evident from animal experiments that when a main branch is suddenly occluded these collateral vessels are not sufficient to maintain the beat of the heart for a long period of time. Death often follows shortly after the ligation of the left coronary according to the experiments of Cohnheim and Porter. Nor do the recent experiments of Hirsch and Spalteholtz, who maintained animals alive for two weeks after ligation of the left coronary vessel, prove the functional sufficiency of this collateral circulation, for Miller and Matthews have subsequently shown that, if allowed to live, animals in which the left coronary had been ligated succumbed within twenty-six to ninety days with symptoms typical of cardiac insufficiency.

The signs following sudden occlusion depend on the size and nature of the vessel involved. Occlusion of the main left coronary artery is often not followed by any immediate change except the production of an occasional extra-systole. The heart rate continues normal but the drop of the maximal and rise of the minimal pressure within the ventricles, as well as the slower rate of systole, give evidence of an impaired cardiac contraction (Porter). Upon ligating the right coronary which supplies the right auricle as well as ventricle, the preliminary extra-systoles are followed by a tachy- 
cardia which is rapid in its onset and may last from a few seconds to thirty-five minutes (Lewis).

These conditions of comparative regularity may persist for a few moments or may extend over hours and days. Then follow signs that the heart is failing. The chambers dilate and ventricular systole becomes incomplete (Porter, Miller and Matthews). Without further signs of irregularity the ventricles suddenly go into fibrillation. The blood-pressure falls rapidly and death results from acute anemia of the respiratory and cerebral centres.

Clinical Manifestation of Coronary Embolus and Thrombosis.Closure of the large coronary vessels is known to occur more frequently from thrombosis than from the lodgement of an embolus. On post mortem a characteristic anemic infarct is found. The symptoms vary considerably. Occlusion of the coronaries is one of the most frequent causes of sudden death after middle age. It is no doubt due to the induction of fibrillation of the ventricles. Death in such cases is characterized by its quiet character, the individual undergoing no convulsive movements such as accompany other forms of asphyxia. In cases not immediately fatal death may be preceded for moments, hours or days by a sensation of unrest and a sense of impending peril, of oppression and air hunger or even by anginoid pains. The heart sounds are weak, the pulse feeble, sometimes rapid and at other times slow (Krehl).

Not all cases of coronary obstruction result fatally, especially if due to a thrombus. Cases in which no cardiac disturbance was suspected have come to autopsy and revealed the fact that the entire obliteration of large vessels like the descending ramus had existed for years (Birch-Hirschfeld).

Dynamic Anemia of the Heart.-Pathological Physiology.-By dynamic anemias we will understand those forms of deficient blood supply which result from a dynamic change in the circulation, as distinguished from the essential anemias resulting from alterations in the composition of the blood.

The quantity of blood passing through the heart is determined by the height of aortic pressure and the total resistance in the coronaries (i.e., vasomotor tonus, ventricular tonus and intraventricular pressure). In addition, however, the heart possesses a more direct mechanism of adapting its blood supply to its immediate needs. This is accomplished through the compression of the intramural vessels which acts to propel blood through the heart at a velocity which is directly proportional to the rate and amplitude of contraction. This is an exceedingly important mechanism for it tends to compensate for changes otherwise tending to reduce the blood supply. This may be shown by passing adrenalin through a perfused heart. When the heart is perfused at a constant pressure with an oxygen-free solution it does not beat. Adrenalin then 
unfailingly causes a decreased flow due to a constriction of the peripheral coronary vessels. When the heart is perfused with an oxygenated Ringer's solution, on the other hand, and is beating, adrenalin increases the blood flow since it simultaneously increases the rate and amplitude of ventricular contraction which is sufficient to overpower the constriction effect on the coronary vessels themselves. The writer has observed, furthermore, that an increased flow may still occur if, while the adrenalin action is at its maximum, the perfusion pressure is somewhat reduced. We have other evidence of the value of this direct mechanical regulating influence on the coronary circuit. When the blood-pressure lowers after hemorrhage the heart may accelerate to such a degree that a larger coronary blood-flow actually occurs in spite of the lower arterial pressure (Wiggers) Fig. 72.

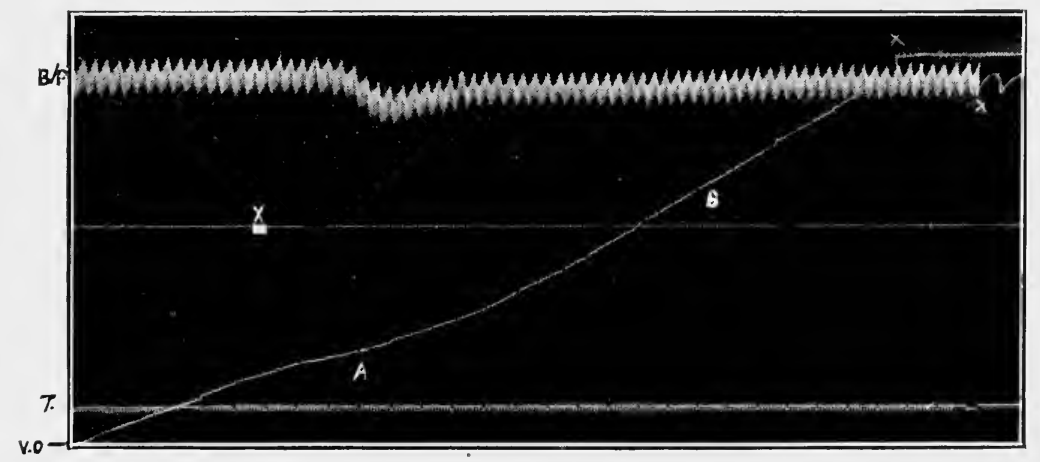

FIG. 72.-Effect of hemorrhage on coronary blood-flow. $\quad V . O$., rate of flow; B. P., carotid mean pressure.

In view of the fact that the heart normally accelerates as the blood-pressure lowers it is questionable whether a dynamic anemia of sufficient degree to modify cardiac functions ever occurs from an alteration of the blood-pressure alone. After severe hemorrhages and in shock the writer has never observed irregularities nor were the functions of conductivity and contractility impaired in experimental animals.

This important protective mechanism depends for its existence upon the elasticity of the coronary vessels and can operate only inadequately when they have become hardened by sclerosis or calcification, or obliterated by endarteritis. Hence, when these conditions supervene, the heart has suffered a great loss. If, now, the arterial pressure lowers or the heart increases in rate, the flow cannot be increased to the needs of the heart. If the arterial pressure rises and the work of the heart is increased no appreciable 
increase in coronary flow can occur. It follows that a dynamic anemia only seriously affects the essential function of the heart when alterations in the vessels themselves occur.

Clinical Manifestations of Coronary Sclerosis.-Sclerosis of the coronary vessels follows many infections or toxic agents but syphilis holds first rank as a causative factor. It is always associated with parenchymatous degeneration that can be only partly accounted for by the altered blood supply. These may themselves give simptoms of toxic myocarditis as later considered. But the best single symptom indicating the existence of coronary impairment is the occurrence of angina pectoris.

Angina pectoris is no longer regarded as a disease but as a symptom indicating that the heart muscle is exhausted. It is perhaps true, as Mackenzie observes, that sclerosis without impairment of the heart is not sufficient to produce angina, at least the fact that many severe cases of sclerosis that come to autopsy showed during life no signs of angina, may be so explained. While sclerosis without angina may thus exist it is very probable that all typical cases of angina are accompanied by some degree of coronary involvement.

The symptom-complex designated as angina pectoris is composed of three elements: the intense pain in the cardiac region sometimes radiating to the left arm, the sensation of ring-like thoracic constriction, and the sensation of fear and of impending dissolution.

The cause of these sensations has been the subject of frequent discussion. It is often assumed, in a very general fashion, that the pain is due to a process similar to that which takes place in skeletal muscles during fatigue or overstrain-that muscular pain is, in short, an evidence of overstrain. This is the statement of a fact rather than an explanation, however. More definitely, it has been suggested that an impaired blood supply is the cause of painful sensations. 'The intense pain resulting from shutting off the blood supply from a limb is cited as a common example. Furthermore, it has been found that the pain and the subsequent paresis which occurs during exercise in the condition known as intermittent claudication (Charcot's) is associated with arteriosclerosis of the limb vessels (Erb). Hence, it has been assumed that, while the blood supply may be sufficient during rest, the increased demand during exercise cannot be supplied and hence the pain occurs. This explanation is not entirely sufficient to account for those anginoid attacks which frequently occur without exertion.

It has also been frequently assumed that a vasoconstriction of the coronaries occurs which further narrows the caliber of the vessels that are already reduced by sclerosis. The demonstration that the coronaries possess vasomotor nerves (Porter, Wiggers) forms a basis for such a theory. 
Admitting that pain is associated with muscular exhaustion, and that this may result from an inadequate blood supply, the exact mechanism through which pain sensations are generated still remains obscure. In regard to this two general theories have arisen. It may be due to the chemical stimulation of waste or fatigue products, or the mechanical pressure of a high degree of tonus on the sensory nerves which presumably leave the heart via the sympathetic and vagus (Müller, Goldschneider, Krehl) or via the depressor nerve (Cyon). Again, others hold that cardiac sensory nerves have not actually been demonstrated and that the heart, in common with other internal organs, is not capable of recording pain. On the other hand, it is able to so modify the irritability of the spinal segments from which its nerves arise that the pain is referred to the superficial sensory nerves having their terminals in the same segments. In this way the reflexes from the heart would involve the lower cervical and upper thoracic nerves and account for the viscero-sensory pain reflex in the chest and left arm during attacks of angina (Mackenzie). In a similar way a viscero-motor reflex may be set up causing the muscles innervated from the same segment to contract. This view attributes the sense of constriction to the tonic contraction of the intercostal muscles.

\section{THE AFFECTIONS OF THE HEART DUE TO EXOGENOUS TOXIC INFLUENCE-CHRONIC ALCOHOLISM.}

The chemical substances that affect the functions of the heart may be classified as exogenous and endogenous. A consideration of the exogenous agents belongs more properly to the province of pharmacology and toxicology. We shall, therefore, deal here, by way of illustration, merely with the changes brought about by chronic alcoholic intoxications.

Chronic Alcoholic Heart.-Pathological Physiology and Histology. -Whatever may be our opinions as to whether the "occasional" or therapeutic use of alcohol causes through reflexes or associated psychical stimulation, an improvement or an impairment of the cardiac functions, it is well established that its immoderate use always causes a depression of the heart as in the case of intravenous injections in animals. In "heavy drinkers" we may assume that the heart is continually bathed in a weak alcoholic solution which tends to depress its activity and reduce its output. If continued for a long time this leads to loss of tonus and results in dilatation. It is this direct toxic influence on the heart muscle which is primarily responsible for its impaired action.

Another influence soon supervenes, however. Alcohol is a food and represents a high caloric equivalent; one gram yielding seven calories, as compared to three and seven tenths calories yielded 
by one gram of sugar. The heart in connection with other organs must take part in its oxidation. If a proportionate number of calories of other food should be withheld, it is possible that the alcohol would be burned completely and in normal fashion. Usually, however, it is added to an already sufficient caloric intake, and then it acts to spare the fat which is stored in various regions. Among these are the connective tissue of the abdomen and that around the heart. This is probably the reason that the fatty heart is most frequently associated with the intake of malted liquors which contain, in addition to their alcohol, other substances of caloric value. Such fatty deposits may further diminish the capacity of the heart for effective work by mechanically interfering with its movements. These deposits occur not only around the heart but penetrate within the connective tissue and cause an infiltration of the myocardium, or fat may also appear within the muscle cells. Virchow taught that this represented a conversion of the muscle protein into fat as a result of a toxic influence or a reduced blood supply, and, following his teaching, such fatty degeneration was to be carefully distinguished from fatty infiltration. According to Lusk, who gives another and a more probable explanation, there is no essential difference between infiltration and degeneration. A reduced local circulation (such as must naturally result during weakened cardiac action under alcohol) may cause an imperfect local oxidation of the lactic acid normally found both within and without the cell. This incomplete oxidation of carbohydrate causes an attraction of a superfluously large quantity of fat to the sugar hungry cell, which is deposited in and around the cell. According to this conception, the intracellular fatty degeneration is not the cause but the result of impaired function.

Clinical Manifestations.-The condition may manifest itself by various signs and symptoms which accompany cardiac dilatation and myocardial insufficiency (see page 306). Inability to indulge in customary muscular activity and pressure sensations in the chest are the first subjective manifestations. These are the only symptoms so long as the heart is able to maintain an effective circulation through the tissues. The medullary centres are the first to respond to any failure in this respect, by increased respiration, dyspnea, and heart acceleration.

As dilatation increases, we may get symptoms on the part of the heart itself-extrasystoles and the presence of functional murmurs. The pulse is always feeble and the blood-pressure usually low. The cardiac area is enlarged, as indicated by percussion and by the orthodiagram. This enlargement may be merely the result of fatty infiltration and be accompanied by an actual atrophy of the muscle cells, or it may be accompanied by some hypertrophy as in the Munich beer heart. The symptoms of hypertrophy are entirely 
obscured, however, by those of injured myocardial function, which may supervene and continue until all the symptoms characterizing heart failure are present (see page 310 ).

\section{THE AFFECTIONS OF THE HEART DUE TO ENDOGENOUS TOXIC INFLUENCES-GOITRE, ANAPHYLAXIS.}

Among endogenous substances may be classed all the splitproducts of digestion and metabolism. There is little positive evidence that such as probably enter the circulation, $e$. g., amino acids, urea, uric acid and xanthin derivatives, creatinine, etc., exert any recognized influence on the functions of the heart or that they are capable of producing histological changes. Of greater significance are the changes in the cardiac functions which accompany disturbances of the ductless glands, notably the thyroid.

\section{THE THYROID HEART.}

Clinical Physiology.-From the thyroid gland Baumann isolated a non-protein, iodine-containing body which he termed iodothyrin. When this substance or an extract of the thyroid gland is injected into experimental animals it causes an increased or decreased heart rate, and a fall of blood-pressure. ${ }^{1}$ This acceleration is apparently due partly to a stimulation of the accelerator mechanism and partly to a direct action on the cardiac muscle, the inhibitory mechanism being apparently unaffected (Cushny). The fall of blood-pressure is due to a peripheral dilatation of the bloodvessels which is, according to $\mathrm{v}$. Cyon, a reflex caused by the stimulation of the depressor endings. Since similar effects have been produced both in animals and man (Hellin) by feeding large doses, and since these, in turn, correspond in a certain manner with the symptoms noticeable in patients suffering from exopthalmic goitre (Graves' disease), it is generally inferred that in this disease the internal secretion is poured into the blood in abnormal amounts which give rise to the clinical conditions associated with the affection (v. Fürth). Further corroboration has recently been added by Sanford and Blackford who showed that a similar effect occurs in animals when the serum of goitre patients is injected into them. The first injection produces tolerance for subsequent ones. The effect is not abolished by atropine, and in this way it differs from the depression of blood-pressure produced by other tissue extracts.

Clinical Manifestations.-The chief cardiac symptom consists in the persistent or easily provoked tachycardia accompanied by palpitation. The tachycardia is usually of the sinus variety, though

\footnotetext{
1 For a recent review of the literature and additional evidence that the depressor effect bears no relation to the iodine content of the extract injected, see Fawcett, Rogers, Rahe, and Beebe, Amer. Jour. Physiol., 1915, xxxvi, 113.
} 
attacks simulating paroxysmal tachycardia have been reported (v. Hoesslin). 'The cardiac area is at first normal but later enlarged. The apex beat is very vigorous and out of proportion to the amplitude of the arterial impulse which seems comparatively weak. Respiratory arrhythmia and extra-systoles may occur. The bloodpressure is variable, a hypotension and a hypertension both having been reported. 'There is no doubt that other attendant pathological processes play an important role when the pressure is elevated. Krause and Friedenthal found the pulse pressure increased.

\section{THE ANAPHYLACTIC HEART.}

If a guinea-pig be sensitized by the peritoneal introduction of one milligram of a foreign serum and ten days later a second dose of five milligrams be given, it becomes uneasy, dyspneic, paralyzed, and dies with lightning rapidity. In the case of the rabbit and dog the symptoms are less severe and recovery often occurs. The symptoms are spoken of as the anaphylactic reaction and are of interest in relation to the serum reaction in man.

The cause of the serious symptoms and rapid death has been variously interpreted, as follows: (1) A paralysis of the vasomotor centre (Richet). (2) A paralysis of the peripheral vasomotor mechanism (Edmunds). (3) A paralysis of the respiratory centre (Rosenau and Anderson, Gay and Southard). (4) A spasm of the bronchial muscles causing asphyxia (Auer). (5) A spasm of all non-striated muscle (Anderson and Schultz).

It has been shown experimentally that the injection of foreign proteins into previously sensitized rabbits causes a rapid rise of blood-pressure, which is maintained at a high level for some time and then falls. The heart shows partial block, e.g., a $3: 1$ rhythm may be produced (Lewis and Auer). Both the block and the rise of blood-pressure are probably due to asphyxia following the bronchial spasm. A partial or complete block has also been substantiated in rabbits, in some of which a fatal and in others a non-fatal outcome resulted. It was present whether the vagi were intact or cut. Its onset occurred very promptly after injection (thirty seconds to three minutes) (Robinson and Auer).

In dogs in which the tonic contraction of the bronchial muscles is less pronounced, hence causing no asphyxia, the anaphylactic reaction expresses itself by a fall of pressure lasting about three and a half minutes. The action resembles that produced by the ingestion of Witte's peptone and also that of the toxic portion of Vaughn's split proteins (Edmunds). The myocardiogram shows a slight weakening effect on the heart (Eisenbrey and Pearce, Edmunds) but experiments indicate that the chief cause of the fall of blood-pressure is due to an accumulation of blood in the liver, presumably in consequence of a vasodilation (Edmunds). 
Recently it has been found that partial or complete block occurs in one-half of the cases examined by the electrocardiograph. The heart is slower and the ventricular complexes change. The $S$ depression is deeper and the $T$ wave exaggerated. On the whole, the curve gives the impression that the right ventricle has suffered most. The cardiac disturbance of conduction is not the result of low blood-pressure nor does central inhibition play a part (Robinson and Auer). It must therefore be concluded that the heart is directly affected in the anaphylactic reaction.

\section{MYOCARDITIS IN ACUTE INFECTIONS.}

Myocarditis may accompany or follow diphtheria, scarlet fever, pneumonia, infections with the staphylococcus, streptococcus and gonococcus, and less frequently typhoid and syphilis. The cause and effect relation of parenchymatous and interstitial changes and the altered function have already been indicated. In toxemic or infectious diseases the efficiency of the heart and circulation may suffer, not only on account of the myocarditis, but also because of the associated elevation of temperature and changes in the peripheral circulation. It is therefore desirable to consider these influences apart from the specific effect of the infectious process.

\section{Differentiation of Effects due to Temperature Changes and} Peripheral Vascular Changes from Myocardial Involvement.Clinical Physiology.-Temperature exerts a direct action upon the heart, for it can be shown in perfusion experiments that an increase in temperature within limits causes not only an increase in rate but also in amplitude as well. The tonus is decreased and the heart dilates. Cardiometer experiments indicate that similar changes occur in the intact heart independent of vagus influence, the systolic output increasing in spite of a more rapid heart rate (Wolf).

The idea that changes in the peripheral circulation can secondarily involve the heart has been widely accepted in recent years. There is distinct evidence that cardiac power itself is not exhausted in death from infectious fevers for, $(a)$ hearts of animals and of man have been revived after death and $(b)$ circulatory failure has often been warded off by the use of adrenalin or abdominal compression. (For literature see MacCallum.) It cannot be doubted, therefore, that the decrease in cardiac efficiency and even its final cessation is aided by the failure of the peripheral circulation. The demonstration that the heart was not alone concerned has by some been regarded as proof that the vasomotor centre necessarily failed. This idea was given impetus by a series of researches published by Romberg and his pupils (1896-1899) in which it was attempted to show that during the course of such illnesses as diphtheria, pyocyaneus and pneumococcus infections, the cause 
of circulatory failure was a paralysis of the vasomotor centre. The sequence of events produced may be summed up as follows: dilatation of the peripheral bloodvessels, an accumulation of blood in the splanchnic areas, a fall of arterial blood-pressure, an impairment of the coronary circulation and, secondarily, an enfeebled heart action. In other words, a complete picture of "toxemic shock."

Recent observations by Porter and his pupils, by more exact methods, have failed to corroborate the fact that the vasomotor centre fails, for in both diphtheria and pneumonia, the vasomotor reaction was normal to the end.

Such observations do not prove that the peripheral circulation does not fail but indicate that another explanation than vasomotor paralysis must be sought to explain toxemic shock. Although open to minor objections, the hypothesis first advanced by Malcolm and more definitely elaborated by Henderson seems most plausible. According to this idea, the infectious agents cause a decrease in the volume of the circulating blood (oligemia) by establishing an excessive osmosis of water to the tissues. The reduced volume of blood causes a deficient return to the heart and so impairs its filling and systolic discharge. Hence, the pulse becomes of small amplitude and the arterial pressure falls in spite of marked vasoconstriction and without any impairment of the cardiac function of contractility.

Clinical Differentiation.-When circulatory disturbances occur during an infectious process it is desirable to differentiate whether the changes are ushered in through the effects of temperature, toxemic shock, or of acute myocarditis. While a satisfactory differentiation may not be possible, certain guiding principles may be followed.

The most prominent change in the heart is the variation in rate. Clinical observations during rapid changes in temperature show that for every degree of temperature rise the heart accelerates from eight to ten beats. When this relation is disturbed some other influence may be suspected (Mackenzie). During a rise in temperature the output is augmented in spite of a shortened diastole, and since the vessels are relaxed the pulse pressure and pulse amplitude increase. A large pulse associated with a normal diastolic pressure, leads us to suspect that the circulatory disturbances may be traced to temperature elevation; whereas, a small pulse with a lower diastolic pressure brings to our minds the thought of peripheral change or myocardial involvement.

Changes in cardiac rhythm show us most unmistakably that we are dealing with myocardial disease. This may be inferred because irregularities seldom occur when the disturbance is peripheral (hemorrhage, shock), nor are they caused by excessive temperature. Furthermore, their clinical occurrence is frequently associated with myocardial lesions found in subsequent post mortems. The 
nature of the irregularity varies with the locality of the lesions, but not necessarily with their extent. Thus, a small area of myocarditis affecting the conducting system of Tawara may lead to extensive disturbances in conductivity, and to marked arrhythmias; while extreme infiltration in other areas may affect the heart action very slightly, if at all. In the majority of cases in which conductivity disturbances are present, however, it may be assumed, on the basis of postmortem experience, that the myocarditis is not limited or circumscribed, but is diffusely distributed throughout the heart.

Not all irregularities are indications of myocardial lesions, however. Thus the occurrence of extra-systoles is of no diagnostic significance and pulsus alternans may occur when the heart is otherwise overburdened. Again, sinus irregularity after acute infections may be regarded as good evidence that the heart has escaped injury (Mackenzie). Heart block of varying degree and the occurrence of auricular fibrillation are, on the other hand, quite definite proofs that the myocardium is diseased. It is apparent, therefore, that the distinction between different forms of arrhythmia is of the first importance in these conditions.

\section{DIPHTHERITIC MYOCARDITIS.}

The many deaths resulting from diphtheria are due largely to its detrimental effect on the circulation, the heart being seriously affected in 10 to 20 per cent. of all cases (Romberg, Schwartz). Circulatory involvement may occur during the course of infection (usually the first or second week) or during convalescence.

It has been found that by the second week the cardiac changes are largely parenchymatous in character though interstitial exudates occur. (See Röhmer, Tanaka, literature.) These may or may not involve the conducting system of Tawara (Tanaka, Röhmer, Leede). This can be gleaned from the following table, which is a partial list of the published reports:

Pathological Changes in the Heart After Diphtheria.

\begin{tabular}{|c|c|c|c|c|c|c|}
\hline Mönckeberg & - & - & . & $\begin{array}{l}\text { Cases. } \\
1\end{array}$ & $\begin{array}{l}\text { Myocardium. } \\
\text { Uniform fatty degen- } \\
\text { eration of all. }\end{array}$ & Conducting system. \\
\hline Amenomeya & . & . & • & 12 & $\begin{array}{l}\text { Fatty and Vacuolar } \\
\text { degeneration of all. }\end{array}$ & \\
\hline Heilhecker & . & . & . & 6 & Fatty degeneration. & No change. \\
\hline Löw · . & . & . & . & 3 & Fatty degeneration. & $\begin{array}{l}\text { Interstitial infiltra- } \\
\text { tion. Fatty change. }\end{array}$ \\
\hline Burger & . & • & - & 12 & Degeneration in all. & \\
\hline Tanaka & - & . & - & 15 & $\begin{array}{l}\text { Fatty degeneration in } \\
\text { all (except one case). }\end{array}$ & \\
\hline Leede . & . & - & . & 3671 & $\begin{array}{l}\text { Fatty, hyaline degen- } \\
\text { eration. }\end{array}$ & $\begin{array}{l}\text { Bundle affected in } \\
\text { part of cases. }\end{array}$ \\
\hline Price and $\mathrm{Ma}$ & ackenzie & & • & 1 & $\begin{array}{l}\text { Cellular infiltration; } \\
\text { fatty degeneration. }\end{array}$ & No change in bundle. \\
\hline
\end{tabular}


The pulse is unusually small and rapid and the blood-pressure is low (Norris), which may be referred either to the peripheral circulation or to the heart itself. In cases with regular hearts the electrocardiogram shows no changes (Röhmer). The circulation may become less and less efficient until death ensues. It is difficult to estimate the share that failure of the peripheral circulation plays in such cases. The blood-pressure is low and the decrease may be sudden and progressive. 'That this is not due to vasomotor failure seems probable both because the experimental evidence of such action is negative and also because the patient gives every appearance of having highly constricted rather than relaxed vessels. The skin is pale and the palpable arteries small. Death must therefore be attributed either to a direct cardiac involvement or to an oligemia. 'The former is more probable since previous to death the ventricular complexes of the electrocardiogram change. The $R$ wave increases in width and may be very low. The $T$ wave may be inverted in all leads and the $P$ wave absent. Apparently death is usually due to a general myocardial degeneration rather than any disturbance of conduction (Röhmer). In some cases the heart is slow and irregular. Studies of these have shown that they are caused by partial or complete block (Fleming and Kennedy, Price and Mackenzie, Hume, etc.) and electrocardiographic studies indicate that in such blocks left- and right-sided ventricular complexes are evident (Röhmer). Other forms of irregularities such as nodal rhythm, premature auricular systole, auricular flutter, and paroxysmal tachycardia have been described. The arrhythmia often changes in type from day to day (Hume). These are undoubtedly brought on by destruction of various parts of the conducting system. Thus, Hume found a granular degeneration of the sinus node and an unaffected $a-v$ node in a case in which nodal rhythm developed on the eighth to tenth day. A number of cases of block have been reported, however, in which no disturbances of the conducting system could be found ( $c f$. Price and Mackenzie), in which case it must be tentatively assumed that the altered ventricular muscle failed to receive the impulses, for evidence is lacking that heart block can be experimentally produced by injection of the toxin alone (Röhmer). In many instances it is impossible to say whether nodal rhythm is due to an increased irritability of the $a-v$ node or to a depression of the sinus node (Hume, Clegg).

According to Leede, death occurs in 54.16 per cent. of cases during the first week, and of these 3.7 per cent. occurred during the first twenty-four hours. Such intensity of toxic action is probably unequaled in any other infectious disease.

The cardiac failure occurring during convalescence is often sudden and unexpected in its onset. It is always associated with acute interstitial myocarditis. The changes evident in the heart 
are either tachycardia or bradycardia. The tachycardia is usually of the sinus variety but cases of true paroxysmal tachycardia have been reported (Krehl, Hume). The heart, however, more often shows irregularities or a slow rate because of partial or complete heart block. Evidences of dilatation are given by the increase in dulness, the onset of functional mitral murmurs, and by the orthodiagram. Dietlen has shown by the use of the orthodiagraph that the dilatation may increase progressively. The arterial pressure falls and the pulse is small. Venous stasis and edema often appear. Evidence seems to assure us that death in such cases is entirely traceable to direct cardiac failure.

In the stage of convalescence, death has been reported as occurring suddenly and without premonitory symptoms. It is probable, however, as Krehl remarks, "that death will occur unannounced less and less often as the physician examines the heart more and more extensively." By the employment of recent methods it is possible to detect early changes in conductivity which presage more extensive disturbances and should warn against the employment of remedies tending to augment such a block (e.g., digitalis).

\section{THE HEART IN ACUTE RHEUMATIC FEVER.}

With the general recognition that acute rheumatic fever so frequently excites an endocarditis, the fact that it causes a myocarditis which may seriously affect the cardiac function has been distinctly underemphasized. This is due, in part, to the fact that it has not been understood that signs regarded as characteristic of endocarditis (e. g., systolic murmurs) may be produced by myocardial dilatation as well. The absence of pathological changes in the heart muscle during the early stages has also favored this view. More recently there has been a tendency to return to the older conception, viz.: that a carditis is produced in which the involvement of the endocardium and myocardium cannot be differentiated by the symptoms.

The signs of acute myocardial lesions may occur early in the course of the infection and are then brought about by the toxic influence on the heart; or they may occur late in the disease, in which case they are accompanied by degenerative changes in the heart cells, by leukocyte infiltration or small cellular foci (Aschoff) which present a very typical appearance. They contain a hyaline centre surrounded by a layer of giant cells and these, in turn, are surrounded by a layer of mononuclear or eosinophile leukocytes.

The characteristic signs of cardiac trouble are three: Dilatation of the heart, presence of systolic mitral murmurs, and changes in the rate and rhythm of the pulse. The cardiac dilatation is indicated by the rapid rate. The sounds are sharp and clear and sometimes 
reduplicated. The dilatation is rarely severe enough to cause discomfort, breathlessness or edema. The occurrence of a soft, blowing systolic murmur may also be due to the muscular insufficiency but in this case it is impossible to say that it is not associated with the endocarditis affecting the valves. More definite evidence of myocardial trouble is given when the heart becomes irregular. Extra systoles occur early in the disease and probably indicate that the toxic action has produced a hyperirritable condition of the cardiac muscle. The writer has known them to occur two days after the onset of acute rheumatic fever. Changes in conductivity appear somewhat later and undoubtedly accompany parenchymatous or interstitial changes in the heart muscle. Heart blocks of various degrees present themselves, both sino-auricular and sino-ventricular block being met with (Mackenzie). It should be borne in mind, however, that during such infections the heart is peculiarly susceptible to drugs producing heart block and the possibility should be remembered that the changes may be the result of medication rather than of myocardial lesions.

Outcome.-The acute myocarditis occurring early during a febrile attack rarely results fatally but the patient only occasionally makes a complete recovery. Most frequently a chronic myocarditis supervenes which grows progressively worse as the years pass. The cellular exudates are absorbed, necrotic tissue disappears, and fibrous tissue forms; but not infrequently the muscle cells continue to show parenchymatous degeneration and a degree of hypertrophy depending on the concomitant valvular defects. Indeed, it is difficult after a number of years to separate the myocardial involvement directly due to the previous infection and that due to impaired function arising from valvular lesions. The irregularities present during a chronic myocarditis may be caused by $(a)$ sclerotic and fibrous changes involving the conducting system and so producing a form of heart block, $(b)$ increased pressure in the auricles producing extra-systoles and perhaps leading to auricular flutter or fibrillation, (c) areas of fibrous and muscular degeneration involving the sinus region and auricle and causing auricular fibrillation. Dilatation readily occurs in such cases. This manifests itself in symptoms of dyspnea, venous engorgement, and edema. The presence of a systolic type of venous pulse due to tricuspid insufficiency, a low arterial pressure and small irregular pulse are also characteristic. The cardiac area itself is enlarged.

\section{MYOCARDITIS ASSOCIATED WITH SEPTIC INFECTIONS, EXANTHEMATOUS DISEASES, ETC.}

The myocardial changes characteristic of the acute stages of rheumatic fever may also be present during septic infections of the 
myocardium with staphylococci, streptococci and gonococci, and as a result of the unknown infection of scarlet fever and occasionally after measles. In the virulent septicemias the accumulation of leukocytes and necrosis are greater than in acute rheumatic fever and often result in abscesses of varying size. The same clinical cardiac symptoms which occur in rheumatic fever are present, in varying degree. Dilatation, tachycardia, mitral murmurs, and irregularities are typical. Because the myocardium is so severely attacked and because of the associated endocarditis, the danger of heart failure during the acute attack is far greater in the septicemias than in rheumatic fever, but after recovery has occurred there is less danger of a subsequent chronic myocarditis.

\section{THE MYOCARDIAL CONDITION IN TYPHOID.}

A study of the pathological and clinical signs during typhoid shows that the myocardium is involved at the height of the affection. In the early stages (first week) such pronounced changes in the circulation occur that they are often diagnostic. The pulse rate accelerates but not in proportion to the fever. It usually reaches the neighborhood of one hundred. Its amplitude is large, the descending limb falls rapidly and is followed by a deep and prominent dicrotic notch which is usually evident to the palpating finger. The systolic blood-pressure is generally lower, but the writer has found no reliable study showing that the diastolic pressure is low during the first week. The pulse-pressure is great, as one should expect. During the existence of these signs no pathological changes are present in the heart. The entire circulatory complex is therefore referable to a peripheral change-apparently a vasodilatation. Further work, however, is required to establish this fact.

The writer has often pointed out to students that every form of cardiac and pulse change observed during the first week or ten days of typhoid infection, can be well illustrated within a few minutes after inhalation of amyl nitrite. The systolic blood-pressure falls, the pulse pressure is increased, the pulse amplitude becomes larger, and the pulse, more collapsing and dicrotic. From a reflex, through the vagus, the heart accelerates and thus causes an abreviation in the length of the pulse wave. If the heart beats too rapidly the dicrotic wave of one oscillation may be mounted on the upstroke of the next ascending pulse giving a superdicrotic effect (Fig. 74, 2).

After the first week or two the character of the pulse changes gradually until it often becomes more rapid while its dicrotism disappears and its amplitude-decreases. Its shape still indicates that the blood-pressure is low, a fact confirmed by the sphygmomanometer. The occurrence of granular degeneration together with 
the fact that systolic murmurs, feebler first sounds or even a gallop) rhythm occur, indicate that the myocardium is weakened during this stage (Fig. 74).

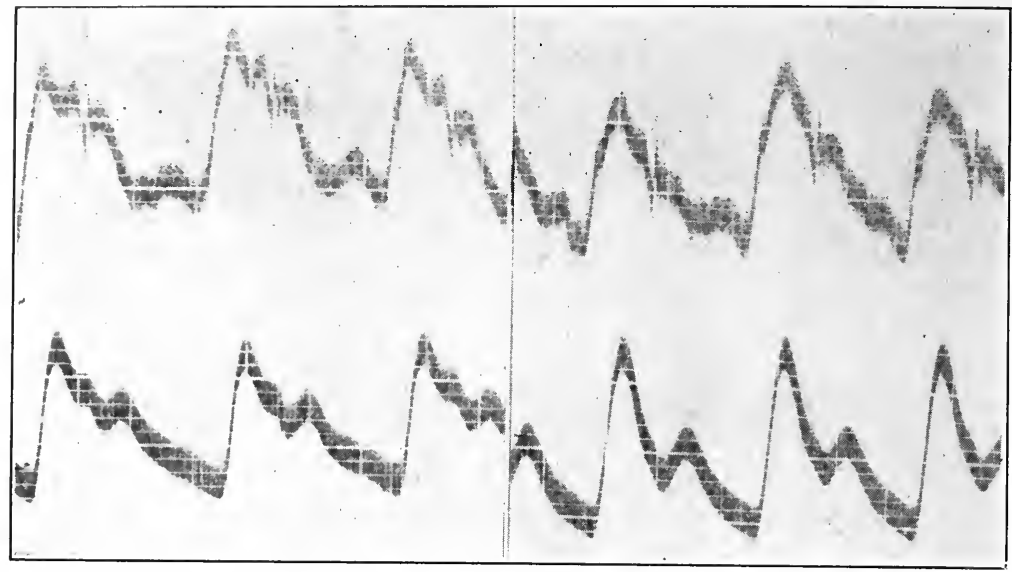

FIG. 73.- Simultaneous tracings of subclavian (upper) and radial (lower) pulses. (a) before and (b) after amyl nitrite.

The pulse rate gradually diminishes with the disappearance of the fever, but the cardiovascular picture is further changed by the frequent occurrence of an extreme bradycardia during conval-

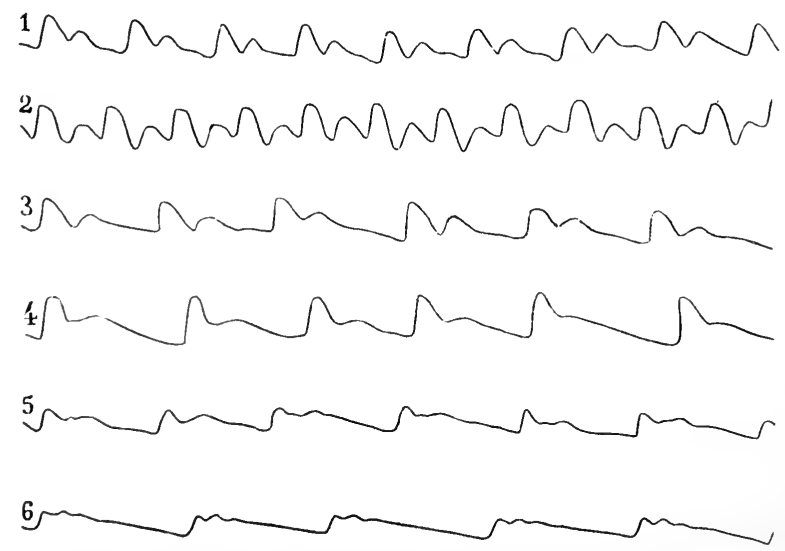

FIG. 74.- Series of sphygmograms taken on successive days in typhoid fever by Marey's sphygmograph. (After Marey.)

escence. The writer, in spite of diligent search, has been unable to find in the literature, any evidence showing whether such slow pulses may be entirely attributed to an augmented vagus influence 
but this seems very probable. Cases have been reported, however, in which partial or complete block supervened and accounted for the slow rhythm. In these the well-known fatty changes and hyaline degeneration of the muscle cells occurring late in typhoid infections probably involved the His-Tawara system of conducting fibers.

\section{THE HEART IN PNEUMONIA.}

The toxic influence exerted by the pneumococcus infection on the heart has not been carefully studied. Clinically there are three periods of the disease in which the circulation appears embarrassed. Before the crisis has been reached the pulse may become small and extremely rapid, sometimes reaching 140 per minute. The blood-pressure may be low, and irregularities such as dropped beats due to block and pulsus alternans may appear (Mackenzie). The heart may become dilated - the first tone impure and venous stasis may be present. From this condition the patient may either recover or succumb with spmptoms of collapse within twelve to thirty-six hours. It is impossible at present to determine the cause of these symptoms. We are confronted with two facts. When death occurs the heart muscle cells show no definite lesions (Lepine, Homburger) and vasomotor failure apparently does not take place (Porter and Newburgh). It is therefore possible to assume with equal evidence $(a)$ a toxic depression of the heart without changes, $(b)$ an interference with the blood supply of the left heart and a damming back of blood into the right heart by the consolidated lung, $(c)$ a concentration of the blood volume and a deficient supply, such as is found in toxemic shock ( $c f$. Sandelowsky).

Very similar symptoms occur at two other stages of the disease, namely, during or after the crises and in convalescence. At these times, however, they seem to be rarely fatal. Their cause is also obscure and subject to further study.

\section{THE HEART IN SYPHILIS.}

When the trepanoma pallidum invades the heart, it causes only slight parenchymatous changes - the cells may show the presence of fat but rarely undergo serious retrograde changes or necrosis. It causes, however, interstitial infiltrations with leukocytes and lymphocytes and an arterial impairment (Mallory). This is probably the reason that the active stages of syphilis are accompanied so rarely by circulatory disturbances. When the blood supply becomes decreased, as a result of endarteritis and sclerosis the proliferation of fibroblasts causes the formation of gummata. A favorite location seems to be in the septum between the auricle 
and ventricle where they may encroach upon the narrow His bundle and so interfere seriously with the heart's action. So it comes about that the serious myocardial involvement often occurs many years after the acute disease has passed off (6-10 years) (Krehl). The effect of the syphilitic infection manifests itself in three directions: (a) the production of sclerosis and gummata interfering with special cardiac functions, as contractility or conductivity, (b) the presence of arteriosclerosis with its attendant symptoms and $(c)$ the presence of aortitis and valvular lesions with their effects. All that has been said in other sections in regard to the signs and symptoms due to factors $(b)$ and $(c)$ applies to the syphilitic involvement of the heart which cannot be separated from pure myocardial involvement.

To avoid repetition, we may limit ourselves to the evidences of direct myocardial change. No infectious process is so commonly associated with disturbances of the conducting function. Varying degrees of sino-ventricular block, as also complete block with the attendant Stokes-Adami complex, are frequent accompaniments of this disease. Auricular fibrillation is also a common result when the sclerosis involves the auricular musculature.

\section{BIBLIOGRAPHY.}

Воокs.

Adami and McCrae. Text-book of Pathology, 1912, Philadelphia and New York. Birch-Hirschfeld. Lehrhuch d. path. Anat., 1894, ii, 4th ed., Leipsic.

Cowan. Diseases of the Heart, 1914, Philadelphia and New York.

Cushny. Pharmacology and Therapeutics, 1910, 5th ed., Philadelphia.

Hirschfelder. Diseases of the Heart and Aorta, 1913, 2d ed., Philadelphia and London.

Krehl. Die Erkrankungen des Herz Muskels, 1913, 2d ed., Wien and Leipsic.

“ Path. Physiologie., 1914, 8th ed., Leipsic.

Krehl-Marchand. Handbuch der Pathology, 1913, Bd. ii, abt. ii, Leipsic.

Mackenzie. Diseases of the Heart, 1913, London.

Mallory. Principles of Pathological Histology, 1914, Philadelphia and London.

Norris. Blood-pressure and its Clinical Applications, 1914, Philadelphia.

Osler and McCrae. Modern Medicine, vol. iv, 1915, Philadelphia.

Strümpell. Lehrbuch der Speciellen Pathologie u. Therapie, 1914, 19th ed., Leipzig.

\section{Papers.}

Articles Dealing with Disturbances in Blood Supply of the Heart.

Aschoff. Brit. Med. Jour., 1906, p. 1103.

Bond. Jour. Exper. Med., 1910, xii, 575.

Cohnheim and v. Schulthess Reichberg. Arch. f. path. Anat., 1881, lxxxv, 503.

Hirsch and Spalteholz. Deutsch. med. Wchnschr., 1907, xxxiii, 790.

Lewis. Heart, 1909, i, 98.

Merkel. Verhandl. d. deutsch. path. (Yesellsch., 1907, p. 127.

Miller and Matthews. Arch. Int. Med., 1909, iii, 476.

Porter. Jour. Exper. Med., 1896, i, 46.

" Jour. of Physiol., 1893, xv, 131. 
Pratt. Am. Jour. Physiol., 1896, i, 86.

Wiggers. Am. Jour. Physiol., 1909, xxiv, 391. Arch. Int. Med., 1914, xiv, 33.

\section{Literature Dealing with Toxic Substances.}

Auer and Lewis. Jour. Exper. Med., 1910, xii, 151, 638.

Cyon. Arch. f. d. ges. Physiol., 1898, lxx, 126; lxxi, 431; lxxiv, 97 ; lxxvii, 215.

Edmunds. Ztschr. f. Immunitätsforschung u. exper. Therap. 1913, xvii, 105.

Esser. Arch. f. exper. Path. u. Pharmakol., 1903, xlix, 190.

v. Fürth. Ergebnisse d. Physiol., 1909, viii, 524.

Hellin. Arch. f. exper. Path. u. Pharmakol., 1897, xl, 121.

Hirschfelder. Jour. Exper. Med., 1910, xii, 586.

v. Hoesslin. München. med. Wchnschr., 1896, xliii, 25.

Kraus and Friedenthal. Berl. klin. Wehnschr., 1908, xlv, 1709.

Oswald. Arch. f. path. Anat., 1902, clxix, 444.

Pearce and Eisenbrey. Jour. Pharmacol. and exper. Therap., 1912, iv, 21. " " Arch. Intern. Med., 1910, vi, 218.

Robinson and Auer. Jour. Exper. Med., 1913, xviii, 450, 556.

Roos. Ztschr. f. physiol. Chem., 1896, xxi, 19; 1897, xxii, 16.

Sanford and Blackford. Jour. Am. Med. Assn., 1914, lxii, 117.

Schultz. Hyg. Lab. Bul., 1912, lxxx.

\section{Literature Dealing with Effects of Infections on the Heart.}

Beck and Stapa. Wien. klin. Wehnschr., 1895, xviii, 323.

Cleghorn. Am. Jour. Physiol., 1899, ii, 273.

Dietlin. München. med. Wchnschr., 1905, i, 683.

Flemming and Kennedy. Heart, 1910-11, ii, 77.

Henderson. Am. Jour. Physiol., 1910, xxvii, 152.

Hume. Heart, 1913, v, 25.

Hume and Clegg. Quart. Jour. of Med., 1914, viii, 1.

Leede. Ztschr. f. klin. Med., 1913, lxxvii, 297.

MacCallum. Am. Jour. Med. Sci., 1914, cxlvii, 37.

Malcolm. Lancet, 1905, ii, 573; 1907, i, 497.

Meyer. Arch. of Exper. Pharmacol., 1909, lx, 208.

Porter and Newburgh. Am. Jour. Physiol., 1914, xxxv, 1.

Porter and Pratt. Am. Jour. Physiol., 1914, xxxiii, 431.

Price and Mackenzie. Heart, 1912, iii, 233.

Rohmer. Ztschr. f. exper. Path. u. Therap., 1912, xi, 426.

Rolly. Arch. f. exper. Path. u. Pharmakol., 1899, xlii, 283.

Sandelousky. Deutsch. Arch. f. klin. Med., 1909, xevi, 445.

Tanaka. Virchow's Arch. f. path. Anat., 1912, cevii, 115.

Wolf. Arch. Int. Med., 1911, viii, 461. 


\section{CHAPTER X VIII.}

\section{THE DIAGNOSIS AND SIGNIFICANCE OF CARDIAC ARRHYTHMIAS.}

Disturbances in the regularity of cardiac action arise when the functions of impulse origination (rhythmicity), impulse conduction (conductivity), or contractility are in some way affected. It is not possible, however, to base a practical classification upon the nature of the disturbance alone, as was at one time hoped, for the simple reason that most forms of irregularity result from the involvement of many functions. For this reason the classifications were at first empirical and then, as our knowledge concerning their causes and relationships grew, they were necessarily varied to conform to scientific and practical standards alike. For the same reason, it may be confidently expected that they will continue to change, not only as to their groupings, but to a certain extent also in their nomenclature. The classification at present best meeting the combined demands of the scientific investigator, the diagnostician and the teacher of medicine seems to be that followed in the following scheme (modified after Hoffmann).

\section{CLASSIFICATION.}

I. Sinus Irregularities.

(a) Sinus tachycardia.

(b) Sinus bradycardia.

(c) Sinus depression with ventricular bradycardia.

(d) Respiratory arrhythmia.

(e) Phasic sinus arrhythmia.

II. Heart block (disturbances of conductivity).

(a) Partial sino-ventricular block (commonly called auriculoventricular block).

(b) Complete sino-ventricular block.

III. Premature contractions (extra-systoles).

(a) Ventricular.

(b) Auricular.

(c) Nodal.

IV. Tachyrhythmias.

(a) Auricular tachyrhythmias.

1. Paroxysmal tachycardia.

2. Auricular flutter. 
(b) Atrioventricular (nodal) tachyrhythmia.

1. Regular.

2. Irregular.

(c) Ventricular.

V. Total arrhythmia (auricular fibrillation).

VI. Alternation of the heart beat.

\section{SINUS IRREGULARITIES.}

The sinus region of the heart is that posterior portion of the auricle which is bounded above by the superior vena cava and below by the coronary sinus and which extends to the intra-auricular septum. It contains a set of peculiar muscle fibers and some nerve cells and fibers collectively forming a club-shaped enlargement spoken of as the sinus node or the node of Keith and Flack.

According to the most recent evidence, it is within this node that the heart beat is rhythmically inaugurated, ${ }^{1}$ and hence it has been designated the pace-maker of the heart (Lewis). In the sinus region terminate the vagus fibers, which normally exert their influence not only to lessen the rate at which impulses are originated, but also to determine what part of the sinus region shall send them forth $^{2}$ (Zahn, Meek, and Eyster).

The degree of vagus influence, which is estimated from the acceleration resulting when the vagi nerves are cut or their terminals are paralyzed by atropine, varies in different animals. Thus, in the cat, vagus section or the action of atropine produces an acceleration, while in the rabbit, the rate is unchanged. In the dog the vagus often exerts a rhythmic rather than a constant tonic action, so that the beats occur more rapidly during inspiration than during expiration, in fact the heart may stop entirely during the latter respiratory phase. This rhythmic variation is abolished by vagus section which proves that it is due to a central vagus influence.

The degree to which the vagus influences the tempo with which impulses are initiated in the sinus region of man likewise varies. The tonic vagus activity constantly increases, which accounts for the progressive slowing of the heart from infancy until adult life. During adolescence, when its power over the heart becomes most pronounced, it is apt to produce a rhythmicity resembling that found in the dog. This rhythmic variation also occurs in the adult though in a lesser degree. It is not always precisely associated with the phases of respiration, however. (See Pretzig.) This is probably because the influence of the respiratory over the cardioinhibitory centre is interfered with by influences from the other centres from the cerebrum, and reflexly. As is well known, swallow- 
ing is associated with an acceleration of the heart and Lombard and Pillsbury believe that the rhythmic action of the vasomotor centre can also affect the cardio-inhibitory centre.

(a) Sinus Tachycardia (Rapid Pulses of Sinus Origin).When the heart accelerates because of fright and anxiety; or after indulgence in exercise or drugs, such as the nitrites or atropine, we are dealing with a physiological form of sinus tachycardia. It results from a removal of normal vagus influence, and shows certain characteristic features. ${ }^{1}$ Its onset is gradual and progressive; it continues while the cause is acting. The shortening of the heart cycles occurs largely at the expense of diastole (Bowen, Krauss). Electrocardiograms show that when acceleration becomes excessive, the relative height of the complexes alters; the $R$ and $T$ waves become larger; the $P$ wave and the $P R$ interval remain practically unchanged. Such tachycardias are normal in every sense.

It happens, however, especially in the type of patient regarded as "neurotic" that an acceleration of the heart may occur from trivial causes. Thus, psychic acceleration is provoked on seeing a mouse or a shadow, smelling a disagreeable odor, hearing a shrill noise, etc. Such tachycardias have long been known to neurologists and psychologists as evidences of nervous instability of which they constitute a sign.

Persistent tachycardia often accompanies disturbances of nutrition or of internal secretion, $e$. g., in Basedow's disease; or it may be a symptom in graver disturbances, as hemorrhage and shock, infections, and fevers. It is, therefore, not entirely devoid of diagnostic import.

(b) Sinus Bradycardia (Slow Pulses of Sinus Origin).-When the vagus influence increases we obtain an abnormally slow pulse. Such slow rates occur normally in old age, convalescence, and during pregnancy. Pathologically, they are found in disturbances affecting the vagus centre, $e . g$. , in toxic icterus, etc. (see page 114.) The arterial curves show only the slow, rhythmic pulse. The jugular pulse and electrocardiogram are characterized by their normal sequence of waves.

(c) Ventricular Bradycardia Associated with Depressed Activity of the Sinus Region. - It is well-known that when the sinus node is removed or destroyed, the rhythm will be set up by some other part of the conducting tissue. In such cases it may happen that the impulse is originated so far down in the conducting system that the ventricle may beat before the auricle (Williams and James, Cohn). Williams and James report a unique case in man in

1 For a consideration dealing with the question of voluntary acceleration of the heart rate, consult Tarchanoff, Arch. f. d. ges. Physiol., 1885, xxxv, 109; Pease, Boston Med. and Surg. Jour., 1889, cxx, 525; Van der Velde, Arch. f. d. ges. Physol., 1897, lxvi, 232; Koehler, Arch. f. d. ges. Physiol, 1914, clviii, 579. 
which such a reversed cardiac action occurred resulting in a regular bradycardia (40 per minute). ' The electrocardiogram showed a $P$ wave occurring 0.18 second after the $R$ wave and the $x$-rays verified the fact that the ventricle contracted before the auricle. Atropine was without effect.

(d) Respiratory Arrhythmia.-When variations in length occur from one pulse cycle to the next and these changes are related to the phases of respiration, we speak of a respiratory arrhythmia. The acceleration, as a rule, coincides more or less accurately with the phase of inspiration, but its occurrence during expiration is not unknown.

Clinical Recognition.-The irregular rhythm of the pulse may often be recognized by palpation alone; or the irregular sequence of heart sounds may be heard and its relation to breathing determined. The irregularity does not usually stop when the breath is held, although its character may be modified. The arterial pulse tracing (Fig. 36, $A$ ) is characterized by a progressive increase and decrease in the cardiac cycles, the chief variation occurring in its diastole which is roughly estimated by the distance from the dicrotic notch to the next systolic rise. The waves vary somewhat in size, the smaller ones following the more rapid beats. This is due largely to the fact that the duration of the heart cycle modifies both the systolic and the diastolic pressures within the artery. As the writer has pointed out (page 71) a shortening of a cardiac cycle, as a rule, increases the diastolic and decreases the systolic pressure of the beat following. Conversely, a lengthening of a cycle causes an elevation of the systolic and a fall of the diastolic pressure.

The venous pulse (Fig. 36) shows a regular sequence of the $a c v$ waves with perhaps additional diastolic waves in the long cycles, or the abolition of the $v$ wave in the shorter cycles. The $a-c$ interval occasionally varies but we cannot be certain to what extent respiratory variations in venous pressure may account for the change. Hence it cannot positively be said that the conductivity is impaired through vagus action. The venous record, by showing the $a c v$ sequence, is occasionally of service in establishing the nature of this form of irregularity, $e . g$., when the contraction becomes so premature as to simulate an extra-systole in the arterial record.

The electrocardiogram adds but little information in interpreting this condition. 'The $P, R$ and $T$ waves show slight variations, probably due to the fact that the position of the heart changes. In slow hearts $R_{1}$ is larger and $T_{1}$ smaller, in the rapid beats $R_{1}$ is smaller and $T_{1}$ larger. The $P-R$ interval is unchanged.

Clinical Significance.-This form of irregularity is frequently present in early youth and has therefore been designated "the youthful type" by Mackenzie. It is usually of no significance and, indeed, its persistence after a febrile attack or an acute infection, 
according to Mackenzie, is a good omen for the very fact that the heart is susceptible to such labile nerve influences is good evidence that it has itself escaped injury.

(e) Phasic Sinus Arrhythmia.-It is frequently found, especially after digitalis medication, that one or more long cycles are interspersed among shorter ones. This may be so pronounced as to be in reality a temporary standstill. The long cycles may even recur periodically but then they bear no relation to respiration. These irregularities are abolished by atropine, hence we may assume that they are vagal in origin and simulate the effects produced in animals by brief vagus stimulation or toxic substances, as morphine (Einthoven, Meek and Eyster).

Clinical Recognition.-The variation in the cardiac cycles is evident both in the arterial tracings and in electrocardiograms. The $a c v$ sequence in the jugular tracing and the $P R T$ relations in the electrocardiogram are usually normal. Occasionally a prolonged standstill occurs which may last four or five seconds and is then accompanied by a brief attack of syncope (Mackenzie, Laslett). The ventricle, during these long pauses, may, as in animal experiments, escape from inhibition and give a purely ventricular beat originating, as Lewis has very recently shown, in the $a-v$ node.

Clinical Significance.-These forms of sinus irregularities have been reported in association with vagus neuritis (Hoover), vagal tumors (Staekle), meningitis (Reyfisch), increased intracranial tension (Hirschfelder), cerebral lesions and contractions of the foramen magnum ( $c f$. Lewis). They are not necessarily indicative of such serious organic disturbances, however, but may merely indicate a highly irritable vagus centre which responds to impulses from neighboring centres or reflexes which are normally ineffective. In this class probably also belong those rare, physiological curiosities who are able to voluntarily stop their hearts. The writer has heard of two such cases, reported to him by authority which he considers reliable. In all cases the danger from possible stoppage of a permanent nature, and liability to sudden death should be borne in mind.

In summing up the significance of sinus irregularities, it may be impressed that they give no evidence of cardiac involvement; but are often a criterion of affections of the nerve supply of the heart. As such they may be used as an index of nervous function much as the heart rate variations are used by the psychologist as a criterion of psychical processes.

\section{HEART BLOCK (DISTURBANCES OF CONDUCTIVITY).}

Clinical Physiology.-It is rendered highly probable by recent work that the sinus node in which the heart beat originates com- 
municates with cells of the His-Tawara system (Koch, Thorel) leading to the ventricle, and that the ventricle receives its impulses from the sinus node without the interposition of auricular tissue (Keith, Eyster and Meek). To follow the impulses originating in the sinus node more definitely, they pass $(a)$ partly to the auricle by the sino-auricular junction, and $(b)$ partly to the ventricle by passing consecutively through (1) the node of Tawara ( $a-v$ node), (2) the His bundle, and (3) the left and right branches of the HisTawara system. The impulses arising in the sinus region are apparently transmitted without delay to the $a-v$ node, in fact this node shows negativity before the auricular muscle itself (Eyster and Meek). A distinct delay occurs, however, before the ventricles - actually respond, a delay usually attributed to the $a-v$ node or the His bundle. Recently more definite evidence has been submitted to show that the $a-v$ node is actually the place where the delay occurs (Lewis, White, and Meakins). The conduction progresses through the two branches to the right and left ventricles so that all parts of the two ventricles are simultaneously excited (Clement, Erfman, Lewis. $C f$. also p. 23).

The conduction time from the sinus to the ventricles can be definitely estimated in experimental animals by recording the primary negativity of the sinus region as compared with that of the ventricles. More commonly the conduction interval is measured even in experimental work by the time difference between the first evidences of auricular and ventricular contractions. Thus, when the contractions of the chambers are recorded by levers or by a myocardiograph, it is the interval between the auricular and the ventricular upstroke, $(i$. $e$., the so-called $A s-V s$ interval). When the jugular pulse is recorded, in animals as well as in man, it is the difference between the $a$ and $c$ waves, $i$. $e$., the $a-c$ interval.

The idea of using the difference between the beginning of auricular and ventricular systoles as an estimate of conduction time was suggested at a time when it was supposed that the ventricle received its stimulus via the auricular musculature. Recent work has rendered this improbable ( $c f$. page 24$)$. The actual relation between the sino-ventricular conduction period and the $A s-V s$ interval is illustrated diagrammatically in Fig. $75, B . A, B$ represents the conduction time from sinus to auricle; $C, D$ the conduction time from sinus to the Tawara node; $D, F$ the conduction time from this node to the ventricular musculature. It is evident that the sinoventricular conduction interval, $C, F$ is longer than the $A s-V s$ interval, $B, F$; and that the $A s-V s$ interval serves as an estimate of the conduction time only when we may presuppose that the conduction from sinus to auricle- $A, B$ is constant. It appears that aside from technical considerations our methods used to estimate the conduction interval are not entirely free from error. It is 
probable that the onset of the $P$ wave (lead $I I)$ of the electrocardiogram marks, not the beginning of auricular systole, but that it is coincident with sinus negativity. Theoretically, the interval from the beginning of the $P_{\text {II }}$ wave to the $R_{\text {II }}$ wave gives us the most reliable indication we are able to obtain of the true conduction time interval. Simultaneous studies of the venous pulse and electrocardiogram indicate that probably the sino-auricular conduction time varies less than the sino-ventricular, for the $P-R$ and $a-c$ intervals usually lengthen together. This by no means occurs without exception, however, and the $a c$ interval should always be accepted guardedly, as evidence of conductivity changes, especially when the variations are slight.

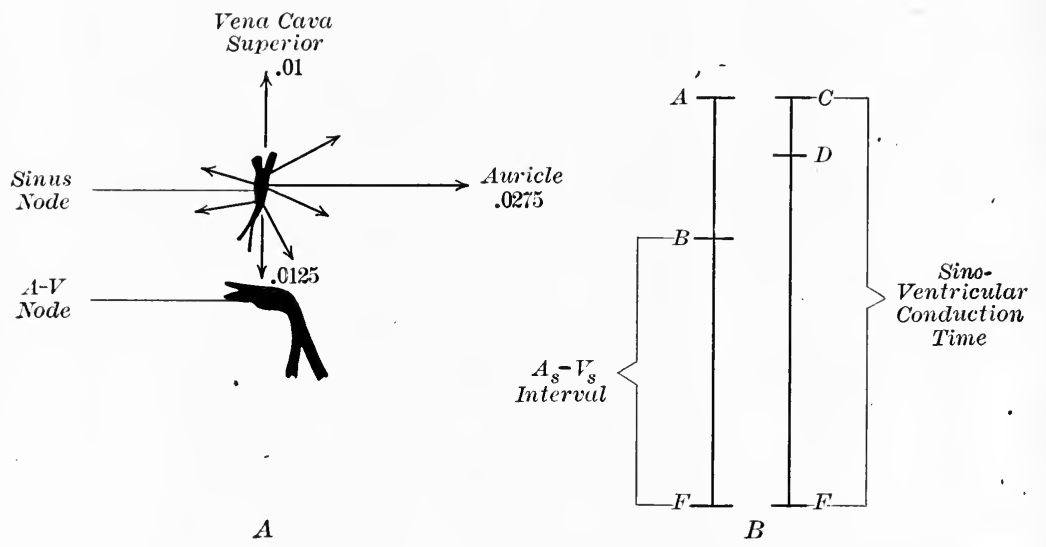

FIg. 75.-Two diagrams illustrating $(A)$ the rate and direction of impulse conduction from sinus node to auricle and to ventricle, and $(B)$ the relation between the $A_{s}-V_{s}$ interval and sino-ventricular conduction time.

The conduction interval from sinus to ventricle, as estimated by the procedure described, may be lengthened by vagus stimulation, asphyxia, chemical action, etc., or through more intense action of these influences, the passage of impulses may be entirely prevented. This condition is usually designated as auriculoventricular block, but in view of more recent studies, it is probably better expressed by the term, sino-ventricular block. In such cases, the Tawara node is apparently the most susceptible region (Lewis, White and Meakins) and it may be inferred that block is established here.

Experimentally, sino-ventricular heart block may also be produced by cutting or compressing the different portions of the His-Tawara system. Such experiments have proven very instructive. Complete transection or ligation of the His bundle causes cessation of all impulse transmission to the ventricle (Humblet, Hering, Erlanger, 
Cohn and Trendelenburg). When this occurs, the ventricle temporarily stops and after a short interval reassumes an independent rhythm. If the bundle is only partially divided or slightly compressed, various stages of block may be produced. These may be summarized as follows:

(a) An increase in the $A s-V s$ and $P-R$ intervals.

(b) An occasional failure of the ventricle to respond to sinus stimuli when the auricle does so.

(c) A regular dropping of ventricular beats, as shown by the fact that the ratio of auricular to ventricular beats such as $10: 9$, $8: 7,4: 3$ is established.

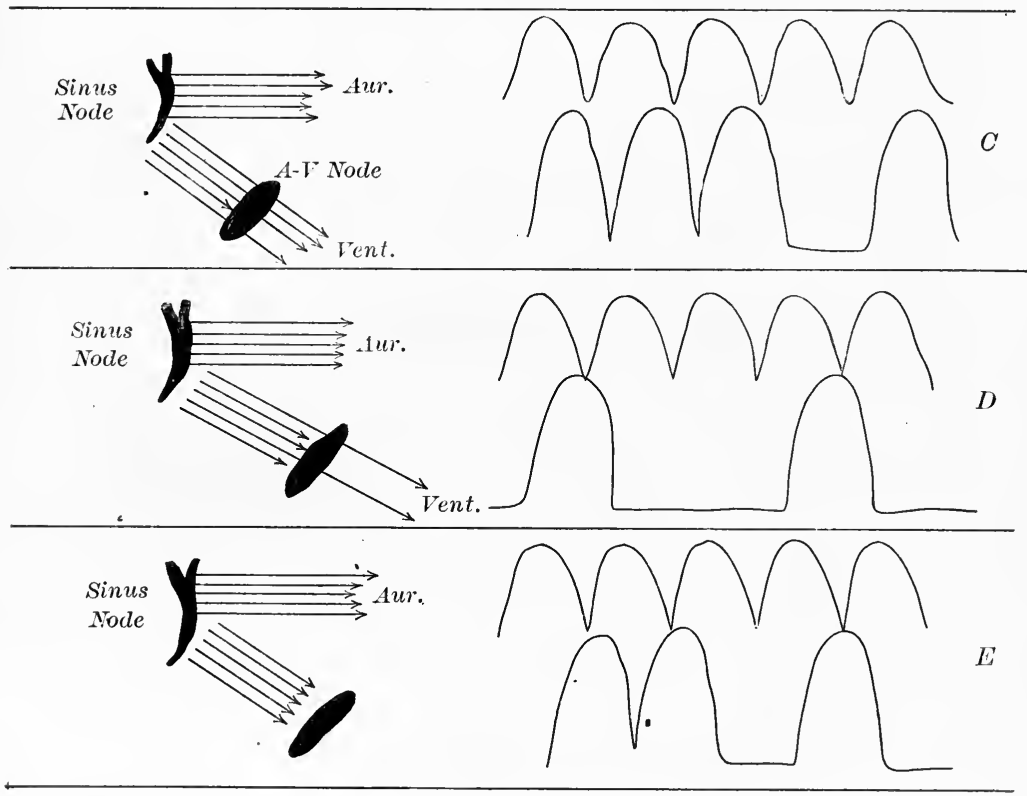

FIG. 76.-Diagram illustrating the nature and cause of $4: 3,3: 1$ rhythm and absolute sino-ventricular block.

(d) A regular ventricular response to every second; third, or fourth sinus impulse, as indicated by the fact that such ratios as $2: 1,3: 1$ or $4: 1$ are established between auricular and ventricular beats.

(e) An entire failure of impulses to reach the ventricle, as shown by the fact that the ventricular rhythm bears no relation to the auricular.

The last three instances are rendered clearer by the aid of Fig. 76 .

If a single branch of the His bundle after its division is severed or crushed, disturbances of a different nature result. Both ven- 
tricles respond, but the ventricle in which the bundle has been divided reacts later than the other, apparently deriving its impulse by way of the ventricle with its bundle intact. In such cases, the contour of the ventricular complexes of the electrocardiograms alters (Rothberger and Eppinger). The changes which occur when the two bundles are cut are shown in Fig. 77. In leads from the esophagus and anus, the ventricular complex changes. The $R$ and $T$ waves are replaced by a large and extended diphasic wave which is first positive and later negative when the branch to the left ventricle is injured, and first negative and then positive when the right branch is severed.
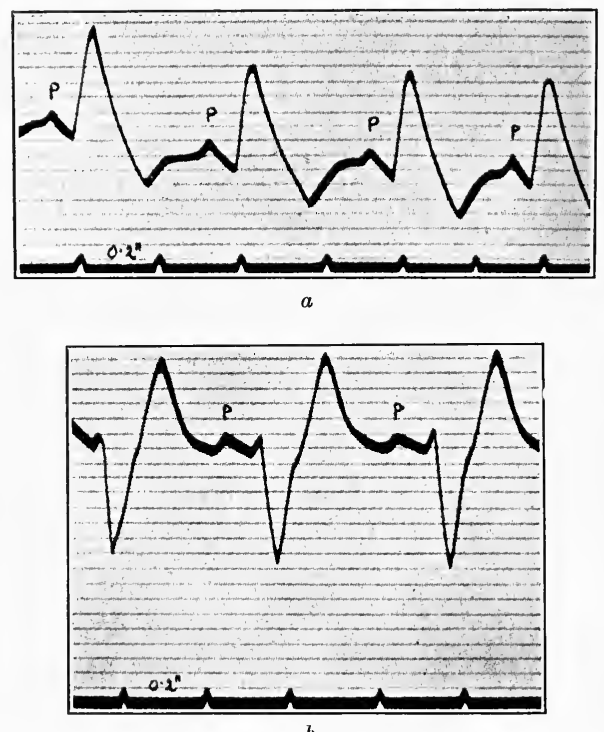

FIG. 77.-Effect of cutting (a) left branch and (b) right branch of the His-Tawara system on electrocardiogram (leads from anus and esophagus). (After Eppinger and Rothberger.)

Clinical Recognition of Heart Block.- (a) Partial Sino-ventricular Block.-Partial heart block may be accompanied by no subjective symptoms other than the existence of a slow pulse or the sensation of an occasional intermission. If the slowing is great, however, it may be accompanied by attacks of syncope similar to those described in complete block.

A scheme for determining the existence of minor degrees of block from the arterial pulse tracings alone was devised by Wenckebach, who diagnosed conduction disturbances, for example, in those forms of intermitting pulses in which the first wave after an intermission is longest, the next shorter, and the last one before 
another intermission longer again. Wenckebach contended that the conduction from auricle to ventricle was progressively decreased during activity so that the ventricle followed the auricle with a progressively longer interval until, finally the passage of the impulse failed altogether causing a pulse intermission. The long rest thus resulting increased the conductivity again and allowed a more rapid impulse conduction to follow.

In such an analysis, no account is taken of the varying transmission time of the pulse which may readily account for the slight variation present. Furthermore, it has been shown that such a periodic reduction in conductivity does not necessarily occur. It is therefore questionable whether disturbances in conduction can be safely determined from the arterial pulse tracings alone.

Either one of two things characterizes the venous pulse (Fig. $36, E)$ in this condition, viz., (a) a variation in the $a-c$ interval, and (b) the presence of $a$ waves followed neither by $c-v$ groups or arterial waves. The variation in the $a-c$ interval may be the only evidence of a disturbed conduction. When this interval becomes long, 0.6-0.7 second, such a disturbance may safely be assumed. The $a-c$ interval may lengthen from beat to beat until an intermission occurs. In many cases, however, no lengthening of this interval occurs. When $a$ waves occur in their regular sequence and are not followed by an $a-c-v$ group and an arterial pulse intermission, a periodic block is very likely present. The intermissions may recur at long or at short intervals, regularly or irregularly. If the arterial wave fails regularly so that two, three or four $a$ waves occur to every arterial wave, the condition is spoken of as a $3: 1$ or a $4: 1$ block (Fig. $36, E$ ). The electrocardiograms show normal $P$ waves recurring in regular sequence. These are followed, except when the ventricle fails to contract, by regular $R-S-T$ complexes. The $P-R$ intervals may not vary or they may increase in length to 0.3 second (normal $0.13-0.18$ ).

(b) Complete Sino-ventricular Block. - Complete heart block gives rise to certain symptoms and signs that make one at once suspicious of the condition. These are: a slow irregular pulse (30 to 40 per minute), signs of dyspnea and weakness on exertion. If these are accompanied by syncopal attacks, muscular twitchings and epileptic seizures (in short the characteristic Stokes-Adams syndrome) the diagnosis is quite certain. The Stokes-Adams syndrome may occur from any cause producing cèrebral anemia, but its association with very slow and irregular heart action is almost diagnostic. These attacks occur during two stages of the disease $(a)$ when block becomes complete and the ventricles stop entirely before inaugurating their independent rhythm, and $(b)$ after its establishment when the ventricular rate is rapid and the contractions small and ineffective in opening the semilunar valves. It does not necessarily 
follow that all forms of heart block are accompanied by a slow, irregular pulse. Cases of complete dissociation are known in which

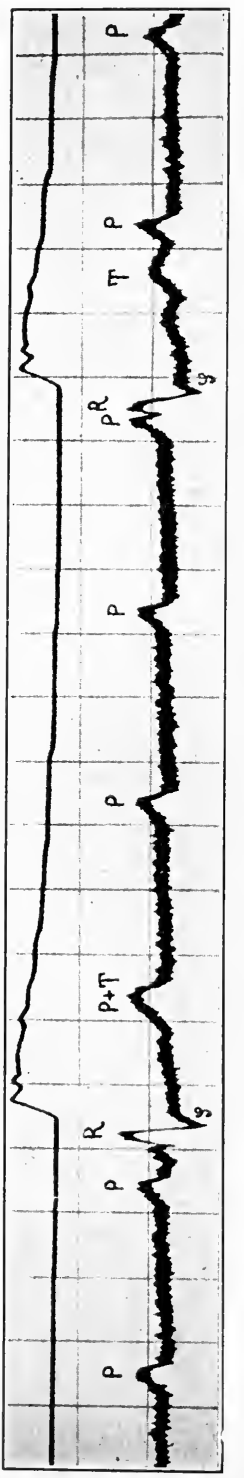

the rhythm is quite regular though slow (Fig. 78). The condition may be definitely recognized by combined arterial and jugular tracings or by the electrocardiogram. The jugular pulse shows a regular sequence of $a$ waves and a haphazard sequence of waves due to ventricular systole, which correspond to the arterial waves. The arterial pulse is always slow and usually irregular (Fig. $36, G$ ).

The electrocardiogram (Fig. 78) shows, at regularly spaced intervals, distinct $P$ waves which may be unusually large. Most of them are isolated but here and there, evidently without relation to the $P$ waves, groups due to ventricular contraction appear. In many cases these are of typical normal type. Occasionally, the typical form of the ventricular complex undergoes minor changes. Thus, $R_{11}$ may be relatively low and $S_{11}$ deep. Another variation consists in a broadening. of the $R$ wave which may stretch over an interval of 0.15 second (normal 0.04). All of these variations may still be regarded as showing that the block occurs above the division of the His bundle and that the new rhythm originates in the His system.

In other cases the ventricular complex resembles curves such as have been obtained on severing the right or left bundle (Fig. 77) and by analogous animal experiments; it has been assumed that the conduction disturbance extends to the branches as well. Rothberger and Winterberg recently pointed out that, inasmuch as the anus-esophagus leads correspond to lead III in man, one-sided block can be diagnosed in man only when leads $I$ and $I I I$ both give the same atypical complexes. If the atypical waves occur in one lead and not in the other, they are probably due to hypertrophy rather than to block (cf. Fig. 87).

Causation and Prognosis of Clinical Heart Block.-It has been established by careful histological study of postmortem cases of heart block that the condition is frequently associated with definite 
lesions of the $a-v$ node, His bundle, or its branches. . It is important to observe that no instance of complete destruction of the bundle has been found without the presence of heart block during life. Inflammatory reactions with edema, cellular infiltrations such as occur during rheumatic fever, influenza, typhoid, pneumonia and syphilis (see Fleming and Kennedy), septic foci, post inflammatory fibrosis, fatty and calcareous degenerations, ulcerations, tumors and, by far the most frequent, gummata, have all been found in cases that succumbed to this condition (see Lewis). When such lesions involvè the bundle, they are not as a rule confined to it but are distributed throughout the myocardium.

On the other hand, several investigators (Fahr, Krumbhaar, Mackenzie, etc.) have reported cases in which no lesion could be found in complete block, and the same condition has been frequently found in partial block. It must be supposed that in these cases either some toxic agent has modified the conductivity before it has had a chance to produce a pathological change, or that the rest of the heart muscle which does show pathological changes is unable to react to the stimuli conducted to it.

The prognosis must be considered from two angles; first, the amount of damage that probably exists in the heart, and second, the ability of the altered heart rate to maintain an efficient circulation.

If the heart block is complete and there is evidence of involvement of the branches as well, it may safely be assumed that extensive degeneration of the heart has taken place and the possibility that the patient's life may terminate at any time must be borne in mind. Mackenzie, who has probably had the widest experience, however, states that some cases may lead a quiet life for ten or fifteen years before death occurs. If the block is incomplete, it is not necessarily an indication of structural impairment of the heart, and the prognosis is more favorable unless the degree of block increases.

The question of immediate importance is whether the beat of the heart during block is capable of maintaining an efficient circulation. A certain minimal rate of beat is necessary to maintain the circulation under normal conditions and this rate may be entirely inadequate when excessive demands are made. The one source of danger in heart block lies in the fact that, during exercise, the adaptive increase in heart rate cannot take place. This is truer in the incomplete than in the complete block, for in the former it is possible that the block lessens on the removal of vagus influence. Hence it frequently happens that, although the circulation is carried on efficiently during repose, the patient suddenly succumbs during exertion.

Other things being equal, a regularly acting heart maintains a more efficient circulation than an irregular one. Of the irregular 
forms of ventricular action the slower rhythms preserve the circulation better than the rapid ones. The reason for this is that, in the slower rate, the majority of the contractions are effective and cause a considerable systolic output; while, in the rapid cases, so many of the weaker contractions fail entirely to open the semi-lunar valves and others produce a very small output so that a low arterial pressure accompanied by a damming back of blood takes place (cf. Fig. 83). The regularity and rate of the pulses are therefore of importance in forming a prognosis as to the immediate liability of circulatory failure.

\section{PREMATURE CONTRACTIONS (EXTRA-SYSTOLES).}

(a) Premature Ventricular Contractions.-Clinical Physiology. (Fig. 79.) - If a mechanical or electrical stimulus is applied to the normally beating ventricle of any vertebrate animal (man

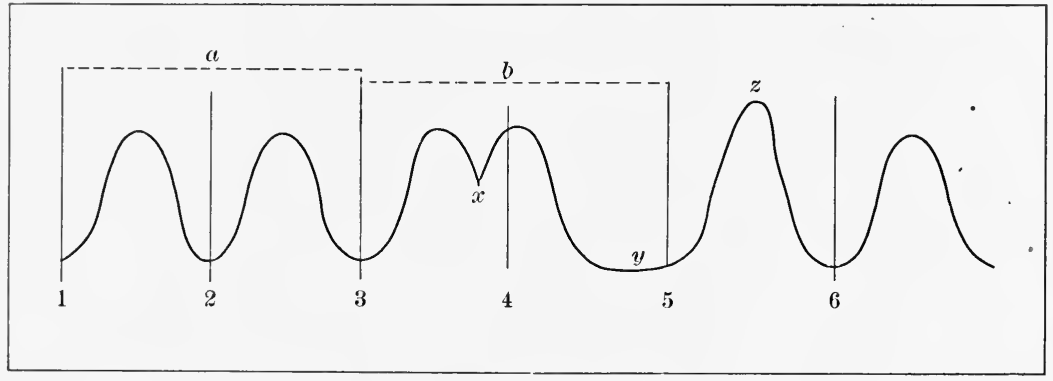

FIG. 79.-Diagram illustrating the nature and cause of the compensatory pause after a premature contraction. Lines 1, 2, 3, etc., represent time of arrival of sinus impulse: $x$, premature contraction in refractory phase of which normal impulse (4) falls. Pcriods $a$ and $b$ are equal.

included) during its diastolic phase, the ventricle will respond with a "premature" or "extra" contraction (Marey, Engleman, Meyer, Cushny and Matthews, etc.). If a similar stimulus is applied during its contraction, however, no response is given. When a contraction is prematurely called out by an external stimulus, the total number of beats is not increased, as a rule, but this premature beat is followed by a pause of such length that it plus the preceding contraction equals approximately an interval of two heart beats (Knoll). This "compensatory pause," as it is termed, exists because the normal sinus impulse which should evoke a ventricular contraction, reaches the ventricle during the refractory phase of the premature contraction. The next ventricular contraction does not occur until the following rhythmic stimulus from the sinus occurs. Careful measurements have shown, however, that this 
compensatory pause is not always exactly equal to two normal periods, but that it falls short of this. When this occurs it is probably due to the fact that the sino-ventricular conduction has improved during the long rest, making the contraction premature. The ventricular systole following that prematurely called out is usually larger than normal, which accounts in part for the larger pulse beats recorded in mamalian experiments.

It is evident that in the above cases, there is no beat gained by the heart; hence the term premature contraction seems preferable to extra-systole, so generally employed. It may happen, however, that true extra-contractions or "interpolated" systoles occur. Thus, when the cardiac rhythm is very slow and the premature contraction is elicited early in diastole, the ventricle may have passed its refractory phase before the next regular sinus impulse arrives.

Clinical Recognition.-Premature systoles of the ventricle may frequently be diagnosed by auscultation of the heart sounds alone. Optical records (Lewis, Gerhartz) indicate that the premature contraction causes two sounds when the semi-lunar valves open. The first sound is of shorter duration and smaller amplitude (35 per cent. less) but of the same vibration frequency as the normal sound. The second sound is also of smaller amplitude (10 per cent. less) and shorter duration but of higher frequency than the normal. Gerhartz quotes a case in which the first sound lasted 0.257 second (normal 0.461 second) and the second sound 0.095 second (normal 0.138 second). When the semi-lunar valves are not opened only a single sound is recorded.

In the main, these records corroborate the auscultation findings. The regular sequence of sounds is interrupted by two short sharp sounds or by only a single sound if the systole is ineffective. According to Mackenzie, it may be accepted that such a sequence during a pulse intermission or small wave is due to a premature contraction. The only confusion arises in cases of partial block with mitral stenosis in which a sound due to the contraction of the auricle may occasionally be produced, but in these cases there is always a presystolic murmur to differentiate.

The arterial pulse (Fig. $36, B$ ) is distinctive. The regular rhythm is interrupted by a premature small wave or the absence of a wave and a long pause. The characteristic feature pointed out by Cushny and Wenckebach, consists in the fact that the small contraction and the compensatory period following are equal approximately to two normal cycles, a form of pulse to which the term full bigeminus has been applied. The only deviation from the rule occurs when a beat is interpolated. In this case, the three beats of a trigeminal group equal two normal beats. The compensation period is followed, as a rule, by a wave of larger amplitude. This is due to the larger pulse pressure which results in part from 
the stronger systole, in part from the more complete diastole following, and in part from the lower diastolic pressure existing at the time.

The venous pulse (Fig. 36, B) is often characterized by the occurrence of a premature $c$ wave at the time of the premature systole. This is followed in its proper sequence by an isolated wave that may be attributed to subsequent auricular contraction (a wave).

The place of origin of the ventricular systole cannot be determined exactly by any of these procedures. It may be guessed at with a fair degree of certainty in certain cases. Thus, in cases of high arterial pressure or in aortic insufficiency, we may surmise that the stimulus arises in the left ventricle; whereas, in mitral lesions, the suspicion is directed toward the right ventricle (Hirschfelder). The electrocardiogram offers a more certain means of differentiating, however, since it is accompanied by distinctive variations for each ventricle. Kraus and Nicolai found that premature contractions elicited from the upper portion of the heart or right ventricle caused a positive variation followed by a negative wave (type $A$ ); whereas, one elicited near the apex or left ventricle caused a prominent negative variation followed by a positive variation (type $B$ ). These results are apparently correct but it was shown by subsequent work ( $c f$. Kahn) that the nature of the electrocardiogram depended largely on the lead. Thus, as is shown in Fig. 45, if the systole arises from the right ventricle, lead $I$ shows a variation of type $B$, while lead $I I I$ shows a variation of type $A$. The same relation obtains when the systole arises in the left ventricle. Here lead $I$ shows a variation of type $A$ and lead $I I I$ a variation of type $B$. (Cf. also Fig. 82.)

(b) Premature Auricular Systoles.-Clinical Physiology.-The auricle, like the ventricle is refractory to stimuli applied during the process of contraction. The reaction of the entire heart to stimuli applied during auricular relaxation depends, to a considerable degree, upon the region in which they are applied. A stimulus applied near the large veins or near the sinus node causes both the auricles and ventricles to respond with premature contractions, then, at an interval almost equal to another beat, both contract normally again. Such premature systoles are sometimes spoken of as sinus extra-systoles.

If, however, a stimulus is applied to the front portion of the auricle, its premature contraction is followed by a somewhat longer pause than the interval required for a normal beat. This pause is not so long, however, that the time interval of the two beats quite equals two normal beats (Fig. 36, C). 'The reason that the sinus region does not reëstablish the rhythm, as in other cases at the proper time has been attributed by Cushny. and Wenckebach 
to the fact that the extra stimulus is conducted back to the pacemaker and annihilates, as it were, the energy-producing substance without eliciting an actual contraction. It then requires an additional interval to rebuild its energy sufficiently to inaugurate a contraction. It is further found that the interval occupied by the two auricular beats is greater than the interval occupied by the corresponding two ventricular contractions. This has been assigned to the fact that the longer rest following the premature systole causes a reduction in the conduction interval from sinus to ventricle. The fact that the interval occupied by a normal plus a premature systole of the ventricle is equal to less than two beats, is used to distinguish premature systoles originating in the auricle from those originating in the ventricle. The degree of shortening of the ventricular beats, however, depends also on the phase of auricular diastole at which the premature contraction is called out. Thus, Hirschfelder and Eyster found that while the interval occupied by two ventricular beats was always less than that of two normal beats when a premature contraction was called out early in auricular diastole, the same interval very nearly equaled two normal beats when the auricular premature contraction took place late in diastole, for, in this case, the sino-ventricular conduction time is probably not increased.

Clinical Recognition.-It is evident from the experimental analysis:

1. That the period of premature contraction of the auricle plus a normal contraction equals less than two auricular periods.

2. That the premature response of the ventricle plus a normal beat usually equals an interval less than two normal periods, but when the premature contraction occurs very late in diastole, the double beats are almost exactly equal to two normal periods.

The arterial pulse is usually characteristic, if carefully studied (Fig. 36, C). 'The regular rhythm is interrupted by a small wave or an intermission, as in ventricular premature contractions. It differs from this condition in that the interval following is not sufficiently long so that it together with the preceding beat equal two normal pulse lengths; that is, in the case of auricular premature contractions, we get a shortened bigeminus. It is further possible to determine whether the premature contraction arose near the sinus region or in some other section of the heart. In sinus extrasystole the distance from the beginning of the premature contraction to the next normal beat equals a normal beat in length. This is not the case in premature systoles arising in other portions of the auricle.

The venous pulse (Fig. 36, $A$ ) shows throughout a normal $a-c-v$ sequence. Where the premature contraction occurs an $a-c-v$ group also appears prematurely. The $a-c$ interval is usually increased in this group, partly because the isometric period of 
contraction is prolonged and the opening of the semilunar valves delayed. The electrocardiogram is supposed, not only to establish the presence of premature systoles, but also to give further evidence as to the region of the auricle in which they arise. In the simplest cases there is merely an early occurrence of a normal $P-R-T$ complex. The $P$ wave may be superimposed on a preceding $T$, making it larger or notched. Occasionally it may be absent. It is usually better recognized in leads $I I I$ and $I$. Experimental work has indicated (Lewis, Ganter and Zahn) that the nature of the $P$ wave is determined largely by the region of the auricle from which the stimulus arises. Thus, while a systole arising in the sinus region gives a premature $P$ wave of normal form, those arising in the region of the coronary sinus or in the left auricle show a negative $P$ wave, presumably because the impulse spreads in the opposite direction. According to Lewis, most electrocardiograms from patients give evidence of a negative wave and so indicate that the stimuli arise in some region of the auricle aside from the normal pace-maker (Lewis). Since the ventricular complexes $R, S, T$ are normal, it must be inferred that the premature stimulus reaches both ventricles simultaneously which could probably occur only as long as it originates in the Purkinje system (Hoffman).

Not all premature auricular contractions are followed by such normal $R-S-T$ complexes in the electrocardiogram, however. According to Hoffman, they are rare. 'Undoubtedly this is because disturbances of conduction are often present in these cases, disturbances which may be due either to organic changes or to insufficient rest. The chief variations met with are: $(a)$ Complexes resembling those arising from a premature contraction of the right ventricle (cf. Fig. 45), or those arising when the branch to the left ventricle is cut (cf. Fig. 77). This may be explained by assuming that a conduction disturbance is present and the stimulus is propagated to the right ventricle before passing to the left.

It is apparent that electrocardiograms of premature auricular contractions may, on account of conduction disturbances, resemble, on casual examination, those arising in the ventricle. This is especially so when, in addition to the "aberrant" complexes, the $P$ wave is obscured by the preceding $T$ wave.

(c) Premature Contractions of Nodal Origin.-Clinical Physiology. -It has been shown in frogs that if the tissue of the $a-v$ junction is stimulated with a needle, a series of premature contractions occur simultaneously in auricle and ventricle. Such simultaneous beats of auricles and ventricles have also been found in cases of aconite poisoning (Cushny) and after ligation of a coronary branch (Lewis), and the assumption seems justifiable that in these cases the impulse arises somewhere in the junctional tissue. Owing to the fact that the impulse may reach either the auricle or the ventricle 
first, it usually happens that the contractions are not absolutely synchronous but that merely a reduced $A s-V s$ interval, or, in case the ventricle contracts first, a short $V s-A s$ interval occurs. This interval is not necessarily reduced when the impulses originate at the $a-v$ node - in fact, such a shortening occurs experimentally, only in those cases in which the impulse originates in the middle region of the node (Zahn).

Clinical Recognition.-The diagnosis of this condition in man hinges largely upon establishing the existence of a reduced $A s-V s$ interval, or the entire abolition of this interval. In this capacity the arterial pulse is of no value for it presents the picture of a ventricular premature contraction with a somewhat shortened compensatory pause. According to Mackenzie and Lewis the condition may be more than suspected $(a)$ when the $a$ and $c$ waves fall together so as to produce a bifurcated systolic wave, $(b)$ when the $a-c$ interval is appreciably reduced (Mackenzie, Wenckebach), and $(c)$ when the $a$ wave definitely follows the $c$ wave.

Too great caution cannot be urged, however, in the interpretation of these cases, for lever oscillations of polygraph tambours or premature ventricular systoles may similarly produce such summated or notched waves; and respiratory variations of venous pressure may apparently reduce the $a-c$ interval. Furthermore, according to Volhard, the occurrence of an $a$ wave after a $c$ wave may result from a retrogression of a ventricular impulse. Finally, we cannot always be sure that a certain wave is due to auricular systole.

The electrocardiogram shows a normal ventricular complex, RST, which indicates that we are not dealing with a ventricular premature systole as might be inferred from the carotid. The $P$ wave is abnormal owing to the opposite direction of the impulse. Its character is usually best seen in leads $I I$ and $I I I$ (Hoffman), when it appears as an inverted or negative wave. According to Ganter and Zahn, if the excitation proceeds from the coronary sinus, $P$ is diphasic. Occasionally, as Herzig points out, we may have a superposition of $P$ waves on the ventricular complex.

Causes and Clinical Significance of Premature Systoles.-Experimentally, premature contractions may be elicited otherwise than by direct stimulation. They have followed an increase in blood pressure (clamping the aorta, adrenalin), ligation of a coronary branch, and the injection of drugs raising the irritability of the heart (digitalis, adrenalin). Compression of the vena cava has been credited with producing them. Although the writer has many times compressed the vena cava in recent work, it has not once caused such a premature systole, nor have they been present when the venous pressure was seriously reduced as in hemorrhage and shock. In fact the tendency to these contractions has not even 
been increased. It seems that decreased general blood-pressure is not particularly favorable to their production.

The first evidence that premature contractions may be produced by nerve stimulation has been brought forward by the experiments of Rothberger and Winterberg, and Ken Kurè. These investigators showed that when the heart was under the influence of $B a$ or $C a$, or undergoing vagus stimulation, simultaneous accelerator stimulation might cause premature systoles.

It is probable that premature contractions may often arise in perfectly normal hearts. The pressing up of the diaphragm by a distended stomach or during pregnancy is sufficient to cause them. Again, they may occur when the pressure is increased by stooping, the abdominal vessels being compressed. More frequently, however, they arise in hearts that are abnormally irritable. It may be assumed in these cases either that the irritability of the heart is increased through toxins, internal secretion, caffein, nicotine, etc., so that it reacts to the tiny, normally ineffective stimuli that bombard it; or that pathological disturbances of structure are actually present. When they occur in advancing age, after infections and in association with hypertrophy and valvular lesions we should incline to the latter diagnosis.

The prognostic import of premature systoles seems to depend largely whether they are the only sign of irregular action or whether they are accompanied by other evidences of disturbed function. In the former case they are probably of little concern, for then, as Mackenzie points out, patients may lead vigorous lives without any other evidences of cardiac impairment. In the latter case they indicate disturbances in the structure and function of the myocardium. Their chief clinical value is that they attract attention to the cardiac condition, which leads to a search for the presence or absence of other symptoms of cardiac impairment.

\section{TACHYRHYTHMIAS.}

(a) Auricular Tachyrhythmia.-Clinical Physiology.-If the auricles of experimental animals are stimulated with a weak tetanizing current, their irritability is raised and they respond suddenly with an increased rate (Hertz and Goodhart, Jolly and Ritchie), often amounting to 300 to 420 per minute. Such a condition may be called auricular tachyrhythmia. Only exceptionally do the ventricles of animals respond to this rapid rate, but when they do we designate the condition as ventricular tachyrhythmia. More often the ventricle responds to every third, fourth or sixth beat and this response may be very irregular; in other words, a partial block arises. The writer has observed a similar relation after large doses of adrenalin both in animals in which the vagi have been previously 
cut and in perfusion experiments. In such cases adrenalin brings about a rapid increase in both auricular and ventricular rates until finally a pace is reached at which the ventricle no longer reacts to each stimulus and a partial block is established. Evidently we may have an accelerated rhythm of the auricle either accompanied or unaccompanied by an increased ventricular rate.

1. Paroxysmal Tachycardia.-This condition has long been recognized clinically by the sudden onset of rapid heart action, accompanied by palpitation, extreme cardiac discomfort and a sense of exhaustion, followed by as sudden a cessation and a resumption of the normal rhythm until the appearance of another attack. These attacks vary in duration from a few seconds to days or months.

The agreement has been reached that this pathological condition is due to an excessive irritability of the auricle causing it to react, first with a few premature contractions and later with a series of very small contractions recurring very rapidly, often at a rate approximately double that of the normal heart.

Clinical Recognition.-The clinical recognition is usually easy without graphic procedures. These, however, make the diagnosis more certain. The arterial pulse presents the typical picture of the paroxysmal attack ushered in by a few premature contractions of auricular origin followed by small contractions at a very rapid rate. The venous pulse, likewise, shows the regular rhythm, at first interrupted by these premature contractions of auricular origin. Later, the normal sequence of $a-c-v$ waves may be retained, or, if the pulse becomes too rapid, the $a$ wave replaces the $v$ wave or is even mounted on the descending limb of the $c$ wave (Fig. 36, $D)$. It is precisely in cases of such rapid action that the ordinary polygraph tambours introduce the greatest errors through lever fling and the addition of excessive waves, and it cannot be expected that they follow the changes in the venous pulse exactly.

The electrocardiogram gives evidence that the extra contractions and paroxysmal beats arise in the same area for the $P$ wave is always of the same character. It may be negative, as in the cases reported by Lewis and Rihl or excessively large, as in cases presented by Hoffman. The $P$ wave is followed by ventricular complexes which differ from the normal merely in that the $P$ wave is often superimposed upon or substituted for the preceding $T$ wave.

Vagus Compression during Tachycardia.-It has long been recognized that a reduction of the tachycardia could be accomplished in certain cases by vagus compression in the neck, while in others, this procedure was entirely without effect. Hoffmann found that when the rate of the ventricle was reduced by such a compression it was not due to any modification of the auricular rate which remained constant, but to the production of a partial vagus heart 
block. The block might be so complete that the ventricular beats entirely ceased, as shown in the electrocardiogram, in which case syncope supervenes. In other words, these cases may often be converted by vagus pressure into the type next considered, that is, those in which the ventricle does not follow each auricular impulse.

2. Auricular Flutter.-This condition was long unrecognized principally because all evidence of the rapid auricular contractions was obscured in the jugular by the irregular action of the ventricle. As a result of electrocardiographic studies, interpreted in the light of animal experiments, it may be said to be a condition of auricular tachyrhythmia imperfectly followed by the ventricle. Several effects may be induced: (a) The block may be partial and of a more or less irregular nature, in which case the ventricle responds to the third, fourth, fifth or eighth impulse from the auricle, or in which it periodically responds in different ratios; $(b)$ the block may be complete, the ventricle assuming an irregular rhythm as in heart block.

Clinical Recognition.-The existence of auricular flutter, according to Lewis, is often satisfactorily revealed by the arterial pulse tracing. This must apply to a very limited number of favorable cases. When complete block is present the heart may for long intervals be perfectly regular. The rate is then usually slow, but, according to Hoffmann, is not necessarily so. More frequently the block is irregular as shown in Fig. 80. In such cases the waves though irregular upon careful measurement, show groupings. Thus, in Fig. 80, one-half the period occupied by waves labeled 2 , is contained five times in the waves labeled 5 and four times in the waves labeled 4 .

The difficulty in analyzing the curves so as to make a diagnosis is very great. They are often complicated by a pulsus alternans (see page 299), which is confusing. Again, the amplitude of the waves is influenced by the variation in the preceding pauses, hence the pulse beats following a 2:1 period are weak and may simulate premature systoles. Lastly, the block is accompanied by $A s-V s$ intervals, presphygmic periods and transmission times which are variable and tend to disturb any exact measurements which should theoretically obtain. Thus, in Fig. 80, the three arterial waves labeled 2 are all of a $2: 1$ rhythm, as shown by accompanying electrocardiograms, yet the length becomes progressively shorter in consecutive waves.

In the venous pulse, the prominence of the $a$ waves is determined by the ventricular rate. When this is rapid with a $2: 1$ block, each auricular wave falls within a period of ventricular contraction and, hence, it is impossible to separate the one wave from the other. With a greater degree of block $(e . g ., 4: 1)$ the $a$ wave is clearer, 
especially toward the end of the diastolic period. In such cases, each venous wave, though probably due to ventricular contraction, may be taken to indicate that auricular systole has occurred. An optical record taken simultaneously with the subclavian pulse is

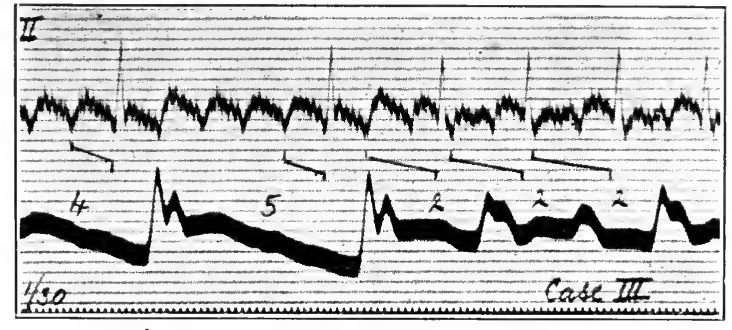

Fig. 80.-Radial curves and electricardiogram in auricular flutter, showing how the $A s-V s$ interval varies when the ventricle responds irregularly. Figures indicate the scheme of regular irregularity present. (After Lewis.)

shown in Fig. 81. The small waves recur during intervals apparently diastolic, at a rate of approximately 300 per minute.

The electrocardiogram (Fig. 80) shows a continuous series of oscillations which are sometimes clearly defined but often small

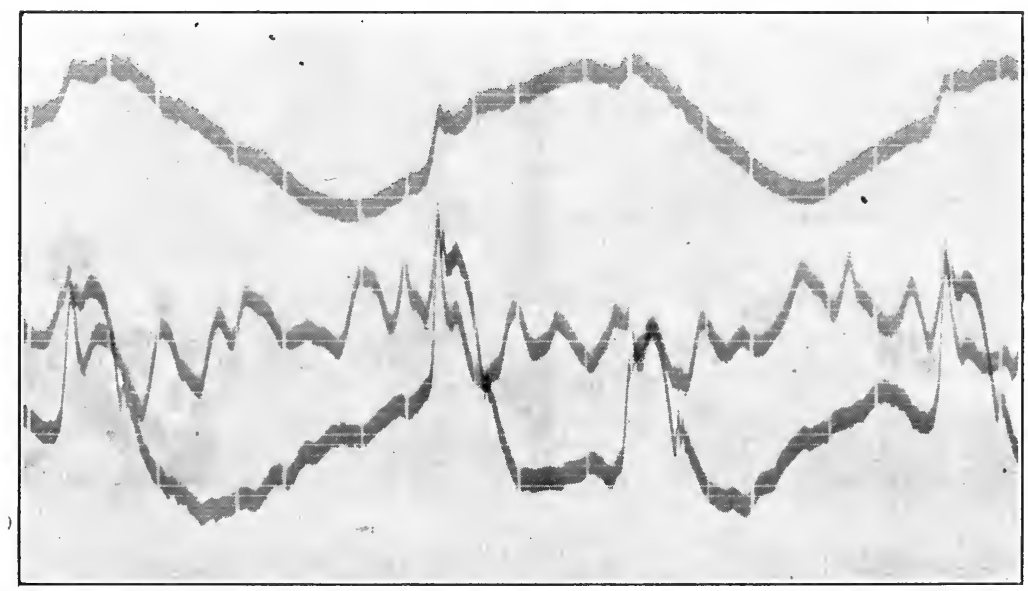

Fig. 81.-Optical tracings from case of auricular flutter. Respiration (upper), supraclavicular venous pulse (middle) and subclavian pulse (lower). Variations in contour of so-called $a$ waves very variable. $S^{1}$, first sound due to ineffective systole.

or pointed, or scarcely recognizable, appearing merely as a wavy line. They recur regularly at extremely rapid rates (120-300) except when replaced at intervals by a ventricular complex of $R-S$ variations. When a regular block occurs, the $R-S$ complex 
is regularly interposed between every third or fourth $P$ wave and its successor; when the block is irregular, however, as in Fig. 80 , these wave groups appear at irregular intervals and are accompanied by irregular $P-R$ intervals. The electrocardiogram indicates by the contour of the $R S T$ complexes that a condition of partial block rather than complete block with ventricular rhythm exists.

Tachyrhythmias of Atrio-ventricular Origin.-Clinical Physiology. - Just as extra-systoles may arise in the $a-v$ junctional tissues and cause a simultaneous contraction of the auricle and ventricle or a shortened $A s-V s$ interval, so it is conceivable that a series of such beats may occur and cause a tachycardia of auriculo-ventricular origin. It has been shown experimentally that the node of Tawara then becomes the pace-maker. Thus, after the application of formalin, or after excision or cooling of the sinus node, the auricles and ventricles may beat in synchronous rhythm (Lohman, Hering, Ganter, Zahn, Brandenburg, Hoffmann), the impulse arriving from the sino-ventricular node. A similar condition results when the sinus is beating rhythmically, as it does after stimulation of the left accelerator (Rothberger and Winterberg), and during asphyxia (Ken Kurè).

A nodal rhythm, then, appears $(a)$ when the sinus node is depressed or poisoned and $(b)$ when the sino-ventricular node or node of Tawara is stimulated. Quite recently Meek and Eyster and Zahn have corroborated the above findings and have proved, by recording the initial point of negativity, that either the auricular or the ventricular poles of the node may inaugurate the rhythm, and, furthermore, that the seat of impulse formation may shift from one portion to another upon vagus stimulation.

Clinical Recognition.--According to Hoffman, tachyrhythmias of nodal origin may be rhythmic or arrhythmic. In the rhythmic form the attacks quite closely simulate those of auricular origin from which they are distinguished only by a study of the electrocardiograms. The arterial pulse is at times small and again large and dicrotic (probably because of imperfect recording sphygmographs). The jugular pulse is characterized by systolic elevations in which $a$ waves are presumably merged. Many of the published records, however, are clearly arterial tracings from the neck.

In the electrocardiogram, the $P$ wave may be lost in the $R$ wave which may be increased or depressed. Sometimes a small preliminary wave on the $R$ indicates that the auricular contraction preceded the ventricular by a brief interval. Perfect regularity persists and the absence of complexes such as are typical for systoles of ventricular origin indicates that the rhythm must have originated in the supra-ventricular region. A shortening of the $P-R$ interval shows its junctional origin. Recently, Meakins (Heart, v, 285) has 
undertaken an experimental study to determine the shape of the electrical auricular complex during nodal rhythm and finds it variable. As a rule, it starts in a downward direction or may be entirely directed downward. Often no carotid pulse is felt upon cessation of this form of tachycardia, but the galvanometer string undergoes violent oscillations. These, upon analysis, resemble extra-systoles of left ventricular origin. After several such oscillation groups, the normal rhythm returns but in a somewhat accelerated fashion (100 per minute) (Hoffmann).

In some patients these irregular types of tachycardias alternate with intervals in which the pulse is regular, presenting no evidence of tachycardia. In others the irregularity is a constant feature. The paroxysms resembles auricular tachycardia, lasting hours or days. They differ in being extremely irregular as well as rapid. The electrocardiogram always shows typical waves such as originate in the Purkinje system which differ from the regular form described by the uneven character of the electrocardiogram. (For illustrations of these forms of electrocardiograms see Hoffmann.)

Tachyrhythmias of Ventricular Origin.-Experimental Physiology. - It has been demonstrated by Lewis that a series of extra-systoles may be elicited from the ventricles of experimental animals thus giving the effect of a tachyrhythmia of ventricular origin. When the ventricle is stimulated by repeated and accurately spaced electrical stimuli, "these stimuli are at first only periodically conducted back to the auricle and so call out only irregular auricular systoles. Gradually the conduction is improved so that more and more premature contractions occur until at last the auricle may accurately follow the pace set by the ventricle. In the dog the rate may reach 300 to 420 per minute. Similar results have been obtained by tying the coronary artery (Lewis), by stimulation after poisoning the heart with $B a$ or $C a$ and, also, by combined stimulation of accelerator and vagus nerves (Rothberger and Winterburg) (Ken Kurè).

Clinical Recognition.-The condition differs from other tachycardias by persisting for a shorter time, usually for from six to thirty beats (Hoffmann). Lewis reports a case lasting five minutes which is the maximum period reported. The rate approximates 210 to 300 per minute. The attacks also recur frequently.

The venous pulse shows a complex mixture of $a$ and $c$ waves, and those so far recorded seem of little value in differentiating the condition. Even the electrocardiograms are uncertain in determining whether or not auricular contractions exist and hence the question whether retrograde changes are present as in the animals is uncertain. According to the origin of the ventricular stimulus, these disturbances may be divided into three classes, as indicated by the electrocardiogram: (a) those in which premature 
contractions arise in the right ventricle exclusively, $(b)$ those in which they arise in the left ventricle exclusively, and $(c)$ those in

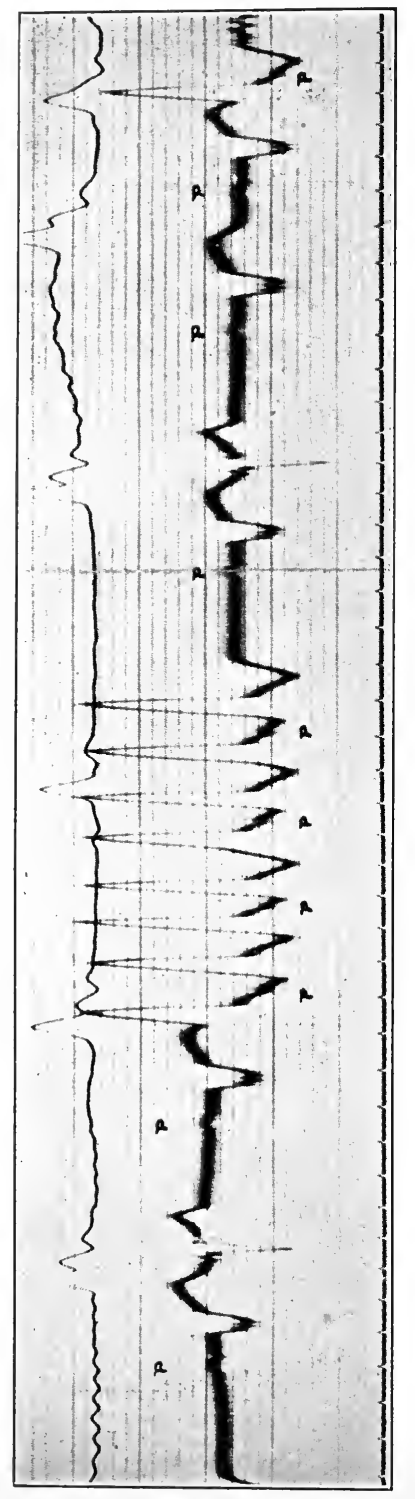
which they originate in both ventricles. As may be anticipated, the characteristic feature consists of 6-30 wave groups resembling complexes characteristic of right and left ventricular premature systoles. In the third group, mixtures of the two occur.

An example of the onset and effect of such a paroxysm of the right basal type is shown in Fig. 82 taken by lead $I I I$. The first beat is clearly a premature one and is followed by others similarly recurring at a rate of 240 per minute. The notch on the descending limb is possibly a $P$ wave due to reverse conduction. Between paroxysms the ventricular beats are often arranged in groups so that normal complexes alternate with premature complexes.

Pathological Significance of Tachycardias.-It is apparent that the paroxysmal tachycardias recognized by the sphygmograph are due to five distinct causes, but that all arise from some portion of the conducting system. In other words, a new pace-maker is developed which submerges the activity of the original. It is most probable that the primary cause must be sought in a fundamental disturbance of the heart. That these paroxysms may be called out through the agency of the central nervous system cannot

be denied in view of recent experimental evidence. It is apparent, nevertheless, that forms of tachycardia cannot now, as formerly, be regarded as without serious significance. 


\section{AURICULAR FIBRILLATION.}

Clinical Physiology.--If the auricle is stimulated with a tetanic current of moderate strength, the period of auricular acceleration first produced passes rapidly into a condition in which the auricle, instead of contracting, in an orderly rhythm, executes a series of minute rapid twitchings which at times merge into irregular but independent wavelets traveling short distances over the auricular surface. As to the cause of this fibrillating state, it has been commonly assumed of late, in accordance with the work of Mac William and Lewis, that incoördination results from the fact that the irritability of the stimulated areas increases so that they become independently and highly rhythmic, or, as it might be expressed, they initiate a rapid series of premature systoles which spread in all directions over the auricle. The inadequacy of this view has recently been pointed out by Garrey, who found that if the excited area is excised, or otherwise separated from the rest of the auricle, it ceases fibrillating while the rest of the auricle continues to do so. Further observations on the fibrillation of strips of various size and the ability of the process to pass small strips convinced this author that the condition is due to the induction of a series of small blocks within the auricular tissue making it impossible for the entire musculature to receive its stimuli at once. 'This makes it possible for the contraction wave to travel from one muscle cell to the other in a series of ring-like circuits of shifting location and of multiple complexity.

The ventricle during auricular fibrillation beats with a very irregular rhythm which, however, is coördinated and sufficient to maintain the circulation. Its rate is usually quite rapid. If, while the auricle is fibrillating, heart block is mechanically produced, through vagus stimulation or by the use of drugs, the ventricular rate slows and becomes more regular, as in an uncomplicated case of heart block (Fredericq, Lewis, Gerhardt). Cushny, in 1899, first suggested that the condition might explain certain types of perpetually irregular pulses in man. The idea appealed to Mackenzie and Wenckebach and its relation to such pulses was rendered more probable by the combined experimental and clinical studies of Lewis and Rothberger and Wintemberg, who showed that the electrocardiogram typical of this condition in the dog was practically reduplicated in man.

Hemodynamics.-The effects of such irregular ventricular sequence have been studied experimentally (Lewis, Wiggers). The efficiency, of the circulation apparently depends on the rate of the ventricular rhythm and the number of ineffective systoles resulting. Two types of cases may consequently exist, viz.: (1) those in which a fairly normal arterial pressure is maintained and 
(2) those in which the arterial pressure falls and venous engorgement ensues. The writer has analyzed the dynamics in these two groups in detail on the basis of optical manometer records obtained from the auricle, ventricle, and subclavian.

In Fig. 83, $A$, is shown a case of the former type. The upper record shows the variations in intraventricular pressure from beat to beat; the lower, the corresponding variations in subclavian pressure.

The isometric period extends from $A$ to $B$, the ejection period from $B$ to $C$. It is evident that systoles varying greatly in strength occur without definite order. The differences in the systoles of different strength manifest themselves in the gradient of the upstroke, in the contour of the ejection period, and in the slope of the relaxation curve. It is obvious that the variations in the upstroke and ejection period do not, as in hearts possessing a normal rhythm, depend much upon the initial tension, but more upon the interval between its onset and the end of the previous contraction. The waves occurring immediately after systole are smallest and the rise is more gradual. Arranging the waves from the smallest to the largest, those numbered $3,5,7,10,2$, and 9 may be placed in a series. When, however, an extra supply of energy is exhausted by two rapidly recurring systoles, the third wave as at 8 and 11 may be very small, even though a longer interval intervened. The rate of relaxation is directly related to the strength of systole; partly, at least, because in the weaker beats more blood is retained in the ventricles; partly also, perhaps, because the inherent rate of muscular relaxation is slower, although no direct evidence for this is obtained.

The pressure of an aortic pulse curve, as well as its shape, depends, to some extent on the strength of the ventricular systole, but is also governed by the height of the diastolic pressure at the end of the isometric period of cardiac contraction. Thus, while the gradient of the ventricular upstroke and the height of the curve in waves 10,13 , and 15 are not materially different, the size and shape of the corresponding arterial curves vary extensively. When the semilunar valves fail to open, as in wave 7 , no arterial wave is found and the second heart sound fails to occur; while in weaker contractions such as 3, 8 , and 11, no sounds can be heard by means of the stethoscope. This shows that the ventricle may contract and give rise to neither arterial waves nor heart sounds.

Quite a different picture is presented, however, by another class of experiments shown in Fig. 83, B. In this experiment, the heart was dilated, the venous pressure high, and the carotid mean pressure averaged $27 \mathrm{~mm}$. of mercury. The upper curve again represents intraventricular pressure; the lower, intra-auricular pressure. Each group of waves in a heart cycle becomes an interesting study 

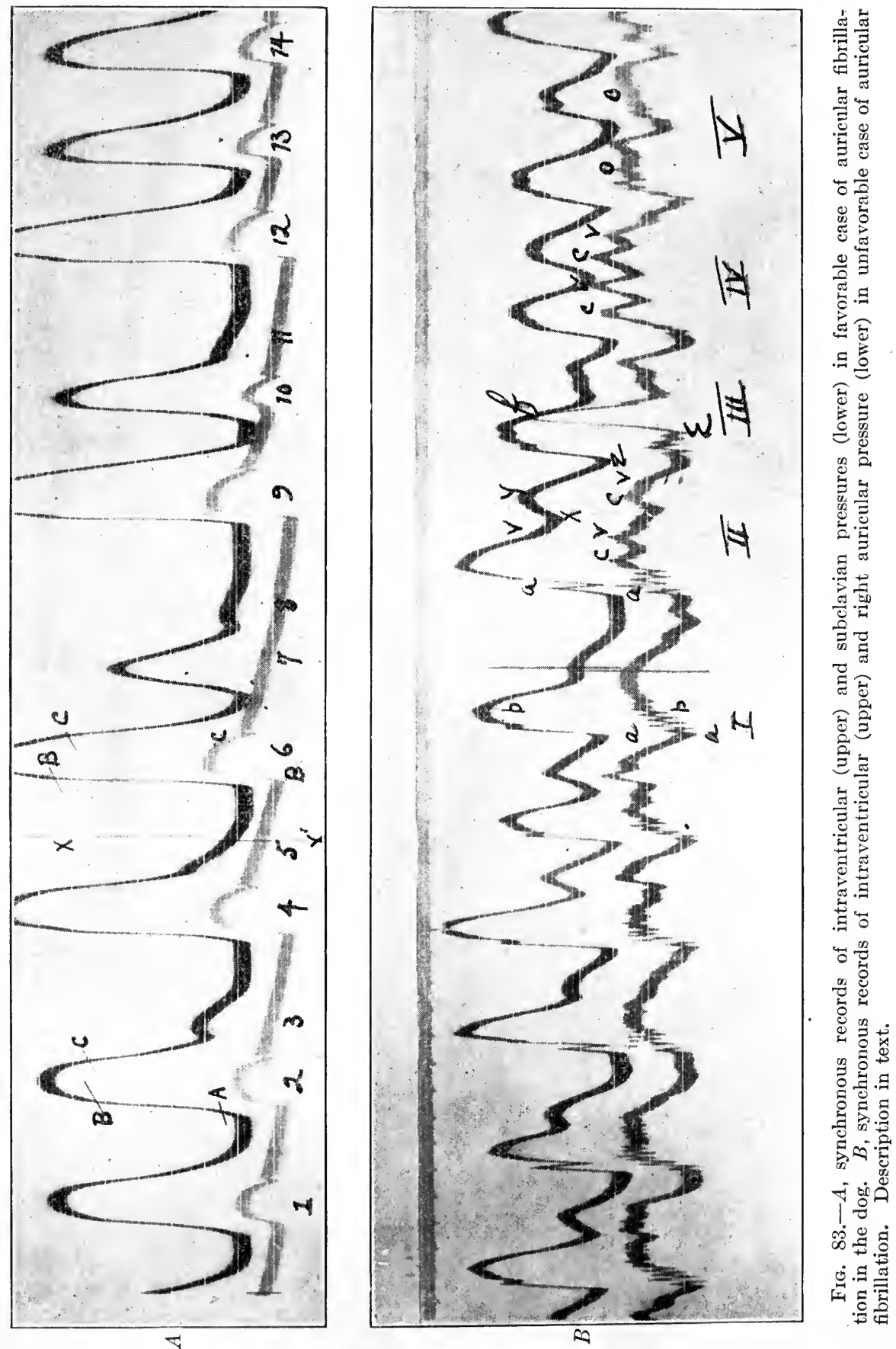
by itself. In group $I$, it is shown that the ventricle is still capable of giving strong beats. This intraventricular curve appears fairly normal in spite of some regurgitation which is made evident in the auricular curve, both by the sudden rise of pressure during ventricular systole and by the murmur vibration created at $a-b$. In group $I I$, a vibration occurs in the ventricle, which corresponds exactly in time with the first vibration in the auricle. Probably not so much regurgitation occurred in this beat. In the auriclés distinct $c$ and $v$ waves are produced. During the attenuated systole, $x, y$, and $z$, waves corresponding to the $a$ rise and $v$ fall are present. Without an intraventricular pressure curve as a guide, these waves might be interpreted as occurring during the diastole: of the previous beat or cycle. The third wave group shows, after a valvular vibration and at the beginning of the ejection period, a sudden rise, $e-f$, apparently indicating that the triscupid regurgitation was suddenly augmented during the height of the ejection period and permitted a sharp regurgitation. In the fourth group marked out, two weak ventricular systoles caused no regurgitation but produced a series of four distinct waves comparable to $c$ and $v$ waves. Similar ventricular contractions may, however, be accompanied by a regurgitation. This occurs in the two waves of group $v$, as shown by the immediate and sustained increase of pressure in the auricle, as well as by the murmur vibration superimposed at $O$. The chief deviation of the intraventricular pressure curve consists in the slow rate of relaxation which might result from a high degree of tonus or be due to a great accumulation of blood within the ventricle because the average systole takes place with too little vigor. Since the vigor of the systole is dependent on the frequency of the ventricular contraction, it is apparent that the most efficient circulation is maintained in auricular fibrillation when the beats are initiated somewhat slowly.

Clinical Recognition.-This condition is not as a rule difficult to diagnose. The arterial pulse alone is often diagnostic. The rate may be fast or slow, although a rapid rate is most frequent, if medicines have not been used. The characteristic feature is the absolute irregularity of beats. There is no sequence to be discovered in the irregularity. Beats of different size follow each other in varied order and it has often been pointed out that, contrary to other conditions, there is apparently no relation between the strength of the pulse beat and the intervening pause. From this the inference is sometimes drawn that the ventricle does not obey the law that the strength of contraction is inversely related to the preceding period of rest. The writer has recently shown, however, that this law is obeyed but that the seeming lack of relation between the size of the pulse and the interval intervening is due to the varying diastolic pressure existing at the time of the ejection. Since there 
is still some doubt whether all such types of completely irregular pulse are due to auricular fibrillation (Hoffman) it is desirable to study other graphic methods. The venous pulse, according to descriptions given by Mackenzie and Lewis are characterized $(a)$ by the entire absence of $a$ waves and $(b)$ by the presence of prominent waves rising synchronously with ventricular systole and often showing a sharp drop or notch in mid-systole. The details are well shown in Fig. 84. When the pause is long diastole is characterized, in addition to the $v$ wave and an $h$ wave, by small movements estimated to recur at the rate of $350-500$ per minute (Rihl, Lewis and Mackenzie). They are irregular in their periods and size as well as their occurrence and are attributed to auricular fibrillations. These ripples are well shown in an optical tracing in Fig. $84, f, f$.

Upon auscultation, characteristic changes in the heart sounds or murmurs develop with the onset of fibrillation. If a presystolic murmur has previously been present, when this disturbance is added to mitral stenosis, that murmur disappears (Mackenzie), while the early diastolic murmur due to ventricular filling persists. This is shown in the sound record of the murmur of Fig. 84 .

The electrocardiogram throws interesting light both upon the diagnosis of the condition and upon the origin of the impulses as well (Fig. 85). The curves are characterized (a) by a lack of waves typical of auricular contraction ( $P$ waves); $(b)$ by the presence, in irregular sequence, of normal $Q-R-S-T$ variations which vary in height; $(c)$ by the presence of small irregular oscillations varying in form and time intervals attributed to auricular fibrillation. They are usually present in this condition and in no other known affection. Some of these waves occasionally form larger waves which resemble in form such as might be due to auricular contractions. They have been attributed both to occasional auricular contractions (Hoffmann) and to tonus changes in the auricle. If present they are not related to ventricular complexes. In some cases the normal ventricular complex is replaced by one resembling that obtained when the impulse reaches one ventricle before the other. It is impossible to decide at present whether these complexes are due to an actual premature systole or to 'an impairment of the conduction through one bundle of the conducting svstem.

The question as to the place where the impulses causing the irregular ventricular contractions arise must remain an open one. That the impulses are of a supraventricular and not, as a rule, of ventricular origin is indicated definitely by the electrocardiogram and by experimental studies. The view was suggested by Lewis that the impulses originated in the auricle aside from the normal pace-maker and were passed to the ventricle in such profusion that it could react to only a small part of them. The suggestion 
298 DIAGNOSIS AND SIGNIFICANCE OF ARRHYTHMIAS
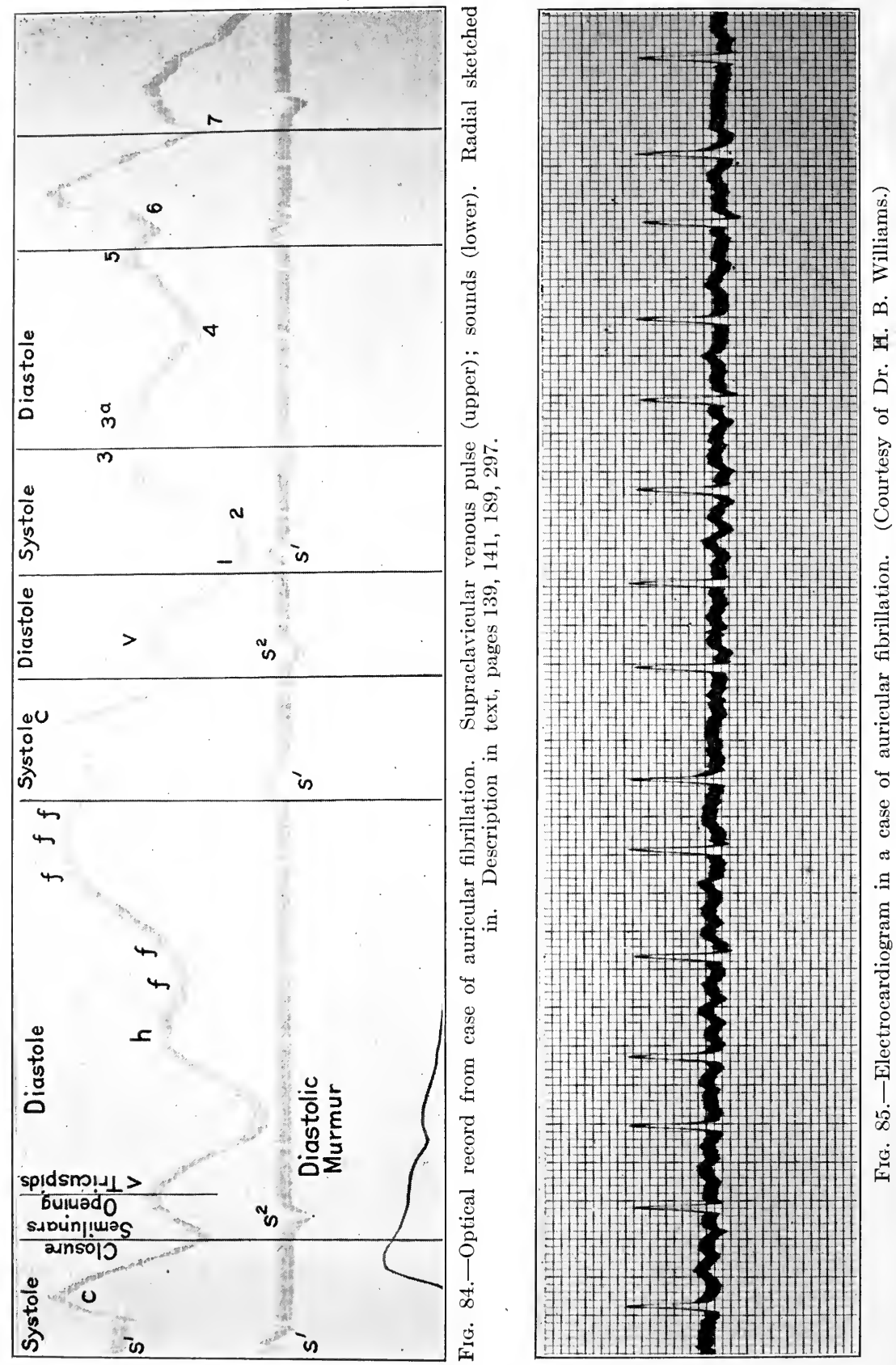
of Edens that the rhythm is given by the coronary sinus or that it may be originated in the $a-v$ node and connecting tissue is not at variance with the evidence at hand. In fact most of the evidence supports the idea that the pace-maker for the ventricle wanders in cases of fibrillation.

The influence of the vagus stimulation and of digitalis to slow the ventricular rhythm may also be explained upon either hypothesis. According to Lewis it increases the block for auricular impulses, but it is equally possible that it causes a shifting of the pace-maker for the ventricular beat.

Prognosis.- Auricular fibrillation is not infrequently associated with the late stages of mitral lesions, so that the irregular pulse has often been called the "mitral pulse." Histological examination of cases has revealed degenerative and inflammatory changes, or a production of fibrous tissue throughout the auricle and the conducting tissue (Koch, Mackenzie, Schönberg, Cohn, etc.). The existence of fibrillation may therefore be regarded as an indication of a general cardiac involvement. The immediate prognosis depends on how efficiently the heart is able to maintain the circulation under the strain of an irregular rhythm. The measurements of the highest and lowest systolic pressures, or the average systolic pressure is therefore capable of giving evidence as to the prognosis. Once present, fibrillation probably persists for the remainder of life. Mackenzie has reported a case in which it was present for thirteen years. Eventually death results through an embarrassment of the circulation. 'There is then a progressive failure of the heart, breathlessness, dropsy, liver enlargement, etc. Death follows after these symptoms have lasted days, weeks, or months.

\section{ALTERNATION OF THE VENTRICULAR CONTRACTIONS.}

Clinical Physiology.-When the intact or isolated heart is subjected to adverse conditions or is frequently stimulated (as is frequently observed in student laboratories) the ventricular beats recur in pairs, one of each pair being weaker than its fellow. They may be perfectly rhythmic or the smaller beat may occupy a shorter time interval. Similar alternation may be produced by depressing drugs, as aconite, glyoxylic acid, and, as the writer has observed, with pituitary extract. The dynamic difference in strength is evidenced by any mechanical form of registration, by the myocardiograph, the intraventricular pressure or the arterial pulse tracings. The study of the latter two shows in the weaker beats a slower rate of contraction, a longer isometric period and, consequently, a reduction in the ejection period and systolic discharge (Kahn and personal observations). 
The electrocardiogram shows normal $P-R-T$ waves. During the feebler beats $T$ and $R$ may decrease in height together, or $T$ alone may be smaller. The cardiac cycle is usually of the same length.

Pulsus alternans has been repeatedly investigated. As to the cause of the smaller beats three general views are held: $(a)$ that owing to local block in the ventricles, the impulses are not received by all the muscle fibers, hence that a maximum contraction of only a portion of the fibers occurs (Gaskell, Muskins, Galli); (b) that, similarly, only a part of the ventricular fibers contract, due to the fact that certain ones have a longer refractory period; $(c)$ that all the fibers contract, but owing to a periodic variation in their con- tractility or state of irritability, they do not contract to a maximum degree during the smaller beats (Hoffmann, Engleman, Wenckebach, Fredericq).

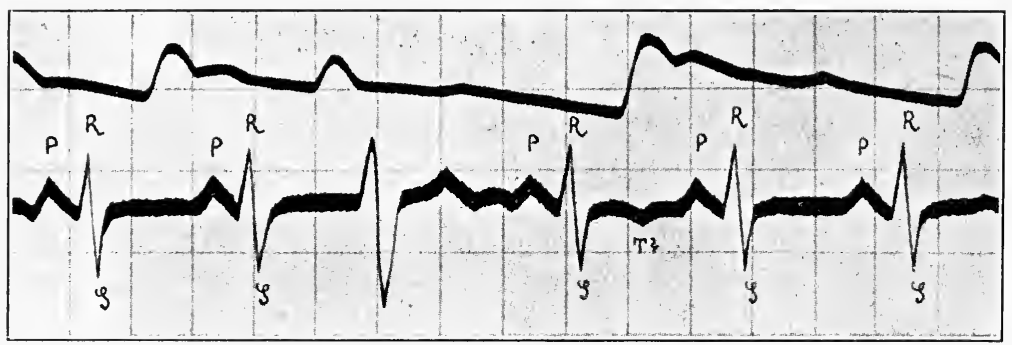

FIG. 86.--Radial pulse and electrocardiogram in case of pulsus alternans. (After Kahn.)

Clinical Recognition.-(Fig. 86). The condition was first recognized by the alternating heights of pulse waves, but was frequently confounded with bigeminal pulses due to recurring premature contractions. These two conditions may be distinguished by the fact that the beats in pulsus alternans recur regularly. If any slight variation does occur, the larger beats may occupy a somewhat longer interval, which probably occurs because the period of isometric tension of the preceding smaller beat is longer. In the case of premature systoles, on the contrary, the intervals are never equal, and the smaller beats occupy the longest interval.

It may happen that the condition is complicated by premature contractions in which case curious complexes are produced (Fig. 86). The electrocardiogram shows a normal sequence of $P, R$, and $T$ waves. Variations in the duration of systole and diastole are usually not present, indicating that the pulse variations may not be a true criterion of the actual length of the cardiac cycles. The waves may show alternations in height which do not always correspond to the pulse variations, however. In many cases no 
variation occurs when the pulse shows the alternation plainly. This suggests that possibly the electrocardiogram waves are an expression of excitation rather than of conduction. At any rate, the electrocardiogram is not a certain means of recognizing this affection. When premature systoles occur, as in the third complex of Fig. 86, they may be readily recognized by their peculiar contour.

Pulsus alternans may also be associated with other forms of irregularities. It frequently accompanies auricular tachyrhythmia (auricular flutter) as well as paroxysmal tachycardia of the whole heart.

Prognosis.-The existence of pulsus alternans indicates a damaged condition of the heart muscle or that the heart is unable to react to a greater strain. The prognosis, therefore, depends upon the associated disturbance. If we find it present during rapid heart action following exercise or during paroxysmal tachycardia it may indicate that the muscle is unable to meet the strain for the time being. It is then not of so serious a nature. If, however, the pulsus alternans comes on during normal action of the heart, it can be due only to extensive degenerative changes, sclerosis or other involvement of the heart muscle. No hopeful prognosis can then be given and the danger is even more pronounced if the pulsus alternans is accompanied by pains of an anginal character.

\section{BIBLIOGRAPHY.}

Books AND Monograms.

Cowan. Diseases of the Heart and Aorta, 1914, London, Philadelphia.

Hirschfelder. Diseases of the Heart and Aorta, 1913, 2d ed., Philadelphia.

Hoffman. Die Electrocardiographie, etc., 1914, Wiesbaden.

Kahn. Das Electrocardiogram, Wiesbaden, 1914.

Kraus and Nicolai. Das Electrocardiogram des gesunden u. kranken Menschen, 1910, Leipzig.

Krehl. Die Erkrankungen des Herzmuskels u. die nervösen Herzkrankheiten, 1913, 2d ed, Wien Leipzig.

Le Clercq. Maladies du cour et de l'Aorta, 1914, Paris.

Lewis. Mechanism of the Heart Beat, 1911, London.

Lewis. Lectures on the Heart, 1915, New York.

Mackenzie. Diseases of the Heart, 1913, 2d ed., London.

Ritchie. Auricular Flutter, 1914, New York.

Wenckebach. Die Arhythmia als Ausdruck bestimter Functionsstorungen des Herzens, Leipsic, 1903.

\section{Pafers.}

\section{Literature Dealing with Sinus Rhythm and Arrhythmia.}

Bowen. Contributions to Medical Research Dedicated to V. C. Vaughan, 1903, p. 462 .

Cohn. Proc. Soc. Exper. Biol and Med., 1912, x, 36.

Eyster and Meek. Heart, 1912-13, iv, 59.

Gasser and Meek. Amer. Jour. Physiol., 1914, xxxiv, 48.

Lombard and Pillsbury. Amer. Jour. of Physiol., 1899, iii, 201.

Meek and Eyster. Heart, 1914, v, 227. 
Putzig. Ztschr. f. exper. Path. u. Therap., 1912, xi,.115.

Reyfisch. Berl. klin. Wchnschr., 1905, xlii, 1468.

Williams and James. Heart, 1914, v, 109.

Zahn. Arch. f. d. ges. Physiol., 1913, cli, 247.

\section{Literature Dealing with Heart Block.}

Cohn. Heart, 1912-13, iv, 7 .

Cohn and Trendelenburg. Arch. f. d. ges. Physiol., 1910, exxxi, 1.

Clement. Ztschr. f. Biol., 1912, lviii, 110.

DeWitt. Physician and Surg., 1910, xxxii, 4.

Eppinger and Rothberger. Ztschr. f. klin. Med., 1910, lxx, 1.

Eppinger and Stoerk. Ztschr. f. klin. Med., 1910, lxxi, 157.

Erfman. Ztschr. f. Biol., 1913, Ixi, 155.

Erlanger. Jour. of Exper. Med., 1906, viii, 58.

Eyster and Meek. Heart, 1914, v, 119.

Fahr. Arch. f. Path. Anat., 1907, clxxxviii, 562.

Garten. Skan. Arch. f. Physiol., 1913, xxix, 114.

Hering. Arch. f. d. ges. Physiol., 1905, cvii, 97; 1905, cviii, 267; 1906, cxi, 298.

Humblet. Arch. internat. de Physiol., 1905 6, iii, 330.

Koch. Med. Klinik., 1911, xii.

Krumbhaar. Bull. of the Ayer Clin. Lab., 1910, vi.

Lewis, White, Meakins. Heart, 1914, v, 289.

Oppenheimer and Williams. Proc. Soc. Exper. Biol. and Med., 1913, x, 86.

Rothberger and Mintemberg. Zentralbl. f. Herz u. Gefässkrank., 1913, v, 206.

Thorel. München. med. Wchnschr., 1909, xlii, 2159.

\section{Literature Dealing with Tachyrhythmias.}

Agassiz. Heart, 1912, iii, 353.

Brandenburg and Hoffman. Med. Klinik, 1912, viii, 16.

Cohn. Heart, 1910, ii, 170.

Cluzet et Rebattu. Jour. de physiol. et path. gén., 1912, xiv, 81, 97.

Cushny. Jour. Exper. Med., 1899, iv, 327.

“ Heart, 1909, i, 1.

Cushny and Mathews. Jour. of Physiol., 1897, xxi, 213.

Dresbach and Mumford. Heart, 1914, v, 197.

Edens. Deutsch. Arch. f. klin. Med., 1910, c, 221.

Engleman. Arch. f. d. ges. Physiol., 1896, lxii, 543; 1894, lix, 309; 1894, lvi, 149.

Ganter and Zahn. Arch. f. d. ges. Physiol., 1912, cxlv, 335.

Hering. Arch. f. d. ges. Physiol., 1911, cxli, 497; 1910, cxxxi, 572.

" Ztschr. f. exper. Path. u. Therap., 1905, ii, 75.

Hering and Rihl. Ztschr. f. exper. Path. u. Therap., 1906, ii, 510

“ “ Arch. f. d. ges. Physiol., 1912, cxlviii, 169.

Herzog. Deutsch. Areh. f. klin. Med., 1912, cv, 235.

Hirschfelder. Johns Hopkins Hosp. Bull., 1908, xix, 322; 1906, xvii, 337.

Hirschfelder and Eyster. Amer. Jour. of Physiol., 1907, xviii, 222.

Hoffmann. München. med. Wchnschr., 1909, xlvi, 2258.

James and Hart. Am. Jour. Med. Sci., 1914, cxlvii, 63.

Jolly and Ritchie. Heart, 1910, ii, 177.

Kahn. Zentralbl. f. Physiol., 1909, xxiii, 444.

Ken Kurè. Ztschr. f. exper. Path. u. Therap., 1913, xii, 389.

" “ Deutsch. Arch. f. klin. Med., 1912, cvi, 33.

Knoll. Wien. Sitzungsb., d. k. Akad. d. Wien., 1892, lxvi, iii, 224.

Laslett. Heart, 1909, i, 83.

Lewis. Heart, 1910, i, 43-98; 1910, ii, 23, 1912-13, iv, 171.

Lohman. Arch. f. Anat. u. Physiol., 1904, 431.

" Arch. f. d. ges. Physiol., 1908, cxxiii, 628.

Marey. Jour. de l'Anat. et de la Physiol., 1877, xiii, 60.

MeWilliam. Jour. of Physiol., 1887, viii, 296; 1888, ix, 345.

Meakins. Heart, 1914, v, 281.

Meek and Eyster. Heart, 1914, v, 227. 
Meyer. Arch. de Physiol., 1893, 184.

Rihl. Ztschr. f. exper. Path. u. Therap., 1913, xiii, 1; 1911, ix, 277.

Ritchie. Quart. Jour. Med., 1913, vii, 1.

Rothberger and Winterberg. Zentralbl. f. Physiol., 1911, xxv, 189. “ “
Arch. f. d. ges. Physiol., 1911, cxlii, 461; 1911, cxli,

$343 ; 1910, \operatorname{cxxxv}, 506$.

Stuart Hart. Heart, 1913, iv, 128.

Turnbull. Heart, 1911, iii, 89.

Volhard. Ztschr. f. klin. Med., 1904, liii, 475.

Zahn. Zentralbl. f. Physiol., 1912, xxvi, 495.

\section{Literature Dealing with Aurucular Fibrillation.}

Cohn. Heart, 1913, iv, 221.

Cohn and Lewis. Heart, 1913, iv, 15.

Cushny and Edmunds. Am. Jour. Med. Sci., 1907, cxxxiii, 66.

Falconer and Dean. Heart, 1912, iv, 87.

Fredericq. Arch. internat. de. physiol., 1904, ii, 281.

Freund. Deutsch. Arch. f. klin. Med., 1912, cvi, 1.

Garrey. Am. Jour. Physiol., 1914, xxxiii, 397.

Gerhardt. Cor., Bl. f. schweiz. Aerzte, 1911, xli, 801.

“ Zentralbl. f. Herz. u. Gefässkrkh., 1910, ii, 339, 373.

Hewlett. Heart, 1910, ii, 107.

Koch. Berl. klin. Wchnschr., 1910, ii, 1108.

Lewis. Heart, 1909-10, i, 306.

“ Jour. Exper. Med., 1912, xvi, 395.

Lewis and Mack. Quart. Jour. Med., 1910, iii, 273.

Mackenzie. Quart. Jour. Med., 1907-08, i, 38.

Rihl. Ztschr. f. exper. Path. u. Therap., 1910, vii, 693; 1910, viii, 446.

Rothberger and Winterberg. Arch. f. d. ges. Physiol., 1910, xxxl, 387. " "

Schönberg. Frankf. Ztschr. f. Path., 1909, ii, 153, 462.

Wenckebach. Arch. f. Anat. u. Physiol., 1907, p. 1.

Wiggers. Arch. Int. Med., 1915, xv, 77.

\section{Literature Dealing with Alteration of the Heart.}

Cushny. Heart, 1909, i, 1.

Engleman. Arch. f. Anat. u. Physiol., 1902, p. 103.

Fredericq. Arch. f. d. ges. Physiol., 1913, clxi, 106.

Gaskell. Phil. Tr. Roy. Soc. London, 1882, clxxiii, Part III, 993.

Hering. Ztschr. f. exper. Path. u. Therap., 1909, vii, 363; 1912, x, 14.

Hoffman. Arch. f. d. ges. Physiol., 1901, lxxxiv, 130.

Kahn. Arch. f. d. ges. Physiol., 1911, cxl, 471.

Kahn u. Starkenstein. Arch. f. d. ges. Physiol., 1910, cxxxiii, 579.

Muskins. Jour. of Physiol., 1907, xxxvi, 104.

Rihl. Ztschr. f. exper. Path. u. Therap., 1906, iii, 274.

Selenin. Zentralbl. f. Herz u. Gefasskr., 1914, vi, p. 57.

Windle. Heart, 1910, ii, 95. 


\section{HYPERTROPHIC COMPENSATION, CARDIAC INSUFFI- CIENCY, AND DILATATION.}

\section{HYPERTROPHIC COMPENSATION.}

Clinical Physiology.-By hypertrophy is meant an increase in size of the muscular elements of the heart. It is an anatomical term. By hypertrophic compensation is meant the increased function possessed by hypertrophied heart muscle. It is a physiological term which implies the existence of hypertrophy. The presence of anatomical hypertrophy, on the other hand, is not necessarily indicative of hypertrophic compensation, for it is quite possible, as we shall see, for such hypertrophied muscle to be below par in function and to exhibit evidence of hypertrophic cardiac insufficiency.

How is hypertrophic compensation brought about? It has been shown by Frank in the case of frog's hearts placed in communication with artificial circulation schemes and, for the heart intact within the body (Wiggers, Straub, Patterson, Piper and Starling) that, when the peripheral circulation is not changed, the height and steepness of the intraventricular pressure curves increase directly as the initial pressure (Fig. 10). This means that the power of the heart to respond increases with the quantity of blood in the ventricles during diastole. The more blood the cardiac chambers receive, the more efficiently they pump it out, thus maintaining an equilibrium in the circulation. There comes a time, however, as Frank has shown, when the heart instead of reacting to an increased supply by an increased output, responds by a diminished contraction. The intraventricular pressure that may be created before this occurs, measures the reserve power of the normal heart. Very much the same process occurs when the normal heart is submitted to augmented strain, as an extreme rise of arterial blood-pressure or during aortic stenosis or aortic insufficiency. Thus, in experimental aortic insufficiency, when a greater volume of blood and a higher pressure exist in the ventricle, the ventricle immediately responds with a greater contraction.

The strength of the cardiac contraction may be modified by chemical agents quite independently of the initial pressure and diastolic volume. Thus, by way of illustration, the height and gradient of the intraventricular pressure curve decrease after 
depression by pituitary extract and increase after stimulation by adrenalin. It is not improbable that a similar effect may be exerted on the heart by toxic and infectious substances. When either or both factors persist for long intervals it can be shown experimentally that an increase in the size or a hypertrophy of heart muscle occurs (cf. Stadler, Stewart). This results not merely from a better blood supply (in fact many conditions with increased blood supply show no hypertrophy) but from the fact that the cells have received some stimulus (mechanical or chemical) which increases their liberation of mechanical energy. By virtue of this their metabolism is placed upon a higher level and so tends to bring about an increase in their size ( $c f$. also Edens).

It is probable that the hypertrophy resulting in man when the heart is placed under additional strain comes about in a similar manner. A great deal of discussion has arisen as to whether the increase in initial tension which serves to cause temporarily the augmented beats is alone responsible for the increase in size. Külbs' experiments, showing that hypertrophy in animals can be produced by the strain of exercise; and the well-known enlargement present in the so-called athletic heart speak in favor of the possibility of a purely mechanical origin of the hypertrophy. The question is, however, more complicated when the hypertrophy follows disease processes. That it is a reaction to increased work is evident since it is often present in cases in which the initial pressure has been increased. That this is not the only cause, however, is indicated by these observations: (1) that usually hypertrophy involves both sides of the heart equally or that the hypertrophy is out of all proportion to that possible from mechanical factors alone; (2) that the hypertrophy is generally accompanied by degenerative changes indicating that a toxic stimulus has acted upon the cells. It is probable, therefore, that the hypertrophy found in diseased conditions at least results from the action of the combined mechanical and toxic stimuli on the cell nutrition.

Hypertrophy of the muscle cells may therefore be looked upon as evidence that the contracting power of the heart has been previously augmented. Functionally, it is apparently a protective process. Although the larger cells react as did the normal cells, there is this difference: The normal heart submitted to continued extra work has nearly exhausted its reserve power, so that the imposition of additional work would result in less efficient beats. Graphically, the intraventricular curve would reach a lower summit, as shown in Fig. 10. The hypertrophied cells on the other hand have acquired with their anatomical enlargement a functional increase of their reserve power, so that the margin of safety is equal or even beyond that of the normal heart beating under the usual conditions (Romberg and Hasenfeld, Külbs). 
Cardiac Insufficiency.-Cardiac insufficiency may be said to occur when the heart fails to discharge as much blood as it receives. From a dynamic aspect, insufficiency occurs as soon as the maximum level of intraventricular pressure and the steepness of the isometric rise no longer increase with a rise of the initial tension in the ventricle (Fig. 10). When this happens the systemic pressures fall and the heart dilates. Dilatation and cardiac insufficiency have therefore become almost synonymous words. This is very unfortunate, however, for we may have dilatation in a perfectly sufficient heart. Thus, a very slow and efficient heart may be dilated owing to the fact pointed out by Henderson that the degree of diastolic distention is a function of cardiac diastole. Such an increased filling is largely equalized, however, by the more efficient systole which follows.

Dilatation may accompany cardiac insufficiency when the elastic resistance of the myocardium is overcome by the higher initial intraventricular pressure and the ventricle is passively distended. Dilatation, however, also occurs when the tonus diminishes-using the word tonus in its restricted sense, that is, a partial state of muscular contraction due to the slowing of the relaxation. Such a dilatation may be designated as active. It is probably a cause rather than an accompaniment of cardiac insufficiency; for, in such a case, the maximal efficiency for any need is not reduced primarily by a defect of the contraction power. On the contrary, it occurs because the lower tonus accommodates more fluid without increasing the initial tension, and so fails to supply the conditions necessary for a stronger beat to expel the blood.

On the other hand, the heart may become inefficient from too high a state of tonus, for then the capacity is so reduced that the output per beat is diminished and the maximum intraventricular pressure becomes lower.

It is clear, then, that insufficiency of the heart may be due to several causes and is not necessarily synonymous with dilatation or loss of tonus. Frequent association with loss of tonus is indicated by the experiments of Hirschfelder, who found that clamping the aorta, for example, caused, first a decreased output and consequently a passive dilatation of the heart. If the heart was in poor condition the dilatation became progressively worse. If, however, the heart was in good condition, the dilatation progressively diminished. It is possible that this was due to the counteracting influence of tonus but the fact has not been excluded that the reduced dilatation was traceable to the larger amplitude of discharge which is clearly evident in the published curves.

It is often difficult to determine which condition predominates when a dilatation is present. We may attempt to analyze a few types of cases in the light of experimental work. In the first place, 
it is commonly agreed that in normal men the pulse accelerates, the arterial pressure rises and the heart outline, as studied by the orthodiagram, diminishes during sudden, vigorous exercise (Dietlen). Whether the diminution in size of the heart is entirely the result of the increased heart rate or whether a true tonus exists cannot be said. If strenuous exercise be persisted in by persons unaccustomed to it, the arterial pressure soon falls and the heart area enlarges, while the patient suffers from acute exhaustion. Why does the heart dilate? It can scarcely be attributed to an increased rate. It is also improbable that the increased venous pressure commonly noticed in such cases is a cause-more probably it is an effect. It must therefore either be assumed that the tonus decreases or that the reserve power is passed and the heart fails to pump out the blood supplied. It is impossible at present to decide which occurs. In either case cardiac insufficiency is clearly present. In pathological cases which are even more complicated the cause and significance of the dilatation cannot be determined. The relative roles played by incomplete contraction and failure of tonus must be considered as not established. As older clinicians held largely to the idea of passive dilatation, so the recent tendency has been to give to tonus the entire responsibility. It may be true that the failure of tonus in certain cases seems alone to account for the condition (Mackenzie) but it is not necessary to assume that all cases are concerned with such a failure. The whole question deserves a careful reinvestigation.

While cardiac insufficiency sometimes occurs in normal hearts, it is always the sequel of hypertrophy.

To the question as to why this inevitable sequence occurs, it may be answered: (1) that the process of hypertrophy may be merely the first effect of a toxic action which finally leads to degenerative changes in the muscle thus impairing its contractile power and tonus, and which brings about a connective tissue proliferation that may develop into a marked myofibrosis (Krehl); (2) that the part of the heart which most needs compensation is not always the one to develop it, since it has often been found post mortem and by the electrocardiograms that the greatest hypertrophy may occur in the right ventricle in aortic disease or in the left in mitral lesions (Lewis); and that finally, (3) irregularities due to associated myocardial lesions often occur which tend to interfere with the efficiency of the circulation.

Clinical Manifestations of Cardiac Hypertrophy.-Clinically, hypertrophy of the ventricle cannot always be reliably distinguished. An enlargement of the heart revealed by percussion or by the use of the orthodiagraph may be due to dilatation alone. In fact, with the exception of the hypertrophy of aortic insufficiency or nephritic hypertension, it is questionable whether the increase in size is 
great enough to be detectable by percussion (Sahli). Certain signs are generally considered not only suggestive, but also indicative of the side involved. Thus, an increase in the deep and relative dulness to the right is usually attributed to right-sided hypertrophy. It may be recalled, however, that the orthodiagrams show the right border to be composed largely of right auricle. It is questionable, therefore, whether a dilatation of the right ventricle can be diagnosed upon such evidence alone. Mackenzie has pointed out that dilatation of the right ventricle pushes the dulness to the left with the apex displaced downward and outward much as in left-sided hypertrophy. The difficulty in distinguishing right- from left-sided hypertrophy by percussion is therefore great. Fluoroscopic examination and a study of the form of the orthodiagram in various lesions is sometimes of assistance (see page 246).

Auscultation is considered of value in establishing the existence of hypertrophy because an augmented second sound in the pulmonic or aortic area is presumably associated with a higher blood-pressure and this, in turn, with a ventricular hypertrophy. It is by no means certain, however, that a higher pressure will cause a louder sound, in fact, Lewis found no increase in amplitude of the sounds on compressing the aorta in animals. If this were the case, however, there are many causes of a high pressure other than hypertrophy. The presence of systolic murmurs reflected to the tricuspid and mitral regions, especially when they appear and disappear, may with more reason be regarded as a sign of dilatation, for when the muscular support is removed from the valve rings, a functional insufficiency probably results.

Changes in the cardiac pulsations are said to be characteristic. 'Thus, a strong localized apex beat associated with a large pulse is attributed to left ventricular hypertrophy; whereas, a diffuse apex beat associated with a small pulse and epigastric pulsation favors right-sided hypertrophy. Little reliance can be placed on a negative apex beat during ventricular systole as a criterion of right-sided involvement. So much depends upon the placement of the tambour, upon the proper time allowance for transmission and upon the recording instruments, that curves such as presented by Mackenzie, for example, are far from convincing.

It is not any single sign so much as a correlation of findings that determines the diagnosis. The most satisfactory means of arriving at a diagnosis is probably by the use of the electrocardiogram, as suggested by Einthoven (Fig. 87). If right-sided hypertrophy predominates, $R_{\mathrm{I}}$ is directed downward and $R_{\mathrm{III}}$ upward. In left-sided hypertrophy, $R_{\mathrm{III}}$ is downward and $R_{\mathrm{I}}$ upward in direction. If the $R$ and $T$ waves are small, dilatation is probable; if they are large, hypertrophy is indicated. The accuracy of our clinical methods in differentiating hypertrophies has lately been 
checked by Lewis who finds the electrocardiograms very reliable but reports that ordinary clinical findings were substantiated in only 70 per cent. of cases.

A

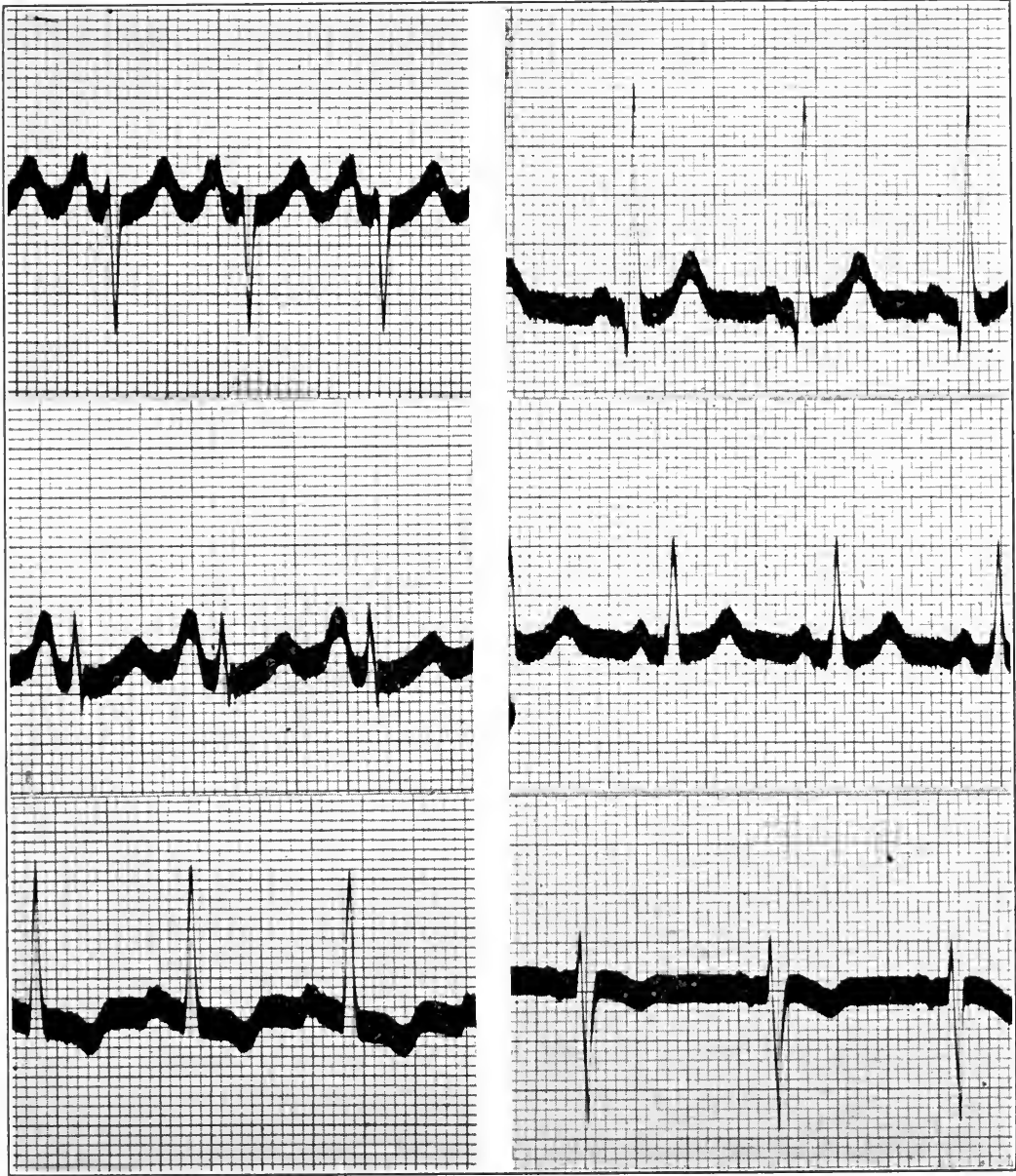

Fig. 87.-Two sets of electrocardiogram of right and left-sided hypertrophy. $A$, hypertrophy of right ventricle following rheumatic endocarditis and mitral stenosis.- Note large $P$ wave in lead $I I . \quad B$, hypertrophy of left ventricle, recent rheumatic endoearditis. Abseiscæ, 1 div. $=0.04$ sec. (Courtesy of H. B. Williams.)

Clinical Manifestations of Cardiac Insufficiency.-The signs of an impaired circulation following dilatation with cardiac insufficiency are well-known and characteristic. Insufficiency of the left heart causes low arterial pressure, a small pulse and a congestion 
of the pulmonary circuit with its attendant symptoms. Insufficiency of the right heart is accompanied by venous engorgement and the symptoms produced from stasis in the liver, stomach, etc. Their details are considered in connection with cardiac insufficiency following valvular lesions.

\section{LITERATURE.}

Fdens. Deut. Arch. f. klin. Med., 1913, cxi, 288.

Hasenfeld and Romberg. Arch. f. exp. Path. u. Pharm., 1897, xxxiii, 333.

Kïlbs. Arch. f. exp. Path. u. Pharm., 1906, lv, 288.

Lewis. Heart, 1914, v, 401.

Riesman. Amer. Jour. Med. Sci., 1913, cxlv, 487.

Stewart. Jour. Exp. Med., 1911, xiii, 187.

“ Jour. Path. and Bact., 1912, xvii, 64. 


\section{THE VALVULAR LESIONS OF THE HEAR'T.}

The normal valvular action on which the heart beat depends for an efficient transfer of blood from the venous to the arterial channels may be interfered with, either by the narrowing of the valves (stenosis) which offers an added resistance to the flow of blood, or by their failure to close completely (insufficiency or incompetency) which brings about a backward leakage.

These changes in the valves are usually the after-effects of an infectious endocarditis following the invasion of the blood stream by streptococci, ${ }^{1}$ staphylococci, pneumococci or gonococci; or they may be an accompaniment of a general arteriosclerosis in which case syphilis predominates as the etiological factor. The changes consist of vegetations, scars, ulcerations, fibrous and atheromatous changes, fusion of cusps during reorganization, etc. Finally, valves may rupture during excessive strain or become insufficient when the ventricles dilate or lose their tonus, in which case the valve rings are stretched and the chordæ tendinæ perhaps become taut before valve closure has been effected. The latter type of lesion is often designated as "functional" as contrasted with "organic lesions" in which permanent morphological changes in the valves are evident.

The presence of fibrosis, atheromatous plaques, rough edges or even vegetations does not necessarily result in insufficiency, for not only smooth sounds but even knotted cords have been introduced experimentally and the valves by their elasticity still adapted themselves to these irregularities (de Santille and Grey).

It is generally recognized that any valve defect is accompanied by a dilatation of the heart cavities which leads to anatomical hypertrophy of the cardiac muscle. It is also assumed that such a hypertrophied heart is capable of exerting a stronger beat and discharging a larger systolic output with less call on its reserve force. This has been termed "compensation." It is not so generally appreciated, however, that the heart is capable of compensating at any time without any increase in muscular development. This is due to the physiologically established fact that the output of the heart per beat or the strength of its stroke is governed by the initial pressure within the ventricle. In experimental work, the sudden production of a lesion is followed immediately by an increase in the 
diastolic pressure in the chamber behind and, during the very next beat, this chamber responds by a more vigorous contraction. It is very questionable, indeed, whether the subsequent increase in muscular tissue is not a result rather than a cause of the greater physiological activity of which each beat is capable ( $c f$. page 304$)$.

\section{AFFECTIONS OF THE TRICUSPID VALVES.}

Pathological lesions of the tricuspid valives are comparatively rare, and, when found, are usually associated with other lesions. The precise reason that the valves of the right heart are more frequently spared in endocarditis is not fully explained. The most common lesion is a functional insufficiency which occurs when the right ventricle is dilated, either because it is unable to empty properly on account of a great "vis a fronte," or because its contractions fail. This type of insufficiency has been looked upon as a safety device protecting the right ventricle from excessive distention and it is often supposed that a relatively small distention is sufficient to cause regurgitation. Observations on experimental animals do not confirm this opinion, however, for their ventricles may be distended under enormous pressures (200 to $250 \mathrm{~mm}$. water during diastole) without causing any regurgitation.

Hemodynamics of Tricuspid Insufficiency.-While tricuspid insufficiency, as recognized clinically, is usually secondary to changes in the left heart or alterations in the pulmonary arterial pressure, the experimental investigations have concerned themselves with the effects of tricuspid insufficiency per se. The results of these experiments can be applied absolutely to only a small per cent. of clinical cases.

When the tricuspid valves are rendered insufficient in animals (Rosenbach, Rihl, MacCallum), the pulmonary mean pressure may fall slightly but the systemic pressure is not appreciably affected unless the lesion is severe and the right heart becomes extremely dilated. The venous pressure rises and the auricular pressure shows a positive wave during systole (Rihl) similar to the ventricular type of venous waves described by Mackenzie. Investigation of tricuspid insufficiency during dilatation of the right heart by sensitive optical manometers shows that this is not a smooth wave, as is commonly assumed, but that the waves of peculiar and varying contour which occur are made up of a series of smaller vibrations which are evidently the cause of the systolic murmur detected 'in such lesions ( $c f$. Fig. $83, B)$. 'They may be explained by assumg that blood is forced through small crevices in the valves and not through a patent opening.

Clinical Manifestations.-The symptoms of tricuspid insufficiency alone are chiefly those of cardiac insufficiency in general. They 
are attributed to venous stasis and a deficient arterial supply to vital organs. The accumulation of blood in the venous reservoirs leads to a stagnation in the liver and portal system as well. This accounts for the symptoms of tenderness over liver, reflex abdominal rigidity, .jaundice, anorexia or even nausea and vomiting. The secretory and motor activity of the entire intestinal canal is apparently interfered with, as is shown by the persistent accumulation of gas. Venous stasis and the slowed current probably account also for the production of edema and ascites because the intracapillary pressure is thereby increased (Starling).

The diminished blood supply in dynamic anemia affects primarily the cerebral and medullary centres giving rise to such symptoms as dizziness, giddiness, forgetfulness, augmented breathing, and dyspnea. The heart rate is accelerated by a depression of the vagus centre. These symptoms are of course not pathognomonic of tricuspid regurgitation but of cerebral anemia of any type.

The renal organs are next affected. The secretion of urine diminishes and its specific gravity becomes high. Albuminuria is frequently present.

Physical Examination.-The facies of the patient is cyanotic, the degree depending on the venous stasis. In extreme cases the tips of the ears and nose may become a livid blue. If congestion is less severe the yellow jaundiced tint may appear in the skin as well as in the sclera. The neck veins are, as a rule, distended and pulsate visibly. A systolic liver pulsation is felt on palpation.

The apex beat is diffuse and may extend to the nipple line. Sometimes the positive beat is replaced by a negative retraction which probably has no special significance. The cardiac dulness increases to the right owing to the distention of the right auricle. Fluoroscopic examination confirms these findings.

Upon auscultation, a soft, blowing, systolic murmur is heard over the tricuspid area or, more frequently, over the lower end of the sternum. The murmur of a relative insufficiency is frequently softer than one due to endocarditic changes, but it should be borne in mind that the latter may become very faint when the cardiac contractions become weak. As far as the writer is aware, 'no records of the murmurs have been reported.

The venous pressure is high and in the arm may easily reach $25 \mathrm{~cm}$. of water when decompensation is extreme (Hooker). The venous pulse shows a typical variation from normal. It is usually stated that the negative systolic wave is replaced by a positive wave giving rise to what Mackenzie has termed the ventricular type of venous pulse. Optical tracings indicate that the.contour of the supraclavicular venous pülse is entirely changed, however; in fact several variations may occur in consecutive order when the heart is beating irregularly (Fig. 88). Their nature is also shown 


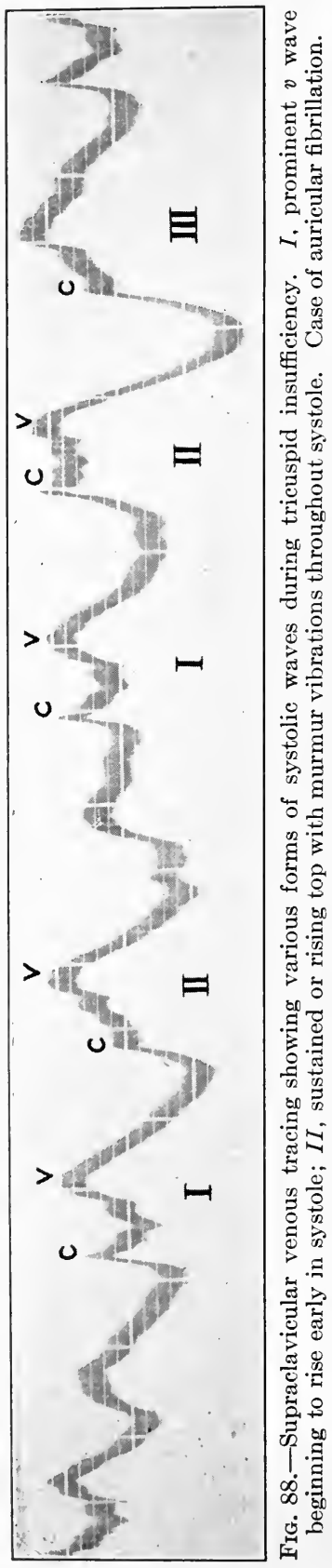

in Figs. 35 and 83. Briefly recounted, the curves rise rapidly early in systole and upon their summit are superimposed a series of vibrations resembling those in experimental animals ( $c f$. Fig. 83, $B$, and Fig. $88, I I)$.

\section{MITRAL INSUFFICIENCY.}

Hemodynamics.-The dynamic changes in mitral insufficiency have been studied both in artificial circulation schemes (Marey) and in animal experiments (MacCallum and McClure). In relatively simple experiments with artificial circulation machines, it can be shown that, when a mitral valve is rendered insufficient, the mean auricular pressure rises and the mean arterial pressure falls. It is evident that this is due to a decreased transfer of blood from the venous to the arterial side which, with an unaltered heart rate, indicates a decreased discharge. This decreased discharge accounts for the fact that the amplitude of the arterial pulse is reduced (Fig. 89). It can further be shown that, if the stroke is artificially increased in such a scheme, thereby resembling the effect of compensation in the body, the venous pressure is again reduced, the arterial pressure rises and the pulse amplitude returns more nearly to the normal. These simple experiments indicate that the circulatory changes are similar in every respect to the effect of a decreased systolic output of the heart ( $c f$. page 68).

These conclusions have, in the main, been confirmed and extended by animal circulation experiments. After cutting or destroying a valve, the carotid pressure falls and the mean left auricular pressure rises. Furthermore, the pressure in the left auricle, instead of decreasing sharply early in systole, now increases very much as is the case in the right 
auricle during tricuspid insufficiency. The mean pressure within the pulmonary artery is altered relatively little. With a slight grade of insufficiency, it rises slightly due to a rise of left auricular pressure, but if the insufficiency is great it falls. This has been interpreted as traceable to a diminished venous return to the heart (MacCallum and McClure). In either case the minute flow as well as the velocity of flow through the lungs is decreased. There is this difference-in the former case, the total blood content of the lungs is increased (pulmonary congestion); whereas, in the latter, blood accumulates elsewhere in the body. Similar results have been obtained in experiments carried out by the writer in conjunction with DuBois. These records, however, show a much smaller rise of mean left auricular pressure after very considerable leaks, a difference evidently due in part to the fact that other investigators used undamped man-
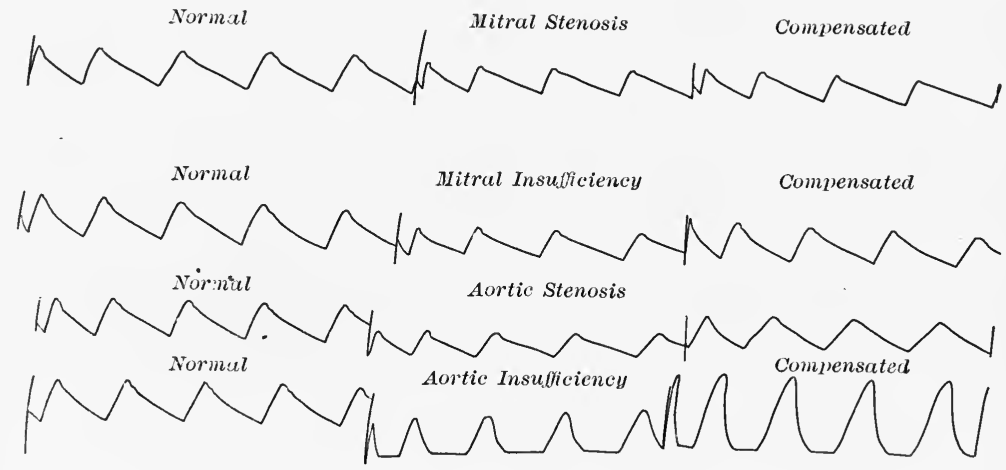

FIG. 89.-Pulse tracings from artificial circulation machine, comparing the effects of different lesions with normal curves.

ometers which were thrown into more violent oscillation during the insufficiency and hence gave the erroneous impression of a great increase in pressure.

Two mechanisms may operate to prevent any extreme increase in left auricular pressure such as occurs in artificial circulation experiments in similar leaks. In the first place, the rise in left auricular pressure increases the effective initial pressure in the ventricle which responds immediately with a greater output. In the second place, it is possible that the greater distention of the left ventricle may crowd the interventricular septum to the right and so encroach upon the right ventricle and reduce the output (Prince and Henderson). It is clear that this question demands further investigation, not only because of its scientific aspects, but also because of its practical bearing on the question, as to whether valvular insufficiency per se can cause a pulmonary congestion and 
back pressure into the right ventricle and veins as is commonly taught. Mackenzie points out in his convincing way that the so-called "back-pressure theory" receives little support from a careful study of any progressive case of valvular lesion. $\mathrm{He}$ believes that a back-pressure occurs only when myocardial insufficiency supervenes.

Clinical Manifestations.-The symptoms and signs of mitral insufficiency depend, to a considerable degree, on the condition of the heart muscle. They may be grouped into compensated and decompensated cases.

In compensated lesions, as those produced in animals, the circulation is not greatly embarrassed. In the mildest cases, the only symptom that is noted is a shortness of breath when attempting tasks that formerly caused no respiratory distress. As time passes, the signs rather than the symptoms alter. The cheeks become pink, flushed, or sometimes even purple. The smaller venules are enlarged. This rosy appearance and bright eyes (mitral facies) give the patient, as viewed by the popular eye, the appearance of excellent health. The radial pulse is often small but shows no important changes from the normal. As long as compensation is good, it remains regular except for an occasional premature systole. The apex tracings show nothing characteristic. The waves may be positive or negative, but no importance can be attributed to a negative record.

It was hoped by clinicians that the registration of esophageal curves might establish the existence of a regurgitant wave into the left auricle. The method is, however, an inconvenient one and the curves thus obtained cannot with certainty be interpreted as showing such a regurgitation (Young, Hewlett, Edens).

The systolic and diastolic pressures are not greatly affected (Norris). Occasionally the systolic pressure is lower and the pulse pressure smaller which seems to be particularly the case when the heart is rapid.

On examination, the apex is found displaced to the left and may reach the anterior axillary line in the fifth $I$. C. S. A systolic thrill is sometimes felt. On percussion, the dulness proves to be increased transversely toward the left axilla. The orthodiagram shows a smooth circular enlargement to the left.

Uncompensated or Decompensated Lesions.-If, for any reason, the ventricle does not augment its output and so compensate for the deleterious effects of valvular lesions; or, if compensation once developed fails, due to dilatation, the symptoms and signs alter. This is because the blood accumulates in the left auricle and pulmonary circuit, and, if the right ventricle similarly fails to perform its function and dilates, a relative tricuspid insufficiency is added. Such cases suffer, therefore, from intense dyspnea, due, perhaps, 
in part, to the pulmonary congestion and, in part, to the diminished blood supply to the medulla. The pulmonary congestion, furthermore; gives rise to a chronic bronchitis in which bloody sputum is raised in coughing paroxysms. The dilatation of the ventricles often manifests itself by pain over the cardiac region. To these symptoms may be added all those already described as characteristic of tricuspid insufficiency.

On auscultation, a systolic murmur obscuring or obliterating the first sound is heard loudest or exclusively at the apex. It is transmitted toward the axilla and, in children, to the back. Its quality is apparently quite variable. Its greater intensity at the apex has been attributed to the fact that the vibrations are conducted by the anterior papillary muscle to the ventricular apex, thus forming the

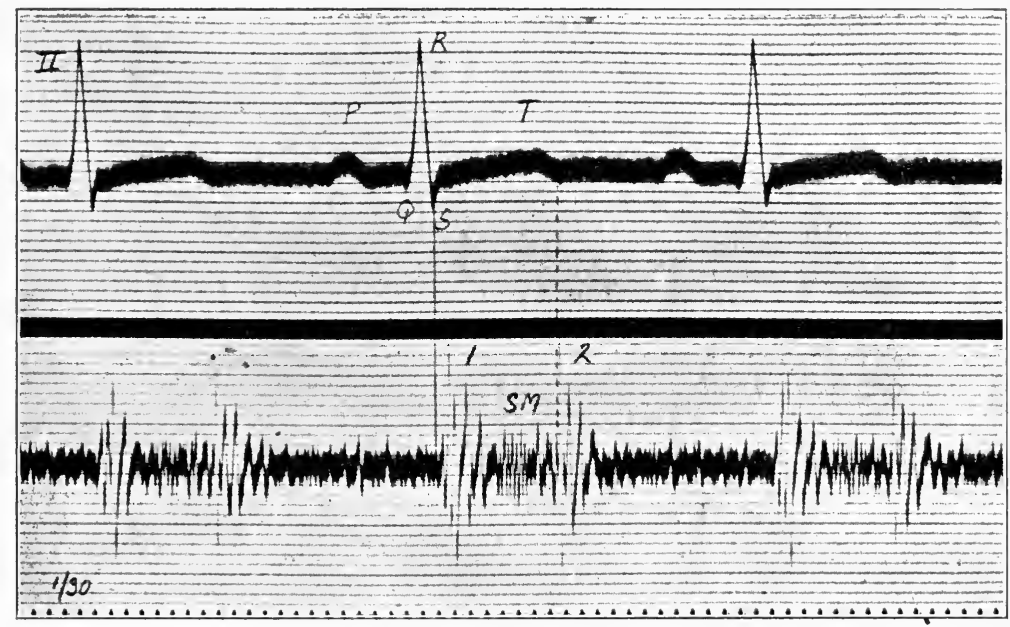

FIG. 90.-Phonocardiogram from case of mitral regurgitation. SM, systolic murmur; 1, 2, heart sounds. (After Lewis.)

closest solid conduction to the chest wall. The murmur accompanying this lesion seems difficult to record (Joachim, Lewis). In the tracings successfully taken with the string galvanometer, it. is indicated either as small vibrations superimposed on those due to the first sound, or occurring after the sound. In the latter case, it has a frequency of 112 to 140 per minute (Lewis) (Fig. 90). Gerhartz has given the murmur a duration of 0.45 seconds when it replaces the first sound.

Percussion shows that the dulness now extends, not only to the left, but also to the right of the sternum. The orthodiagram shows a uniform enlargement making the shadow appear like a poorly rounded circle, as shown in Fig. 91. The right auricular border is distinctly enlarged to the right and the pulmonary artery dilated. 
The second pulmonic sound is accentuated but the proof that this exemplifies a high pressure in the pulmonary artery is by no means established ( $c f$. Lewis).

During extreme dilatation the auricles may give extra-contractions or go into fibrillation giving rise to an absolutely irregular arterial pulse. For the details of its interpretation by the aid of the supraclavicular venous pulse and electrocardiogram, other chapters must be consulted. The electrocardiogram is of additional value, however, in showing the existence of a right-sided hypertrophy, since the $R$ wave in lead $I$ is directed downward instead of upward, as is normally the case (Fig. $87, A$ ).

The arterial pulse waves are not only irregular, but also unequal in height in such cases. The systolic and diastolic pressures are therefore constantly varying and it becomes difficult, if not
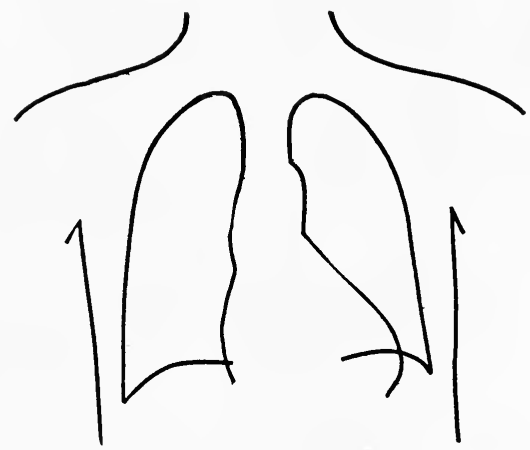

FIG. 91.-Orthodiagram in mitral insufficiency. (After Claytor and Merrill.)

impossible, to determine them even approximately. The method proposed by James and Hart may then be utilized or the extremes of systolic and diastolic pressures stated ( $c f$. page 216$)$.

\section{MITRAL STENOSIS.}

Hemodynamics.-It has long been recognized (Vieussens, 1715) that a narrowing of the mitral orifice increases the resistance to the flow from the auricle to the ventricle, thereby limiting the filling and reducing the output. These facts can readily be demonstrated by an adequate artificial circulation machine. After the production of a stenosis the mean venous pressure rises, the mean arterial pressure falls, and the amplitude of the pulse wave is reduced (Fig. 89). It can further be shown that, if the stroke is artificially increased, no material changes are effected.

Animal experiments have corroborated these findings. If the condition is simulated by tightening a ligature placed around the 
auriculo-ventricular ring, or invaginating the auricle into the ventricle with one's finger, or by inserting a small inflated balloon, similar changes occur. The mean arterial pressure falls while the left auricular and pulmonary pressures rise definitely (MacCallum and McClure). It has been sought to determine, experimentally, the exact effect of stenosis on ventricular filling, by applying a cardiometer to the two ventricles (Hirschfelder). The conclusions and schematic diagrams probably represent essentially the changes in filling that actually take place. Thus, with mild stenosis, the inflow during active diastole is slower but is compensated, to some extent, by the more vigorous impact of auricular systole. In more severe lesions the filling occurs very slowly during the entire period of diastole and the total inflow and, consequently, the output during the next systole are reduced. The records published can scarcely be accepted as experimental evidence of these conclusions, however, nor is it probable that the volume changes of the left ventricle can be accurately deduced from volume changes of both ventricles when their action differs. These conclusions may therefore be accepted as theoretical or probable rather than as experimentally demonstrated.

The increase in pressure within the pulmonary artery is commonly supposed to augment the pressures within the right ventricle, to cause its distention and, if it becomes great enough to produce a dilatation of the tricuspid ring and so bring about a regurgitation. Under these conditions only is the venous pressure raised (Kornfeld), otherwise it-falls due to the lower arterial pressure. The total quantity of blood within the lungs is apparently increased more than in mitral stenosis but the minute flow and velocity of flow are reduced.

Clinical Manifestations.-The symptoms due to mitral stenosis are caused by the pulmonary congestion, dilatation of the ventricle and often relative tricuspid insufficiency. They therefore resemble those already described in mitral insufficiency plus, in later stages, those characteristic of tricuspid regurgitation. They make their appearance relatively early in the disease owing to the fact that the lesion cannot be so effectively compensated by increased muscular action.

Percussion shows a slight increase in cardiac dulness to the left, especially upward. In $x$-ray plates and orthodiagrams, the heart shadow resembles an oval with a vertical axis (Fig. 92). The enlarged left auricle is prominent on the left margin and in severe cases the pulmonary shadow bulges giving the entire left border a step-like contour.

On palpation at the apex, a-rough presystolic thrill terminating in a sharp systolic shock is felt. This sign is so characteristic that a diagnosis may in some cases be made from it alone. This 
palpable thrill and shock correspond in time and are probably due to the same factors that produce the audible presystolic rumble which increases in intensity and terminates in the snapping first
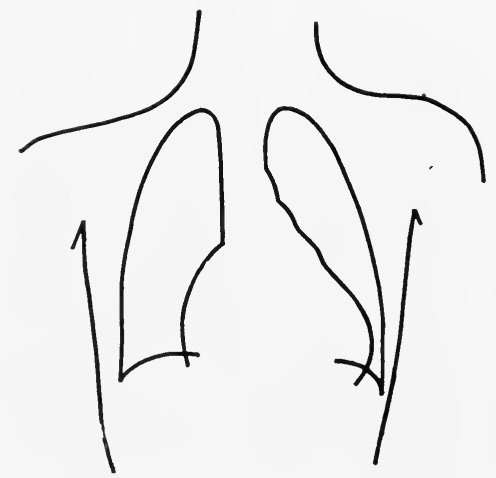

Fif. 92.-Mitral stenosis after prolonged loss of compensation. (Claytor and Merrill.)

sound. The presystolic murmur which is recorded relatively easily by the string galvanometer has a higher frequency, 41-107 per second, than those making up the first sound and the amplitude

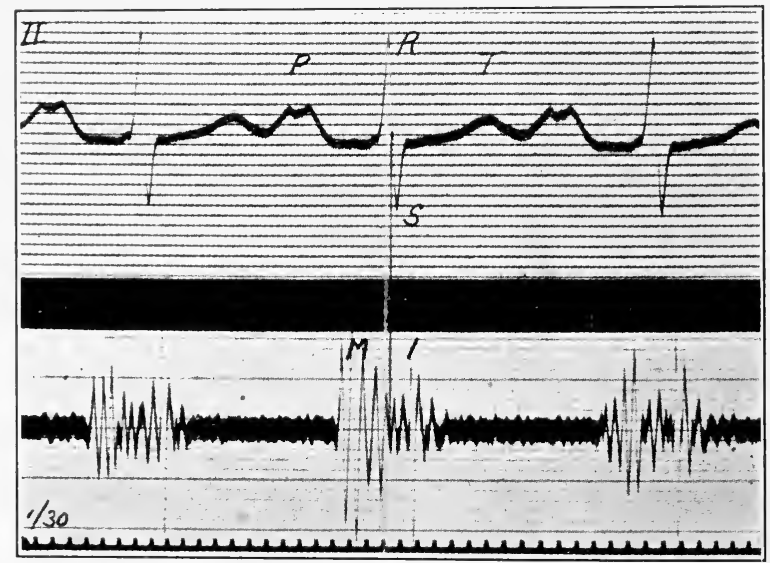

FIG. 93.-Electrocardiogram and phonocardiogram in mitral stenosis, showing notched $P$-wave and presystolic murmur. (After Lewis.)

of its vibrations is larger (Fig. 93). Comparisons with the waves of the electrocardiogram show that it extends during the interval covered by the $P$ and $T$ waves but it may begin before the $P$ wave 
starts. Indeed, the murmur may occur in the records during the entire period of diastole. According to Lewis, the duration of the murmur before ventricular systole is determined by the degree of stenosis. If this is slight and the rhythm regular, a presystolic murmur alone is present. If the stenosis is moderate, an early diastolic murmur separated from the next presystolic murmur also occurs due to the inrush of blood to the relaxing ventricle. If stenosis is extreme and especially if the heart is rapid, the entire period of diastole is filled with murmurs which pass into the period of presystole.

The characteristic signs revealed by palpation and auscultation change when compensation fails. When auricular contraction becomes weak, the presystolic murmur and thrill may disappear and the systolic shock alone remain. When auricular fibrillation supervenes and auricular contraction is absent, the presystolic murmur, strictly speaking, disappears (Mackenzie), or better stated, perhaps, no murmur precedes the first heart sound when the rest of diastole is free from murmurs (Lewis). Diastolic murmurs are frequently present during a portion or throughout diastole (Fig. 84). Owing to the varying length of diastole, the character of these murmurs changes from beat to beat. 'The shorter cycles may be filled with murmurs; while in long cycles they often occur only in the early portion. These murmurs are distinguished from the diastolic murmurs due to aortic insufficiency by their slower frequency (lower pitch) and the fact that a short interval exists between the second sound and the murmur in mitral stenosis (Lewis).

When auricular fibrillation develops, the arterial pulse is extremely irregular and the beats vary in amplitude. The temporal changes of the venous pulse and electrocardiogram accompanying, this condition have been analyzed elsewhere. ${ }^{1}$ 'The electrocardiogram, it may be mentioned, shows typical changes in form in mitral stenosis before disturbances in rhythm occur. 'The $P$ wave is frequently several times as large as normal, ${ }^{2}$ often it is prolonged and has a flattened or bifurcated top, ${ }^{3}$ signs of a left auricular hypertrophy. The hypertrophy of the right ventricle accompanying such cases is indicated by the direction of the electrocardiogram waves. The $R$ wave is normally directed upward in all three leads; whereas, in mitral stenosis, $R_{\mathrm{I}}$ is directed downward, while the $R$ wave of lead $I I$ is smaller than that of lead $I I I$. 'This may be of value in differentiating it from those cases in which a diastolic as well as a presystolic murmur occurs from aortic insufficiency for a Flint murmur, in the latter condition is accompanied by a leftsided hypertrophy (Lewis). 


\section{AORTIC STENOSIS.}

Aortic stenosis may exist as a simple lesion when the cusps undergo atheromatous degeneration but retain sufficient flexibility to close during diastole. When the entire valves are involved, in the sclerotic process, however, or their edges fused a certain degree of insufficiency is associated with the stenosis. This combination is not uncommon (40 per cent., Hirschfelder).

Hemodynamics.- Stenosis produced in an artificial circulation apparatus causes a marked elevation of intraventricular pressure during systole while both systolic and diastolic pressures fall in the arterial system. The pulse wave recorded from the rubber tube furthermore shows a characteristic slow ascent and a rounded top (Fig. 89) which persist after compensation. The changes in animal experiments are apparently similar (Luderitz, de Heer). The pressure within the ventricle rises steeper during the isometric period and the ejection begins at a higher pressure level. The

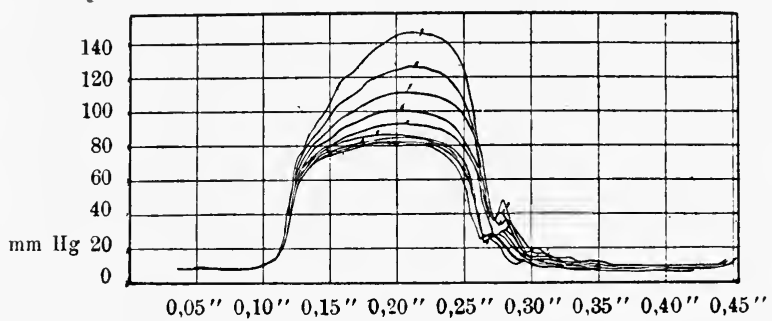

FIG. 94.-Intraventricular pressure curves in different degrees of aortic stenosis. (After de Heer.)

duration of this period, however, remains unchanged (Fig. 94). The intraventricular pressure rises to a very great height when the stenosis is severe. In mild cases, the rise may not much exceed the normal (de Heer). It appears from these observations that a considerable narrowing must occur before the resistance increase at all compares to the total resistance normally offered by the blood. The period of ejection is, however, prolonged and in this way a fairly normal quantity of blood is ejected. According to de Heer, the stenosis may be extremely great before the volume curve shows any appreciable reduction in output or a fall of mean blood-pressure exceeding 10-30 per cent. Apparently the prolongation of systole is of a protective nature to the conservation of arterial pressure. The contour of the pressure curve in the aorta shows essentially the same features as in artificial circulation experiments.

As long as systemic arterial pressure is sustained, the pulmonary arterial pressure is unaffected, but, as soon as the former falls, 
the latter rises (MacCallum). This takes place in spite of a fall in venous pressure and in spite of a reduced output from the right heart, hence it must be referred to a backing up of blood into the pulmonary circuit. To what extent this is due to the mechanical back-pressure effect of valve stenosis, and, to what extent to dilatation and failure of the ventricle have not been clearly separated.

Clinical Manifestations.-The earliest subjective symptoms are probably those arising from increased intraventricular tension and perhaps the impeded blood flow through the heart. They consist of palpitation, a sense of constriction, substernal pain or anginal attacks at first recurring only after excitement or exercise, but later being present without apparent exciting cause. Still later when the right ventricle dilates, symptoms due to pulmonary engorgement and often tricuspid insufficiency supervene. Upon inspection, the apex beat may not be visible or there may be a slow, heaving impulse. The dulness is enlarged to the left and downward. Orthodiograms show an enlargement of the heart shadow to the left. The lower portion gives the appearance of a horizontal oval above which the shadows of the pulmonary artery and aorta are visible. The shadow, though, as a rule, smaller, resembles in shape that of aortic insufficiency. The enlargement is evidently due to hypertrophy of the left ventricle. In confirmation of this conclusion, it is found that the $R$ wave of the electrocardiogram is directed down in lead $I I I$.

Palpation often discovers an intense systolic thrill over the aortic region and a loud, rough, systolic murmur is also heard here. It is transmitted upward to the large vessels and may be registered from the lower neck. On registration, it consists of a series of irregular vibrations occurring at the rate of about eighty per second, a diminuendo beginning a short interval after the first sound (Weiss and Joachim) (cf. also vibrations on subclavian pulse recorded by Frank's capsules, Fig. 97).

The second sound is usually weak or may be entirely absent; while, if the valves fail to close, it may be replaced by a diastolic murmur.

The radial pulse is usually small and its rise is slow. The tracings sometimes show an anacrotic limb and occasionally a pulse with a typical slow rise. It is questionable, however, whether reliable tracings of this condition have ever been reported ( $c f$. page 118 for a discussion of this question). Whether a characteristic peripheral pulse is found with reliable apparatus must abide further investigation. For, aside from the fact that these variations are imperfectly transmitted to the periphery, the condition is so frequently complicated by some degree of insufficiency or arteriosclerosis which serve to modify the form of the pulse. It is frequently arrhythmic; 
premature systoles and pulsus alternans being the most frequent forms of irregularity. The electrocardiogram by its typical complexes indicates that the premature systoles usually arise in the left ventricle or auricle.

\section{AORTIC INSUFFICIENCY.}

Hemodynamics.-The dynamic effects of aortic regurgitation have been analyzed both in artificial circulation apparatus experiments (Marey, Moritz) and in animal experiments (Cohnheim, Rosenbach, Becker, de Jager, Kornfeld, Romberg and Hasenfeld, Stewart, '/ollinger). It can be readily shown on an artificial circulation apparatus that the production of an aortic insufficiency causes a fall of diastolic pressure in the artery, while the systolic pressure either falls slightly or remains unchanged. ${ }^{2}$ The maximum pressure within the ventricle also falls slightly, but the diastolic pressure rises just before the next systole, although some discrepancies occur that can probably be attributed to experimental technic. Animal experiments have also shown that, after the production of a lesion, the systolic pressure remains unchanged or falls slightly, while the diastolic and consequently the mean pressure fall greatly (Kornfeld, MacCallum, Stewart). Whenever these changes occur, the pressure curve in the arteries and also the pulse alters ( $c f$. Fig. 95); its contour in that the amplitude becomes greater and the descending limb more rapid in its fall. This change has usually been attributed to the fact that a considerable quantity of blood regurgitates into the ventricle during diastole causing an augmented amount to be thrown out during the next systole. This suggestion was supported by the results of Kornfeld who found that the left auricular pressure often rose after the lesion, while the systemic pressure fell. Stewart, however, pointed out that this could not be the correct explanation for: (1) the rapid fall always occurs before the dicrotic notch, and, hence, during ventricular systole; (2) no appreciable regurgitation is shown in the volume curves of the heart. He therefore explains the rapid drop as the effect of a reflex vasodilation, since, in the first place, this accounts for all the changes in pressure and pulse contour and, in the second, the normal contour returns in spite of the lesion when the peripheral vessels are constricted during aortic insufficiency, as by aortic compression, or the use of adrenalin. This conclusion, in turn, was not supported either by MacCallum or Hewlett, but neither of these investigators attempted to explain the results of Stewart. 
For these reasons, the writer has recently restudied the dynamics of this condition by recording simultaneously the pressure changes in the left ventricle and the subclavian artery. ${ }^{1}$ This study was facilitated by the use of a method for producing a temporary insufficiency. In this manner, insufficiency and normal records can be taken alternately for comparison and the immediate effect of an insufficiency studied. When, by such a special procedure, lesions are temporarily produced and normal relations reëstablished, the changes in pressure take place within a single heart cycle, that is, in a time interval far shorter than is necessary to cause a reflex vasomotor response. The changes produced in an efficiently beating heart are shown in Fig. 95. The normal intraventricular pressure curve shown in $I$ has all the characteristics of the curves

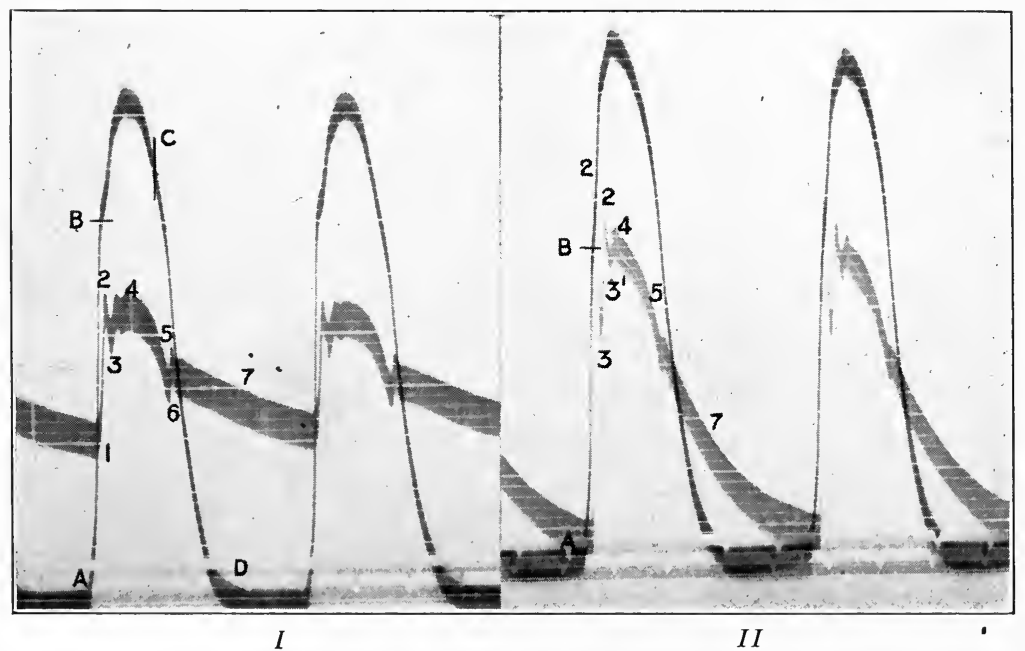

Fici. 95.- The subclavian and intraventricular pressure curves' $I$. normal: $I I$, after aortic insufficiency. (Description in text.)

found in an efficiently contracting ventricle. It may be divided into the isometric period $(A-B)$, the ejection period $(B-C)$, the period of active relaxation $(C-D)$ and the period of passive filling $(D-A)$, that is, diastasis. The arterial curve contains all the details of the arterial pulse except that the preliminary oscillations are not so marked. The variations consist of the primary oscillations $(1,2,3)$, the systolic summit $(4)$, the incisura (5) with an aftervibration (6), and the slow diastolic fall (7). After the production of an aortic insufficiency in curve $I I$ the following changes are observed: The initial pressure in the ventricle (at $A$ ) is higher.

1 A detailed report of this investigation will appear in the Arch. Int. Med., 1915; a preliminary report ean be found in Proc. Soc. Biol. and Med., 1914, xl, 55. 
The ejection begins earlier (at $B$ ) and the curve reaches a higher summit. This is probably in reaction to the greater initial distending pressure. As a result of this more vigorous contraction and ejection, a larger quantity of blood is thrown out during the first half of systole resulting in several pronounced primary oscillations $\left(2-3,2^{\prime}-3^{\prime}\right)$ in the arterial curve. The systolic summit (4) is . sharper and the systolic fall and incisura (5) more rapid. Although the cycles are exactly the same length, the chief drop (7) responsible for the low diastolic pressure comes in diastole and not, as Stewart believed, in systole. Furthermore, on removing the insufficiency, the curves shown in $I$ were reproduced within the time interval of a single beat. A careful consideration of these curves and others in the process of publication, indicates that the large wave and the extreme fall of arterial pressure cannot possibly be due to a peripheral dilatation of vessels. These changes must be attributed to a back leak of pressure into the ventricle, with or without an appreciable back-flow of blood. That this back-flow is very little, at least after the first beat following suddenly produced insufficiency, is indicated both by Stewart's cardiometer experiments and by the facts that the rate of ventricular relaxation is absolutely unaltered $(C-D)$ and that the pressure in the left auricle at the end of systole drops precisely as it did before the insufficiency occurred, showing that the normal influx of blood takes place.

The general pressure in the left auricle and in the pulmonary circuit remains unchanged. It appears that even in the most sudden and the most marked lesion of the aortic valves, a normal volume of blood is ejected and no back pressure effect is produced as long as the ventricle does not fall below its normal condition.

Clinical Manifestations.-As long as a deficiency in cardiac action does not exist, the chief symptoms are probably the result of variations in arterial pressure. The arterial throbbing in the neck, chest, and abdomen may be quite disturbing. Cerebral symptoms, headache, roaring in the ears, hallucinations of sight, hearing and smell are frequently present, but it seems never to have been shown whether they are the result of aortic insufficiency or of the same influence which is responsible for the insufficiency. The dull or anginoid pains over the heart and palpitation are probably due to the high pressures within the heart.

When physiological compensation fails and pulmonary congestion sets in, dyspnea, cough, cardiac asthma, etc., occur.

Patients with aortic insufficiency have a very characteristic appearance (aortic facies) which on casual inspection resembles somewhat that of exophthalmic goitre. The complexion is pale and sallow, the cheeks are sunken, the pupils dilated, and the palpebral fissures are wide. The peripheral vessels throb and a capillary pulse is often observed in the finger nails. 
Upon examination, the apex beat is displaced downward and to the left, sometimes extending in the fifth or sixth interspace to the anterior axillary line. A heavy impulse is usually visible. A pulsation of the aorta may be visible in the second right interspace.

Percussion shows, in cases of long standing, an immense increase in cardiac dulness, chiefly to the left and downward. The orthodiagram shows an outline which, while resembling that of stenosis, is very much larger (Fig. 96). The lower portion is a horizontal oval while the entire figure is often described as shoe-shaped.

On palpation, one or more thrills may be felt, but are neither constant nor diagnostic. They may be systolic, diastolic or presystolic in time.

Auscultation of the cardiac base reveals a blowing, hissing or musical murmur, early in diastole, closely following or replacing the second sound. Its greatest intensity is over the sternum opposite

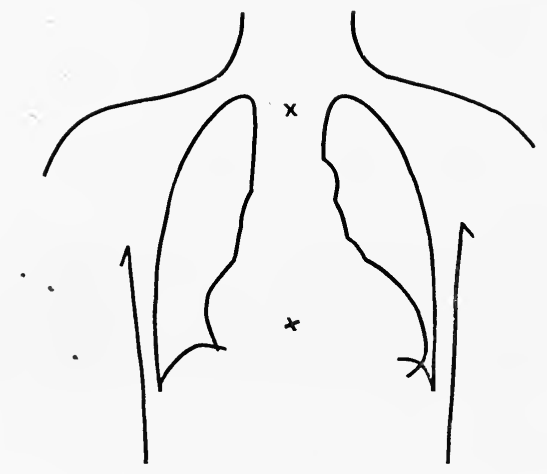

FIf. 96.-Orthodiagram of aortic insufficiency.

the third rib, or in the second right intercostal space. The first sound is sometimes clear and of a snapping quality but at others is replaced by a soft systolic murmur transmitted upward to the neck where it may be recorded (Fig. 97). A presystolic murmur (Flint) is generally present at the apex. Its cause is still in doubt and will probably remain so until actual experiments supplant theoretical speculation. Flint ascribed it to a functional stenosis produced when the mitral valves are floated into position by the marked regurgitation.

The recorded aortic murmur varies with its auditory quality. the rough to-and-fro murmurs which are both systolic and diastolic in time are made up of vibrations.having a frequency of eighty per second, and are crescendo in character. The musical murmurs are entirely diastolic, last, on an average, 0.24 second and are made up of uniform vibrations, recurring at the rate of 138 per 
second (Lewis) (cf. Fig. 50). Their amplițde is larger than that of the first heart sound vibrations. The systolic murmur associated with this lesion is particularly well transmitted to the neck and recorded as a series of superimposed vibrations of the central pulse. Records taken from two different cases in Bellevue Hospital with Frank's capsules are shown in Fig. 97. It is still questionable whether this murmur is associated with some complicating stenosis or is due to a mechanical vibration of the blood currents.

In typical cases of aortic regurgitation, the radial pulse gives the palpating finger a sensation of sudden impact and rapid recoil, or collapse. It has therefore been termed, since its description

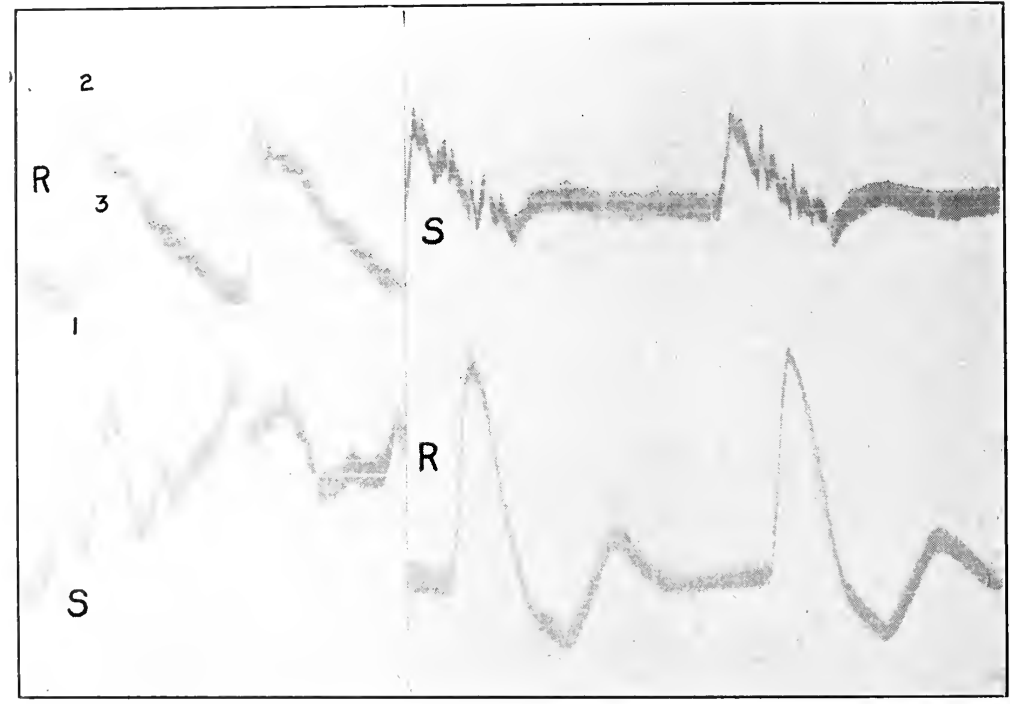

FIG. 97.-Radial pulse $(R)$ and subclavian pulse $(S)$ in two cases of aortic insufficiency in which a "collapsing pulse" was diagnosed by palpation and the Jacquet sphygmograph. Observe the vibrations due to the systolic murmur superimposed on the subclavian pulse.

by Corrigan (1832) as a "collapsing pulse" or a "water-hammer pulse." . Physicians have repeatedly been impressed by the fact, however, that when the palpating finger receives the sensation of a collapsing pulse, the sphygmogram often fails to corroborate it. This has frequently been ascribed to the mechanical errors of the sphygmographs employed and with great reason, for it is often possible to obtain for the same patient entirely different pulse forms by using different instruments or by merely different pressures in the same instrument. The pulses shown in Fig. 97 were obtained from cases in which the pulse was described as "collapsing" to the touch. The first two curves gave with the Jacquet a curve of large 
amplitude and collapsing character; the last showed no deviation from normals recorded with that instrument. Comparisons with the optical tracings of Fig. 97 show that none of the sphygmographs recorded the form correctly nor did the palpating finger appreciate the true character. The sharp primary oscillation $(1,2,3)$ in left hand record of Fig. 97, apparently transmitted to the radial from the central pulse, gave the sensation of a sharp impact. A true collapsing pulse, on the other hand, is present in the second radial tracing. The collapsing type of pulse is associated, as Hewlett and Van Zwaluenburg have demonstrated by means of the pulse-flow curve, with an actual backward movement of blood from the arm, but whether this excess of flow is accommodated in the central arteries or actually regurgitates into the ventricle does not seem proven by their work.

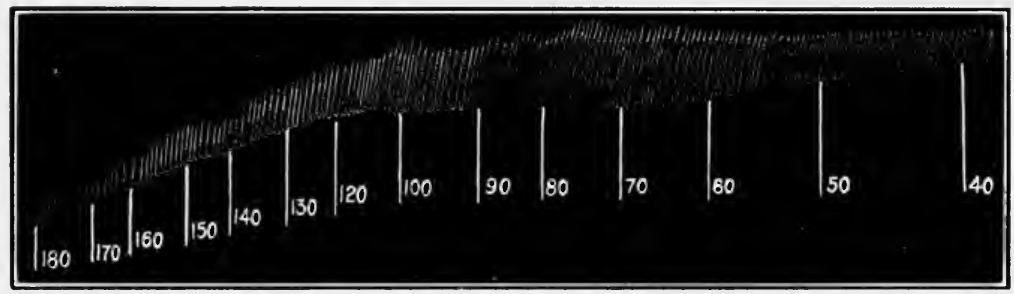

FIG. 98.-Erlanger sphygmomanometer tracing from case of aortic insufficieney. Diastolic reading about $58^{\circ}$, systolic, indefinite.

Nearly all investigators find that the systolic pressure is normal or high (180-200) and the diastolic pressure low (60-30) (Huchard, Lian, Baj, Tausig and Cook, etc.). It is questionable whether it is necessary to call in the presence of arteriosclerosis to explain the high systolic pressure. With the Erlanger sphygmomanometer the change to smaller pulsations is usually sharp and distinct, permitting an accurate determination of diastolic pressure (Fig. 98). In employing the auscultatory method, it is necessary to take the fourth phase as the criterion since the sounds do not entirely disappear at very low pressures in the bag. This plan corresponds quite accurately with the diminution in amplitude of oscillations. In determining systolic pressure in cases in which a collapsing pulse is present difficulty is met in utilizing Erlanger's criterion, for the bases of waves do not separate as in a normal pulse ( $c f$. Figs. 57, 58, and 98). The auscultatory or palpatory method must therefore be resorted to. 


\section{LITERATURE.}

\section{Books Dealing with Valvolar Lesions.}

v. Basch. Allg. Physiol. u. Path. des. Kreislaufs, Wien, 1892.

Cowan. Diseases of the Heart, 1914, Philadelphia.

Cohnheim. Vorlesungen über allgemeine Pathologie, ii, Aufl. 38.

Gerhardt, D. Herzklappenfehler, Wien, 1913.

Gibson. Diseases of the Heart and Aorta, Edinburgh and London, 1898.

Hirschfelder. Diseases of the Heart and Aorta, Philadelphia, 1913, 2d ed.

Mackenzie. Diseases of the Heart, 1913, London.

Marey. La Circulation du Sang, Paris, 1881.

Osler. The Principles and Practice of Medicine, 1912, 8th ed.

Osler and McCrae. Modern Medicine, Philadelphia, 1915, iv.

Strümpel. Lehrbuch d. speciellen, Path u. Therapie, 1914, 19th ed., Lieipzig.

For references dealing with heart sounds, venous and arterial pulses, and eleclrocardiograms, see end of chapters dealing in detail with these subjects.

\section{Articles Dealing with Experimental Dynamics.}

Becher. Arch. f. Ophth., 1872, xviii, 206.

Gerhardt. Arch. f. exper. Path. u. Pharm., 1901, xlv, 186.

Hasenfeld and Romberg. Arch. exper. Path. u. Pharm., 1897, xxxix, 333.

de Heer. Arch. f. d. ges. Physiol., 1912, cxlviii, 1.

Henderson and Prince. Heart, 1914, v, 217.

Hirschfelder. Johns Hopkins Hosp. Bul., 1908, xix, 318.

de Jager. Arch. f. d. ges. Physiol., 1883, xxxi, 215.

Kornfeld. Ztschr. f. klin. Med., 1896, xxix, 91, 344.

Luderitz. Ztschr. f. klin. Med., 1892, xx, 373.

MacCallum. Johns Hopkins Hosp. Bul., 1911, xxii, 197.

MacCallum and McClure. Johns Hopkins Hosp. Bul., 1906, xvii, 260.

Moritz. Verhandl. d. Cong. f. inn. Med., 1895, xiii, 395.

“ Deutsch. Arch. f. klin. Med., 1899, lxvi, 349.

Rihl. Ztschr. f. physik. Diätet, 1907, xi, 298.

“ Berl. klin. Woehnschr., 1907, xliv, 825.

Rosenbach. Arch. f. exper. Path. u. Pharm., 1878, ix, 1.

de Santelle and Grey. Arch. Int. Med., 1911, viii, 734.

Starling. Lancet, 1897, i, 569, 652, 723.

Stewart. Arch. Int. Med., 1908, i, 102.

Wiggers. Proc. Soc. Exper. Biol. and Med., 1914, xi, 55.

Wiggers and Du Bois. Proc. Soc. Exper. Biol. and Med., 1913, x, 87.

Zollinger. Arch. f. exper. Path. u. Pharm., 1909, Ixi, 193.

\section{Articles Dealing with Clinical Problems of Lesions.}

Baj. Med. Klin., 1914, x, 284.

Corrigan. Edinburgh Med. and Surg. Jour., 1832, xxxvii, 225.

Hewlett and Van $Z$ waluenberg. Arch. Int. Med., 1913, xii, 18 .

Hill and Rowlands. Heart, 1912, iii, 219.

Lewis. Brit. Med. Jour., 1912, p. 1700.

“ Heart, 1913, iv, 241.

Lian. Presse Med., 1913, xlv, 445.

Rolleston. Heart, 1912, iv, 83.

Stewart. Proc. Soc. Exper. Biol. and Med., 1910, viii, 13.

Taussig and Cook. Arch. Int. Med., 1913, xi, 542. 


\section{CHAPTER XXI. \\ DISTURBANCES DUE TO CHANGES IN THE CIRCULATING VOLUME.}

\section{PLETHORA.}

An increase in the total volume of circulating blood (plethora) may result either from an increase in the water constituents alone so that the volume per cent. of proteins and cellular elements is decreased (hydremic plethora) or from a proportionate increase in all the blood constituents (true plethora).

Pathological Physiology. - The effects of introducing large quantities of saline intravenously have been carefully studied in laboratory experiments. Such experiments indicate that such introductions cause a very slight elevation of mean arterial pressure. Thus, Cohnheim and Lichtheim introduced a quantity of saline equal to 44 per cent. of the animal's weight, and Dastre and Loyè increased the blood volume four-fold without any appreciable effect on arterial pressure. Similar observations have been repeatedly made.

Johanson and Tigerstedt showed that both the systolic discharge and minute output were increased after venous infusion, and the writer has recently shown that such an increase is still possible when the effective venous pressure is raised far above normal, so that the results cannot be accounted for upon the assumption that the experiments were carried out under conditions in which abnormal venous pressures existed. ${ }^{1}$

It might be asked why this greater output does not increase the arterial pressure. In the first place, the statement that arterial pressure is not affected is not strictly accurate. The systolic pressure is raised appreciably but the diastolic pressure remains unchanged or falls somewhat, thereby greatly increasing the pulse pressure. Hence, the mean pressure, as measured by the mercury manometer, indicates only a very inconsiderable rise or none at all. The rise of systolic pressure is clearly the effect of the greater systolic discharge. The fact that the diastolic pressure remains unaltered or drops can be accounted for only by a reduced peripheral resistance in which two factors, a reflex vaso-dilation (Sewall and

\footnotetext{
1 The fact that the heart can react with a greater volume output after pressures greater than those regarded as critical by Henderson, has also been recently corroborated by Patterson and Starling.
} 
Stenier) and a reduced viscosity of the blood, play a part. By these compensatory mechanisms the arterial pressure is prevented from mounting to an appreciable degree.

If the infusion of saline is slow, the increased cardiac output effectively removes the surplus fluid from the veins and the venous pressure rises only slightly; but if the saline is introduced at a rapid rate, the venous pressure rises and the blood accumulates in the liver (Stolnikow).

When the infusion is stopped, the excess of fluid is rapidly excreted by the glands of the digestive tract and by the kidney so that soon the volume of circulating blood returns to normal (see Tigerstedt). So, also, it has generally been thought that an osmotic hydremia induced, $e . g$. , by intravenous injection of sugar, was only temporary, for, as water passed from the tissues to the blood, it was accompanied by an immediate increase in urinary secretion (Brasol, Starling). A restudy of the question on unanesthetized dogs and for shorter periods of time by Fisher and Wishart demonstrated that during the second hour after the alimentary ingestion of sugar, the hemoglobin fell 10-20 per cent., indicating a plethora, but this was accompanied by an actual diminution in urinary secretion. This observation goes far to show that a condition of plethora due to osmotic influences alone may be maintained even under physiological conditions.

All of the above mentioned experiments are concerned with the establishment of a hydremic plethora. According to Plesch, however, a true plethora may be induced in animals during experimental uranium nephritis. Here the blood volume may equal 16 per cent. of the body weight instead of 5.4 per cent. as normally. This condition is invariably accompanied by a proportionate increase in red cells and hemoglobin as well as by marked cardiac hypertrophy.

The dynamic effect of a true plethora on the blood-pressure and heart may be studied in an experiment by introducing blood from the artery of one animal into the vein of a second. When this is done the effect on the cardiac output and intraventricular tension resembles very much that of saline infusion, but both the systolic and diastolic pressures increase, owing probably to the fact that the viscosity of the blood is not reduced as it is in the case of saline infusion. It is evident that the condition of true plethora, on account of the rise of diastolic pressure, increases the load against which the heart has to work more than does hydremic plethora, and hence may be expected to lead to cardiac hypertrophy and dilatation sooner than the latter condition.

Clinical Manifestations.-It has been long suspected and recently more certainly demonstrated by a gasometric estimation ${ }^{1}$ of the 
ratio of blood volume to body weight that hydremic plethora is the accompaniment of certain functional disturbances. Apparently it occurs under three conditions: (1) when an excessive osmosis of fluid from the tissues without a corresponding increase in urinary secretion occurs; (2) when $a_{1}$, deficient urinary secretion due to kidney disease exists; and (3) when an excessive absorption of fluid from the alimentary tract occurs.

An excessive osmotic attraction for water may possibly explain the greater volume of blood supposed to be found in heavy and continuous eaters. It is possible, indeed, that in these cases, as indicated by the experiments of Fischer and Wishart, the blood is continually surcharged with products of digestion exerting a high osmotic tension without inducing a corresponding urinary secretion. it has been questioned whether the lower capillary pressure in cases of cardiac decompensation may not favor a passage of fluid to the blood stream and so cause a condition of plethora. The fact that the specific gravity of the blood is reduced favors the view that such a hydremic plethora exists, but Starling and Fawcett who showed that the hemoglobin and red cells are actually reduced believe that this alone may account for the lower specific gravity without the assumption of plethora.

In nephritis, when albumin is eliminated and the excretion of water is interfered with, Plesch found that the blood volume equaled 10.8 per cent. of the body weight, as compared with the normal average of 5.3 per cent., when edema was not present.

The third cause of plethora, $i$. $e$., increased absorption of fluid from the alimentary tract, an absorption in excess of the excretion, may be responsible for the plethora supposed to be present in excessive beer drinkers.

The clinical manifestations of hydremic plethora are not always definite and the diagnosis cannot be made from appearance alone, the so-called "plethoric habit" being no criterion of the circulation volume. It is generally assumed, however, that hydremic plethora exists when corpulence, ruddy complexion, full and dilated superficial vessels are associated with an enlarged heart, a pulse of large amplitude, and some elevation of systolic pressure. The reliability of this assumption is undoubtedly increased when the history of the patient indicates an excessive consumption of fluid, or when evidences of nephritis exist.

The diagnosis can be definitely settled only when the actual relation of blood volume to body weight is determined, as by the gasometric method (Haldane and Smith, Plesch). This involves a knowledge of the relation of these factors under normal conditions.

It is generally stated after Welcker that the blood volume is equal to one thirteenth (7.6 per cent.) of the body weight. It appears, however, upon more recent investigation that the normal 
blood volume equals more nearly one nineteenth or (5.3 per cent.) of the body weight, and that the hemoglobin equals 0.7 per cent. of the body weight (Plesch). In chlorosis, posthemorrhagic anemias and nephritis, the volume has been found to increase in proportion to the body weight. In chlorosis, Smith found that in cases having less than 50 per cent. hemoglobin, the blood volume equaled 6.4 per cent. Plesch has corroborated his results. After hemorrhage the increase is not great and is sometimes not present at all. It also varies with the interval elapsing after the hemorrhage before the results are obtained. In nephritis, the highest volumes are obtained, Plesch having reported cases in which the blood volume varied from 7.87 per cent. to 10.8 per cent. of the body weight.

It still remains undetermined whether a condition of true plethora, $i$. e., one in which hyperglobinemia and normal specific gravity are associated with an increased blood volume, occurs. Plesch found such a condition in experimental uranium nephritis in dogs, but, so far as the author is aware, it has never been satisfactorily demonstrated in man.

\section{OLIGEMIA.}

Acute Oligemia and Toxemic Shock.-Oligemia is a condition in which the volume of circulating fluids is diminished. It occurs acutely during a progressive hemorrhage and at times when water is rapidly abstracted from the blood by osmotic or secretory influences. The abstraction of water during serious diarrhea has long been recognized. Rogers recently showed that in cholera 64 per cent. of the blood serum may be lost. A concentration of blood has also been found in pneumonia (Sandelowsky) and in scarlet fever (Oppenheimer and Reiss). Such a concentration of blood and reduction in volume also seems to accompany traumatic shock (Sherrington and Coperan, Lyon, Henderson), and, according to Henderson, is largely responsible for the changes in the circulation in toxemic as well as traumatic shock.

Pathological Physiology.-When the volume of circulating blood is so far reduced that the increased respiration and possibly the venomotor control is unable to maintain a normal effective venous pressure in the right auricle, it usually happens that the effective pressure in the left auricle also falls to some extent. In consequence the output of the left heart diminishes and tends to lower the arterial pressure. Such a tendency usually prevails, although counteracted by a reflex peripheral constriction. Hence, both systolic and diastolic pressures are low and the pulse pressure becomes smaller.

Clinical Manifestation.-The symptoms and an analysis of the blood concentration both indicate that acute oligemia is the direct cause of death in many cases of toxemic shock (Henderson). The 
pulse is rapid and feeble and the heart sounds are weak thus suggesting "acute heart failure." These signs occur not because the myocardium is primarily affected but because it is unable to maintain a sufficient discharge. Saline infusions are of only temporary benefit, as a rule, for the same factors which are responsible for the original withdrawal of fluid from the blood probably continue to eliminate the fluid injected.

According to the experiments of Romberg and Passler, circulatory failure in these conditions is partly, at least, due to failure of the vasomotor centre and the onset of peripheral dilatation. They showed, for example, that the reflex rise or fall of blood-pressure obtained upon stimulation of an afferent nerve is not as great as normally obtained in animals affected by pneumonia or diphtheria; but the researches of Porter and his pupils have recently shown that the percentile rise of blood-pressure, which is the true criterion of vasomotor centre action, is not impaired up to and even immediately after death. Experimental evidence, therefore, indicates that circulatory failure in toxemic shock is due neither to a direct toxic action on the heart nor to a vasomotor paralysis, but to an acute oligemia.

\section{SHOCK AND HEMORRHAGE.}

Traumatic and Surgical Shock.- "Shock" may be defined as a functional disturbance of the circulation which follows severe trauma or surgical operation and which progresses into a severe condition, or even results in death. Upon postmortem examination, no pathological changes can be discerned to account for it.

Clinical Manifestations.-When an individual is injured, as by extensive burns, fractures, mutilations, concussions, etc., the period of extreme pain and hyperexcitability is followed frequently by one of apathy, reduced sensibility, motor weakness, rapid, weak pulse, and irregular gasping respirations. To this symptom-complex the term "traumatic shock" is given. It follows more easily after injuries to the brain, testicles and abdomen than after those of other organs. A condition resembling traumatic shock in every way often supervenes after surgical operations and it is this surgical shock which is responsible for the failure to recover in many surgical procedures. It is therefore not surprising that a large amount of energy has been directed toward discovering the nature and cause of surgical and traumatic shock.

Although, as Meltzer points out, clinical shock involves the functions of many systems, the failure of the circulation is of the greatest interest, since upon it depends the continuance of life.

Experimental Physiology.- It is impossible at the present day to reach any conclusion as to the nature and cause of shock by review- 
ing the work of any single, or, in fact, that of any group of investigators. The only logical method is to review first the circulatory conditions as they exist in shock and then the theories devised to explain them.

Changes in the Circulation during Shock.-We may inquire first what changes occur in the circulation during shock experimentally produced. First in importance is the low blood-pressure. The experiments of Crile indicate that cutting, tearing, burning or mutilating peripheral parts of the body, as the arms and legs cause a great elevation of blood-pressure, while crushing, cutting of the testicle, opening the abdomen, handling the intestines, etc., usually cause a fall. Stimulation of peripheral nerves causes at first a rise of blood-pressure followed by a fall.

It has been quite generally assumed from the signs present that the heart suffers directly during shock so that its contractions are feeble and its output small. It was also believed that this progressive weakening was the cause of death. Crile, however, has shown that the heart is not fundamentally injured, for if saline is injected or the abdominal vessels compressed by a pneumatic bag, the heart is capable of resisting an elevated pressure. The condition of the heart in shock was more directly studied by Howell, who found that in a certain share of cases it alone suffered, while in others the peripheral circulation was also disturbed. The changes in the heart consisted in an increase in rate, and a decrease in amplitude.

The cardiometer experiments of Henderson practically corroborated these findings and indicated, in addition, that the chief cause of the reduced output lay in an incomplete diastolic relaxation, which, in turn, was brought on by a decreased auricular pressure.

To summarize, there is practically no difference of opinion as to what the circulatory changes are in shock: The "effective right auricular pressure" is low, the ventricular filling is diminished, the ventricular relaxation incomplete, and the output per beat consequently small. The heart rate is rapid but the minute volume is diminished. The arterial blood-pressure is low and the peripheral flow is slowed.

The main contention in accounting for these circulatory changes, it appears, centres around the questions: "Is the peripheral circulation primarily altered and responsible for the secondary changes in the cardiac output?" "If so, through what mechanism is the peripheral change brought about?" or "Is the change in the output of the heart primarily responsible for the lower arterial pressure?"

It is conceivable that a progressive vasodilation due to a failure, depression or inhibition of the vasomotor centre occurs, which, like cutting the splanchnic or stimulating the depressor, so increases the capacity of the abdominal and portal vessels that a large quan- 
tity of the circulating blood stagnates. In consequence of this the return flow to the heart and its output would be secondarily reduced. The experiments of Porter, however, indicate that such a failure of the vasomotor centre does not occur in shock and hence cannot be the primary cause of the low pressure. The writer (in unreported experiments) has sought to solve the question by simultaneously recording the right auricular pressure and the flow of perfused fluid through a kidney left attached to the central nervous system, after the method suggested by Sollman and Pilcher. When shock supervened due to handling the intestines, the right auricular and arterial pressures both fell, while after a considerable interval (40-80 seconds) the vessels of the kidney constricted, as evidenced by the decreased flow. Using a similar method, Morrison and Hooker have very recently reached the same conclusion in regard to the vessels of the kidney, intestines, and limbs. A similar constriction in the limb vessels has been reported by a number of observers (Seelig and Lyon, Mann, Muns).

It would seem, therefore, that dilation of the arterioles is responsible neither for the decreased return of blood to the heart nor for the fall of arterial pressure.

It is conceivable, also, that the stagnation of blood occurs from a loss of venous tonus or support due to some local influence. Thus, Henderson found that the loss of $\mathrm{CO}_{2}$ in the blood (acapnia) is associated with extreme congestion of the intestinal areas and with an inhibition of movements and a relaxation of the non-striated muscles of the intestines. He, therefore, suggested that the support offered by the non-striated muscles in tonus is removed in acapnia and that stagnation is thus brought about. Hooker's experiments made on strip preparations indicate, however, that the presence of $\mathrm{CO}_{2}$ and not its absence relaxes vascular and rhythmically-acting non-striated muscle, and Janeway and Ewing obtained abdominal congestion and shock upon handling the intestines when the $\mathrm{CO}_{2}$ content of the blood was, by special means, not appreciably reduced. The latter investigators suggest, as an explanation, that a local paralysis of the vessels and intestines occurs which accounts for the stasis. The recent experiments of Morrison and Hooker seem to indicate that the vascular stasis and increase in weight occurring in intestinal loops is due to a reduction of venous tonus.

The results of Lyon indicate, however, that an actual accumulation of blood, such as is generally accredited to the splanchnic areas does not take place in the abdominal organs. If these findings can be corroborated it will be unnecessary to evolve further hypotheses concerning the stasis of blood in the splanchnic area and the reduced flow to the heart must be attributed to an oligemia.

The manner in which such an oligemia can arise in traumatic or surgical shock forms a separate and distinct chapter of the subject. 
Apparently two views have been suggested. Malcolm believes that the fluid is mechanically pressed out of the smaller vessels when they constrict due to intense pain. Henderson has assigned to $\mathrm{CO}_{2}$ the role of maintaining the normal fluid volume of the blood. He pictures the following series of events as initiating circulatory failure: After trauma, pain causes deep and rapid respiration and so eliminates an excessive quantity of $\mathrm{CO}_{2}$ from the blood. During the administration of anesthetics a similar process occurs. To this must be added, in abdominal operation, the direct and rapid loss of $\mathrm{CO}_{2}$ from the intestinal and mesenteric surfaces. In these ways a state of $\mathrm{CO}_{2}$ deficiency, or acapnia, results. A reduced $\mathrm{CO}_{2}$ content of the blood causes a greater passage of $\mathrm{CO}_{2}$ from the tissues. For every $\mathrm{CO}_{2}$ ion passing, two $\mathrm{Cl}$ ions migrate to the tissue spaces and in this way increase the osmotic attraction for water and induce the oligemia.

Hemorrhage.-Pathological Physiology.-The moment that an artery of considerable size ruptures, the total resistance against which blood is discharged is reduced. Consequently, a fall in diastolic pressure results. The systolic pressure also decreases though not to the same extent as the diastolic. Hence the pulse pressure increases. The amplitude of the pulse, which is determined by the pulse pressure, accordingly becomes large and the shape of the peripheral pulse, which is determined by the low resistance, assumes the collapsing form.

While the left heart is thus still maintaining fully a normal discharge and the pulse pressure is greater, the peripheral flow of blood into the veins is almost immediately reduced. The right auricular pressure is not immediately affected, probably because the reduced volume of venous blood from one region is compensated by an increased flow from another. When the luxus blood-content of the veins has become somewhat reduced, however, this balance of venous flow is lost and the "effective right auricular pressure" is decreased. Almost simultaneously the volume curve of the entire heart diminishes in amplitude (Fig. 14), pulmonary arterial and left auricular pressures fall and the systolic pressure becomes rapidly smaller until the pulse pressure is less than normal.

'The flow through the organs of the body is slowed and the centres in the medulla are stimulated by the reduced blood flow. While these dynamic changes are taking place, a series of important chemical alterations occur in the blood. The loss of red blood cells from the circulation and the consequent reduction of the oxygen-carrying power, aided by the slowed circulation in the peripheral capillaries lead, first, to a decrease in the volume per cent. of oxygen in the renous and later in the arterial blood, while in neither is the percentage of carbon dioxide materially altered.

This reduction in the oxygen percentage of arterial blood operates 
with the reduced flow to increase the activity of the respiratory centre. At first, the respirations increase in rate and depth, giving rise, for a considerable interval, to hyperpnea. 'The greater depth and rate of respirations thus instituted cause a better average pressure in both auricles and, to judge from the variations in pulse pressure, a greater average discharge of the left ventricle.

The altered circulation through the medulla affects also the cardio-inhibitory and vasomotor centres. A depression of the cardioinhibitory centre causes an acceleration of the heart but this is not equally pronounced in all cases, depending on the initial tonus of the cardio-inhibitory centre, and on the average rate of blood loss. Stimulation of the vasomotor centre causes an increased contraction of the peripheral bloodvessels during hemorrhage.

Such a tendency to increase the total resistance by constriction is antagonized by the reduction in the viscosity of the blood. Cope has shown that, in spite of this, the total resistance remains much increased. Little more than inference seems to support the commonly held idea that the arterial walls contract through vasomotor influence and thus adapt their caliber to the reduced volume of blood. The demonstration of such an arteriomotor reaction by faultless methods would be of the greatest importance. The accommodative diminution in the larger arteries is more probably due to an elastic recoil of the vessels, which is even more favorable, for while the vessels draw together when the pressure lowers, no increased resistance to the flow results, such as would be induced to a general vasoconstriction.

To sum up, the fall of arterial pressures is counteracted by the augmented breathing, an accelerated heart, an accommodative contraction of the larger vessels and by a peripheral constriction leading to an increased resistance and diminished blood flow in spite of the reduction in viscosity.

The Mechanism of Hemorrhage Cessation.-Every hemorrhage, except one coming from a very large vessel, has a natural tendency to be checked long before the loss of blood becomes dangerous. The duration of bleeding depends, not so much on the size of the vessel wounded as on the facility with which an efficient clot forms over the opening. When blood is shed, it begins to coagulate on the tissues at a distance from the opening. Having formed a point of adhesion to the tissues, it is added to by accretion until it reaches and covers the wound. 'The factors which determine the distance of the first clot formation and its development are, therefore, directly concerned with the duration of the hemorrhage. They may be enumerated as follows:

1. Other factors being equal, the pressure at the arterial opening determines the distance that the blood will be ejected before the force is sufficiently spent to permit the coagulum to form. A clot 
once formed is called on to resist, not the mean, but the maximal pressure that exists in the artery.

2. The surface on which blood is shed determines the extent of its spread. Thus the smooth surface upon which the intestinal and uterine vessels rupture compares unfavorably with the reticular lung parenchyma and subcutaneous connective tissue. So, also, the spread may be limited by an ulceration or an excavation.

3. The formation of clots may be retarded or prevented by muscular contractions of organs. The regular cardiac contraction, as a rule, absolutely prevents the formation of clots over wounds of the heart and forms the only serious element in such wounds. The rhythmic peristalsis of the stomach and intestines is very unfavorable to clot formation.

Events Following the Cessation of Hemorrhage.-When hemorrhage terminates favorably by coagulation, the volume of blood returned to the right auricle increases with surprising rapidity. This is shown by the ventricular volume curves as well as by the increase in venous pressure quickly following the cessation of hemorrhage. The immediate cause of this increase is probably found in the prompt rise of arterial pressure following clot formation or the application of a hemostat together with the rapid absorption of lymph to replete the blood volume. This dilution of the blood decreases the number of red cells and diminishes the percentage of hemoglobin (Dawson), thus explaining the posthemorrhagic decrease in both for several days.

In consequence of the better venous supply and greater output of both ventricles, the pulse pressure increases, for there is little alteration in the peripheral resistance and the increase in the volume elasticity coefficient remains almost a negligible factor. Both systolic and diastolic pressures gradually rise, owing to the augmented cardiac output. The assumption is generally made that this post-hemorrhagic increase in mean pressure is further assisted by an additional vasoconstriction; indeed, this is often quoted as the chief or only cause for the pressure recovery. All experimental observations indicate, however, that vasoconstriction is a gradual process, starting while hemorrhage is still in progress and continuing after its cessation. Neither experiments nor $a$ priori reasoning, however, warrant the assumption that a greater vasomotor reaction after hemorrhage is at all likely to occur, nor is such an assumption necessary to explain this secondary rise.

The rise of arterial pressure, together with the continued dilution of the blood, tends to increase the flow both in the peripheral tissues and in the medulla. The overstimulation of the respiratory centre gradually ceases and the respirations become normal again. The heart continues rapid, however, and the peripheral vessels 
remain contracted until sufficient regeneration of the red blood cells has taken place to reëstablish normal conditions.

Events in the Terminal Stages.-If coagulation fails to occur, the time soon comes when the reduction in blood volume exceeds the possibilities of lymph absorption to such an extent that the mechanical function of the circulation cannot be efficiently carried out. The amplitude of cardiac contraction then decreases, largely because fluid is lacking to feed the pump.

The lack of oxygen in the blood soon changes the previous stimulation of the respiratory centre to a depression. Slow and shallow breathing, occasionally marked by a deeper gasp, leads to complete cessation. The "pressor influence" of augmented breathing is removed and the blood-pressure falls rapidly. Asphyxial changes in the circulation terminate the case. The heart-beats are slow but of great amplitude and vigor, thus making a last attempt to elevate the pressure, which is partly successful but soon fails for want of oxygen.

Clinical Manifestations.-From a clinical point of view hemorrhages may be classified as accessible and inaccessible. In the former class belong external hemorrhages, as from the mouth, nose, throat, wounds, etc., and such internal hemorrhages as can be reached or inspected by special methods, namely, those from the uterus, urethra, bladder, rectum, etc. In the latter class may be listed such internal hemorrhages as intestinal, pulmonary, renal, cardiac, and cerebral. In all of these forms, aside from the loss of blood and the dynamic changes in the circulation, the functions of all organs suffer in varying degree. The signs and symptoms, therefore, common to all forms of hemorrhage are due, partly to changes in the circulation itself and partly to altered functions of various organs. In addition there are often special symptoms resulting from an extreme interference with the function of the organ in which the hemorrhage occurs.

The general symptoms may be considered in four groups: (1) those immediately following a hemorrhage, (2) those occurring during its progress, (3) those following cessation, and (4) those preceding a fatal termination.

Immediately after the occurrence of an obscure, internal hemorrhage of moderate degree, the patient may have few symptoms. If the hemorrhage is large, however, and the fall of arterial bloodpressure sudden, the attendant cerebral anemia gives rise to various psychic phenomena. These may be: nausea, vertigo, or faintness, sensations of flashes of light, ringing in the ears, etc. The pulse is rapid and of large amplitude.

As hemorrhage continues, the interval depending on the amount of blood lost, other symptoms make their appearance. The skin and mucous membranes are pale and the body feels cold. These 
are partly attributable to the lower pressure, but partly also to an increased peripheral constriction directing the blood flow internally. Muscular weakness now comes on, tremors appear, and the voice is weaker. The breathing is increased in rate and depth and a cold sweat appears. Meanwhile the blood-pressure has fallen, the pulse has become small and increasingly rapid and the heart sounds, especially the second, have weakened. Blood examination shows a decrease in red cells and hemoglobin percentage, also a lessened specific gravity.

If the hemorrhage ceases at this stage, the symptoms may remain for a time practically unaltered, which makes it difficult to decide whether or not the hemorrhage is continuing. The pulse is still rapid with no discernible change in amplitude. Augmented respirations may continue or they may become slower and more shallow, and it is impossible to determine whether this latter condition signifies recovery or that a fatal end is approaching. The hemoglobin and red cells continue to diminish for several days due to the greater dilution of the blood by incoming tissue fluids. The pressure, however, begins a steady rise and the pulse pressure increases. The writer has suggested this pressure rise as a sign of prognostic significance in hemorrhage. In animals, indeed, it is possible to determine accurately by consecutive blood-pressure readings at what point hemorrhage ceases. In practice, however, it is not always so easy to determine systolic and diastolic pressures because of muscular tremors and excessive muscular respiratory movements.

If, instead of ceasing, the hemorrhage continues to a fatal termination, certain symptoms generally precede death. The skin becomes yellow and dry, the eyes lustreless, and the patient sinks into a semi-comatose condition. The secretion of urine stops and the rectal temperature falls. The pulse becomes extremely weak, the respiration slow and interspersed with irregular gasps. Convulsive movements may supervene. The heart then becomes very slow, due to asphyxia, and the pulse beats become larger. This is soon followed by a state of ventricular fibrillation which marks the end.

When the functions of important organs are seriously interfered with, special features often obscure by their prominence these general symptoms. Thus, in cerebral hemorrhages, the entrance of blood into the cranial cavities causes an increase in intracranial pressure with its attendant symptoms of slow heart, high pressure and Cheyne-Stokes breathing, associated, if the motor areas are involved, with hemiplegia.

In hemorrhage from the lungs the coughing of blood and respiratory difficulty, together with the flooding of the lungs with blood and the psychic reaction invariably attendant upon hemoptysis, 
often cause an extreme acceleration of the heart and a rise of arterial pressure.

In wounds of the heart the accumulation of blood in the pericardium leads rapidly to an embarrassment of the cardiac filling similar to that found in cases of pericardial effusions. As soon as the extracardial pressure is great enough to impede the inflow of venous blood, the neck veins fill, the pulse becomes small, and syncope rapidly follows.

\section{BIBLIOGRAPHY.}

Books and Monograms Dealing with Plethora.

Krehl. Pathologische Physiol., 1914, 8th ed., Leipzig.

“ Marschand-Erkrankungen des Herzmuskels, 1913, 2d ed., Wien, Leipzig.

Tigerstedt. Physiologie des Kreislaufes, 1893, Leipzig.

\section{Papers Dealing with Plethora.}

Brasol. Arch. f. Physiol., 1884, p. 211.

Cohnheim and Lichtheim. Arch. f. path. Anat., 1877, lxix, 106.

Dastre and Loyè. Compt. rend. de soc. de Biol., 1889, i, 261.

$$
\text { “ " Arch. de physiol., 1889, p. } 280 .
$$

Fischer and Wishart. Jour. of Biol. Chem., 1912, xiii, 49.

Johansson and Tigerstedt. Skan. Arch. f. Physiol., 1889, i, 331.

Patterson and Starling. Jour. of Physiol., 1914, xlviii, 357.

Plesch. Ztschr. f. exper. Path. u. Therap., 1909, vi, 462.

Sewall and Steiner. Jour. of Physiol., 1885, vi, 162.

Starling. Lancet,' $1896, \mathrm{i}, 652$.

“ Jour. of Physiol., 1899, xxiv, 317.

Stolmikow. Arch. f. d. ges. Physiol., 1882, xxviii, 255.

Wiggers. Amer. Jour. of Physiol., 1914, xxxiii, 13.

\section{Papers Dealing with Oligemia, Shock, and Hemorrhage.}

Crile. An Experimental Inquiry into Surgical Shock, Philadelphia, 1899.

Henderson. Amer. Jour. of Physiol., 1910, xxvii, 152.

Hooker. Amer. Jour. of Physiol., 1912, xxxi, 47.

Howell. Contributions to Medical Research, Victor Vaughan, 1903, p. 51.

Janeway and Ewing. Annals of Surgery, 1914, lix, 158; Proc. Soc. Biol. and Med., 1915, xii, 83.

Mann. Johns Hop. Hosp, Bul., 1914, xxv, 245.

Meltzer. Arch. Int. Med., 1908, i, 571.

Morison and Hooker. Amer. Jour. Physiol., 1915, xxxvii, 86.

Muns. Proc. Soc, Biol. and Med., 1915, xii, 87.

Porter and Pupils. Amer. Jour. of Physiol., 1907, xx, 399, 444, 500.

Sollman and Pilcher. Amer. Jour. of Physiol., 1910, xxvi, 233.

Wiggers. Arch. Int. Med., 1914, xiv, 33. 


\section{MECHANICAL IMIPAIRMENT OF CARDIAC AC'TION.}

\section{INFLUENCES AFFECTING THE POSITION OF THE HEART.}

TuE heart is suspended in the thorax by its large vessels and its long axis is prevented from assuming a vertical position only by the fact that it rests upon the diaphragm below and is supported by the lungs laterally. It is inclosed within the pericardium, a relatively inelastic structure which is attached to the large vessels at the base, continued upward with the cervical fascia and merged with the central tendon of the diaphragm below. It is further anchored to the sternum and vertebral column by bands of connective tissue which are often sufficiently well defined to merit the term "ligaments." Owing to the intimate relation existing between the heart and the diaphragm the position of the former is determined by the movements and height of the latter. A change occurs even during respiration. The inspiratory descent of the diaphragm causes the axis to become more vertical, as electrocardiographic and $x$-ray studies show. The apex and base both move down, the heart shadow elongates, while the right and left borders move toward the right (Fig. 4).

Pathological Changes in the Position of the Heart.-Aside from congenital variations and the pressure of intrathoracic tumors, the position of the heart may be altered, either as a result of an abnormal position of the diaphragm or of traction by pathological adhesions.

When the position of the diaphragm is very low, as frequently occurs in emphysema (Fig. 99), in enteroptosis with loose abdominal walls, etc., the heart occupies a median position, while its contour is long and narrow. Because the larger vessels are stretched, the actual boundaries of the heart may be displaced below the fourth intercostal space.

When the entire diaphragm is pushed upward, as in cases of ascites, pregnancy, meteorism, etc., the heart assumes a more horizontal position. This is essentially an exaggeration of what normally occurs during expiration. If only the left dome of the diaphragm is pushed upward (as in cases of gastric inflation, tumors of the fundus, etc.), the apex is raised, and when the left dome rises higher than the right, the heart is pushed toward the 
right. Ascent of the left dome due to a retraction of the left lung, such as takes place in incipient tuberculosis, often causes the two domes to be equal in height and results in a median placement of the heart. The elevation of the right dome, $e . g$., by an enlarged liver, pushes the heart horizontally to the left side.

The position of the heart may be influenced by intrathoracic processes which exert a traction or pressure in a certain direction. Thus, any extensive atalectasis, pneumothorax, bronchostenosis, etc., of one side causes a movement of the heart toward that side. On the other hand, pleural effusions, aneurysms, etc., cause by pressure a displacement of the heart in the opposite direction. Adhesions between pleura and pericardium also tend to draw the

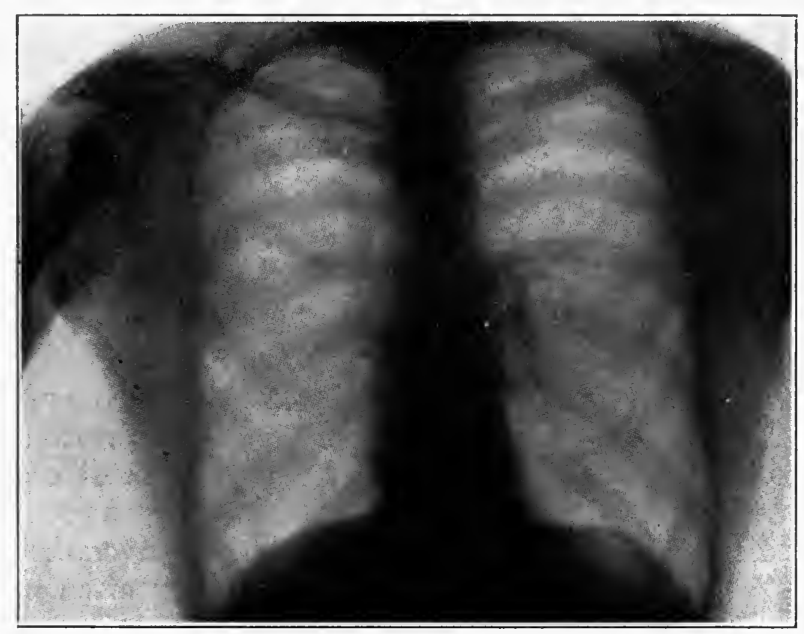

FIG. 99.-Radiogram showing the effect of a low diaphragm on the position of the heart. (After Grödel.)

heart to the side upon which they occur. Furthermore, adhesions between the lungs and diaphragm may secondarily affect the position and respiratory movements of the heart. The writer has demonstrated in dogs that the posterior portion of the heart and venæ cavæ descend more than the anterior aspects of the base during inspiration, while the anterior aspect of the apex moves forward and, consequently, slightly upward. The descent of the base is largely due to a traction on the ligamentum pulmonale (a double fold of the pleura continuous with the pleura pulmonalis, and passing downward from the roots of the lung to its vertebral and diaphragmatic attachments), which causes the roots of the lung, the pulmonary vessels and through them the base of the heart to move downward. In man, the ligamentum pulmonale is not continuous 
with the diaphragmatic pleura, but adhesions between pleura and diaphragm are frequently seen in the dissecting-room which must act in a similar manner in drawing down the large pulmonary vessels and base of the heart.

Clinical Manifestations.-A low position of the diaphragm and a vertical position of the heart are attended by clinical symptoms which indicate that the output of the heart is deficient. The patients suffer from dizziness and tire easily. The extremities are cold and the pulse is small, rapid, and weak. These symptoms may be associated with a lax abdominal wall and definite evidence of enteroptosis. Or, they may be found associated with long thoraces, and emphysematous changes in which the diaphragmatic excursion is markedly diminished. In some cases, careful observation shows that the abdominal wall is actually drawn in during inspiration (Wenckebach). In these cases, the heart is often hanging, as it were, by the large vessels and constitutes the so-called "hanging heart." Owing to the traction that each systole then has on the trachea, the patient may experience a distinct systolic tracheal tug resembling that obtained in aneurysm, or, since in some cases the condition is more pronounced during inspiration, the tug may be felt only during that phase. There may also be an inspiratory filling of the neck veins, as in adhesive pericarditis, or the arterial pulse may be small during inspiration due to a traction on the aorta.

A high position of the diaphragm causes the heart to lie more horizontally, hence the heart dulness is often increased and the apex displaced to the left. The pulse is also small and poorly filled, but there are no signs of edema, venous engorgement, etc. The condition is sometimes associated with tachycardia and the writer is convinced that premature systoles are often associated with it.

\section{CONDITIONS AFFECTING THE PULMONARY CIRCULATION.}

The resistance offered to the blood flow in the pulmonary circuit affects not only the work of the right ventricle, but may also determine the output of the left heart and the arterial pressure to a certain degree. It has been generally supposed ('Tigerstedt) that a large share of the pulmonary circuit can be occluded without affecting the pulmonary arterial or the left auricular pressure. This has been accounted for as due to a compensatory opening up of the more peripheral pulmonary vessels which are normally collapsed to a certain extent. The writer has demonstrated, however, by more delicate manometers that the clamping of a single branch of the pulmonary artery in animals causes an increase in the maximal pressure both within the right ventricle and in the pulmonary 
artery. The initial tension and steepness of the intraventricular pressure rise remain unchanged but the ejection period begins and ends progressively later and the summit is reached more slowly. These experiments indicate that wherever an increased resistance occurs, such as must follow pulmonary embolus, sclerosis and probably infiltration in pneumonia, the right heart having a greater after-load to overcome, must respond with a more vigorous contraction. If this persists it can be readily appreciated how the right-sided hypertrophy so generally associated with this condition arises.

A similar increase in vascular resistance probably occurs in chronic pneumonia, tuberculosis, atalectasis and compression from pleural exudates, accounting in part for the hypertrophy of the right ventricle (Hirsch).

The degree of lung expansion itself determines the resistance to the blood flow, as well as the capacity of the vessels. Cloetta has pointed out that two forces act upon the intrapulmonary bloodvessels when the lungs expand: (1) Since the vessels are situated between alveoli, there is at first a tendency to exert a radical traction and so expand them, as is shown in Fig. 24, but, as enlargement continues and the air spaces become inflated to their polygonal shape, they tend to compress the vessels contained in the spaces between their corners (Fig. 24); (2) in addition, as the lungs expand, a linear traction tends to elongate the vessels and so narrow their lumens. It is apparent from such observations that the effect on the resistance and flow of blood is the resultant of several factors. When the lungs are moderately inflated the flow is improved. This may account for the fact that the pulmonary arterial pressure falls in inspiration. When inflation becomes very great, according to Cloetta, the resistance increases, and this may explain why the pulmonary arterial pressure, to judge from the augmented second pulmonic sound, is increased and the right ventricle often hypertrophied in conditions of emphysema and certain stages of asthma.

It is not necessary to assume that the filling and output of the left heart are interfered with in all conditions in which the resistance is increased and the right heart action augmented. Thus, it is found that ligation of a section of pulmonary artery causes, synchronous with the elevation of pulmonary arterial pressure, no diminution in the left auricular pressure. This is partly because the velocity of flow is increased through the channels still open. This is in accordance with clinical findings, namely, that the bloodpressure is not low and the flow through the heart is not diminished or may be even increased (Stewart) in cases of advanced tuberculosis and in pulmonary emphysema. 


\section{PERICARDIAL EFFUSIONS.}

Pathological Physiology.-A small quantity of fluid is normally present within the pericardial sac, but this is without effect on the circulation. It has been demonstrated repeatedly, however (Cohnheim, Starling), that a marked increase in pericardial fluid causes a decrease in arterial pressure and a rise of venous pressure because fluid is prevented from entering the heart. The results of Starling indicate that other reactions are indirectly called into play. This investigator allowed varying quantities of a bland oil to enter the pericardium and observed the simultaneous effects of different quantities on the arterial, venous, and portal pressures. These effects are indicated in the following table:

\begin{tabular}{cccc}
$\begin{array}{c}\text { Amount of oil } \\
\text { introduced, c.c. }\end{array}$ & $\begin{array}{c}\text { Arterial pressure, } \\
\text { mm. of mercury. }\end{array}$ & $\begin{array}{c}\text { Portal venous } \\
\text { pressure, mm. } \\
\text { MgSO }\end{array}$ & $\begin{array}{c}\text { Inferior vena } \\
\text { cava, mm. } \\
\text { IIgSO }\end{array}$ \\
\hline 20 & 90 & 128 & 36 \\
40 & 90 & 128 & 36 \\
60 & 90 & 128 & 40 \\
70 & Slight fall & 128 & 58 \\
90 & 90 & 134 & 76 \\
100 & 56 & 160 & 124 \\
Heart stopped & 26 & 180 & 170 \\
Removed fluid to 0 & 15 & 215 & 215 \\
Ten minutes later & 146 & 322 & 36 \\
\end{tabular}

A perusal of this table indicates that, though the venous pressure is raised, as much as 70 c.c. can be injected without affecting the arterial pressure. Starling believes that the decreased output is compensated for by a vaso-constriction. The portal pressure also rises due to a similar vaso-constriction and to the higher venous pressure. When more than 70 c.c. are injected, arterial pressure rapidly falls, for the reduction in output is then so great that it cannot be counterbalanced by any further peripheral vaso-constriction. The portal pressure rises steadily with the venous pressure. When the pericardial fluid is removed, the arterial pressure rises suddenly to a much greater height than before and the portal pressure rises still higher, indicating that a great constriction has occurred (Starling).

Clinical Signs. - Patients with pericardial effusions may show few symptoms or they may be afflicted with fainting attacks. Upon examination, the interspaces are often found to bulge if the effusion is great. The impulse is either diffuse or absent. The auscultated sounds are dull, muffled, or indistinguishable. Under fluoroscopic examination all differentiation between auricle, ventricle and aorta are lost and $x$-ray plates give a characteristic triangular shadow with its base down which corresponds to the dulness made out by percussion. The lung areas are dark owing to their congestion 
(Fig. 100). After the removal of the exudate the heart pulsations appear normal and the shadows regain their clearer outlines. A study of the circulation when effusions are present reveals a high venous pressure, a positive venous pulse, and is often accompanied by an enlarged liver. The arterial pulse is small, the arterial bloodpressure low, and the pulse pressure small. All of these symptoms are usually relieved by tapping the pericardium.

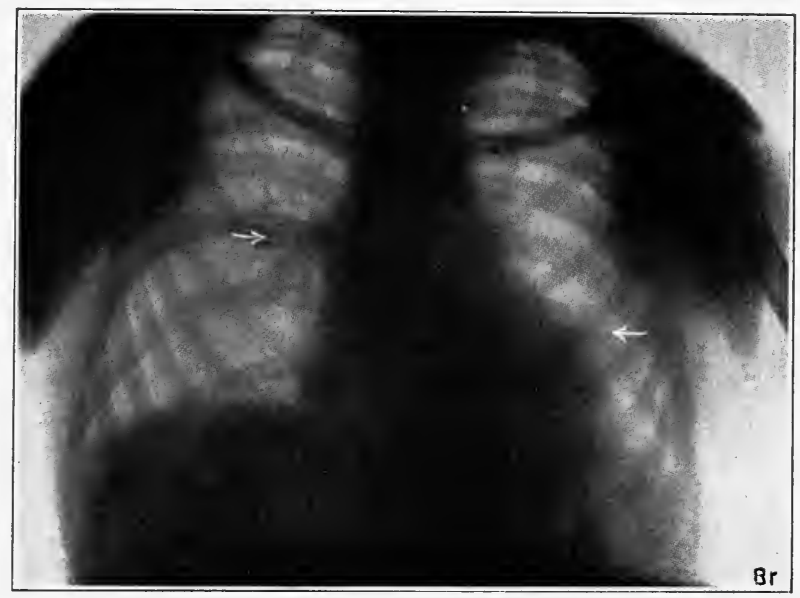

F1G. 100.-Radiogram from case of pericardial effusion. (After Grödel.)

\section{PERICARDIAL ADHESIONS.}

In place of a total reabsorption of the exudate and fibrin, it not infrequently happens that the latter undergoes organization and forms strong elastic bands anchoring the epicardium to the pericardium. These bands may pass: (a) posteriorly to the mediastinal tissue, $(b)$ below to the diaphragm, $(c)$ anteriorly to the sternum and $(d)$ laterally to the pleura.

Clinical Manifestions.-The clinical signs and symptoms are as varied as the nature of the adhesions and consequently the diagnosis is most unsatisfactory. Many cases pass undiscovered to the postmortem table. The symptoms and disturbances created depend less on the number and extent of the adhesions than on their location and anchorage (Riegel). Disturbances arise when the movements and normal contractions of the heart are interfered with or when the entrance or exit of blood is mechanically blocked.

According to Riegel, the adhesions may be divided, clinically, into two groups, namely, those showing general disturbances and those showing definite physical signs. The general disturbances are essentially those of cardiac insufficiency, palpitation, dyspnea, 
cyanosis, and sometimes edema. They offer nothing distinctive upon which to base a diagnosis. The cases in which definite signs are present are also difficult to diagnose since the signs vary so much in different cases. It is desirable, therefore, to limit ourselves to the typical effects of different adhesions.

The character of the apex beat is sometimes altered when anterior adhesions occur. It may not be displaced, as normally, with a change in body position, or there may be a strong systolic retraction of the interspaces. When the heart is absolutely fixed to the surrounding structures of the posterior mediastinum, the anterior wall and to the diaphragm, there is with each systole a retraction of the lateral and lower interspaces opposite which the diaphragm is inserted (Broadbent's sign). This is of greater significance when the cartilages and lower end of the sternum are drawn in as well during each cardiac systole. In some cases an inspiratory inward movement of this region occurs (Wenckebach).

Posterior adhesions usually encircle or compress either the vena cava or aorta in inspiration. If the former is the case then the extrathoracic veins fill in inspiration and the output of the heart decreases so that the arterial pulse becomes small in that phase. An entire wave may be dropped in inspiration when the adhesion causes a traction on the aorta and produces a stenosis or kinking (Kussmaul's pulse).

If the adhesions are firmly attached to the lungs, their deflation may cause a kinking of the artery during expiration and so account for the dropping of an expiratory beat (Riegel's phenomenon).

If the adhesions interfere mainly with efficient contraction of the right side of the heart, venous engorgement is prominent, while, if the left ventricle is affected, dyspnea and pulmonary edema are often brought on.

\section{AUGMENTATION OF INTRATHORACIC PRESSURE.}

An augmentation of intrathoracic pressure occurs under all conditions in which the egress of air is interfered with in expiration, either through a contraction of bronchioles, as presumably occurs in asthma and anaphylaxis or in some forms of laryngeal stenosis.

Clinical Physiology.-If the intrathoracic pressure of an animal is increased by rendering its free expiration more difficult, it results, first, in a rise and later, a fall of the arterial pressure. The rise of arterial pressure can readily be shown to be accompanied by an increased cardiac output, the subsequent fall to a decreased discharge of the heart. Since the venous pressure in the extrathoracic veins rises and the effective right auricular pressure falls (personal 
observation) in the latter case, the fall of arterial pressure is associated with an impeded venous return occasioned by the high intrathoracic pressure.

Clinical Significance.-A similar effect on the circulation results in paroxysms of asthma. The pulse is rapid and dicrotic, the veins congested, and arterial blood-pressures low. For purposes of study a similar reaction can be demonstrated in normal man by carrying out the well-known experiment of Valsalva, which consists in making a forced and prolonged expiratory effort after a preliminary deep inspiration. The radial pulse tracing then shows (Fig. 101, E) that the base line at first rises and later, if expiration be maintained, falls somewhat, the pulse wave at the same time becoming hyperdicrotic. The rise has been shown by Lewis to be due to an actual increase in arterial pressure and not, as sometimes supposed, to a congestion of the venæ comites under the button (Hill, Barnard and Sequiera). The fall of the curve associated with hyperdicrotic waves following the rise of pressure is usually explained as indicating a fall of arterial pressure due to the reduced venous return to the heart and a subsequently decreased systolic discharge. This has been questioned by Lewis who contends that, owing to a simultaneous contraction of abdominal muscles, the filling of the heart in man is never impaired, and the arterial pressure remains high. Upon this point, however, his experiments are not convincing. Tracings taken with optical apparatus indicate that the pulse amplitude becomes smaller at the same time that the entire curve falls and the dicrotic notch becomes larger.-- It seems more plausible, therefore, to regard the prolonged rise of intrathoracic

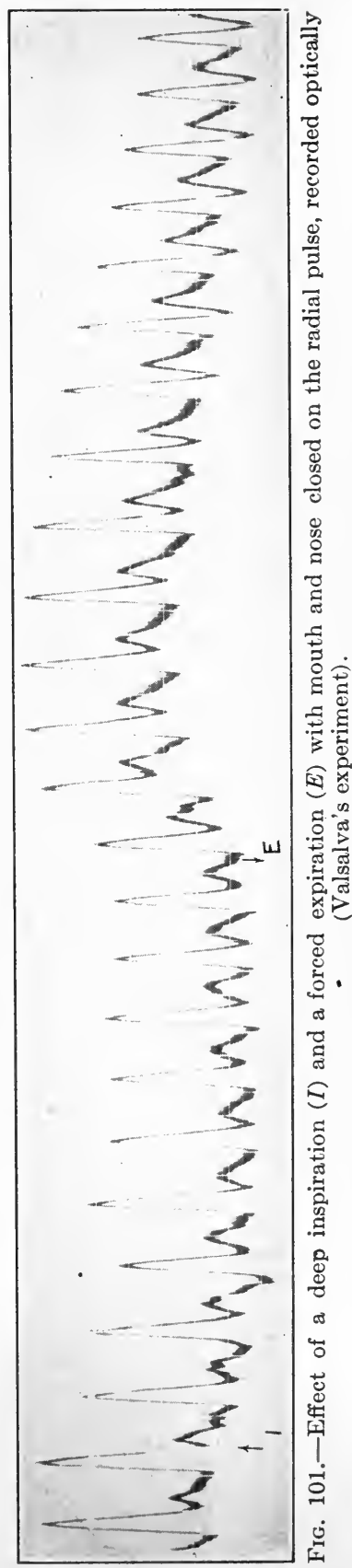




\section{pressure as interfering with the venous return and so reducing the output and the arterial pressure.}

\section{BIBLIOGRAPHY.}

Books.

Gibson. Diseases of the Heart and Aorta, Edinburgh and London, 1898.

Hirschfelder. Discases of the Heart and Aorta, Philadelphia.

Janeway. The Clinical Study of the Blood-pressure, New York and London, 1904.

Krehl. Erkrankungen des Herzmuskels, 1914, 2d ed., Wien and Leipzig.

Norris. Blood-pressure and its Clinical Application, Philadelphia and London, 1914.

Russell. Arterial Hypotonus, Sclerosis and Blood-pressure, Philadelphia and Edinburgh, 1908.

Wenckebach. Path. Beziehungen zwichen Athmung u. Kreislauf. Saml. klin. Vorträge, Volkman, Leipzig, 1907, H. F., No. 465, Inn. Med.

\section{PAPERS.}

Achelis. Deut. Arch. f. klin. Med., 1914, exv, 419.

Edens and Förster. Deut. Arch. f. klin. Med., 1914, cxv, 290.

Förster. Inaug. Disscrt., Beiträg. zur. Diagnose. der Herzbeutelverwachsungen. Hill, Barnard, and Sequeira. Jour. Physiol., 1897, xxi, 147.

Kusmaul. Berl. klin. Wehschr., 1873, pp. 433, 445, 461.

Lewis. Jour. Physiol., 1906, xxxiv, 391.

Riegel. Samml. klin. Vorträge, Volkmann, No. 177.

Roy and Adami. Practitioner, 1890, xliv, 5 .

Starling. Lancet, 1897, i, 569, 652, 723.

Tigerstedt. Ergebn. der Physiol., 1903, $\mathrm{II}_{2}, 558$.

Wenckebach. Ztsch. f. klin. Med., 1910, lxxi, 71, 402.

Wiggers. Proc. Soc. exper. Biol. and Med., 1914, xi, 107. 


\section{CHAPTER XXIII.}

\section{AFFECTIONS OF ARTERIES.}

\section{ANEURYSM OF THE THORACIC AORTA.}

By an arterial aneurysm we understand the cylindrical, saccular or globular enlargement of an artery that follows the diminished resistance of its walls to the internal arterial pressure. Although aneurysms may develop in any artery, we shall confine ourselves to those affecting the intrathoracic aorta, both because this vessel is most frequently attacked and because an aneurysm in this situation produces the most pronounced changes in the circulation.

Pathological Physiology.-An aneurysm never develops without some weakening of the arterial wall. Apparently, the primary cause is found in an inflammation of the vasovasorum which travels inward toward the media. Because of the deficient blood supply and toxic influences, a degeneration of the elastic tissue and muscle fibers occurs which diminishes the elasticity of the vessels. Hence, with every arterial impact there is less and less return to the normal condition and the artery undergoes a progressive expansion.

The shape of the aneurysm is determined primarily by the area involved, much as in glass blowing the protrusion upon blowing becomes globular, cylindrical, sımmetrical or asymmetrical, in accordance with the area softened. The shape is sometimes secondarily altered by contact with external structures which distort it, or by irregular and secondary arterial necrosis which often gives a somewhat lobulated character to the sac.

The progressive bulging is counteracted by two forces. The intima devoid of bloodvessels undergoes a compensatory thickening which may be so extensive in small aneurysms as to strengthen the wall adequately and "cure" the condition. In larger aneurysms this process is rarely sufficient. In these cases a deposition of fibrin layers frequently occurs which serves to strengthen the arterial wall locally.

The dynamic effects of aortic aneurysms have been studied experimentally and the results applied to clinical cases by Marey and François Franck. These investigators concluded that the introduction of an elastic diverticulum into a periodic circulation scheme causes a damping of the pulse wave in the nearest arteries and, hence, makes the pulse smaller in these vessels. Thus, they 
believed that if an elastic and expansile aneurysm were situated in the innominate artery or in the aortic arch near the origin of the innominate, the right radial pulse would be smaller and the systolic pressure lower. If, on the other hand, the aneurysm involved the aorta near the origin of the left subclavian, or affected this vessel itself, the pulse and blood-pressure in the left arm would be smaller. The pulsus differens, as the difference in the two radial pulses has been termed, has therefore attained a certain diagnostic significance. Associated with the smaller pulse beat is a slower rise. This is due to the fact that the arterial system which, from a dynamic point of view, represents a poor system for transmitting the details of the central pressure variations to the periphery, becomes even less efficient when its volume elasticity coefficient $\left(\frac{d p}{d v}\right)$ is further decreased by the introduction of an elastic sac. It is difficult to understand, however, why an aneurysm below the origin of the innominate should not affect the height of the pulse in the entire arterial system, rather than, as François Franck maintained, the pulse in the right radial artery alone. If it be recalled that the velocity of the pulse wave is directly proportional to the elasticity coefficient and the thickness of the arterial wall, it can readily be seen that the rise of the pulse wave will be delayed in the vessels beyond the aneurysm. 'Thus, if the aneurysm be situated on the ascending aorta, the delay will be equal in both radials; if it affects the innominate, the right radial will be delayed; while, if it affects the subclavian or its origin, the left radial will be delayed. This delay is of greater diagnostic value than any change in size and contour.

The expansile pulsation of the aneurysm itself occurs in two stages, as published graphic records show. The rise of such tracings is at first very steep, then becomes more gradual. There is no doubt that optical tracings would reveal even more important details, but such records, to the writer's knowledge, have not yet been reported.

The effect that an aneurysmal sac has on the volume flow of blood and the velocity in the vessels peripheral to the aneurysm is of even greater importance. Since the output of the heart is unaltered, the total peripheral flow is unchanged. Artificial circulation experiments indicate that, if the aneurysm is situated on the ascending arch of the aorta, the flow in the branches corresponding to the two radials is equal but the stream is more constant. The pulse tends to be anacrotic. During systole more blood is accommodated in the more capacious arterial system and during diastole the flow is greater. If the aneurysm affects the innominate, the volume flow through the branches corresponding to the right radial is reduced and the flow is less intermittent than 
in the left. The reverse occurs when the aneurysm is at the origin of the left subclavian.

Clinical Manifestations.--The clinical manifestations of aortic aneurysm are due almost entirely to pressure and so depend largely on their location; hence, it is desirable to consider separately the symptoms and signs of aneurysms involving $(a)$ the ascending aorta, $(b)$ the innominate or its origin, $(c)$ the aortic arch and $(d)$ the descending aorta.

(a) Aneurysm of the Ascending Aorta.-If the aneurysm occurs within the pericardial sac, it manifests itself by many symptoms. Attacks of breathlessness and cardiac asthma associated with precordial pains of anginoid character usually occur. They are largely traceable to the fact that the pericardial space is encroached upon and the heart action mechanically interfered with. If the aneurysm is large, the entrance of venous blood is impeded. Hence, signs of venous engorgement and edema occur in the neck, arms and hand which may become considerably swollen. Evidence of a diminished ventricular output is shown by the smaller pulse and pulse pressure in both arms. A pulsation may be visible in the second or third interspace. The $x$-ray plates may show a normal shadow, but when taken in the left posterior to the right anterior direction a racket-shaped enlargement appears.

Aneurysm of the ascending aorta occurring external to the pericardium manifests itself in an entirely different way. The symptoms are less pronounced and occur only when the tumor becomes very large. The compression of the superior vena cava causes a venous engorgement of the face and neck particularly on the right side. Dyspnea from encroachment upon the intrathoracic space, and pain, due to erosion, are then present.

The physical signs are very definite. The enlargement occurs to the right and forward and by displacing the vena cava and lung makes its appearance in the second and third interspaces to the right of the sternum. In the earlier stages, when the aneurysm comes in contact with the chest wall only, palpation over this region reveals a shock synchronous with the first sound and, more rarely, a diastolic shock synchronous with the second sound. Upon auscultation, a systolic murmur is heard in addition to the heart sounds. If the aneurysm is adherent to the trachea, the wellknown systolic tracheal tug described by Oliver can be elicited. When enlargement continues so that a forward pressure occurs, and especially when erosion of bone and cartilage has begun, the pulsation becomes visible. On palpation, its forcible and expansile character are plainly perceived. The delay in the radial pulses is uniform but the right radial is often of smaller size. Röntgen ray plates show an enlargement to the right of the sternum in the second to the fourth interspaces. 
(b) Aneurysm of the Innominate Artery or its Origin.-The signs and symptoms resemble closely those associated with aneurysm of the ascending aorta with the exception that the pulsating tumor makes its appearance higher, $i$. e., under the right clavicle. Extension backward may cause a compression of the recurrent laryngeal nerve and give rise to cough and paralysis of the right vocal cord, which gives the cough a peculiar brassy quality. Pressure upon the sympathetic may perhaps cause the dilatation of the right pupil often present though it is not certain that the accompanying effects of syphilis are not accountable for this change (Babinski). The right radial pulse is smaller, more gradual in its rise, and delayed more than the left. The systolic blood-pressure may be from 10-30 $\mathrm{mm}$. lower in the right arm.

(c) Aneurysm of the Transverse Aortic Arch.-Inasmuch as little space is available without encroaching upon vital structures in the region where the aortic arch curves backward, aneurysms in this portion are attended by severe symptoms even when not very large. Broadbent has designated this type "the aneurysm of symptoms" as contrasted with "the aneurysm of physical signs" which applies to those arising from the ascending aorta.

The s!mptoms are due largely to pressure upon the surrounding structures. Pressure upon the trachea, bronchi and veins causes dyspnea, especially when the patient is reclining and the tumor mass gravitates backward. Pressure upon the esophagus causes dysphagia; upon the recurrent laryngeal, paralysis of the vocal cords with a change in voice and cough of a peculiar, brassy character. Pressure on the nerves backward produces agonizing, localized pain, while ssmpathetic involvement causes the pupils to become unequal, the left being usually dilated. Compression of the veins on the left causes the veins of the left arm to become dilated and swollen. As the tumor makes its way forward, it appears medially in the suprasternal notch or lifting and eroding the manubrium (Fig. 102). The characteristic heaving, expansile impulse, the sudden shock and thrill characteristic of aneurysm can be felt a short interval after the first sound. The $x$-ray shadow is very definite. The long, narrow shadow normally cast by the intrathoracic vessels is replaced by a broad shadow superimposed on that of the heart (Fig. 103). The pulses differ on the two sides, being delayed and smaller on the left. Stewart, who measured the blood flow in such cases, found it almost equal in the two hands, a fact which indicates that, although an aneurysm distorts the shape of a pulse curve, it does not diminish the volume flow.

(d). Aneurysm of the Descending Aorta.-The aneurysms of the descending aorta cause their symptoms chiefly by pressure on nerves emerging from the spinal cord. Thus may be explained the lancinating pains either in the shoulders, sides or abdomen, depend- 
ing on the region affected and the areas of hyperesthesia. The pulsation may be visible posteriorly to the left of the spinal column or the shock and sounds may be heard only in this region.

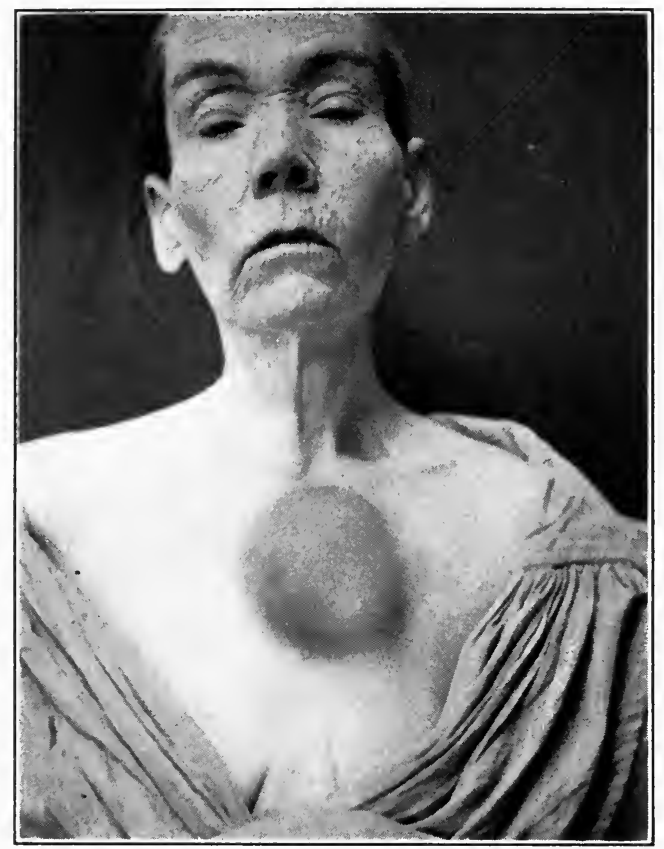

Fig. 102.-Photograph from case of aneurysm of arch of aorta. (After Adami and McCrae.)

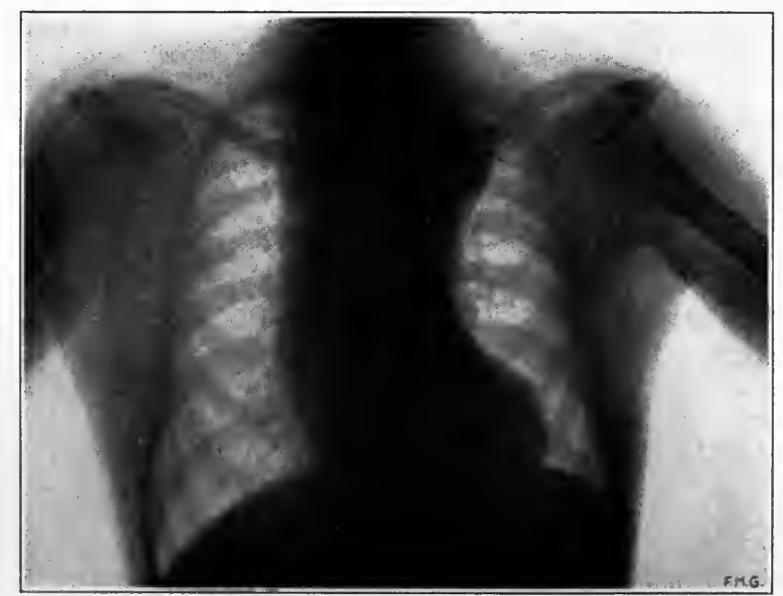

FıG. 103.- Radiogram from case of aneurysm of aortic arch. (After Grödel.) 


\section{ARTERIOSCLEROSIS.}

Functionally, arteriosclerosis may be considered as a condition in which the elasticity and distensibility of the arterial wall are reduced or abolished by proliferative and degenerative changes in the intima, media, and adventitia. It is still debatable whether the intimal proliferation is a primary inflammatory one, or a compensatory thickening (Virchow, Thoma); whether it is entirely secondary to changes in the media and adventitia (Koster, Marchand, etc.); or whether these occur independently though simultaneously (Ophüls).

Pathological Physiology.-As far as the effects of arteriosclerosis on the circulation are concerned, we may divide them into two classes, viz.: those that affect the large elastic arteries (aorta, carotid, brachial, radial, femoral, etc.), and those that involve the peripheral arteries and arterioles in the media of which muscle fibers predominate.

The more nearly the semi-elastic vessels approach the condition of inelastic tubes the more rapidly the pulse is propagated to the periphery. This occurs because physically they form a more perfect conducting system.

As a consequence of the reduced distensibility, the quantity of ejected blood cannot be accommodated in the aorta and, hence, a larger onward displacement occurs during systole. During the subsequent diastole, however, less elastic or potential energy is available to move the blood onward, hence the flow and pressure both diminish rapidly. The result is a tendency toward an intermittent flow at the periphery and a rapid drop of the pulse wave in diastole. It is apparent that, through this factor, the peripheral pulse, in addition to its early rise and small amplitude, changes its shape, in that the descending limb is rapid (cf. page 120).

When the large elastic vessels undergo sclerotic changes, their distensibility for equal pressure increments diminishes. The difference from normal vessels is schematically indicated in Fig. 104. In a normal relaxed vessel the relative increase in cubic contents, as shown by the width of the lines, decreases with an increase of pressure. Hence, a pulse pressure of $20 \mathrm{~mm}$. would give a greater excursion between $40-60$ than between 100-120, while this, in turn, would be greater than the excursion between 180 and 200. If, however, an artery is tonically contracted, the lower pressures do not cause as great an extension as moderate ones sufficient to overcome the tonicity of the arterial wall. Thus, a pulse pressure of $20 \mathrm{~mm}$. might cause a larger excursion between 100 and 120 than between 40 and 60 , although at still higher press- 
ures the amplitude would be diminished, for example, between 180 and 200 (MacWilliam, Fürst and Soetbeer, etc.). In arteriosclerotic vessels the expansion also diminishes progressively with increments of pressure provided the muscular tonus is absent. When contracted tonically, however, the expansion may be irregular. In either case the expansion for corresponding increments of pressure will be less than in the normal arteries. Thus, in the arteriosclerotic artery the expansion between 40 and 60 , between 100 and 120 , and between 180 and 200 will always be less than at corresponding pressures in the normal arteries.

We may now inquire what effect a peripheral sclerosis has on the arterial circulation. Inasmuch as the endarteritis causes
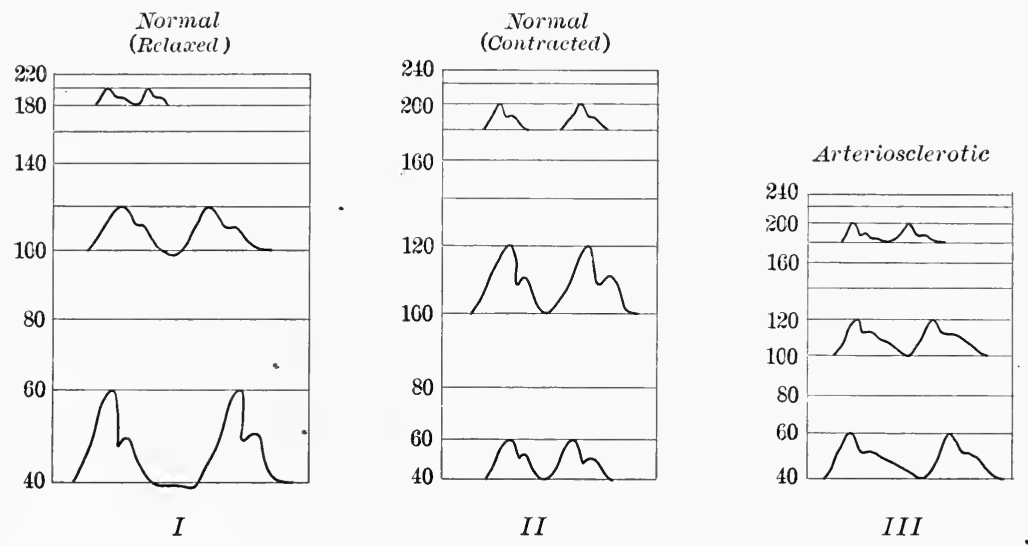

FIg. 104.- Three diagrams illustrating the effect of equal intra-arterial pressure changes on the pulse amplitude at different diastolic pressures. I, normal arteries, relaxed; $I I$, normal arteries under tonus; $I I I$, arteriosclerotic arteries.

a reduction in the lumen of the peripheral arterioles, the effect resembles an increase in peripheral resistance such as accompanies intense vasoconstriction. The effect on the arterial presure is well known. The decreased peripheral flow from the arteries to the capillaries causes an accumulation of blood in the arterial circuit and so elevates both systolic and diastolic pressures. The pulse pressure is decreased and the descending limb of the pressure wave becomes more nearly horizontal. The pulse is also decreased in amplitude and corresponds to the high tension pulses with gradual descent.

The dynamic effect of sclerosis limited to the large distributing arteries and to the terminal arteries and arterioles is summarized in the following diagram: 
Sclerosis of Distributing Vessels.

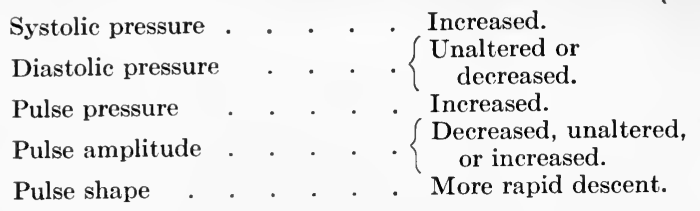

Blood flow from arteries to cap- $\int$ Increased in systole. illaries
Sclerosis of Peripheral Vessels.

Increased.

Increased.

Decreased.

Increased.

More gradual ascent and descent.

Decreased in systole. Decreased in diastole.

Clinical Manifestations.-Although the sclerotic process may involve the vessels of one region or organ more severely or exclusively, the process is rarely restricted to either the central or the peripheral vessels alone, hence the effect on the circulation, governed partly by the severity of the process, is the resultant of the areas involved. It seems to be generally agreed that the systolic pressure is not altered so long as the large splanchnic region remains unaffected, even when sclerosis is palpable in the large peripheral vessels. When these vessels are affected, the systolic pressure is raised and the pulse pressure increases to $50-60 \mathrm{~mm}$. (normal $30-40 \mathrm{~mm}$.). The rate of transmission of the pulse is always increased and may vary from 10.1 to 23 meters per second (Freiberger, Münzer).

The peripheral pulse may be large or small, depending on the relative prominence of the factors before analyzed and also on the degree of cardiac hypertrophy usually accompanying arteriosclerosis.

The contours of both the central and peripheral pulses have been studied by the optical capsules of Frank (Freiberger, Veil). It appears that the central pulse (carotid) has a rounded or flat top and that the preliminary vibrations have disappeared. The peripheral pulse shows a steep rise and either a rounded top or a plateau due to the fact that the form of the central pulse is transmitted better to the periphery. The rapid rise is accounted for by the rapid transmission of pressure in the rigid tubes. Secondary waves are often superimposed high on the descending limb, perhaps because they are more rapidly reflected from the periphery ( $\mathrm{v}$. Frey). Very frequently, however, there is no trace of dicrotism. The pulses obtained are not to be differentiated from those obtained when the tonus alone is augmented ( $i . e$. , ice application, digitalis).

When the splanchnic vessels are involved or extensive sclerosis of the aorta above the diaphragm occurs, the heart hypertrophies, but whether in reaction to a greater blood-pressure alone or as a result of a simultaneous toxic influence, has been discussed elsewhere. The apex is dislocated to the left and the impulse is often heaving and forcible. The sounds are loud and clear, especially the second aortic sound which is accentuated. 
The radial arteries often reveal a palpable thickening which is either diffusely distributed or arranged as nodular buttons or ringlike bands. This is of particular diagnostic significance, since it seems that in "alcoholics" these vessels are the first to be attacked. Other more superficial vessels, as the temporal, show a tortuous course, which, upon palpation gives the impression of a stiff cord. Examination by the $x$-rays often establishes the existence of an arteriosclerotic vessel beyond doubt.

In addition to these general symptoms, other signs and symptoms occur when the vessels of special organs, for example, the heart, the brain, the kidneys and the limbs are affected. For their details, previous chapters and other text-books must be referred to.

\section{BIBLIOGRAPHY.}

Books.

Gibson. Diseases of the Heart and Aorta, Edinburgh and London, 1898.

Hirschfelder. Diseases of the Heart and Aorta, 2d ed., Philadelphia and London, 1913.

Janeway. The Clinical Study of Blood-pressure, New York and London, 1904.

Norris. Blood-pressure and its Clinical Application, Philadelphia and London, 1914.

Osler. Practice of Medicine, New York and London, 1912.

Osler and McCrae. Modern Medicine, iv, 2nd ed., Philadelphia and London, 1915.

Romberg. Krankheiten des Herzens u. Blutgefässe, Stuttgart, 2d ed., 1909.

Russell. Arterial Hypotonus, Sclerosis and Blood-pressure. Philadelphia and Edinburgh, 1908.

\section{Articles Dealing with Arteriosclerosis.}

Freiberger. Deutsch. Arch. f. klin. Med., 1912, cvii, 280.

Fürst and Soetbeer. Deutsch. Arch. f. klin. Med., 1907, xc, 190.

Hasenfeld. Deutsch. Arch. f. klin. Med., 1897, lix, 193.

Hirsch. Deutsch. Arch. f. klin. Med., 1900, Ixviii, 55.

Münzer. Kong. inn. Med., 1912, xxix, 431.

Ophüls. Arch. Int. Med., 1912, ix, 158.

Veiel. Deutsch. Arch. f. klin. Med., 1912, cv, 249.

\section{Articles Dealing with Aneurysm.}

Baetger. Johns Hopkins Hosp. Bul., 1906, xvii, 24.

François Franck. Jour. de l'anat. et de la physiol., 1878, xiv, 113; 1879, xv, 97.

Marey. La Circulation du Sang, Paris, 1881.

v. Ziemmsen. Deutsch. Arch. f. klin. Med., 1891, xlvi, 288. 



\section{N D EX.}

\section{A}

A-C interval, 141, 273

$\mathrm{A}_{\mathrm{s}}-\mathrm{V}_{\mathrm{s}}$ interval, 17, 273

Aberrant waves in electrocardiogram, 170

Acapnia as cause of shock, 337

Accelerator nerves, 27, 29, 30

Acids, amino-, in heart beat, 33, 34

Adhesions of pericardium, 245, 349

arterial pulse in, 118

blood-pressure in, 216 position of heart and, 345

Adrenalin, action of, on cerebral vessels, 80

on coronary vessels, 81

on heart, $26,34,285$

on pulmonary vessels, 93 pulse and, 120

Alcohol, dilatation of ventricles and, 254

heart and, 253

Alcoholism, chronic, heart in, 253, 254

"All or none," law of, 25

Allorrhythmia, arterial pulse in, 115

Alternation of heart, 117, 299

arterial pulse in, 117

clinical physiology of, 299 recognition of, 300 prognosis of, 301

Alveoli, shape of, pulmonary resistance and flow and, 93, 347

Amino-acid in heart beat, 33, 34

Amplitude, pulse. See Pulse.

Amyl nitrite, pulse and, 120, 263

Anacrotic pulse, 119

Anaphylaxis, arrhythmia in, 257 circulation in, 256

electrocardiograph in, 257

heart in, 34, 256

theories of, 256

Anemia, blood flow in, 232

heart in, 250,338

dynamic, 250

Aneroid manometers, 199

Aneurysm, cough in, 356

cyanosis in, 355

of thoracic aorta, 353

Roentgen rays in, 355 , 356

Angina pectoris, 252
Angina pectoris, referred pain in, 253

Anticathode rays, 239

Aorta, aneurysm of, 353

curve of arterial pressure in, 53 pressure in, 53, 66

Aortic facies, 326

insufficiency, arterial pulse in, 120, 328

capillary pulse in, 326

clinical manifestations of, 326

hemodynamies of, 324

intraventricular pressure in, 325

murmurs in, 327

orthodiagrams in, 327

phonocardiograms in, 192, 327

radial pulse in, 328

symptoms of, 326

systolic pressure in, 329

$x$-rays in, 327

stenosis, arterial pulse in, 118, 323 clinical manifestations of, 323

hemodynamics of, 322

intraventricular pressure in, 322

phonocardiogram in, 191

radial pulse in, 323

Roentgen rays in, 246, 323 327

symptoms of, 323

Apex beat, 43, 150

segment capsule for registering, 151

Arm, blood flow in, 225

pieces of blood-pressure apparatus, 195

Arrhythmia in anaphylaxis, 257 .

arterial pulse in, 115

blood flow in, 232

pressure in, 216

classification of, 115, 268

in coronary occlusion, 249

diagnostic significance of, 258

in diphtheria, 260

electrocardiograms in, 169, 268

in goitre, 256

of heart, 268

phasic sinus, 272

radial pulse in, 115, 268

respiratory, 271 arterial pulse in, 116 
Arrhythmia, respiratory, clinical recognition of, 271

in rheumatic fever, 262

sinus, 269

clinical importance of, 269,272 recognition of, 272

pulse in, arterial, 115 venous, 143

significanee of, 272

supraclavicular, venous pulse in,

143,268 et seq.

in syphilis, 266

$x$-ray examination in, 245

Arterial manometer, Frank's, 50 pressure, 66

blood supply and, 79

curve of, in aorta, 53

in pulmonary artery, 89

cycle of heart and, 71

diastolic, 65,68

in man, measurement of, 208

pulse waves and, 71,119 , 120,293

during plethora, 331

in goitre, 256

heart-sounds and, 189

hemorrhage and, 341

human, clinical value of, 214

estimation of, 195

variations of, 214

manometers for recording, 50

maximal, 70

minimal, 70

pulmonary, 87

influences modifying, 91

maximal, 88

minimal, 88

pathological changes in, 346

respiratory variations of, 95 , 99,216

in smaller vessels, 71

systolie, 66,68

in aortic insufficiency, 329

average, 216

discharge and, 68

in man, measurement of, pulse, 101 204

in adhesions of pericardium, 118

in allorrhythmia, 115

in alternation of heart, 117

in aortic insufficiency, 120,

- 122,328

stenosis, 118, 323

in arrhythmia, 115, 268 et seq.

respiratory, 116, 271

sinus, 115,269

in auricular fibrillation, 117, 296
Arterial pulse in auricular flutter, 116, 288

in heart block, 116, 117, 276

in nephritis. 122

in paroxysmal tachycardia, 116,287

in premature contractions of auricle, 116, 282

of ventriele, 116,281

in typhoid fever, 121, 263

Arteries, distensibility of, on bloodpressure, 66

on estimation of human pressure, 204

on pulse amplitude, 358

pulse in, central, 101, 102, 103

sclerosis of, 358

velocity of blood flow in, 72, 74, 225

Arterioles, pressure in, 72

Arteriosclerosis, blood flow in, 232 clinical manifestations of, 360 orthodiagram of heart in, 246 pathological physiology of, 358 pulse in, $122,124,359$

Roentgen rays in, 361

Asphyxia, blood-pressure and, 256 heart and, 256

Asthma, circulation and, 245, 350, 351

Auricle, contractions of, premature, 282 clinieal physiology of, 282 recognition of, 283 significanee of, 285

pulse in, arterial, 116, 282 venous, 143,283

fibrillation of, 293

clinical importance of, 296

physiology of, 293

recognition of, 296

electrocardiogram in, 169, 297

intraventricular pressure in, 293

mitral stenosis and, 321

pulse in, arterial, 117, 296 venous, 144, 297

flutter of, 288

clinical physiology of, 286 recognition of, 288

electrocardiogram in, 169, 289 .

pulse in, arterial, 116, 288 venous, 144,288

hypertrophy of, 304

elinical signs of, 308

electrocardiogram in, 170, 309

Roentgen rays in, 246

impulse spread in, 24

right, function of, 63

pressure in, 53, 62, 99

systole of, $40,53,63,128$

tachycardia of, 286, 287

tachyrhythmia of, 286

iculo-ventricular block, 272 et seq.

bundle and node, 23,273 
Auriculo-ventricular bundle, relation of Blood pressure, intensity of heart extracardiac nerves to, 29,273

floor, movement of, 59

tachyrhythmia, 290

Auscultation, diastolic blood-pressure and, 211

of heart sounds, 175

systolic blood-pressure and, 205

\section{B}

BACterial toxins on heart, 248

Bathmotrophic effects on heart, 30

Bigeminal pulse, 116, 281, 283

Bigeminus, full, 116, 281

shortened, 283

Bile, effects of, on heart, 34

Blood, defibrinated, in perfusion, 32 flow in anemia, 232

in arm, 225

in arrhythmia, 232

in arteriosclerosis, 232

determination of, clinical importance of, 231

muscular contraction and, 79, 250

peripheral resistance and, 67

in valvular affections of heart, 231

velocity of, in arteries, 72,74 , 225

in diastole, 74

in systole, 74

viscosity in, 79

mass movement of, 229

pressure, 66

in animals, Valsalva's experiment on, 350

apparatus, arm pieces of, 195

in arrhythmia, 216

asphyxia and, 256

blood supply of organs and, 79

in capillaries, 72

in circle of Willis, 71

curve of, in aorta, 53

in pulmonary artery, 89

determination of, clinical importance of, 214

diastolic, 66,68

auscultation and, 211

measurement of, in man, 208

CAlcium in heart beat, $33,36,37$

distensibility of arteries on, 66

effective, in man, 237

in right auricle, 61,77

fever and, 217

heart rate and, 68,96

human, clinical significance of, 214

estimation of, 195

variations of, 214

rhythmic, 215

sounds and, 189

Korotkoff's method of determining, 205, 211

maximal, 70

in pulmonary circuit, 89

minimal, 70

in pulmonary circuit, 89

occillatory method of estimation of, 206

respiratory variations of, 95, 216

in smaller vessels, 71

systolic, 66,68 auscultation and, 205 average, 70,216

measurement of, in man, 204

in valvular affections of heart, 217

supply, arterial pressure and, 79

of brain, 78

of head, 78

of heart, 78

of liver, 84

of thyroid gland, 78

velocity of, 72

volume of, 75,231

determination of, 225

flow of, 74, 225

heart rate and, 76

in man, determination of, 227

in pathological conditions, 231, 232, 331

per cent. of oxygen in, 228, 229

venous pressure and, 77

Bloodvessels of brain, 80

carbon dioxide and, 337

Body currents, compensation for, $15 \%$

Bradycardia, sinus, 269

Bradycardias, 114, 270

Brain, blood supply of, 78

bloodvessels of, 80

perfusion of, 80

Broadbent's sign, 350

Bulbo-spiral fibers of heart, 42

Bundle of His, 23, 273

Calorimetric method for determining volume flow, 226

Canalis auricularis, 21

Capillaries, blood-pressure in, 72

Capillary electrometer, 154 pulse in aortic insufficiency, 326

Capsules, optical, 101

Carbon dioxide, bloodvessels and, 337

cardiac tonus and, 26

heart and, 337 
Carbon dioxide, heart beat and, 34

Cardiac action, infiltrations and, 248

affections, dyspnea in, 313,316

function, retrograde changes and, 248

hemorrhage, 343

insufficiency, 306

symptoms of, 310

tonus of heart and, 306

movements, pericardium and, 43 , 345

tonus, carbon dioxide and, 26

valves, stenosis of, 311

Cardiogram, 43, 150

clinical importance of, 152

negative, 152

waves of, 151

Cardiometer, 56

Cardiopneumatic variations in pleural cavities, 58

Cardioptosis, 345

Carditis in rheumatic fever, 261 in septicemia, 262

Cerebral hemorrhage, 342 vessels, action of adrenalin on, 80 circulation in, 80 vasomotors of, 80,82

Chlorosis, volume flow in, 232

Christen's energometer, 221

Circle of Willis, anastomosis of, 82 blood-pressure in, 71

Circulation in anaphylaxis, 34, 256 asthma and, $245,350,351$

cerebral, in sleep, 83

in coronary vessels, 80

in diphtheria, $257,259,335$

emphysema in, 344,347

exogenous toxic influences in, 253

extracts of thyroid gland and, 255

normal, hemodynamics of, 45,66 in pneumonia, 258, 263

portal, 84

pulmonary, hemodynamics of, 54 , 87

syphilis and, 265

in typhoid fever, 263

Coefficient of elasticity, 66

Compensation of heart, 311

hypertrophic, 304, 308

Compensatory pause, 280

Compressed air manometers, 197

Compression of vagus nerves in tachyeardia, 287

Conduction, cocain and, 36 disturbances of, 273

in heart, $17,23,25,272,273$

nerves and, 29

temperature and, 32

Contractility, nerves and, 30 temperature and, 32

Contractions of auricle, premature, 282 of papillary muscles, 42
Contractions, premature, clinical importance of, 281, 283, 285, 286

nodal, 284,285

of ventricles, 41

premature, 280

Coronary arteries, embolism of, 249 symptoms of, 250

occlusion of, 249 arrhythmia in, 249

intraventricular pressure and, 250

sclerosis of, 252

symptoms of, 252

thrombosis of, 249

symptoms of, 250

vagus nerves and, 82

flow, heart beat and, 250

intraventricular pressure and, 79

systole of ventricle and, 84

vessels, action of adrenalin on, 81 vasomotor nerves and, 81

Corrigan pulse, 328

Cough in aneurysm, 356

Critical pressure, 61,77

Current of action, 154

Cyanosis in aneurysm, 355

in valvular disease, 313, 317, 319

Cybulski's method of measuring velocity flow, 72

Cycle of heart, 40

arterial pressure and, 71

pulmonary pressure and, 89

\section{D}

Depressants of heart, 248

Dextrose, heart beat and, 33

Diaphragm, abnormal positions of, heart and, 44

descent of, heart and, 44

Diastasis, 59

Diastole, $40,52,59$

velocity of blood flow in, 74

Diastolic blood-pressure, 66,68 auscultation and, 211

murmurs, 192

waves of venous pulse, 136

Dicrotic waves, 103, 104

Dicrotism, significance of, 120

in typhoid fever, 263

Differential manometer, 73

Dilatation of heart, 306 of right ventricle, 306

of ventricles, alcohol and, 254 clinical importance of, 306 electrocardiogram in, 170 $x$-rays in, 246

Diphasic current, 154

Diphtheria, arrhythmia in, 260 circulation in, 257, 259, 335 
Diphtheria, dilatation of heart in, 261 electrocardiogram in, 260

heart in, 261

murmurs in, 261

Dromotrophic effect on heart, 29

Ductless glands, heart and, 255

Dudgeon sphygmograph, 105, 107

Dynamic anemia of heart, 280

Dynamics of heart beat, 46

Dyspnea in cardiac affections, 313,316

\section{$\mathbf{E}$}

Effective pressure in left auricle, 91 in man, 237 in right auricle, 61,77

Effusions, pericardial, 349

Einthoven's phonocardiograph, 177

Elasticity, coefficient of, 66 in man, 122

Electrocardiogram in anaphylaxis, 256 in arrhythmia, 169, 268 in auricular fibrillation, 169, 297 flutter, 169,289

clinical importance of, 166

in dilatation of ventricles, 170

in diphtheria, 260

in exophthalmic goitre, 171

of exposed heart, 20,162

in heart block, 170, 278

in hemorrhage, 171

in hypertrophy of auricle, 170, 172, 309

of ventricle, 170, 172, 309

interpretation of, 159

in mitral stenosis, $170,320,321$

in premature contractions of ventricle, 170, 382

respiration and, 168

time relations of, 159

in valvular affections of heart, 171 lesions, $317,320,321,322,327$

variations of, normal, 166 pathological, 169

waves of, 158

Electrometer, capillary, 154

Embolism of coronary arteries, 249

$$
\text { heart and, } 250
$$

Emphysema on circulation, 344, 347

Endocarditis, 261, 311

Endogenous toxic impulses, 255

Energometer, 221

Enteroptosis of heart, 344

Epinephrin. See Adrenalin.

Erlanger's criterion for systolic pressure, 206

sphygmomanometer, 200

Esophageal pulse, 146

venous pulse and, 131

waves of, 147

Esophagrams, clinical importance of, 148
Esophagrams, interpretation of, 147

Exercise, heart rate and, 115, 269

orthodiagram and, 245,246

venous pressure after, 235

Exogenous toxic influences on circulation, 253

Exophthalmic goitre, 255 electrocardiogram in, 171

heart in, 115, 255

pulse in, 115

tachycardia in, 255

volume flow in, 233

Extrasystole, nodal, 284

sinus, 282

\section{$\mathbf{F}$}

FACIEs, aortic, 326

mitral, 316

Fatigue of heart, 252

Feddé oscillometer, 201

Fever, blood-pressure and, 217

heart and, 31, 257

Fibrillation of auricle, 117, 144, 169 , 293, 296, 337

of ventricle, 250

Filling of ventricles, $60,61,63$

Finger plethysmograph, 111

First sound, cause of, 186, 187 nature of, 183 quality of, 174 registration of, 184 significance of, 189

Flint murmur, 193, 327

Fluoroscopic examinations in valvular affections, 244

Flutter of auricle, 116, 144, 169, 286, 288

Frank-Petter sphygmograph, 105, 107

Frank's arterial manometer, 50

differential manometer, 73

segment capsules, 101

for recording heart sounds, 179

Full bigeminus, 116

\section{G}

Gaertner's method of determining venous pressure, 232

Gallop rhythm, 174, 190

Galvanometer of Einthoven, 154 for recording heart sounds, 177

Ganglia of heart, 25, 35

Gasometric method of determining volume flow, 227

Gerhartz's apparatus for recording of heart sounds, 181

Goitre, arrhythmia in, 256 arterial pressure in, 256 exophthalmic, 255

Gonococcus infections on heart, 257, 263,311 
H

"Hanging heart," 346

Head, blood supply of, 78

Heart, abnormal positions of diaphragm and, 344

action of adrenalin on, $26,34,285$ of vagus nerves on, 29, 269

in acute infections, $257-266$

alcohol and, 253

alcoholic, symptoms of, 254

alternation of, 117,299

in anaphylaxis, 34,256

in anemia, 250, 338

arrhythmias of, 268

asphyxia and, 256

asthma and, 245, 350, 351

augmentor fibers of, 30

bacterial toxins on, 248

bathmotrophic effects on, 30

beat, alternation of, clinical importance of, 300

amino-acids in, 33, 34

calcium in, $33,36,37$

carbon dioxide and, 34

cause of, 35

coronary flow and, 250

dextrose and, 33

dynamies of, 46

influences modifying, 31-34, 248

intraventricular pressure and, 32

mechanism of, 63

myogenic theory of, 35

neurogenic theory of, 35

potassium and, 33,36

proteins in, 33

sodium and, 33,36

superimposability of, 60

viscosity in, 33

block, causes of, 278

clinical importance of, 276280

physiology of, 272-275

recognition of, 276,277

complete, 275,277

electrocardiogram in, 170,278

partial, 274, 276

production of, 274

prognosis of, 278

pulse in, arterial, 116, 117 venous, 143,144

blood supply of, 78

bulbo-spiral fibers of, 42

carbon dioxide and, 337

chromotropic effects on, 29

in chronic alcoholism, 253, 254

compensation of, 311

conduction in, $17,23,25,272,273$

coronary embolus and, 250

occlusion and, 250

cycle of, 40
Heart, cycle of, blood-pressure and, pulmonary, 89 systemic, $71,97,98$

pulse amplitude and, 118

pulse pressure and, 66

depressants of, 248

descent of diaphragm and, 44

dilatation of, 306

in alcoholism, 253, 254

in diphtheria, 261

in rheumatic fever, 261

displacements of, $x$-rays in, 345

distribution of vagus nerves in, 27,29

drugs and, 248

ductless glands and, 255

dynamic anemia of, 280

effects of bile on, 34

ejection period of, 52

enteroptosis of, 344

examination of, $x$-rays in, 245

in exophthalmic goitre, 115,255

exposed, electrocardiogram of, 20 , 162

failure, 306

symptoms of, 252

fatigue of, 252

fever and, 31,257

foreign proteins in, 34

ganglia of, 25,35

"hanging," 346

in hemorrhage, 338

indol and, 34

infections in, 257-266.

infiltration of, 248

inotropic influences on, 30

insufficiency of, myocardial, 306 symptoms of, 310 valvular, 311 et seq.

irregularity of, 268 et seq.

irritability of, 17,25

nerves and, 30

temperature and, 32

isolated, temperature in, 31

isometric period of, 52

in man, determination of, 102,123

isotonic curve of, 56

mechanical energy of, 66 impairment of, 344

movements of, 40,42

murmurs, 191

muscle, properties of, 17,21

nerves of, $26,27,29,30,37,269$

nutrition of, 248

orthodiagrams of, in arteriosclerosis, 246

output of, $60-63,74-77$

overstrain of, 306

perfusion of, 31,81

effect of serum on, 32, 34

pituitary extract and, 34,248

position of, adhesions and, 345 
Heart, position of, respiration and, 43 presystole of, 40

rate, blood-pressure and, 68, 96

in exercise, 115,265

hemorrhage and, 339,340

in man, influences affecting, 114

minute volume and, 62,76

nerves and, 29, 269

pulmonary pressure and, 92

in shock, 335

systolic discharge and, 60

temperature and, 32

in typhoid fever, 263

in uncomplicated acute fevers, 258

reflex pain and, 252, 253

refractory period of, 25

relaxation period of, 52

reserve power of, 304

respiration and, 44, 58, 70

retrograde changes in, 248

in rheumatic fever, 261

salts and, 32

sclerosis of, 248

in shock, 336

sino-spiral fibers of, 42

size of, variations in, 245

skatol and, 34

sounds, 174

apparatus for recording, 177

arterial pressure and, 189

auscultation of, 175

cause of, 185, 190

closure of valves of heart and, 187

determination of intensity of, 189

intensity of, 189

blood-pressure and, 189

Ohm's gelatin membranes for recording, 180

in premature contractions, 281 recording, Frank's segment capsule for, 179

galvanometer for, 177

gelatin membrane for, 180

Gerhartz's apparatus for, 181

reduplication of, 190

registration of, 177

segment capsule for registering, 178

string galvanometer for registering, 177

stimulants of, 248

study of, methods of, 17

sympathetic fibers to, 26, 28, 29

temperature and, 31,257

Thebesian vessels of, 84, 249

thyroid, symptoms of, 255

tonus of, 17, 26, 31, 32, 63, 306 -

cardiac insufficiency and, 306
Heart, tonus of, nerves and, 31

temperature and, 32

volume curve and, 63

toxic substances and, 34, 253 et seq.

urea and, 34

valves of, action of, 46

affections of, 311

blood flow in, 231

blood-pressure in, 217

electrocardiogram in, 171

phonocardiogram in, 191

$x$-ray examination in, 245

closure of, 64

heart sounds and, 187

insufficiency of, 311 et seq.

lesions of, 311

mechanism of, 46

opening of, 64

vibrations of, in venous pulse, 135

Witte's peptone and, 34, 256

wounds of, 343

Hemodromograph, 72

Hemodynamics of aortic insufficiency, 324

stenosis, 322

of mitral insufficiency, 314

stenosis, 318

of normal systemic circulation, 45,66

of pulmonary circulation, 54, 87

of tricuspid insufficiency, 312

Hemorrhage, absence of irregularity in, 285

arterial pressure and, 341

cardiac, 343

cerebral, 342

cessation of, mechanism of, 339

clinical manifestations of, 341

electrocardiogram in, 171

events following, 340

from lungs, 342

heart in, 338 rate and, 339,340

pathological physiology of, 338

pulmonary, 342

terminal events in, 341

Hepatic vessels, vasomotor control of, 85

Hewlett and v. Zwaluenburg's method of determining volume flow in arm, 225

His, bundle of, 23, 273

conductivity in, 24

crushing of, 25,274

in disease, 258, 259

nerves and, 29, 273

His-Tawara, node of, 23, 273

system, 23, 273

pathological changes in, 279

Hooker and Eyster's method of determining venous pressure, 233

Hydremic plethora, 332 
Hypertension, causes of, 215

Hypertrophic compensation, clinical importance of, 304 manifestations of, 308 physiology of, 304

Hypertrophy of auricles, 170, 246 of ventricle, $170,172,246,304,309$

Hypotension, causes of, 215

I

INCISURA, 53, 103

Indol, heart and, 34

Infections, acute, heart in, 257, 266

Infiltration of heart, 248 cardiac action and, 248

Inflation of lungs, pulmonary circuit and, 93

Initial tension in ventricles, 55

Inotropic influences on heart, 30

Insufficiency, aortic, 324

mitral, 316

myocardial, 306

tricuspid, $311 \mathrm{et} \mathrm{seq.}$

Intersystolic period, 40

Intra-auricular pressure, intraventricular pressure and, 55

mimute volume and, 77 systolic discharge and, 77 variations of, 54,132 venous pulse and, 131, 132 ventricle pressure curve and, 55

volume curve and, 61

Intracardial manometer, Wiggers', 51 nerves, 22,27

Intracranial pressure, 79

Intrathoracic pressure, auricular pressure and, 62,99

pathological disturbances of, 350

pulmonary resistance and, 93 respiration and, 58

venous pressure and, 62, 99 ventricular contraction and, 58,99

Intraventricular pressure in aortic insufficiency, 325

stenosis, 322

in auricular fibrillation, 293

coronary flow and, 79 occlusion and, 250

curve of, 52

heart beat and, 32

influences modifying, 55

intra-auricular pressure and, 55

Irregularity of heart, 268

maniometer for recording, 51

Irritability of heart, 17,25

nerves and, 30

temperature and, 32
Isometric period of heart, 52

in man, 102, 123

Isotonic curve of heart, 56

\section{J}

JACQUET polygraph, 109 sphygmograph, 105, 107

Jugular pulse, segment capsule for registering, 134

\section{$\mathbf{K}$}

Keith-Flack node, 21

Kent, right lateral bundle of, 25

Korotkoff's method of determining blood-pressure, 205, 211

Kussmaul's pulse, 350

\section{L}

LAW of "all or none," 25 of uniformity of behavior, 60

Liver, blood supply of, 84 venomotor nerves of, 85

Locke's solution, 33

Lungs, hemorrhage from, 342

infiltration of, pulmonary circuit and, 93

\section{M}

Mackenzie polygraph, 109

Manometers, aneroid, 199

arterial, Frank's, 50

compressed air, 197

differential, Frank's, 73

intracardial, Wiggers', 51

optical, 50,51

for recording arterial pressure, 50 intraventricular pressure, 51

theory of, 48

types of, on sphygmomanometers, 196

vibration frequency test of, 49

Marey's transmission sphygmograph, 109

wrist sphygmograph, 105, 107

Mass movement of blood, 229

Microphone in sound registration, 177

Minute volume, 75,231

in man, 227

pathological conditions and, 231,232

Mitral facies, 316

insufficiency, 316

hemodynamics of, 314

murmurs in, $37,192,317$

orthodiagrams in, 317 
Mitral insufficiency, phonocardiograms in, 192,317

radial pulse in, 315

symptoms of, 316

$x$-rays in, $245,246,317$

pulse, 299, 318

regurgitation, hemodynamics of, 314

stenosis, 319

auricular fibrillation and, 321

electrocardiogram in, 170, 320, 321

hemodynamics of, 318

phonocardiogram in, 192, 320

radial pulse in, 321

symptoms of, 319

$x$-rays in, $245,246,319$

Moritz and Tabora's method of estimating venous pressure, 235

Murmurs, 190

in aortic insufficiency, 192, 327 stenosis, 191, 323

diastolic, 192

, in diphtheria, 261

in mitral insufficiency, 192, 317 stenosis, 192,320

presystolic, 192

registration of, 190

in rheumatic fever, 263

systolic, 191

in tricuspid stenosis, 312

Muscles, papillary, contraction of, 42

Muscular contraction, blood flow and, 79,250

Myocardial insufficiency of heart, 306

Myocardiograph, $19^{\circ}$

Myocarditis in acute infections, 257265

Myogenic theory of heart beat, 35

\section{$\mathrm{N}$}

Nephritis, arterial pulse in, 122 plethora in, 333,334

Nerves, accelerator, 26, 27, 29, 30, 37 bundle of His and, 29

of heart, $26,27,29,30,37,269$ rate and, 29,269

intracardial, 22,27

splanchnic vasomotor, of liver, 85

vagus, action of, on heart, 29, 269 compression of, in tachycardia, 287

coronaries and, 82

cutting of, 269

distribution of, in heart, 27,29

vasomotor, action of, 79,80 cerebral vessels and, 80 coronary vessels and, 81 in disease, 252

pulmonary vessels and, 93

Neurogenic theory of heart beat, 35
Nodal origin, extrasystole, 284 premature contractions of, 284, 285 rhythm, 270, 290 tachycardia, 290

Node, sinus, 21, 22, 269 of Tawara, 23, 274

\section{o}

Occlusion of coronaries, 249

heart and, 250

intraventricular pressure and, 250

Ohm's heart sound capsules, 180

Oligemia, 334

Optical capsules, 101

manometers, 50, 51

transmission sphygmographs, 109

Orthodiagrams, 242

in aortic insufficiency, 327 stenosis, 323

in exercise, 245,246

of heart in arteriosclerosis, 246

in mitral insufficiency, 317 stenosis, 319

in tricuspid insufficiency, 313

Orthodiagraph, 241

Oscillometers, 201

Feddé's, 201

of sphygmomanometers, 202

Oxygen, minute consumption of, 229

percentage volume of, in blood, 228

rhythmicity and, 32

tonus and, 26

\section{$\mathbf{P}$}

P-R interval, 21, 161, 167, 274

Pacemaker, the, 22, 23, 269

Pain, referred, in angina, 253 reflex, heart and, 252,253

Palpation of pulse, 112 of radial artery, 112

Papillary muscles, contraction of, 42

Paroxysmal tachycardia, 116, 143, 287

Perfusion of brain, 80

of fluids, 32

of heart, 31,81

Pericardial effusions, pathological physiology of, 348

symptoms of, 348 $x$-rays in, 348

Pericardium, adhesions of, 245,349 arterial pulse in, 118

blood-pressure in, 216 $x$-rays in, 245

cardiac movements and, 43,345

Peripheral pulse, 101, 103 resistance, blood flow and, 67 on flow through organs, 79 in infectious diseases, 257 
Peripheral resistance on pressure and Pulmonary circuit, maximum bloodblood flow, 67 in pulmonary circuit, 93 vasomotor nerves in, 79 vessels, sclerosis of, 359

Phasic sinus arrhythmia, 272

Phonendoscope, 176

Phonocardiograms, 183

in aortic insufficiency, 192, 327 stenosis, 191

clinical importance of, 189 significance of, 187

in mitral insufficiency, 192, 317 stenosis, 192, 320

time relations of, 184

in valvular affections of heart, 191

Phonocardiograph, Einthoven's, 177

Phonoscope, Weiss', 182

Photokymograph, 102

Pitot's tubes, 72

Pituitary extract, heart and, 34, 248

Plethora, arterial pressure during, 331 clinical manifestations of, 333 hydremic, 332

in nephritis, 333,334

pathological physiology of, 331 venous pressure in, 332

Plethysmograph, 73 finger, 111

method of determining venous pressure, 235

volume flow, 225

Pleural cavities, cardiopneumatic variations in, 58

Pneumonia, circulation in, 258, 263

Polygraphs, efficiency of, 109, 136

Jacquet, 109

Mackenzie, 109

Zimmerman's, 109

Portal vessels, pressure in, 85 vasomotor control of, 85

Potassium, heart beat and, 33, 36

Premature contractions of auricle, 116, 143,282

nodal, 284,285

of ventricle, $116,143,170,190$, 280,281

Presphygmic period, 123

Pressure, arterial, 53, 66, 79, 95, 195

auricular, 53, 62, 99 curve, venous pressure and, 55 intracranial, 79

intrathoracic, 58, 62, 93, 99

intraventricular, 52,55

pulmonary arterial, $87,89,91$

venous, 5.5, 77, 132

Presystole of heart, 40

Presystolic.murmurs, 192

wave of venous pulse, 135,139

Proteins, foreign, on heart, 34

Pulmonary arterial pressure, 87 curve of, 89

circuit, inflation of lungs and, 93

pressure in, 89

minimum blood-pressure in, 89

peripheral resistance and, 93 circulation, hemodynamics of, 54 , 87

hemorrhage, 342

pressure curve, respiration and, 91 cycle of heart and, 89

heart rate and, 92

respiratory variations of, 89

systolic discharge and, 92

resistance, intrathoracic pressure and, 93

vessels, action of adrenalin on, 93 vasomotor nerves and, 93

Pulse, adrenalin and, 120

amplitude, distensibility of arteries on, 358

heart cycle and, 118

respiration and, 118

amyl nitrite and, 120, 263

anacrotic, 119

arterial, 101

in adhesions of pericardium, 118

in allorrhythmia, 115

in alternation of heart, 117,300

in aortic insuffici ncy, 120, 328

in arrhythmia, 115, 268

respiratory, 115,271

sinus, 115,269

in auricular fibrillation, 117, 256

flutter, 116, 288

in heart block, 116, 117, 276, 277

palpation of, 112

in paroxysmal tachycardia, 116,287

in premature contractions of aúricle, 116,283

of ventricle, 116, 281

recording of, 105

in typhoid fever, 121

velocity of, 122

in arteriosclerosis, 122, 124, 359

bigeminal, 116, 281, 283

capillary, in aortic insufficiency, 326

Corri an, 328

esophageal, 146

waves of, 147

in exophthalmic goitre, 115

flow in man, 266

jugular, segment capsule for registering, 134

Kussmaul's, $3 \dot{50}$

mitral, 299, 318

palpation of, 112

peripheral, 101, 103

pressure, 66 
Pulse pressure, average, 70

heart cycle and, 71 significance of, $68,70,217$

radial, 101

in aortic insufficiency, 328 stenosis, 323

in arrhythmia, 115, 268

clinical significance of, 113

collapsing, 120

conformation of, 117, 118

deficit in, 113

in low resistance, 120, 263

in mitral insufficiency, 315 ' stenosis, 321

registration of, 105

rhythm of, 115

subclavian, 104

supraclavicular venous, 126

in arrhythmia, 143, 268 et seq.

stasis in, 139

tricuspid regurgitation and, 313

waves of, 128,130

tracings, radial, clinical importance of, 113

by sphygmoscope, 111

transmission time of, 122

trigeminal, 282

unequal, 117

Valsalva's experiment on, 351 venous, 126

in arrhythmia, 143, 268

respiration, 126

sinus, 143,269

in auricular fibrillation, 144, 296

flutter, 144, 288

diastolic waves of, 136

esophageal pulse and, 131

in heart block, 143, 144, 276, 277

intra-auricular pressure and, 131, 132

in paroxysmal tachycardia, 143,287

positive, 126

in premature contraction of auricles, 143, 283 of ventricle, 143, 281

presystolic wave of, 135 , 139

systolic wave of, 135,138

vibrations of valves of heart in, 135

volume, 75

in man, measurement of, 208

wave, velocity of, 101, 122

Pulsus alternans, 117, 300

differens, 354

inequalis, 117
$\mathbf{R}$

RADIAL artery, palpation of, 112 sclerosis of, 361 pulse, 101

Radiograms, 238, 240

Recoil curve of body, 229

Reduplication of heart sounds, 190

Referred pain in angina, 253

Reflex pain, heart and, 252, 253

Regurgitation, aortic, 324

mitral, 314

tricuspid, 312

Respiration, electrocardiogram and, 168 heart and, 44, 58, 70

intrathoracic pressure and, 58 position of heart and, 43

pressor effects of, on pulmonary pressure, 89

on systemic blood-pressure, 70, 341

pulmonary pressure curve and, 91 pulse amplitude and, 118

superimposability of volume curve and, 62

venous pulse and, 126

Respiratory arrhythmia, 271

variations in arterial pressure, 95, 216

of blood-pressure, 95, 216

of pulmonary pressure, 89

Retrograde changes, cardiac functions and, 248

Rheumatic fever, arrhythmia in, 262 carditis in, 261

dilatation of heart in, 261

murmurs in, 263

Rhythm, nodal, 270, 290

of pulse, 115

radial, 115

Rhythmic variations in human bloodpressure, 215

Rhythmicity, 17, 21

disturbances of, 269

nerves and, 29

oxygen and, 32

temperature and, 32

Riegel's phenomenon, 350

Right auricle. See Auricle, right.

lateral bundle of Kent, 25

ventricle. See Ventricle, right.

Ringer's solution, 33

Riva Rocci's method of estimating systolic pressure, 204 sphygmomanometer, 195

Roentgen rays. See also X-rays. in aortic insufficiency, 246, 327 stenosis, 246, 323

in arteriosclerosis, 361 clinical value of, 242 et seq. in displacement of heart, 345 in hypertrophy of auricle, 246 of ventricle, 246 
Roentgen rays in mitral insufficiency, Sphygmographs, Frank-Petter, 105, 317

stenosis, 319

in pericardial effusions, 348

physics of, 238

technic of, 240

in thoracic aneurysm, 355,356

in tricuspid insufficiency, 313

use of, 240

\section{$\mathbf{S}$}

SAHLI's sphygmobolograph, 220

Saline solutions in normal circulation, 331

in perfusion, 32

in shock, 335

Sclerosis of arteries, 358

of coronaries, 252

of heart, 248

of peripheral vessels, 359

of radial artery, 361

Segment capsule, 101, 102

for registering apex beat, 151 heart sounds, 178 jugular pulse, 134

Serum, effect of, on perfused heart, 32 , 34

foreign, effect of, on circulation, 256

Septicemia, carditis in, 262

Shock, heart in, 336

rate in, 335

saline solutions in, 335

surgical, 335 clinical manifestations of, 335 experimental physiology of, 335

toxemic, 334

vasomotor centre in, 258

traumatic, 335

clinical manifestations of, 335

experimental physiology of, 335

Sino-spiral fibers of heart, 42

Sino-ventricular block, 272

node, 23,274

Sinus arrhythmia, 269 clinical importance of, 269 ,
272

bradycardia, 269

extrasystole, 282

node, $21,22,269$

tachycardia, 270

venous, function of, 25

Skatol, heart and, 34

Sleep, cerebral circulation in, 83

Sodium, heart beat and, 33, 36

Sounds, heart, 174 et seq.

Sphygmobolograph, 220

Sphygmograms, technic of taking, 108

Sphygmographs, 105 et seq.

Dudgeon, 105, 107

107

Jacquet, 105, 107

transmission, 109

optical, 109

vibration frequency test of, 106

v. Frey's, 105, 107

wrist, 105,107

Sphygmomanometer, 195 et seq.

arm pieces of, 195

Erlanger's, 200

manometers of, 196

oscillometers of, 202

Riva Rocci's, 195

Sphygmoscope as oscillometer, 201

pulse tracings by, 111

Stannius ligature, 21

Stasis in supraclavicular venous pulse, 139

Stenosis, aortic, hemodynamics of, 322 intraventricular pressure in, 322

murmurs in, 191, 323

orthodiagrams in, 323

of cardiac valves, 311

mitral, 319

auricular fibrillation and, 321

hemodynamics of, 318

murmurs in, 192, 320

orthodiagrams in, 319

Roentgen rays in, 319

tricuspid murmurs in, 312

Stethoscope, efficiency of, 176

Stokes-Adams syndrome, 277

String galvanometer, 154

for recording heart sounds, 177

Strohmuhr, 72, 75

Subclavian pulse, 104

Supraclavicular venous pulse, 126

Surgical shock, 335 waves of, 128, 130

ympathetic innervation of heart, 26 , 28,29

Syphilis, arrhythmia in, 266

circulation and, 265

Systole, 40, 52, 59

of auricle, 40,53,63, 128

velocity of blood flow in, 74

of ventricle, $40,52,64$ coronary flow and, 84

Systolic arterial pressure, 66, 68

blood-pressure, 66,68 auscultation and, 205 average, 70,216

discharge, arterial pressure and, 68 heart rate and, 60 intra-auricular pressure and, 77

pulmonary pressure and, 92

rate and, 60

venous pressure and, 61

ventricular tonus and, 63 
Systolic murmurs, 191

pressure, 66,68

Erlanger's criterion for, 206

Riva Rocci's method of estimating, 204

v. Recklinghausen's criterion for, 206

wave of venous pulse, 135, 138

\section{$\mathbf{T}$}

TACHOGRAPH, 71

Tachycardia, auricular, 286, 287

clinical significance of, 292

compression of vagus nerve in, 287

in exophthalmic goitre, 255

nodal, 290

paroxysmal, 287

pulse in, arterial, 116 venous, 143

sinus, 270

vagus compression during, 287

ventricular, 291

Tachygraphic method of v. Kriess, 74

Tachyrhythmia, auricular, 286

auriculo-venticular, 290

clinical importance of, 287, 288, $289,290,291$

Tachyrhythmias, 286

Tawara, node of, 23, 274

Teleradiograms, 241

Thebesian vessels of heart, 84,249

Thoracic aorta, aneurysm of, 353

clinical manifestation of, 355 .

pathological physiology of, 353

Roentgen rays in, 355,356

Thrombosis of coronaries, 249

Thyroid gland, blood supply of, 78 extracts of, circulation and, 255

Tonus of heart, 17,26

of organs, total resistance and

flow and, 79

oxygen and, 26

Toxemic shock, 334

vasomotor centre in, 258

Transmission sphygmograph, 109

Traumatic shock, 335

Tricuspid insufficiency, clinical manifestations of, 312

fluoroscopic examination of, 245

hemodynamies of, 312

orthodiagrams in, 313

physical signs in, 313

relative, 312,316

Roentgen rays in, 313

venous pulse in, 138,313

Trigeminal pulse, 282

Typhoid fever, arterial pulse in, 121
Typhoid fever, circulation in, 263

dicrotism in, 263

heart rate in, 263

split product of, on heart, 248

\section{U}

UNEQUAL pulse, 117

Uniformity of behavior, law of, 60

Urea, heart and, 34

\section{V}

VAGUs nerve, action of, on heart, 29, 269 mode of, 37

compression of, in tachycardia, 287

coronaries and, 82

distribution of, in heart, 27,29

severing of, in different animals, 269

Valsalva's experiment on blood-pressure in animals, 350

on pulse, 351

$x$-ray examination in, 245

Valves of heart. See Heart, valves of.

Valvular affections of heart, 311

diseases, cyanosis in, 313, 317, 319

insufficiency of heart, $311 \mathrm{et} \mathrm{seq} \mathrm{.}$

Vascularity of organs, 77

Vasomotor centre in toxemic shock, 258,334

control of cerebral vessels, 82 of hepatic vessels, 85

of portal vessels, 85

nerves of cerebral vessels, 80

of coronary vessels, 81

in disease, 252

in peripheral resistance, 79

of pulmonary vessels, 93

Veins, pressure in, 72, 237

Velocity of blood flow in arteries, 72 , 74,225

of pulse wave, 101,122

Venomotor nerves of liver, 85

Venous pressure, blood volume and, 77 critical, 61,77

in exercise, 235

Gaertner's method of determining, 232

gauges, 234

Hooker and Eyster's method of determining, 233

intrathoracic pressure and, 62,99

in man, measurement of, 232 measurements, clinical importance of, 235

Moritz and Tabora's method of estimating, 235

output and minute volume and, 77 
Venous pressure in plethora, 331 plethysmograph method determining, 235

pressure curve and, 55 systolic discharge and, 61 variations of, 132

v. Recklinghausen's method of determining, 233

pulse, 126

in arrhythmia in, 143, 268

respiration, 126 sinus, 143

in auricular fibrillation, 144 flutter, 144, 288

diastolic waves of, 136

esophageal pulse and, 131

in heart block, 143, 144, 276

intra-auricular pressure and, 131,132

negative, 126

in paroxysmal tachycardia, 143,287

positive, 126

in premature contractions of auricle, 143, 283

of ventricle, 143,281

presystolic wave of, 135, 139

supraclavicular, 126

systolic wave of, 135, 138

tracings, clinical importance of, 136

in tricuspid insufficiency, 138, 313

stasis, 313

vibrations of valves of heart in, 135

Ventricles, alternation of, 299

contraction of, 41,42

premature, clinical physiology of, 280,282

recognition of, 281 significance of, 285

electrocardiograms in, 170

pulse in, arterial, 116, 300 venous, 143

sounds in, 190

dilatation of, alcohol and, 254

clinical importance of, 306

electrocardiogram in, 170 $x$-rays in, 246

excitation wave of, 24

fibrillation of, 250

hypertrophy of, 304

electrocardiogram in, 170, 172, 309

Roentgen rays in, 246

musculature of, 42

right, dilatation of, 306

pressure in, 55

systole of, $40,52,64$

volume curves of, 56,59

Ventricular contractions, intrathoracic pressure and, 58, 99
Ventricular tachycardia, 291

tonus, systolic discharge and, 63

Ventril tubes, 239

Vibration frequency test of levers, 18 of manometers, 49 of sphygmographs, 106

Viscosity in blood flow, 79 in heart beat, 33

Volume curves of ventricles, 56, 59 flow of blood, 74, 225

plethysmograph method of determining, 225

pulse in man, 110,227

v. Frey's sphygmograph, 105, 107

v. Kriess' tachygraphic method, 74

v. Recklinghausen's criterion for systolic pressure, 206

method of determining venous pressure, 233

\section{W}

Waves of cardiogram, 151

dicrotic, 103, 104

of electrocardiogram, 158

of esophageal pulse, 147

of supraclavicular venous pulse, 128,130

Weiss' phonoscope, 182

Wiggers' intracardial manometer, 51

Witte's peptone, heart and, 34,256

Wounds of heart, 343

Wrist sphygmographs, 105, 107

$\mathbf{x}$

X-rays. See also Roentgen rays.

in adhesions of pericardium, 245

in aortic insufficiency, 327 stenosis, 327

in diagnosis, 238

in dilatation of ventricles, 246

in displacements of heart, 345

examination in arrhythmia, 245 of heart, 245

in Valsalva's experiment, 245

in valvular affections of heart, 245

in mitral insufficiency, 245, 317

stenosis, 245, 246, 319

in pericardial effusions, 348

physics of, 238

technic of use of, 240

in thoracic aneurysm, 355,356

in tricuspid insufficiency, 313

tubes, inverse current of, 239

$\mathbf{Z}$

Zimmerman's polygraph, 109 
.

.

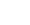

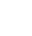

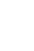




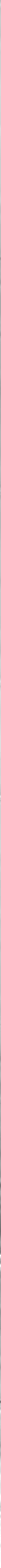


tis

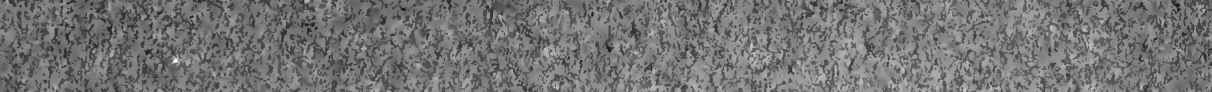

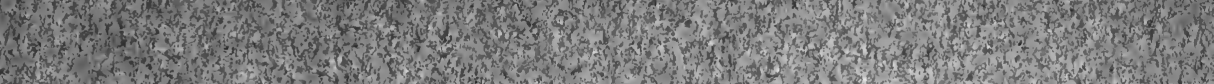

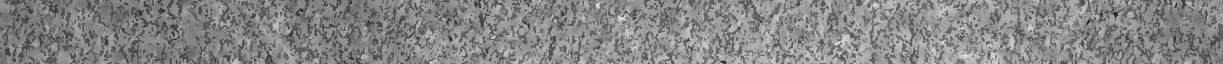

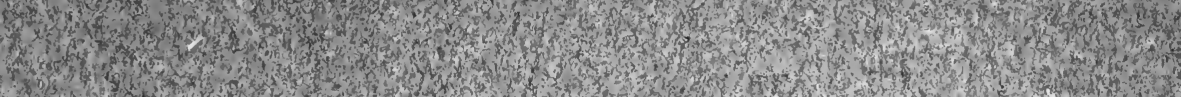

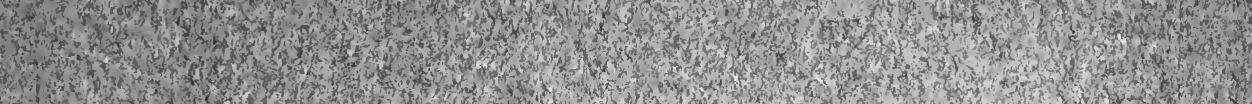
W.t.

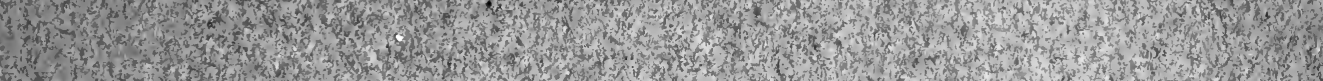
and

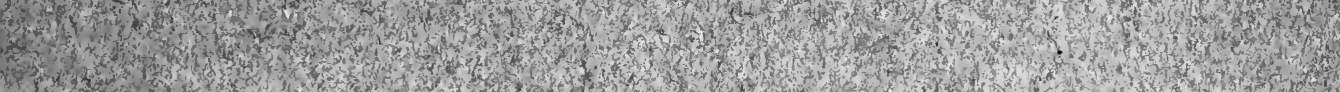

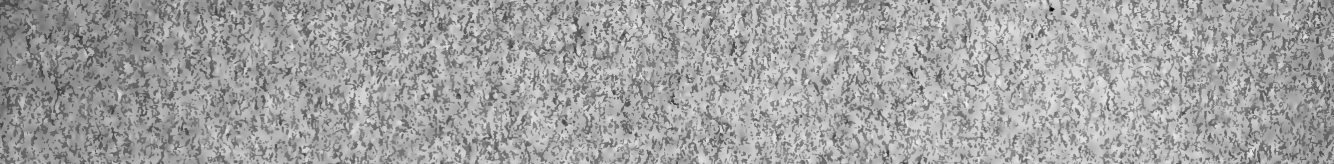

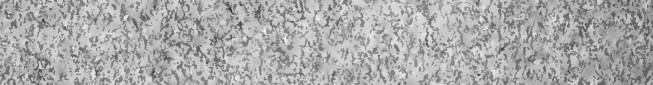
How

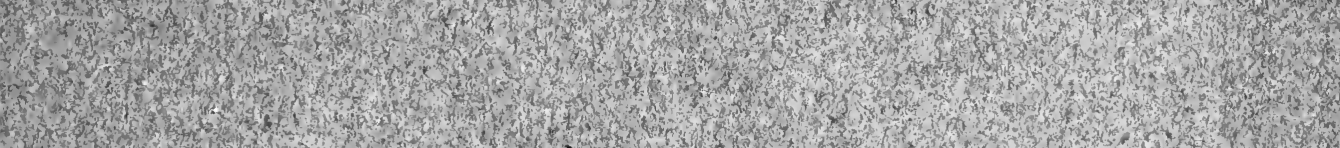

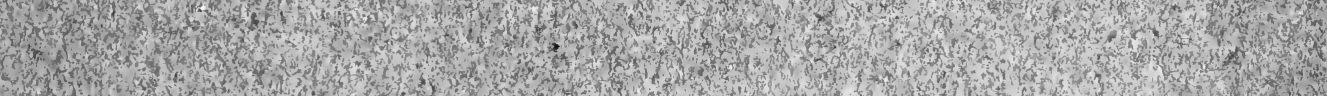

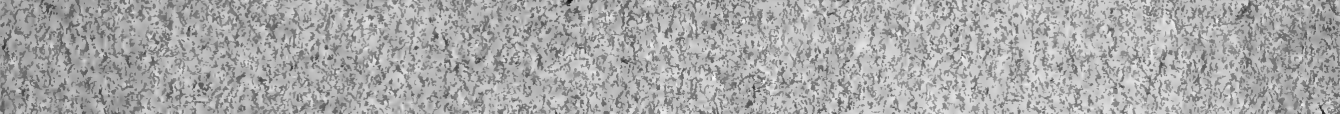

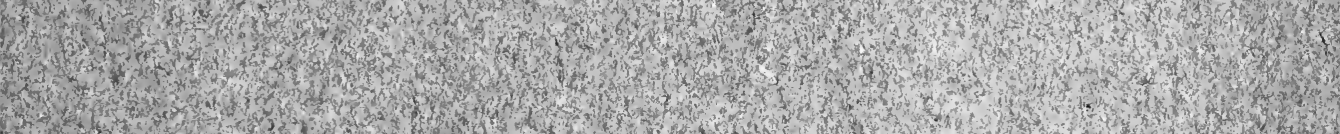

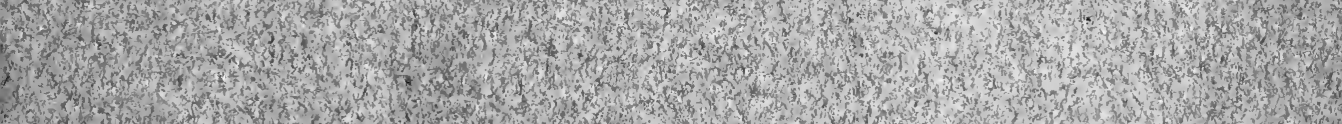
P. 130.

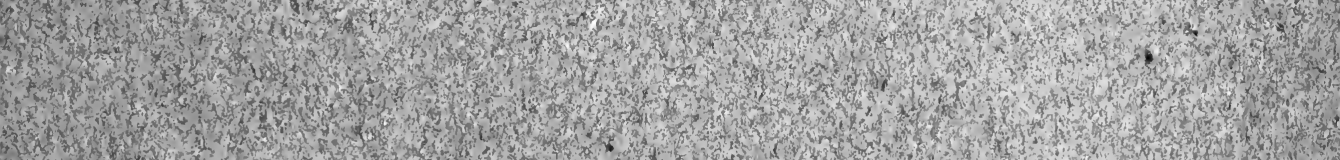

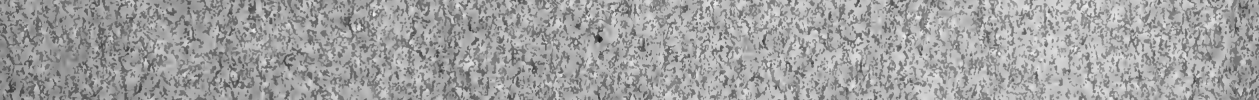

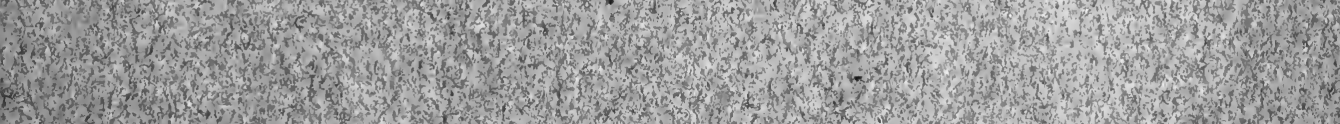

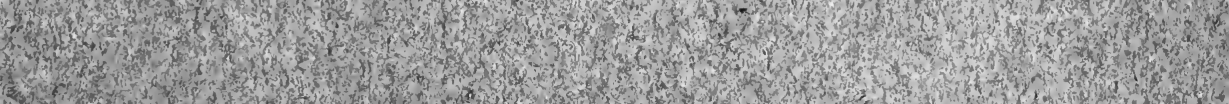

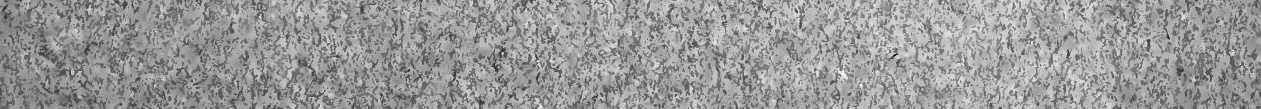

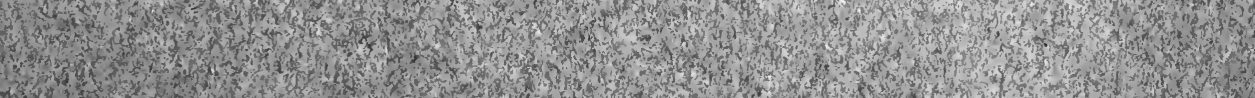

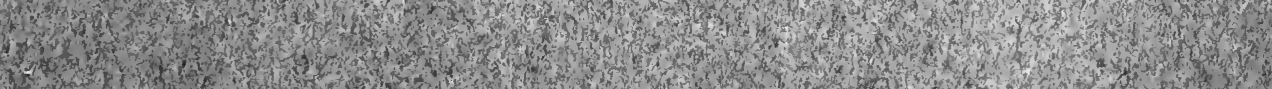

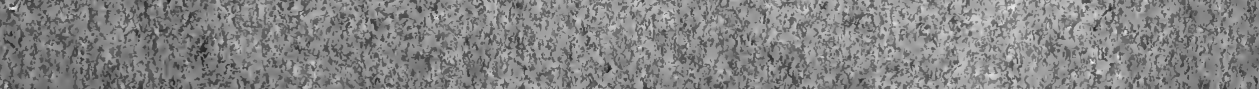

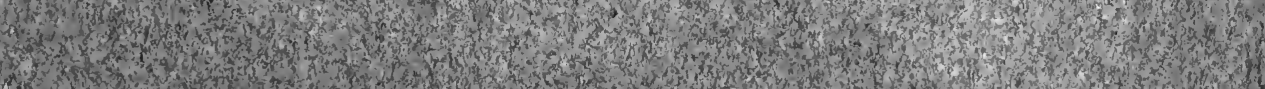
Nom

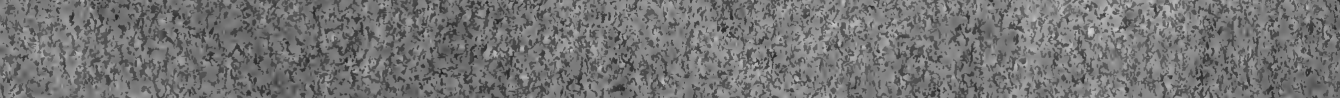

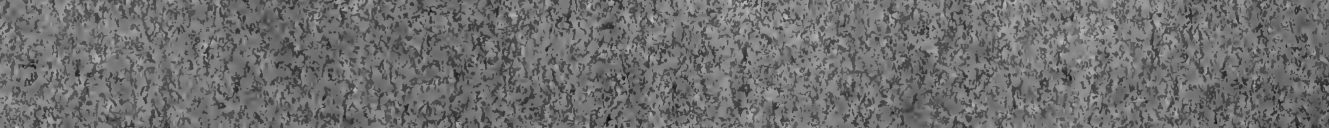

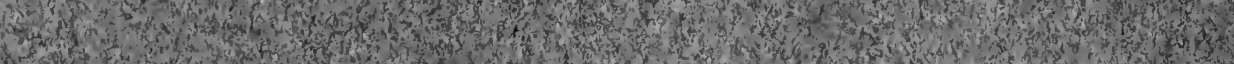

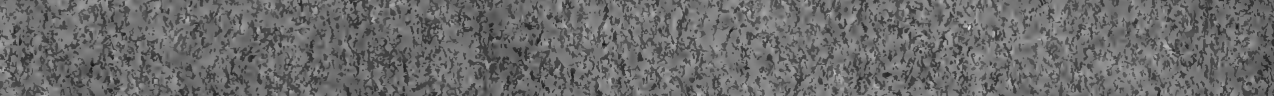

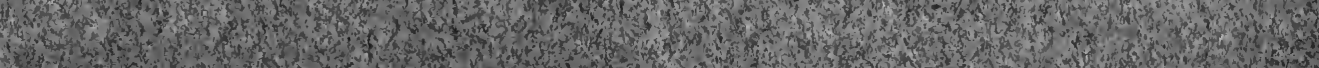





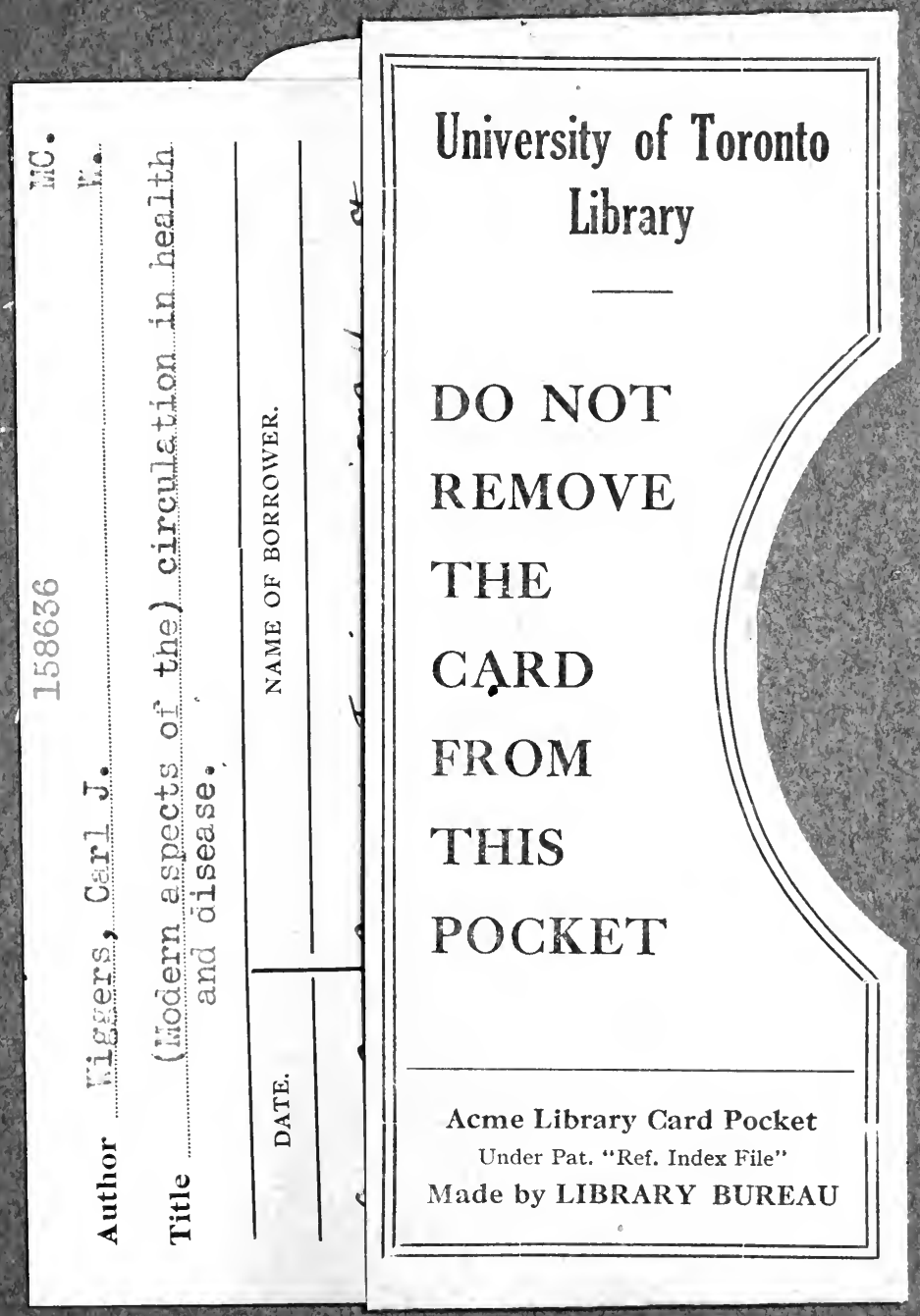




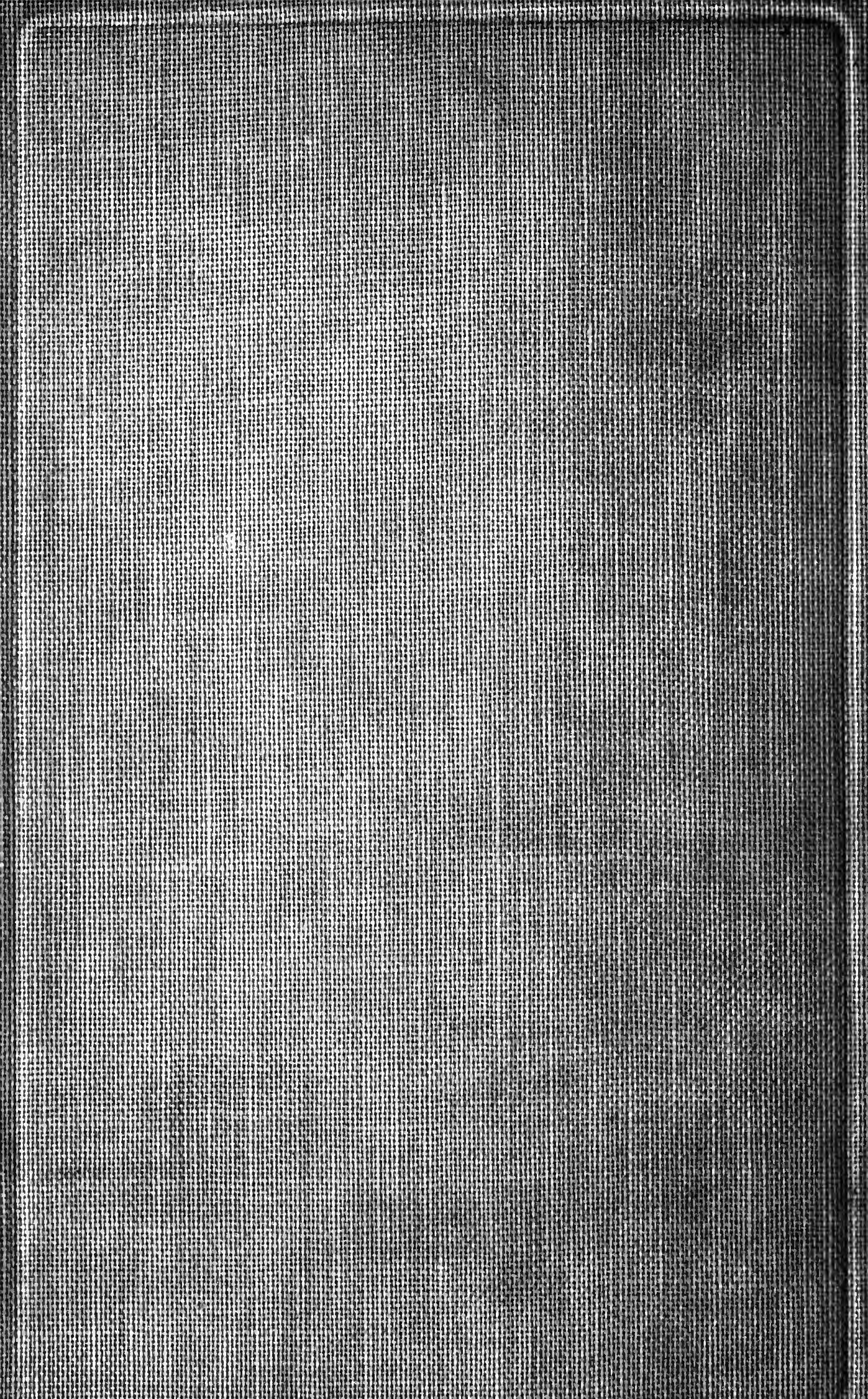

